\title{
STATISTICALLY DESIGNED STUDY OF THE VARIABLE AND PARAMETERS OF CARBON DIOXIDE EQUATIONS OF STATE
}

Final Report

\author{
By \\ Marc D. Donohue \\ Daniel Q. Naiman \\ Gang Jin \\ Joseph R. Loehe
}

May 1991

Work Performed Under Contract No. DE-AC22-89BC14200

Prepared for

U.S. Department of Energy

Assistant Secretary for Fossil Energy

Jerry Casteel, Project Manager

Bartlesville Project Office

P. O. Box 1398

Bartlesville, OK 74005

Prepared by

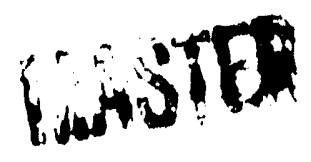

The Johns Hopkins University

Baltimore, MD 21218 


\begin{abstract}
Carbon dioxide is used widely in enhanced oil recovery (EOR) processes to maximize the production of crude oil from aging and nearly depleted oil wells. Carbon dioxide also is encountered in many processes related to oil recovery. Examples include: purification of carbon dioxide obtained from underground oil and gas reservoirs, custody transfer operations, control of carbon dioxide pipeline operations, enhanced oil recovery by miscible and immiscible techniques, wellbores, separation and purification of carbon dioxide dissolved in oil, recycling, and numerical reservoir simulations. It should be noted that, in most process streams, carbon dioxide is present at concentrations larger than any other component. Therefore, accurate representations of the properties of carbon dioxide, and its mixtures with hydrocarbons, play a critical role in a number of enhanced oil recovery operations.

One of the first tasks of this project was to select an equation of state to calculate the properties of carbon dioxide and its mixtures. In doing this, the equation's simplicity, accuracy, and reliability in representing phase behavior and thermodynamic properties of mixtures containing carbon dioxide with hydrocarbons at corditions relevant to enhanced oil recovery were taken into account. We also have determined the thermodynamic properties that are important to enhanced oil recovery and the ranges of temperature, pressure and composition that are important. After reviewing the technical literature and consulting with experts in both industry and academia, we chose twelve equations of state for preliminary studies to be evaluated against these criteria. All of these equations were tested for pure carbon dioxide and eleven were tested for pure alkanes and their mixtures with carbon dioxide. Considering industrial practice and comparing the calculated results with available experimental data, two equations of state - not one, the ALS equation and the ESD equation, were selected for detailed statistical analysis.
\end{abstract}




\section{TABLE OF CONTENTS}

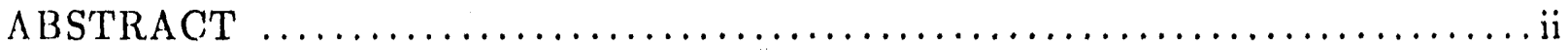

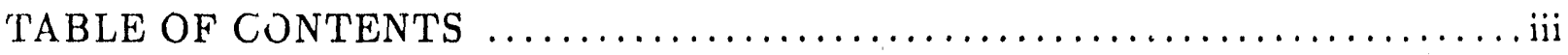

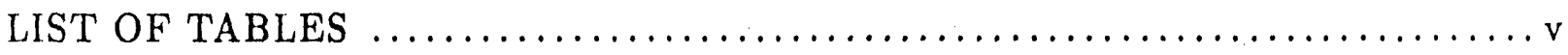

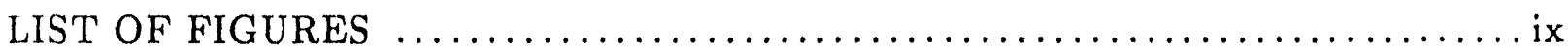

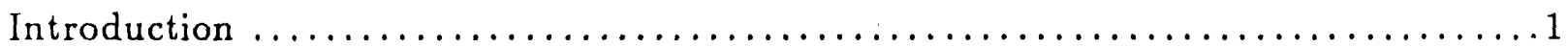

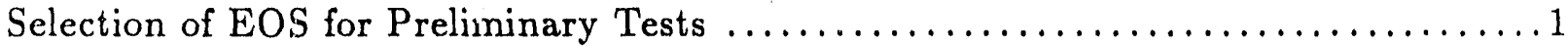

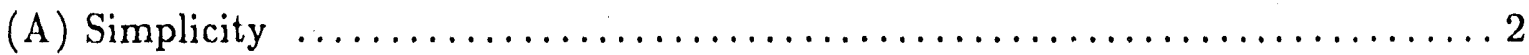

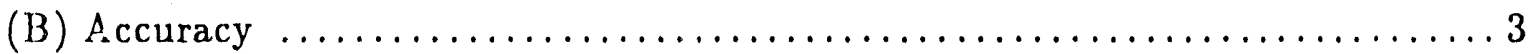

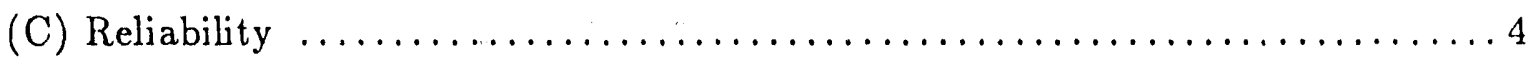

(D) Number of Parameters,

Availability of Parameters and Mixing Rules $\ldots \ldots \ldots \ldots \ldots \ldots \ldots \ldots$

Selection of Temperature and Pressure Ranges

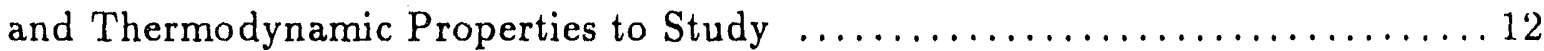

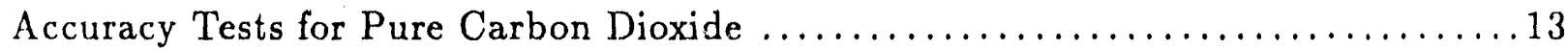

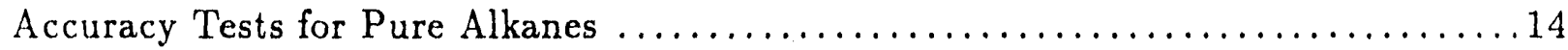

Accuracy Tests for Mixtures of Carbon Dioxide and Alkanes $\ldots \ldots \ldots \ldots \ldots \ldots \ldots$

Reliability Tests (Mixture Critical Point Calculations) $\ldots \ldots \ldots \ldots \ldots \ldots \ldots \ldots 16$

Selection of Equations of State for The Statistical Studies $\ldots \ldots \ldots \ldots \ldots \ldots \ldots \ldots$

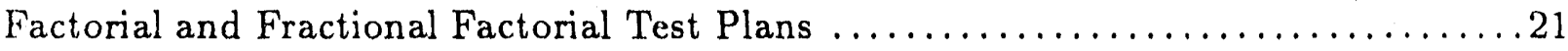

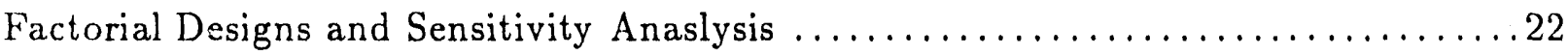

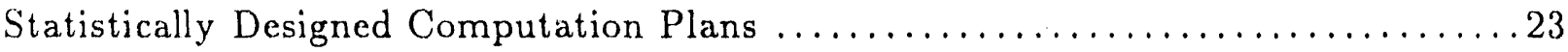

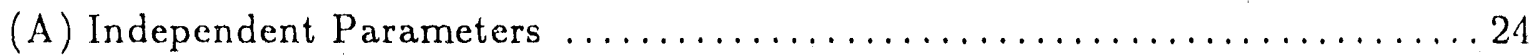

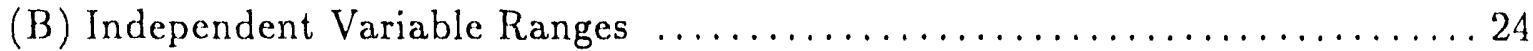

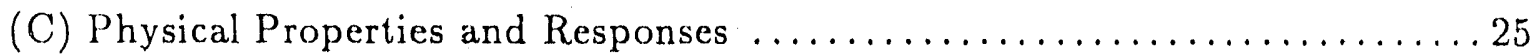




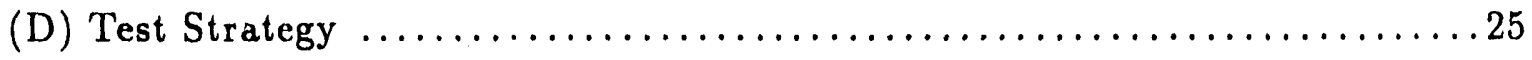

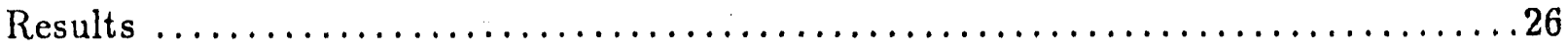

(A) Evaluation of the Responses - Convergence and Divergence ............27

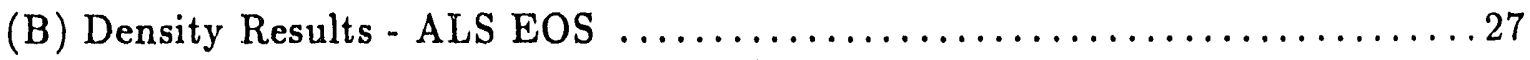

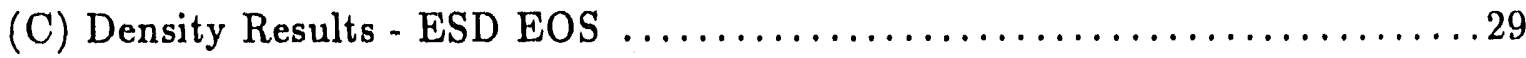

(D) Critical Line Locus Results - ALS EOS $\ldots \ldots \ldots \ldots \ldots \ldots \ldots \ldots \ldots \ldots \ldots \ldots$

(E) Critical Line Locus Results - ESD EOS $\ldots \ldots \ldots \ldots \ldots \ldots \ldots \ldots \ldots \ldots \ldots \ldots \ldots \ldots \ldots \ldots$

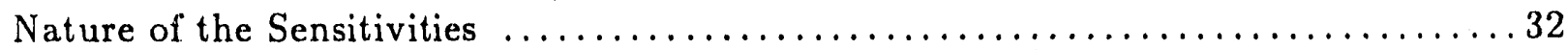

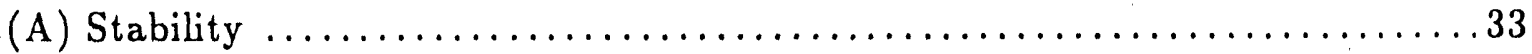

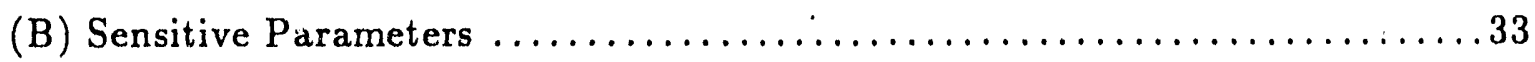

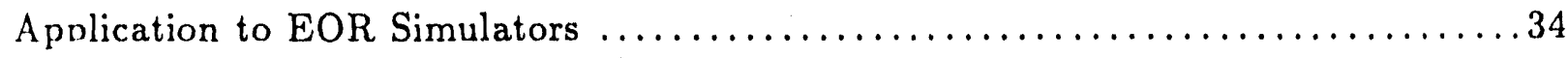

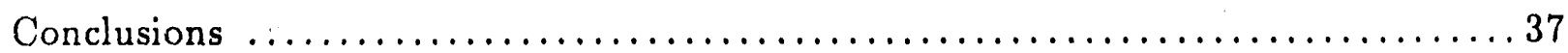

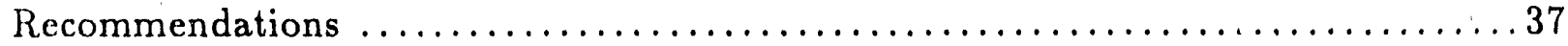

Tables ....................................................... 39

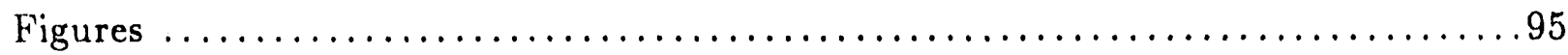

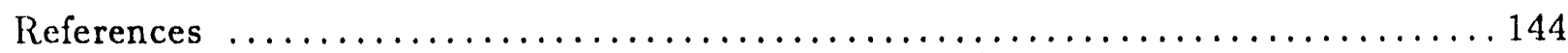

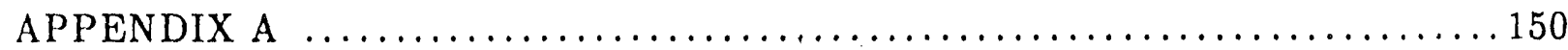

APPENDIX B

The Peng-Robinson-Bokis (PRB) Equation of State $\ldots \ldots \ldots \ldots \ldots \ldots \ldots \ldots \ldots$ APPENDIX C

The Elliott-Suresh-Donohue (ESD) Equation of State .................155 APPENDIX D

The Heidemann-Khalil-Michelsen (HKM) Algorithm and

The Derivatives of Helmholtz Free Energy Using EOS ................ 157 APPENDIX E

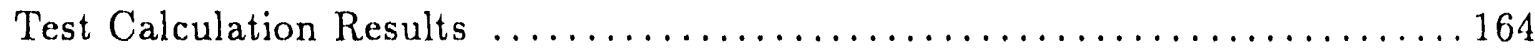
APPENDIX F

FORTRAN Source Code 


\section{LIST OF TAB $L E S$}

Table 1. Average percentage errors of molar volume calculations for pure $\mathrm{CO}_{2}$ for

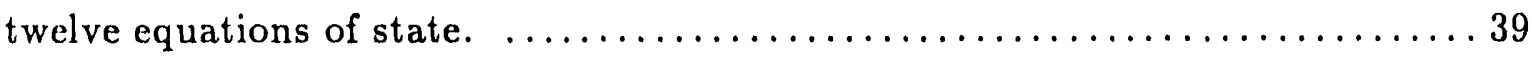

Table 2. Average percentage errors of molar volume calculations for pure $n-C_{4}$ for

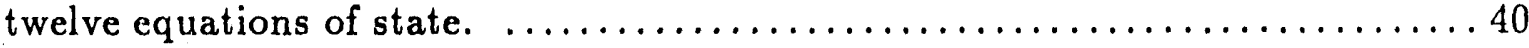

Table 3. Average percentage errors obtained from volume calculations for nine pure alkanes for eleven equations of state.

Table 4. Average percentage errors of bubble point calculations and flash calculations for $\mathrm{CO}_{2} / \mathrm{n}-\mathrm{C}_{4}(\mathrm{~T}: 273-410 \mathrm{~K}, \mathrm{P}: 0.7-75.5 \mathrm{bar})$ for ten equations of state. $\ldots 42$

Table 5. Average percentage errors of bubble point calculations and flash calculations for $\mathrm{CO}_{2} / \mathrm{n}-\mathrm{C}_{6}(\mathrm{~T}: 313-393 \mathrm{~K}, \mathrm{P}: 7.7-116.0 \mathrm{bar})$ for ten equations of state. $\ldots 42$

Table 6. A comparison between experimental data (Ekiner and Thodos, 1966; Etter and Kay, 1961) and calculated critical temperatures and critical pressures using the RKS, PR, ALS and ESD equations of state for multicomponent mixtures. ....43

Table 7. Average percentage errors of mixture critical point calculations using four equations of state (RKS, PR, ALS and ESD). . . . . . . . . . . . . . . . 44

Table 8. Accuracy rankings for the eleven equations of state used in the volume calculations for pure $\mathrm{CO}_{2}$.

Table 9. Average overall errors of the predictive capability for eleven equations of state.

Table 10. Overall Ranking of the eleven equations of state studied in the preliminary tests.

Table 11. Independent parameters for the ALS equation of state. $\ldots \ldots \ldots \ldots 47$

Table 12. Independent parameters for the ESD equation of state. $\ldots \ldots \ldots \ldots 48$ 
Table 13. Test pattern in scaled factors - ALS EOS ................... 49

Table 14. Test pattern in scaled factors - ESD EOS $\ldots \ldots \ldots \ldots \ldots \ldots \ldots \ldots \ldots$

Table 15 A. Liquid density sensitivities for $n-C_{4} / \mathrm{CO}_{2}-$ ALS EOS - Average absolute

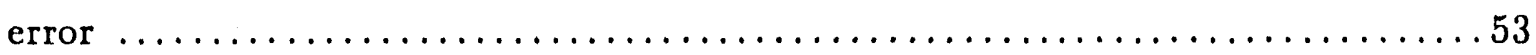

Table $15 \mathrm{~B}$. Liquid density sensitivities for $\mathrm{n}-\mathrm{C}_{4} / \mathrm{CO}_{2}$ - ALS EOS - Bias ....54

Table 15 C. Liquid density sensitivities for $n-C_{4} / \mathrm{CO}_{2}-\mathrm{ALS}$ EOS - CPU time ..55

Table 16 A. Liquid density sensitivities for $n-C_{6} / \mathrm{CO}_{2}-$ ALS EOS - Average absolute error .56

Table 16 B. Liquid density sensitivities for $n-C_{6} / \mathrm{CO}_{2}-$ ALS EOS - Bias .....57

Table 16 C. Liquid density sensitivities for $n-C_{6} / \mathrm{CO}_{2}-\mathrm{ALS}$ EOS - CPU time ..58

Table 17 A. Liquid density sensitivities for $n-C_{8} / \mathrm{CO}_{2}-$ ALS EOS - Averagc absolute

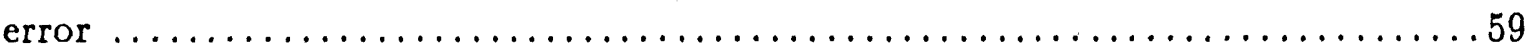

Table $17 \mathrm{~B}$. Liquid density sensitivities for $\mathrm{n}-\mathrm{C}_{8} / \mathrm{CO}_{2}-\mathrm{ALS}$ EOS - Bias $\ldots \ldots 60$

Table 17 C. Liquid density sensitivities for $n-C_{8} / \mathrm{CO}_{2}-\mathrm{ALS}$ EOS - CPU time . .61

Table 18 A. Liquid density sensitivities for $n-C_{10} / \mathrm{CO}_{2}-\mathrm{ALS}$ EOS - Average absolute error 62

Table $18 \mathrm{~B}$. Liquid density sensitivities for $n-\mathrm{C}_{10} / \mathrm{CO}_{2}$ - ALS EOS - Bias ....63

Table $18 \mathrm{C}$. Liquid density sensitivities for $\mathrm{n}-\mathrm{C}_{10} / \mathrm{CO}_{2}$ - ALS EOS - CPU time .64

Table $19 \mathrm{~A}$. Liquid density sensitivities for $\mathrm{n}-\mathrm{C}_{4} / \mathrm{CO}_{2}-\mathrm{ESD}$ EOS - Average absolute

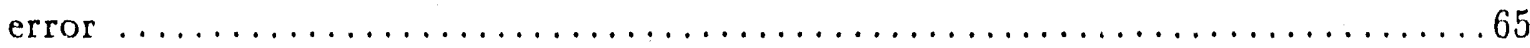

Table 19 B. Liquid density sensitivities for $n-C_{4} / \mathrm{CO}_{2}-\mathrm{ESD}$ EOS - Bias .....66

Table 18 C. Liquid density sensitivities for $\mathrm{n}-\mathrm{C}_{4} / \mathrm{CO}_{2}-\mathrm{ESD}$ EOS - CPU time .667 Table 20 A. Liquid density sensitivities for $n-C_{6} / \mathrm{CO}_{2}-$ ESD EOS - Average absolute error .68 
Table $20 \mathrm{~B}$. Liquid density sensitivities for $\mathrm{n}-\mathrm{C}_{6} / \mathrm{CO}_{2}-\mathrm{ESD} \mathrm{EOS}-\mathrm{Bias}$ .69

Table $20 \mathrm{C}$. Liquid density sensitivities for $\mathrm{n}-\mathrm{C}_{6} / \mathrm{CO}_{2}-\mathrm{ESD}$ EOS - CPU time ..70

Table 21 A. Liquid density sensitivities for $n-C_{8} / \mathrm{CO}_{2}-$ ESD EOS - Average absolute

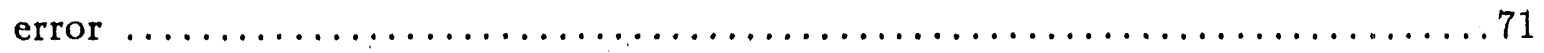

Table 21 B. Liquid density sensitivities for $n-C_{8} / \mathrm{CO}_{2}-$ ESD EOS - Bias $\ldots \ldots .72$

Table 21 C. Liquid density sensitivities for $n-C_{8} / \mathrm{CO}_{2}-$ ESD EOS - CPU time $\ldots 73$

Table 22 A. Liquid density sensitivities for $n-C_{10} / \mathrm{CO}_{2}$ - ESD EOS - Average absolute

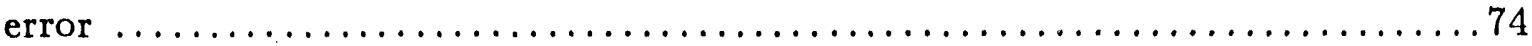

Table $22 \mathrm{~B}$. Liquid density sensitivities for $\mathrm{n}-\mathrm{C}_{10} / \mathrm{CO}_{2}-\mathrm{ESD}$ EOS - Bias $\ldots \ldots 75$

Table 22 C. Liquid density sensitivities for $n-C_{10} / \mathrm{CO}_{2}-$ ESD EOS - CPU time .76

Table 23 A. Critical line locus sensitivities for $\mathrm{n}-\mathrm{C}_{4} / \mathrm{CO}_{2}$ - ALS EOS - Average

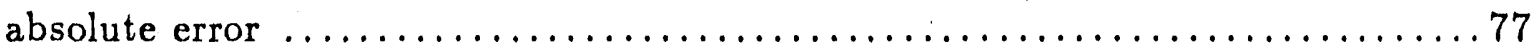

Table $23 \mathrm{~B}$. Critical line locus sensitivities for $\mathrm{n}-\mathrm{C}_{4} / \mathrm{CO}_{2}-\mathrm{ALS}$ EOS - Bias $\ldots 78$

Table 23 C. Critical line locus sensitivities for $n-C_{4} / \mathrm{CO}_{2}-\mathrm{ALS}$ EOS - CPU time 79

Table 24 A. Critical line locus sensitivities for $n-C_{6} / \mathrm{CO}_{2}$ - ALS EOS - Average

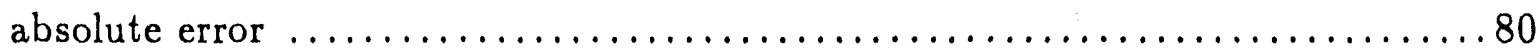

Table 24 B. Critical line locus sensitivities for $n-C_{6} / \mathrm{CO}_{2}-$ ALS EOS - Bias $\ldots 81$

Table $24 \mathrm{C}$. Critical line locus sensitivities for $\mathrm{n}-\mathrm{C}_{6} / \mathrm{CO}_{2}-\mathrm{ALS}$ EOS - CPU time 82

Table 25 A. Critical line locus sensitivities for $n-C_{4} / \mathrm{CO}_{2}-$ ESD EOS - Average

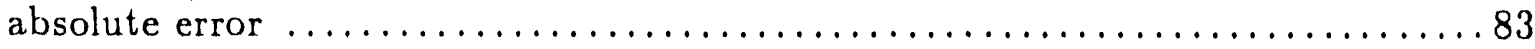

Table $25 \mathrm{~B}$. Critical line locus sensitivities for $\mathrm{n}-\mathrm{C}_{4} / \mathrm{CO}_{2}-$ ESD EOS - Bias $\ldots 84$

Table $25 \mathrm{C}$. Critical line locus sensitivities for $\mathrm{n}-\mathrm{C}_{4} / \mathrm{CO}_{2}-\mathrm{ESD}$ EOS - CPU time 85

Table 26 A. Critical line locus sensitivities for $n-C_{6} / \mathrm{CO}_{2}-$ ESD EOS - Average

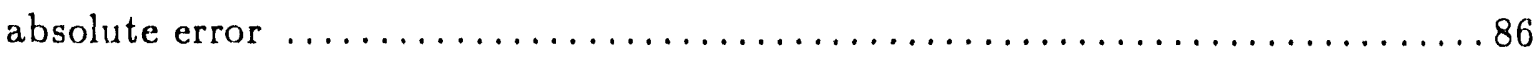


Table 26 B. Critical line locus sensitivities for $n-C_{6} / \mathrm{CO}_{2}-$ ESD EOS - Bias $\ldots 87$

Table 26 C. Critical line locus sensitivities for $\mathrm{n}-\mathrm{C}_{6} / \mathrm{CO}_{2}-\mathrm{ESD}$ EOS - CPU time 88

Table 27. Maximum sensitivities - Liquid density calculations - ALS EOS ......89

Table 28. Migration of the largest five sensitivities across Mixtures - Mixture Liquid

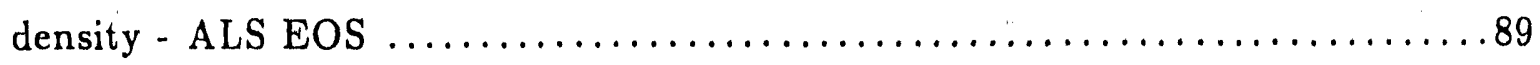

Table 29. Migration of the largest five sensitivities across Mixtures - Mixture Liquid

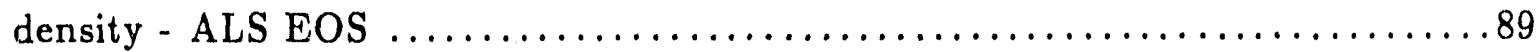

Table 30. Number of the five most sensitive factors in the Higher level parameters ALS EOS; Mixture Liquid density - Error responses . . . . . . . . . . . . 90

Table 31. Maximum sensitivities - Liquid density calculations - ESD EOS ......91

Table 32. Migration of the largest five sensitivities across Mixtures - Mixture Liquid

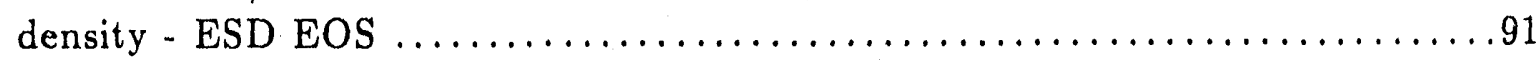

Table 33. Migration of the largest five sensitivities across Mixtures - Mixture Liquid

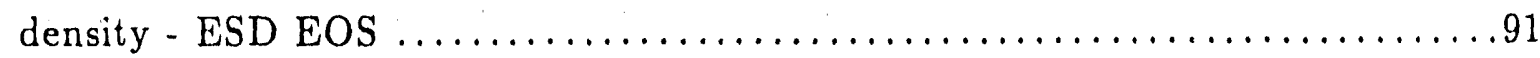

Table 34. Number of the five most sensitive factors in the Higher level parameters ESD EOS; Mixture Liquid density - Error responses ................... 92

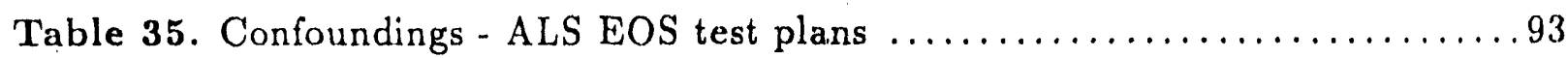

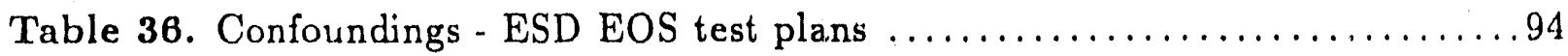




\section{LIST OF FIGURES}

Figure 1. A comparison with experimental values of calculated pressure for $\mathrm{CO}_{2}$ using

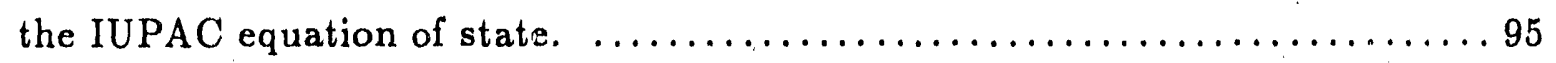

Figure 2. A comparison with experimental values of calculated volume for $\mathrm{CO}_{2}$ using

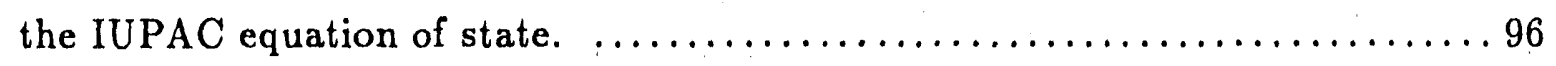

Figure 3. A comparison with experimental values of calculated volume for $\mathrm{CO}_{2}$ using

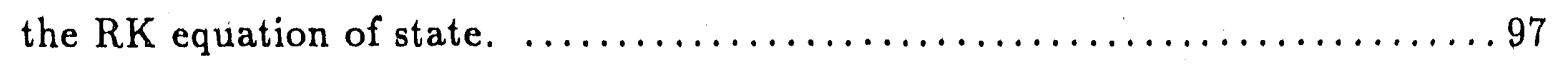

Figure 4. A comparison with experimental values of calculated volume for $\mathrm{CO}_{2}$ using

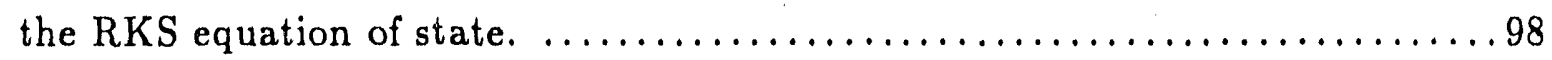

Figure 5. A comparison with experimental values of calculated volume for $\mathrm{CO}_{2}$ using

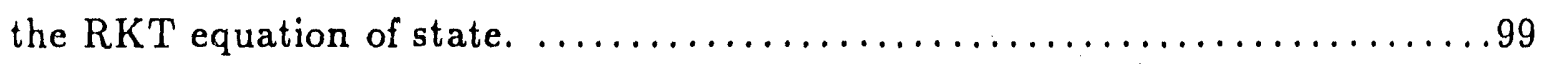

Figure 6. A comparison with experimental values of calculated volume for $\mathrm{CO}_{2}$ using the PR equation of state.

Figure 7. A comparison with experimental values of calculated volume for $\mathrm{CO}_{2}$ using the PRB equation of state. .101

Figure 8. A comparison with experimental values of calculated volume for $\mathrm{CO}_{2}$ using

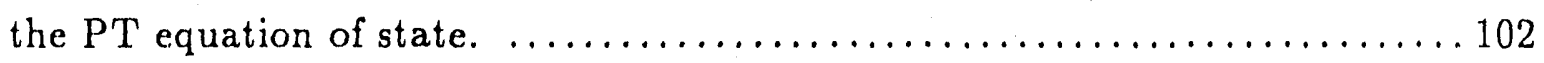

Figure 9. A comparison with experimental values of calculated volume for $\mathrm{CO}_{2}$ using the ALS equation of state. .103

Figure 10. A comparison with experimental values of calculated volume for $\mathrm{CO}_{2}$ using

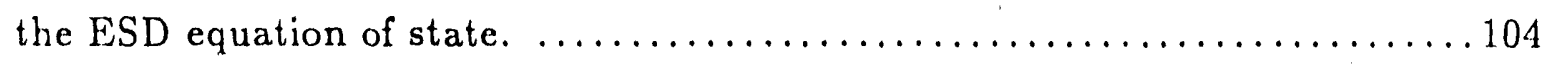

Figure 11. A comparison with experimental values of calculated volume for $\mathrm{CO}_{2}$ using

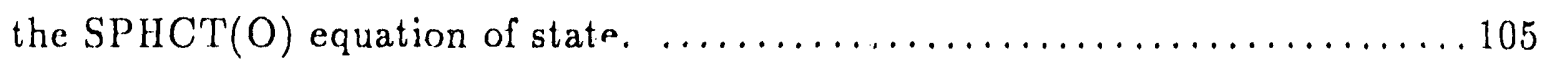

Figure 12. A comparison with experimental values of calculated volume for $\mathrm{CO}_{2}$ using

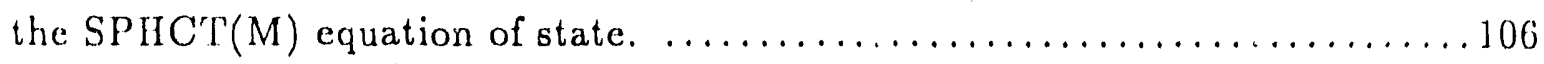


Figure 13. A comparison with experimental values of calculated volume for $\mathrm{CO}_{2}$ using the PACT equation of state.

Figure 14. A comparison with experimental values of calculated pressure for $n-C_{4}$ using the RK equation of state. 108

Figure 15. A comparison with experimental values of calculated volume for $n-C_{4}$ using the RKS equation of state. 109

Figure 16. A comparison with experimental values of calculated volume for $n-C_{4}$ using the RKT equation of state. 110

Figure 17. A comparison with experimental values of calculated volume for $n-C_{4}$ using the PR equation of state. 111

Figure 18. A comparison with experimental values of calculated volume for $n-C_{4}$ using the PRB equation of state.

Figure 19. A comparison with experimental values of calculated volume for $n-C_{4}$ using the PT equation of state.

Figure 20. A comparison with experimental values of calculated volume for ${ }_{1}-\mathrm{C}_{4}$ using the ALS equation of state. 114

Figure 21. A comparison with experimental values of calculated volume for $n-C_{4}$ using the ESD equation of state. .115

Figure 22. A comparison with experimental values of calculated $v$ ume for $n-C_{4}$ using the $\operatorname{SPHCT}(\mathrm{O})$ equation of state.

Figure 23. A comparison with experimental values of calculated volume for $n-C_{4}$ using the $\operatorname{SPHCT}(\mathrm{M})$ equation of state. .117

Figure 24. A comparison with experimental values of calculated volume for $n-C_{4}$ using the PACT equation of state. 
Figure 25. The results of bubble point calculations for $\mathrm{CO}_{2} / \mathrm{n}-\mathrm{C}_{4}$ using $\mathrm{RKS}, \mathrm{PR}$, PRB, ESD and PACT equations compared with experimental data (Knapp, et al, 1982).

Figure 26. The results of bubble point calculations for $\mathrm{CO}_{2} / \mathrm{n}-\mathrm{C}_{4}$ using PT and ALS equations compared with experimental data (Knapp, et al., 1982).

Figure 27. The results of flash calculations for $\mathrm{CO}_{2} / \mathrm{n}-\mathrm{C}_{4}$ using $\mathrm{RK}, \mathrm{RKS}, \mathrm{PR}$ and PRB equations compared with experimental data (Knapp, et al., 1982). .... 121

Figure 28. The results of flash calculations for $\mathrm{CO}_{2} / \mathrm{n}-\mathrm{C}_{4}$ using $\mathrm{ESD}, \operatorname{SPHCT}(\mathrm{O})$, SPHCT(M) and PACT equations compared with experimental data (Knapp, et al., 1982). .122

Figure 29. The results of flash calculations for $\mathrm{CO}_{2} / \mathrm{n}-\mathrm{C}_{4}$ using PT and ALS equations compared with experimental data (Knapp, et al., 1982). ............... 123

Figure 30. The results of bubble point calculation for $\mathrm{CO}_{2} / \mathrm{n}-\mathrm{C}_{6}$ using $\mathrm{RKS}$, PR, PRB, ESD and PACT equaiions compared with experimental data (Li,

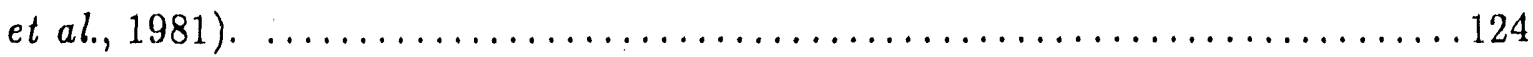

Figure 31. The results of bubble point calculation for $\mathrm{CO}_{2} / \mathrm{n}-\mathrm{C}_{6}$ using $\mathrm{PT}$ and ALS equations compared with experimental data $(\mathrm{Li}$, et al., 1981) . . . . . . . . 125

Figure 32. The results of flash calculation for $\mathrm{CO}_{2} / \mathrm{n}-\mathrm{C}_{6}$ using $\mathrm{RK}, \mathrm{RKS}, \mathrm{PR}$ and PRB equations compared with experimental data (Li, et al., 1981). ........ 126

Figure 33. The results of flash calculation for $\mathrm{CO}_{2} / \mathrm{n}-\mathrm{C}_{6}$ using $\mathrm{ESD}, \operatorname{SPHCT}(\mathrm{O})$, SPHCT(M) and PACT equations compared with experimental data (Li, et al., 1981). 127

Figure 34. The results of flash calculation for $\mathrm{CO}_{2} / \mathrm{n}-\mathrm{C}_{0}$ using $\mathrm{PT}$ and $\mathrm{ALS}$ equations compared with experimental data (Li, et al., 1981).

Figure 35. A comparison between calculated critical temperatures and critical pressures of $\mathrm{C}_{4} \mathrm{H}_{10}(1) / \mathrm{C}_{8} \mathrm{H}_{18}(2)$ using the RKS, PR, ALS and ESD equations of state and experimental data (Hicks and Young, 1975). 
Figure 36. A comparison between calculated critical temperatures and critical pressures of $\mathrm{C}_{6} \mathrm{H}_{14}(1) / \mathrm{C}_{14} \mathrm{H}_{30}(2)$ using the RKS, PR, ALS and ESD equations of state and experimental data (Hicks and Young, 1975). ................... 130

Figure 37. A comparison between calculated critical temperatures and critical pressures of five binary mixtures containing $\mathrm{CO}_{2}$ using the RKS, PR, ALS and ESD equations of state and experimental data (Hicks and Young, 1975). ....... 131

Figure 38 A. Average Absolute Errors Among Mixtures (Liquid Density) - ESD EOS

Figure 38 B. Bias Among Mixtures (Liquid Density) - ESD EOS ........... 133

Figure 38 C. CPU Time Among Mixtures (Liquid Density) - ESD EOS .......134

Figure 39 A. Average Absolute Errors Among Mixtures (Liquid Density) - ALS EOS

Figure 39 B. Bias Among Mixtures (Liquid Density) - ALS EOS ........... 136

Fig.ıre 39 C. CPU Time Among Mixtures (Liquid Density) - ALS EOS ........137

Figure 40 A. Average Absolute Error Among Mixtures (Critical Line Locus) - ESD EOS

Figure 40 B. Bias Among Mixtures (Critical Line Locus) - ESD EOS ......... 139

Figure 40 C. CPU Time Among Mixtures (Critical Line Locus) - ESD EOS . . . 140

Figure 41 A. Average Absolute Error Among Mixtures (Critical Line Locus) - ALS EOS

Figure 41 B. Bias Among Mixtures (Critical Line Locus) - ALS EOS ........ 142

Figure 41 C. CPU Time Among Mixtures (Critical Line Locus) - ALS EOS ... 143 


\section{Introduction}

The principle task of this project was to perform a statistically designed computer analysis of selected equations of state relating to enhanced oil recovery. The purpose was to determine the importance and sensitivity of thermodynamic variables and of the parameters in the equations of state. An important aspect of this was to determine how the accuracy and/or precision of these parameters affects the agreement with experimental data.

This research project was initiated by reviewing technical literature and consulting with experts to select the type of equations of state to study, to determine the main thermodynarnic jroperties to study, and to set the temperature and pressure ranges for study. Several generalized mathematical algorithms and computer programs have been developed to calculate thermodynamic properties for pure $\mathrm{CO}_{2}$ and alkanes and their mixtures including critical points of the mixtures. In the initial screening, twelve equations of state were tested for pure $\mathrm{CO}_{2}$ and eleven of them were studied for pure alkanes and their binary mixtures with carbon dioxide. Considering industrial practice and comparing the calculated results with experimental data, two equations of state were selected for statistical analysis. The details of how these selections were made are described in the following sections.

In the final sections of this report, we describe the results of the fractional factorial test plans that were made for the selected equations of state. We also discuss the characteristics of these equations revealed by this analysis. Finally, we present the significance of these methods for future work, especially involving enhanced oil recovery simulations.

\section{Selection of Equations of State for Preliminary Tests}

One of the first tasks in this research project was to choose which particular equations of state to study. To do this, we reviewed much of the literature relevant to the thermodynamics of enhanced oil recovery processes, consulted with numerous experts 
in academia and in industry (see Appendix A), and did our own preliminary analysis of the accuracy of several different equations of state. From this, we established four criteria that should be considered in choosing equations of state for enhanced oil recovery applications. These are simplicity, accuracy, reliability, and availability of parameters. Each of these is discussed below. In considering these criteria, we chose twelve equations of state for preliminary tests. The rationale for choosing these equ itions is discussed as well.

\section{(A) Simplicity}

Though there literally are hundreds of equations of state in literature, only a few of them are used widely for calculations of thermodynamic properties in industry. Those that are used were chosen as a compromise between simplicity and other considerations such as accuracy and reliability. Since calculations of thermodynamic properties are in the "inner loops" of EOR reservoir simulations, the use of a simple eq"ation of state, and preferably a cubic equation of state, becomes paramount (Froning, et o.l, 1982; O'Connell, 1989; Teja, 1989; Peng, 1989; Chao, 1989; Nagarajan, 1989; Katz and Lee, 1990). Therefore, we considered simplicity to be the first criterion for selecting an equation of state for enhanced oil recovery calculations.

Because the roots can be determined analytically, cubic equations of state are used for both petroleum reservoir simulation studies and the design of various processes encountered in enhanced oil recovery. The Redlich-Kwong (RK) equation (Redlich and Kwong, 1948) and the Peng-Robinson (PR) equation (Peng and Robinson, 1976) are the most commonly used equations of state for calculations of thermodynamic properties for oil and gas processing. Although both the original RK and PR equations give good results in vapor-liquid equilibrium calculations, they often give large errors in the evaluation of other thermodynamic properties iuch as compressibility factor, density, and enthalpy departure. Various modifications to the original RK equation have been made to try to reduce the errors in these other properties (Zudkevich and Joffe, 1970; 
Chang and Lu, 1970; Joffe et al., 1970; Soave, 1972). Of these, the Redlich-KwongSoave (RKS) equation is the most widely used in the petroleum industry. All three of these equations (RK, RKS and PR) are two-parameter equations of state. The values of these parameters for each pure component are obtained from the critical temperature, critical pressure and acentric factor. The mixing rules for these parameters also are specified for each equation. Because of their simplicity and availability of parameters and mixing rules, we chose the RK, the RKS and the PR equations as the first three equations of state to study.

\section{(B) Accuracy}

The results of EOR reservoir simulation depend strongly on the accuracy of the equation of state used in calculations of thermodynamic properties. The accuracy of an equation of state affects not only the results of the simulation, but also the choice and application of the methods used for the solutions of the large sets of nonlinear algebraic equations involved in the EOR simulation. Obviously, if an accurate representation of properties can be obtained with a simple equation of state, then such an equation of state will offer a significant advantage over a more complex equation.

It has been common practice to compare the accuracy of cubic equations of state by noting how well they predict vapor pressures and saturated liquid densities for pure components through the equality of liquid and gas fugacities (Martin, 1979; Walas, 1985; Trebble and Bishnoi, 1986). By reviewing the literature that describes the accuracy of these various cubic equations of state, we selected two additional equations, the PatelTeja (PT) equation (Patel and Teja, 1982) and the Adachi-Lu-Sugie (ALS) equation (Adachi, et al., 1983), for our preliminary test because they appeared to have particular promise for enhanced oil recovery operations.

However, vapor pressures and saturated liquid densities are not the only properties of interest in enhanced oil recovery simulations. In particular, compressed liquid densities also are important and there are modifications to the RK and PR equations 
which have been made specifically to improve the volumetric behavior of these equations. Other modifications to the RK and PR equations have been made which attempt to improve the accuracy even further by using more adjustable parameters. To test the accuracy of these modifications, here, we include the Peng-Robinson-Bokis (PRB) equation, a modified PR equation (Bokis, et al., 1989), and the Redlich-Kwong-Turek (RKT) equation, (Morris and Turek, 1985), in our study. In the PRB equation, a correction term, $\Delta v$, is added to the molar volume, $v$, in the $\mathrm{PR}$ equation. It improves the results of volumetric calculations (see Appendix B). In the RKT equation of state, both $a$ and $b$ parameters in the original RK equation of state are optimized for carbon dioxide and alkanes using literature PVT data. The regressed values of $a$ and $b$ are fitted as functions of temperature. These temperature-dependent parameters significartly improve the accuracy of volumetric calculations.

\section{(C) Reliability}

In critically selecting equations of state for EOR processes, one should realize the complex nature of petroleum reservoir fluids and the complicated phase behavior that is observed at elevated temperatures and pressures in enhanced oil recovery processes (Yarborough, 1979; Vogel, et al., 1983; Pederson, et al., 1983; Gani and Fredenslund, 1986; Charback and Turek, 1986). This complex behavior arises because of the wide variety of compounds encountered. These are mostly non-spherical molecules, such as the heavy alkanes, aromatic hydrocarbons, naphthenes as well as polar non-hydrocarbons (carbon dioxide and hydrogen sulfide). The phase behavior is complicated because the high pressures observed in enhanced oil recovery processes make the heavier hydrocarbons highly soluble in the $\mathrm{CO}_{2}$-rich gas phase and the supercritical components such as $\mathrm{CO}_{2}$ highly soluble in the oil-rich liquid phase. This results in near-critical and retrograde behavior in mixtures. Therefore, in selecting equations of state for enhanced oil recovery processes, the reliability of the equation is considered as important as its accuracy (O'Connell, 1989; Chao, 1989; Nagarajan, 1989). In other words, the equation of state should have the ability to predict certain properties quantitatively in certain 
phase regions and also must be able to qualitatively describe the complex behavior of fluid mixtures over wide ranges of conditions.

Furthermore, real mixtures encountered in enhanced oil recovery almost always contain some water. Therefore, modelling phase behavior in the petroleum reservoir must include liquid-liquid immiscibilities which are common for oil mixtures containing water. LLE is much more difficult to represent with an equation of state than VLE. The cubic equations of state described above are not able to predict liquid-liquid immiscibilities without significant empirical modification (Chaback and Turek, 1986), especially when significant amounts of water are present. It therefore was felt that more accurate equations should be examined, despite their complexity. Recently, a semi-empirical cubic equation of state, the Elliott-Suresh-Donohue (ESD) equation (Elliott, et al., 1989), has been developed to describe non-spherical molecules and mixtures which contain water (see Appendix C). It was decided to include this equation of state in the preliminary screening as well.

(D) Number of Parameters, Availability of Parameters and Mixing Rules

In addition to the criteria of simplicity, accuracy and reliability, there are practical considerations which must be taken into account. They are: the number of parameters in an equation of state, the ease of parameter estimation, the accuracy of the mixing rules, and the potential for future refinement and generalization.

The scientific literature abounds with cubic equations of state which were developed in attempts to obtain a simple equation of state with only a few parameters which could accurately predict thermodynamic behavior of complex molecules such as polar molecules and chain molecules. The generic form of these pressure-explicit equations of state that are cubic in volume can be written as

$$
P=\frac{R T}{v-b_{1}}-\frac{a(T)}{v^{2}+b_{2} v+b_{3}}
$$

where $P$ is the total pressure of system; $T$ is temperature; $v$ is molar volume; $R$ is the universal gas constant; $a(T)$ is an interaction parameter which is a function of 
temperature; and $b_{1}$ to $b_{3}$ are volumetric parameters which are usually constants for each component. By specializing the parameters $a(T)$ and $b_{i}$ and by straightforward algebraic rearrangement, many well-known forms of cubic equations of state can be be obtained from equation (1). Below the equations of state discussed above are presented in this form.

\section{Redlich-Kwong (RK)}

For

$$
\begin{gathered}
a(T)=a_{(\mathrm{RK})}(T) \\
b_{1}=b_{2}=b_{(\mathrm{RK})} \\
b_{3}=0
\end{gathered}
$$

where

$$
\begin{aligned}
a_{(\mathrm{RK})}(T) & =0.4278 \frac{R^{2} T_{c}^{2.5}}{P_{c} T^{0.5}} \\
b_{(\mathrm{RK})} & =0.0867 \frac{R T_{c}}{P_{c}}
\end{aligned}
$$

equation (1) gives the RK equation of state (Redlich and Kwong, 1948):

$$
P=\frac{R T}{v-b_{(\mathrm{RK})}}-\frac{a_{(\mathrm{RK})}(T)}{v\left(v+b_{(\mathrm{RK})}\right)}
$$

Redlich-Kwong-Soave (RKS)

For

$$
\begin{gathered}
a(T)=a_{(\mathrm{RKS})}(T) \\
b_{1}=b_{2}=b_{(\mathrm{RKS})} \\
b_{3}=0
\end{gathered}
$$

where

$$
a_{(\mathrm{RKS})}(T)=0.42747 \frac{R^{2} T_{c}^{2}}{P_{c}}\left[1+\left(0.48+1.574 \omega-0.176 \omega^{2}\right)\left(1-T_{r}^{0.5}\right)\right]^{2}
$$




$$
b_{(\mathrm{RKS})}=0.08664 \frac{R T_{\mathrm{c}}}{P_{c}}
$$

we have the RKS equation of state (Soave, 1972):

$$
P=\frac{R T}{v-b_{(\mathrm{RKS})}}-\frac{a_{(\mathrm{RKS})}(T)}{v\left(v+b_{(\mathrm{RKS})}\right)}
$$

\section{Peng-Robinson (PR)}

For

$$
\begin{gathered}
a(T)=a_{(\mathrm{PR})}(T) \\
b_{1}=b_{(\mathrm{PR})} \\
b_{2}=2 b_{(\mathrm{PR})} \\
b_{3}=-b_{(\mathrm{PR})}^{2}
\end{gathered}
$$

where

$$
\begin{gathered}
a_{(\mathrm{PR})}(T)=0.45724 \frac{R^{2} T_{c}^{2}}{P_{c}}\left[1+\left(0.37464+1.54226 \omega-0.26992 \omega^{2}\right)\left(1-T_{r}^{0.5}\right)\right]^{2} \\
b_{(\mathrm{PR})}=0.0778 \frac{R T_{c}}{P_{c}}
\end{gathered}
$$

equation (1) yields the PR equation of state (Peng and Robinson, 1976):

$$
P=\frac{R T}{v-b_{(\mathrm{PR})}}-\frac{a_{(\mathrm{PR})}(T)}{v\left(v+b_{(\mathrm{PR})}\right)+b_{(\mathrm{PR})}\left(v-b_{(\mathrm{PR})}\right)}
$$

\section{Patel-Teja (PT)}

For

$$
\begin{gathered}
a(T)=a_{(\mathrm{PT})}(T) \\
b_{1}=b_{(\mathrm{PT})} \\
b_{2}=b_{(\mathrm{PT})}+c_{(\mathrm{PT})} \\
b_{3}=-b_{(\mathrm{PT})} c_{(\mathrm{PT})}
\end{gathered}
$$


where

$$
\begin{gathered}
a_{(\mathrm{PT})}(T)=\Omega_{a}\left[1+\left(0.452413+1.30982 \omega-0.295937 \omega^{2}\right)\left(1-T_{r}^{(0.5}\right)\right]^{2} \\
b_{(\mathrm{PT})}=\Omega_{b} \frac{R T_{c}}{P_{c}} \\
c_{(\mathrm{PT})}=\Omega_{c} \frac{R T_{c}}{P_{c}}
\end{gathered}
$$

with

$$
\begin{gathered}
\Omega_{a}=3 \zeta^{2}+3(1-2 \zeta) \Omega_{b}+\Omega^{2}+1-3 \zeta \\
\Omega_{b}^{3}+(2-3 \zeta) \Omega_{b}^{2}+3 \zeta^{2} \Omega-\zeta^{3}=0 \\
\Omega_{c}=1-3 \zeta \\
\zeta=0.329032-0.076799 \omega+0.0211947 \omega^{2}
\end{gathered}
$$

One obtains the PT equation of state (Patel and Teja, 1982):

$$
P=\frac{R T}{v-b_{(\mathrm{PT})}}-\frac{a_{(\mathrm{PT})}(T)}{v\left(v+b_{(\mathrm{PT})}\right)+c_{(\mathrm{PT})}\left(v-b_{(\mathrm{PT})}\right)}
$$

\section{Adachi-Liu-Schugi (ALS)}

For

$$
\begin{gathered}
a(T)=a_{(\mathrm{ALS})}(t) \\
b_{1}=b_{1(\mathrm{ALS})} \\
b_{2}=b_{3(\mathrm{ALS})}-b_{2(\mathrm{ALS})} \\
b_{3}=-b_{2(\mathrm{ALS})} b_{3(\mathrm{ALS})}
\end{gathered}
$$

where

$$
\begin{gathered}
a_{(\mathrm{ALS})}(T)=\Omega_{a}\left[1+\left(0.407+1.3787 \omega-0.2933 \omega^{2}\right)\left(1-T_{r}^{0.5}\right)\right]^{2} \\
b_{1(\mathrm{ALS})}=\frac{R T_{c}}{P_{c}}\left(0.08974-0.03452 \omega+0.0033 \omega^{2}\right) \\
b_{2(\mathrm{ALS})}=\frac{R T_{c}}{P_{c}}\left(0.03686+0.00405 \omega-0.01073 \omega^{2}+0.00157 \omega^{3}\right)
\end{gathered}
$$




$$
\begin{aligned}
& b_{3(\mathrm{ALS})}=\frac{R T_{c}}{P_{c}}\left(0.154+0.14122 \omega-0.00272 \omega^{2}-0.00484 \omega^{3}\right) \\
& \Omega_{a}=\frac{R^{2} T_{c}^{2}}{P_{c}}\left(0.44869+0.04024 \omega+0.01111 \omega^{2}-0.00576 \omega^{3}\right)
\end{aligned}
$$

equation (1) becomes the ALS equation of state (Adachi, et al., 1983):

$$
P=\frac{R T}{v-b_{1(\mathrm{ALS})}}-\frac{a_{(\mathrm{ALS})}(T)}{\left(v-b_{2(\mathrm{ALS})}\right)\left(v+b_{3(\mathrm{ALS})}\right)}
$$

Many examples other than the five presented above also can be obtained from equation (1) (van der Waals, 1873; Martin, 1967; Usdin and McAuliffe, 1976; Lin, et al., 1983).

\section{ESD, PRB, and RKT}

Beca ase the derivations of the PRB equation and the ESD equation are different from the cubic equations of state given above, they cannot be obtained directly from equation (1). Nevertheless, these two equations of state also contain few parameters, three for the PRB equation and four for the ESD equation. These parameters also can be calculated using critical temperature $T_{c}$, critical pressure $P_{c}$ and acentric factor $\omega$. These two equations of state are described in Appendices B and C.

In the RKT equation, instead of using critical properties to estimate parameters $a$ and $b$ in original RK equation, both $a$ and $b$ are correlated with temperature using PVT data for each component. For example, the parameters $a$ and $b$ for carbon dioxide are given as

$$
a=a_{0} \Delta T^{3}+a_{1} \Delta T^{2}+a_{2} \Delta T+a_{3}+a_{4}\left(a_{5}-\Delta T\right)^{a_{6}}+a_{7} /\left(a_{8}-\Delta T\right)
$$

and

$$
b=b_{0} \Delta T^{3}+b_{1} \Delta T^{2}+b_{2} \Delta T+b_{3}+b_{4}\left(b_{5}-\Delta T\right)^{b_{6}}+b_{7} /\left(b_{8}-\Delta T\right)
$$

where $a_{i}$ and $b_{i}$ are constants for each component and $\Delta T$ is defined as

$$
\Delta T \equiv \frac{T}{T_{c}}-1
$$

For alkanes, different forms of correlations are given for parameters $a$ and $b$. 
For the seven cubic equations of state selected (RK, RKS, RKT, PR, PRB, P'l and ALS), the same mixing rules are used for the parameters $a_{m i x}$ and $b_{m i x}$. For mixture interaction parameter $a_{m i x}$, the geometric mean is used

$$
a_{m i x}=\sum_{i} \sum_{j} x_{i} x_{j} a_{i j}
$$

where $x_{i}$ is the mole fraction of component $i$, and the cross interaction parameter $a_{i j}$ is given by

$$
a_{i j}=\left(a_{i i} a_{j j}\right)^{0.5}\left(1-k_{i j}\right)
$$

where $k_{i j}$ is an additional binary interaction parameter for each pair of components $i$ and $j$. The arithmetic mean is used to calculate mixture volumetric parameter $b_{m i x}$

$$
b_{\operatorname{mix}}=\sum_{i} x_{i} b_{i}
$$

\section{PACT, PHCT, SPHCT(O), SPHCT(M)}

While simplicity is a key issue, we felt it important to examine some of the more theoretical non-cubic equations of state in our preliminary tests to determine if their accuracy or reliability outweighed the increased computation time associated with their complexity. Since these theoretical equations are based on physical models, the parameters in these equations can be related to the physical properties of the components such as molecular size, shape and intermolecular forces. These equations of state generally represent the phase behavior more accurately over a wide range of conditions than cubic equations of state. The main disadvantage of non-cubic equations of state is that they require more computer time to calculate the phase volume secause an iterative procedure must be used. However, this disadvantage is becoming less important with the ever increasing speed of computers.

Therefore, despite the fact that most industrial simulations use cubic equations of state, it was decided that it would be useful to test three non-cubic equations of state, the original Simplified-Perturbed-Hard-Chain Theory [SPHCT(O)] (Kim, et al., 1986), 
the modified Simplified-Perturbed-Hard-Chain Theory [SPHCT(M)] (Vimalchand and Donohue, 1989) and the Perturbed-Anisotropic-Chain Theory (PACT) (Vimalchand, et al., 1985; Vimalchand 1986), were included in the preliminary tests. This was done to give an estimate of how much accuracy could be reasonably expected from an equation of state.

The PACT is developed by adding polar interaction terms to the Perturbed-HardChain Theory (PHCT) (Donohue and Prausnitz, 1978). The PHCT was developed specifically for the natural gas, petroleum and related industries. The PACT, which is derived by a rigorous statistical mechanical treatment, encompasses into one comprehensive equation several, previously available, but separate equations for configurational properties of fluids. The PACT can be used to calculate properties of both pure fluids and mixtures containing molecules of differing sizes (from simple molecule such as methane to polymers), different shapes (spherical and non-spherical), and different interaction forces (non-pciar and polar interactions) at all densities, from ideal-gas to compressed liquids. Further, the computer programs for the original PHCT are being converted to an engineering computer program (Hohmann, 1979) for wide use in the petroleum industry for process design calculations. An improved version of the PHCT has been shown to accurately model the properties of $\mathrm{CO}_{2}$-oil mixtures at petroleum reservoir operating conditions (Cotterman and Prausnitz, 1986). The SPHCT(O) and SPHCT(M) are the two simplified versions of PHCT.

As a result, eleven equations of state, eight cubic equations and three non-cubic equations were chosen and screened for accuracy. They are RK, RKS, RKT, PR, PRB, PT, ALS, ESD, SPHCT(O), SPHCT(M) and PACT. In the next section, we discuss our accuracy tests for both pure components (carbon dioxide and alkanes) and their mixtures. 


\section{Selection of Temperature and Pressure Ranges and Thermodynamic Properties to Study}

In enhanced oil recovery operations, various solvents are used to mobilize oil by a multiple-contact miscible displacement. Currently, enriched gas (methane plus liquefied petroleum gas), carbon dioxide, methane, nitrogen, and flue gas are considered to be efficient and economical for oil recovery. A solvent is selected according to economic conditions, solvent availability, and reservoir pressure and temperature. The solvent must be tested to ensure that it can displace the reservoir oil miscibly. Therefore, solvent properties, oil properties, mixture properties, and the operating temperature and pressure are important parameters in EOR design and simulation.

As a general rule, for higher pressure ( $>280$ bar) reservoirs, methane, nitrogen and flue gas usually are used as solvent. Carbon dioxide has the greatest potential in reservoirs at moderate temperature $\left(<323^{\circ} \mathrm{K}\right)$ and pressure $(65-280$ bar) (Froning, et al., 1982). Therefore, in both our preliminary screening and in the detailed statistical analysis we have planned, we intend to focus on these ranges of temperature and pressure.

Based on discussions with both industrial and academic experts (see Appendix A), we believe that the four physical properties, molar volume (density), the the number of phases present, viscosity, and interfacial tension, are the most important in enhanced oil recovery processes. Among these four properties, molar volume and number of phases are the only ones that can be calculated using an equation of state (Froning, et al., 1982; Morris and Turek, 1985; O'Connell, 1989; Nagarajan, 1989). Although the density calculation is a small part of a whole simulation program, density and fugacity calculations significantly effect the final results of the EOR design and simulation (Morris and Turek, 1985; Chaback and Turek, 1985). Therefore, as discussed before, in order to reduce the computer time and minimize errors in thermodynamic calculations, simplicity and accuracy of an equation of state are the two primary considerations in selecting equations of state. 
Under the temperature and pressure conditions set above, the complex nature of petroleum reservoir fluids leads to complicated phase behavior in EOR processes (Chaback and Turek, 1985). This complex behavior arises not only because of the wide variety of compounds encountered but also because the pressures observed in EOR processes make the heavier hydrocarbons highly soluble in the $\mathrm{CO}_{2}$-rich gas phase and the supercritical components (such as $\mathrm{CO}_{2}$ ) highly soluble in the oil-rich liquid phase. This results in near-critical and retrograde behavior in mixtures. Therefore, in addition to mixture molar volumes, mixture critical points also are an important thermodynamic variable to study.

Hence, we determined that the thermodynamic variables that are most appropriate to test in our statistical analysis are molar volumes and mixture critical points. Accuracy tests for pure components in both molar volume and critical state have been completed for all eleven equations selected in the previous section. While we believe that mixture density and mixture critical points are the most important properties to study, because of the ready availability of $K$-factor data for binary mixtures, mixture $K$-factors were used in our preliminary screening of equations.

\section{Accuracy Tests for Pure Carbon Dioxide}

The accuracy of the estimations of thermodynamic properties is cne of the controlling factors in petroleum reservoir simulations. Having an accurate equation of state for carbon dioxide, which would allow prediction of densities, phase behavior, and enthalpies, is especially important. In this section, the eleven equations of state were tested for their ability to represent experimental densities of carbon dioxide over a wide range of conditions $\left(T / T_{c}: 0.71-3.62 ; P / P_{c}: 0.01-13.73\right)$. In the tests, an empirical equation of state provided by the International Union of Pure and Applied Chemistry. (IUPAC) (Angus, et al., 1976) is used for comparison. This empirical equation of state was developed by a very thorough and critical study of all available experimental data for $\mathrm{CO}_{2}$ over the entire phase diagram. It gives very accurate results in both pressure 
and density calculations. Figures 1 and 2 show the high accuracy of pressure and density calculations using the IUPAC equation. The calculated results are compared with 284 experimental data points. Figures 3 to 13 compare calculated volumes using the cleven equations of state with experimental data. An error comparison for volumetric calculations among the twelve equations of state is given in Table 1. One can see that all of the cubic equations give reasonable accuracies in the supercritical and subcritical vapor regions but that larger errors are obtained in the subcritical liquid and critical regions. The comparison shows that the smallest overall error is obtained from ALS equation.

\section{Accuracy Tests for Pure Alkanes}

Using the eleven equations of state (RK, RKS, RKT, PR, PRB, PT, ALS, ESD, $\operatorname{SPHCT}(\mathrm{O}), \operatorname{SPHCT}(\mathrm{M})$ and $\mathrm{PACT}$ ), volumetric calculations for nine alkanes (methane, ethane, propane, n-butane, i-butane, pentane, hexane, heptane and octane) were carried out over wide ranges of temperature and pressure, including the critical regions. In addition, these equations were studied separately in four phase regions, supercritical, critical, subcritical vapor and subcritical liquid regions for n-butane to test their accuracy over entire phase diagram. The calculated values of molar volume using these equations are compared with 187 experimental PVT and liquid-density data points. The average percentage errors obtained from the calculations using these equations in the rifferent phase regions of pure n-butane are given in Table 2. Again, one sees that all of the cubic equations of state give reasonable accuracy for supercritical and subcritical vapor regions while larger deviations are obtained in the subcritical liquid and the critical regions. Conversely, the errors given by PACT in the liquid phase are smaller than the errors obtained in the vapor phase. Comparisons with experimental data are shown for the eleven equations in Figures 14 to 24, respectively.

The average percentage errors for the volume determinations for nine alkanes are listed in Table 3. For small molecules (carbon number is less than 4), all the equations 
give fairly good accuracy. However, as carbon number increases, the errors increase for all the cubic equations of state except the ESD equation. This occurs because the first seven equations are van der Waals-type of equations which do not take molecular shape into account correctly. However, the ESD and the three non-cubic equations were developed by considering segment-segment interactions for non-spherical molecules, and hence the errors given by these equations do not increase as the size of the alkane increases. Tests for pentane, hexane, heptane and octane were not carried out using the RKT equation because parameters were not available. As in the tests for pure carbon dioxide, the error comparisons shown in Tables 2 and 3 indicate that ALS equation gives the most accurate results over the whole phase region for the pure alkanes.

\section{Accuracy Test for Mixtures of Carbon Dioxide and Alkanes}

Using ten equations of state (RK, RKS, PR, PRB, PT, ALS, ESI), SPHCT(O), SPHCT(M) and PACT), vapor-liquid equilibrium (VLE) calculations were carried out for two binary mixtures, $\mathrm{CO}_{2}(1)-\mathrm{C}_{4} \mathrm{H}_{10}(2)$ and $\mathrm{CO}_{2}(1)-\mathrm{C}_{6} \mathrm{H}_{14}(2)$. We did not use the RKT equation for mixture calculations because the mixture parameters in the RKT equation are not avaliable. We concentrated on two types of calculations to test thermodynamic properties at vapor-liquid equilibria: bubble point calculations and flash calculations. In the bubble point calculations, system pressure $P$ and vapor compositions $y_{i}$ are calculated at given temperature $T$ and given liquid phase compositions $x_{i}$. In the flash calculations, compositions in boih vapor and liquid phases are computed using the equations of state at given temperature and pressure. Figures (25), (26), (30) and (31) show the results of the bubble point calculations for both binary mixtures. Figures (27) to (29) and (32) to (34) give calculated $K$ factors $\left(K_{i}=y_{i} / x_{i}\right)$ obtained from flash calculations. All these results are compared with experimental data given by Knapp, et al. (1982) and Li, et al., (1981).

In all calculations, a binary interaction parameter, $k_{1,2}$, was used. Average errors obtained from the calculations are given by Tables 4 and 5 . The reasonably small errors 
and the small values of $k_{1,2}$ show that the non-cubic equations have the most promise for making a priori predictions mixture behavior. Among the seven cubic equations of state, the ALS equation gives the best results with reasonable values of $k_{1,2}$ for both mixtures of $\mathrm{CO}_{2}-\mathrm{C}_{4} \mathrm{H}_{10}$ and $\mathrm{CO}_{2}-\mathrm{C}_{6} \mathrm{H}_{14}$.

\section{Reliability Tests (Mixture Critical Point Calculations)}

Since the operating temperature and pressure in a petroleum reservoir are always near or above the critical temperature of the fluid mixture and since the phase behavior of fluid mixtures in petroleum reservoir is complex at these operating conditions (O'Connell, 1989; Nagarajan, 1989), an equation of state used in EOR design and simulation must be reliable in its description of phase behavior of $\mathrm{CO}_{2}$ mixtures near the mixture critical points. Therefore, to test each equation's reliability, mixture critical points also were selected as a thermodynamic variable to study.

Many correlations have been proposed for predicting the critical properties of mixtures (Spencer, et al., 1976). But, most of these correlations are empirical and they are limited in the types of systems which they can represent. Therefore, the calculation of critical points using equations of state by applying thermodynamic criteria has become more common.

The rigorous thermodynamic definition of a mixture critical point was originally given by Gibbs (1876). The definition shows that a critical state must obey two restrictions. In the literature, these restrictions are presented several different forms. Nevertheless, the independent variables most frequently used in these forms are pressure, temperature and compositions in the Gibbs energy approach and volume, temperature and compositions in the Helmholtz free energy approach (Hicks and Young, 1975). For example, for a binary system, the two restrictions are written in terms of Gibbs energy $G$ as

$$
\left(\frac{\partial^{2} G}{\partial x_{2}^{2}}\right)_{T, P}=0
$$


and

$$
\left(\frac{\partial^{3} G}{\partial x_{2}^{3}}\right)_{T, P}=0
$$

The restrictions also can be given in terms of Helmoholtz free energy $A$ as

$$
\left(\frac{\partial^{2} A}{\partial x^{2}}\right)_{T, V}-W^{2}=0
$$

and

$$
\left(\frac{\partial^{3} A}{\partial x^{3}}\right)_{T, V}-3 W\left(\frac{\partial^{3} A}{\partial x^{2} \partial v}\right)_{T}+3 W^{2}\left(\frac{\partial^{3} A}{\partial x \partial v^{2}}\right)_{T}-W^{3}\left(\frac{\partial^{3} A}{\partial v^{3}}\right)_{T, x}=0
$$

where

$$
W=\frac{\left(\frac{\partial^{2} A}{\partial x \partial v}\right)_{T}}{\left(\frac{\partial^{2} A}{\partial v^{2}}\right)_{T, x}}
$$

where $x$ is mole fraction. Since all equations of state are written in an explicit form for pressure and give several roots for volume at given temperature and pressure, it is more convenient to use the Helmholtz approach in which molar volume $v$ and temperature $T$ are used as the independent variables.

Peng and Robinson (1977) evaluated their equation of state for mixture critical points directly by using the thermodynamic definition of the mixture critical state given by Gibbs (1876). Heidemann and Khalil (1980) and Michelsen and Heidemann (1981) developed an improved algorithm which was considerably more rapid and more robust than its predecessors. Recently, a thorough evaluation of equation of state method for calculating mixture critical points was given by Elliott and Daubert (1987). In our test, the Heidemann-Khalil-Michelsen (HKM) algorithm was used.

In the HKM approach, the two restrictions of critical state for a multicomponent mixture can be written as

$$
\operatorname{det}|\mathbf{Q}|=0
$$

and

$$
C=\sum_{i} \sum_{j} \sum_{k}\left(\frac{\partial^{3} A}{\partial n_{i} \partial n_{j} \partial n_{k}}\right)_{T, V} \Delta n_{i} \Delta n_{j} \Delta n_{k}=0
$$


where $n_{i}$ is moles of component $i, \Delta n_{i}$ is change of moles of component $i$ at $T_{c}+\Delta T$, and the matrix $\mathbf{Q}$ in equation (54) is given with elements

$$
Q_{i j}=\left(\frac{\partial^{2} A}{\partial n_{i} \partial n_{j}}\right)_{T, V}
$$

Both the second and the third derivative of the Helmholtz free energy $A$ in equations (56) and (55) can be written in terms of fugacity $f$ using a thermodynamic relation

$$
\left(\frac{\partial A}{\partial n_{i}}\right)_{T, V}=R T \ln f_{i}
$$

These derivatives are given as

$$
\begin{gathered}
\left(\frac{\partial^{2} A}{\partial n_{i} \partial n_{j}}\right)_{T, V}=R T n_{T}\left(\frac{\partial \ln f_{i}}{\partial n_{j}}\right)_{T, V} \\
\left(\frac{\partial^{3} A}{\partial n_{i} \partial n_{j} \partial n_{k}}\right)_{T, V}=\operatorname{RTn}_{T}^{2}\left(\frac{\partial^{2} \ln f_{i}}{\partial n_{j} \partial n_{k}}\right)_{T, V}
\end{gathered}
$$

where $n_{T}$ is total number of moles in system and $f_{i}$ is fugacity of component $i$ which can be calculated using an equation of state by

$$
\ln f_{i}=\frac{1}{R T} \int_{v}^{\infty}\left[\left(\frac{\partial P}{\partial n_{i}}\right)_{T, V}-\frac{R T}{V}\right] \mathrm{d} V+\ln \frac{R T n_{i}}{V}
$$

By solving equations (54) and (55), two unknowns, critical temperature $T_{c}$ and critical volume $v_{c}$ can be obtained. The critical pressure $P_{c}$ then can be calculated using the equation of state.

The strategy of the HKM algorithm is to interate on $T_{c}$ and $v_{c}$ in a nested manner instead of performing iterations on both variables simultaneously as in a typical solution of multiple nonlinear equations. Based on an initial guess of $v_{c}$, value of $T_{c}$ can be obtained by iterating equation (54). Closure of equation (55) then is checked, a new estimate for $v_{c}$ is generated, and the iteration on $T_{c}$ is carried out again. The advantage of this strategy is that it leads to convergence on the desired root much more often than by use of simultaneous iteration. Iteration on $T_{c}$ and $v_{c}$ by the secant method was 
found to be satisfactory for most calculations performed in this study. A more detailed description of this algorithm is given in Appendix D.

By reviewing the simplicity and the results of the accuracy test presented in the previous sections, we decided to test the ALS equation for these critical point calculation. Since the ESD equation is the only cubic equation which is based on molecular theory, we decided to include this equation in this test as well. Also, because of the wide industrial use of the RKS and PR equations, they were tested also as well. Using the four cubic equations of state (RKS, PR, ALS and ESD equations), we calculated critical temperatures and critical pressures for a number of muliple component mixtures at different compositions. These systems include five alkane-alkane binary mixtures, five $\mathrm{CO}_{2}$-alkane binary mixtures, two ternary alkane mixtures and one mixture containing six components of alkanes.

Figures 35 and 36 compare the calculated critical points with experimental data at different compositions for butane-octane and hexane-tetradecane mixtures, respectively. A comparison between calculated critical points and experimental data for five binary mixtures containing carbon dioxide is given in Figure 37. One can see that the results obtained using the four equations of state are comparable, and the ALS equation always gives the best predictions. The results of critical point calculations for mulicomponent systems are listed in Table 6 . The average percentage errors obtained from our calculations for all the systems tested are given in Table 7.

One should notice that calculations are presented only for the ESD equation for the system decane-carbon dioxide in Figure 37. While these results are not good, the other equations (RKS, PR and ALS) fail to predict the critical properties even qualitatively. This is because the repusive terms used in these equations of state are based on the van der Waals theory which assumes that the molecules are spherical. Table 7 shows that the ESD equation of state gives better results for mixtures containing molecules which differ greatly in size. Again, among the three van der Waals type of equations of state 
tested here, the ALS equation gives better results than those obtained from the other two.

\section{Selection of Equations of State for The Statistical Studies}

In the previous sections, accuracy and reliability tests have been carried out for pure $\mathrm{CO}_{2}$, pure alkanes and their mixtures using eleven equations of state of which eight are cubic in form. Based on the results of these calculations, two equations were selected for the statistically designed studies.

The average percentage errors obtained from molar volume calculation for pure carbon dioxide was presented in Table 1 both globally and in various regions of the phase diagram. Table 8 gives the accuracy rankings for the eleven equations used in the calculations. For example, in the volume calculation for the entire phase region of $\mathrm{CO}_{2}$, the smallest error is obtained using the ALS equation and the largest error is given by the RK equation. Thus, the error sequence numbers for the ALS equation and the RK equation are given as (1) and (11), respectively.

Accuracy rankings also were made for molar volume calculations for pure alkanes, VLE calculations for mixtures and mixture critical point calculations. The results are shown in Table 9. In Table $9,\left\langle E>_{(C n \leq 4)}\right.$ and $\left\langle E>_{(C n \geq 4)}\right.$ are the average overall errors in molar volume calculations over all pure alkanes of which carbon numbers equal or are less than 4 and of which carbon numbers are greater than 4 , respectively; $\langle E\rangle_{(m i x)}$ is the average overall error in prediction of phase behavior (bubble point calculation and flash calculations) for both $\mathrm{CO}_{2}-\mathrm{C}_{4} \mathrm{H}_{10}$ and $\mathrm{CO}_{2}-\mathrm{C}_{6} \mathrm{H}_{14}$ binary mixtures; and $\langle E\rangle_{c(\text { mix })}$ is the average overall error in mixture critical point calculations for all tested multicomponent systems. By analyzing all of this data, overall rankings of the relative accuracies of the eleven equations of state were obtained. These are given in 'Table 10.

Considering equation's simplicity, accuracy, reliability, and availability of parameters, we feel that the ALS equation is the most appropriate for the statistical studies. 
Ilowever, because the ESD equation is the only cubic equation of state tested which worked well for very large molecules, we feel it should be more reliable for enhanced oil recovery calculations. We have included this equation of state in the statistical studies as well.

\section{Factorial and Fractional Factorial Test Plans}

Factorial and fractional factorial test plans are members of a class of test plans called statistical experimental designs. These test series are designed to provide accurate approximations of the response function which characterizes some feature of the system under study over the chosen range of the tested variables (factors). They share a common trait - each type of design permits evaluation of the relative importance of a series of factors using relatively few tests, thereby gaining more information for less cost than traditional methods. Further, they are orthogonal designs. That is, the individual factors are tested in a pattern which assures their independence, and yields an unambiguous assignment of factors to changes in system responses (effects). For full factorial designs in which $k$ factors are evaluated at 2 levels in $2^{k}$ tests, all of the linear and interaction effects, from the product of each possible pair through the product of the $k$-tuple, can be independently assessed. In the case of fractional factorial designs, the interactions which can be examined independently depend on the resolution of the design. For $k$ factors at two levels, a properly constructed design requiring $2^{k-1}$ tests (a half-fractional factorial, resolution $\mathrm{V}$ design) can yield the effects of the linear factors and their two factor interactions without confounding any of them. For higher fractionals, information can be obtained on fewer and fewer interactions until in a saturated resolution III design using $2^{k-p}$ tests, only the effects associated with $2^{k}-1$ linear factors can be determined independently of each other.

In this section of the final report we review the construction, analysis, advantages, and problems associated with the use of factorial and fractional factorial designs to perform sensitivity analyses on equations of state. We discuss the strategy we have used 
in this study for testing the effects of the parameters, variables, and their interactions on the accuracy of EOS representations of experimental data. Specifically, we discuss the choice of computational designs, the choice of responses, and catalogue the sucesses and difficulties encountered in the calculations which we have completed. The results of the calculations, their significance in understanding the nature of the equations of state, and their importance for larger scale sensitivity studies are discussed in later sections.

\section{Factorial Designs and Sensitivity Analysis}

The objective of this study was to determine the important parameters and variables in a $\mathrm{CO}_{2}$ EOS relating to Enhanced Oil Recovery (EOR), and to deduce to what degree their accuracy and/or precision affect the calculated results and agreement with experimental results. These goals are accomplished through a sensitivity analysis of the EOS using a statistically designed :omputer study.

Sensitivity analyses have been performed on simulation models using factorial computational plans for nearly 20 years (Close, 1967). Box et al., (1978) describes the use of a 16 test, 2-level fractional factorial to sort out and rank the linear effects of 8 variables and the 7 sets of confounded two factor interactions (2FI) between those variables on the results of an air traffic control simulation. In that study, the choice of the test program was dictated by the expense of running the simulator and by the desire to examine the linear effects and (at most) 2FI's. Since the simulator included sampling from waiting time distributions, it was possible to obtain slightly different answers from each execution of the program for a given set of conditions. This led to an estimate of "experimental error" which was then used to rank and assess the statistical significance of the various effects. A statistical test design was chosen for that analysis because the model could not be solved analytically and because the simulation results were inherently noisy. 
In assessing the relative importance of parameters and variables in a $\mathrm{CO}_{2} \mathrm{EOS}$ relating to enhanced oil recovery, we have chosen two complex equations of state containing parameters which are functions of the absolute temperature and composition. While it may have been possible to perform a partial senitivity analysis by examining the matrix of derivatives of the EOS with respect to the parameters and the variables, such an array is at best large and unwiel-ty.

In this situation, approximation of the EOS residuals surface for selected properties with empirical functions efficiently derived from fractional factorial computation plans is an intelligent and effective way to achieve the study objectives. Since there is no variation in the results of any computation repeated at the same values of the parameters and variables, each effect will be significant. The size of each effect will then be a direct measure of the sensitivity of the residual surface to changes in the value of the tested parameter or variable.

\section{Statistically Designed Computation Plans}

This portion of the report discusses the computation strategy for determining the sensitivity of the selected mixture properties calculated by the equations of state of interest to the various parameters within those equations. The discussion is divided into three sections:

(1) Identification of independent parameters and appropriate test ranges within the EOS

(2) Identification of physical properties (responses) to be studied and

(3) Testing strategy for these equations and properties.

This discussion outlines the statistical studies which have been undertaken in this project. It will relate the general ideas presented in the technical proposal to the actual work which was completed in the statistical studies under this contract. 


\section{(A) Independent parameters}

Two equations of state were selected for sensitivity analysis of their parameters - the four parameter Adachi-Lu-Sugie EOS and the four parameter Elliott-Suresh-Donohue COS. As is usually the case with sophisticated equations of state, the major parameters in the EOS lie at the top of a hierarchy of equations. Examination of the form of the equations in this heirarchy (Appendix C) shows the primary EOS parameters to be functions of the accentric factor, absolute temperature, and critical temperature and pressure. These functions, either polynomials or quotients of polynomials, make it impossible to vary the major parameters in the EOS independently. The mathematically independent variables required for the sensitivity analysis are to be found in the lowest levels of the parameter equation heirarchy. They include the pure-component properties and the best-fit values of the coefficients used to relate the pure-component properties to the major EOS parameters. They are listed for the ALS EOS in Table 11, and for the ESD EOS in Table 12.

As can be scen in Tables 11 and 12, there are 18 low level fitting parameters plus $3 n$ physical properties for each n component mixture to be studied by the ALS EOS, and 17 low level fitting parameters plus $3 n$ physical properties for each $n$ component mixture plus $m$ binary interaction parameters for the $m$ binary pairs in the mixture modelled by the ESD EOS.

\section{(B) Independent variable ranges}

The studies conducted under this contract have used two levels of the the independent variables. 'The low level is the accepted value of the independent variable. The high level is the accepted value increased by either $0.5 \%$ or $0.25 \%$ of the accepted value. This narrow range helped to preserve the inherent characteristics of the EOS under study, and minimized mathematical stability problems, especially in the critical locus calculations. 


\section{(C) Physical Properties and Responses}

As discussed in the Thermodynamics Section, two types of mixture properties have been studied as responses which are appropriate to Enhanced Oil Recovery modelling.

(1) Mixture liquid density and

(2) Locus of the critical line as a function of pressure, temperature, and composition.

Two responses were calculated from these properties - average absolute error and bias. These responses were calculated from the difference between calculated and either observed or base-case properties. Base-case properties are those calculated from the EOS using the accepted values of the independent parameters.

For mixture liquid density values, this difference is the scalar distance between the calculated and observed or base-case value of the density. For the critical line locus, this distance is the vector sum of the difference between the observed or base-case and calculated critical temperalure and pressure at the given mixture composition.

The average absolute error is the average of the absolute values of the differences between the observed or base-case value of the property and the calculated value of the property.

The bias is the sum of the signed value of the differences between the observed or base-case values of the property and the calculated value of the property.

In addition to the responses generated from the physical properties, the elapsed CPU time for the evaluation of the property was captured and evaluated as a response.

\section{(D) Test Strategy}

The objective of the test series was to rank the parameters in the EOS chosen for study in the order of decreasing influence on the mixture property and computation time. The use of statistically designed computation plans assures that this goal will be met with the least number of computations.

As noted above, the two equations chosen for study each have more than 20 independent parameters which could have an influence on the predicted mixture properties and computation time. Since the equations have different mathematical.forms, each one 
has been analyzed by its own set of computation plans. The discussion which follows applies to both of the equations in this study. Each of the test plans was applied to each of the equations in turn.

The analysis of the EOS sensitivity has been carried out on a series of hydrocarbon $/ \mathrm{CO}_{2}$ mixtures. The mixture liquid density work was completed for the even numbered $\mathrm{n}$-alkane $/ \mathrm{CO}_{2}$ mixtures with carbon numbers between 4 and 10. The critical locus effort was conducted on $\mathrm{n}-\mathrm{C}_{4}$ and $\mathrm{n}-\mathrm{C}_{0} / \mathrm{CO}_{2}$ mixtures.

The test strategy consisted of computation plans designed to unambiguously rank the EOS parameters and aliased $2 \mathrm{FI}$ in order of decreasing influence on the three responses. The first plan is a resolution IV fractional factorial containing 64 tests. The test patterns for the ALS EOS tests in Table 13, and those for the ESD EOS studies are given in Table 14. The aliased 2 FI resulting from these tests appear in Tables 35 and 36 .

\section{Results}

The results obtained for each of the test programs are presented in Tables 15 through 22 for the mixture liquid density calculations and in Tables 23 through 26 for the critical locus computations. These tables contain the effect on the response expressed as the ratio of the percent change in the response to the percent change in the independent variable. For example, assume that the effect of a $0.5 \%$ change in accentric factor for $\mathrm{CO}_{2}$ changed the value of the calculated average absolute crror of the liquid volume from $4.0 \%$ to $6.0 \%$ in the $\mathrm{n}-\mathrm{C}_{4} / \mathrm{CO}_{2}$ mixture. Then the effect associated with that change would be $+2 \%$, and the effect listed in in the table for the $\mathrm{CO}_{2}$ accentric: factor would be $(2 / 4) / 0.005=100$. This value can be interpreted to mean that each percent change in the accentric factor for $\mathrm{CO}_{2}$ changes the average absolute error by $100 \%$. Thus, the effects listed in Tables 15 through 26 are direct measures of the sensitivity of the responses to changes in the individual factors or $2 \mathrm{FI}$. 
For reference, the values of the effects in their raw form are presented in Appendix $\mathrm{E}$ for each of the test mixtures.

\section{(A) Evaluations of the responses - Convergence and Divergence}

Both EOS calculated liquid densities without problems for the range of data studied. That is, the density calculations converged on a physically reasonable value for the mixture liquid density. This was true not only for the base-case and observed data comparisons, but also for each of the 512 test cases calculated. Thus the sensitivies listed in Tables 15 through 22 are based on deviations from errors in which every value of each data set is estimated from a converged calculation. Such is not the case for the critical locus calculations.

In the case of the critical line calculations, the algorithm failed to converge in some fashion on a physically reasonable value for at least some of the compositions in the data sets at some of the test conditions. This tendency was managed in the calculations by trapping the error condition, and assigning a large "signal" value to the response in question. Thus the sensitivities in Tables 23 through 26 reflect not only changes due to converged values of the responses but also instabilities in the calculation of those responses. In cases where many of the test compositions failed to yield converged answers, the sensitivity is a measure of the instability, rather than of the error in the predicted value.

\section{(B) Density Results - ALS Equation}

Comparison of the results listed in the Appendix $\mathrm{E}$ for the $4 \mathrm{n}$-alkane $/ \mathrm{CO}_{2}$ mixtures leads to several interesting observations. The average absolute error and bias track each other for the $n-C_{4}$ mixture, and oscillate regularly among three different values - approximately 6, approximately 13 and approximately 20 . The bias is always positive. Neither the tracking nor the single sign belavior is present in the $n-C_{0}$ nor $\mathrm{n}-\mathrm{C}_{8}$ mixture test results, although the biases are all negative in the $\mathrm{n}-\mathrm{C}_{10} / \mathrm{CO}_{2}$ case. These results have produced some curious trends in the calculated sensitivities. 
The absolute values of the sensitivities for Density are plotted for each response in Figures 39a,b,c. Each figure contains the sensitivities for the given response for each of the mixtures tested for a given EOS.

For the ALS equation, the marked plateau apparent for the average absolute error and the bias sensitivities in the $\mathrm{nC}_{4}$ mixture disappears as the carbon number increases. There is, however, a steeper decline between the largest two values in the $n-C_{4}$ and n$\mathrm{C}_{10}$ mixtures than in the intermediate two sets. While the disappearance of the plateau also occurs for the Bias sensitivities, the relative change between the highest two values remains more or less constant.

The CDU time responses vary only slightly within mixtures. In each case, there is a gradual decline in the absolute value of the sensitivies, although there is a marked drop between highest and second highest in the $\mathrm{n}^{-\mathrm{C}_{4}}$ set. This may be due to slower convergence in those tests with the largest errors.

The range of the maximum sensitivities, listed in Table 27 is an order of magnitude for average absolute error and a factor of 6 for the bias sensitivities. CPU time sensitivities are much smaller (by a factor of 50) and range themselves from 7.8 to 2.8 .

For the average absolute error sensitivities, it is interesting to note that none of the factors and aliased $2 \mathrm{FI}$ having the largest 5 sensitivities (in absolute valıe) in the $n-\mathrm{C}_{4}$ set are present in the top 5 in the $\mathrm{n}-\mathrm{C}_{10}$ set. This is illustrated in Tabl 28 along with the placement of the top 5 from each end mixture in the sorted factor lists of the $n-\mathrm{C}_{0}$ and $n-\mathrm{C}_{8} / \mathrm{CO}_{2}$ mixtures. It is noteworthy, however, that the top 3 factors or aliased $2 \mathrm{Fl}$ for the $\mathrm{n}-\mathrm{C}_{10}$ mix remain in the top 10 for $\mathrm{n}-\mathrm{C}_{0}$ and $\mathrm{n}-\mathrm{C}_{8}$, as do the top 2 from the $\mathrm{n}-\mathrm{C}_{4}$ sensitivities list.

For the bias sensitivities (Table 29) the results are even more striking - while the highest value in the $n-C_{4}$ group is the highest in the $n-C_{6}$ and $n-C_{8}$, it is number 42 in the $n-\mathrm{C}_{10}$ sensitivity results. The others wander about much more. Meanwhile, the most sensitive factor in the $n-\mathrm{C}_{10}$ density tests drops through the top 10 into 13 th by the $n-\mathrm{C}_{4}$ results. 
A list of the 5 most sensitive factors in each of the mixtures has 12 unique entries for the average absolute error response, and 15 for the bias response. These two lists share 4 factors and 3 aliased 2 FI. Of those 7 common factors, 5 are shared in the $n-C_{4}$ mixture, leaving 2 in common for the remaining mixtures.

By dividing the tested parameters into groups as in Tables 11 and 12 we find (Table 30) that the most sensitive factors are in the expressions for $a(T)$ and $b_{1}$, with one set of $n$-alkane critical parameters (for $n-C_{10}$ ) important for average absolute error and nearly all the critical properties important for sensitivity to bias, as well as those fitting parameters in the expressions for $b_{1}$ and $a(T)$. It is amazing that neither average absol ${ }^{4} \mathrm{e}$ error nor bias is sensitive to $k_{i j}$. Elapsed CPU time for $\mathrm{n}-\mathrm{C}_{4}$ is sensitive to $k_{i j}$ however.

\section{(C) Density Results - ESD Equation}

Inspection of the test calculations for the mixture liquid density using the ESD EOS reveals a high apparent positive correlation between the average absolute error and the bias in the $n-C_{4} / \mathrm{CO}_{2}$ mixture, a decreasing correlation through the $\mathrm{n}-\mathrm{C}_{6}$ and $\mathrm{n}-\mathrm{C}_{8}$ results, and no correlation in the $\mathrm{n}-\mathrm{C}_{10}$ mixture results. As in the case of the ALS equation, the variation in the elapsed CPU time is small.

Plots of the sensitivities (Figures 38a,b,c) in decreasing absolute value show a gradwal decline through all the values for average absolute error (90\% of the sensitivity range exhausted within the first 20 to 25 factors and aliased 2FI). A smooth but somewhat steeper decline is evident in the bias sensitivities.

The elapsed CPU time sensitivities start out small and decline very slowly. However, there is only a small range observed, as in the results for the ALS equation.

The maximum sensitivities for the liquid density tests on the ESD EOS range over approximately an order of magnitude for both the average absolute error and bias responses, but only over a factor of three for the elapsed CPU cime (Table 31). The largest maximum sensitivity of these test mixtures occurs in the $\mathrm{n}-\mathrm{C}_{8} / \mathrm{CO}_{2}$ mixture 
for all three responses. As in the response data itself, the maximum sensitivities for average absolute error do not track those for bias.

A comparison of the ranking of the largest 5 sensitivities (in absolute value) for average absolute error reveals a surprising consistency compared to the ALS equation results. The top 5 in the $n-C_{4}$ results stay in the top 10 (with two exceptions, in two individual cases) through the $n-C_{0}$ and $n-C_{8}$ results, and are replaced in the $n-C_{10}$ mixture results by three aliased 2FI containing the product of two of the original set (Table 32). This behavior is even more striking in the sensitivities for the Bias response (Table 33) for liquid densities from the ESD EOS. Here, the top 5 for the $n-\mathrm{C}_{4}$ mixture stay in the top 10 throughout the series, while the 5 most sensitive factors in the $n-C_{10}$ mixture are in the top 10 list for all but two individual cases.

Of all the sensitive factors, one factor (c16) is the most sensitive for average absolute error in 2 of the 4 mixtures and 3 of the 4 in the case of bias.

A look at the list of the top 5 across all the mixtures reveals 11 unique factors for the average absolute error (out of 20 possible) and only 9 in the list for bias sensitivities. While the bias and absolute average error do no track each other, nonetheless, these consolidated lists of the most sensitive share 8 common members! All of the aliased $2 \mathrm{FI}$ in the lists appear in the absolute average error group, and as mentioned above, contain products of the single factors which are shared by both responses and many of the mixtures.

Examination of the distribution of the five most sensitive factors among the high level factors for the ESD EOS as shown in Tables 11 and 12 reveals each high-level factor except $c_{i}$ to contain at least one sensitive lower level factor (Table 34). While average absolute error and bias are sensitive to changes in the critical properties of $\mathrm{CO}_{2}$ for the $\mathrm{n}-\mathrm{C}_{6}$ and $\mathrm{n}-\mathrm{C}_{8}$ mixtures, they are senitive to changes in the critical properties of the $\mathrm{n}$-alkanes in the case of $\mathrm{n}-\mathrm{C}_{4}$ and $\mathrm{n}-\mathrm{C}_{10}$ mixtures. While the $\epsilon_{i i}$ term is important in the bias sensitivity results, it is only in the top 5 sensitivities for the $\mathrm{n}-\mathrm{C}_{4}$ average absolute error set. 


\section{(D) Critical Line Locus Results - ALS Equation}

The results of the test calculations on the ALS EOS are dominated in the case of the $\mathrm{n}-\mathrm{C}_{4}$ mixture by the outcome of two tests (numbers 17 and 51 ) in which all three responses were far larger in magnitude than the others. Some investigative work revealed the source not to be a complete failure of the algorithm for locating the critical line, but rather convergence on another local extremum in the vicinity of the global extremum. This behavior could not be changed by decreasing the interval of the parameter perturbations - even a perturbation as small as $.0001 \%$ produced the same results. Such results have produced sensitivities at essentially 2 levels, rendering unproductive comparison of the five most sensitive results. As occured in several of the density studies, the bias results track the average absolute error results in absolute value. All of the biases calculated from the test factor settings are negative.

The $\mathrm{n}-\mathrm{C}_{6}$ mixture results, on the other hand, are dominated by the signal values generated by recovery of the tests from mathematical instabilities. In contrast to the results for the $\mathrm{n}-\mathrm{C}_{4} / \mathrm{CO}_{2}$ mixtures, these sensitivities decreased more gradually (Figures $41 \mathrm{a}, \mathrm{b}, \mathrm{c}$ ), with $90 \%$ of the range being reached between the 38 th and 39 th entry in the sorted list, although the top $2 / 3$ of the range lies between the 1 st and 15 th value. The decline in sensitivity value is about as gradual in the bias response results; while $90 \%$ of the sensitivity range lies in the top 40 , the $66 \%$ point is reached at factor 15 . Virtually the same behavior is evident in the CPU time response.

Comparison of the consolidated lists of the highest five factors for each of the two mixtures studied reveal nine unique entries out of ten possible, but 7 entries in common between average absolute error and bias.

\section{(E) Critical Line Locus Results - ESD Equation}

Six of the 64 tests calculated for the $\mathrm{n}-\mathrm{C}_{4} / \mathrm{CO}_{2}$ mixture using the ESD EOS resulted in mathematical instabilities, and invocation of the signal value for the average absolute error and bias responses. As was the case in the ALS calculations, this resulted in several discrete plateaus in the sensitivity results for the three responses 
(Figures $40 \mathrm{a}, \mathrm{b}, \mathrm{c}$ ). The significant breaks in the average absolute error and bias senitivity distribution curves occur between the $2 \mathrm{~d}$ and $3 \mathrm{~d}$ factors, the 18 th and 19 th factors, and the 45 th and 46 th factors. The decline in sensitivity is more regular in the CPU time sensitivities, although the first $50 \%$ of the sensitivity range is traversed in the top 5 values. Forty-seven of the 64 sets calculated for the $n-C_{6}$ data encountered mathematical instabilities in the calculation of the responses. This again led to some significant plateaus in the sensitivity results, this time occuring between the $2 \mathrm{~d}$ and $3 \mathrm{~d}, 15 \mathrm{th}$ and $16 \mathrm{th}$, and $32 \mathrm{~d}$ and $33 \mathrm{~d}$ values of the sorted list of factors (figures $40 \mathrm{a}, \mathrm{b}, \mathrm{c}$ ). As in the $\mathrm{n}^{-} \mathrm{C}_{4}$ mixture results, the bias sensitivities tracked the average absolute error sensitivities, but the CPU time sensitivities decreased more regularly. Not unexpectedly, the consolidated lists of the top five sensitivities for the error responses for the two mixtures have ten unique entries, and share 6 in common.

The remainder of the discussion is divided into two sections. The first deals with the nature of the sensitivities of the two equations of state which were studied in this project. The second addresses the relevance of this effort to a broader class of problems - namely how to study the sensitivity of the various parameters in more complicated models and important systems such as those described by EOR simulation models. It is in the study of EOR models, we believe, that statistical test plans can be put to the best use.

\section{Nature of the Sensitivities}

The equations of state studied in this work represent two types of EOS - the ALS is an empirical equation, while the ESD is a theoretically based equation. Both equations cuntain a large number of "fitting" parameters, presented as constants in a hierarchy of lower level equations. Both require critical constants and accentric factors as part of the required input for mixtures, and both contain a binary interaction parameter for each hinary pair in the mixture. This study has revealed an interesting number of likenesses and differences between these two equations of state. 


\section{(A) Stability}

(1) Both equations produce very stable liquid density test series. Observed sensitivities are typically less than 500, and all of the calculated cases converge to some physically reasonable value.

(2) In contrast, both EOS are extremely sensitive to changes in the mixture, or in the parameters embedded in the EOS. The ALS equation fails to produce converged estimates of Temperatures and Pressures for some compositions in the base case calculations of the $\mathrm{n}-\mathrm{C}_{6} / \mathrm{CO}_{2}$ mixtures. This behavior is also observed in base-case calculations of $\mathrm{n}-\mathrm{C}_{7}, \mathrm{n}-\mathrm{C}_{8}$ and $\mathrm{n}-\mathrm{C}_{10} / \mathrm{CO}_{2}$ mixtures. The ESD equation performs somewhat more usefully in these mixtures - failing at $n-C_{8}$ and $n-C_{10}$, but not before. While there were no mathematical instabilities observed in the test cases run on the $\mathrm{n}_{4} \mathrm{C}_{4} / \mathrm{CO}_{2}$ mixtures using the ALS EOS, there were two instances in which the calcuated temperature and pressure converged on some local extremum rather than the more physically realistic one. Faced with similar perturbations in the parameters, the ESD EOS chooses to diverge at times for this mixture. All in all, these are not the versatile EOS which the experts claim them to be for EOR representation - at least not when they are used with the HKM (appendix D) algorithm for critical locus calculations.

\section{(B) Sensitive Parameters}

(1) One of the more astonishing results of these tests is the lack of sensitivity of either of the error responses (average absolute error or bias) to changes in the binary interaction parameter. This is true in both the mixture liquid density calculations and the critical locus results. Whether this is true in general for mixture calculations involving these EOS, or whether it is only true for the mixture liquid density and the critical locus can not be determined from these results.

(2) Another interesting, though not entirely unexpected result, is the variety of sensitive factors in the ALS equation, and the relatively small set of sensitive parameters in the ESD equation. In view of the desire to determine which parameters have to be 
determined to the highest degree of precision, the ESD equation is the much more economical. Since the results of its calculations are most sensitive to the smaller number of parameters from mixture to mixture, less effort will need to be spent determining the values of those parameters to the required precision.

(3) There is evidence from some of the exploratory work done in connection with this project, that the sensitivities do not vary linearly with the perturbations in the parameters. That is, the observed effect associated with a $0.5 \%$ change in the parameter size is not necessarily twice the effect of a $0.25 \%$ change. This implies curvature in the surfaces which were approximated by linear surfaces in this study, and could be explored with the help of experimental designs structured to define this behavior further.

\section{Application to EOR Simulators}

An cxample of a class of large, complex, expensive-to-evaluate but extremely important models is the EOR simulator. Typically containing hundreds (or even thousands) of parameters, these models contain hydrological, geological, thermodynamic, and cost modules. They can run for an hour or more on super-computers. They are used to predict the potential production of oil from fields which have been worked out by primary methods. They can be used to evaluate the relative effectiveness and economics of several different EOR technologies for a given site.

The accuracy of their output is important not only to larger firms which may decide to recover oil from marginal fields with EOR techniques, but to small "wildcatters" whose livelihood can depend on an extremely small profit margin. Gaining the confidence of this latter group in these models requires that they be as accurate as possible. Making the simulators as accurate as possible is an expensive proposition, but one which will be made more efficient by knowing which of the subsystems in the model have the greatest effect on its predictions. In addition, the desire to use these models to encourage small operators to work marginal fields implies a need to compress the 
models into a form that can run in a reasonable amount of time on affordable computing platforms. What better way than to identify the most important sections of the models and to reduce the computational load required by standardizing the less significant sections? Such effort could be exceptionally well-guided by the results of statistically designed computational plans for the models.

Naturally, such an effort would not proceed in precisely the same fashion as the one undertaken in this study. For a large, expensive model, especially one which contains stochastic as well as deterministic terms, we would recommend an outside-in approach. That is, the effects of those high order sections of the model which can be evaluated independently would be tested to determine the sensitivity of the model results to those inputs. In this phase, care should be taken to elucidate the effects of 2 FI made from members of this set, since this is the highest level of the analysis. Once the importance of these factors and their interactions had been evaluated, work could begin in earnest on the lower level, independently variable parameters.

As examples of the type of factors which could be manipulated in the outer level, we refer to the compositional EOR model used by Warner to describe the history and project the production of the Arco Block 31 Middle Devonian reservoir (Warner, 1979). Outer level factors which could be varied in a medium resolution fractional factorial include:

(1) EOS with parameters adjusted to experimental data for the stripping gas and crude actually to be used compared with an EOS containing parameters fit to some similar system.

(2) High resolution calculation grid in time and space compared with a lower recolution calculation grid.

(3) Multi-dimensional (in space) flow calculations compared with 2 dimensional flow calculations adjusted for three-dimensional flow effects through manipulation of the gas/oil permeability. 
(4) Highly detailed geological description of the field matching the grid in spatial resolution compared with less detailed geological description of the field.

(5) Complete modelling of the production well separation equipment performance compared with approximate modelling of the well separation train.

(6) Local variation in the porosity and permeability compared with spatially averaged porosity and permeability.

Note that these particular factors all have an impact on either the quantity of lab and field data that have to be accumulated to run the simulator, or on the computational time and platform required.

In addition the to these 6 factors, two different sites could be included as high and low levels in a seventh factor to test the effect of site characteristics and crude make-up. These 7 factors and their 2FI could be economically studied in a level IV resolution design in 16 model evaluations. The results of this analysis would yield directly the sensitivity of the well production economics to the tested techniques, which appear as main effects in the design. In addition, the interaction of the tested factors would be aliased in 7 groups which could be untangled with a reasonable number of further tests, were it deemed necessary to untangle them.

Once the important factors (and interactions) are found, their are at least two possible courses open. The first is to delve deeper into the techniques which were tested (which parameters in the EOS are important, which grouping of the crude components is critical, which of the geological features must be included, etc.) The second course is to optimize the model with respect to a particular objective using repetitions of simple designs or higher order designs based on the first set of studies to get the most accuracy for the least computing cost. The repetition of simple, limited factor range designs to climb to the peak of the objective function is analogous to the EVOP technique used to optimize industrial processes. While not particularly cost effective, it would permit cvaluation of the model close to physically reasonable factor values which have already been studied. 
The second approach, which is very cost-effective, permits approximation of the objective function with a quadratic, and can lead to the desired "high-ground" with a minimum of effort. Such an approach has the advantage of being built on the earlier effort. It would be appropriate for factors listed as (1) through (6) above but not for discrete factors such as site type.

\section{Conclusions}

This study has shown the versatility and value of using statistically designed computational plans for determining the sensitivity of two physical properties important in the simulation of EOR operations to changes in the parameters of the EOS used to calculate them. This effort has demonstrated clearly that

(1) The construction and analysis of the computation plans can be extensively automated.

(2) Such plans are very versatile, can be used to quantify the relative importance of both empirical and theoretically based model parameters (see discussion above).

(3) This approach is ideally suited for determining the sensitivity of the calculated results to the model parameters for models that are expensive to evaluate, must be solved numerically, and which contain a large number of component parts.

\section{Recommendations}

(1) We recommend application of these statistical techniques to the analysis of EOR simulators. We believe they provide a cost-effective method for simultaneously paring down and maintaining the accuracy of the simulators. They are, in our opinion, exceptionally well-suited for such work, providing quantitative, unambiguous and statistically reliable results, which would be difficult or impossible to obtain with equal confidence using traditional methods. 
(2) We recommend establishment of a program to study the applications of these techniques to EOR simulators which are currently in use, to determine the minimum size model required to predict the economics and recoveries for an existing field.

(3) We recommend that these studies begin with an "outside-in" approach, studying the sensitivity of the model predictions to the results calculated by the largest subdivisions of the model. When the most important modules are identified, they could be studied in more detail, while the less important modules could be simplified for eventual implementation on less expensive computing platforms.

We believe that simpler and yet reliable EOR simulators would result from statistically designed sensitivity studies of these models. The availability and reliability of such models, in our opinion, certainly would encourage small and large operators to idratify and work those marginal fields which can be exploited profitably. 


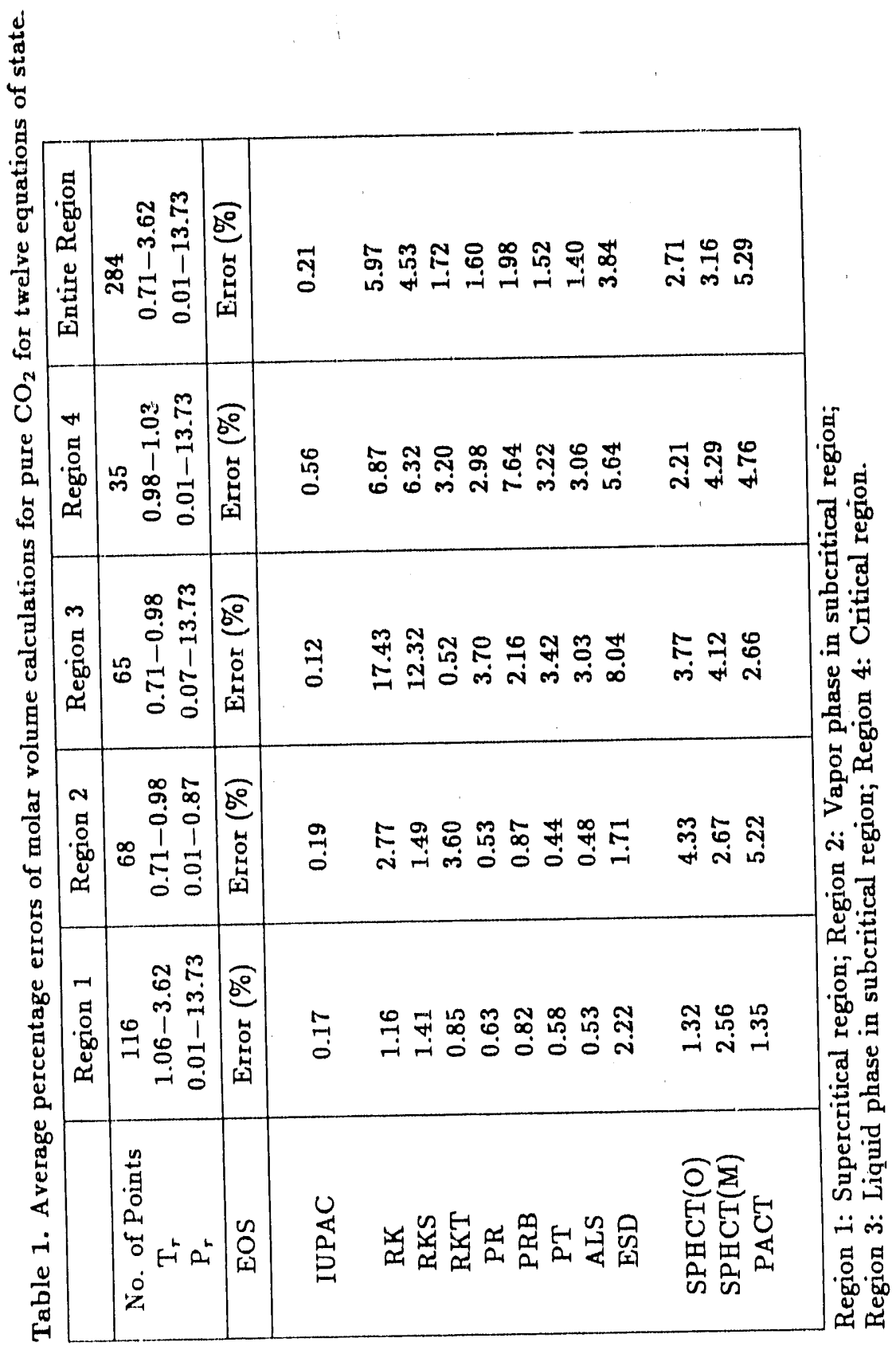




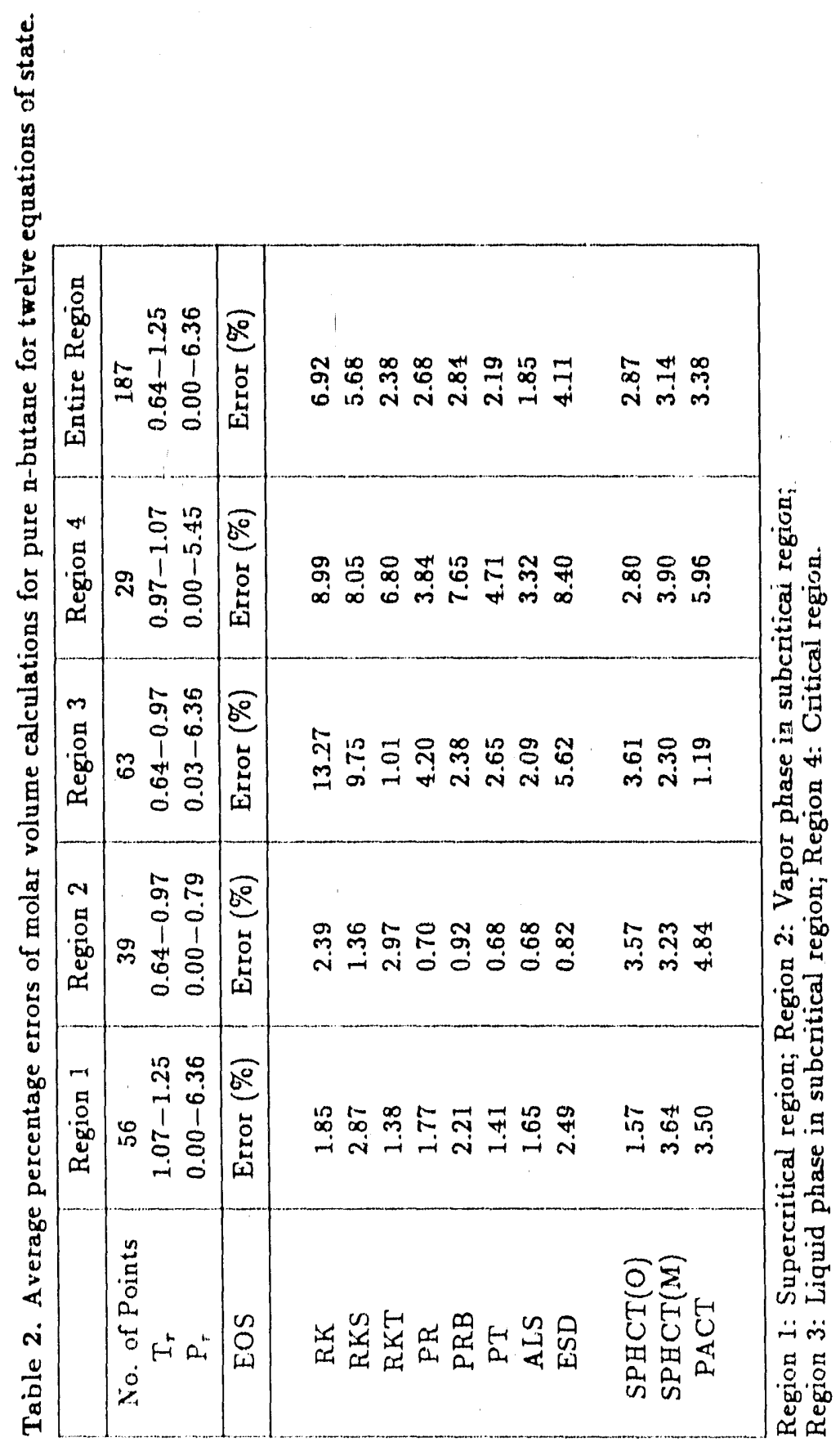




\begin{tabular}{|c|c|c|c|c|c|}
\hline & ن. & 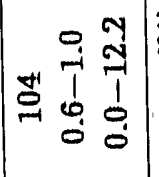 & 可 & 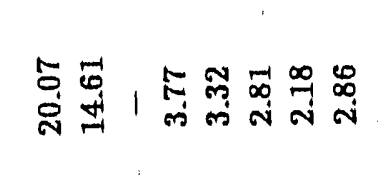 & 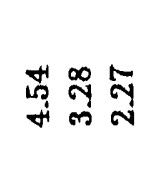 \\
\hline & ن & 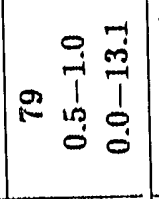 & 可 & 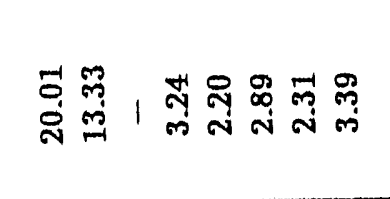 & 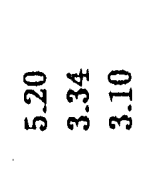 \\
\hline & 章 & 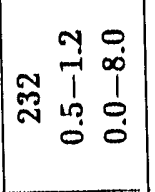 & 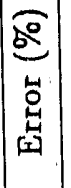 & 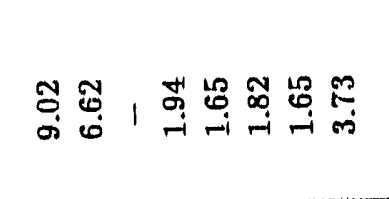 & 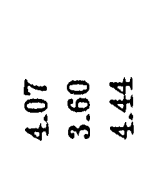 \\
\hline & 告 & 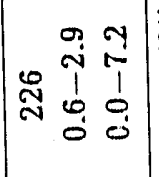 & 吾 & 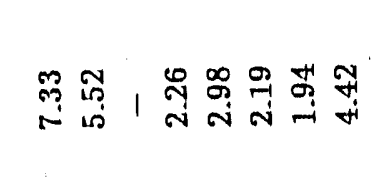 & 婴落 \\
\hline & 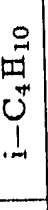 & 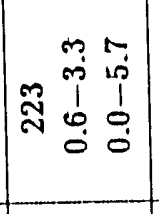 & 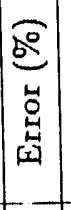 & 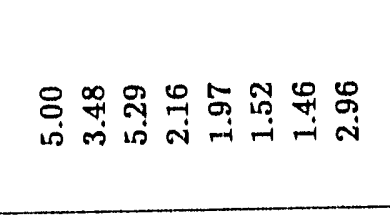 & $\begin{array}{l}\text { Ni } \\
\text { in } \\
\text { in } \\
\text { Ni }\end{array}$ \\
\hline & 辛 & 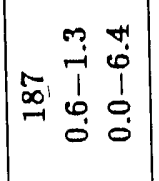 & 可 & 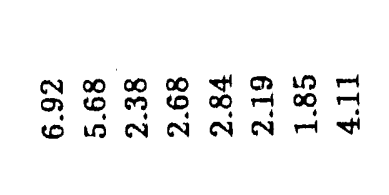 & 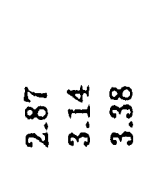 \\
\hline & $\begin{array}{c}\infty \\
0 \\
0 \\
0\end{array}$ & 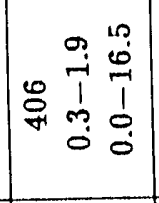 & 呑 & 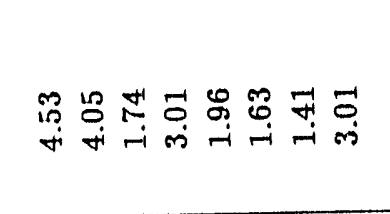 & 旡 突 \\
\hline & 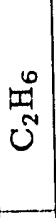 & 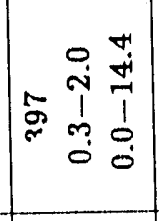 & 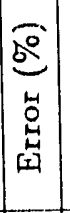 & 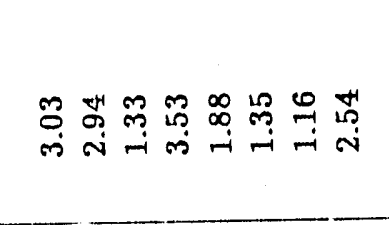 & 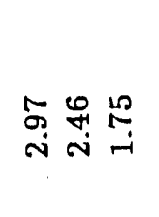 \\
\hline & 㻤 & 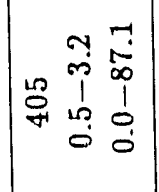 & 总 & 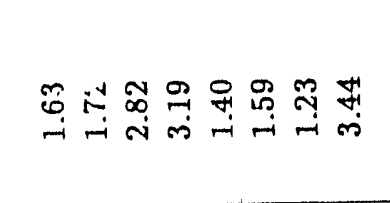 & 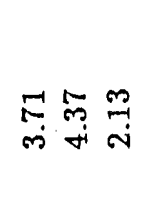 \\
\hline & & 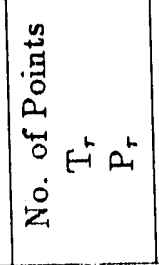 & & 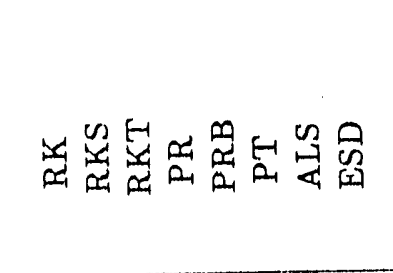 & 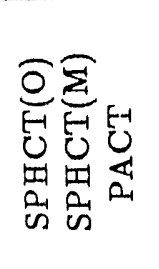 \\
\hline
\end{tabular}


Table 4. Average percentage errors of bubble point calculations and flash calculations for $\mathrm{CO}_{2}(1)-\mathrm{C}_{4} \mathrm{H}_{10}(2)\left(\mathrm{T}: 273-410^{\circ} \mathrm{K}, \mathrm{P}: 0.7-75.5 \mathrm{bar}\right.$ ) for ten equations of state.

\begin{tabular}{|c|c|c|c|c|c|}
\hline EOS & $\% \mathrm{Er}^{b}$ in $\mathrm{P}$ & $\% \mathrm{Er}^{b}$ in $\mathrm{y}_{1}$ & $\% \mathrm{Er}^{f}$ in $\mathrm{x}_{1}$ & $\% \mathrm{Er}^{f}$ in $\mathrm{y}_{1}$ & $k_{1, ?}$ \\
\hline & 16.58 & 19.71 & 46.32 & 38.20 & 0.08198 \\
RK & 2.53 & 8.82 & 12.42 & 10.06 & 0.12100 \\
$\mathrm{RKS}$ & 2.62 & 7.39 & 8.17 & 3.19 & 0.11724 \\
$\mathrm{PR}$ & 2.50 & 6.94 & 7.89 & 3.14 & 0.11968 \\
$\mathrm{PRB}$ & 2.23 & 4.83 & 7.67 & 4.19 & 0.13500 \\
$\mathrm{PT}$ & 2.48 & 4.56 & 7.17 & 3.60 & 0.13700 \\
$\mathrm{ALS}$ & 3.72 & 5.24 & 8.52 & 4.93 & 0.17200 \\
$\mathrm{ESD}$ & & & & & \\
$\mathrm{SPHCT}(\mathrm{O})$ & 584.15 & 9.96 & 62.02 & 26.73 & 0.07000 \\
$\mathrm{SPHCT}(\mathrm{M})$ & 4.00 & 21.91 & 8.26 & 20.26 & 0.14500 \\
$\mathrm{PACT}$ & 3.16 & 11.01 & 6.63 & 11.14 & 0.05728 \\
& & & & & \\
\hline
\end{tabular}

$\% \mathrm{Er}^{b}$ : errors from bubble point calculation, $\% \mathrm{Er}^{f}$ : errors from flash calculation

Table 5. Average percentage errors of bubble point calculations and flash calculations for $\mathrm{CO}_{2}(1)-\mathrm{C}_{0} \mathrm{H}_{14}(2)\left(\mathrm{T}: 313-393^{\circ} \mathrm{K}, \mathrm{P}: 7.7-116.0 \mathrm{bar}\right)$ for ten equations of state.

\begin{tabular}{|c|c|c|c|c|c|}
\hline $\mathrm{EOS}$ & $\% \mathrm{Er}^{b}$ in $\mathrm{P}$ & $\% \mathrm{Er}^{b}$ in $\mathrm{y}_{1}$ & $\% \mathrm{Er}^{f}$ in $\mathrm{x}_{1}$ & $\% \mathrm{Er}^{f}$ in $\mathrm{y}_{1}$ & $k_{1,2}$ \\
\hline & 15.30 & 11.30 & 22.32 & & \\
RK & 6.14 & 1.53 & 7.52 & 0.50 & 0.09974 \\
RKS & 2.71 & 0.92 & 3.33 & 0.71 & 0.11375 \\
PR & 2.32 & 0.69 & 2.87 & 0.61 & 0.12449 \\
PRB & 2.00 & 0.78 & 2.47 & 0.66 & 0.12700 \\
PT & 1.96 & 0.85 & 2.41 & 0.74 & 0.12800 \\
ALS & 7.24 & 1.22 & 8.52 & 1.20 & 0.17200 \\
ESD & & & & & \\
SPHCT(O) & 737.94 & 6.18 & 55.23 & 3.15 & 0.13000 \\
SPHCT(M) & 18.02 & 3.76 & 12.34 & 3.40 & 0.26600 \\
PACT & 2.31 & 2.01 & 2.67 & 2.11 & 0.06304 \\
& & & & & \\
\hline
\end{tabular}

$\% \mathrm{Er}^{b}$ : errors from bubble point calculation, $\% \mathrm{Er}^{f}$ : errors from flash calculation 


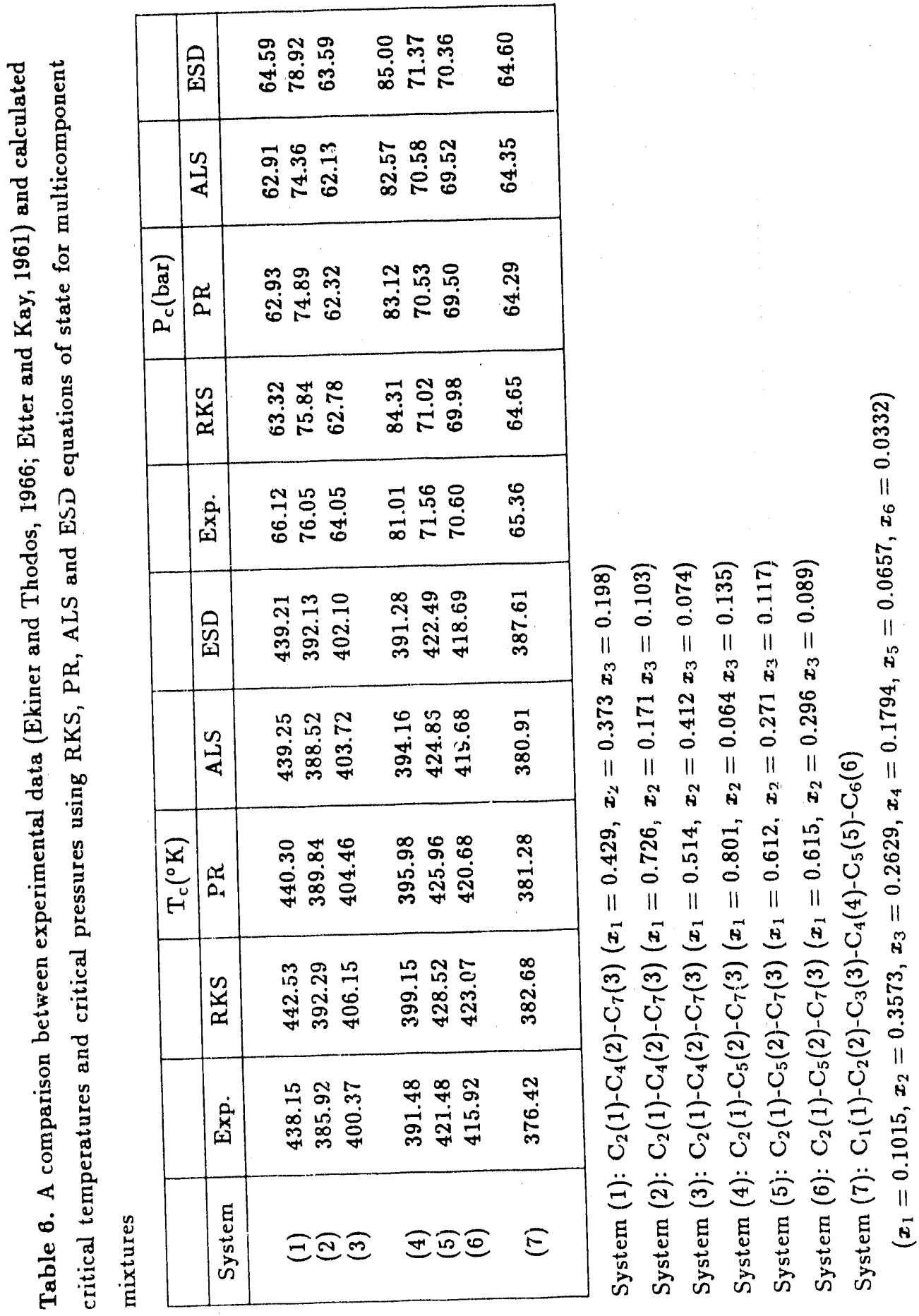




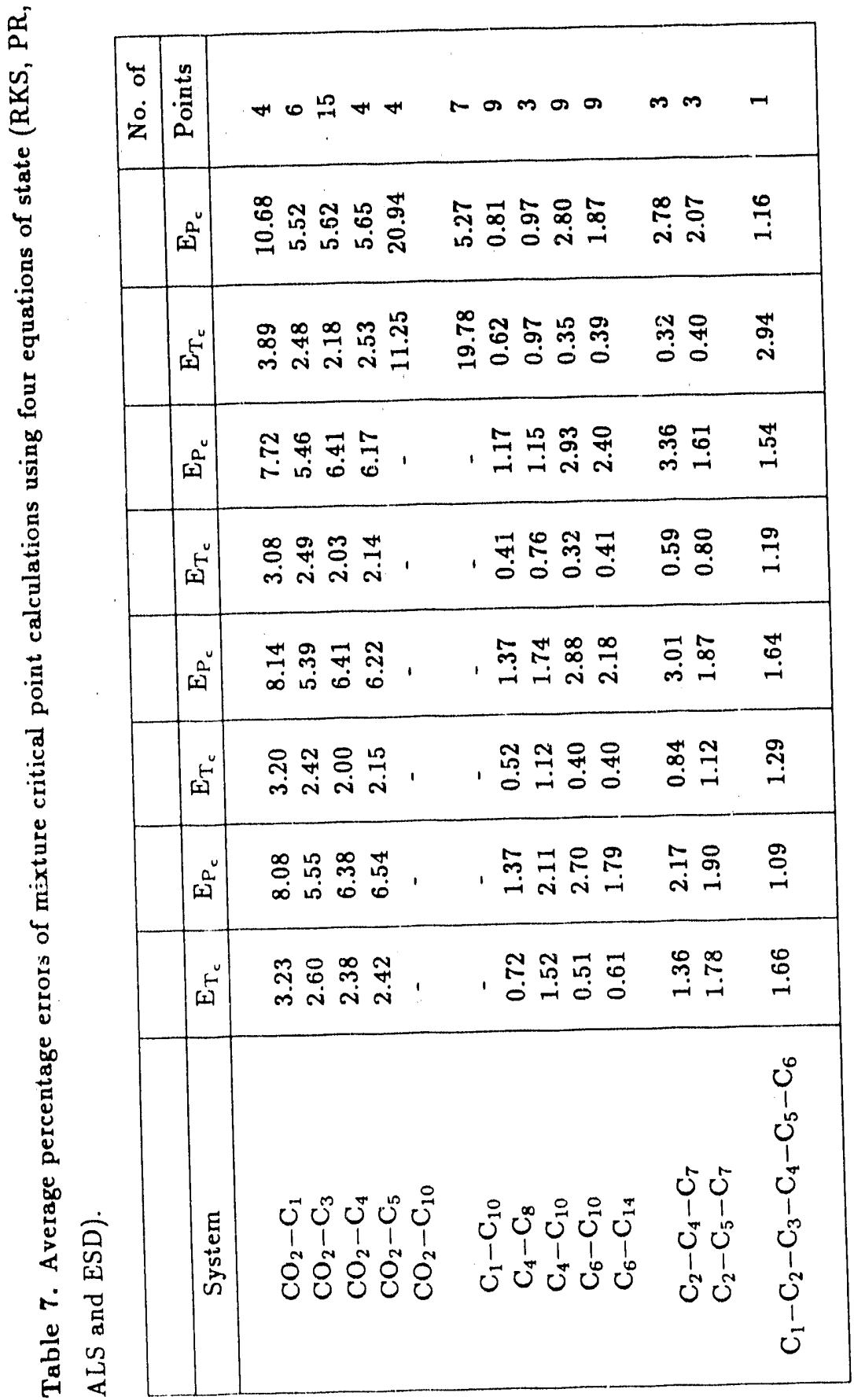


Table 8. Accuracy rankings for the eleven equations of state used in the volume calculations for pure $\mathrm{CO}_{2}$.

\begin{tabular}{|c|c|c|c|c|c|}
\hline EOS & Region 1 & Region 2 & Region 3 & Region 4 & Entire Region \\
\hline RK & (6) & (8) & (11) & (9) & (11) \\
\hline RKS & (9) & (5) & (10) & (10) & $(9)^{\prime}$ \\
\hline $\mathrm{RKT}$ & (5) & (9) & (1) & (4) & (4) \\
\hline PR & (3) & (3) & (6) & (2) & (3) \\
\hline PRB & (4) & (4) & (2) & (11) & (5) \\
\hline $\mathrm{PT}$ & (2) & (1) & (5) & (5) & (2) \\
\hline ALS & (1) & (2) & (4) & (3) & (1) \\
\hline ESD & (10) & (6) & (9) & (8) & (8) \\
\hline $\mathrm{SPHCT}(\mathrm{O})$ & $(7)$ & (10) & (7) & (1) & (6) \\
\hline $\operatorname{SPHCT}(\mathrm{M})$ & (11) & (7) & (8) & (6) & (7) \\
\hline PACT & $(8)$ & (11) & (3) & (7) & (10) \\
\hline
\end{tabular}

Region 1: Supercritical region; Region 2: Vapor phase in subcritical region; Region 3: Liquid phase in subcritical region; Region 4: Critical region.

Table 9. Average overall errors of the predictive capability for eleven equations of state.

\begin{tabular}{|c|c|c|c|c|}
\hline EOS & $\begin{array}{c}<E\rangle_{(C n \leq 4)} \\
(\%)\end{array}$ & $\begin{array}{c}<E\rangle_{(C n \geq 4)} \\
(\%)\end{array}$ & $\begin{array}{c}<E\rangle_{(\text {mix })} \\
(\%)\end{array}$ & $\begin{array}{c}<E\rangle_{c(\text { mix })} \\
(\%)\end{array}$ \\
\hline RK & 4.22 & 14.11 & 22.11 & - \\
RKS & 3.57 & 10.02 & 6.19 & 2.58 \\
RKT & 2.71 & - & - & - \\
PR & 2.91 & 2.80 & 3.63 & 2.48 \\
PRB & 2.01 & 2.54 & 3.37 & - \\
PT & 1.66 & 2.43 & 3.10 & - \\
ALS & 1.42 & 2.02 & 2.97 & 2.38 \\
ESD & 3.21 & 3.60 & 5.07 & - \\
& & & & - \\
SPHCT(O) & 3.72 & 4.27 & 185.67 & - \\
SPHCT(M) & 3.18 & 3.45 & 11.49 & \\
PACT & 2.45 & 3.33 & 5.13 & \\
\hline
\end{tabular}




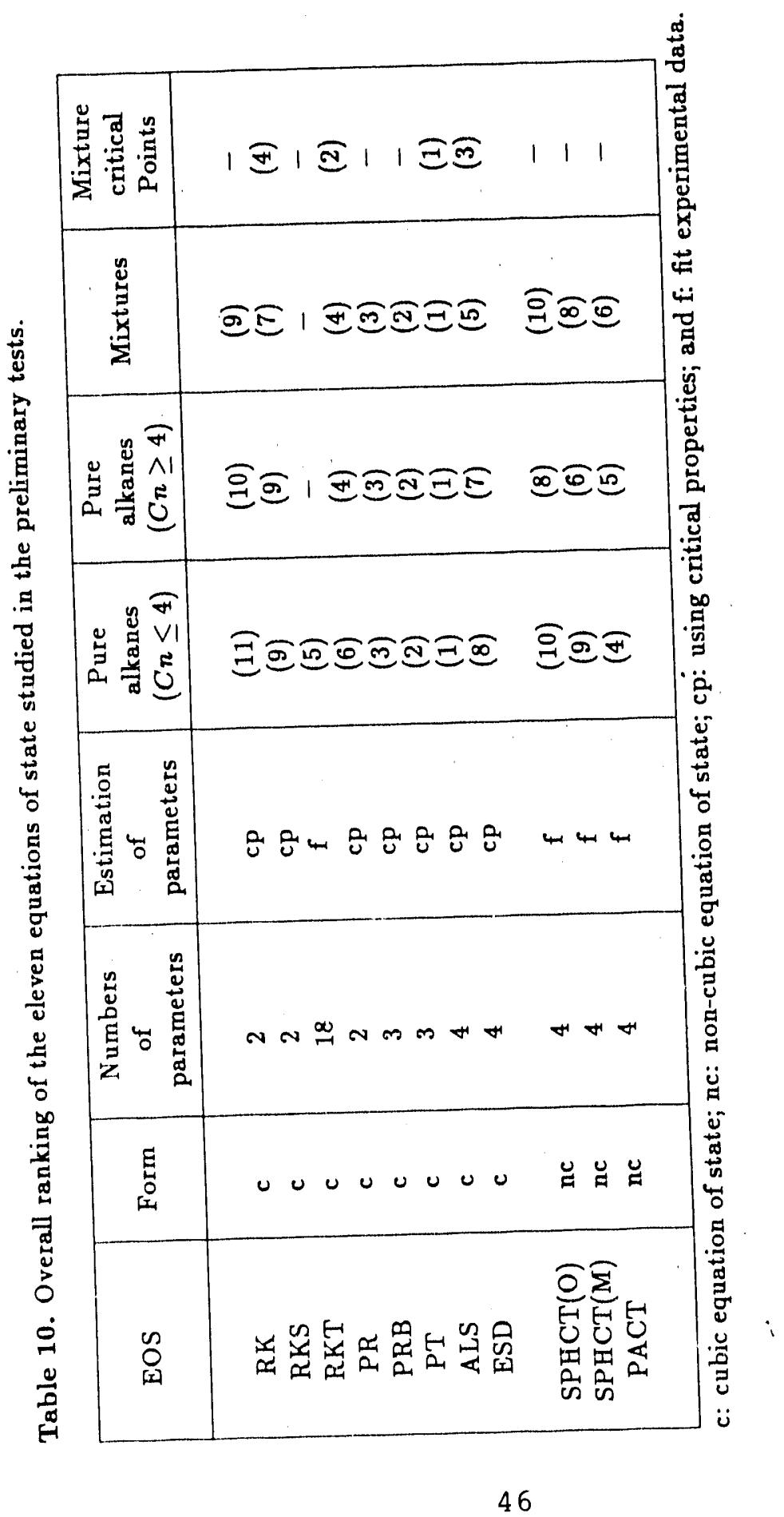


Table 11. Independent parameters for the ALS equations of state.

\section{EOS Parameter}

$b_{1}$

$b_{2}$

$b_{3}$

$a$

$\alpha(T)$
Fitting Parameter

c1

c2

c3

c4

c5

c6

c7

c8

c9

c10

c11

c12

c13

c14

c15

c16

c17

c18
Best-fit Value

0.08974

$-0.03452$

0.00330

0.03686

0.00405

$-0.01073$

0.00157

0.15400

0.14122

$-0.00272$

$-0.00484$

0.44869

0.04024

0.01111

$-0.00576$

0.40700

1.37870

$-0.29330$

Other independently variable parameters: $P_{c}, T_{c}$ and $\omega$ for each component. 
Table 12. Independent parameters for the ESD equations of state.

\begin{tabular}{|c|c|c|}
\hline EOS Parameter & Fitting Parameter & Best-fit Value \\
\hline \multirow[t]{3}{*}{$c_{i}$} & c1 & 1.00000 \\
\hline & c2 & 3.53500 \\
\hline & c3 & 0.53300 \\
\hline \multirow[t]{6}{*}{$\epsilon_{i i}$} & c4 & 1.00000 \\
\hline & c5 & 0.94500 \\
\hline & c6 & 0.13400 \\
\hline & c7 & 1.02300 \\
\hline & c8 & 2.22500 \\
\hline & c9 & 0.47800 \\
\hline \multirow[t]{6}{*}{$v_{i}^{*}$} & c10 & 0.03120 \\
\hline & c11 & 0.08700 \\
\hline & c12 & 0.00800 \\
\hline & c13 & 1.00000 \\
\hline & c14 & 2.45500 \\
\hline & c15 & 0.73200 \\
\hline$Y_{i j}$ & c16 & 1.06170 \\
\hline$q_{i}$ & c17 & 1.90476 \\
\hline
\end{tabular}

Other independently variable parameters: $P_{c}, T_{c}$ and $\omega$ for each component, and $k_{i j}$ for each binary pair. 
$|\overrightarrow{\tilde{a}} 0|+11+1++11++1+11+1++1+1+1++1+1+1++1+1+$ 疋

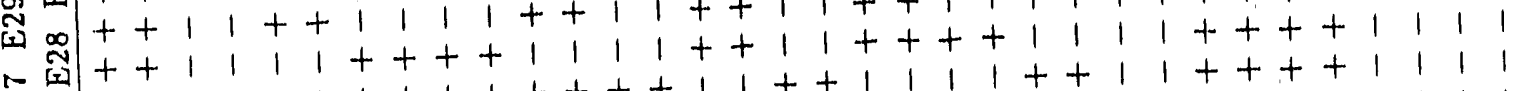

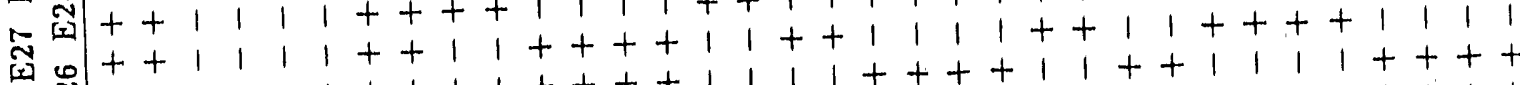

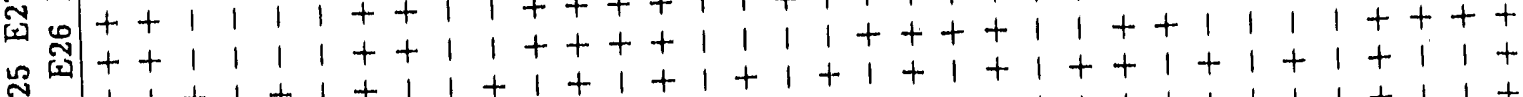
$\approx=1+1+1+1+1+1+1+1+1+1+1+1+1+1+1+1+1+1+1+1+1+$ $\approx \approx=1+11+1+1+1+1+1+1+1+1+1+1+1+1+1+1+1+1+1+$

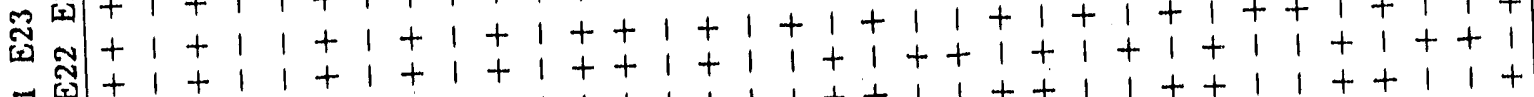

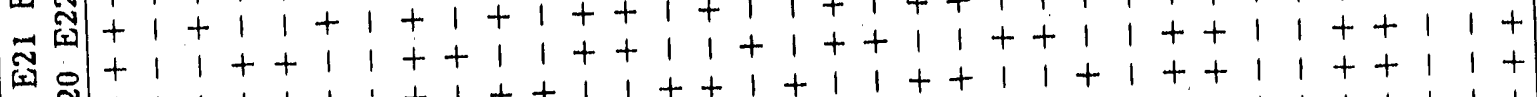

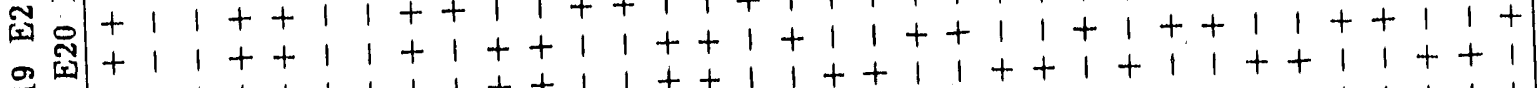

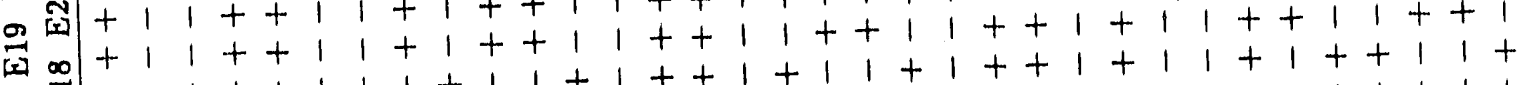

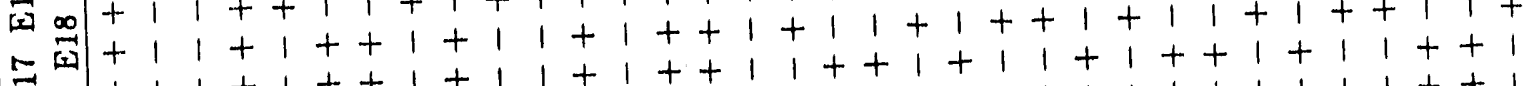
$\Leftrightarrow 00+1+1++1+11+1++1+1++1+1+1+1+1+1+1+1+1$

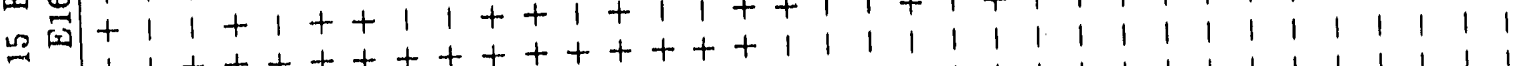
四 $\begin{array}{llllllllllll}t+t+t+t+t+t+t+t+t+t+t & t+t & t & t & 1 & 1 & 1 & 1 & 1 & 1 & 1\end{array}$

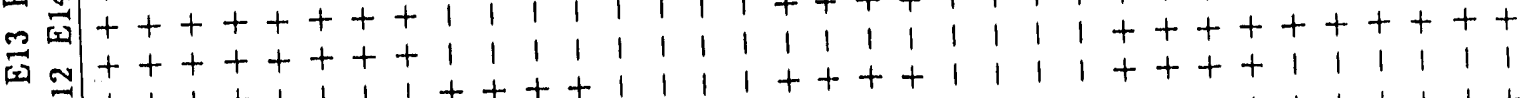

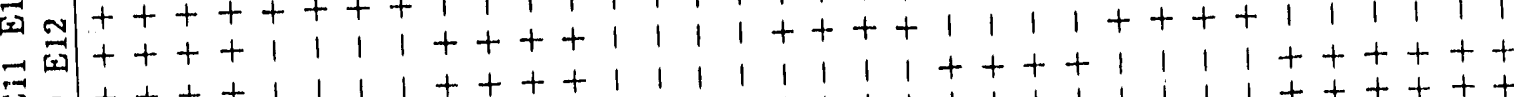

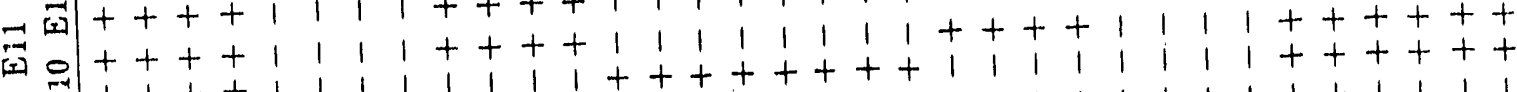
$t+t+1$
0

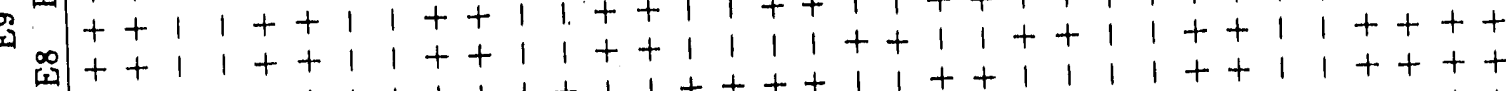

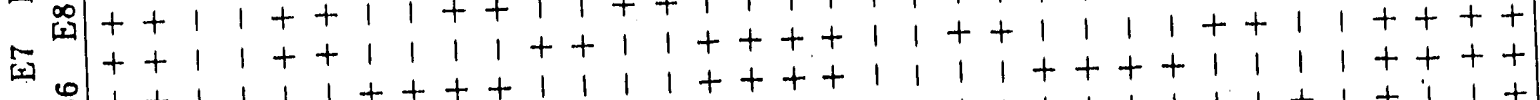

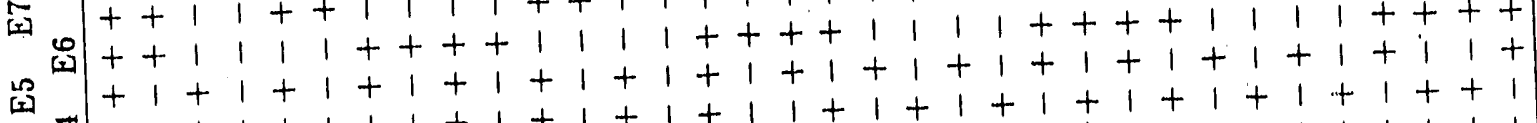
$\vec{a}+1+1+1+1+1+1+1+1+1+1+1+1+1+1+1+1+1+1$ $+1+1+1+1+1+1+1+1+1+10$ $1+1+$

$+1++1+$

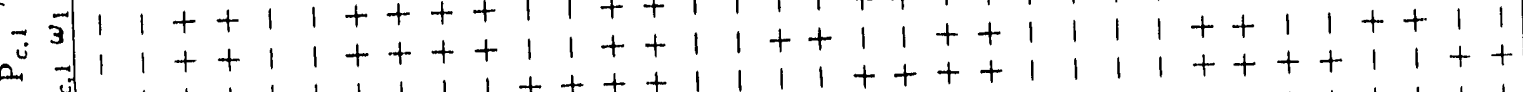

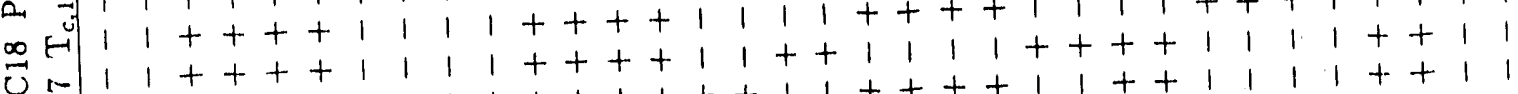

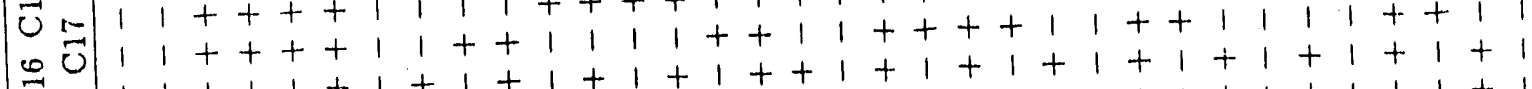

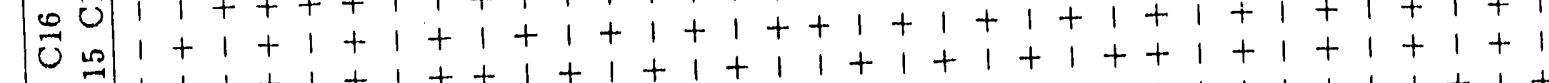

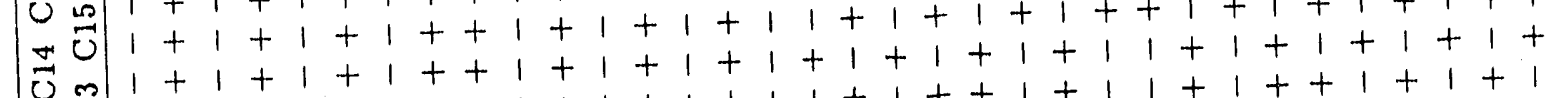

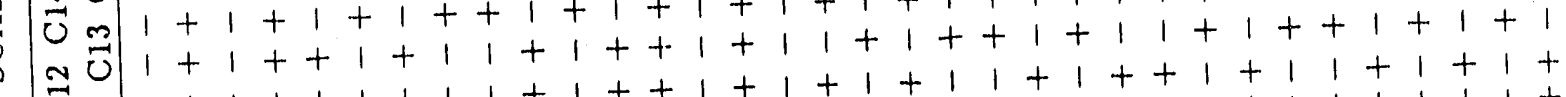

$1++11++11++11++11++11++1+1+1+1+1+1$ $1++11++11++11++1+11++11++1+1+1+1+$ $1++11++1+11++11+1++11++1+1+1+1+1+$ 禹

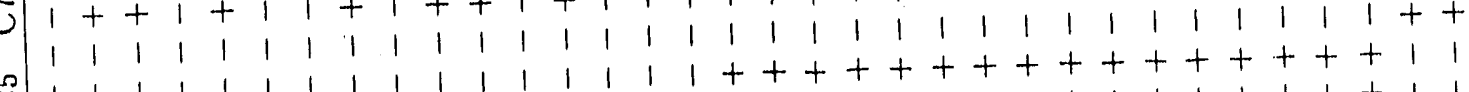

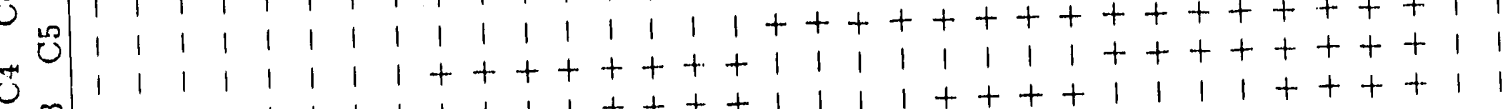

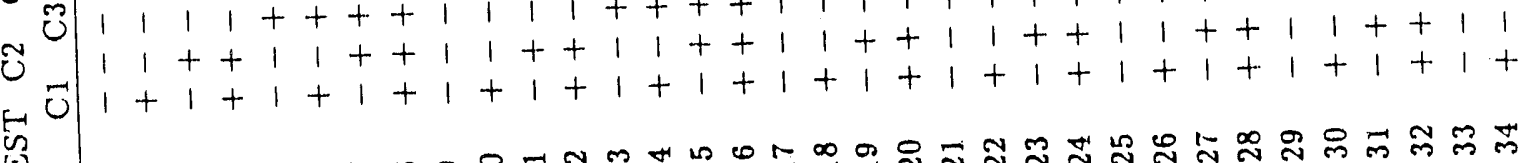




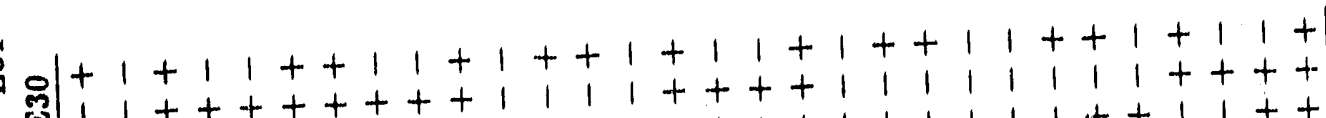

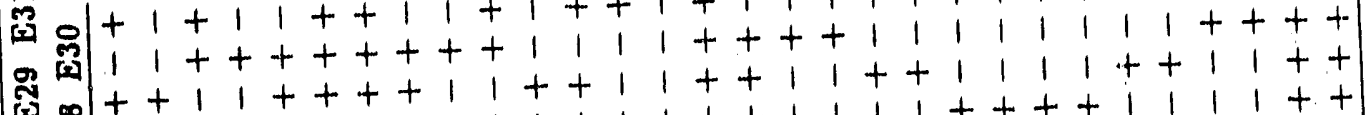

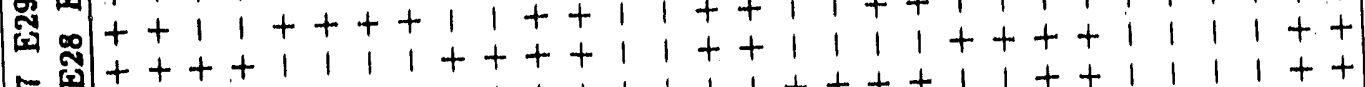

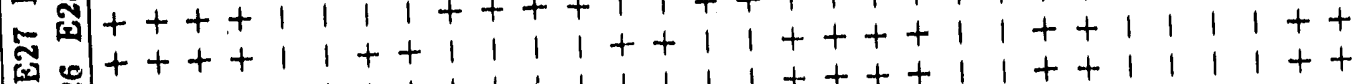

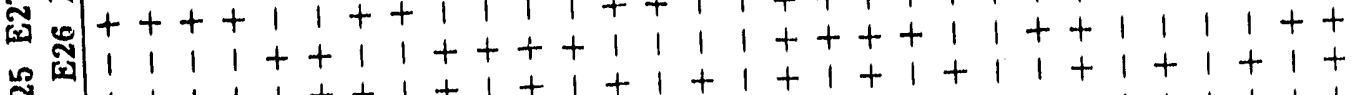
令 $1+1+1++1+1+1+1+1+1+1+1+1+1+1+1+$ $\approx$ 寻 $1++1+11+1++1+1+1+1+1+1++1+1+1+$

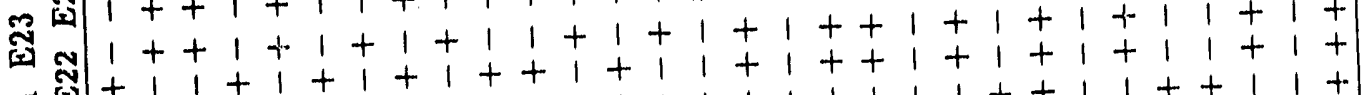

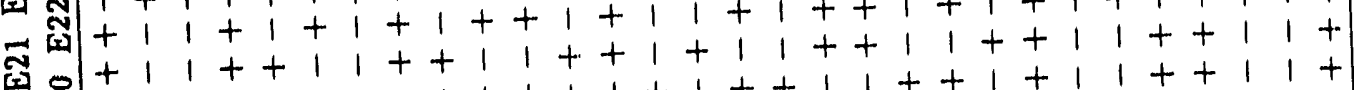
可 $+11++1+11++11+1++11++1+1+1+1+1+$ [ख⿻ $\infty++11+1++11++11++11++1+1+1+1+$

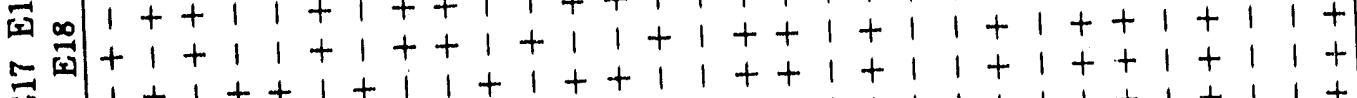

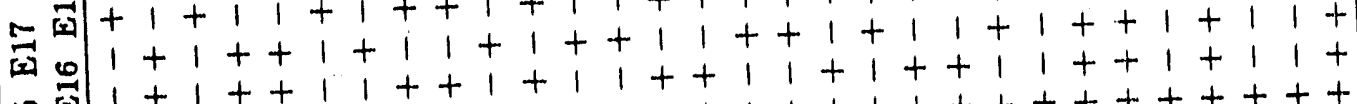

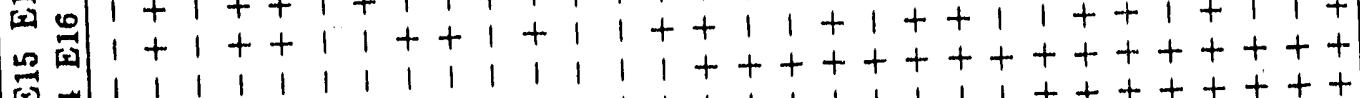

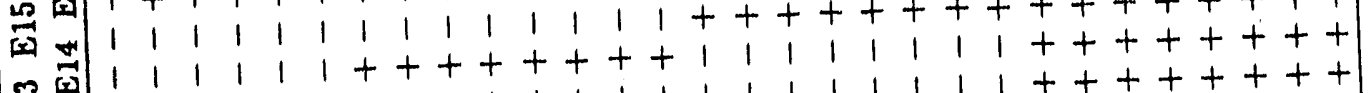

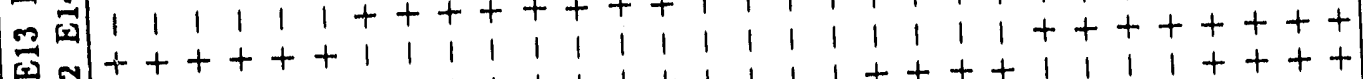

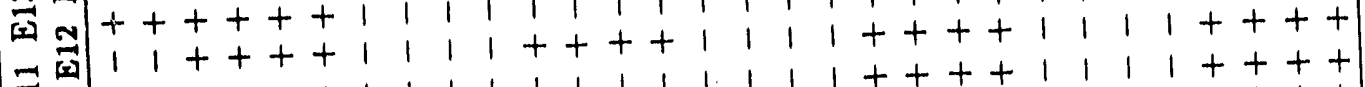

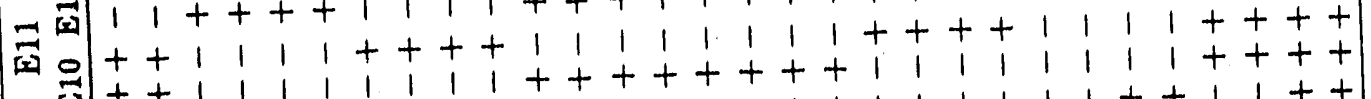
品 $1+1+1++1++1+1+1+1+1+1+1+1+1+t$

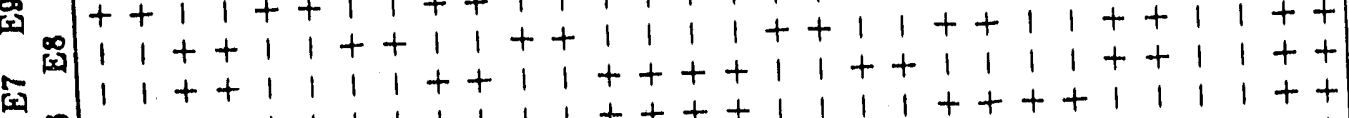

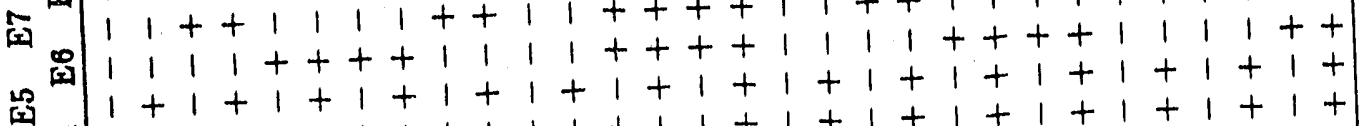

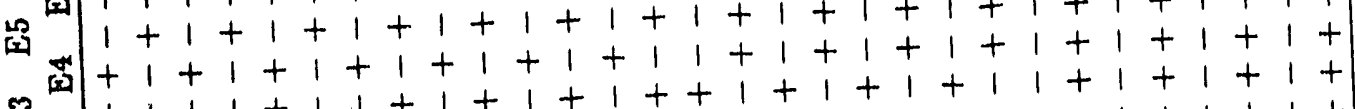
m $2+1+1+1+1+1+1++1+1+1+1+1+1+1+1+$

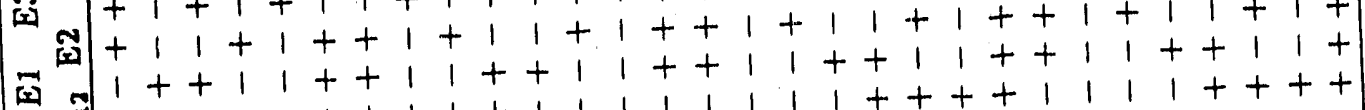

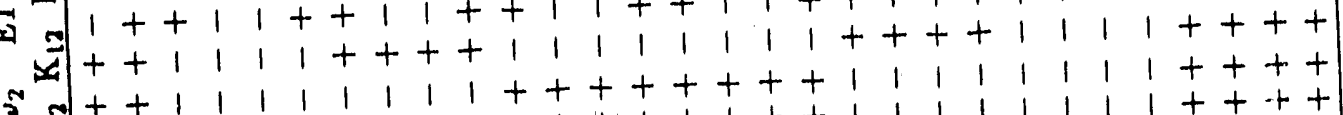

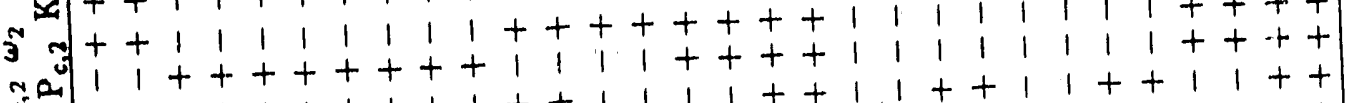

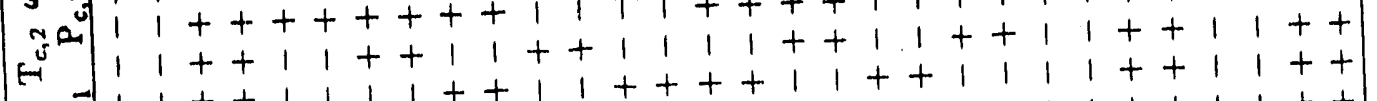

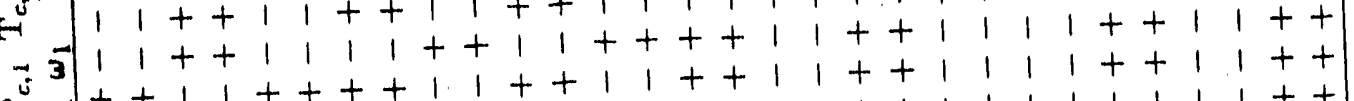

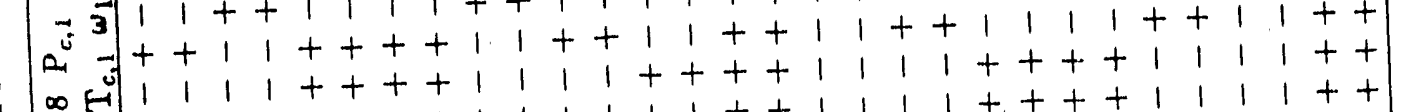

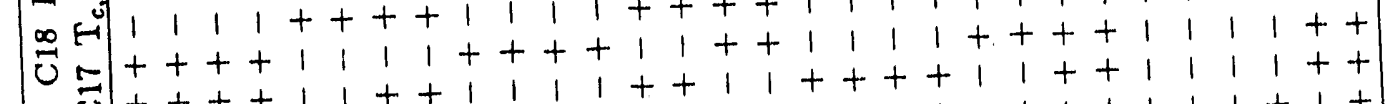

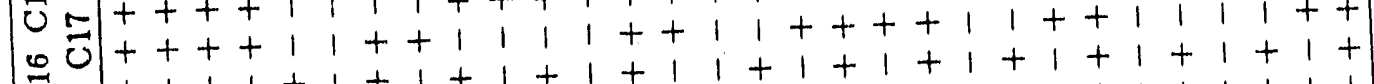

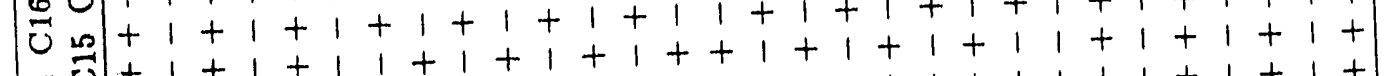

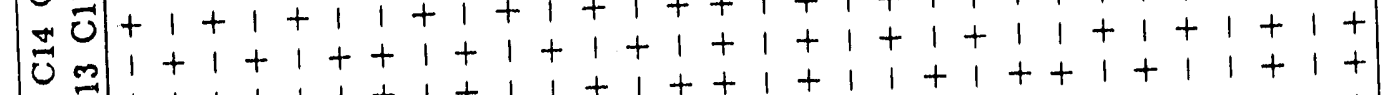

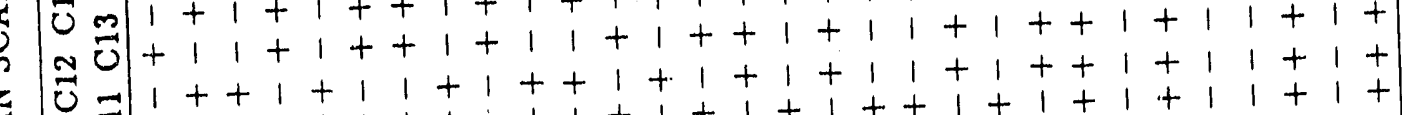

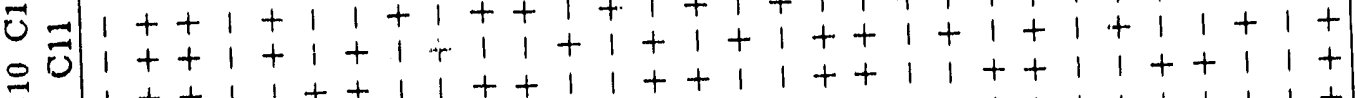

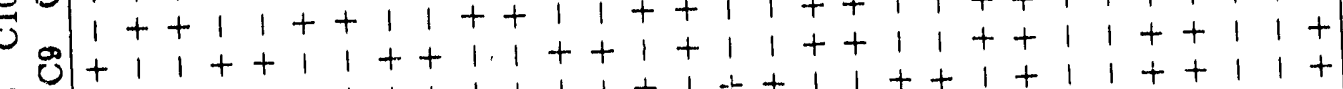
$0+1+1+1+1+1+1+1+1+1+1+1+1+1+$

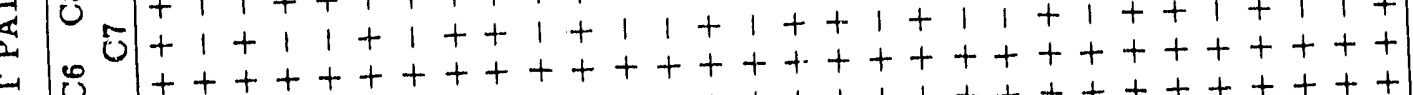
(x)

is (1)

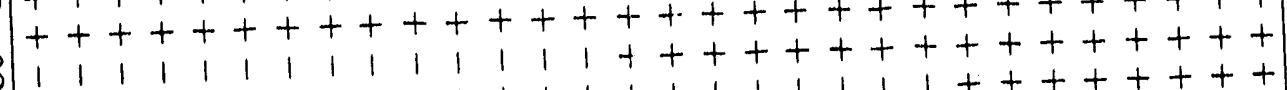

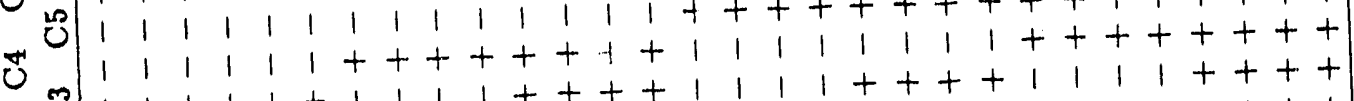

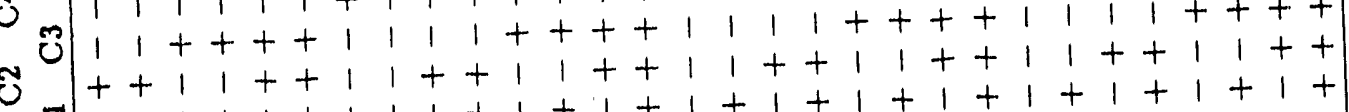
0 $1+1+1+1+1+1+1+1+1+1+1+1+1+1+1+$

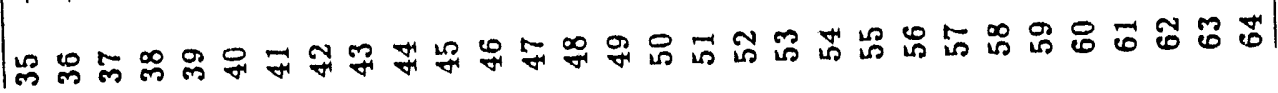


昜 $+11+1++11++1+11+1++1+1+1+1+1+1+1+1+$ 运

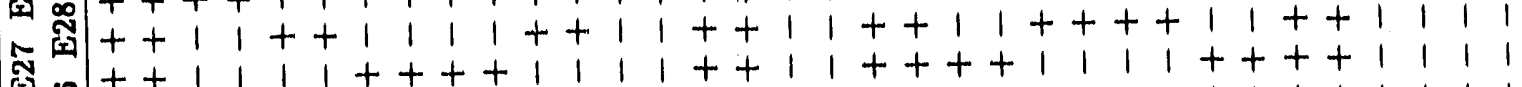

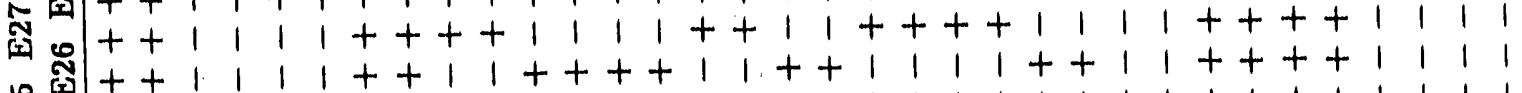

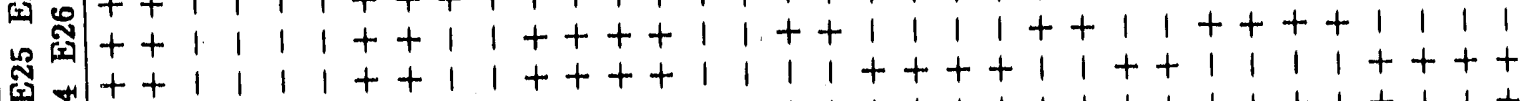
ชิ

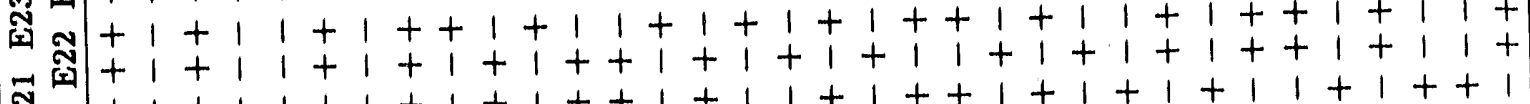
出 0 it $1+1+1+1+1++1+1+1+1+1+1+1+1+1+1+1$ g $⿴ 囗 十)+1++11++11++1+1++1+1++1+1+1+1+1+1+$

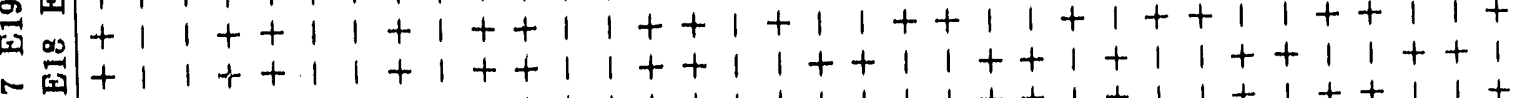
ज्ञ 0 - $1+1++1+1+1+1+1+1+1+1+1+1+1+1+1+$ 细 $11+1++1+1+1++1+1+1+1+1+1+1+1+1+1$ $\rightarrow+11+1++11++1+11++11+1++1+1+1+1+1+1$

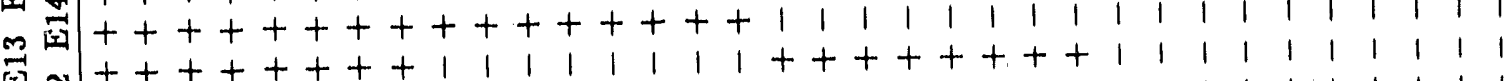

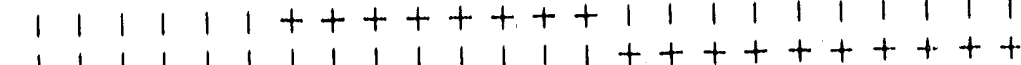
国 $00+t+t+1 \quad 1 \quad 1 \quad 1+t+t+1 \quad 1 \quad 1+t+t+1 \quad 1 \quad 1+t+t+1$

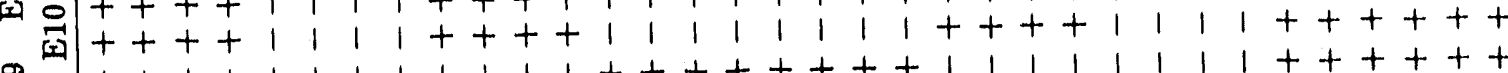
or

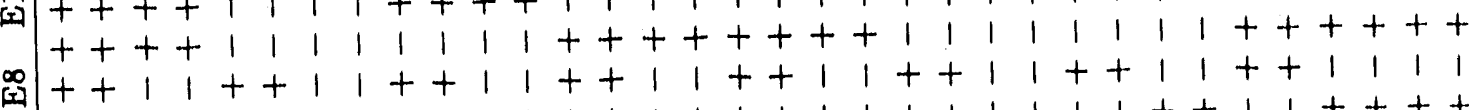

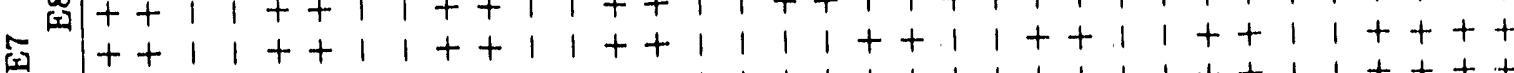

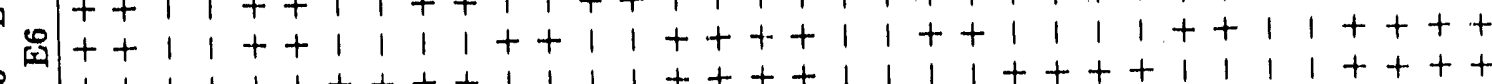
L

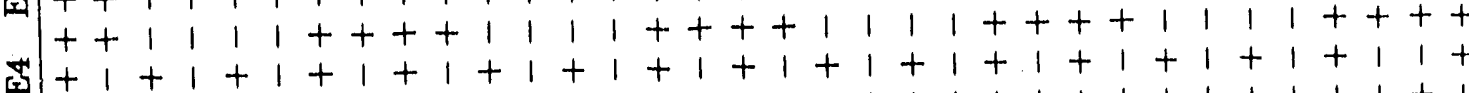
$+1+1+1+1+1+1+1+1+1+1+1+1+1+1+1+1+1+1$ $+1+1+1+1+1+1+1+1++1+1+1+1+1+1+1+1+1+1$

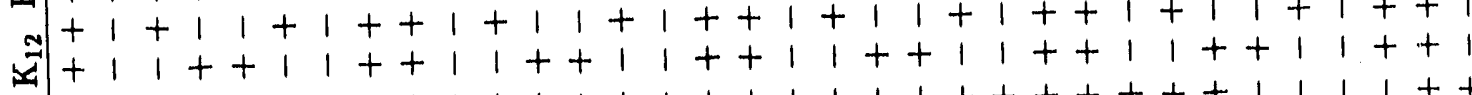
รี

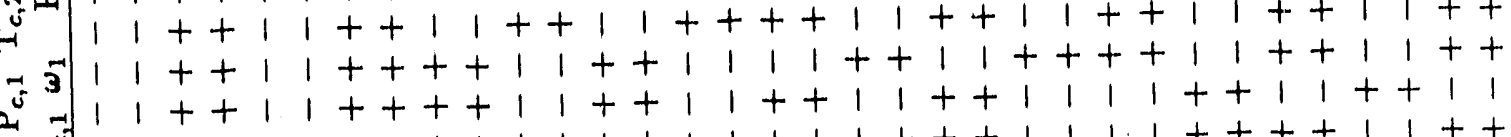

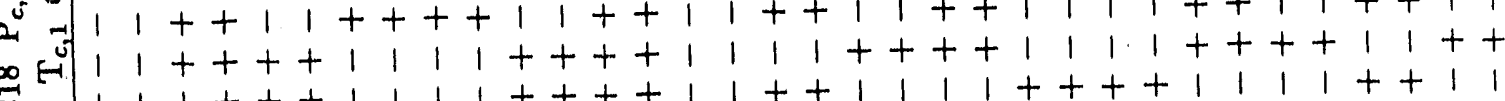

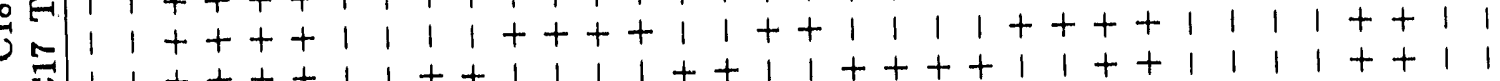
$0011+t++11++1111+t 11+t++11++1111+t 11$ 0 o $1+1+1+1+1+1+1+1++1+1+1+1+1+1+1+1+1$ * $1+1+1+1++1+1+1+11+1+1+1++1+1+1+1+1$ $0 \mathrm{~m} 1+1+1+1++1+1+1+1+1+1+1+1+1+1+1+1+$ $\simeq \overrightarrow{0} 1+1++1+11+1++1+11+1++1+11+1++1+1+1$ $\bar{U}=1+1++1+11+1++1+1+1+11+1++1+11+1+1+$ $001+1++1+1+1+11+1+1+1++1+1+1+11+1+1+$ $01++11++11++11++11++11++11++11++1+1$ $1++11++11++11++1+11++11++11++1+1+$ $51++11++1+11++11+1++11++1+11++1+1+$

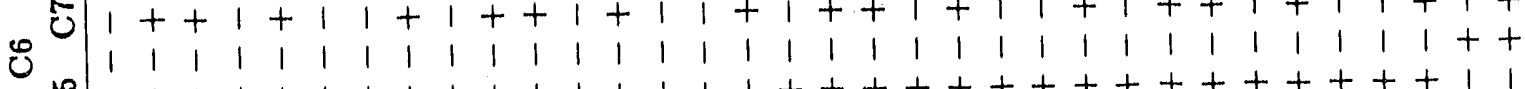
$J$
0

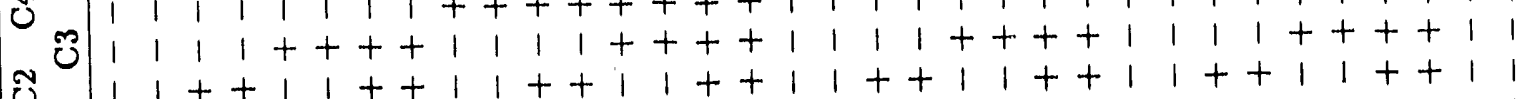
$\mathcal{E} J 1+1+1+1+1+1+1+1+1+1+1+1+1+1+1+1+1+$

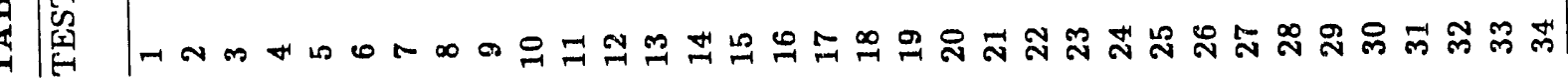


总 $+1+11++11+1++1+11+1++1+1+1+1+$

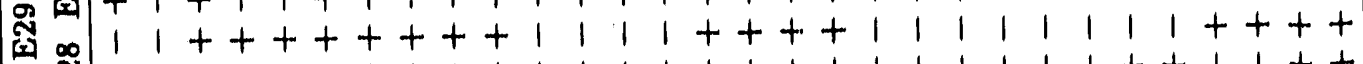

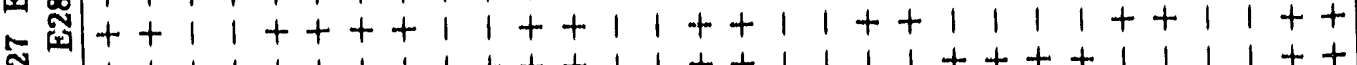

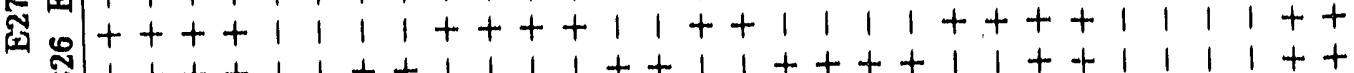

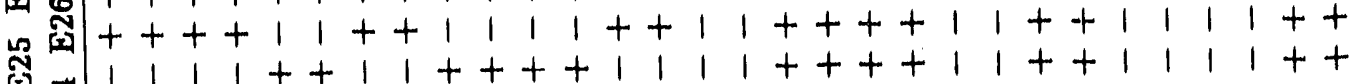

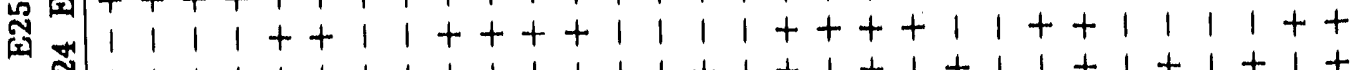
? $1+1+1++1+1+1+1+1+1+1+1+1+1+1+1+$ 丽 $1++1+11+1++1+1+1+1+1+1+1+1+1+$ ก $1++1+1+1+1+1+1+1+1++1+1+1+1+1+1+$ 舲 $+1+1+1+1+1+1+1+1+1+1+1+1+1+$ a $1+11+t 1++11++1+1+1++1++1+1+1+$ $\rightarrow \infty+1+t+1+11++1+1++1+1+1+1+1+1+$ $=1++11+1++11++11++11++1+1+1+1+1+$ G $=0+1+11+1++1+1+1+1+1+1+1+1+1+1+$ 电 $1+1++1+11+1++1+1+1+1+1+1+1+1+$ a $1+1++11++1+11++11+1++1+1+1+1+$ $\rightarrow \quad \begin{array}{lllllllllllllll}a & 1 & 1 & 1 & 1 & 1 & \mid & 1 & 1 & 1 & \mid & \mid & 1 & 1 & t+t+t+t+t+t+t+t+t+t\end{array}$

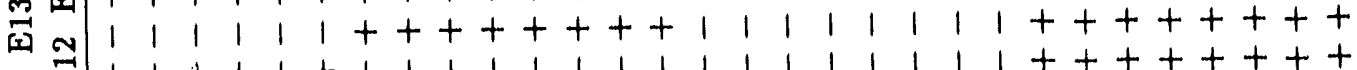

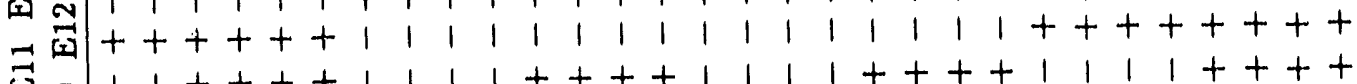

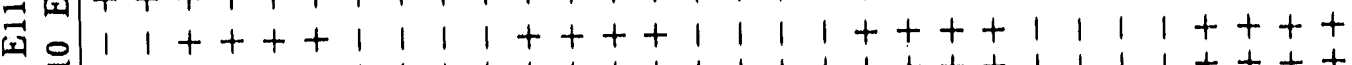
o

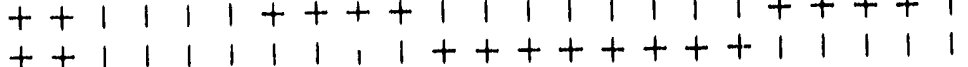

$\infty++11++11++11++11++11+t+1+t+1+t$

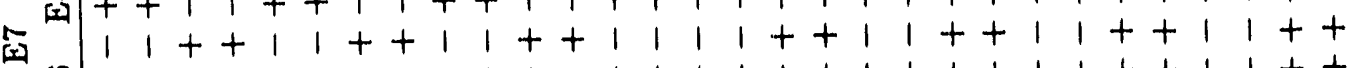

[x] $\infty 1+t|111+t| 11+t+t \mid 1+t+111++11+t$ $1+1+t+1111+t+t 111+1+t+1+11+t$
$1+1+1+1+1+1+1+1+1+1+1+1+1+1+1+$ a $1+1+1+1+1+1+1+1+1+1+1+1+1+1+1+$ 年 $+1+1+11+1+1+1++1+1+1+1+1+1+1+$ ज $-1+1+1+1+11+1++1+1+1++1+1+1+$ $1++11++11++11++11++11++1+1+1+1+$

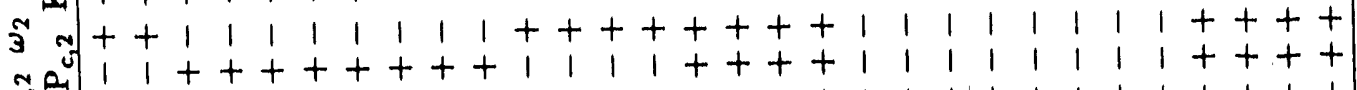

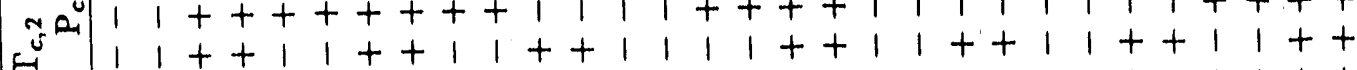

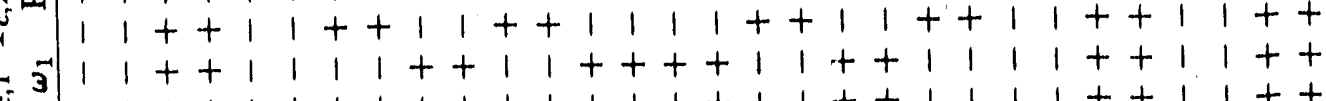

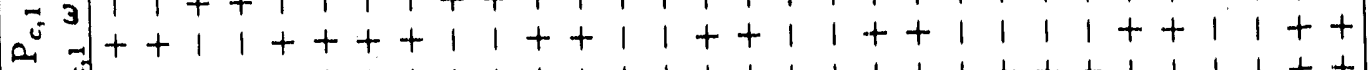

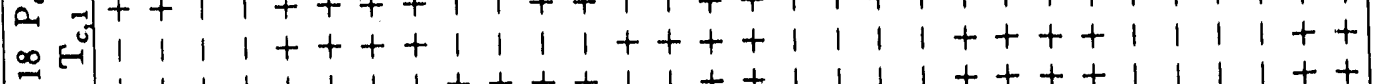

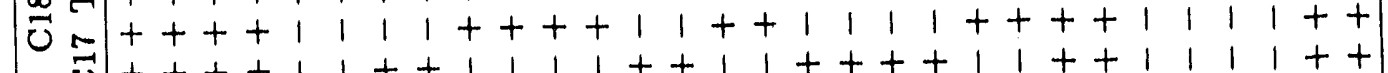

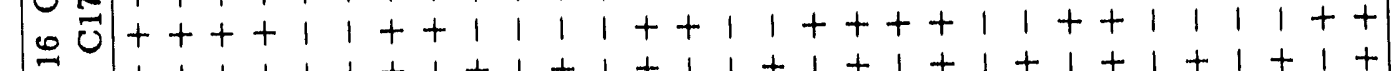
$000+1+1+1+1+1+1+11+1+1+1+1+1+1+1+$ $\pm 0+1+1+1+1+1+1+1+1+1+1+1+1+1+1+$ $\sim \widetilde{U}+1+1+1++1+1+1+1+1+1+1++1+1+1+$ $\overline{0}=1++1+11+1++1+1+1+1+1+1++1+1+1+$ $g+11++11++11++1+1+1+11++11++1+1+$ $\tilde{0}+11++1+11++1+1+1+1+1+1+1+1+1+$ 0
0

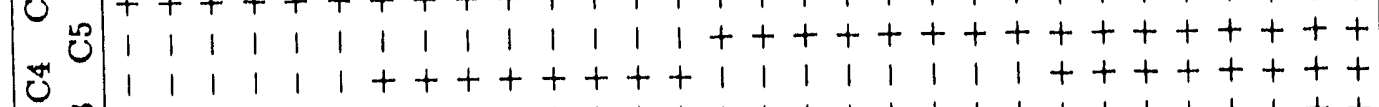

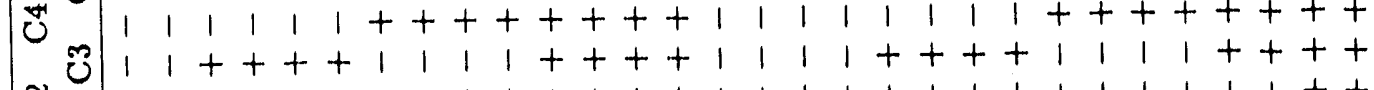
$\mathcal{U}++11++11++11++11++11++11++1+1+$ $\int_{\text {G }} \circlearrowright 1+1+1+1+1+1+1+1+1+1+1+1+1+1+1+$ (1)

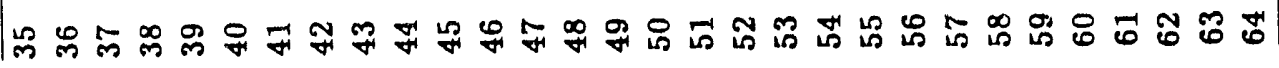


Table 15 A. Liquid Density Sensitivities $n-\mathrm{C}_{4} / \mathrm{CO}_{2}$ ALS EOS - Average Absolute Error

\begin{tabular}{|c|c|c|c|}
\hline Factor & Avg Abs Err & Factor & Avg Abs Err \\
\hline $\mathrm{C} 1$ & $0.4969 \times 10^{3}$ & E5 & 0.2620 \\
\hline E1 & $-.3857 \times 10^{2}$ & $\mathrm{C} 10$ & 0.2515 \\
\hline $\mathrm{E} 20$ & $-.3847 \times 10^{2}$ & E10 & 0.2481 \\
\hline C3 & $-.3822 \times 10^{2}$ & $\mathrm{C} 11$ & 0.2477 \\
\hline E27 & $-.3818 \times 10^{2}$ & E9 & 0.2459 \\
\hline C15 & $-.3818 \times 10^{2}$ & C5 & 0.2102 \\
\hline $\mathrm{C} 7$ & $-.3815 \times 10^{2}$ & E12 & 0.2092 \\
\hline $\mathrm{E} 2$ & $-.3815 \times 10^{2}$ & E16 & 0.1955 \\
\hline $\mathrm{E} 23$ & $-.3815 \times 10^{2}$ & $\mathrm{C} 6$ & 0.1830 \\
\hline E14 & $-.3815 \times 10^{2}$ & E15 & -.1789 \\
\hline E6 & $-.3815 \times 10^{2}$ & E22 & 0.1431 \\
\hline$\omega_{2}$ & $-.3800 \times 10^{2}$ & $k_{12}$ & -.1305 \\
\hline$\omega_{1}$ & $-.3708 \times 10^{2}$ & E25 & 0.1267 \\
\hline $\mathrm{C} 2$ & $-.3609 \times 10^{2}$ & E30 & 0.1091 \\
\hline $\mathrm{C} 12$ & $0.1627 \times 10^{2}$ & E29 & $0.9742 \times 10^{-1}$ \\
\hline$P_{c, 1}$ & $0.1266 \times 10^{2}$ & E31 & $0.9412 \times 10^{-1}$ \\
\hline$T_{c, 2}$ & $0.1119 \times 10^{2}$ & $\mathrm{E} 17$ & $0.9149 \times 10^{-1}$ \\
\hline $\mathrm{C} 8$ & $-.6723 \times 10$ & $\mathrm{C} 18$ & $0.8609 \times 10^{-1}$ \\
\hline $\mathrm{C} 4$ & $0.5086 \times 10$ & $\mathrm{E} 4$ & $0.6287 \times 10^{-1}$ \\
\hline E18 & $-.3414 \times 10$ & E13 & $-.5626 \times 10^{-1}$ \\
\hline$P_{c, 2}$ & $0.2883 \times 10$ & $\mathrm{E} 28$ & $-.5452 \times 10^{-1}$ \\
\hline E11 & $-.1649 \times 10$ & E24 & $-.4330 \times 10^{-1}$ \\
\hline E19 & $0.1313 \times 10$ & C14 & $-.3879 \times 10^{-1}$ \\
\hline $\mathrm{C9}$ & $-.1251 \times 10$ & E26 & $-.1591 \times 10^{-1}$ \\
\hline E7 & $0.1120 \times 10$ & & \\
\hline $\mathrm{E} 21$ & -.7970 & & \\
\hline$T_{c, 1}$ & 0.6340 & & \\
\hline $\mathrm{C} 13$ & 0.5559 & & \\
\hline E3 & -.5328 & & \\
\hline $\mathrm{C} 17$ & 0.5075 & & \\
\hline C16 & 0.3783 & & \\
\hline E8 & 0.2709 & & \\
\hline
\end{tabular}


Table 15 B. Liquid Density Sensitivities n-C $4 / \mathrm{CO}_{2}$ ALS EOS - Bias

\begin{tabular}{|c|c|c|c|}
\hline Factor & Bias & Factor & Bias ' \\
\hline $\mathrm{C} 1$ & $0.4969 \times 10^{3}$ & E5 & 0.2543 \\
\hline E1 & $-.3857 \times 10^{2}$ & $\mathrm{C} 10$ & 0.2438 \\
\hline $\mathrm{E} 20$ & $-.3850 \times 10^{2}$ & E10 & 0.2404 \\
\hline C3 & $-.3822 \times 10^{2}$ & $\mathrm{C} 11$ & 0.2399 \\
\hline $\mathrm{E} 27$ & $-.3818 \times 10^{2}$ & $\mathrm{E} 9$ & 0.2381 \\
\hline C15 & $-.3818 \times 10^{2}$ & $\mathrm{C5}$ & 0.2179 \\
\hline $\mathrm{C} 7$ & $-.3818 \times 10^{2}$ & $\mathrm{E} 12$ & 0.2015 \\
\hline $\mathrm{E} 2$ & $-.3815 \times 10^{2}$ & E16 & 0.1877 \\
\hline E23 & $-.3815 \times 10^{2}$ & $\mathrm{C} 6$ & 0.1753 \\
\hline E14 & $-.3815 \times 10^{2}$ & E15 & -.1711 \\
\hline $\mathrm{E} 6$ & $-.3815 \times 10^{2}$ & E22 & 0.1508 \\
\hline$\omega_{2}$ & $-.3800 \times 10^{2}$ & $\mathrm{E} 25$ & 0.1344 \\
\hline$\omega_{1}$ & $-.3708 \times 10^{2}$ & $k_{12}$ & -.1227 \\
\hline $\mathrm{C} 2$ & $-.3609 \times 10^{2}$ & E30 & 0.1169 \\
\hline $\mathrm{C} 12$ & $0.1628 \times 10^{2}$ & E29 & 0.1052 \\
\hline$P_{c, 1}$ & $0.1267 \times 10^{2}$ & E31 & 0.1019 \\
\hline$T_{c, 2}$ & $0.1120 \times 10^{2}$ & E17 & $0.9924 \times 10^{\cdots 1}$ \\
\hline $\mathrm{C} 8$ & $-.6731 \times 10$ & $\mathrm{C} 18$ & $0.9384 \times 10^{-1}$ \\
\hline $\mathrm{C} 4$ & $0.5079 \times 10$ & $\mathrm{E} 4$ & $0.7061 \times 10^{-1}$ \\
\hline E18 & $-.3421 \times 10$ & E13 & $-.4852 \times 10^{-1}$ \\
\hline$P_{c, 2}$ & $0.2890 \times 10$ & E28 & $-.4681 \times 10^{-1}$ \\
\hline E11 & $-.1641 \times 10$ & E24 & $-.3555 \times 10^{-1}$ \\
\hline E19 & $0.1321 \times 10$ & $\mathrm{C} 14$ & $-.3104 \times 10^{-1}$ \\
\hline C9 & $-.1243 \times 10$ & $\mathrm{E} 26$ & $-.8180 \times 10^{-2}$ \\
\hline E7 & $0.1112 \times 10$ & & \\
\hline $\mathrm{E} 21$ & -.7892 & & \\
\hline$T_{c, 1}^{\prime}$ & 0.6265 & & \\
\hline C13 & 0.5484 & & \\
\hline E3 & -.5406 & & \\
\hline $\mathrm{C} 17$ & 0.4997 & & \\
\hline $\mathrm{C} 16$ & 0.3861 & & \\
\hline $\mathrm{E} 8$ & 0.2786 & & \\
\hline
\end{tabular}


Table 15 C. Liquid Density Sensitivities n- $\mathrm{C}_{4} / \mathrm{CO}_{2}$ ALS EOS - CPU Time

$\begin{array}{llll}\text { Factor } & \text { CPU Time } & \text { Factor } & \text { CPU Time } \\ \text { E13 } & -.7331 \times 10 & \text { E12 } & 0.8489 \\ \text { C1 } & -.4862 \times 10 & \omega_{2} & -.6946 \\ k_{12} & 0.3780 \times 10 & \text { E17 } & -.6946 \\ \text { C4 } & 0.3627 \times 10 & \text { E23 } & 0.6946 \\ \text { E15 } & 0.3627 \times 10 & \text { E25 } & 0.6946 \\ \text { E11 } & -.3472 \times 10 & \text { E28 } & -.6946 \\ \text { E14 } & 0.3163 \times 10 & \text { E4 } & 0.5402 \\ \text { C12 } & -.2701 \times 10 & \text { E5 } & 0.5402 \\ P_{c, 2} & 0.2392 \times 10 & \text { E6 } & 0.5402 \\ \text { E19 } & 0.1929 \times 10 & \text { E8 } & 0.5402 \\ \text { E27 } & 0.1929 \times 10 & \text { C2 } & 0.3859 \\ \text { C9 } & -.1775 \times 10 & \text { C7 } & 0.3859 \\ \text { E29 } & 0.1620 \times 10 & \omega_{1} & -.3859 \\ T_{c, 2} & -.1466 \times 10 & \text { E1 } & -.3859 \\ \text { E9 } & 0.1466 \times 10 & \text { E2 } & -.3859 \\ \text { E16 } & -.1466 \times 10 & \text { E7 } & 0.3859 \\ \text { E21 } & 0.1466 \times 10 & \text { E18 } & -.3859 \\ \text { E30 } & -.1466 \times 10 & \text { E20 } & 0.3859 \\ \text { C11 } & -.1312 \times 10 & \text { C3 } & -.2315 \\ T_{c, 1} & -.1158 \times 10 & \text { C14 } & 0.2315 \\ \text { E22 } & -.1158 \times 10 & \text { E26 } & -.2315 \\ \text { E24 } & 0.1158 \times 10 & \text { C17 } & -.7716 \times 10^{-1} \\ \text { C5 } & 0.1003 \times 10 & P_{c, 1} & -.7716 \times 10^{-1} \\ \text { C6 } & 0.1003 \because 10 & \text { E10 } & 0.7716 \times 10^{-1} \\ \text { C10 } & 0.1003 \times 10 & & \\ \text { C13 } & 0.1003 \times 10 & & \\ \text { C15 } & -.1003 \times 10 & & \\ \text { C18 } & 0.1003 \times 10 & & \\ \text { E3 } & 0.1003 \times 10 & & \\ \text { E31 } & 0.1003 \times 10 & & \\ \text { C8 } & 0.8489 & & \\ \text { C16 } & -.8489 & & \end{array}$


Table 16 A. Liquid Density Sensitivities $n-C_{0} / \mathrm{CO}_{2}$ ALS EOS - Average Absolute Error

\begin{tabular}{|c|c|c|c|}
\hline Factor & Avg Abs Err & Factor & Avg Abs Err \\
\hline $\mathrm{C} 1$ & $-.2155 \times 10^{3}$ & E24 & $-.8259 \times 10$ \\
\hline E19 & $-.1921 \times 10^{3}$ & C5 & $-.7593 \times 10$ \\
\hline $\mathrm{C} 12$ & $0.1398 \times 10^{3}$ & $\mathrm{C} 16$ & $0.7529 \times 10$ \\
\hline$T_{c, 2}$ & $0.1219 \times 10^{3}$ & $\mathrm{C} 13$ & $0.7146 \times 10$ \\
\hline E18 & $0.8699 \times 10^{2}$ & E27 & $-.6822 \times 10$ \\
\hline E11 & $-.7146 \times 10^{2}$ & E23 & $0.6808 \times 10$ \\
\hline$T_{c, 1}$ & $0.6278 \times 10^{2}$ & E9 & $0.6445 \times 10$ \\
\hline $\mathrm{C} 8$ & $-.5487 \times 10^{2}$ & C6 & $-.5821 \times 10$ \\
\hline E1 & $-.4395 \times 10^{2}$ & C14 & $0.5255 \times 10$ \\
\hline $\mathrm{C} 4$ & $0.4361 \times 10^{2}$ & E10 & $-.4614 \times 10$ \\
\hline E7 & $0.4357 \times 10^{2}$ & C15 & $0.3884 \times 10$ \\
\hline E3 & $-.3621 \times 10^{2}$ & E17 & $-.3726 \times 10$ \\
\hline$P_{c, 1}$ & $0.3458 \times 10^{2}$ & $\mathrm{C9}$ & $-.3540 \times 10$ \\
\hline $\mathrm{E} 8$ & $0.3069 \times 10^{2}$ & $\omega_{2}$ & $0.3251 \times 10$ \\
\hline E22 & $-.2810 \times 10^{2}$ & E25 & $0.3231 \times 10$ \\
\hline $\mathrm{F} 20$ & $-.2719 \times 10^{2}$ & $\mathrm{E} 2$ & $-.3195 \times 10$ \\
\hline E30 & $-.2550 \times 10^{2}$ & $\mathrm{C} 18$ & $0.3160 \times 10$ \\
\hline$P_{c, 2}$ & $0.2376 \times 10^{2}$ & C11 & $0.2926 \times 10$ \\
\hline E16 & $0.2220 \times 10^{2}$ & $\mathrm{C} 10$ & $0.2790 \times 10$ \\
\hline $\mathrm{C} 17$ & $0.2053 \times 10^{2}$ & E12 & $-.1914 \times 10$ \\
\hline $\mathrm{E} 6$ & $0.1787 \times 10^{2}$ & E15 & $0.1675 \times 10$ \\
\hline E4 & $-.1762 \times 10^{2}$ & $\mathrm{C} 3$ & $-.1076 \times 10$ \\
\hline E21 & $-.1602 \times 10^{2}$ & $k_{12}$ & -.8252 \\
\hline $\mathrm{C} 2$ & $0.1322 \times 10^{2}$ & E31 & 0.5456 \\
\hline E5 & $-.1209 \times 10^{2}$ & & \\
\hline E14 & $-.1109 \times 10^{2}$ & & \\
\hline $\mathrm{E} 29$ & $-.1073 \times 10^{2}$ & & \\
\hline E28 & $0.1000 \times 10^{2}$ & & \\
\hline E26 & $-.9989 \times 10$ & & \\
\hline$\omega_{1}$ & $0.9940 \times 10$ & & \\
\hline $\mathrm{C} 7$ & $0.8976 \times 10$ & & \\
\hline E13 & $0.8337 \times 10$ & & \\
\hline
\end{tabular}


Table 16 B. Liquid Density Sensitivities $n-\mathrm{C}_{6} / \mathrm{CO}_{2} \mathrm{ALS}$ EOS - Bias

\begin{tabular}{|c|c|c|c|}
\hline Factor & Bias & Factor & Bias \\
\hline $\mathrm{C} 1$ & $-.5619 \times 10^{3}$ & $\mathrm{C} 14$ & 0.4065 \\
\hline$P_{c, 1}$ & $0.2564 \times 10^{3}$ & E1 & -.3850 \\
\hline $\mathrm{C} 12$ & $0.2246 \times 10^{3}$ & E8 & 0.3112 \\
\hline$T_{c, 2}$ & $0.1213 \times 10^{3}$ & $\mathrm{E} 4$ & -.3029 \\
\hline $\mathrm{C} 8$ & $-.1053 \times 10^{3}$ & E5 & 0.2961 \\
\hline $\mathrm{C} 4$ & $0.8763 \times 10^{2}$ & E7 & 0.2589 \\
\hline $\mathrm{C} 2$ & $0.6031 \times 10^{2}$ & $\mathrm{E} 20$ & -.2274 \\
\hline$P_{c, 2}$ & $0.5948 \times 10^{2}$ & E24 & 0.2101 \\
\hline$T_{c, 1}$ & $-.5711 \times 10^{2}$ & $\mathrm{C} 10$ & 0.1485 \\
\hline$\omega_{1}$ & $0.4434 \times 10^{2}$ & E3 & 0.1480 \\
\hline $\mathrm{C} 9$ & $-.2627 \times 10^{2}$ & E12 & -.1350 \\
\hline $\mathrm{C} 17$ & $0.1439 \times 10^{2}$ & E15 & -.1336 \\
\hline $\mathrm{C} 16$ & $0.1317 \times 10^{2}$ & E31 & $-.8657 \times 10^{-1}$ \\
\hline $\mathrm{C} 13$ & $0.5386 \times 10$ & E28 & $0.7231 \times 10^{-1}$ \\
\hline$\omega_{2}$ & $0.4320 \times 10$ & $\mathrm{C} 11$ & $0.6929 \times 10^{-1}$ \\
\hline $\mathrm{C} 5$ & $0.2648 \times 10$ & $\mathrm{C} 7$ & $0.6626 \times 10^{-1}$ \\
\hline E11 & $-.2233 \times 10$ & E30 & $0.5807 \times 10^{-1}$ \\
\hline $\mathrm{C} 6$ & $-.1972 \times 10$ & $\mathrm{C} 15$ & $-.5497 \times 10^{-1}$ \\
\hline E18 & $-.1921 \times 10$ & E13 & $-.5205 \times 10^{-1}$ \\
\hline C3 & $-.1624 \times 10$ & E29 & $0.4116 \times 10^{-1}$ \\
\hline E19 & $-.1281 \times 10$ & $\mathrm{E} 2$ & $0.3637 \times 10^{-1}$ \\
\hline $\mathrm{E} 21$ & $-.1145 \times 10$ & E10 & $-.3322 \times 10^{-1}$ \\
\hline E25 & $0.1093 \times 10$ & E9 & $0.2346 \times 10^{-1}$ \\
\hline E26 & $0.1012 \times 10$ & $k_{12}$ & $-.3481 \times 10^{-2}$ \\
\hline C18 & -.9614 & & \\
\hline E22 & -.9195 & & \\
\hline $\mathrm{E} 23$ & 0.7912 & & \\
\hline E27 & 0.6607 & & \\
\hline E16 & 0.5423 & & \\
\hline E14 & 0.4904 & & \\
\hline E6 & 0.4289 & & \\
\hline E17 & -.4090 & & \\
\hline
\end{tabular}


Table 16 C. Liquid Density Sensitivities n- $\mathrm{C}_{6} / \mathrm{CO}_{2}$ ALS EOS - CPU Time

\begin{tabular}{|c|c|c|c|}
\hline Factor & CPU Time & Factor & CPU Time \\
\hline E14 & $0.5809 \times 10$ & C18 & -.9560 \\
\hline $\mathrm{C} 6$ & $-.5367 \times 10$ & E18 & 0.9560 \\
\hline E15 & $0.5073 \times 10$ & E12 & 0.8089 \\
\hline E13 & $-.3896 \times 10$ & E16 & 0.8089 \\
\hline $\mathrm{C} 5$ & $0.3751 \times 10$ & E26 & 0.8089 \\
\hline $\mathrm{E} 27$ & $-.3602 \times 10$ & E31 & 0.8089 \\
\hline E30 & $0.3602 \times 10$ & C15 & 0.6619 \\
\hline $\mathrm{C} 17$ & $-.3456 \times 10$ & $\mathrm{E} 2$ & -.6619 \\
\hline E9 & $0.3456 \times 10$ & E3 & -.6619 \\
\hline$\omega_{2}$ & $-.3014 \times 10$ & $\mathrm{E} 4$ & 0.6619 \\
\hline $\mathrm{C} 13$ & $-.2868 \times 10$ & $\mathrm{C} 8$ & 0.5148 \\
\hline E11 & $-.2720 \times 10$ & $\mathrm{C} 11$ & 0.5148 \\
\hline$T_{c, 1}$ & $-.2426 \times 10$ & $\mathrm{E} 6$ & -.5148 \\
\hline$\omega_{1}$ & $-.2132 \times 10$ & E22 & -.5148 \\
\hline$k_{12}$ & $0.2132 \times 10$ & $\mathrm{C} 9$ & 0.3678 \\
\hline $\mathrm{E} 20$ & $0.2132 \times 10$ & $\mathrm{C} 16$ & -.3678 \\
\hline E7 & $-.1985 \times 10$ & E5 & 0.3678 \\
\hline $\mathrm{C} 12$ & $-.1691 \times 10$ & E19 & 0.3678 \\
\hline $\mathrm{C} 4$ & $0.1544 \times 10$ & $\mathrm{C} 10$ & -.2206 \\
\hline$P_{c, 1}$ & $0.1397 \times 10$ & E21 & 0.2206 \\
\hline$T_{c, 2}$ & $-.1397 \times 10$ & $\mathrm{C} 2$ & $0.7353 \times 10^{-1}$ \\
\hline E10 & $-.1397 \times 10$ & $\mathrm{C} 7$ & $-.7353 \times 10^{-1}$ \\
\hline $\mathrm{E} 24$ & $0.1397 \times 10$ & $\mathrm{E} 8$ & $-.7353 \times 10^{-1}$ \\
\hline$P_{c, 2}$ & $-.1250 \times 1.0$ & E28 & $0.7353 \times 10^{-1}$ \\
\hline E1 & $-.1250 \times 10$ & & \\
\hline E17 & $0.1250 \times 10$ & & \\
\hline E25 & $-.1250 \times 10$ & & \\
\hline E29 & $0.1250 \times 10$ & & \\
\hline C3 & $-.1103 \times 10$ & & \\
\hline E23 & $-.1103 \times 10$ & & \\
\hline $\mathrm{C} 1$ & -.9560 & & \\
\hline C14 & 0.9560 & & \\
\hline
\end{tabular}


Table 17 A. Liquid Density Sensitivities $n-C_{8} / \mathrm{CO}_{2}$ ALS EOS - Average Absolute Frror

\begin{tabular}{|c|c|c|c|}
\hline Factor & Avg Abs Err & Factor & Avg Abs Err \\
\hline E19 & $-.3743 \times 10^{3}$ & $\mathrm{C} 13$ & $0.1332 \times 10^{2}$ \\
\hline $\mathrm{C} 1$ & $-.2109 \times 10^{3}$ & $\mathrm{E} 26$ & $-.1242 \times 10^{2}$ \\
\hline E18 & $0.2068 \times 10^{3}$ & $\mathrm{E} 17$ & $-.1186 \times 10^{2}$ \\
\hline E11 & $-.1080 \times 10^{3}$ & $\mathrm{C} 2$ & $0.1182 \times 10^{2}$ \\
\hline $\mathrm{C} 12$ & $0.1016 \times 10^{3}$ & E12 & $-.1182 \times 10^{2}$ \\
\hline $\mathrm{E} 1$ & $-.1012 \times 10^{3}$ & $\mathrm{E} 23$ & $0.1145 \times 10^{2}$ \\
\hline $\mathrm{E} 20$ & $-.7172 \times 10^{2}$ & $\mathrm{C} 6$ & $-.1036 \times 10^{2}$ \\
\hline$T_{c, 2}$ & $0.6830 \times 10^{2}$ & $\omega_{1}$ & $0.9775 \times 10$ \\
\hline E3 & $-.6643 \times 10^{2}$ & $\mathrm{C} 15$ & $0.9652 \times 10$ \\
\hline E7 & $0.6456 \times 10^{2}$ & $\mathrm{C} 18$ & $0.9376 \times 10$ \\
\hline E30 & $-.6444 \times 10^{2}$ & $\mathrm{E} 9$ & $0.9311 \times 10$ \\
\hline$T_{c, 1}$ & $0.5480 \times 10^{2}$ & $\mathrm{C} 5$ & $-.9220 \times 10$ \\
\hline E8 & $0.5328 \times 10^{2}$ & E15 & $0.8615 \times 10$ \\
\hline $\mathrm{E} 22$ & $-.4814 \times 10^{2}$ & $\mathrm{C} 11$ & $0.8490 \times 10$ \\
\hline $\mathrm{C} 4$ & $0.4206 \times 10^{2}$ & $\mathrm{C} 16$ & $0.6873 \times 10$ \\
\hline $\mathrm{C} 8$ & $-.4205 \times 10^{2}$ & $\omega_{2}$ & $0.5947 \times 10$ \\
\hline$P_{c, 2}$ & $0.3987 \times 10^{2}$ & E31 & $-.4991 \times 10$ \\
\hline $\mathrm{E} 6$ & $0.3233 \times 10^{2}$ & $\mathrm{E} 25$ & $0.4613 \times 10$ \\
\hline$P_{c, 1}$ & $0.2909 \times 10^{2}$ & $\mathrm{C} 10$ & $0.3342 \times 10$ \\
\hline $\mathrm{E} 4$ & $-.2842 \times 10^{2}$ & C14 & $0.2797 \times 10$ \\
\hline E16 & $0.2775 \times 10^{2}$ & $\mathrm{E} 2$ & $-.2501 \times 10$ \\
\hline $\mathrm{C} 17$ & $0.2601 \times 10^{2}$ & $k_{12}$ & $-.1959 \times 10$ \\
\hline E5 & $-.2413 \times 10^{2}$ & C3 & $-.1073 \times 10$ \\
\hline E29 & $-.2367 \times 10^{2}$ & $\mathrm{C} 9$ & -.7789 \\
\hline E14 & $-.2296 \times 10^{2}$ & & \\
\hline $\mathrm{E} 28$ & $0.2044 \times 10^{2}$ & & \\
\hline E13 & $0.1987 \times 10^{2}$ & & \\
\hline E21 & $-.1842 \times 10^{2}$ & & \\
\hline E24 & $-.1697 \times 10^{2}$ & & \\
\hline E10 & $-.1559 \times 10^{2}$ & & \\
\hline $\mathrm{E} 27$ & $-.1499 \times 10^{2}$ & & \\
\hline $\mathrm{C} 7$ & $0.1335 \times 10^{2}$ & & \\
\hline
\end{tabular}


Table 17 B. Liquid Density Sensitivities n-C $\mathrm{C}_{8} / \mathrm{CO}_{2}$ ALS EOS - Bias

\begin{tabular}{|c|c|c|c|}
\hline Factor & Bias & Factor & Bias \\
\hline $\mathrm{C} 1$ & $-.9343 \times 10^{3}$ & $\mathrm{E} 25$ & 0.4472 \\
\hline$P_{c, 1}$ & $0.4889 \times 10^{3}$ & E18 & 0.4205 \\
\hline $\mathrm{C} 12$ & $0.2413 \times 10^{3}$ & E7 & 0.4110 \\
\hline$T_{c, 1}$ & $-.2391 \times 10^{3}$ & E30 & -.3576 \\
\hline $\mathrm{C} 2$ & $0.1299 \times 10^{3}$ & $\mathrm{E} 4$ & -.3230 \\
\hline $\mathrm{C} 4$ & $0.1231 \times 10^{3}$ & E14 & 0.3006 \\
\hline $\mathrm{C} 8$ & $-.1203 \times 10^{3}$ & $\mathrm{C} 10$ & 0.2719 \\
\hline$P_{r, 2}$ & $0.1059 \times 10^{3}$ & $\mathrm{C} 7$ & 0.2578 \\
\hline$\omega_{1}$ & $0.1047 \times 10^{3}$ & E29 & -.2310 \\
\hline$T_{c, 2}$ & $0.5267 \times 10^{2}$ & E24 & 0.2165 \\
\hline $\mathrm{C} 9$ & $-.3837 \times 10^{2}$ & E5 & 0.2092 \\
\hline $\mathrm{C} 17$ & $0.3180 \times 10^{2}$ & E17 & -.2020 \\
\hline C16 & $0.2209 \times 10^{2}$ & E12 & -.1827 \\
\hline$\omega_{2}$ & $0.9204 \times 10$ & $\mathrm{C} 11$ & 0.1809 \\
\hline $\mathrm{C} 13$ & $0.7265 \times 10$ & $\mathrm{C} 15$ & -.1375 \\
\hline C5 & $0.4778 \times 10$ & E28 & 0.1218 \\
\hline C6 & $-.4689 \times 10$ & E13. & 0.1052 \\
\hline C3 & $-.4663 \times 10$ & $\mathrm{E} 9$ & $0.7547 \times 10^{-1}$ \\
\hline C18 & $-.2791 \times 10$ & $\mathrm{E} 2$ & $0.7434 \times 10^{-1}$ \\
\hline E19 & $-.2016 \times 10$ & E10 & $-.7400 \times 10^{-1}$ \\
\hline E11 & $-.1286 \times 10$ & E31 & $-.4692 \times 10^{-1}$ \\
\hline E21 & -.9937 & E15 & $0.8280 \times 10^{-2}$ \\
\hline $\mathrm{E} 1$ & -.8657 & E27 & $0.4335 \times 10^{-2}$ \\
\hline E22 & -.8061 & $k_{12}$ & $-.4492 \times 10^{-3}$ \\
\hline C14 & 0.7192 & & \\
\hline E26 & 0.6401 & & \\
\hline E3 & -.6256 & & \\
\hline $\mathrm{E} 23$ & 0.6157 & & \\
\hline $\mathrm{E} 20$ & -.6138 & & \\
\hline E6 & 0.5627 & & \\
\hline E16 & 0.4608 & & \\
\hline E8 & 0.4505 & & \\
\hline
\end{tabular}


Table 17 C. Liquid , Density Sensitivities n-C $8 / \mathrm{CO}_{2}$ ALS EOS - CPU Time

\begin{tabular}{|c|c|c|c|}
\hline Factor & CPU Time & Factor & CPU Time \\
\hline C6 & $-.3750 \times 10$ & C16 & -.4688 \\
\hline C5 & $-.2813 \times 10$ & C18 & -.4688 \\
\hline E11 & $-.2188 \times 10$ & $T_{c, 2}$ & 0.4688 \\
\hline E12 & $-.2188 \times 10$ & $\mathrm{E} 4$ & 0.4688 \\
\hline E15 & $-.2188 \times 10$ & E8 & 0.4688 \\
\hline E29 & $0.2031 \times 10$ & E9 & 0.4688 \\
\hline E17 & $0.1875 \times 10$ & E23 & 0.4688 \\
\hline$\omega_{1}$ & $0.1719 \times 10$ & E24 & 0.4688 \\
\hline E10 & $0.1563 \times 10$ & E26 & 0.4688 \\
\hline $\mathrm{E} 20$ & $0.1563 \times 10$ & C3 & -.3125 \\
\hline E6 & $-.1406 \times 10$ & $\mathrm{C} 8$ & -.3125 \\
\hline E7 & $0.1406 \times 10$ & $\mathrm{C} 10$ & 0.3125 \\
\hline E28 & $-.1406 \times 10$ & $\omega_{2}$ & 0.3125 \\
\hline E14 & $-.1250 \times 10$ & E1 & 0.3125 \\
\hline C11 & $-.1094 \times 10$ & E18 & -.3125 \\
\hline $\mathrm{C} 17$ & $0.1094 \times 10$ & E31 & 0.3125 \\
\hline E3 & $-.1094 \times 10$ & $\mathrm{C} 1$ & 0.1563 \\
\hline $\mathrm{E} 27$ & $0.1094 \times 10$ & $\mathrm{C} 12$ & -.1563 \\
\hline $\mathrm{C} 4$ & 0.9375 & $\mathrm{C} 14$ & 0.1563 \\
\hline $\mathrm{C9}$ & 0.9375 & E5 & 0.1563 \\
\hline E30 & 0.9375 & E22 & 0.1563 \\
\hline C15 & 0.7813 & $\mathrm{C} 7$ & 0.0000 \\
\hline$T_{c, 1}$ & -.7813 & $P_{c, 2}$ & 0.0000 \\
\hline$P_{c, 1}$ & 0.7813 & $k_{12}$ & 0.0000 \\
\hline $\mathrm{E} 2$ & 0.7813 & & \\
\hline E25 & -.7813 & & \\
\hline E13 & -.6250 & & \\
\hline E16 & 0.6250 & & \\
\hline E19 & 0.6250 & & \\
\hline $\mathrm{E} 21$ & -.6250 & & \\
\hline $\mathrm{C} 2$ & -.4688 & & \\
\hline $\mathrm{C} 13$ & 0.4688 & & \\
\hline
\end{tabular}


Table 18 A. Liquid Density Sensitivities n-C $\mathrm{C}_{10} / \mathrm{CO}_{2}$ ALS EOS - Average Absolute Error

\begin{tabular}{|c|c|c|c|}
\hline Factor & Avg Abs Err & Factor & Avg Abs Err \\
\hline$T_{c, 2}$ & $-.3124 \times 10^{2}$ & E24 & -.2841 \\
\hline E18 & $0.1438 \times 10^{2}$ & $\mathrm{C} 16$ & -.2256 \\
\hline $\mathrm{C} 12$ & $-.9597 \times 10$ & C18 & 0.1364 \\
\hline$P_{c, 2}$ & $0.7140 \times 10$ & E13 & $0.7917 \times 10^{-1}$ \\
\hline E21 & $-.7080 \times 10$ & E9 & $0.7341 \times 10^{-1}$ \\
\hline $\mathrm{C} 1$ & $0.5850 \times 10$ & E14 & $0.7302 \times 10^{-1}$ \\
\hline E25 & $-.4636 \times 10$ & C10 & $-.6812 \times 10^{-1}$ \\
\hline $\mathrm{E} 27$ & $-.2660 \times 10$ & E16 & $0.5367 \times 10^{-1}$ \\
\hline E11 & $-.2612 \times 10$ & $\mathrm{C} 17$ & $0.4924 \times 10^{-1}$ \\
\hline$T_{c, 1}$ & $-.2067 \times 10$ & C14 & $-.4881 \times 10^{-1}$ \\
\hline $\mathrm{C} 8$ & $0.1942 \times 10$ & $\mathrm{E} 20$ & $-.4593 \times 10^{-1}$ \\
\hline$P_{c, 1}$ & $-.1829 \times 10$ & $\mathrm{C} 13$ & $0.4540 \times 10^{-1}$ \\
\hline$\omega_{2}$ & $-.1721 \times 10$ & E19 & $-.4332 \times 10^{-1}$ \\
\hline E7 & $0.1634 \times 10$ & C3 & $0.4058 \times 10^{-1}$ \\
\hline $\mathrm{E} 22$ & $0.1622 \times 10$ & $\mathrm{E} 4$ & $-.3266 \times 10^{-1}$ \\
\hline E3 & $-.1519 \times 10$ & E31 & $0.3070 \times 10^{-1}$ \\
\hline $\mathrm{C} 4$ & $-.1444 \times 10$ & E12 & $-.2478 \times 10^{-1}$ \\
\hline $\mathrm{E} 23$ & $-.1350 \times 10$ & E30 & $-.2249 \times 10^{-1}$ \\
\hline E26 & $-.1336 \times 10$ & $\mathrm{C} 11$ & $0.1588 \times 10^{-1}$ \\
\hline E29 & $0.1249 \times 10$ & E28 & $0.1570 \times 10^{-1}$ \\
\hline E5 & -.9795 & $\mathrm{E} 2$ & $0.1142 \times 10^{-1}$ \\
\hline E15 & 0.9286 & E10 & $0.9286 \times 10^{-2}$ \\
\hline E17 & 0.6353 & $\mathrm{C} 15$ & $-.7183 \times 10^{-2}$ \\
\hline $\mathrm{C} 2$ & -.5430 & $\mathrm{C} 5$ & $0.5155 \times 10^{-2}$ \\
\hline $\mathrm{E} 1$ & -.4858 & & \\
\hline $\mathrm{C} 9$ & 0.4097 & & \\
\hline$\omega_{1}$ & -.3932 & & \\
\hline$k_{12}$ & -.3909 & & \\
\hline C6 & 0.3601 & & \\
\hline $\mathrm{C} 7$ & -.3595 & & \\
\hline E6 & -.3182 & & \\
\hline $\mathrm{E} 8$ & 0.3113 & & \\
\hline
\end{tabular}


Table 18 B. Liquid Density Sensitivities n-C $10 / \mathrm{CO}_{2}$ ALS EOS - Bias

\begin{tabular}{llll} 
Factor & Bias & Factor & Bias \\
$T_{c, 2}$ & $-.1060 \times 10^{3}$ & E12 & $0.9981 \times 10^{-1}$ \\
C12 & $-.5714 \times 10^{2}$ & E14 & $-.9356 \times 10^{-1}$ \\
C1 & $0.4632 \times 10^{2}$ & E23 & $-.9279 \times 10^{-1}$ \\
C8 & $0.1830 \times 10^{2}$ & E17 & $-.8491 \times 10^{-1}$ \\
$P_{c, 2}$ & $0.1196 \times 10^{2}$ & E19 & $0.8280 \times 10^{-1}$ \\
C4 & $-.9716 \times 10$ & E5 & $0.81 .88 \times 10^{-1}$ \\
$P_{c, 1}$ & $-.7645 \times 10$ & E13 & $-.4522 \times 10^{-1}$ \\
C2 & $-.5305 \times 10$ & C10 & $-.4117 \times 10^{-1}$ \\
C9 & $0.4481 \times 10$ & E16 & $-.3517 \times 10^{-1}$ \\
$T_{c, 1}$ & $-.2681 \times 10$ & E4 & $0.3470 \times 10^{-1}$ \\
$\omega_{1}$ & $-.2493 \times 10$ & E20 & $0.1899 \times 10^{-1}$ \\
E21 & $0.1331 \times 10$ & C11 & $0.1689 \times 10^{-1}$ \\
E11 & $0.1253 \times 10$ & C16 & $0.1314 \times 10^{-1}$ \\
C13 & $-.1014 \times 10$ & E2 & $-.1265 \times 10^{-1}$ \\
E27 & -.9241 & E24 & $-.1133 \times 10^{-1}$ \\
C17 & -.7597 & E18 & $-.1024 \times 10^{-1}$ \\
$\omega_{2}$ & -.6992 & C18 & $0.1019 \times 10^{-1}$ \\
E29 & -.5776 & E31 & $-.9933 \times 10^{-2}$ \\
E26 & 0.4603 & E30 & $-.8866 \times 10^{-2}$ \\
E7 & -.4546 & E10 & $-.8558 \times 10^{-2}$ \\
E25 & 0.4403 & E9 & $-.7045 \times 10^{-2}$ \\
C6 & 0.3675 & E28 & $0.3370 \times 10^{-2}$ \\
C5 & -.3136 & C7 & $-.2939 \times 10^{-2}$ \\
E22 & -.3049 & C15 & $0.1969 \times 10^{-2}$ \\
E15 & -.2654 & & \\
C3 & 0.1724 & & \\
E3 & 0.1713 & & \\
E1 & 0.1689 & & \\
$k_{12}$ & -.1109 & & \\
C14 & -.1107 & & \\
E8 & -.1059 & & \\
E6 & $0.9995 \times 10^{-1}$ & & \\
& & & \\
\hline
\end{tabular}


Table $18 \mathrm{C}$. Liquid Density Sensitivities $n-C_{10} / \mathrm{CO}_{2}$ ALS EOS - CPU Time

\begin{tabular}{|c|c|c|c|}
\hline Factor & CPU Time & Factor & CPU Time \\
\hline$\omega_{1}$ & $0.2823 \times 10$ & $\mathrm{C} 18$ & 0.6048 \\
\hline$T_{c, 2}$ & $-.2419 \times 10$ & $P_{c, 2}$ & 0.6048 \\
\hline E12 & $0.2419 \times 10$ & E15 & 0.6048 \\
\hline E13 & $0.2218 \times 10$ & E16 & -.6048 \\
\hline E22 & $-.2218 \times 10$ & E6 & 0.4032 \\
\hline $\mathrm{C} 4$ & $0.2016 \times 10$ & E21 & 0.4032 \\
\hline E19 & $-.2016 \times 10$ & $\mathrm{E} 25$ & -.4032 \\
\hline E14 & $0.1815 \times 10$ & E28 & 0.4032 \\
\hline E10 & $-.1613 \times 10$ & E30 & 0.4032 \\
\hline E11 & $0.1613 \times 10$ & $\mathrm{C} 1$ & -.2016 \\
\hline $\mathrm{C} 8$ & $-.1411 \times 10$ & $\mathrm{C} 3$ & 0.2016 \\
\hline$T_{c, 1}$ & $0.1411 \times 10$ & $\mathrm{C} 16$ & -.2016 \\
\hline$k_{12}$ & $-.1411 \times 10$ & C17 & -.2016 \\
\hline E2 & $0.1411 \times 10$ & $\mathrm{E} 7$ & 0.2016 \\
\hline E17 & $-.1411 \times 10$ & $\mathrm{E} 8$ & -.2016 \\
\hline $\mathrm{C} 7$ & $-.1210 \times 10$ & E9 & -.2016 \\
\hline E1 & $0.1210 \times 10$ & E18 & 0.2016 \\
\hline E4 & $-.1210 \times 10$ & E23 & -.2016 \\
\hline E5 & $0.1210 \times 10$ & E24 & -.2016 \\
\hline E27 & $-.1210 \times 10$ & E29 & -.2016 \\
\hline C9 & $-.1008 \times 10$ & $\mathrm{C} 2$ & 0.0000 \\
\hline C10 & $-.1008 \times 10$ & C13 & 0.0000 \\
\hline $\mathrm{C} 14$ & $-.1008 \times 10$ & $P_{c, 1}$ & 0.0000 \\
\hline $\mathrm{C} 15$ & $0.1008 \times 10$ & E26 & 0.0000 \\
\hline$\omega_{2}$ & $0.1008 \times 10$ & & \\
\hline E31 & $-.1008 \times 10$ & & \\
\hline $\mathrm{C} 5$ & 0.8065 & & \\
\hline $\mathrm{C} 6$ & 0.8065 & & \\
\hline $\mathrm{C} 11$ & -.8065 & & \\
\hline $\mathrm{C} 12$ & 0.8065 & & \\
\hline E3 & 0.8065 & & \\
\hline E20 & 0.8065 & & \\
\hline
\end{tabular}


Table 19 A. Liquid Density Sensitivities $n-C_{4} / \mathrm{CO}_{2}$ ESD EOS - Average Absolute Error

$\begin{array}{llll}\text { Factor } & \text { Avg Abs Err } & \text { Factor } & \text { Avg Abs Err } \\ \text { C16 } & -.1826 \times 10^{2} & \text { C12 } & -.5350 \\ \text { C8 } & -.1126 \times 10^{2} & \text { E22 } & -.5334 \\ \text { C4 } & 0.1075 \times 10^{2} & \text { E18 } & -.4896 \\ \text { C17 } & 0.9865 \times 10 & \omega_{1} & -.4767 \\ T_{c, 2} & 0.9658 \times 10 & \text { E16 } & -.4342 \\ \text { C5 } & 0.8197 \times 10 & \text { E10 } & 0.3837 \\ \text { C7 } & -.6505 \times 10 & \text { E17 } & -.3671 \\ T_{c, 1} & 0.5606 \times 10 & \text { E27 } & 0.2982 \\ \text { C11 } & -.3244 \times 10 & \text { E3 } & -.2982 \\ P_{c, 1} & 0.2650 \times 10 & \mathrm{E} 4 & -.2357 \\ \text { C14 } & 0.2354 \times 10 & k_{12} & 0.2241 \\ P_{c, 2} & 0.2308 \times 10 & \text { E21 } & -.2037 \\ \text { C9 } & -.2235 \times 10 & \mathrm{C} 15 & 0.1242 \\ \text { E19 } & -.1682 \times 10 & \text { C2 } & 0.1160 \\ \text { E5 } & -.1552 \times 10 & \text { E20 } & -.1158 \\ \text { E29 } & 0.1533 \times 10 & \text { C1 } & -.1147 \\ \text { C10 } & -.1405 \times 10 & \text { E7 } & 0.8959 \times 10^{-1} \\ \text { C13 } & 0.1211 \times 10 & \text { C3 } & 0.8510 \times 10^{-1} \\ \text { E25 } & -.1204 \times 10 & \text { E14 } & 0.7355 \times 10^{-1} \\ \text { C6 } & 0.9746 & \text { E11 } & -.6997 \times 10^{-1} \\ \text { E2 } & -.9303 & \text { E24 } & -.2107 \times 10^{-1} \\ \text { E31 } & -.9163 & \text { E8 } & 0.1597 \times 10^{-1} \\ \text { E1 } & -.9127 & \text { E15 } & -.1015 \times 10^{-1} \\ \text { E6 } & 0.9098 & & \\ \text { E30 } & 0.8788 & & \\ \text { E28 } & 0.8492 & & \\ \text { E9 } & 0.8422 & & \\ \text { E13 } & 0.8319 & & \\ \omega_{2} & 0.7782 & & \\ \text { E26 } & 0.7648 & & \\ \text { E23 } & -.6767 & & \\ \text { E12 } & 0.5459 & & \end{array}$


Table 19 B. Liquid Density Sensitivities n-C $4 / \mathrm{CO}_{2}$ ESD EOS - Bias

$\begin{array}{llll}\text { Factor } & \text { Bias } & \text { Factor } & \text { Bias } \\ \text { C16 } & -.2535 \times 10^{2} & \text { E16 } & -.4346 \\ \text { C8 } & -.1622 \times 10^{2} & \text { E23 } & -.4298 \\ \text { C4 } & 0.1565 \times 10^{2} & \text { E1 } & -.4264 \\ \text { C17 } & 0.1472 \times 10^{2} & \text { E22 } & -.4018 \\ \text { C5 } & 0.1174 \times 10^{2} & \omega_{1} & -.3956 \\ T_{c, 2} & 0.1112 \times 10^{2} & \text { E30 } & 0.3588 \\ \text { C7 } & -.9537 \times 10 & \text { E4 } & -.3456 \\ T_{c, 1} & 0.7232 \times 10 & \text { E3 } & -.3017 \\ P_{c, 1} & 0.7152 \times 10 & \text { C2 } & 0.2960 \\ \text { C11 } & -.6890 \times 10 & \text { E10 } & 0.2439 \\ \text { C14 } & 0.5638 \times 10 & \text { E21 } & -.2327 \\ P_{c, 2} & 0.3539 \times 10 & \text { E17 } & -.2268 \\ \text { C10 } & -.3220 \times 10 & \text { E11 } & 0.2011 \\ \text { C9 } & -.3046 \times 10 & k_{12} & 0.1724 \\ \text { C13 } & 0.2946 \times 10 & \text { E14 } & 0.1562 \\ \text { E19 } & -.1545 \times 10 & \text { E27 } & 0.1134 \\ \text { C6 } & 0.1365 \times 10 & \text { C3 } & 0.9186 \times 10^{-1} \\ \text { E5 } & -.1279 \times 10 & \text { E20 } & -.7406 \times 10^{-1} \\ \text { E29 } & 0.1272 \times 10 & \text { E8 } & 0.6488 \times 10^{-1} \\ \text { C15 } & 0.9346 & \text { E7 } & 0.5567 \times 10^{-1} \\ \text { E25 } & -.9323 & \text { C1 } & 0.5342 \times 10^{-1} \\ \text { E9 } & 0.8749 & \text { E15 } & -.3251 \times 10^{-1} \\ \omega_{2} & 0.8658 & \text { E24 } & -.1908 \times 10^{-1} \\ \text { E31 } & -.8025 & & \\ \text { C12 } & -.7931 & & \\ \text { E26 } & 0.7135 & & \\ \text { E13 } & 0.7041 & & \\ \text { E6 } & 0.5997 & & \\ \text { E12 } & 0.5473 & & \\ \text { E18 } & -.5387 & & \\ \text { E28 } & 0.5319 & & \\ \text { E2 } & -.4817 & & \\ & & & \end{array}$


Table 19 C. Liquid Density Sensitivities $n-\mathrm{C}_{4} / \mathrm{CO}_{2}$ ESD EOS - CPU Time

$\begin{array}{llll}\text { Factor } & \text { CPU Time } & \text { Factor } & \text { CPU Time } \\ \text { C6 } & -.4912 \times 10 & \text { E22 } & 0.5952 \\ \text { E11 } & 0.4912 \times 10 & \text { E23 } & 0.5952 \\ \omega_{2} & -.4614 \times 10 & \text { C4 } & 0.4464 \\ \text { C17 } & -. .571 \times 10 & k_{12} & 0.4464 \\ \text { E15 } & -.2829 \times 10 & \text { E10 } & 0.4464 \\ P_{c, 1} & 0.2083 \times 10 & \text { E16 } & 0.4464 \\ \omega_{1} & -.2083 \times 10 & \text { E19 } & 0.4464 \\ \text { E9 } & 0.2083 \times 10 & \text { C1 } & 0.2976 \\ \text { E27 } & 0.2083 \times 10 & \text { C11 } & 0.2976 \\ \text { E30 } & -.1935 \times 10 & \text { C12 } & -.2976 \\ \text { C2 } & -.1786 \times 10 & P_{c, 2} & -.2976 \\ \text { C3 } & 0.1637 \times 10 & \text { E4 } & 0.2976 \\ T_{c, 2} & -.1488 \times 10 & \text { E8 } & -.2976 \\ \text { E17 } & 0.1339 \times 10 & \text { E29 } & 0.2976 \\ \text { E3 } & -.1190 \times 10 & \text { C10 } & -.1488 \\ \text { E26 } & -.1190 \times 10 & \text { E1 } & -.1488 \\ \text { E28 } & 0.1190 \times 10 & \text { E12 } & -.1488 \\ \text { C9 } & -.1042 \times 10 & \text { E31 } & 0.1488 \\ \text { E13 } & 0.1042 \times 10 & \text { C14 } & 0.0000 \\ \text { E14 } & 0.1042 \times 10 & \text { E2 } & 0.0000 \\ \text { E18 } & -.1042 \times 10 & \text { E6 } & 0.0000 \\ \text { E21 } & 0.1042 \times 10 & \text { E7 } & 0.0000 \\ \text { C13 } & -.8929 & \text { E25 } & 0.0000 \\ \text { C15 } & 0.8929 & & \\ \text { C16 } & -.8929 & & \\ \text { E24 } & 0.8929 & & \\ \text { C5 } & -.7440 & & \\ \text { C7 } & 0.7440 & & \\ \text { C8 } & 0.7440 & & \\ \text { E20 } & 0.7440 & & \\ T_{c, 1} & 0.5952 & & \\ \text { E5 } & 0.5952 & & \\ & & & \end{array}$


Table 20 A. Liquid Density Sensitivities n- $\mathrm{C}_{6} / \mathrm{CO}_{2} \mathrm{ESD}$ EOS - Average Absolute Error

$\begin{array}{llll}\text { Factor } & \text { Avg Abs Err } & \text { Factor } & \text { Avg Abs Err } \\ \text { C16 } & -.1484 \times 10^{3} & \text { E29 } & 0.1146 \times 10^{2} \\ P_{c, 1} & 0.1342 \times 10^{3} & \text { E16 } & -.1141 \times 10^{2} \\ \text { C14 } & 0.1256 \times 10^{3} & \text { E13 } & 0.1140 \times 10^{2} \\ \text { C17 } & 0.1187 \times 10^{3} & \text { E12 } & 0.1067 \times 10^{2} \\ \text { C11 } & -.1145 \times 10^{3} & \text { E26 } & 0.9997 \times 10 \\ \text { C8 } & -.1077 \times 10^{3} & \text { E14 } & 0.8551 \times 10 \\ \text { C5 } & 0.8435 \times 10^{2} & \text { E7 } & 0.8330 \times 10 \\ \text { C4 } & 0.8099 \times 10^{2} & \omega_{2} & 0.5843 \times 10 \\ P_{c, 2} & 0.6106 \times 10^{2} & \text { E9 } & 0.4885 \times 10 \\ \text { C13 } & 0.5958 \times 10^{2} & \text { C2 } & 0.4843 \times 10 \\ \text { C15 } & 0.5527 \times 10^{2} & \text { E31 } & -.4663 \times 10 \\ \text { C7 } & -.5511 \times 10^{2} & \text { E4 } & 0.3362 \times 10 \\ \text { E2 } & -.3784 \times 10^{2} & \text { E15 } & -.3190 \times 10 \\ \text { C10 } & -.3477 \times 10^{2} & \text { E18 } & 0.2671 \times 10 \\ \text { E30 } & 0.3260 \times 10^{2} & \text { C6 } & 0.2404 \times 10 \\ \text { E20 } & -.3145 \times 10^{2} & \text { E19 } & -.1846 \times 10 \\ \text { E6 } & 0.2696 \times 10^{2} & \text { E8 } & 0.1654 \times 10 \\ \text { E1 } & -.2350 \times 10^{2} & \text { E24 } & -.1440 \times 10 \\ \text { C9 } & -.2311 \times 10^{2} & \text { C3 } & 0.1405 \times 10 \\ T_{c, 2} & 0.2198 \times 10^{2} & \text { C12 } & 0.9782 \\ T_{c, 1} & -.2181 \times 10^{2} & \omega_{1} & 0.6747 \\ \text { E23 } & -.1977 \times 10^{2} & \text { E3 } & 0.5191 \\ \text { E27 } & 0.1974 \times 10^{2} & \text { E10 } & 0.2452 \\ \text { E22 } & -.1734 \times 10^{2} & & \\ \text { E21 } & -.1694 \times 10^{2} & & \\ \text { E28 } & 0.1688 \times 10^{2} & & \\ \text { E25 } & -.1557 \times 10^{2} & & \\ \text { E5 } & -.1540 \times 10^{2} & & \\ \text { C1 } & 0.1512 \times 10^{2} & & \\ k_{12} & -.1458 \times 10^{2} & & \\ \text { E11 } & -.1369 \times 10^{2} & & \\ \text { E17 } & -.1270 \times 10^{2} & & \\ & & & \end{array}$


Table 20 B. Liquid Density Sensitivities $n-C_{6} / \mathrm{CO}_{2}$ ESD EOS - Bias

\begin{tabular}{|c|c|c|c|}
\hline Factor & Bias & Factor & Bias \\
\hline $\mathrm{C} 16$ & $-.1815 \times 10^{3}$ & E31 & 0.6295 \\
\hline$P_{c, 1}$ & $0.1727 \times 10^{3}$ & E9 & -.5822 \\
\hline $\mathrm{C} 11$ & $-.1663 \times 10^{3}$ & E12 & -.5475 \\
\hline $\mathrm{C} 17$ & $0.1476 \times 10^{3}$ & E6 & -.4996 \\
\hline $\mathrm{C} 8$ & $-.1416 \times 10^{3}$ & E16 & 0.4043 \\
\hline C14 & $0.1408 \times 10^{3}$ & E28 & -.3867 \\
\hline $\mathrm{C} 4$ & $0.1148 \times 10^{3}$ & $\mathrm{E} 27$ & 0.3088 \\
\hline C5 & $0.1078 \times 10^{3}$ & E2 & 0.2853 \\
\hline$P_{c, 2}$ & $0.6795 \times 10^{2}$ & $\mathrm{E} 23$ & -.2718 \\
\hline $\mathrm{C} 7$ & $-.6584 \times 10^{2}$ & E15 & -.1878 \\
\hline C10 & $-.5853 \times 10^{2}$ & E21 & -.1862 \\
\hline $\mathrm{C} 13$ & $0.5643 \times 10^{2}$ & E20 & -.1729 \\
\hline C15 & $0.4338 \times 10^{2}$ & E1 & 0.1521 \\
\hline $\mathrm{C} 9$ & $-.3070 \times 10^{2}$ & E10 & 0.1228 \\
\hline$T_{c, 1}$ & $-.2473 \times 10^{2}$ & E14 & 0.1112 \\
\hline$T_{c, 2}$ & $0.2218 \times 10^{2}$ & E11 & -.1025 \\
\hline $\mathrm{C} 1$ & $0.1638 \times 10^{2}$ & E7 & $-.7468 \times 10^{-1}$ \\
\hline $\mathrm{C} 2$ & $0.1599 \times 10^{2}$ & E17 & $-.7010 \times 10^{-1}$ \\
\hline $\mathrm{C} 12$ & $-.1586 \times 10^{2}$ & E24 & $-.5622 \times 10^{-1}$ \\
\hline C6 & $0.1551 \times 10^{2}$ & 푸 & $-.4420 \times 10^{-1}$ \\
\hline$\omega_{1}$ & $0.1284 \times 10^{2}$ & E3 & $0.4196 \times 10^{-1}$ \\
\hline$\omega_{2}$ & $0.4501 \times 10$ & $k_{12}$ & $0.2212 \times 10^{-1}$ \\
\hline E19 & $0.1619 \times 10$ & E8 & $0.3440 \times 10^{-2}$ \\
\hline E29 & $-.1559 \times 10$ & & \\
\hline E5 & $0.1505 \times 10$ & & \\
\hline E18 & $0.1485 \times 10$ & & \\
\hline E25 & $0.1115 \times 10$ & & \\
\hline E13 & $-.1099 \times 10$ & & \\
\hline E26 & -.9961 & & \\
\hline C3 & 0.6763 & & \\
\hline E22 & 0.6542 & & \\
\hline $\mathrm{E} 4$ & 0.6432 & & \\
\hline
\end{tabular}


Table 20 C. Liquid Density Sensitivities $n-C_{6} / \mathrm{CO}_{2}$ ESD EOS - OPU Time

\begin{tabular}{|c|c|c|c|}
\hline Factor & CPU Time & Factor & CPU Time \\
\hline E30 & $0.1843 \times 10$ & E5 & 0.4008 \\
\hline $\mathrm{C} 13$ & $-.1683 \times 10$ & E6 & 0.4008 \\
\hline E19 & $0.1523 \times 10$ & E21 & 0.4008 \\
\hline $\mathrm{C} 12$ & $0.1362 \times 10$ & E25 & 0.4008 \\
\hline E14 & $-.1362 \times 10$ & E28 & -.4008 \\
\hline$P_{0,1}$ & $-.1202 \times 10$ & $\mathrm{C} 11$ & -.2404 \\
\hline E3 & $0.1202 \times 10$ & C14 & -.2404 \\
\hline E12 & $0.1202 \times 10$ & $T_{c, 1}$ & 0.2404 \\
\hline C5 & $-.1042 \times 10$ & $T_{c, 2}$ & -.2404 \\
\hline $\mathrm{C} 10$ & 0.8815 & $k_{12}$ & -.2404 \\
\hline E9 & 0.8815 & $\mathrm{E} 1$ & 0.2404 \\
\hline E10 & -.8815 & E16 & 0.2404 \\
\hline E11 & -.8815 & E17 & 0.2404 \\
\hline $\mathrm{C} 2$ & -.7213 & $\mathrm{E} 20$ & -.2404 \\
\hline C3 & -.7213 & E26 & -.2404 \\
\hline C6 & 0.7213 & E27 & 0.2404 \\
\hline $\mathrm{C} 7$ & 0.7213 & E29 & 0.2404 \\
\hline $\mathrm{C} 17$ & -.7213 & $P_{c, 2}$ & $0.8013 \times 10^{-1}$ \\
\hline E22 & -.7213 & $\mathrm{E} 4$ & $-.8013 \times 10^{-1}$ \\
\hline E23 & 0.7213 & E7 & $0.8013 \times 10^{-1}$ \\
\hline E31 & 0.7213 & E8 & $0.8013 \times 10^{-1}$ \\
\hline $\mathrm{C} 1$ & -.5610 & E13 & $0.8013 \times 10^{-1}$ \\
\hline $\mathrm{C} 4$ & 0.5610 & E24 & $0.8013 \times 10^{-1}$ \\
\hline $\mathrm{C} 9$ & -.5610 & & \\
\hline $\mathrm{C} 16$ & 0.5610 & & \\
\hline $\mathrm{E} 2$ & 0.5610 & & \\
\hline E15 & 0.5610 & & \\
\hline E18 & -.5610 & & \\
\hline $\mathrm{C} 8$ & 0.4008 & & \\
\hline C15 & -.4008 & & \\
\hline$\omega_{1}$ & -.4008 & & \\
\hline$\omega_{2}$ & -.4008 & & \\
\hline
\end{tabular}


Table 21 A. Liquid Density Sensitivities $n-C_{8} / \mathrm{CO}_{2}$ ESD EOS - Average Absolute Error

$\begin{array}{llll}\text { Factor } & \text { Avg Abs Err } & \text { Factor } & \text { Avg Abs Err } \\ P_{c, 1} & 0.1894 \times 10^{3} & \omega_{1} & 0.1467 \times 10^{2} \\ \text { C14 } & 0.1674 \times 10^{3} & \text { E16 } & -.1463 \times 10^{2} \\ \text { C11 } & -.1644 \times 10^{3} & \text { C12 } & -.1421 \times 10^{2} \\ \text { C16 } & -.1379 \times 10^{3} & \text { E14 } & 0.1293 \times 10^{2} \\ \text { C17 } & 0.1370 \times 10^{3} & k_{12} & -.1156 \times 10^{2} \\ \text { C8 } & -.1142 \times 10^{3} & \text { E5 } & -.1141 \times 10^{2} \\ \text { C5 } & 0.9699 \times 10^{2} & \text { E19 } & 0.1131 \times 10^{2} \\ \text { C15 } & 0.8218 \times 10^{2} & \text { E26 } & 0.1037 \times 10^{2} \\ P_{c, 2} & 0.8130 \times 10^{2} & \text { E25 } & -. .8754 \times 10 \\ \text { C4 } & 0.7260 \times 10^{2} & \text { E7 } & 0.7962 \times 10 \\ \text { C13 } & 0.6679 \times 10^{2} & \text { E10 } & -.7485 \times 10 \\ T_{c, 1} & -.6038 \times 10^{2} & \text { E13 } & 0.6501 \times 10 \\ \text { C7 } & -.4845 \times 10^{2} & \text { E12 } & 0.5760 \times 10 \\ \text { E20 } & -.4791 \times 10^{2} & \text { C3 } & 0.5481 \times 10 \\ \text { E2 } & -.3907 \times 10^{2} & \text { E29 } & 0.5284 \times 10 \\ \text { E30 } & 0.3900 \times 10^{2} & \omega_{2} & 0.5027 \times 10 \\ \text { C9 } & -.3641 \times 10^{2} & \text { E3 } & 0.3087 \times 10 \\ \text { C10 } & -.3415 \times 10^{2} & \text { E31 } & 0.2711 \times 10 \\ \text { E23 } & -.2820 \times 10^{2} & \text { C6 } & 0.2344 \times 10 \\ \text { E6 } & 0.2773 \times 10^{2} & \text { E4 } & 0.1933 \times 10 \\ \text { E15 } & -.2711 \times 10^{2} & \text { E24 } & -.1634 \times 10 \\ \text { E27 } & 0.2485 \times 10^{2} & \text { E8 } & 0.1209 \times 10 \\ \text { E1 } & -.3463 \times 10^{2} & \text { E9 } & -.3658 \\ \text { E21 } & -.2255 \times 10^{2} & & \\ \text { E28 } & 0.2066 \times 10^{2} & & \\ \text { E11 } & -.1990 \times 10^{2} & & \\ \text { C2 } & 0.1925 \times 10^{2} & & \\ \text { E17 } & -.1878 \times 10^{2} & & \\ \text { C1 } & 0.1823 \times 10^{2} & & \\ \text { E22 } & -.1721 \times 10^{2} & & \\ \text { E18 } & 0.1685 \times 10^{2} & & . \\ T_{c, 2} & 0.1620 \times 10^{2} & & \end{array}$


Table 21 B. Liquid Density Sensitivities n- $\mathrm{C}_{8} / \mathrm{CO}_{2}$ ESD EOS - Bias

$\begin{array}{llll}\text { Factor } & \text { Bias } & \text { Factor } & \text { Bias } \\ P_{c, 1} & 0.2629 \times 10^{3} & \text { E31 } & 0.4831 \\ \text { C11 } & -.2314 \times 10^{3} & \text { E23 } & -.4389 \\ \text { C14 } & 0.1889 \times 10^{3} & T_{c, 2} & 0.3757 \\ \text { C16 } & -.1757 \times 10^{3} & \text { E6 } & -.3590 \\ \text { C17 } & 0.1692 \times 10^{3} & \text { E9 } & -.3537 \\ \text { C8 } & -.1498 \times 10^{3} & \text { E27 } & 0.3278 \\ \text { C5 } & 0.1213 \times 10^{3} & \text { E15 } & -.3083 \\ \text { C4 } & 0.1049 \times 10^{3} & \text { E20 } & -.2331 \\ T_{c, 1} & -.9538 \times 10^{2} & \text { E21 } & -.2172 \\ P_{c, 2} & 0.8101 \times 10^{2} & \text { E14 } & 0.2099 \\ \text { C15 } & 0.7529 \times 10^{2} & \text { E2 } & 0.1882 \\ \text { C10 } & -.6557 \times 10^{2} & \text { E17 } & -.1785 \\ \text { C13 } & 0.6141 \times 10^{2} & \text { E16 } & 0.1744 \\ \text { C7 } & -.5753 \times 10^{2} & \text { E28 } & -.1543 \\ \text { C9 } & -.4155 \times 10^{2} & \text { E30 } & 0.1469 \\ \omega_{1} & 0.3394 \times 10^{2} & \text { E10 } & 0.1215 \\ \text { C2 } & 0.3373 \times 10^{2} & \text { E7 } & -.8174 \times 10^{-1} \\ \text { C12 } & -.2866 \times 10^{2} & \text { E11 } & -.3878 \times 10^{-1} \\ \text { C1 } & 0.2578 \times 10^{2} & \text { E24 } & -.2936 \times 10^{-1} \\ \text { C6 } & 0.2260 \times 10^{2} & \text { E8 } & -.2798 \times 10^{-1} \\ \omega_{2} & 0.3667 \times 10 & \text { E3 } & -.2013 \times 10^{-1} \\ \text { C3 } & 0.1942 \times 10 & k_{12} & 0.9677 \times 10^{-2} \\ \text { E18 } & 0.1421 \times 10 & \text { E1 } & -.1978 \times 10^{-2} \\ \text { E19 } & 0.1195 \times 10 & & \\ \text { E29 } & -.1187 \times 10 & & \\ \text { E5 } & 0.1186 \times 10 & & \\ \text { E13 } & -.8937 & & \\ \text { E25 } & 0.8299 & & \\ \text { E26 } & -.7296 & & \\ \text { E22 } & 0.5651 & & \\ \text { E12 } & -.4997 & & \\ \text { E4 } & 0.4899 & & \end{array}$


Table 21 C. Liquid Density Sensitivities $n-C_{8} / \mathrm{CO}_{2}$ ESD EOS - CPU Time

\begin{tabular}{|c|c|c|c|}
\hline Factor & CPU Time & Factor & CPU Time \\
\hline C6 & $-.6927 \times 10$ & E31 & $0.1014 \times 10$ \\
\hline E10 & $0.4054 \times 10$ & C10 & 0.8446 \\
\hline E27 & $-.4054 \times 10$ & E28 & 0.8446 \\
\hline $\mathrm{C} 2$ & $0.3886 \times 10$ & C7 & -.6757 \\
\hline E3 & $0.3549 \times 10$ & $\mathrm{E} 4$ & -.6757 \\
\hline E7 & $0.3549 \times 10$ & E9 & -.6757 \\
\hline E13 & $-.3549 \times 10$ & E15 & -.6757 \\
\hline$k_{12}$ & $0.3211 \times 10$ & E16 & -.6757 \\
\hline E11 & $-.2873 \times 10$ & E22 & 0.6757 \\
\hline $\mathrm{C} 17$ & $-.2534 \times 10$ & C11 & 0.5068 \\
\hline C15 & $-.2365 \times 10$ & $P_{c, 2}$ & 0.5068 \\
\hline C5 & $-.2196 \times 10$ & E18 & 0.5068 \\
\hline $\mathrm{C} 16$ & $0.2196 \times 10$ & E20 & 0.5068 \\
\hline$\omega_{2}$ & $0.2196 \times 10$ & $\mathrm{C} 8$ & 0.3378 \\
\hline E14 & $0.2196 \times 10$ & C13 & 0.3378 \\
\hline E25 & $0.2196 \times 10$ & $\omega_{1}$ & 0.3378 \\
\hline C14 & $-.2027 \times 10$ & E2 & -.1689 \\
\hline E26 & $-.2027 \times 10$ & E6 & 0.1689 \\
\hline $\mathrm{C} 1$ & $0.1858 \times 10$ & E24 & 0.1689 \\
\hline$T_{c, 1}$ & $-.1689 \times 10$ & E29 & -.1689 \\
\hline E23 & $-.1689 \times 10$ & $\mathrm{C} 4$ & 0.0000 \\
\hline E12 & $-.1520 \times 10$ & $P_{\mathrm{c}, 1}$ & 0.0000 \\
\hline E17 & $0.1520 \times 10$ & $\mathrm{E} 1$ & 0.0000 \\
\hline C3 & $0.1351 \times 10$ & & \\
\hline E5 & $-.1351 \times 10$ & & \\
\hline E21 & $-.1351 \times 10$ & & \\
\hline E30 & $-.1351 \times 10$ & & \\
\hline C9 & $0.1182 \times 10$ & & \\
\hline E19 & $0.1182 \times 10$ & & \\
\hline $\mathrm{C} 12$ & $-.1014 \times 10$ & & \\
\hline$T_{c, 2}$ & $-.1014 \times 10$ & & \\
\hline $\mathrm{E} 8$ & $0.1014 \times 10$ & & \\
\hline
\end{tabular}


Table 22 A. Liquid Density Sensitivities n-C $10 / \mathrm{CO}_{2}$ ESD EOS - Average Absolute Error

\begin{tabular}{|c|c|c|c|}
\hline Factor & Avg Abs Err & Factor & Avg Abs Err \\
\hline$T_{c, 2}$ & $-.1089 \times 10^{2}$ & E2 & $-.1050 \times 10$ \\
\hline E19 & $-.1080 \times 10^{2}$ & $\omega_{2}$ & -.9585 \\
\hline E5 & $-.7366 \times 10$ & E11 & -.9457 \\
\hline E29 & $0.7053 \times 10$ & $\mathrm{C} 1$ & 0.8697 \\
\hline C16 & $0.5477 \times 10$ & E24 & 0.7681 \\
\hline E9 & $0.4428 \times 10$ & $k_{12}$ & -.6867 \\
\hline$P_{c, 2}$ & $0.4225 \times 10$ & C3 & 0.6317 \\
\hline E25 & $-.4076 \times 10$ & E30 & -.6004 \\
\hline $\mathrm{C} 5$ & $-.3881 \times 10$ & E1 & -.5540 \\
\hline $\mathrm{C} 11$ & $-.3543 \times 10$ & E15 & -.5459 \\
\hline E31 & $-.3360 \times 10$ & E18 & 0.4980 \\
\hline $\mathrm{C} 8$ & $0.3314 \times 10$ & E10 & 0.3509 \\
\hline$T_{c, 1}$ & $0.3258 \times 10$ & C6 & -.3368 \\
\hline E12 & $0.2893 \times 10$ & E14 & -.2396 \\
\hline C14 & $0.2318 \times 10$ & E20 & 0.2268 \\
\hline E6 & $0.2311 \times 10$ & C17 & -.1678 \\
\hline E28 & $0.2303 \times 10$ & E21 & 0.1623 \\
\hline $\mathrm{C} 9$ & $0.2107 \times 10$ & E7 & 0.1447 \\
\hline C12 & $0.2075 \times 10$ & E26 & 0.1348 \\
\hline E23 & $-.2064 \times 10$ & E13 & -.1239 \\
\hline $\mathrm{C} 4$ & $-.2036 \times 10$ & E27 & $-.5894 \times 10^{-1}$ \\
\hline $\mathrm{C} 13$ & $0.2028 \times 10$ & $\mathrm{C} 2$ & $-.3328 \times 10^{-1}$ \\
\hline E16 & $-.1419 \times 10$ & E17 & $-.2370 \times 10^{-1}$ \\
\hline E3 & $-.1331 \times 10$ & & \\
\hline $\mathrm{E} 22$ & $0.1296 \times 10$ & & \\
\hline $\mathrm{C} 15$ & $0.1282 \times 10$ & & \\
\hline E8 & $-.1261 \times 10$ & & \\
\hline $\mathrm{C} 7$ & $0.1144 \times 10$ & & \\
\hline$\omega_{1}$ & $0.1119 \times 10$ & & \\
\hline C10 & $-.1109 \times 10$ & & \\
\hline $\mathrm{E} 4$ & $-.1085 \times 10$ & & \\
\hline$P_{c, 1}$ & $-.1058 \times 10$ & & \\
\hline
\end{tabular}


Table 22 B. Liquid Density Sensitivities n- $\mathrm{C}_{10} / \mathrm{CO}_{2}$ ESD EOS - Bias

$\begin{array}{llll}\text { Factor } & \text { Bias } & \text { Factor } & \text { Bias } \\ \text { C16 } & 0.1542 \times 10^{3} & \text { E16 } & -.7266 \\ T_{c, 2} & -.1395 \times 10^{3} & \text { E22 } & 0.6976 \\ \text { C8 } & 0.8732 \times 10^{2} & \text { E8 } & -.6325 \\ \text { C4 } & -.7519 \times 10^{2} & \text { C1 } & -.6034 \\ \text { C17 } & -.7466 \times 10^{2} & \text { E1 } & 0.5151 \\ \text { C5 } & -.6596 \times 10^{2} & \text { E26 } & 0.4731 \\ \text { C7 } & 0.4466 \times 10^{2} & k_{12} & 0.4696 \\ \text { C9 } & 0.1873 \times 10^{2} & \text { E4 } & -.4690 \\ P_{c, 1} & -.1029 \times 10^{2} & \text { C10 } & -.3990 \\ \text { C6 } & -.8799 \times 10 & \text { E20 } & 0.3428 \\ \text { E19 } & -.7137 \times 10 & \text { E27 } & -.2971 \\ P_{c, 2} & 0.6633 \times 10 & \text { E2 } & -.2645 \\ T_{c, 1} & -.5872 \times 10 & \text { E13 } & -.1944 \\ \text { E5 } & -.4888 \times 10 & \text { E15 } & -.1456 \\ \text { E29 } & 0.4363 \times 10 & \text { E10 } & -.1301 \\ \text { E9 } & 0.2534 \times 10 & \text { C3 } & 0.1217 \\ \text { C12 } & 0.2296 \times 10 & \text { E17 } & -.1069 \\ \text { E25 } & -.2249 \times 10 & \text { E7 } & -.9412 \times 10^{-1} \\ \text { C15 } & -.2000 \times 10 & \text { E6 } & 0.7764 \times 10^{-1} \\ \text { E12 } & 0.1793 \times 10 & \text { E21 } & 0.7591 \times 10^{-1} \\ \text { E30 } & -.1725 \times 10 & \text { E3 } & -.6216 \times 10^{-1} \\ \text { E31 } & -.1641 \times 10 & \text { E11 } & -.5322 \times 10^{-1} \\ \text { C11 } & 0.1486 \times 10 & \text { E14 } & 0.3018 \times 10^{-1} \\ \text { C14 } & -.1406 \times 10 & & \\ \text { C2 } & -.1212 \times 10 & & \\ \text { E28 } & 0.1130 \times 10 & & \\ \text { C13 } & 0.1072 \times 10 & & \\ \text { E18 } & 0.1051 \times 10 & & \\ \omega_{1} & -.9866 & & \\ \text { E23 } & -.9118 & & \\ \omega_{2} & -.9068 & & \\ \text { E24 } & 0.8168 & & \end{array}$


Table 22 C. Liquid Density Sensitivities n- $\mathrm{C}_{10} / \mathrm{CO}_{2}$ ESD EOS - CPU Time

$\begin{array}{llll}\text { Factor } & \text { CPU Time } & \text { Factor } & \text { CPU Time } \\ \text { C6 } & -.5745 \times 10 & \text { C4 } & -.5042 \\ k_{12} & 0.4939 \times 10 & \text { C16 } & -.5042 \\ \text { E15 } & 0.4535 \times 10 & T_{c, 1} & 0.5042 \\ \text { E10 } & -.3326 \times 10 & P_{c, 2} & -.5042 \\ \text { E14 } & 0.3326 \times 10 & \text { E7 } & 0.5042 \\ \text { C5 } & 0.3125 \times 10 & \text { E8 } & 0.5042 \\ T_{c, 2} & 0.2722 \times 10 & \text { E26 } & -.5042 \\ \text { E12 } & 0.2722 \times 10 & \text { C1 } & 0.3024 \\ \text { E6 } & -.2520 \times 10 & \text { C7. } & -.3024 \\ \text { E28 } & 0.2319 \times 10 & \text { C8 } & 0.3024 \\ \text { E4 } & 0.2117 \times 10 & \text { C9 } & 0.3024 \\ \text { E31 } & 0.1915 \times 10 & \text { E23 } & 0.3024 \\ \omega_{1} & -.1714 \times 10 & \text { E24 } & 0.3024 \\ \text { E20 } & 0.1714 \times 10 & \text { E27 } & 0.3024 \\ \text { E21 } & 0.1714 \times 10 & \text { E29 } & -.3024 \\ \omega_{2} & -.1512 \times 10 & \text { E30 } & 0.3024 \\ \text { E9 } & 0.1311 \times 10 & \text { C2 } & -.1008 \\ \text { E13 } & 0.1311 \times 10 & \text { C3 } & 0.1008 \\ \text { E16 } & -.1311 \times 10 & \text { C10 } & -.1008 \\ \text { E25 } & 0.1311 \times 10 & \text { C12 } & 0.1008 \\ \text { C13 } & 0.9074 & \text { C17 } & 0.1008 \\ \text { C14 } & -.9074 & \text { E5 } & 0.1008 \\ P_{c, 1} & 0.9074 & \text { E17 } & -.1008 \\ \text { E2 } & -.9074 & & \\ \text { E11 } & -.9074 & & \\ \text { C11 } & -.7058 & & \\ \text { C15 } & 0.7058 & & \\ \text { E1 } & -.7058 & & \\ \text { E3 } & -.7058 & & \\ \text { E18 } & 0.7058 & & \\ \text { E19 } & -.7058 & & \\ \text { E22 } & 0.7058 & & \end{array}$


Table 23 A. Critical Line Locus Sensitivities $\mathrm{n}-\mathrm{C}_{4} / \mathrm{CO}_{2}$ ALS EOS - Average Absolute Error

\begin{tabular}{|c|c|c|c|}
\hline Factor & Avg Abs Err & Factor & Avg Abs Err \\
\hline$T_{c, 2}$ & $0.3119 \times 10^{3}$ & E30 & $\begin{array}{l}0.7499 \times 10^{2} \\
-7499 \times 10^{2}\end{array}$ \\
\hline $\begin{array}{l}T_{c, 1} \\
\mathrm{C} 4\end{array}$ & $-.3069 \times 10^{3}$ & E1 & \\
\hline $\begin{array}{l}\text { C4 } \\
\text { E11 }\end{array}$ & $-.3051 \times 10^{3}$ & E8 & $0.7496 \times 10^{2}$ \\
\hline $\begin{array}{l}\text { E11 } \\
\omega_{1}\end{array}$ & $-.3023 \times 10^{3}$ & $\mathrm{C} 6$ & $0.7496 \times 10^{2}$ \\
\hline $\begin{array}{l}\omega_{1} \\
\text { E27 }\end{array}$ & $-.3022 \times 10^{3}$ & E6 & $-.7496 \times 10^{2}$ \\
\hline $\begin{array}{l}\mathrm{E} 27 \\
\mathrm{E} 4\end{array}$ & $0.3022 \times 10^{3}$ & E17 & $0.7496 \times 10^{2}$ \\
\hline $\begin{array}{l}\text { E4 } \\
\text { E10 }\end{array}$ & $-.3021 \times 10^{3}$ & C15 & $0.7493 \times 10^{2}$ \\
\hline E10 & $0.3021 \times 10^{3}$ & E5 & $-.7493 \times 10^{2}$ \\
\hline C11 & $-.3021 \times 10^{3}$ & C7 & $0.7493 \times 10^{2}$ \\
\hline $\mathrm{C} 10$ & $-.3021 \times 10^{3}$ & E15 & $0.7493 \times 10^{2}$ \\
\hline E2 & $0.3021 \times 10^{3}$ & E25 & $0.7493 \times 10^{2}$ \\
\hline E9 & $0.3021 \times 10^{3}$ & C18 & $-.7493 \times 10^{2}$ \\
\hline C14 & $0.3021 \times 10^{3}$ & E14 & $-.7490 \times 10^{2}$ \\
\hline E28 & $-.3021 \times 10^{3}$ & $\mathrm{C} 13$ & $0.7490 \times 10^{2}$ \\
\hline E20 & $0.3021 \times 10^{3}$ & E19 & $0.7490 \times 10^{2}$ \\
\hline E31 & $-.3021 \times 10^{3}$ & E16 & $-.7490 \times 10^{2}$ \\
\hline $\mathrm{C} 3$ & $-.3021 \times 10^{3}$ & E12 & $-.7490 \times 10^{2}$ \\
\hline E13 & $-.3021 \times 10^{3}$ & E24 & $0.7490 \times 10^{2}$ \\
\hline E29 & $-.3021 \times 10^{3}$ & E7 & $-.7480 \times 10^{2}$ \\
\hline C5 & $0.3021 \times 10^{3}$ & E26 & $0.7477 \times 10^{2}$ \\
\hline E3 & $0.3021 \times 10^{3}$ & $\omega_{2}$ & $0.7465 \times 10^{2}$ \\
\hline E22 & $-.3021 \times 10^{3}$ & $\mathrm{C} 17$ & $0.7449 \times 10^{2}$ \\
\hline E21 & $-.3018 \times 10^{3}$ & C9 & $-.7428 \times 10^{2}$ \\
\hline E18 & $0.3018 \times 10^{3}$ & C2 & $0.7425 \times 10^{2}$ \\
\hline$P_{c, 2}$ & $0.3003 \times 10^{3}$ & & \\
\hline $\mathrm{C} 12$ & $0.2999 \times 10^{3}$ & & \\
\hline $\mathrm{C} 1$ & $-.2928 \times 10^{3}$ & & \\
\hline$P_{c, 1}$ & $-.8100 \times 10^{2}$ & & \\
\hline $\mathrm{C} 8$ & $0.7867 \times 10^{2}$ & & \\
\hline $\mathrm{C} 16$ & $-.7551 \times 10^{2}$ & & \\
\hline E23 & $-.7511 \times 10^{2}$ & & \\
\hline$k_{12}$ & $-.7499 \times 10^{2}$ & & \\
\hline
\end{tabular}


Table 23 B. Critical Line Locus Sensitivities $n-\mathrm{C}_{4} / \mathrm{CO}_{2}$ ALS EOS - Bias

$\begin{array}{llll}\text { Factor } & \text { Bias } & \text { Factor } & \text { Bias } \\ T_{c, 2} & 0.4211 \times 10^{3} & \text { C6 } & 0.1000 \times 10^{3} \\ T_{c, 1} & -.4186 \times 10^{3} & \text { E1 } & -.1000 \times 10^{3} \\ \text { C4 } & -.4108 \times 10^{3} & \text { E8 } & 0.9998 \times 10^{2} \\ \omega_{1} & -.4038 \times 10^{3} & \text { E25 } & 0.9998 \times 10^{2} \\ \text { E11 } & -.4038 \times 10^{3} & \text { E6 } & -.9998 \times 10^{2} \\ \text { E27 } & 0.4036 \times 10^{3} & \text { C15 } & 0.9998 \times 10^{2} \\ \text { E4 } & -.4033 \times 10^{3} & k_{12} & -.9998 \times 10^{2} \\ \text { E10 } & 0.4033 \times 10^{3} & \text { E17 } & 0.9994 \times 10^{2} \\ \text { C11 } & -.4033 \times 10^{3} & \text { C7 } & 0.9994 \times 10^{2} \\ \text { C10 } & -.4033 \times 10^{3} & \text { E5 } & -.9994 \times 10^{2} \\ \text { E2 } & 0.4033 \times 10^{3} & \text { E14 } & -.9994 \times 10^{2} \\ \text { E9 } & 0.4033 \times 10^{3} & \text { E15 } & 0.9994 \times 10^{2} \\ \text { E28 } & -.4033 \times 10^{3} & \text { E16 } & -.9990 \times 10^{2} \\ \text { E20 } & 0.4033 \times 10^{3} & \text { C18 } & -.9990 \times 10^{2} \\ \text { C14 } & 0.4033 \times 10^{3} & \text { E19 } & 0.9990 \times 10^{2} \\ \text { E31 } & -.4033 \times 10^{3} & \text { E24 } & 0.9990 \times 10^{2} \\ \text { E3 } & 0.4033 \times 10^{3} & \text { E12 } & -.9990 \times 10^{2} \\ \text { E13 } & -.4033 \times 10^{3} & \text { C13 } & 0.9977 \times 10^{2} \\ \text { C3 } & -.4033 \times 10^{3} & \text { E26 } & 0.9973 \times 10^{2} \\ \text { E22 } & -.4032 \times 10^{3} & \text { E7 } & -.9973 \times 10^{2} \\ \text { E29 } & -.4032 \times 10^{3} & \omega_{2} & 0.9957 \times 10^{2} \\ \text { C5 } & 0.4031 \times 10^{3} & \text { C17 } & 0.9903 \times 10^{2} \\ \text { E21 } & -.4027 \times 10^{3} & \text { C2 } & 0.9808 \times 10^{2} \\ \text { E18 } & 0.4026 \times 10^{3} & \text { C9 } & -.9804 \times 10^{2} \\ P_{c, 2} & 0.3954 \times 10^{3} & & \\ \text { C12 } & 0.3935 \times 10^{3} & & \\ \text { C1 } & -.3791 \times 10^{3} & & \\ \text { C8 } & 0.1103 \times 10^{3} & & \\ P_{c, 1} & -.1091 \times 10^{3} & & \\ \text { C16 } & -.1013 \times 10^{3} & & \\ \text { E23 } & -.1002 \times 10^{3} & & \\ \text { E30 } & 0.1000 \times 10^{3} & & \\ & & & \end{array}$


Table 23 C. Critical Line Locus Sensitivities n-C $4 / \mathrm{CO}_{2}$ ALS EOS - CPU Time

$\begin{array}{llll}\text { Factor } & \text { CPU Time } & \text { Factor } & \text { CPU Time } \\ \text { C5 } & 0.1009 \times 10^{2} & \text { E16 } & -.2714 \times 10 \\ \text { E11 } & -.9291 \times 10 & \text { E23 } & 0.2216 \times 10 \\ \omega_{1} & -.8940 \times 10 & \text { C16 } & -.2167 \times 10 \\ \text { E10 } & 0.8494 \times 10 & \text { E8 } & 0.2167 \times 10 \\ \text { C14 } & 0.7395 \times 10 & \text { C8 } & 0.2116 \times 10 \\ \text { E31 } & -.7346 \times 10 & \text { E1 } & -.2067 \times 10 \\ \text { E9 } & 0.7296 \times 10 & \text { C2 } & 0.1917 \times 10 \\ \text { C10 } & -.724 .3 \times 10 & \text { E7 } & -.1868 \times 10 \\ \text { E4 } & -.7240 \times 10 & \text { E26 } & 0.1818 \times 10 \\ \text { E2 } & 0.7196 \times 10 & \text { E5 } & -.1618 \times 10 \\ T_{c, 2} & 0.6649 \times 10 & \text { C6 } & 0.1370 \times 10 \\ \text { E28 } & -.6449 \times 10 & \text { E17 } & -.1370 \times 10 \\ \text { C1 } & -.6399 \times 10 & \text { E24 } & 0.1370 \times 10 \\ \text { E29 } & -.6200 \times 10 & \text { E15 } & -.1171 \times 10 \\ \text { E21 } & -.6100 \times 10 & k_{12} & -.1120 \times 10 \\ P_{c, 2} & 0.5852 \times 10 & \text { E6 } & -.1120 \times 10 \\ \text { E18 } & 0.5852 \times 10 & \text { C18 } & -.1071 \times 10 \\ \text { E27 } & 0.5752 \times 10 & P_{c, 1} & 0.7720 \\ \text { C13 } & 0.5503 \times 10 & \text { C9 } & -.4233 \\ \text { E3 } & 0.5453 \times 10 & \text { E19 } & -.4233 \\ \text { C12 } & 0.5354 \times 10 & \text { C15 } & 0.3735 \\ \text { C3 } & -.5254 \times 10 & \text { E12 } & -.3735 \\ \text { E20 } & 0.5204 \times 10 & \text { C17 } & -.2241 \\ \omega_{2} & 0.5104 \times 10 & \text { C7 } & 0.1743 \\ \text { E14 } & -.4806 \times 10 & & \\ \text { E22 } & -.4806 \times 10 & & \\ \text { E25 } & 0.4806 \times 10 & & \\ \text { C4 } & -.4507 \times 10 & & \\ T_{c, 1} & -.4208 \times 10 & & \\ \text { E13 } & -.3959 \times 10 & & \\ \text { C11 } & -.2963 \times 10 & & \\ \text { E30 } & 0.2764 \times 10 & & \\ & & & \end{array}$


Table 24 A. Critical Line Locus Sensitivities n- $\mathrm{C}_{6} / \mathrm{CO}_{2}$ ALS EOS - Average Absolute Error

\begin{tabular}{|c|c|c|c|}
\hline Factor & Avg Abs Err & Factor & Avg Abs Err \\
\hline E7 & $0.4663 \times 10^{3}$ & $\mathrm{C} 8$ & $-.8242 \times 10^{2}$ \\
\hline $\mathrm{C} 4$ & $0.3764 \times 10^{3}$ & $\omega_{1}$ & $-.7879 \times 10^{2}$ \\
\hline E16 & $-.3522 \times 10^{3}$ & E29 & $-.6740 \times 10^{2}$ \\
\hline $\mathrm{C} 2$ & $0.3098 \times 10^{3}$ & E30 & $0.5306 \times 10^{2}$ \\
\hline C13 & $0.3031 \times 10^{3}$ & E15 & $-.5068 \times 10^{2}$ \\
\hline E18 & $0.2991 \times 10^{3}$ & $\mathrm{C} 18$ & $0.5043 \times 10^{2}$ \\
\hline $\mathrm{C} 7$ & $-.2898 \times 10^{3}$ & E27 & $-.4663 \times 10^{2}$ \\
\hline $\mathrm{E2}$ & $-.2874 \times 10^{3}$ & E17 & $0.4563 \times 10^{2}$ \\
\hline E23 & $0.2761 \times 10^{3}$ & $\mathrm{E} 21$ & $0.4296 \times 10^{2}$ \\
\hline C11 & $-.2619 \times 10^{3}$ & $\mathrm{C} 14$ & $0.3969 \times 10^{2}$ \\
\hline$\omega_{2}$ & $-.2249 \times 10^{3}$ & $\mathrm{C} 17$ & $-.3027 \times 10^{2}$ \\
\hline E10 & $-.2082 \times 10^{3}$ & E1 & $0.2891 \times 10^{2}$ \\
\hline E28 & $0.2055 \times 10^{3}$ & $P_{c, 2}$ & $0.2875 \times 10^{2}$ \\
\hline E13 & $-.1838 \times 10^{3}$ & $\mathrm{E} 4$ & $-.2520 \times 10^{2}$ \\
\hline$T_{c, 2}$ & $-.1816 \times 10^{3}$ & $\mathrm{C} 15$ & $0.2501 \times 10^{2}$ \\
\hline E11 & $0.1801 \times 10^{3}$ & E22 & $0.2478 \times 10^{2}$ \\
\hline E14 & $-.1629 \times 10^{3}$ & E20 & $0.2445 \times 10^{2}$ \\
\hline E26 & $0.1586 \times 10^{3}$ & E25 & $0.2224 \times 10^{2}$ \\
\hline$k_{12}$ & $0.1552 \times 10^{3}$ & E24 & $-.1925 \times 10^{2}$ \\
\hline $\mathrm{C} 1$ & $-.1494 \times 10^{3}$ & $\mathrm{C} 5$ & $0.1687 \times 10^{2}$ \\
\hline C3 & $-.1418 \times 10^{3}$ & C9 & $0.1646 \times 10^{2}$ \\
\hline E6 & $-.1401 \times 10^{3}$ & $\mathrm{C} 12$ & $0.7541 \times 10$ \\
\hline$P_{c, 1}$ & $-.1400 \times 10^{3}$ & E19 & $-.4697 \times 10$ \\
\hline $\mathrm{E} 12$ & $-.1162 \times 10^{3}$ & D16 & $0.1566 \times 10$ \\
\hline $\mathrm{E} 8$ & $-.1143 \times 10^{3}$ & & \\
\hline$T_{c, 1}$ & $-.1095 \times 10^{3}$ & & \\
\hline E9 & $-.1041 \times 10^{3}$ & & \\
\hline C6 & $-.1025 \times 10^{3}$ & & \\
\hline $\mathrm{C} 10$ & $0.9535 \times 10^{2}$ & & \\
\hline E3 & $0.9293 \times 10^{2}$ & & \\
\hline E5 & $-.8742 \times 10^{2}$ & & \\
\hline E31 & $-.8738 \times 10^{2}$ & & \\
\hline
\end{tabular}


Table 24 B. Critical Line Locus Sensitivities n- $\mathrm{C}_{6} / \mathrm{CO}_{2}$ ALS EOS - Bias

\begin{tabular}{|c|c|c|c|}
\hline Factor & Bias & Factor & Bias \\
\hline E7 & $0.6855 \times 10^{3}$ & $\mathrm{C} 10$ & $0.1199 \times 10^{3}$ \\
\hline $\mathrm{C} 4$ & $0.5745 \times 10^{3}$ & E5 & $-.1193 \times 10^{3}$ \\
\hline E16 & $-.5294 \times 10^{3}$ & $\mathrm{C} 8$ & $-.1049 \times 10^{3}$ \\
\hline $\mathrm{C} 2$ & $0.4798 \times 10^{3}$ & C18 & $0.9524 \times 10^{2}$ \\
\hline E18 & $0.4515 \times 10^{3}$ & E27 & $-.8230 \times 10^{2}$ \\
\hline $\mathrm{C} 13$ & $0.4449 \times 10^{3}$ & E17 & $0.7372 \times 10^{2}$ \\
\hline E23 & $0.4388 \times 10^{3}$ & E30 & $0.7359 \times 10^{2}$ \\
\hline E2 & $-.4310 \times 10^{3}$ & E15 & $-.7173 \times 10^{2}$ \\
\hline $\mathrm{C} 7$ & $-.4255 \times 10^{3}$ & E21 & $0.6159 \times 10^{2}$ \\
\hline C11 & $-.3934 \times 10^{3}$ & E22 & $0.5704 \times 10^{2}$ \\
\hline$\omega_{2}$ & $-.3271 \times 10^{3}$ & $P_{c, 2}$ & $0.5492 \times 10^{2}$ \\
\hline E10 & $-.3008 \times 10^{3}$ & $\mathrm{C} 17$ & $-.5344 \times 10^{2}$ \\
\hline E28 & $0.2824 \times 10^{3}$ & $\mathrm{E} 4$ & $-.5116 \times 10^{2}$ \\
\hline E13 & $-.2687 \times 10^{3}$ & C14 & $0.5050 \times 10^{2}$ \\
\hline E11 & $0.2681 \times 10^{3}$ & E1 & $0.4832 \times 10^{2}$ \\
\hline$T_{c, 2}$ & $-.2660 \times 10^{3}$ & C9 & $0.3695 \times 10^{2}$ \\
\hline E14 & $-.2554 \times 10^{3}$ & C15 & $0.3072 \times 10^{2}$ \\
\hline E26 & $0.2514 \times 10^{3}$ & E20 & $0.2995 \times 10^{2}$ \\
\hline$k_{12}$ & $0.2391 \times 10^{3}$ & E24 & $. .2940 \times 10^{2}$ \\
\hline$P_{c, 1}$ & $-.2085 \times 10^{3}$ & $\mathrm{C} 16$ & $-.2518 \times 10^{2}$ \\
\hline E6 & $-.2074 \times 10^{3}$ & E25 & $0.1963 \times 10^{2}$ \\
\hline $\mathrm{C} 3$ & $-.2034 \times 10^{3}$ & E19 & $-.8610 \times 10$ \\
\hline E8 & $-.2027 \times 10^{3}$ & C5 & $-.6874 \times 10$ \\
\hline $\mathrm{C} 1$ & $-.2004 \times 10^{3}$ & $\mathrm{C} 12$ & $0.4875 \times 10$ \\
\hline$T_{c, 1}$ & $-.1854 \times 10^{3}$ & & \\
\hline E9 & $-.1743 \times 10^{3}$ & & \\
\hline E12 & $-.1729 \times 10^{3}$ & & \\
\hline $\mathrm{C} 6$ & $-.1472 \times 10^{3}$ & & \\
\hline $\boldsymbol{\omega}_{1}$ & $-.1359 \times 10^{3}$ & & \\
\hline E31 & $-.1216 \times 10^{3}$ & & \\
\hline E3 & $0.1209 \times 10^{3}$ & & \\
\hline E29 & $-.1201 \times 10^{3}$ & & \\
\hline
\end{tabular}


Table 24 C. Critical Line Locus Sensitivities n- $\mathrm{O}_{6} / \mathrm{CO}_{2}$ ALS EOS - OPU Time

\begin{tabular}{|c|c|c|c|}
\hline Factor & CPU Time & Factor & OPU Time \\
\hline E9 & $0.9357 \times 10^{2}$ & E12 & $0.1674 \times 10^{2}$ \\
\hline C5 & $0.7239 \times 10^{2}$ & E29 & $0.1642 \times 10^{2}$ \\
\hline E28 & $0.5307 \times 10^{2}$ & E4 & $-.1590 \times 10^{2}$ \\
\hline E15 & $0.5192 \times 10^{2}$ & $\mathrm{C} 4$ & $0.1567 \times 10^{2}$ \\
\hline E3 & $0.4649 \times 10^{2}$ & E6 & $0.1523 \times 10^{2}$ \\
\hline E16 & $-.4506 \times 10^{2}$ & E14 & $0.1250 \times 10^{2}$ \\
\hline E30 & $-.4423 \times 10^{2}$ & $\omega_{1}$ & $0.1171 \times 10^{2}$ \\
\hline E23 & $-.4071 \times 10^{2}$ & $\mathrm{C} 16$ & $-.1139 \times 10^{2}$ \\
\hline E22 & $-.4067 \times 10^{2}$ & $\mathrm{C} 2$ & $-.1119 \times 10^{2}$ \\
\hline $\mathrm{C} 13$ & $-.4063 \times 10^{2}$ & C6 & $-.1111 \times 10^{2}$ \\
\hline C10 & $-.4059 \times 10^{2}$ & $\mathrm{C} 12$ & $-.1056 \times 10^{2}$ \\
\hline C9 & $-.3976 \times 10^{2}$ & E1 & $-.9135 \times 10$ \\
\hline$\omega_{2}$ & $-.3960 \times 10^{2}$ & E17 & $0.8856 \times 10$ \\
\hline $\mathrm{C} 1$ & $-.3900 \times 10^{2}$ & $P_{c, 1}$ & $-.8615 \times 10$ \\
\hline C14 & $0.3821 \times 10^{2}$ & E5 & $0.8222 \times 10$ \\
\hline E18 & $0.3576 \times 10^{2}$ & C11 & $-.7981 \times 10$ \\
\hline E27 & $0.3552 \times 10^{2}$ & $k_{12}$ & $-.7867 \times 10$ \\
\hline E24 & $-.3520 \times 10^{2}$ & C3 & $0.4457 \times 10$ \\
\hline E8 & $0.3449 \times 10^{2}$ & E13 & $0.4457 \times 10$ \\
\hline E19 & $-.3390 \times 10^{2}$ & $\mathrm{C} 17$ & $-.3863 \times 10$ \\
\hline$T_{c, 1}$ & $0.3267 \times 10^{2}$ & $\mathrm{C} 18$ & $0.1327 \times 10$ \\
\hline E11 & $0.2732 \times 10^{2}$ & E26 & -.8120 \\
\hline$T_{c, 2}$ & $0.2490 \times 10^{2}$ & E2 & -.3764 \\
\hline $\mathrm{C} 8$ & $-.2403 \times 10^{2}$ & E7 & 0.3764 \\
\hline $\mathrm{C} 15$ & $-.2236 \times 10^{2}$ & & \\
\hline $\mathrm{C} 7$ & $-.2185 \times 10^{2}$ & & \\
\hline$P_{c, 2}$ & $0.2177 \times 10^{2}$ & & \\
\hline E10 & $0.2141 \times 10^{2}$ & & \\
\hline E25 & $-.2042 \times 10^{2}$ & & \\
\hline E31 & $-.2015 \times 10^{2}$ & & \\
\hline $\mathrm{E} 21$ & $-.1821 \times 10^{2}$ & & \\
\hline E20 & $0.1717 \times 10^{2}$ & & \\
\hline
\end{tabular}


Table 25 A. Critical Line Locus Sensitivities n-C $4 / \mathrm{CO}_{2}$ ESD EOS - Average Absolute Error

\begin{tabular}{|c|c|c|c|}
\hline Factor & Avg Abs Err & Factor & Avg Abs Err \\
\hline C14 & $-.2483 \times 10^{3}$ & E11 & $0.8221 \times 10^{2}$ \\
\hline E22 & $-.2465 \times 10^{3}$ & C5 & $0.8208 \times 10^{2}$ \\
\hline$P_{0,1}$ & $-.1673 \times 10^{3}$ & $\mathrm{C} 8$ & $-.8200 \times 10^{2}$ \\
\hline $\mathrm{E} 25$ & $-.1659 \times 10^{3}$ & $\mathrm{C} 2$ & $0.8195 \times 10^{2}$ \\
\hline E15 & $0.1657 \times 10^{3}$ & $\mathrm{C} 17$ & $0.8186 \times 10^{2}$ \\
\hline E20 & $-.1657 \times 10^{3}$ & E13 & $-.8154 \times 10^{2}$ \\
\hline C9 & $-.1651 \times 10^{3}$ & $\omega_{2}$ & $-.8121 \times 10^{2}$ \\
\hline C10 & $-.1649 \times 10^{3}$ & E31 & $0.8083 \times 10^{2}$ \\
\hline $\mathrm{C} 4$ & $0.1648 \times 10^{3}$ & E14 & $-.8067 \times 10^{2}$ \\
\hline E3 & $-.1648 \times 10^{3}$ & E21 & $0.8065 \times 10^{2}$ \\
\hline E19 & $-.1648 \times 10^{3}$ & $k_{12}$ & $-.8065 \times 10^{2}$ \\
\hline E8 & $0.1648 \times 10^{3}$ & E5 & $0.8059 \times 10^{2}$ \\
\hline$\omega_{1}$ & $0.1646 \times 10^{3}$ & $\mathrm{C} 13$ & $0.7951 \times 10^{2}$ \\
\hline E2 & $0.1641 \times 10^{3}$ & $T_{0,2}$ & $0.4622 \times 10$ \\
\hline C3 & $0.1637 \times 10^{3}$ & $\mathrm{C} 16$ & $-.3030 \times 10$ \\
\hline E24 & $0.1632 \times 10^{3}$ & C11 & $0.2676 \times 10$ \\
\hline E30 & $-.1632 \times 10^{3}$ & E9 & $0.1629 \times 10$ \\
\hline E27 & $-.1632 \times 10^{3}$ & C1 & $-.1143 \times 10$ \\
\hline E29 & $0.8357 \times 10^{2}$ & E18 & $-.1099 \times 10$ \\
\hline C15 & $-.8352 \times 10^{2}$ & $T_{c, 1}$ & 0.1574 \\
\hline E12 & $0.8325 \times 10^{2}$ & E26 & $0.8968 \times 10^{-1}$ \\
\hline E28 & $0.8324 \times 10^{2}$ & E10 & $-.5197 \times 10^{-1}$ \\
\hline$P_{c, 2}$ & $0.8322 \times 10^{2}$ & E17 & $0.3835 \times 10^{-1}$ \\
\hline E23 & $-.8321 \times 10^{2}$ & & \\
\hline $\mathrm{C} 6$ & $0.8314 \times 10^{2}$ & & \\
\hline E1 & $-.8257 \times 10^{2}$ & & \\
\hline E4 & $-.8248 \times 10^{2}$ & & \\
\hline E7 & $0.8244 \times 10^{2}$ & & \\
\hline $\mathrm{C} 7$ & $-.8237 \times 10^{2}$ & & \\
\hline $\mathrm{C} 12$ & $-.8233 \times 10^{2}$ & & \\
\hline E6 & $0.8230 \times 10^{2}$ & & \\
\hline E16 & $-.8222 \times 10^{2}$ & & \\
\hline
\end{tabular}


Table 25 B. Critical Lino Locus Sensitivities $\mathrm{n}-\mathrm{C}_{4} / \mathrm{CO}_{2}$ ESD EOS - Bias

\begin{tabular}{|c|c|c|c|}
\hline Factor & Bias & Factor & Bias \\
\hline $\mathrm{C} 14$ & $-.3506 \times 10^{3}$ & $\mathrm{C} 7$ & $-.1130 \times 10^{3}$ \\
\hline E22 & $-.3443 \times 10^{3}$ & $\omega_{2}$ & $-.1128 \times 10^{3}$ \\
\hline$P_{c, 1}$ & $-.2337 \times 10^{3}$ & E13 & $-.1126 \times 10^{3}$ \\
\hline E20 & $-.2329 \times 10^{3}$ & $P_{c, 2}$ & $0.1125 \times 10^{3}$ \\
\hline E25 & $-.2327 \times 10^{3}$ & C5 & $0.1117 \times 10^{3}$ \\
\hline E15 & $0.2318 \times 10^{3}$ & E31 & $0.1117 \times 10^{3}$ \\
\hline E8 & $0.2313 \times 10^{3}$ & F21 & $0.1116 \times 10^{3}$ \\
\hline$\omega_{1}$ & $0.2310 \times 10^{3}$ & $\mathrm{C} 8$ & $-.1115 \times 10^{3}$ \\
\hline E19 & $-.2305 \times 10^{3}$ & E5 & $0.1113 \times 10^{3}$ \\
\hline E3 & $-.2302 \times 10^{3}$ & $k_{12}$ & $-.1105 \times 10^{3}$ \\
\hline C9 & $-.2300 \times 10^{3}$ & E14 & $-.1104 \times 10^{3}$ \\
\hline C10 & $-.2295 \times 10^{3}$ & C17 & $0.1095 \times 10^{3}$ \\
\hline C3 & $0.2285 \times 10^{3}$ & $\mathrm{C} 13$ & $0.1079 \times 10^{3}$ \\
\hline E2 & $0.2281 \times 10^{3}$ & $T_{c, 2}$ & $0.1462 \times 10^{2}$ \\
\hline E27 & $-.2271 \times 10^{3}$ & C11 & $0.8161 \times 10$ \\
\hline $\mathrm{C} 4$ & $0.2270 \times 10^{3}$ & $T_{c, 1}$ & $-.6946 \times 10$ \\
\hline E30 & $-.2267 \times 10^{3}$ & E9 & $0.3598 \times 10$ \\
\hline E24 & $0.2258 \times 10^{3}$ & C1 & $-.3155 \times 10$ \\
\hline $\mathrm{C} 15$ & $-.1178 \times 10^{3}$ & E18 & $-.2480 \times 10$ \\
\hline E29 & $0.1177 \times 10^{3}$ & C16 & $-.2215 \times 10$ \\
\hline E28 & $0.1176 \times 10^{3}$ & E10 & $0.1011 \times 10$ \\
\hline E12 & $0.1172 \times 10^{3}$ & E26 & 0.8929 \\
\hline $\mathrm{C} 6$ & $0.1169 \times 10^{3}$ & E17 & 0.1878 \\
\hline E4 & $-.1165 \times 10^{3}$ & & \\
\hline E1 & $-.1163 \times 10^{3}$ & & \\
\hline E23 & $-.1163 \times 10^{3}$ & & \\
\hline E16 & $-.1159 \times 10^{3}$ & & \\
\hline $\mathrm{C} 12$ & $-.1158 \times 10^{3}$ & & \\
\hline E6 & $0.1153 \times 10^{3}$ & & \\
\hline E7 & $0.1152 \times 10^{3}$ & & \\
\hline E11 & $0.1147 \times 10^{3}$ & & \\
\hline $\mathrm{C} 2$ & $0.1146 \times 10^{3}$ & & \\
\hline
\end{tabular}


Table 25 C. Critical Line Locus Sensitivities n-C 4 / $\mathrm{CO}_{2}$ ESD EOS - CPU Time

$\begin{array}{llll}\text { Factor } & \text { CPU Time } & \text { Factor } & \text { CPU Time } \\ \text { E14 } & -. .9840 \times 10 & \text { C17 } & 0.2132 \times 10 \\ \text { E22 } & -.8160 \times 10 & \text { E29 } & 0.1927 \times 10 \\ \text { E5 } & 0.7896 \times 10 & \text { C12 } & 0.1868 \times 10 \\ \text { E9 } & -.6899 \times 10 & \text { E16 } & -.1809 \times 10 \\ P_{c, 1} & -.6781 \times 10 & \text { E18 } & -.1779 \times 10 \\ \text { E6 } & 0.5868 \times 10 & k_{12} & -.1485 \times 10 \\ \text { E24 } & 0.5751 \times 10 & \text { E8 } & 0.1485 \times 10 \\ \text { E13 } & -.5336 \times 10 & \text { C10 } & 0.1397 \times 10 \\ \text { C9 } & -.5280 \times 10 & \text { E11 } & 0.1397 \times 10 \\ \text { C14 } & -.5280 \times 10 & \text { E26 } & 0.1221 \times 10 \\ \text { E28 } & 0.5134 \times 10 & T_{c, 1} & 0.1191 \times 10 \\ \text { E3 } & -.5073 \times 10 & \text { E30 } & -.1103 \times 10 \\ \text { E19 } & -.4955 \times 10 & \text { C7 } & -.1073 \times 10 \\ \text { E15 } & 0.4927 \times 10 & \text { E1 } & 0.1044 \times 10 \\ \text { E23 } & -.4720 \times 10 & \omega_{2} & -.9266 \\ \text { E31 } & 0.4632 \times 10 & \text { E10 } & -.6028 \\ \text { C13 } & 0.4250 \times 10 & \text { E20 } & -.5148 \\ \text { E27 } & -.4044 \times 10 & \text { C6 } & 0.4852 \\ \text { C4 } & 0.3868 \times 10 & \text { E7 } & 0.4852 \\ \text { E25 } & -.3750 \times 10 & \text { C5 } & 0.3971 \\ \text { C8 } & 0.3720 \times 10 & \text { E17 } & -.3971 \\ \text { C15 } & 0.3632 \times 10 & \text { E21 } & 0.2500 \\ T_{c, 2} & -.3574 \times 10 & \text { C1 } & 0.1324 \\ \text { C2 } & 0.3280 \times 10 & & \\ \text { C16 } & 0.3221 \times 10 & & \\ \omega_{1} & 0.3044 \times 10 & & \\ \text { C3 } & 0.3015 \times 10 & & \\ \text { E12 } & 0.2838 \times 10 & & \\ P_{c, 2} & -.2691 \times 10 & & \\ \text { E2 } & 0.2368 \times 10 & & \\ \text { E4 } & -.2309 \times 10 & & \\ \text { C11 } & -.2250 \times 10 & & \end{array}$


Table 26 A. Critical Line Locus Sensitivities n- $\mathrm{C}_{6} / \mathrm{CO}_{2}$ ESD EOS - Average Absolute Error

$\begin{array}{llll}\text { Factor } & \text { Avg Abs Err } & \text { Factor } & \text { Avg Abs Err } \\ \omega_{1} & 0.1455 \times 10^{8} & \text { E30 } & 0.1643 \times 10^{7} \\ \text { C3 } & -.1138 \times 10^{8} & \text { E19 } & -.1639 \times 10^{7} \\ \text { E2 } & -.8153 \times 10^{7} & \text { E23 } & -.1637 \times 10^{7} \\ \text { E27 } & -.8131 \times 10^{7} & \text { E1 } & -.1636 \times 10^{7} \\ P_{c, 2} & 0.8120 \times 10^{7} & \text { E28 } & -.1634 \times 10^{7} \\ \text { E14 } & -.8114 \times 10^{7} & \text { E22 } & -.1633 \times 10^{7} \\ \text { E16 } & 0.8114 \times 10^{7} & \text { E24 } & -.1626 \times 10^{7} \\ \text { C15 } & 0.8109 \times 10^{7} & k_{12} & 0.1626 \times 10^{7} \\ \text { E20 } & -.8097 \times 10^{7} & P_{c, 1} & -.1624 \times 10^{7} \\ \text { E7 } & 0.8092 \times 10^{7} & \text { E4 } & 0.1622 \times 10^{7} \\ \text { E11 } & 0.8064 \times 10^{7} & \omega_{2} & 0.1619 \times 10^{7} \\ \text { C2 } & 0.8064 \times 10^{7} & \text { C4 } & -.1607 \times 10^{7} \\ \text { E13 } & -.8064 \times 10^{7} & \text { E6 } & -.1599 \times 10^{7} \\ \text { E31 } & 0.8059 \times 10^{7} & \text { E3 } & 0.1591 \times 10^{7} \\ \text { E18 } & 0.8037 \times 10^{7} & \text { E8 } & 0.1591 \times 10^{7} \\ \text { E10 } & -.4921 \times 10^{7} & \text { E26 } & -.1590 \times 10^{7} \\ \text { E25 } & -.4918 \times 10^{7} & \text { E17 } & 0.1590 \times 10^{7} \\ T_{c, 1} & 0.4911 \times 10^{7} & \text { C10 } & -.1589 \times 10^{7} \\ \text { C17 } & 0.4908 \times 10^{7} & \text { E21 } & -.1587 \times 10^{7} \\ T_{c, 2} & -.4889 \times 10^{7} & \text { E5 } & 0.1586 \times 10^{7} \\ \text { E12 } & -.4885 \times 10^{7} & \text { C16 } & 0.1566 \times 10^{7} \\ \text { C9 } & 0.4870 \times 10^{7} & \text { C12 } & -.1564 \times 10^{7} \\ \text { C8 } & 0.4870 \times 10^{7} & \text { C13 } & -.1557 \times 10^{7} \\ \text { E9 } & -.4868 \times 10^{7} & & \\ \text { E15 } & 0.4868 \times 10^{7} & & \\ \text { C5 } & 0.4865 \times 10^{7} & & \\ \text { C6 } & 0.4854 \times 10^{7} & & \\ \text { C11 } & 0.4847 \times 10^{7} & & \\ \text { C1 } & -.4845 \times 10^{7} & & \\ \text { E29 } & -.4832 \times 10^{7} & & \\ \text { C7 } & -.4822 \times 10^{7} & & \\ \text { C14 } & -.4811 \times 10^{7} & & \\ & & & \end{array}$


Table 26 B. Critical Line Locus Sensitivities $\mathrm{n}-\mathrm{C}_{6} / \mathrm{CO}_{2}$ ESD EOS - Bias

\begin{tabular}{|c|c|c|c|}
\hline Factor & Bias & Factor & Bias \\
\hline$\omega_{1}$ & $0.1733 \times 10^{8}$ & C16 & $0.2145 \times 10^{7}$ \\
\hline C3 & $-.1344 \times 10^{8}$ & E21 & $-.2048 \times 10^{7}$ \\
\hline E11 & $0.9738 \times 10^{7}$ & $\mathrm{C} 13$ & $-.2012 \times 10^{7}$ \\
\hline C15 & $0.9738 \times 10^{7}$ & $P_{c, 1}$ & $-.2010 \times 10^{7}$ \\
\hline E7 & $0.9683 \times 10^{7}$ & $\mathrm{C} 4$ & $-.2009 \times 10^{7}$ \\
\hline C2 & $0.9674 \times 10^{7}$ & $\mathrm{C} 12$ & $-.1998 \times 10^{7}$ \\
\hline E20 & $-.9674 \times 10^{7}$ & E22 & $-.1983 \times 10^{7}$ \\
\hline E18 & $0.9637 \times 10^{7}$ & E8 & $0.1964 \times 10^{7}$ \\
\hline E31 & $0.9610 \times 10^{7}$ & E19. & $-.1939 \times 10^{7}$ \\
\hline E13 & $-.9610 \times 10^{7}$ & E24 & $-.1937 \times 10^{7}$ \\
\hline E2 & $-.9600 \times 10^{7}$ & $\mathrm{C} 10$ & $-.1927 \times 10^{7}$ \\
\hline$P_{c, 2}$ & $0.9573 \times 10^{7}$ & E23 & $-.1926 \times 10^{7}$ \\
\hline E16 & $0.9536 \times 10^{7}$ & $\omega_{2}$ & $0.1919 \times 10^{7}$ \\
\hline E14 & $-.9527 \times 10^{7}$ & E5 & $0.1915 \times 10^{7}$ \\
\hline E27 & $-.9472 \times 10^{7}$ & E1 & $-.1915 \times 10^{7}$ \\
\hline $\mathrm{C} 8$ & $0.5857 \times 10^{7}$ & E30 & $0.1900 \times 10^{7}$ \\
\hline C11 & $0.5848 \times 10^{7}$ & E6 & $-.1898 \times 10^{7}$ \\
\hline E15 & $0.5825 \times 10^{7}$ & E3 & $0.1890 \times 10^{7}$ \\
\hline C14 & $-.5813 \times 10^{7}$ & E17 & $0.1882 \times 10^{7}$ \\
\hline C9 & $0.5810 \times 10^{7}$ & E26 & $-.1873 \times 10^{7}$ \\
\hline $\mathrm{E} 29$ & $-.5798 \times 10^{7}$ & $k_{12}$ & $0.1850 \times 10^{7}$ \\
\hline $\mathrm{E} 25$ & $-.5798 \times 10^{7}$ & E4 & $0.1793 \times 10^{7}$ \\
\hline $\mathrm{C} 7$ & $-.5736 \times 10^{7}$ & E28 & $-.1780 \times 10^{7}$ \\
\hline E12 & $-.5735 \times 10^{7}$ & & \\
\hline$T_{c, 2}$ & $-.5706 \times 10^{7}$ & & \\
\hline $\mathrm{C} 1$ & $-.5704 \times 10^{7}$ & & \\
\hline C6 & $0.5701 \times 10^{7}$ & & \\
\hline E9 & $-.5693 \times 10^{7}$ & & \\
\hline C5 & $0.5666 \times 10^{7}$ & & \\
\hline E10 & $-.5617 \times 10^{7}$ & & \\
\hline $\mathrm{C} 17$ & $0.5569 \times 10^{7}$ & & \\
\hline$T_{c, 1}$ & $0.5562 \times 10^{7}$ & & \\
\hline
\end{tabular}


Table 26 C. Critical Line Locus Sensitivities $n-C_{6} / \mathrm{CO}_{2}$ ESD EOS - CPU Time

$\begin{array}{llll}\text { Factor } & \text { CPU Time } & \text { Factor } & \text { CPU Time } \\ \text { E28 } & 0.2436 \times 10^{2} & \text { E17 } & -.9933 \times 10 \\ \text { C15 } & -.2253 \times 10^{2} & \text { E22 } & -.9684 \times 10 \\ k_{12} & 0.2238 \times 10^{2} & \text { C8 } & 0.8846 \times 10 \\ \text { C12 } & -.2236 \times 10^{2} & \text { E24 } & 0.8747 \times 10 \\ P_{c, 1} & 0.2209 \times 10^{2} & \text { C4 } & 0.8549 \times 10 \\ \omega_{1} & 0.2038 \times 10^{2} & T_{c, 2} & -.8399 \times 10 \\ \text { E19 } & 0.2011 \times 10^{2} & \text { E8 } & 0.7609 \times 10 \\ \text { C5 } & 0.2008 \times 10^{2} & \text { E31 } & 0.7312 \times 10 \\ \text { E2 } & -.1796 \times 10^{2} & \text { C13 } & 0.6672 \times 10 \\ \text { E26 } & -.1794 \times 10^{2} & \text { C7 } & -.6522 \times 10 \\ \text { E20 } & -.1761 \times 10^{2} & \text { E6 } & 0.5360 \times 10 \\ \text { C10 } & -.1759 \times 10^{2} & \text { C1 } & 0.5289 \times 10 \\ \text { E7 } & 0.1722 \times 10^{2} & \text { C6 } & 0.4992 \times 10 \\ \text { E25 } & -.1705 \times 10^{2} & \text { E1 } & -.4866 \times 10 \\ \text { E29 } & -.1586 \times 10^{2} & \text { C11 } & -.3508 \times 10 \\ \text { E10 } & -.1544 \times 10^{2} & \text { E12 } & -.2792 \times 10 \\ \text { C3 } & -.1512 \times 10^{2} & \text { E27 } & 0.2693 \times 10 \\ \text { C17 } & -.1512 \times 10^{2} & \text { E23 } & 0.2545 \times 10 \\ \text { E14 } & -.1494 \times 10^{2} & \text { E4 } & -.1235 \times 10 \\ \text { C9 } & 0.1450 \times 10^{2} & \text { E15 } & -.8893 \\ \text { E5 } & -.1440 \times 10^{2} & \text { E16 } & 0.5190 \\ \text { C16 } & -.1406 \times 10^{2} & T_{c, 1} & 0.3706 \\ \omega_{2} & -.1322 \times 10^{2} & \text { E11 } & 0.4941 \times 10^{-1} \\ \text { E13 } & -.1270 \times 10^{2} & & \\ \text { E18 } & 0.1233 \times 10^{2} & & \\ \text { C2 } & 0.1166 \times 10^{2} & & \\ \text { E21 } & -.1151 \times 10^{2} & & \\ \text { E30 } & 0.1122 \times 10^{2} & & \\ \text { E3 } & 0.1119 \times 10^{2} & & \\ \text { C14 } & 0.1109 \times 10^{2} & & \\ \text { E9 } & -.1089 \times 10^{2} & & \\ P_{r, 2} & -.1525 \times 10^{2} & & \\ & & & \end{array}$


Table 27. Maximum Sensitivities - Liquid Density Calculations - ALS EOS

\begin{tabular}{|l|c|c|c|c|}
\hline Response & $\mathrm{CO}_{2} / \mathrm{n}-\mathrm{C}_{4}$ & $\mathrm{CO}_{2} / \mathrm{n}-\mathrm{C}_{6}$ & $\mathrm{CO}_{2} / \mathrm{n}-\mathrm{C}_{8}$ & $\mathrm{CO}_{2} / \mathrm{n}-\mathrm{C}_{10}$ \\
\hline Ave Abs Error & 497. & 215. & 374. & 31. \\
Bias & 497. & 562. & 93. & 106. \\
CPU Time & 7.3 & 5.8 & 3.8 & 2.8 \\
\hline
\end{tabular}

Table 28. Migration of the Largest Five Sensitivities across Mixtures - Mixture Liquid Density - ALS EOS

Average Absolute Error

\begin{tabular}{|l|c|c|c|c|}
\hline Factor & $\mathrm{CO}_{2} / \mathrm{n}-\mathrm{C}_{4}$ & $\mathrm{CO}_{2} / \mathrm{n}-\mathrm{C}_{6}$ & $\mathrm{CO}_{2} / \mathrm{n}-\mathrm{C}_{8}$ & $\mathrm{CO}_{2} / \mathrm{n}-\mathrm{C}_{10}$ \\
\hline $\mathrm{C} 1$ & 1 & 1 & 2 & 6 \\
$\mathrm{E} 1$ & 2 & 9 & 5 & 26 \\
$\mathrm{E} 20$ & 3 & 16 & 6 & 43 \\
$\mathrm{C} 3$ & 4 & 55 & 56 & 46 \\
$\mathrm{E} 27$ & 5 & 37 & 32 & 8 \\
& & & & \\
$\mathrm{~T}_{c, 2}$ & 19 & 4 & 8 & 1 \\
$\mathrm{E} 18$ & 22 & 5 & 3 & 2 \\
$\mathrm{C} 12$ & 15 & 3 & 5 & 3 \\
$\mathrm{P}_{c, 2}$ & 23 & 18 & 16 & 4 \\
$\mathrm{E} 21$ & 26 & 23 & 28 & 5 \\
\hline
\end{tabular}

Table 29. Migration of the Largest Five Sensitivities across Mixtures - Mixture Liquid Density - ALS EOS

Bias

\begin{tabular}{|l|c|c|c|c|}
\hline Factor & $\mathrm{CO}_{2} / \mathrm{n}-\mathrm{C}_{4}$ & $\mathrm{CO}_{2} / \mathrm{n}-\mathrm{C}_{6}$ & $\mathrm{CO}_{2} / \mathrm{n}-\mathrm{C}_{8}$ & $\mathrm{CO}_{2} / \mathrm{n}-\mathrm{C}_{10}$ \\
\hline $\mathrm{C} 1$ & 1 & 1 & 1 & 42 \\
$\mathrm{E} 1$ & 2 & 35 & 22 & 17 \\
$\mathrm{E} 20$ & 3 & 40 & 30 & 32 \\
$\mathrm{C} 3$ & 4 & 20 & 19 & 43 \\
$\mathrm{E} 27$ & 5 & 29 & 55 & 20 \\
& & & & \\
$\omega_{1}$ & 13 & 10 & 9 & 1 \\
$\mathrm{~T}_{c, 2}$ & 17 & 4 & 10 & 2 \\
$\mathrm{E} 12$ & 39 & 43 & 45 & 3 \\
$\mathrm{E} 13$ & 52 & 51 & 49 & 4 \\
$\mathrm{E} 22$ & 43 & 26 & 24 & 5 \\
\hline
\end{tabular}


Table 30. Number of the Five Most Sensitive Factors in the Higher Level Parameters

- ALS EOS; Mixture Liquid Density - Error Responses

Average Absolute Error

\begin{tabular}{|l|c|c|c|c|}
\hline Parameter & $\mathrm{CO}_{2} / \mathrm{n}-\mathrm{C}_{4}$ & $\mathrm{CO}_{2} / \mathrm{n}-\mathrm{C}_{6}$ & $\mathrm{CO}_{2} / \mathrm{n}-\mathrm{C}_{8}$ & $\mathrm{CO}_{2} / \mathrm{n}-\mathrm{C}_{10}$ \\
\hline $\mathrm{a}(\mathrm{T})$ & 0 & 1 & 1 & 1 \\
$\mathrm{~b}_{1}$ & 2 & 1 & 1 & 1 \\
$\mathrm{~b}_{2}$ & 0 & 0 & 0 & 0 \\
$\mathrm{~b}_{3}$ & 0 & 0 & 0 & 0 \\
\hline
\end{tabular}

Critical Properties

\begin{tabular}{|l|c|c|c|c|}
\hline & $\mathrm{CO}_{2} / \mathrm{n}-\mathrm{C}_{4}$ & $\mathrm{CO}_{2} / \mathrm{n}-\mathrm{C}_{6}$ & $\mathrm{CO}_{2} / \mathrm{n}-\mathrm{C}_{8}$ & $\mathrm{CO}_{2} / \mathrm{n}-\mathrm{C}_{10}$ \\
\hline n-alkane & 0 & 0 & 0 & 0 \\
$\mathrm{CO}_{2}$ & 0 & 1 & 0 & 2 \\
$\mathrm{k}_{i j}$ & 0 & 0 & 0 & 0 \\
\hline
\end{tabular}

Bias

\begin{tabular}{|l|c|c|c|c|}
\hline Parameter & $\mathrm{CO}_{2} / \mathrm{n}-\mathrm{C}_{4}$ & $\mathrm{CO}_{2} / \mathrm{n}-\mathrm{C}_{6}$ & $\mathrm{CO}_{2} / \mathrm{n}-\mathrm{C}_{8}$ & $\mathrm{CO}_{2} / \mathrm{n}-\mathrm{C}_{10}$ \\
\hline $\mathrm{a}(\mathrm{T})$ & 0 & 1 & 1 & 1 \\
$\mathrm{~b}_{1}$ & 2 & 1 & 2 & 1 \\
$\mathrm{~b}_{2}$ & 0 & 0 & 0 & 0 \\
$\mathrm{~b}_{3}$ & 0 & 1 & 0 & 1 \\
\hline
\end{tabular}

Critical Properties

\begin{tabular}{|l|c|c|c|c|}
\hline & $\mathrm{CO}_{2} / \mathrm{n}-\mathrm{C}_{4}$ & $\mathrm{CO}_{2} / \mathrm{n}-\mathrm{C}_{6}$ & $\mathrm{CO}_{2} / \mathrm{n}-\mathrm{C}_{8}$ & $\mathrm{CO}_{2} / \mathrm{n}-\mathrm{C}_{10}$ \\
\hline n-alkane & 0 & 1 & 2 & 0 \\
$\mathrm{CO}_{2}$ & 0 & 1 & 0 & 2 \\
$\mathrm{k}_{i j}$ & 0 & 0 & 0 & 0 \\
\hline
\end{tabular}


Table 31. Maximum Sensitivities - Liquid Density Calculations - ESD EOS

\begin{tabular}{|l|c|c|c|c|}
\hline Response & $\mathrm{CO}_{2} / \mathrm{n}-\mathrm{C}_{4}$ & $\mathrm{CO}_{2} / \mathrm{n}-\mathrm{C}_{6}$ & $\mathrm{CO}_{2} / \mathrm{n}-\mathrm{C}_{8}$ & $\mathrm{CO}_{2} / \mathrm{n}-\mathrm{C}_{10}$ \\
\hline Ave Abs Error & 18. & 148. & 189. & 11. \\
Bias & 25. & 182. & 263. & 154. \\
CPU Time & 4.9 & 1.8 & 6.9 & 5.7 \\
\hline
\end{tabular}

Table 32. Migration of the Largest Five Sensitivities across Mixtures - Mixture Liquid Density - ESD EOS

Average Absolute Error

\begin{tabular}{|l|c|c|c|c|}
\hline Factor & $\mathrm{CO}_{2} / \mathrm{n}-\mathrm{C}_{4}$ & $\mathrm{CO}_{2} / \mathrm{n}-\mathrm{C}_{6}$ & $\mathrm{CO}_{2} / \mathrm{n}-\mathrm{C}_{8}$ & $\mathrm{CO}_{2} / \mathrm{n}-\mathrm{C}_{10}$ \\
\hline $\mathrm{C} 16$ & 1 & 1 & 4 & 5 \\
$\mathrm{C} 8$ & 2 & 6 & 6 & 12 \\
$\mathrm{C} 4$ & 3 & 8 & 10 & 21 \\
$\mathrm{C} 17$ & 4 & 4 & 5 & 48 \\
$\mathrm{~T}_{c, 2}$ & 5 & 20 & 32 & 1 \\
& & & & \\
$\mathrm{~T}_{c, 2}$ & 5 & 20 & 32 & 1 \\
$\mathrm{E} 19$ & 14 & 48 & 29 & 2 \\
$\mathrm{E} 5$ & 15 & 28 & 25 & 3 \\
$\mathrm{E} 29$ & 16 & 33 & 52 & 4 \\
$\mathrm{C} 16$ & 1 & 1 & 4 & 5 \\
\hline
\end{tabular}

Table 33. Migration of the Largest Five Sensitivities across Mixtures - Mixture Liquid Density - ESD EOS

Bias

\begin{tabular}{|l|c|c|c|c|}
\hline Factor & $\mathrm{CO}_{2} / \mathrm{n}-\mathrm{C}_{4}$ & $\mathrm{CO}_{2} / \mathrm{n}-\mathrm{C}_{6}$ & $\mathrm{CO}_{2} / \mathrm{n}-\mathrm{C}_{8}$ & $\mathrm{CO}_{2} / \mathrm{n}-\mathrm{C}_{10}$ \\
\hline $\mathrm{C} 16$ & 1 & 1 & 4 & 1 \\
$\mathrm{C} 8$ & 2 & 5 & 6 & 3 \\
$\mathrm{C} 4$ & 3 & 7 & 8 & 4 \\
$\mathrm{C} 17$ & 4 & 4 & 5 & 5 \\
$\mathrm{C} 5$ & 5 & 8 & 7 & 6 \\
& & & & \\
$\mathrm{C} 16$ & 1 & 1 & 4 & 1 \\
$\mathrm{~T}_{c, 2}$ & 6 & 16 & 36 & 2 \\
$\mathrm{C} 8$ & 2 & 5 & 6 & 3 \\
$\mathrm{C} 4$ & 3 & 7 & 8 & 4 \\
$\mathrm{C} 17$ & 4 & 4 & 5 & 5 \\
\hline
\end{tabular}


Table 34. Number of the Five Most Sensitive Factors in the Higher Level Parameters

- ESD EOS; Mixture Liquid Density - Error Responses

Average Absolute Error

\begin{tabular}{|l|c|c|c|c|}
\hline Parameter & $\mathrm{CO}_{2} / \mathrm{n}-\mathrm{C}_{4}$ & $\mathrm{CO}_{2} / \mathrm{n}-\mathrm{C}_{6}$ & $\mathrm{CO}_{2} / \mathrm{n}-\mathrm{C}_{8}$ & $\mathrm{CO}_{2} / \mathrm{n}-\mathrm{C}_{10}$ \\
\hline $\mathrm{c}_{i}$ & 0 & 0 & 0 & 0 \\
$\epsilon_{i i}$ & 2 & 0 & 0 & 0 \\
$\mathrm{v}_{i i}$ & 0 & 2 & 2 & 0 \\
$\mathrm{Y}_{i j}$ & 1 & 1 & 1 & 1 \\
$\mathrm{q}_{i}$ & 1 & 1 & 1 & 0 \\
\hline
\end{tabular}

Critical Properties

\begin{tabular}{|l|c|c|c|c|}
\hline & $\mathrm{CO}_{2} / \mathrm{n}-\mathrm{C}_{4}$ & $\mathrm{CO}_{2} / \mathrm{n}-\mathrm{C}_{6}$ & $\mathrm{CO}_{2} / \mathrm{n}-\mathrm{C}_{8}$ & $\mathrm{CO}_{2} / \mathrm{n}-\mathrm{C}_{10}$ \\
\hline n-alkane & 0 & 1 & 1 & 0 \\
$\mathrm{CO}_{2}$ & 0 & 0 & 0 & 1 \\
$\mathrm{k}_{i j}$ & 0 & 0 & 0 & 0 \\
\hline
\end{tabular}

Bias

\begin{tabular}{|l|c|c|c|c|}
\hline Parameter & $\mathrm{CO}_{2} / \mathrm{n}-\mathrm{C}_{4}$ & $\mathrm{CO}_{2} / \mathrm{n}-\mathrm{C}_{6}$ & $\mathrm{CO}_{2} / \mathrm{n}-\mathrm{C}_{8}$ & $\mathrm{CO}_{2} / \mathrm{n}-\mathrm{C}_{10}$ \\
\hline$\epsilon_{i i}$ & 3 & 1 & 1 & 2 \\
$\mathrm{v}_{i i}$ & 0 & 1 & 1 & 0 \\
$\mathrm{Y}_{i j}$ & 1 & 1 & 1 & 1 \\
$\mathrm{q}_{i}$ & 1 & 1 & 1 & 1 \\
\hline
\end{tabular}

Critical Properties

\begin{tabular}{|l|c|c|c|c|}
\hline & $\mathrm{CO}_{2} / \mathrm{n}-\mathrm{C}_{4}$ & $\mathrm{CO}_{2} / \mathrm{n}-\mathrm{C}_{6}$ & $\mathrm{CO}_{2} / \mathrm{n}-\mathrm{C}_{8}$ & $\mathrm{CO}_{2} / \mathrm{n}-\mathrm{C}_{10}$ \\
\hline n-alkane & 0 & 1 & 1 & 0 \\
$\mathrm{CO}_{2}$ & 0 & 0 & 0 & 1 \\
$\mathrm{k}_{i j}$ & 0 & 0 & 0 & 0 \\
\hline
\end{tabular}


Table 35. Confoundings - ALS EOS test plans

\begin{tabular}{|c|c|c|c|c|c|c|c|c|c|c|c|c|}
\hline $\mathrm{E} 1$ & $=$ & $1 * 2$ & $7 * 3$ & $8 * 4$ & $9 * 5$ & $10^{*} 6$ & $17^{*} 11$ & $18^{*} 12$ & $19^{*} 13$ & $20 * 14$ & $21^{*} 15$ & $22^{*} 16$ \\
\hline $\mathrm{E} 2$ & $=$ & $1^{*} 3$ & $7 * 2$ & $11^{*} 4$ & $12^{*} 5$ & $13^{*} 6$ & $17^{*} 8$ & $18 * 9$ & $19^{*} 10$ & $23^{*} 14$ & $24^{*} 15$ & $25^{*} 16$ \\
\hline$=$ & $=$ & $1^{*} 4$ & $8^{*} 2$ & $11^{*} 3$ & $14^{*} 5$ & $15^{*} 6$ & $17^{* 7}$ & $20 * 9$ & $21^{*} 10$ & $23^{*} 12$ & $24 * 13$ & \\
\hline 4 & $=$ & $1 * 5$ & $9 * 2$ & $12 * 3$ & $14^{*} 4$ & $16^{*} 6$ & $18^{*} 7$ & $20 * 8$ & $22 * 10$ & $23^{*} 11$ & $25^{*} 13$ & \\
\hline & $=$ & $1 * 6$ & $10^{*} 2$ & $13^{*} 3$ & $15^{*} 4$ & $16^{*} 5$ & $19^{*} 7$ & $21^{*} 8$ & $22 * 9$ & $24^{*} 11$ & $25 * 12$ & \\
\hline & $=$ & $2^{*} 3$ & $7^{*} 1$ & $11^{*} 8$ & $12 * 9$ & $13^{*} 10$ & $17^{*} 4$ & $18 * 5$ & $19 * 6$ & $23^{*} 20$ & $24 * 21$ & $25^{*} 22$ \\
\hline $7=$ & $=$ & $2 * 4$ & $8 * 1$ & $11^{* 7}$ & $14^{*} 9$ & $15^{*} 10$ & $17^{*} 3$ & $20 * 5$ & $21 * 6$ & $23^{*} 18$ & $24^{*} 19$ & \\
\hline & $=$ & $2 * 5$ & $9 * 1$ & $12^{*} 7$ & $14 * 8$ & $16^{*} 10$ & $18 * 3$ & $20 * 4$ & $22^{*} 6$ & $23^{*} 17$ & $25 * 19$ & \\
\hline 9 & $=$ & $2^{*} 6$ & $10^{*} 1$ & $13^{*} 7$ & $15^{*} 8$ & $16^{*} 9$ & $19^{*} 3$ & $21 * 4$ & $22 * 5$ & $24^{*} 17$ & $25^{*} 18$ & \\
\hline E10 & $=$ & $3 * 4$ & $8 * 7$ & $11^{*} 1$ & $14^{*} 12$ & $15^{*} 13$ & $17^{*} 2$ & $20 * 18$ & $21^{*} 19$ & $23^{*} 5$ & $24^{*} 6$ & \\
\hline $11=$ & $=$ & $3 * 5$ & $9^{*} 7$ & $12^{*} 1$ & $14^{*} 11$ & $16^{*} 13$ & $18^{*} 2$ & $20^{*} 17$ & $22^{*} 19$ & $23^{*} 4$ & $25^{*} 6$ & \\
\hline E12 & $=$ & $3^{*} 6$ & $10^{*} 7$ & $13^{*} 1$ & $15^{*} 11$ & $16^{*} 12$ & $19^{*} 2$ & $21 * 17$ & $22^{*} 18$ & $24^{*} 4$ & $25^{*} 5$ & \\
\hline E13 & $=$ & $4^{* 5}$ & $9 * 8$ & $12^{*} 11$ & $14^{*} 1$ & $16^{*} 15$ & $18^{*} 17$ & $20 * 2$ & $22 * 21$ & $23^{*} 3$ & $25^{*} 24$ & \\
\hline E14 & $=$ & $4^{*} 6$ & $10 * 8$ & $13^{*} 11$ & $15 * 1$ & $16^{*} 14$ & $19 * 17$ & $21 * 2$ & $22 * 20$ & $24^{*} 3$ & $25 * 23$ & \\
\hline E15 & $=$ & $5^{*} 6$ & $10^{*} 9$ & $13^{*} 12$ & $15^{*} 14$ & $16^{* 1}$ & $19 * 18$ & $21 * 20$ & $22 * 2$ & $24^{*} 23$ & $25^{*} 3$ & \\
\hline E16 & $=$ & $1^{*} 2^{*} 3^{*} 4$ & $7 * 4$ & $8 * 3$ & $11 * 2$ & $17^{*} 1$ & $18^{*} 14$ & $19^{*} 15$ & $20 * 12$ & $21^{*} 13$ & $23^{*} 9$ & $24^{*} 10$ \\
\hline E17 & $=$ & $1 * 2 * 3 * 5$ & $7^{*} 5$ & $9 * 3$ & $12^{*} 2$ & $17^{*} 14$ & $18^{*} 1$ & $19^{*} 16$ & $20^{*} 11$ & $22^{*} 13$ & $23^{*} 8$ & $25 * 10$ \\
\hline E18 & $=$ & $1 * 2 * 3 * 6$ & $7^{*} 6$ & $10^{*} 3$ & $13^{*} 2$ & $17^{*} 15$ & $18^{*} 16$ & $19^{*} 1$ & $21^{*} 11$ & $22^{*} 12$ & $24^{*} 8$ & $25 * 9$ \\
\hline E19 & $=$ & $1 * 2 * 4^{*} 5$ & $8 * 5$ & $9 * 4$ & $14^{*} 2$ & $17^{*} 12$ & $18^{*} 11$ & $20^{*} 1$ & $21^{*} 16$ & $22^{*} 15$ & $23^{*} 7$ & \\
\hline $\mathrm{E} 20$ & $=$ & $1^{*} 2^{*} 4^{*} 6$ & $8 * 6$ & $10^{*} 4$ & $15^{*} 2$ & $17^{*} 13$ & $19^{*} 11$ & $20^{*} 16$ & $21^{* 1}$ & $22^{*} 14$ & $24^{*} ?$ & \\
\hline E21 & $=$ & $1 * 2 * 5 * 6$ & $9 * 6$ & $10^{*} 5$ & $16^{*} 2$ & $18^{*} 13$ & $19^{*} 12$ & $20 * 15$ & $21^{*} 14$ & $22^{*} 1$ & $25^{*} 7$ & \\
\hline $\mathrm{E} 22$ & $=$ & $1^{*} 3^{*} 4^{*} 5$ & $11^{*} 5$ & $12^{*} 4$ & $14^{*} 3$ & $17^{*} g$ & $18^{*} 8$ & $20 * 7$ & $23^{*} 1$ & $24^{*} 16$ & $25 * 15$ & \\
\hline E23 & $=$ & $1^{*} 3^{*} 4^{*} 6$ & $11^{*} 6$ & $13^{*} 4$ & $15^{*} 3$ & $17^{*} 10$ & $19^{*} 8$ & $21^{* 7}$ & $23^{*} 16$ & $24^{*} 1$ & $25^{*} 14$ & \\
\hline E24 & $=$ & $1^{*} 3^{*} 5^{*} 6$ & $12^{*} 6$ & $13^{*} 5$ & $16 * 3$ & $18^{*} 10$ & $19^{*} 9$ & $22 * 7$ & $23^{*} 15$ & $24^{*} 14$ & $25^{*} 1$ & \\
\hline E25 & $=$ & $1 * 4^{*} 5^{*} 6$ & $14^{*} 6$ & $15^{*} 5$ & $16^{*} 4$ & $20^{*} 10$ & $21 * 9$ & $22^{*} 8$ & $23^{*} 13$ & $24^{*} 12$ & $25^{*} 11$ & \\
\hline E26 & $=$ & $2 * 3 * 4^{*} 5$ & $11^{*} 9$ & $12 * 8$ & $14 * 7$ & $17^{*} 5$ & $18^{*} 4$ & $20 * 3$ & $23^{*} 2$ & $24^{*} 22$ & $25^{*} 21$ & \\
\hline $\mathrm{E} 27$ & $=$ & $2 * 3 * 4 * 6$ & $11^{*} 10$ & $13^{*} 8$ & $15^{*} 7$ & $17^{*} 6$ & $19^{*} 4$ & $21^{*} 3$ & $23^{*} 22$ & $24^{*} 2$ & $25^{*} 20$ & \\
\hline E28 & $=$ & $2^{*} 3^{*} 5^{*} 6$ & $12^{*} 10$ & $13^{*} 9$ & $16^{* 7}$ & $18 * 6$ & $19^{*} 5$ & $22 * 3$ & $23^{*} 21$ & $24^{*} 20$ & $25^{*} 2$ & \\
\hline E29 & $=$ & $2^{*} 4^{*} 5^{*} 6$ & $14^{*} 10$ & $15^{*} 9$ & $16^{*} 8$ & $20 * 6$ & $21^{*} 5$ & $22^{*} 4$ & $23^{*} 19$ & $24^{*} 18$ & $25^{*} 17$ & \\
\hline E30 & $=$ & $3^{*} 4^{*} 5^{*} 6$ & $14^{*} 13$ & $15^{*} 12$ & $16^{*} 11$ & $20^{*} 19$ & $21 * 18$ & $22^{*} 17$ & $23^{*} 6$ & $24 * 5$ & $25 * 4$ & \\
\hline 31 & $=$ & $1^{*} 2^{*} 3^{*} 4^{*} 5^{*} 6$ & $17^{*} 16$ & $18^{*} 15$ & $19^{*} 14$ & $20^{*} 13$ & $21^{*} 12$ & $22^{*} 11$ & $23^{*} 10$ & $24 * 9$ & $25^{*} 8$ & \\
\hline
\end{tabular}


Table 35. Confoundings - ESD EOS test plans

\begin{tabular}{|c|c|c|c|c|c|c|c|c|c|c|c|c|}
\hline E1 & $=$ & $1 * 2$ & $7^{*} 3$ & $8 * 4$ & $9 * 5$ & $10 * 6$ & $17^{*} 11$ & $18^{*} 12$ & $19 * 13$ & $20 * 14$ & $21^{*} 15$ & $22 * 16$ \\
\hline $\mathrm{E} 2$ & $=$ & $1 * 3$ & $7^{*} 2$ & $11^{*} 4$ & $12^{*} 5$ & $13^{*} 6$ & $17^{*} 8$ & $18 * 9$ & $19^{*} 10$ & $23^{*} 14$ & $24^{*} 15$ & \\
\hline E3 & $=$ & $1 * 4$ & $8 * 2$ & $11^{*} 3$ & $14^{*} 5$ & $15^{*} 6$ & $17^{*} 7$ & $20 * \theta$ & $21^{*} 10$ & $23^{*} 12$ & $24^{*} 13$ & \\
\hline $\mathrm{E} 4$ & $=$ & $1 * 5$ & $9 * 2$ & $12 * 3$ & $14^{*} 4$ & $16^{*} 6$ & $18 * 7$ & $20 * 8$ & $22^{*} 10$ & $23^{*} 11$ & & \\
\hline E5 & $=$ & $1 * 6$ & $10 * 2$ & $13^{*} 3$ & $15 * 4$ & $\therefore 5$ & $19 * 7$ & $21^{*} 8$ & $22 * 9$ & $24^{*} 11$ & & \\
\hline E6 & $=$ & $2 * 3$ & $T^{*} 1$ & $11 * 8$ & $12 * 9$ & $13^{*} 10$ & $17^{*} 4$ & $18^{*} 5$ & $19^{*} 6$ & $23^{*} 20$ & $24^{*} 21$ & \\
\hline E7 & $=$ & $2 * 4$ & $8^{*} 1$ & $11^{*} 7$ & $14^{*} 9$ & $15^{*} 10$ & $17 * 3$ & $20^{*} 5$ & $21 * 6$ & $23^{*} 18$ & $24^{*} 19$ & \\
\hline E8 & $=$ & $2 * 5$ & $9 * 1$ & $12^{*} 7$ & $14^{*} 8$ & $16^{*} 10$ & $18 * 3$ & $20 * 4$ & $22^{*} 6$ & $23^{*} 17$ & & \\
\hline E9 & $=$ & $2^{*} 6$ & $10^{*} 1$ & $13^{*} 7$ & $15 * 8$ & $16 * 9$ & $19 * 3$ & $21^{*} 4$ & $22^{*} 5$ & $24 * 17$ & & \\
\hline E10 & $=$ & $3^{*} 4$ & $8^{*} 7$ & $11^{* 1}$ & $14^{*} 12$ & $15^{*} 13$ & $17^{*} 2$ & $20^{*} 18$ & $21 * 19$ & $23^{*} 5$ & $24^{*} 6$ & \\
\hline 11 & $=$ & $3^{*} 5$ & $9 * 7$ & $12 * 1$ & $14^{*} 11$ & $16^{*} 13$ & $18 * 2$ & $20 * 17$ & $22 * 19$ & $23^{*} 4$ & & \\
\hline E12 & $=$ & $3^{*} 6$ & $10^{*} 7$ & $13^{*} 1$ & $15^{*} 11$ & $16^{*} 12$ & $19^{*} 2$ & $21 * 17$ & $22 * 18$ & $24^{*} 4$ & & \\
\hline E13 & $=$ & $4^{*} 5$ & $9 * 8$ & $12^{*} 11$ & $14^{*} 1$ & $16^{*} 15$ & $18^{*} 17$ & $20 * 2$ & $22^{*} 21$ & $23 * 3$ & & \\
\hline E14 & $=$ & $4^{*} 6$ & $10^{*} 8$ & $13^{*} 11$ & $15^{*} 1$ & $16^{*} 14$ & $19^{*} 17$ & $21^{*} 2$ & $22 * 20$ & $24 * 3$ & . & \\
\hline E15 & $=$ & $5 * 6$ & $10 * 9$ & $13^{*} 12$ & $15^{*} 14$ & $16^{*} 1$ & $19^{*} 18$ & $21 * 20$ & $22^{*} 2$ & $24^{*} 23$ & & \\
\hline E16 & $=$ & $1 * 2 * 3 * 4$ & $7 * 4$ & $8 * 3$ & $11^{*} 2$ & $17^{*} 1$ & $18^{*} 14$ & $19^{*} 15$ & $20^{*} 12$ & $21^{*} 13$ & $23^{*} 9$ & $24 * 10$ \\
\hline E 17 & $=$ & $1 * 2 * 3 * 5$ & $7 * 5$ & $9 * 3$ & $12^{*} 2$ & $17^{*} 14$ & $18^{*} 1$ & $19^{*} 16$ & $20^{*} 11$ & $22^{*} 13$ & $23 * 8$ & \\
\hline E18 & $=$ & $1 * 2 * 3 * 6$ & $7 * 6$ & $10 * 3$ & $13^{*} 2$ & $17^{*} 15$ & $18 * 16$ & $19^{*} 1$ & $21^{*} 11$ & $22^{*} 12$ & $24 * 8$ & \\
\hline E19 & $=$ & $1^{*} 2^{*} 4^{*} 5$ & $8 * 5$ & $9 * 4$ & $14^{*} 2$ & $17^{*} 12$ & $18^{*} 11$ & $20 * 1$ & $21^{*} 16$ & $22^{*} 15$ & $23 * 7$ & \\
\hline E20 & $=$ & $1^{*} 2^{*} 4^{*} 6$ & $8^{*} 6$ & $10 * 4$ & $15 * 2$ & $17^{*} 13$ & $19^{*} 11$ & $20^{*} 16$ & $21^{*} 1$ & $22^{*} 14$ & $24^{*} 7$ & \\
\hline $\mathrm{E} 21$ & $=$ & $1^{*} 2^{*} 5^{*} 6$ & $9 * 6$ & $10 * 5$ & $16^{*} 2$ & $18^{*} 13$ & $19^{*} 12$ & $20^{*} 15$ & $21^{*} 14$ & $22^{*} 1$ & & \\
\hline E22 & $=$ & $1^{*} 3^{*} 4^{*} 5$ & $11^{*} 5$ & $12^{*} 4$ & $14^{*} 3$ & $17^{*} 9$ & $18 * 8$ & $20 * 7$ & $23^{*} 1$ & $24 * 16$ & & \\
\hline $\mathrm{E} 23$ & $=$ & $1^{*} 3^{*} 4^{*} 6$ & $11^{*} 6$ & $13^{*} 4$ & $15^{*} 3$ & $17^{*} 10$ & $19 * 8$ & $21^{*} 7$ & $23^{*} 16$ & $24^{*} 1$ & & \\
\hline E24 & $=$ & $1^{*} 3^{*} 5 * 6$ & $12^{*} 6$ & $13^{*} 5$ & $16^{*} 3$ & $18^{*} 10$ & $19^{*} 9$ & $22 * 7$ & $23^{*} 15$ & $24^{*} 14$ & & \\
\hline E25 & $=$ & $1^{*} 4^{*} 5^{*} 6$ & $14^{*} 6$ & $15^{*} 5$ & $16^{*} 4$ & $20 * 10$ & $21^{*} 9$ & $22 * 8$ & $23^{*} 13$ & $24^{*} 12$ & & \\
\hline $\mathrm{E} 26$ & $=$ & $2 * 3 * 4^{*} 5$ & $11^{*} 9$ & $12 * 8$ & $14^{*} 7$ & $17^{*} 5$ & $18^{*} 4$ & $20 * 3$ & $23^{*} 2$ & $24 * 22$ & & \\
\hline $\mathrm{E} 27^{\prime}$ & $=$ & $2 * 3^{*} 4^{*} 6$ & $11^{*} 10$ & $13^{*} 8$ & $15^{* 7}$ & $17^{*} 6$ & $19^{*} 4$ & $21^{*} 3$ & $23^{*} 22$ & $24^{*} 2$ & & \\
\hline E28 & $=$ & $2^{*} 3^{*} 5^{*} 6$ & $12^{*} 10$ & $13^{*} 9$ & $16 * 7$ & $18^{*} 6$ & $19^{*} 5$ & $22 * 3$ & $23^{*} 21$ & $24 * 20$ & & \\
\hline E29 & $=$ & $2^{*} 4^{*} 5^{*} 6$ & $14^{*} 10$ & $15^{*} 9$ & $16^{*} 8$ & $20 * 6$ & $21^{*} 5$ & $22 * 4$ & $23^{*} 19$ & $24^{*} 18$ & & \\
\hline E30 & $=$ & $3 * 4 * 5 * 6$ & $14^{*} 13$ & $15^{*} 12$ & $16^{*} 11$ & $20^{*} 19$ & $21^{*} 18$ & $22^{*} 17$ & $23^{*} 6$ & $24^{*} 5$ & & \\
\hline 1 & $=$ & $1^{*} 2^{*} 3^{*} 4^{*} 5^{*} 6$ & $17^{*} 16$ & $18 * 15$ & $19^{\star} 14$ & $20^{*} 13$ & $21^{*} 12$ & $22^{*} 11$ & $23^{*} 10$ & $24 * 9$ & & \\
\hline
\end{tabular}


PRESSURE CALCULATIONS FOR CARBON DIOXIDE

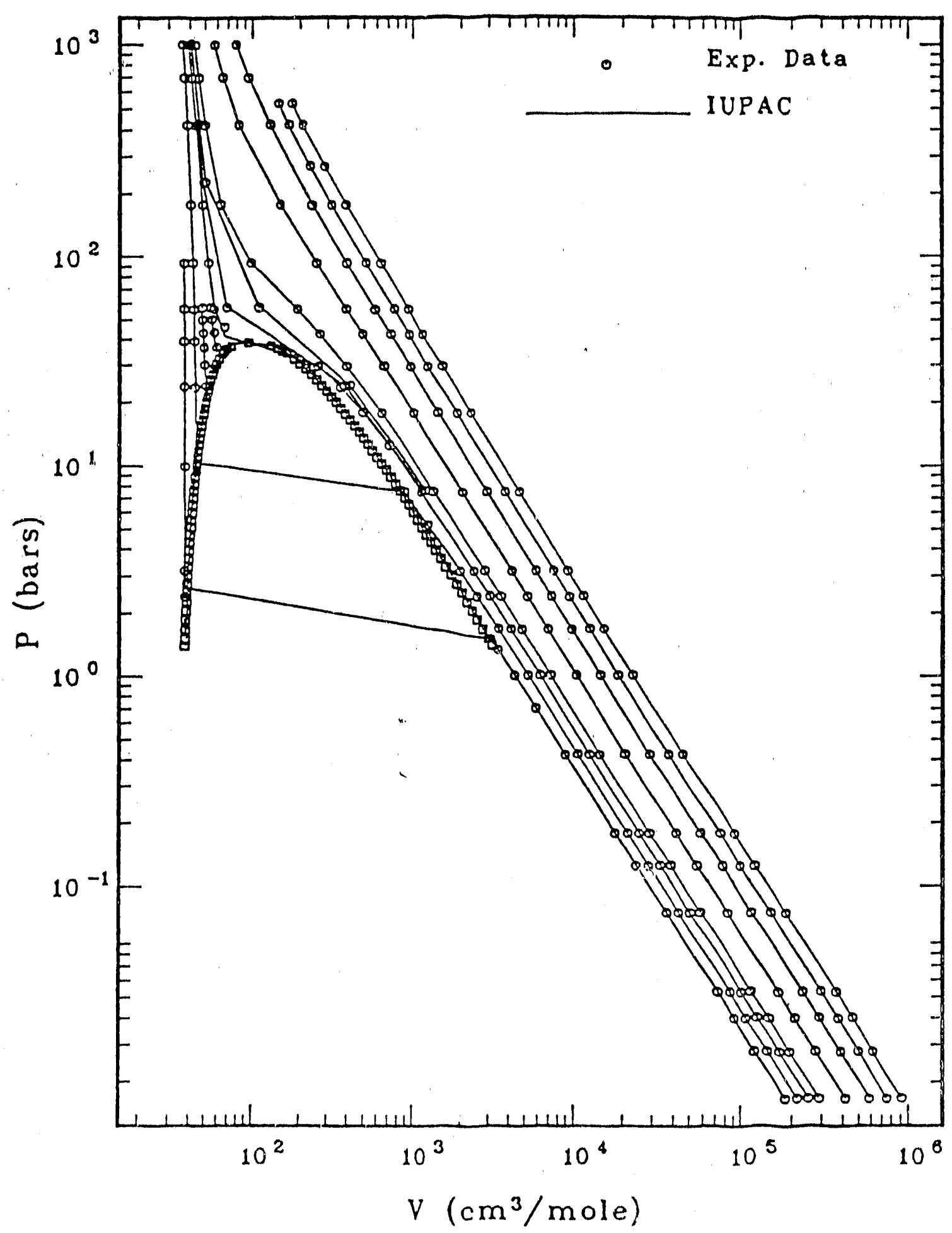

Figure 1. A comparison with experimental values of calculated pressure for carbon dioxide using the IUPAC equation of state. 


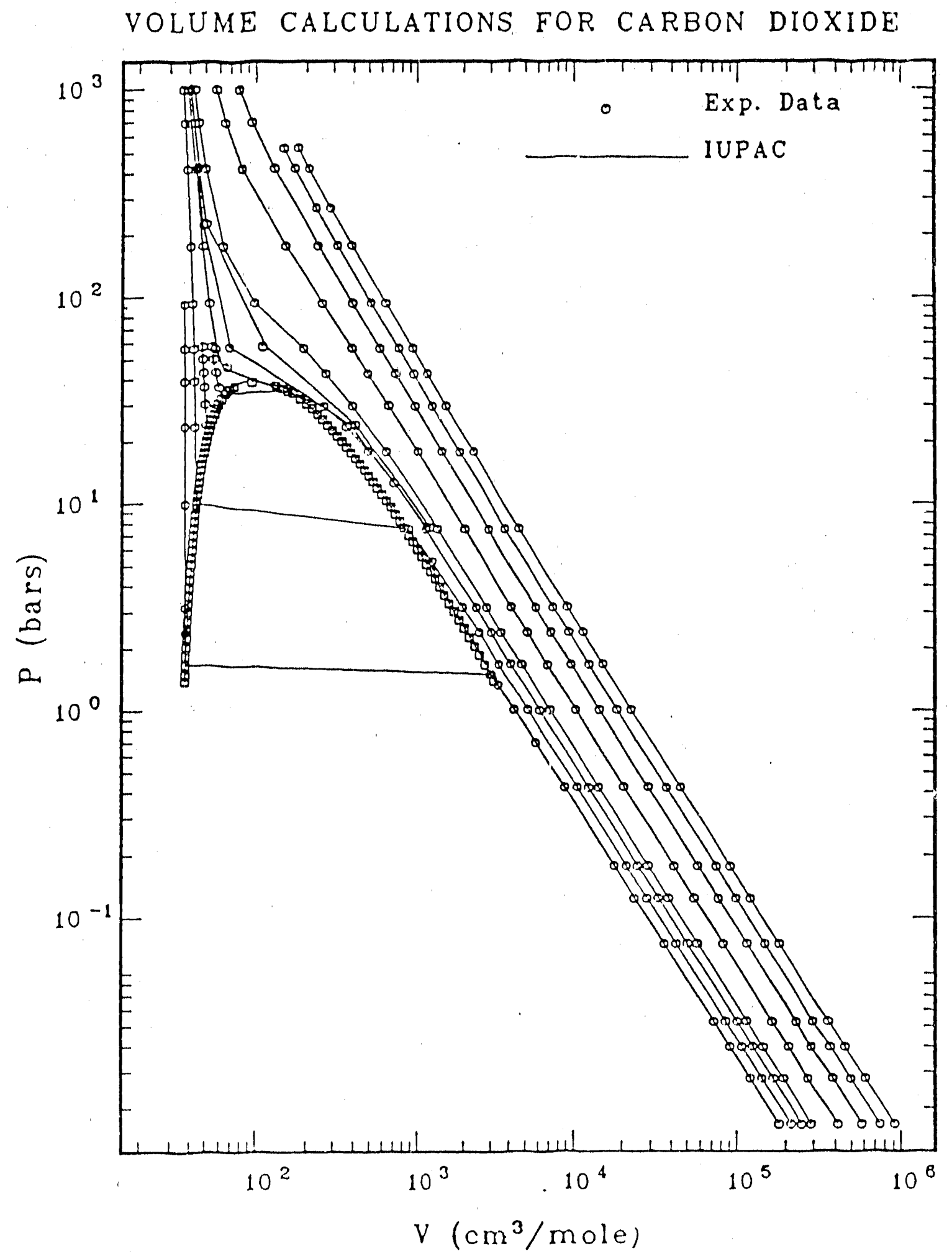

Figure 2. A comparison with experimental values of calculated volume for carbon dioxide using the IUPAC equation of state. 


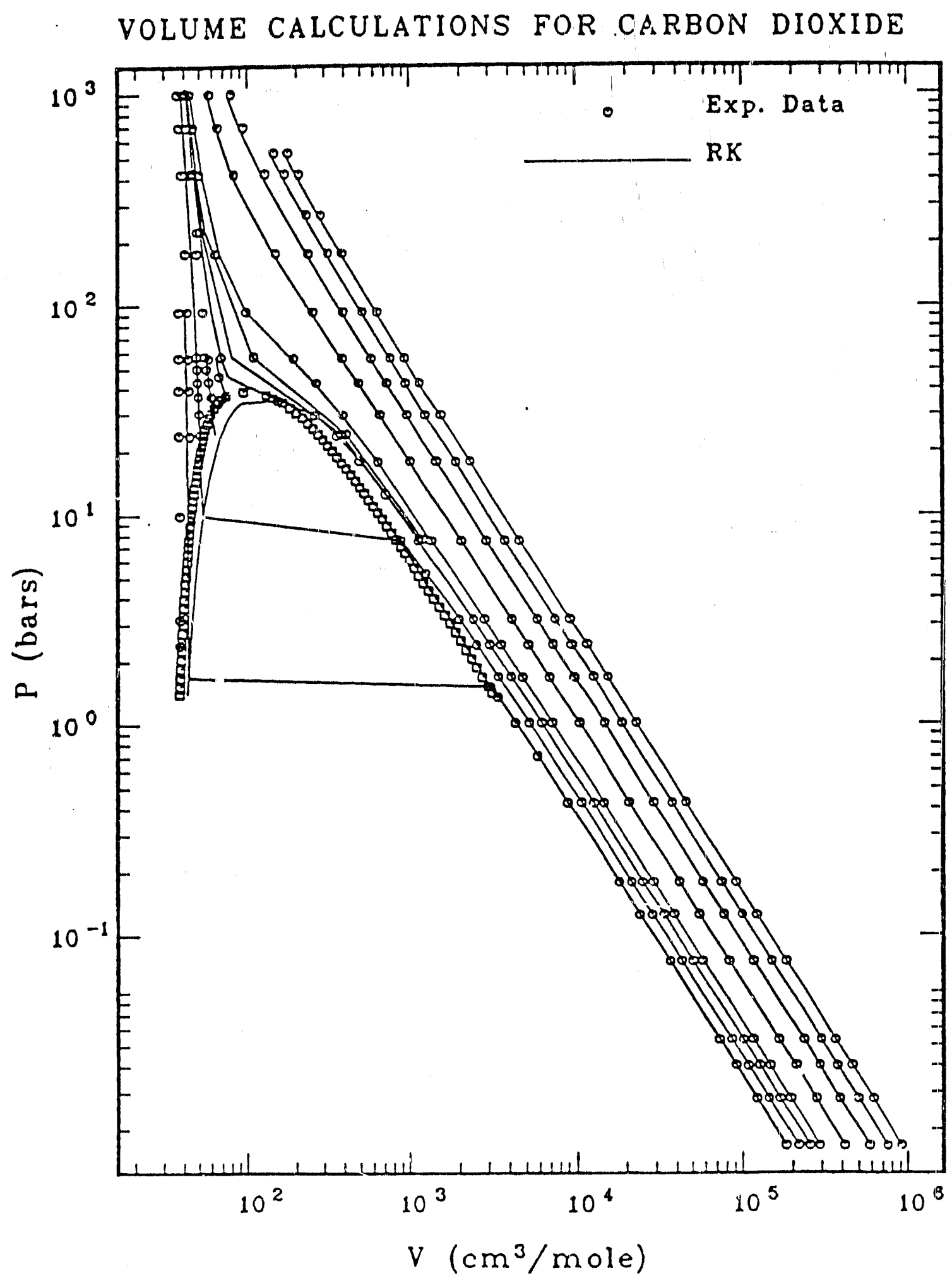

Figure 3. A comparison with experimental values of calculated volume for carbon dioxide using the RK equatic state. 




Figure 4. A comparison with experimental values of calculated volume for carbon dioxide using the RKS equation of state. 


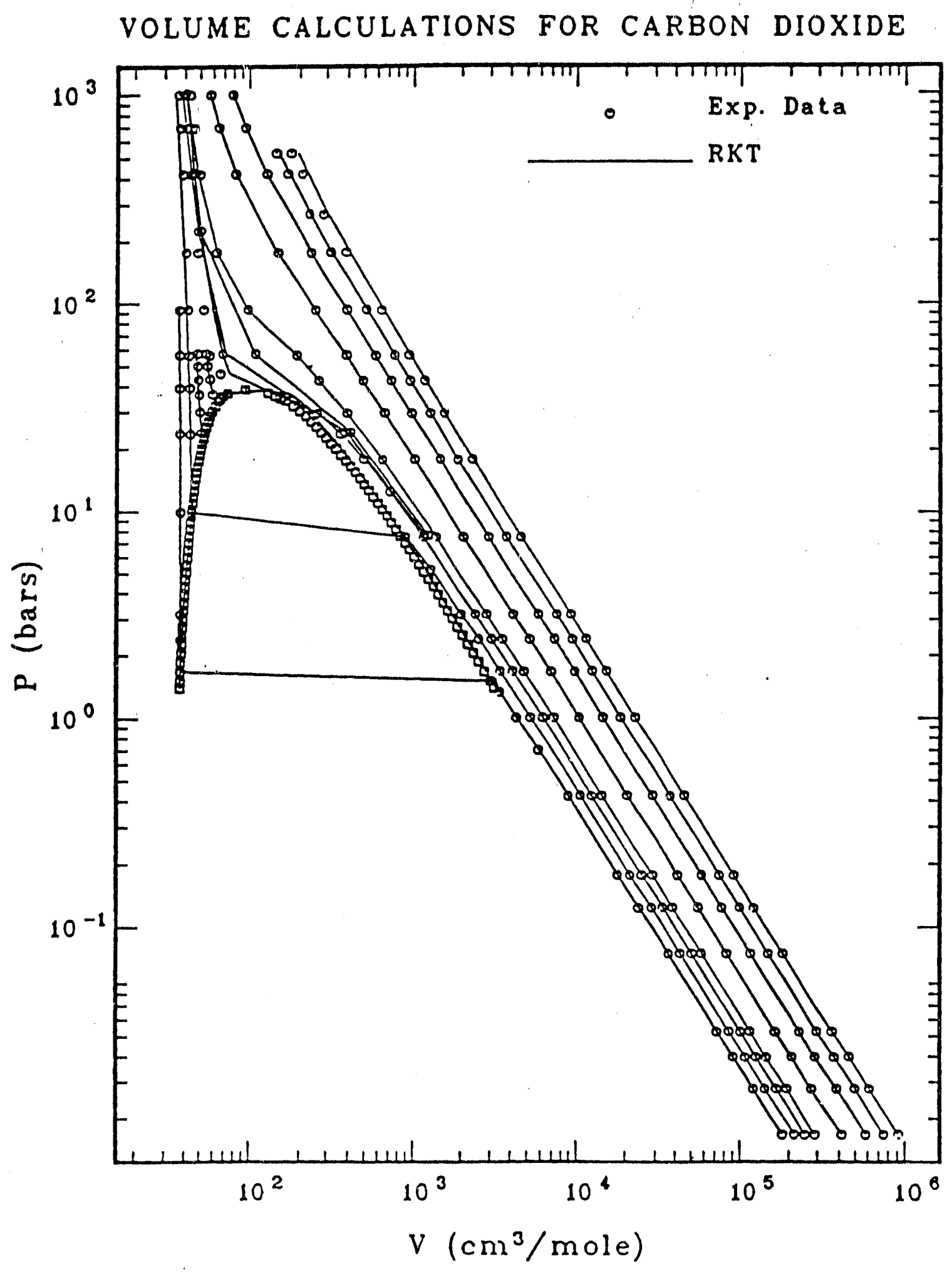

Figure 5. A comparison with experimental values of calculated volume for carbon dioxide using the RKT equation of state. 


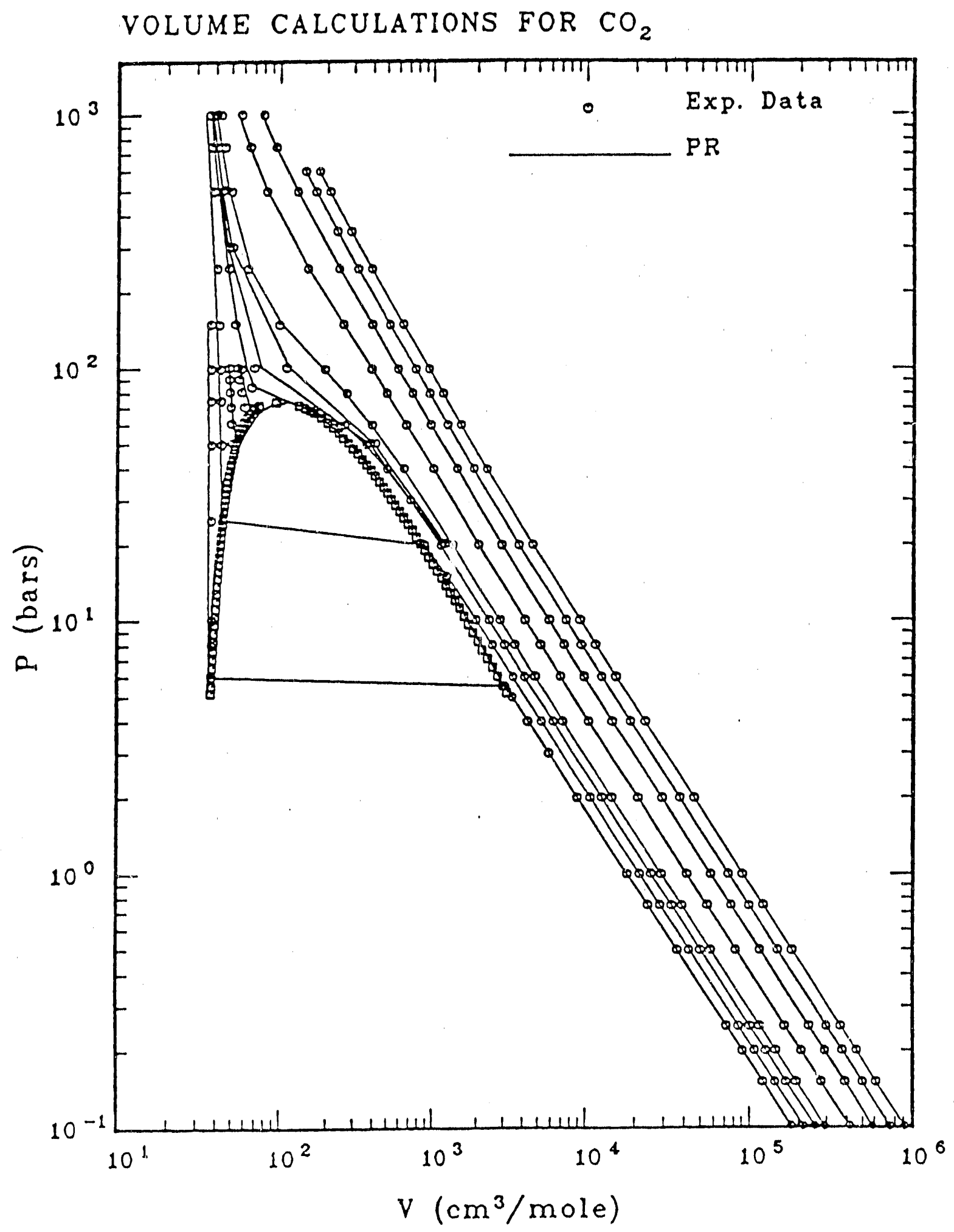

Figure 6. A comparison with experimental values of calculated volume for carbon dioxide using the PR equation of state. 


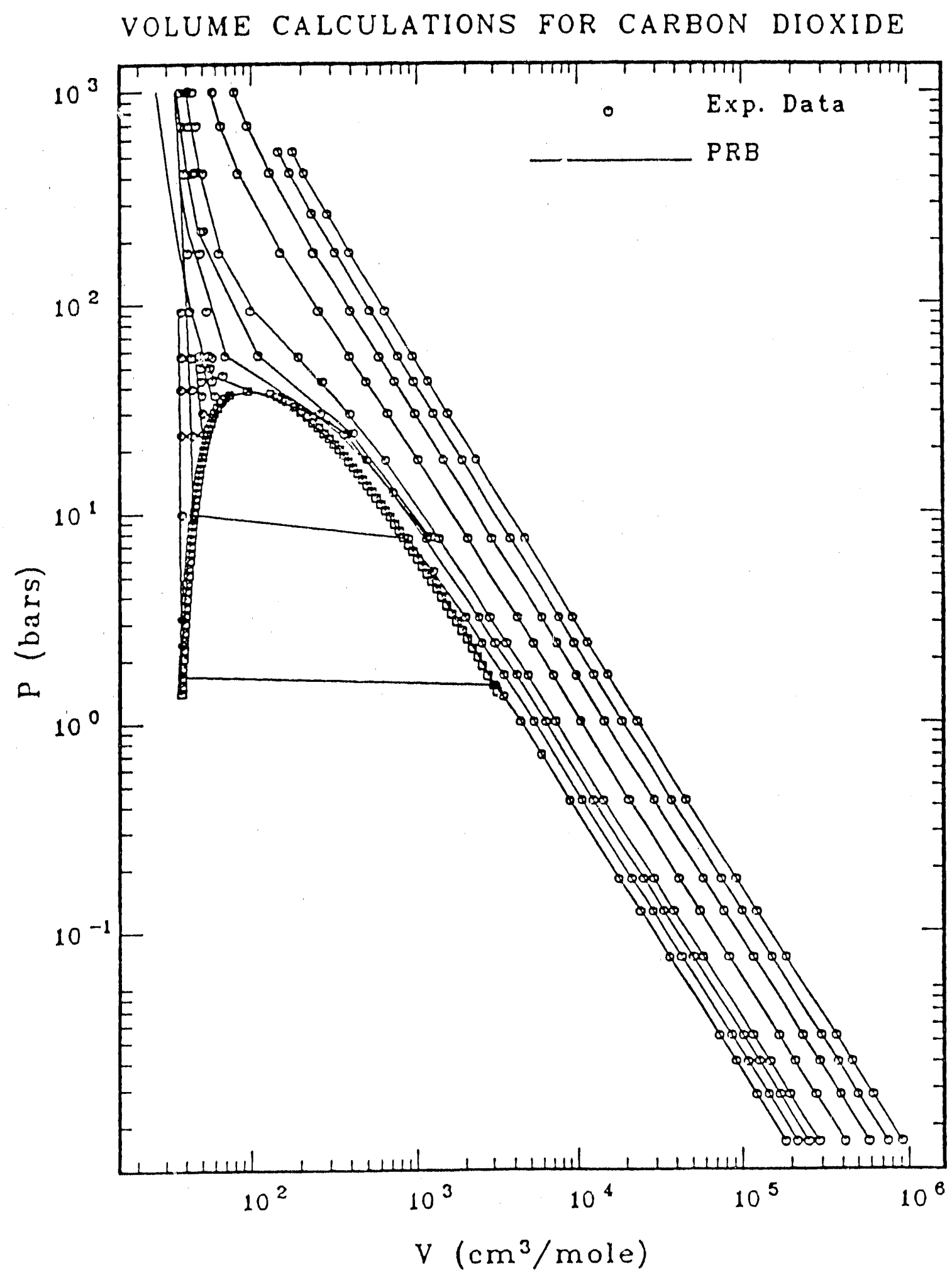

Figure 7. A comparison with experimental values of calculated volume for carbon dioxide using the $\mathrm{PRB}$ equation of state. 


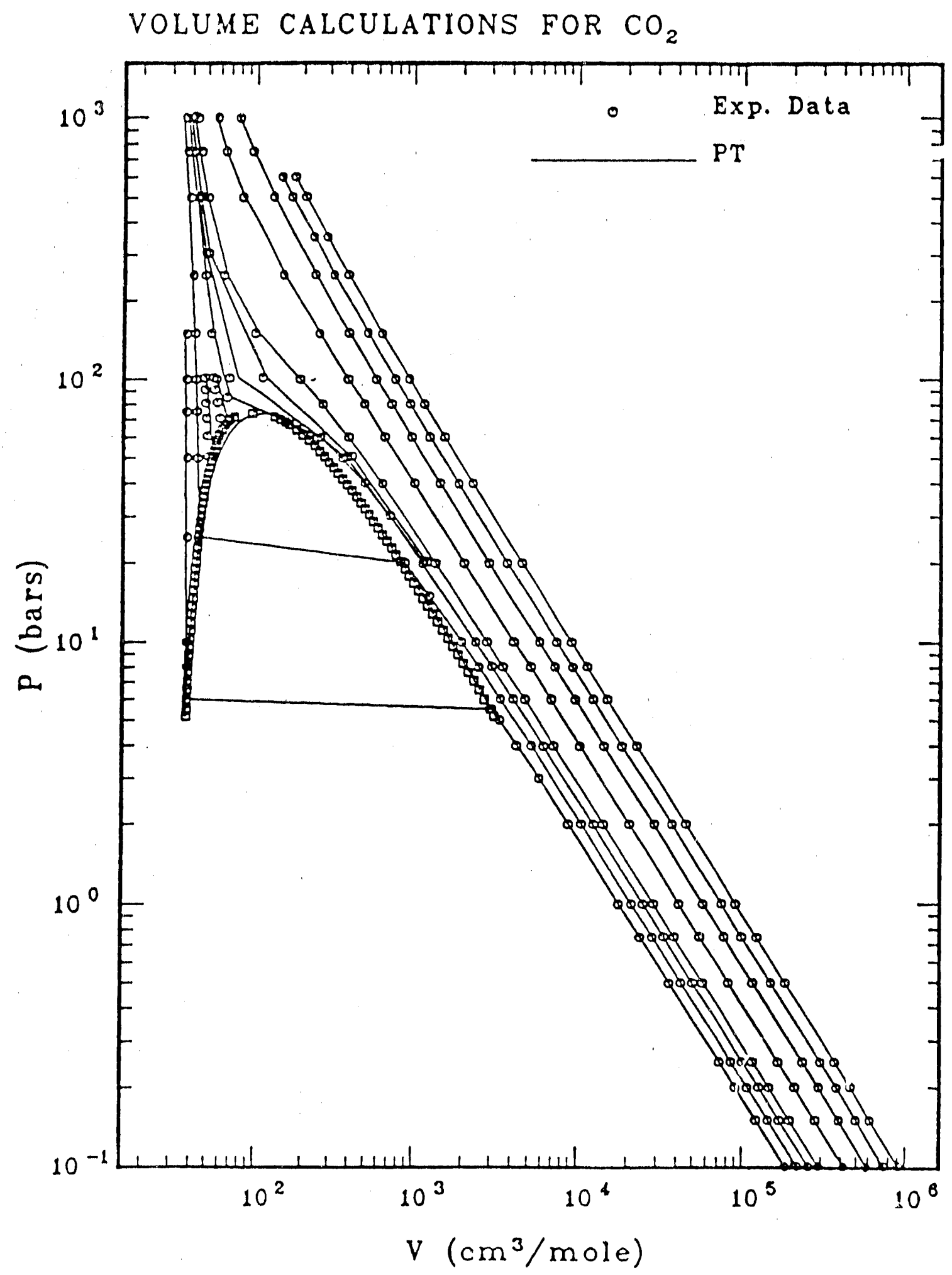

Figure 8. A comparison with experimental values of calculated volume for carbon dioxicie using the PT equation of state. 


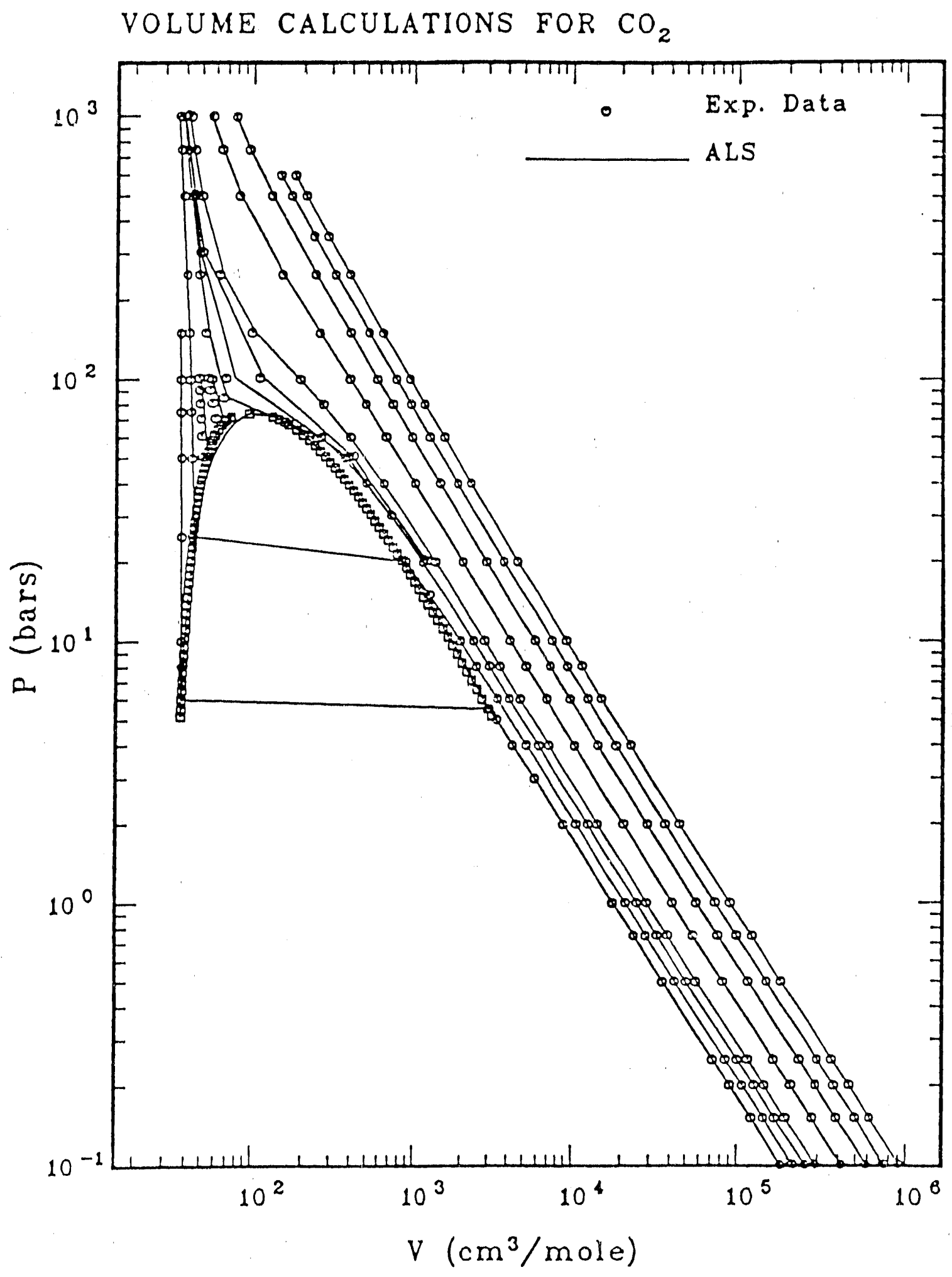

Figure 9. A comparison with experimental values of calculated volume for carbon dioxide using the ALS equation of state. 


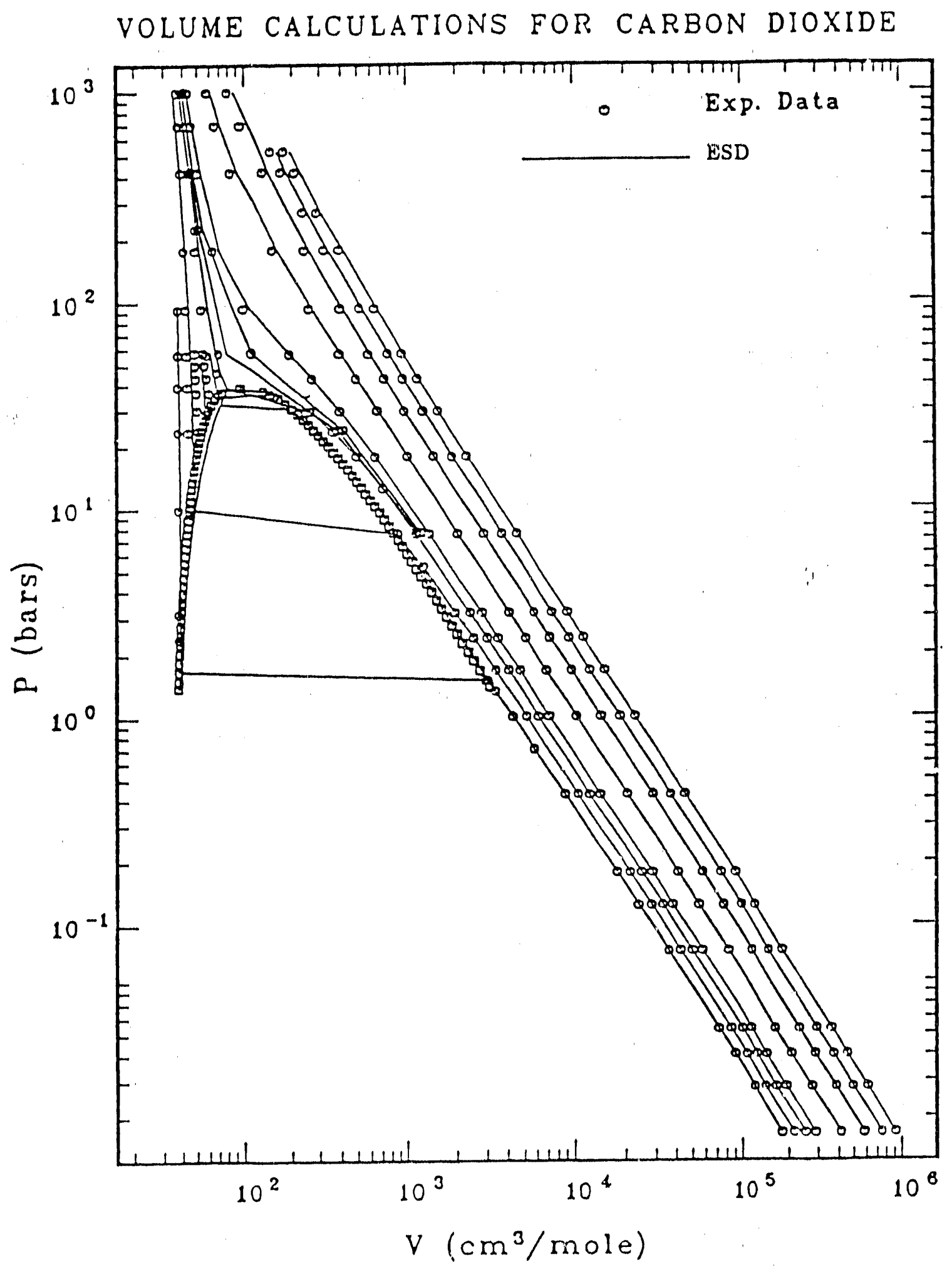

Figure 10. A comparison with experimental values of calculated volume for carbon dioxide using the ESD equation of state. 


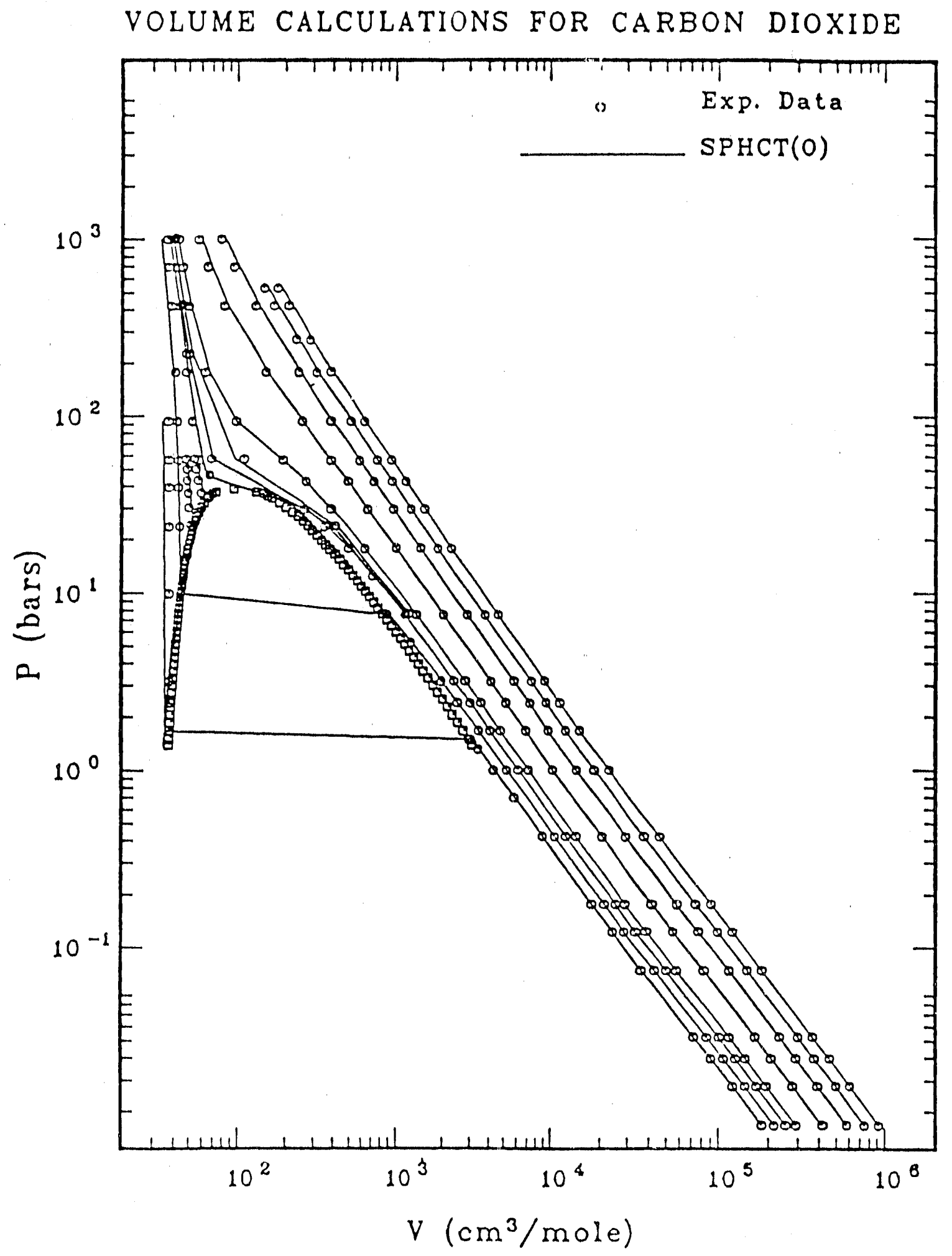

Figure 11. A comparison with experimental values of calculatec volume for carbon dinxide using the $\operatorname{SPHCT}(0)$ equation $f$ state. 


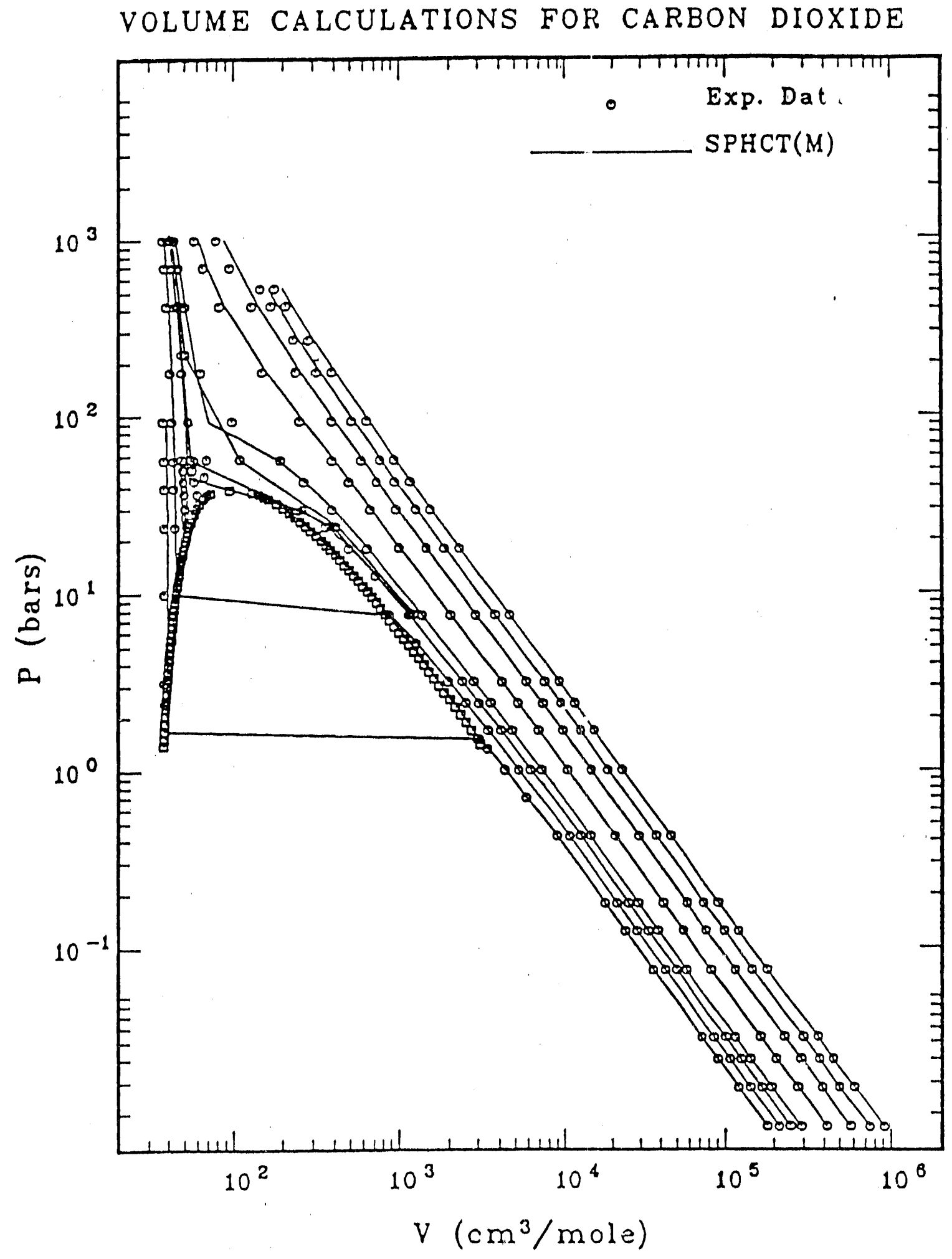

Figure 12. A comparison with experimental values of calculated volume for carbon dioxide using the SPHCT(M) equation of state. 


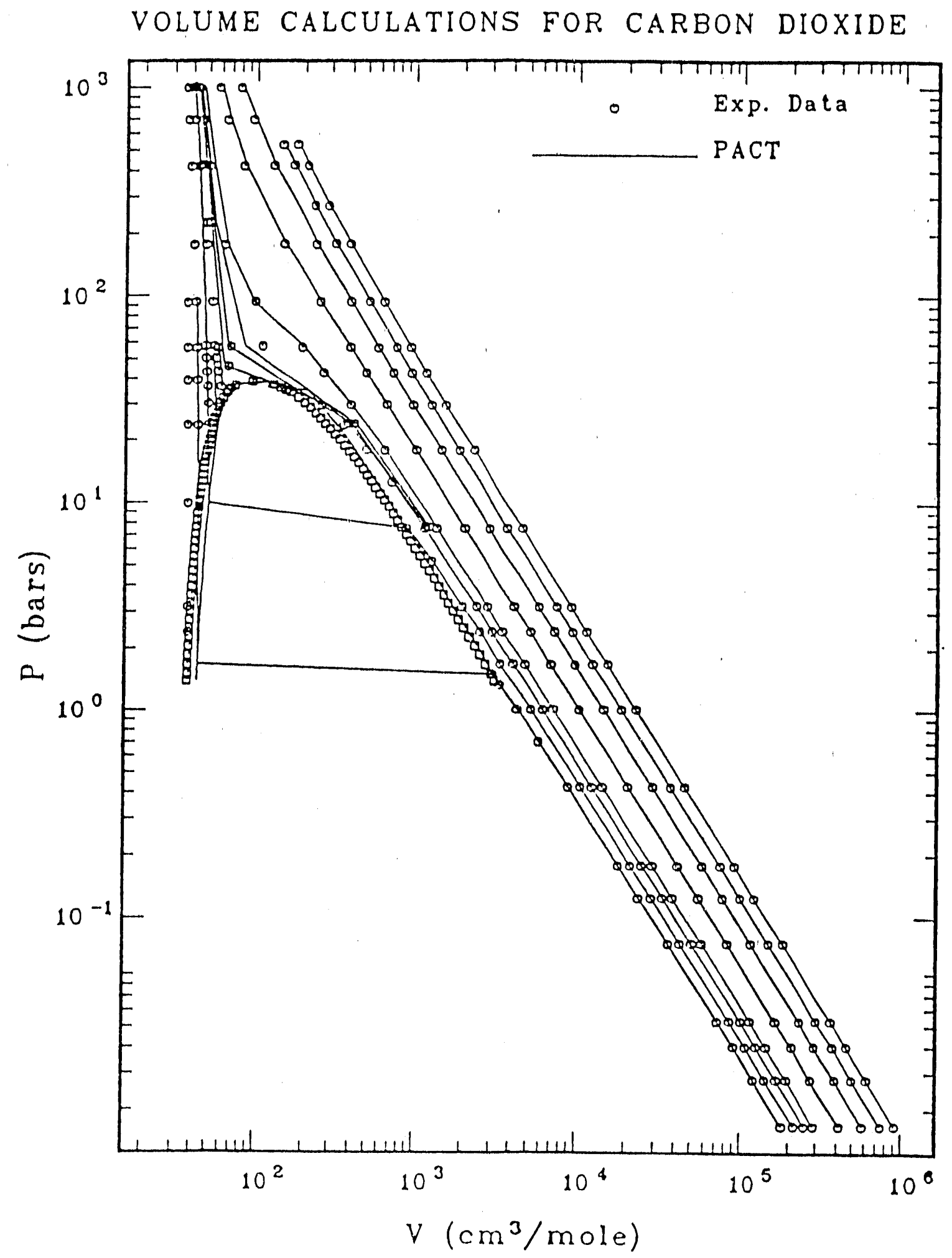

Figure 13. A comparison with experimental values of calculated volume for carlon dioxide using the PACT equation of state. 


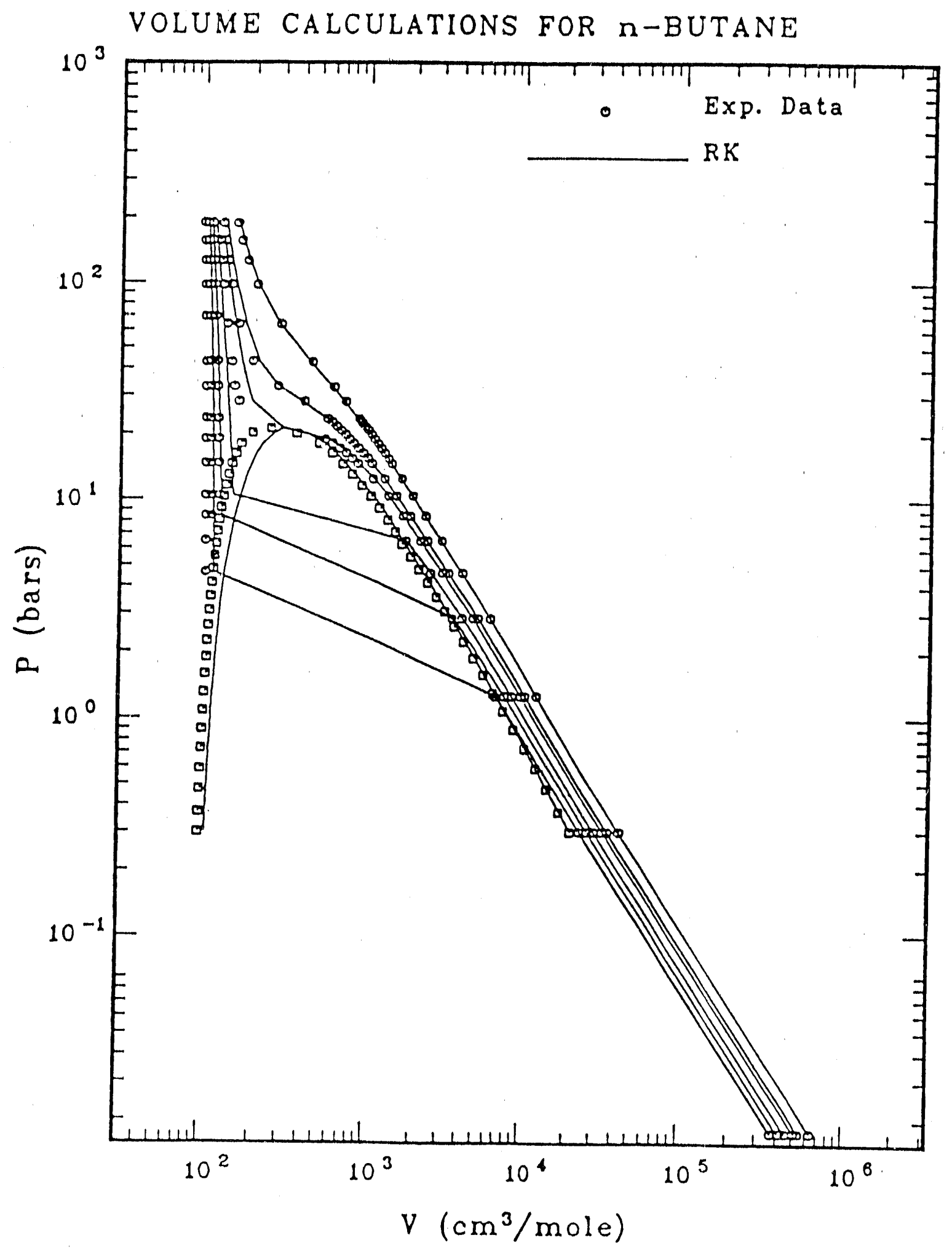

Figure 14. A comparison with experimental values of calculated pressure for $n$-butane using the RK equation of state. 


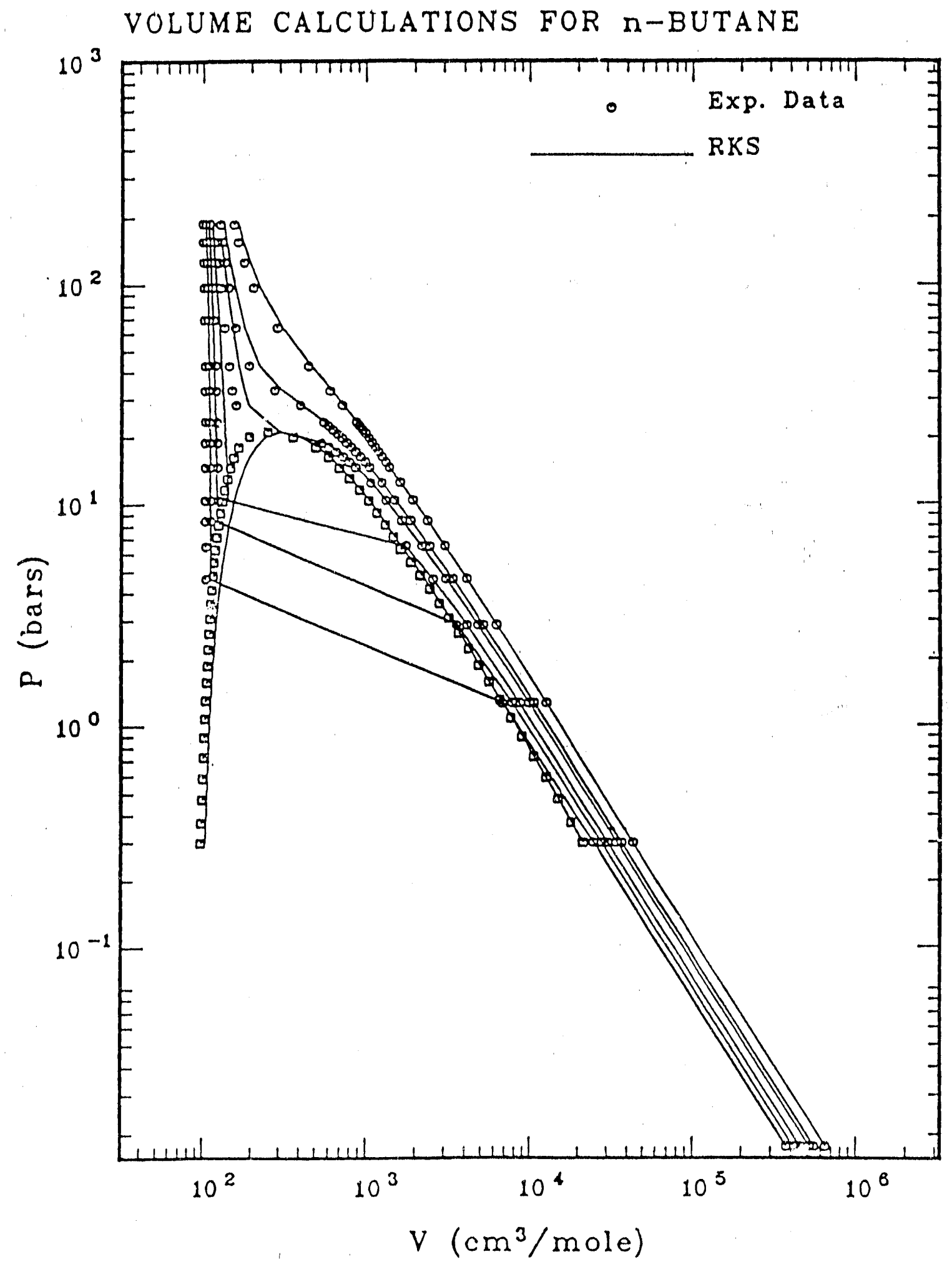

Figure 15. A comparison with experimental values of calculated volume for $\mathrm{n}$-butane using the RKS equation of state. 


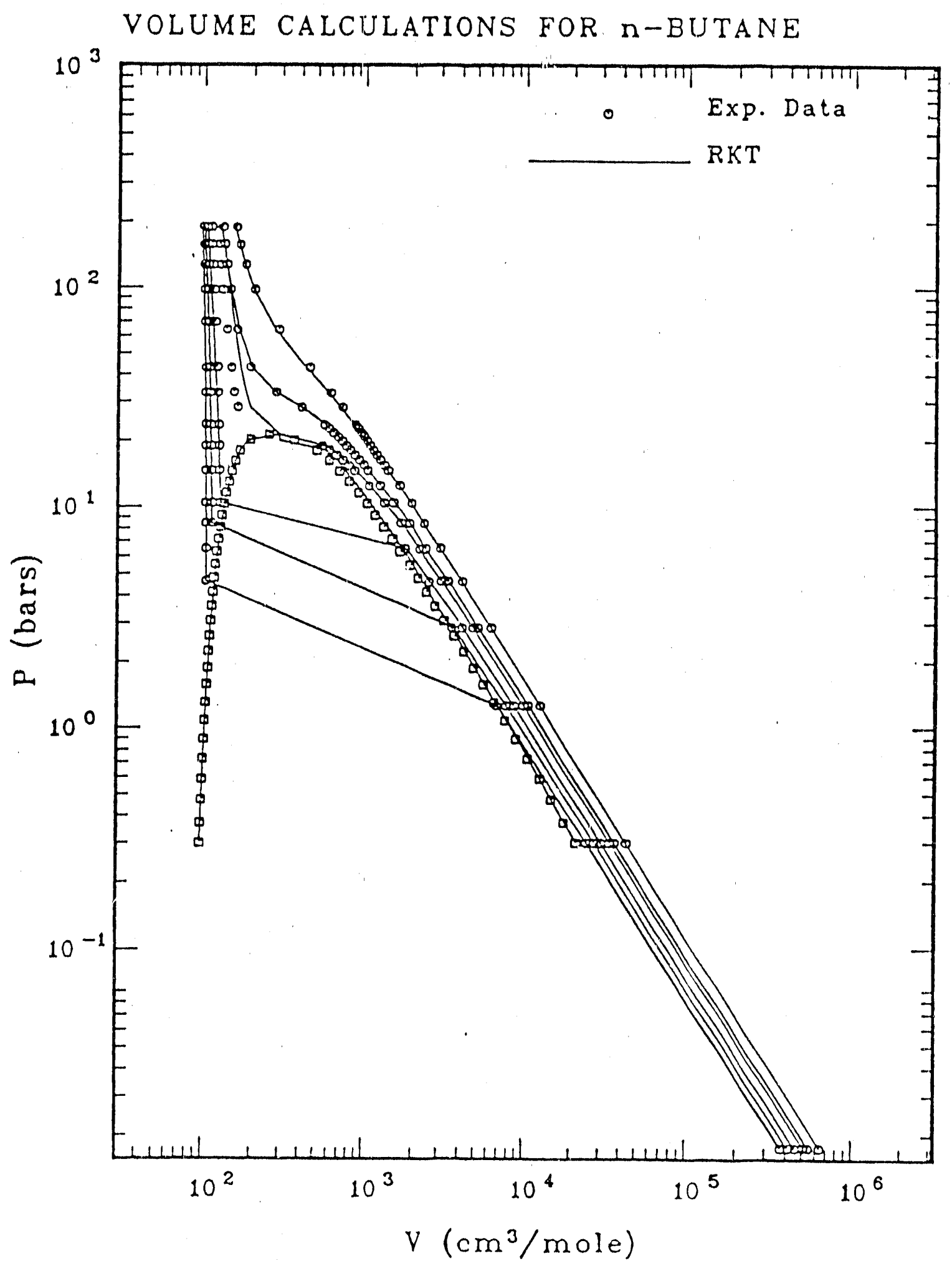

Figure 18. A comparison with experimental values of calculated volume for $n$-butane using the RKT equation of state. 


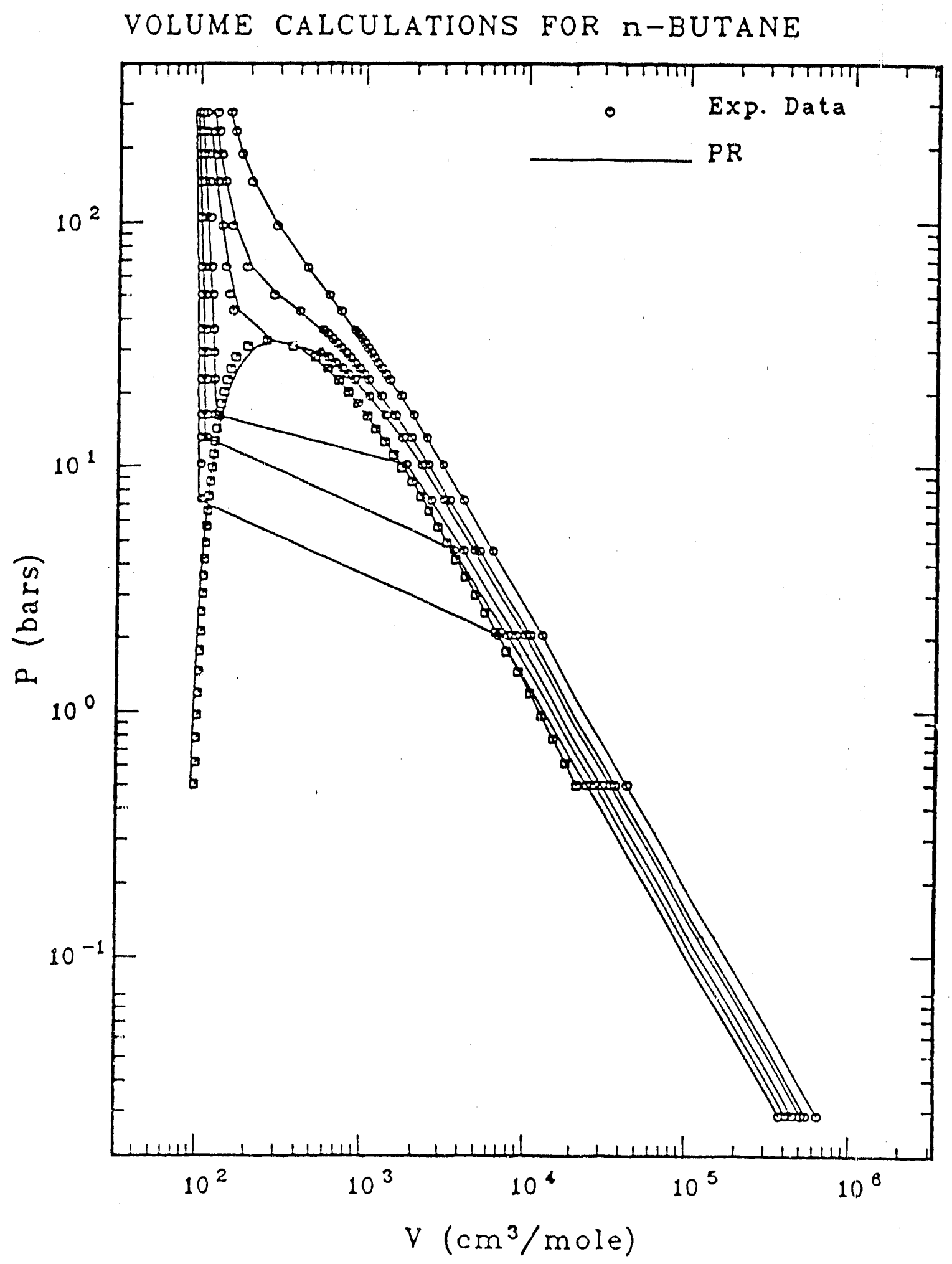

Figure 17. A comparison with experimental values of calculated volume for n-butane using the PR equation of state. 


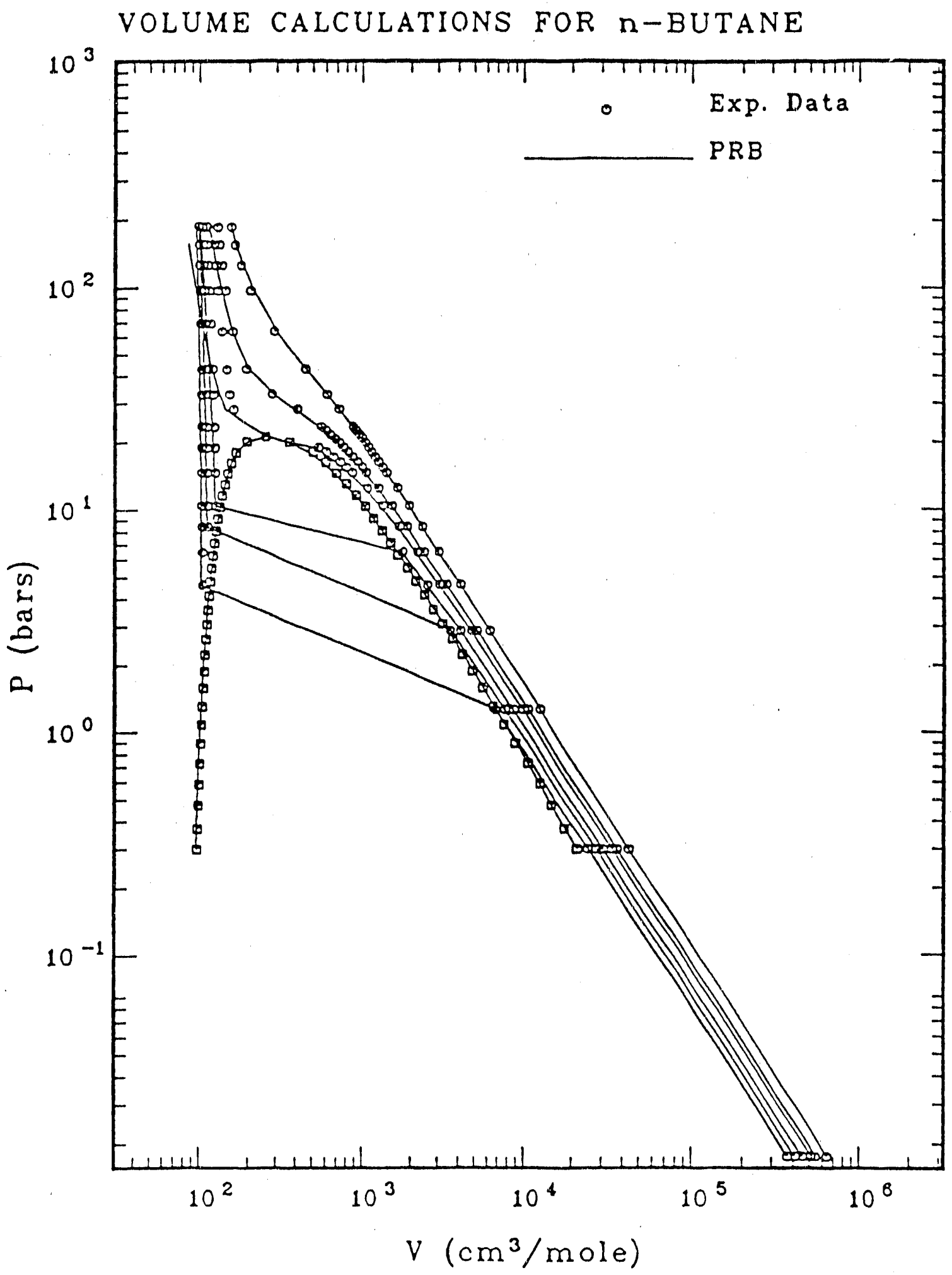

Figure 18. A comparison with experimental values of calculated volume for $n$-butane using the PRB equation of state. 


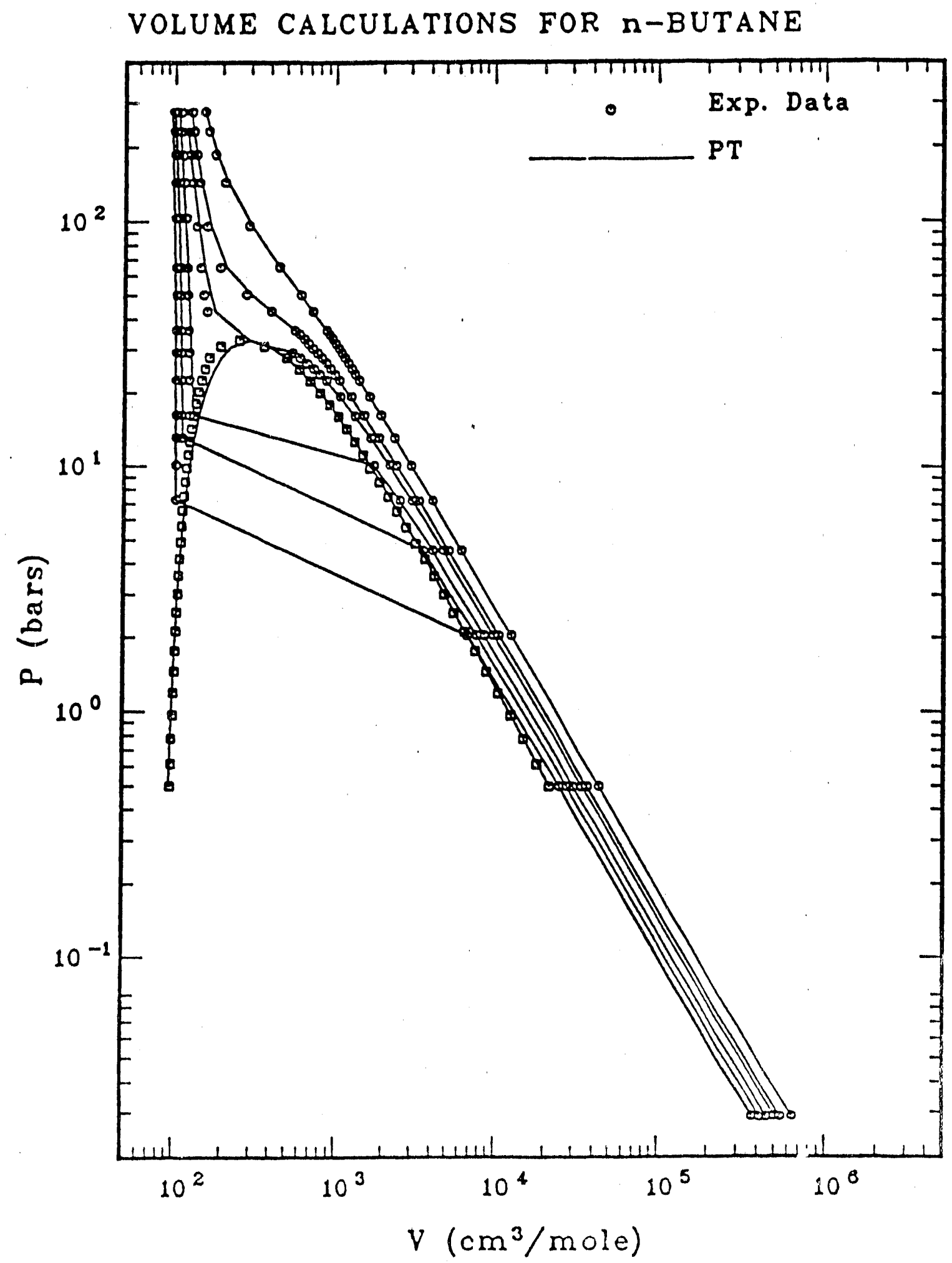

Figure 18. A comparison with experimental values of calculated volume for $n$-butane using the PT equation of state. 


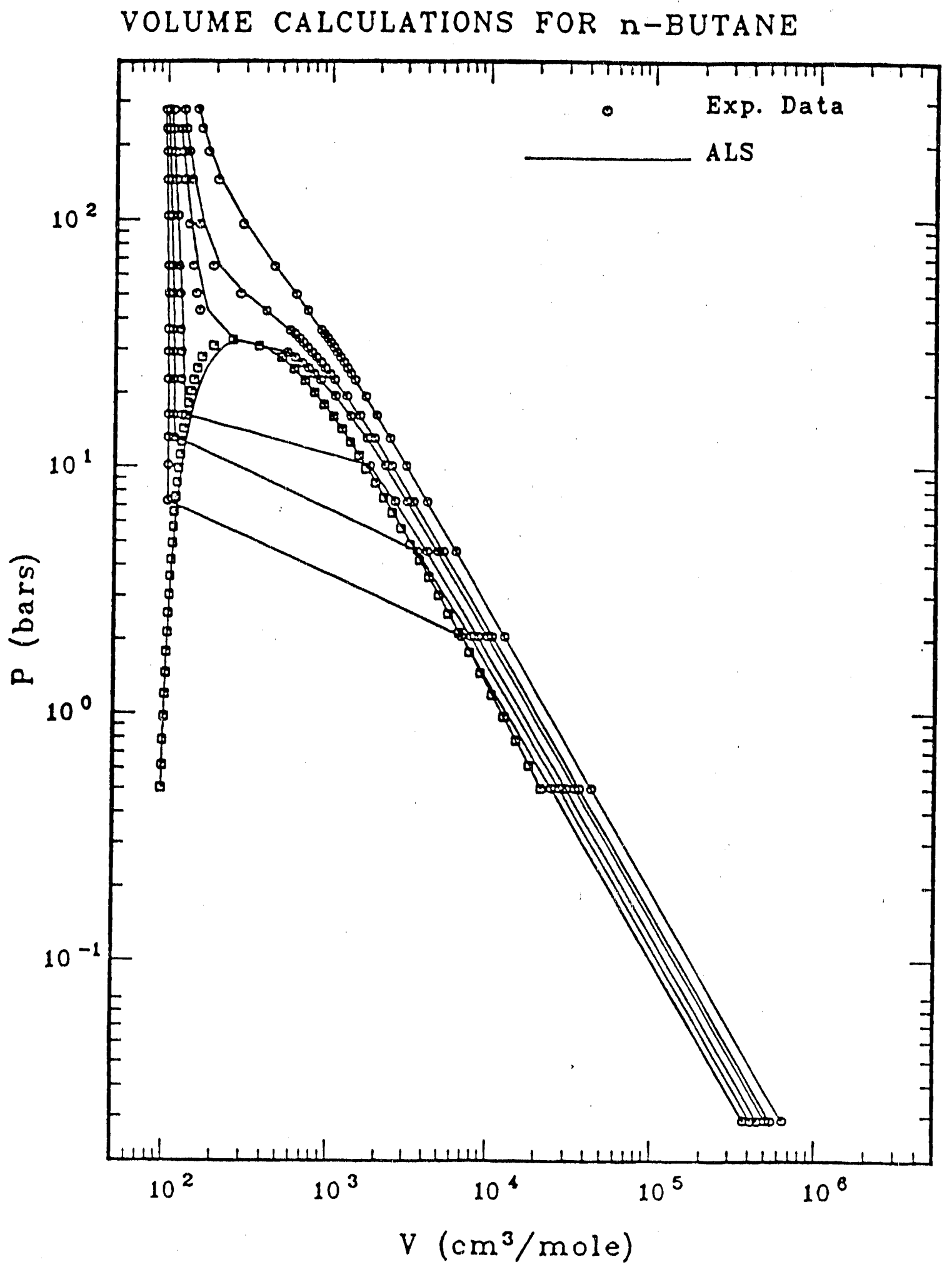

Figure 20. A comparison with experimental values of calculated volume for $n$-butane using the ALS equation of state. 


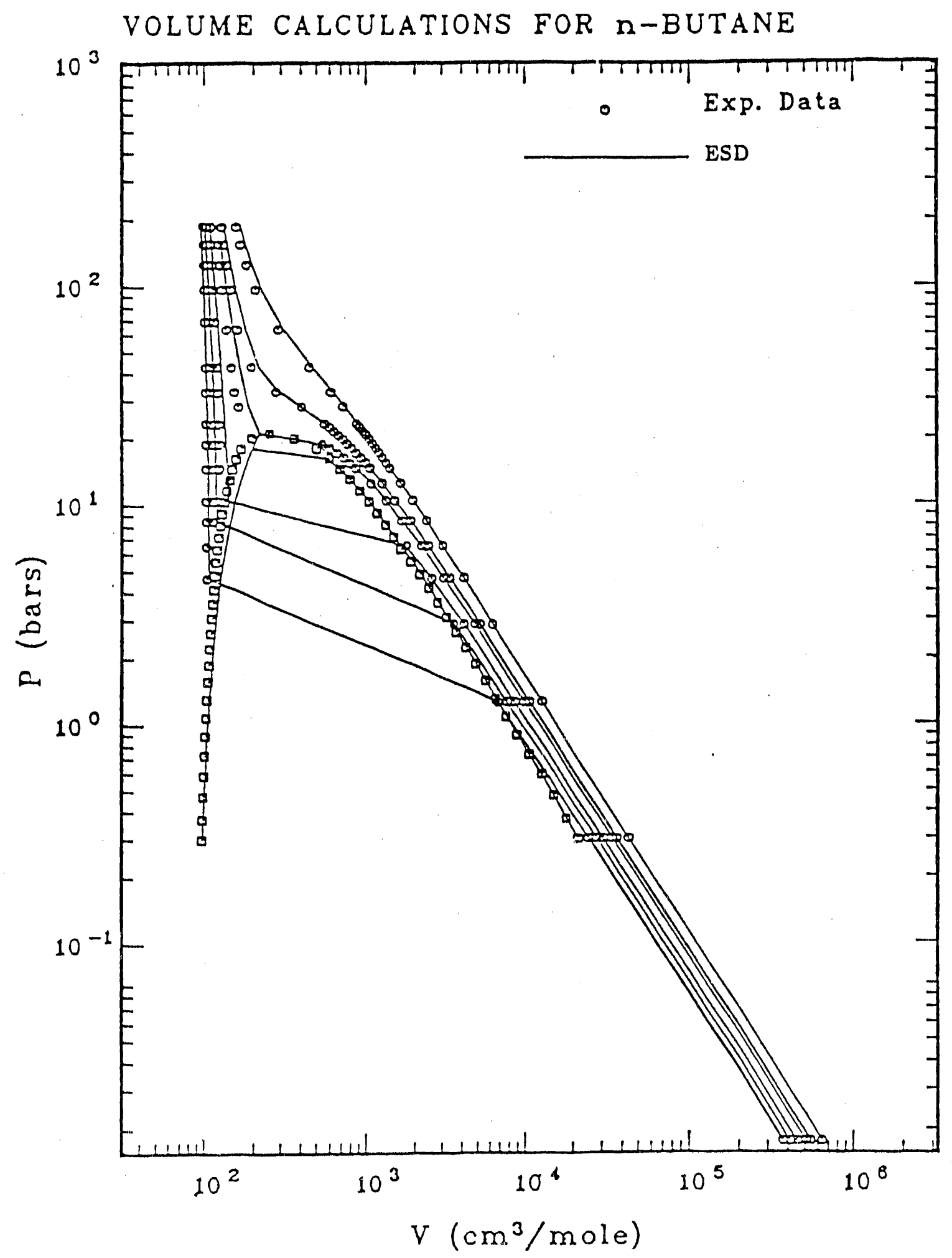

Figure 21. A comparison with experimental values of calculated volume for n-butane using the ESD equation of state. 


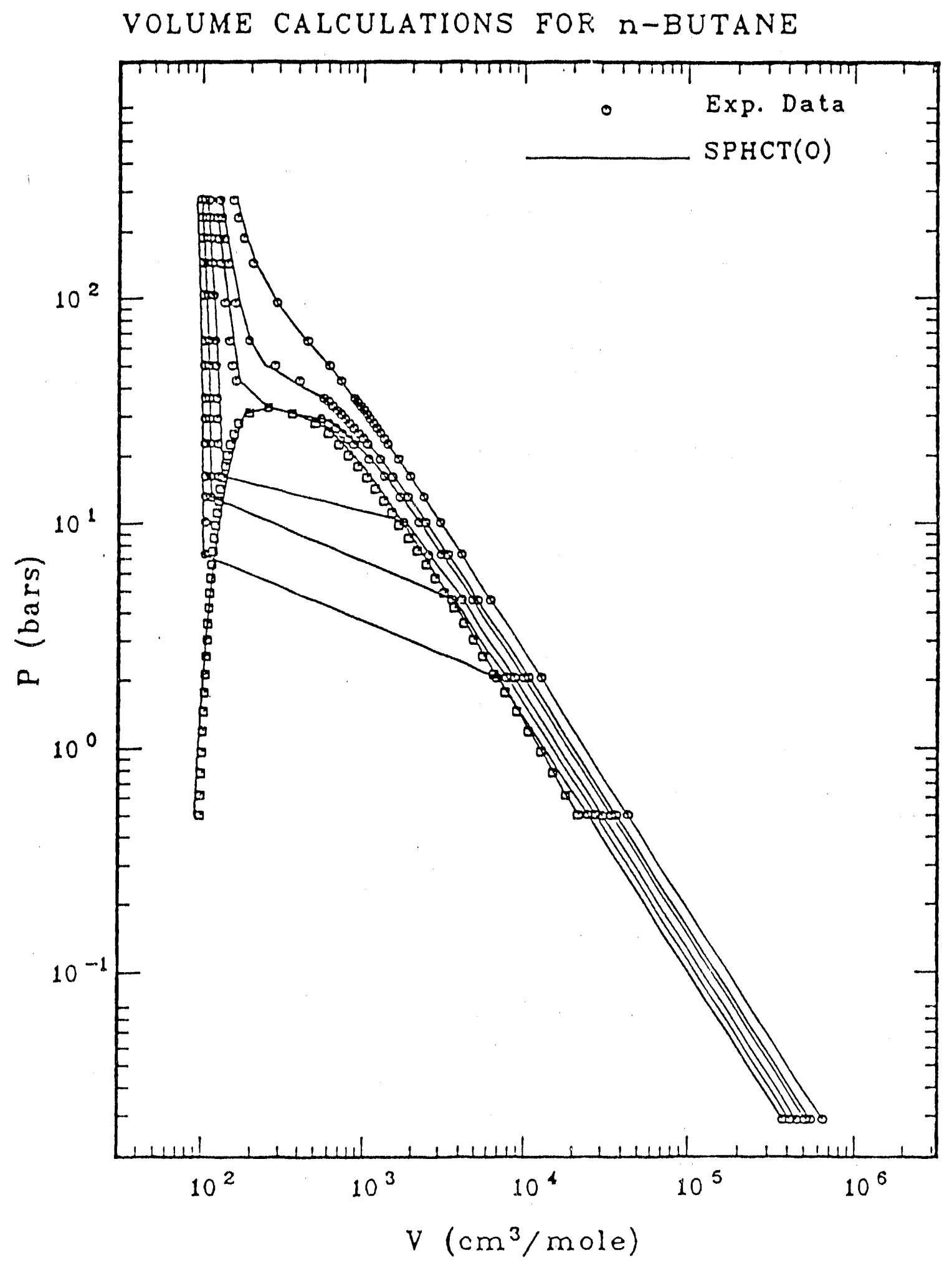

Figure 22. A comparison with experimental values of calculated volume for $\mathrm{n}$-butane using the $\mathrm{SPHCT}(\mathrm{O})$ equation of state. 


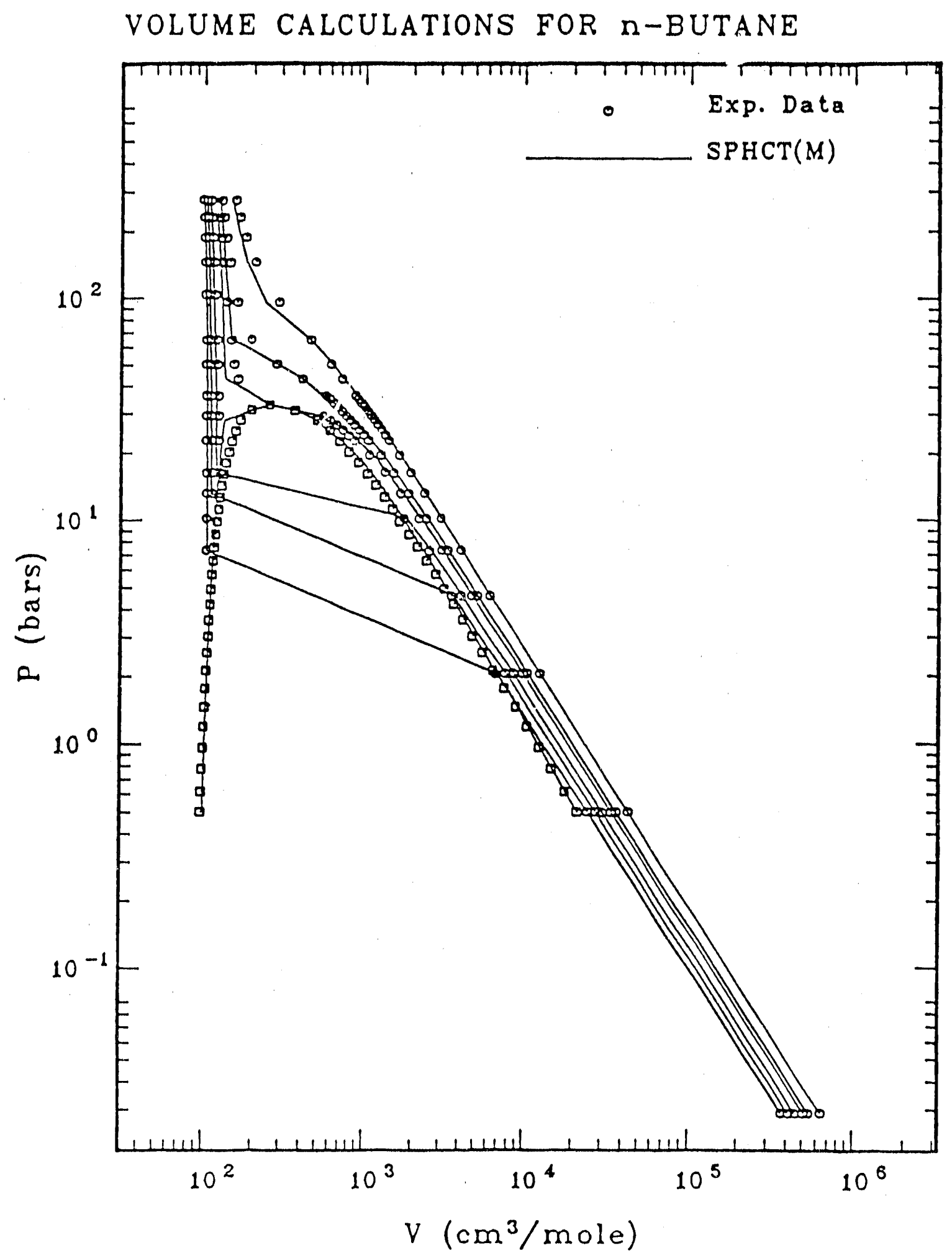

Figure 23. A comparison with experimental values of calculated volume for $n$-butane using the SPHCT(M) equation of state. 


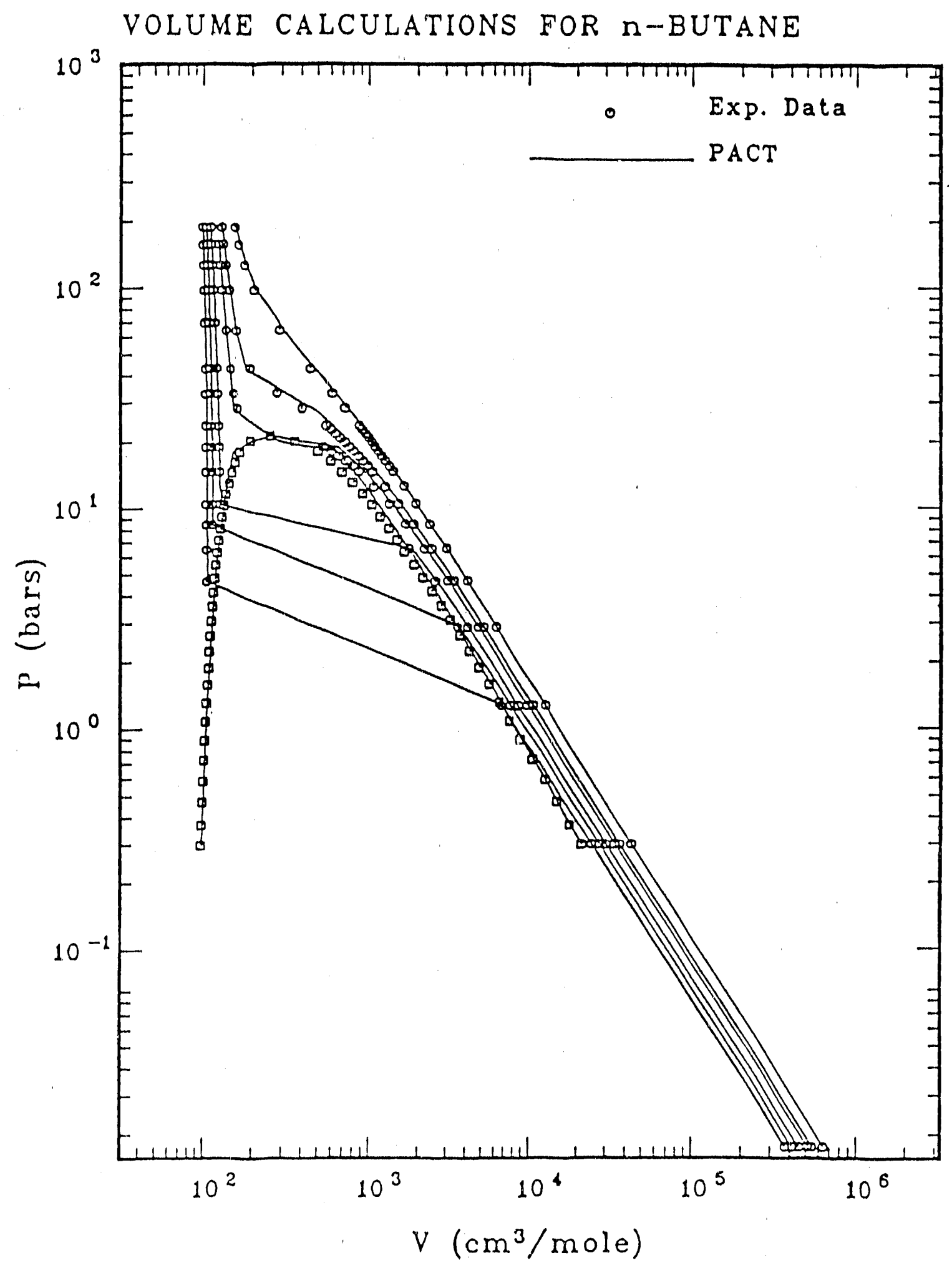

Figure 24. A comparison with experimental values of calculated volume for n-butane using the PACT equation of state. 


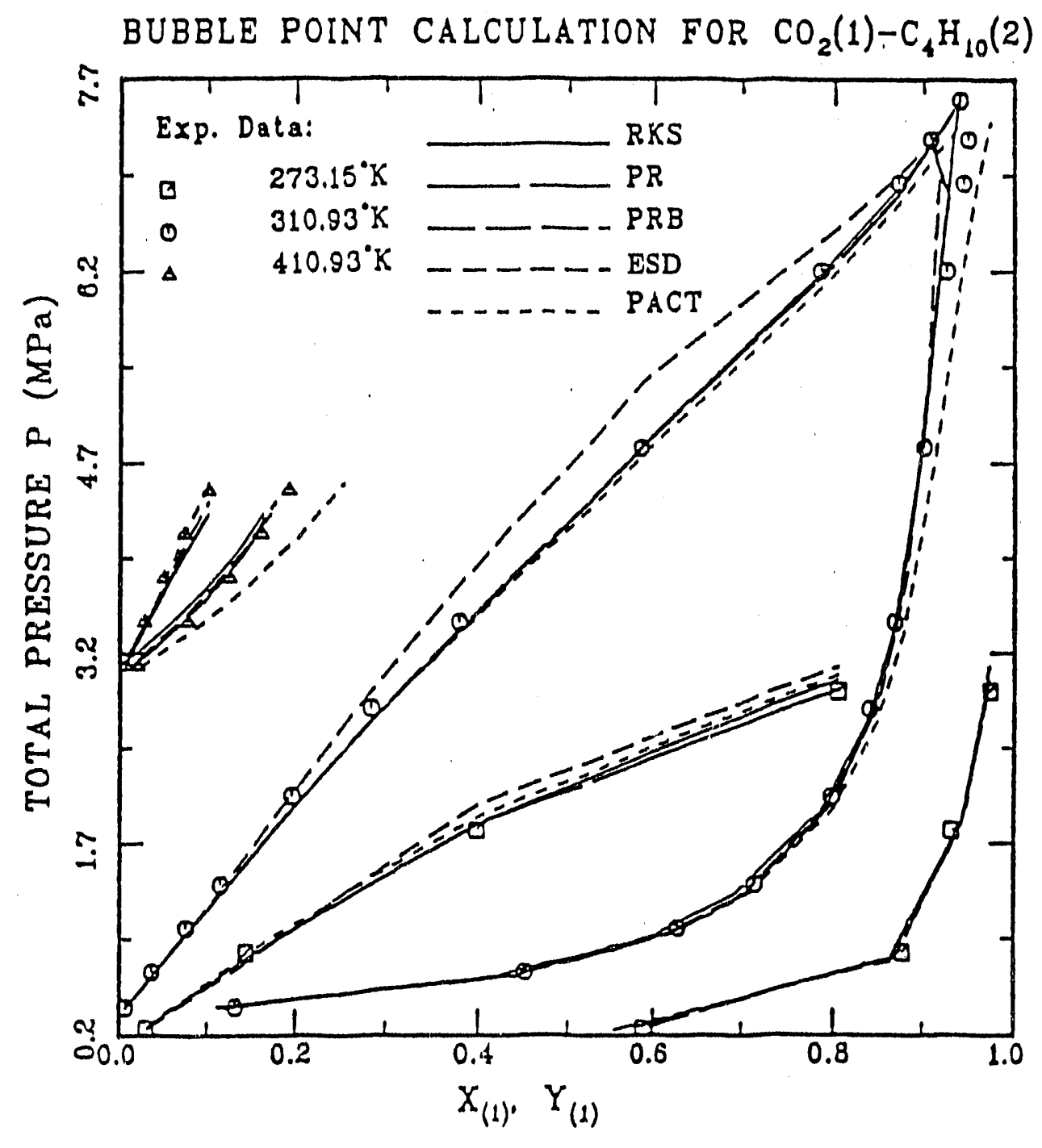

Figure 25. The results of bubble point calculations for carbon dioxide- $n$-butane using RKS, PR, PRB, ESD and PACT equations compared with experimental data (Knapp, et al., 1982). 


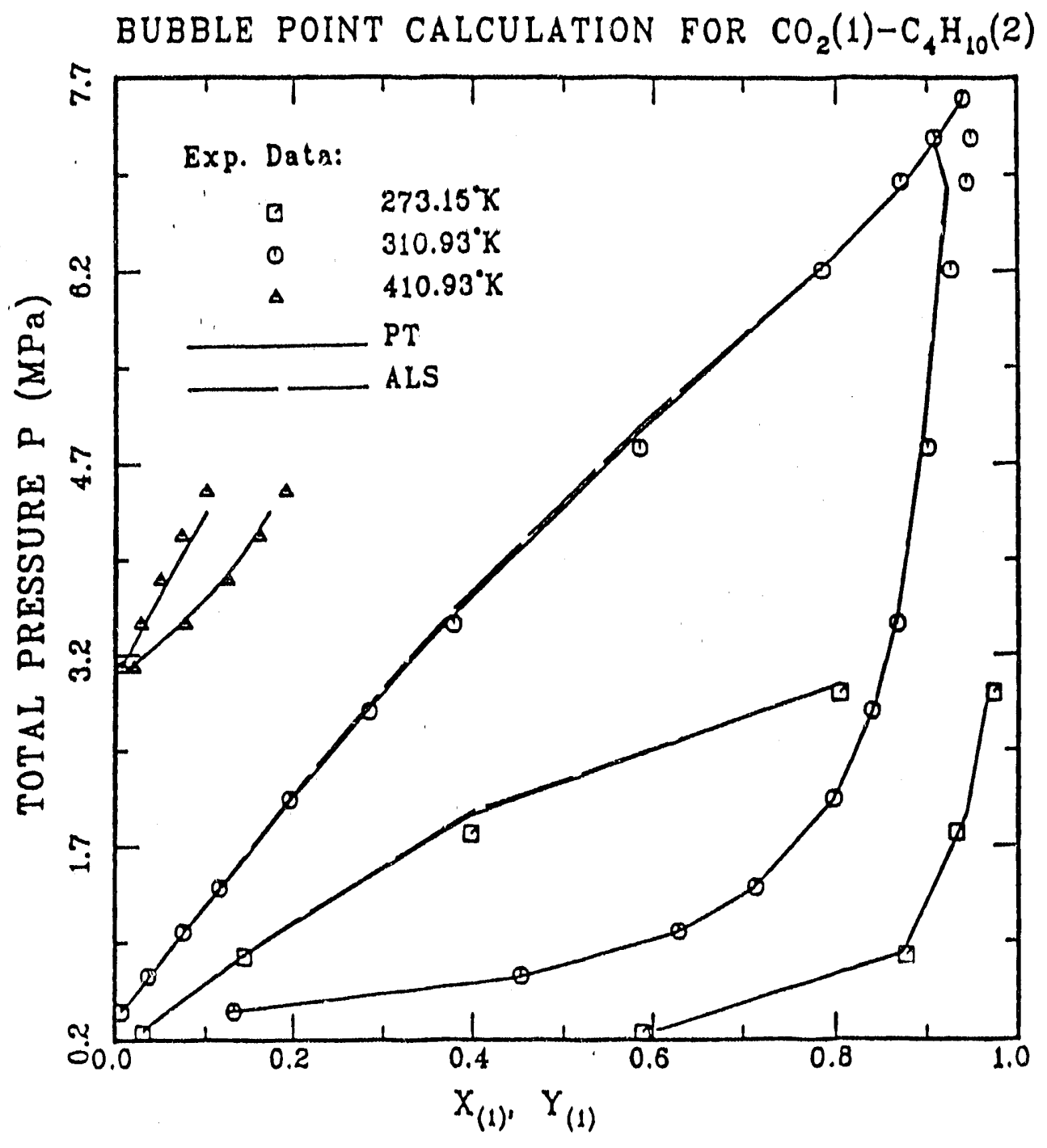

Figure 28. The results of bubble point calculations for carbon dioxide-n-butane using $\mathrm{PT}$ and ALS equations compared with experimental data (Knapp, et al., 1982). 


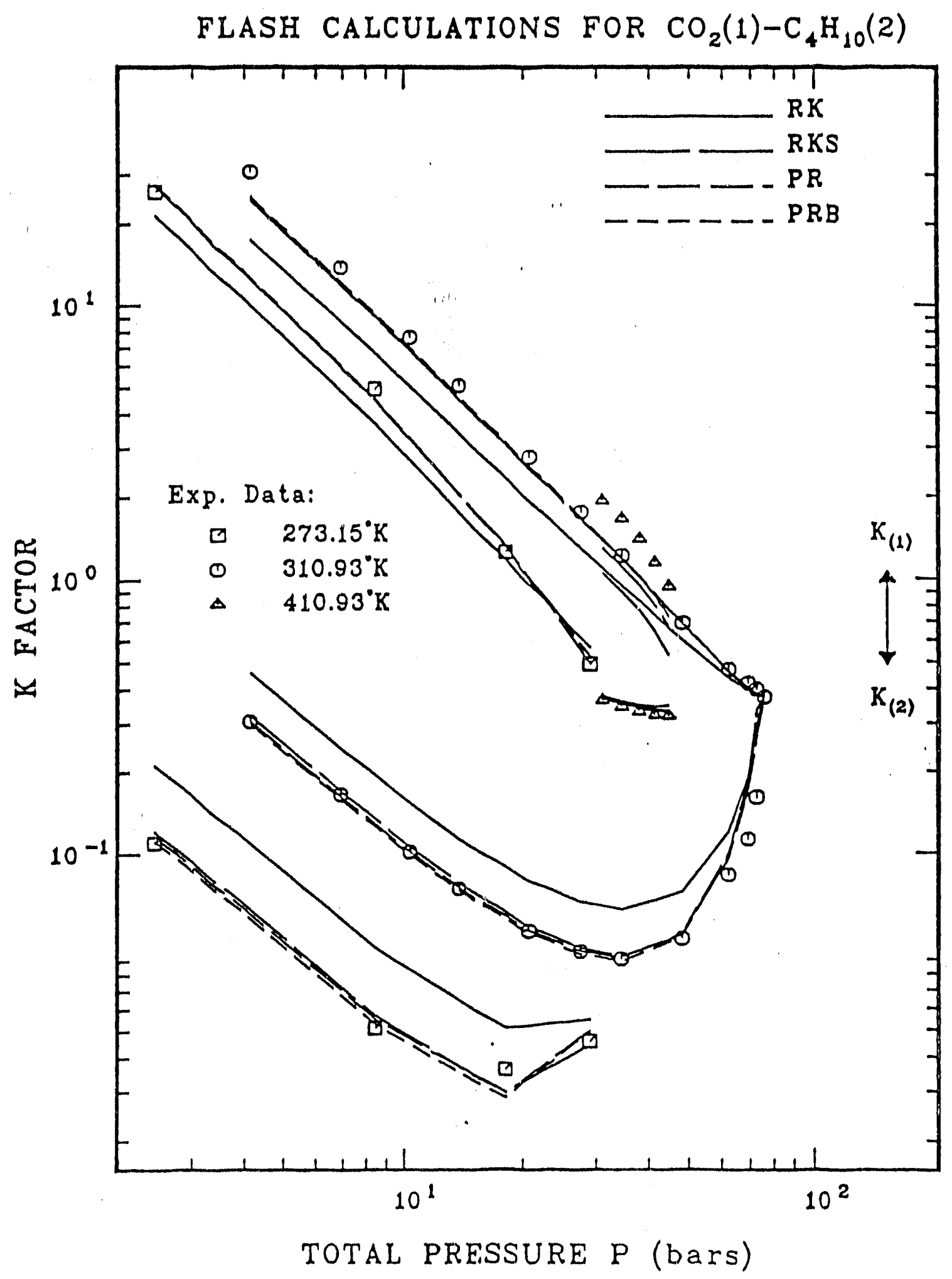

Figure 27. The results of flash calculations for carbon dioxide-n-butane using RK, RKS, PR and PRB equations compared with experimental data (Knapp, et al., 1982). 


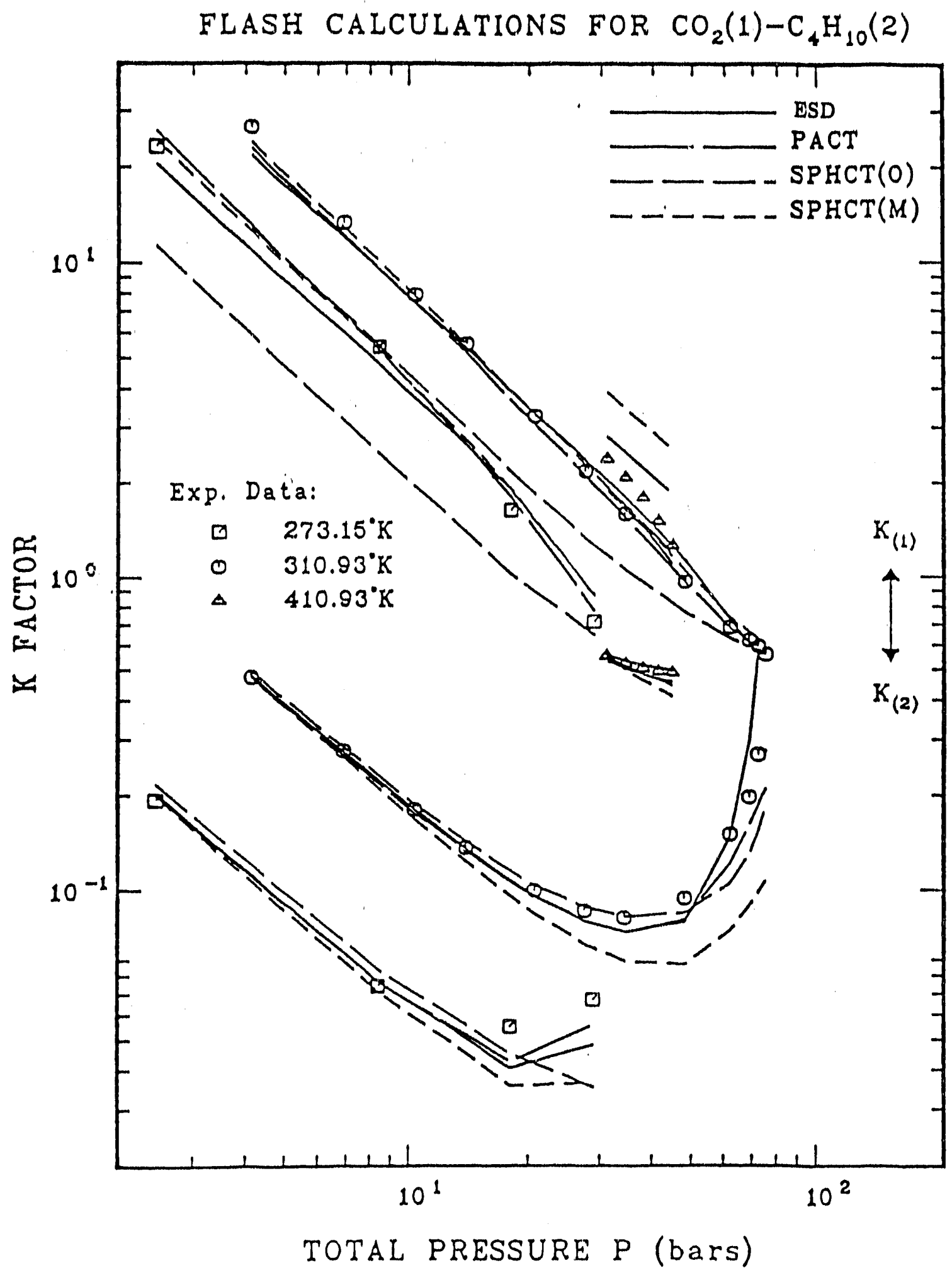

Figure 28. The resu'ts of flash calculations for carbon dioxide-n-butane using ESD, SPHCT $(O), \operatorname{SPHCT}(M)$ and PACT equations compared with experimental data (Krapp, et al., 1982). 


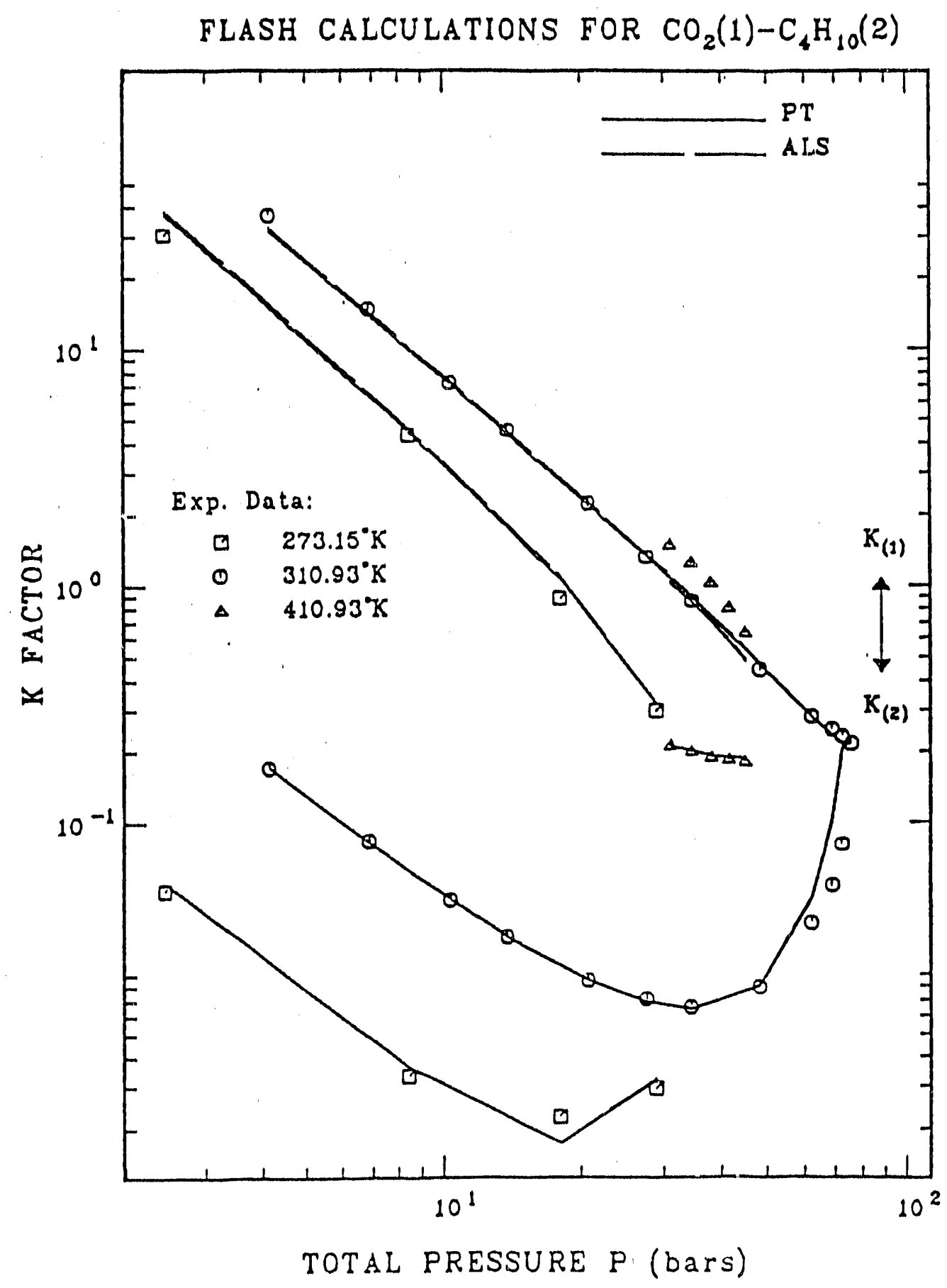

Figure 29. The results of flash calculations for carbon dioxide-n-butane using PT and ALS equations compared with experimental daia (Knapp, et al., 1982). 


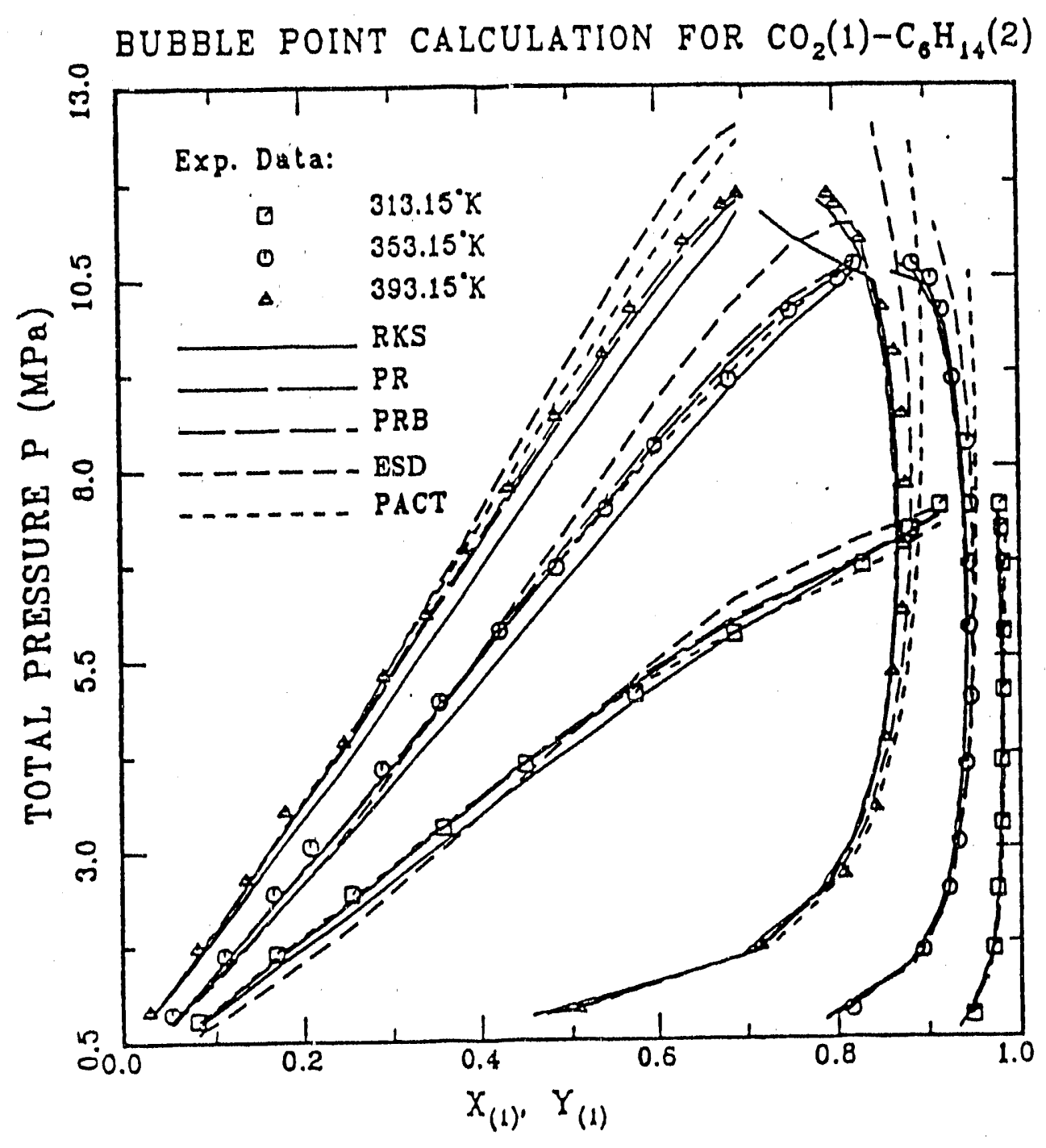

Figure 30. The results of bubble point calculation for carbon dioxide-hexane using RKS $, \mathrm{PR}, \mathrm{PRB}, \mathrm{ESD}$ and PACT equations compared with experimental data ( $\mathrm{Li}$, et al., 1981). 


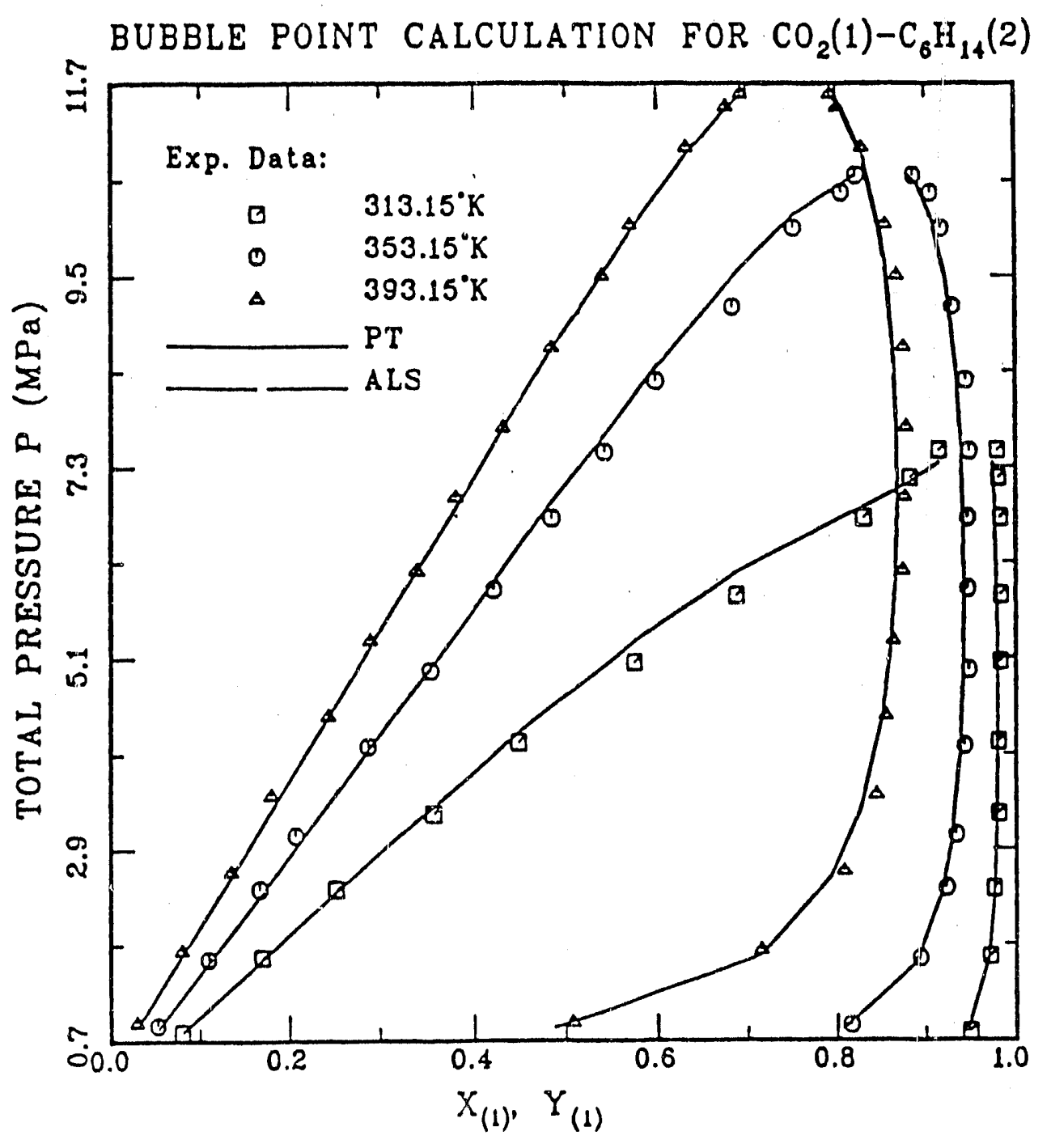

Figure 31. The results of bubble point calculation for carbon dioxide-hexane using $\mathrm{PT}$ and ALS equations compared with experimental data (Li, et al., 1981). 


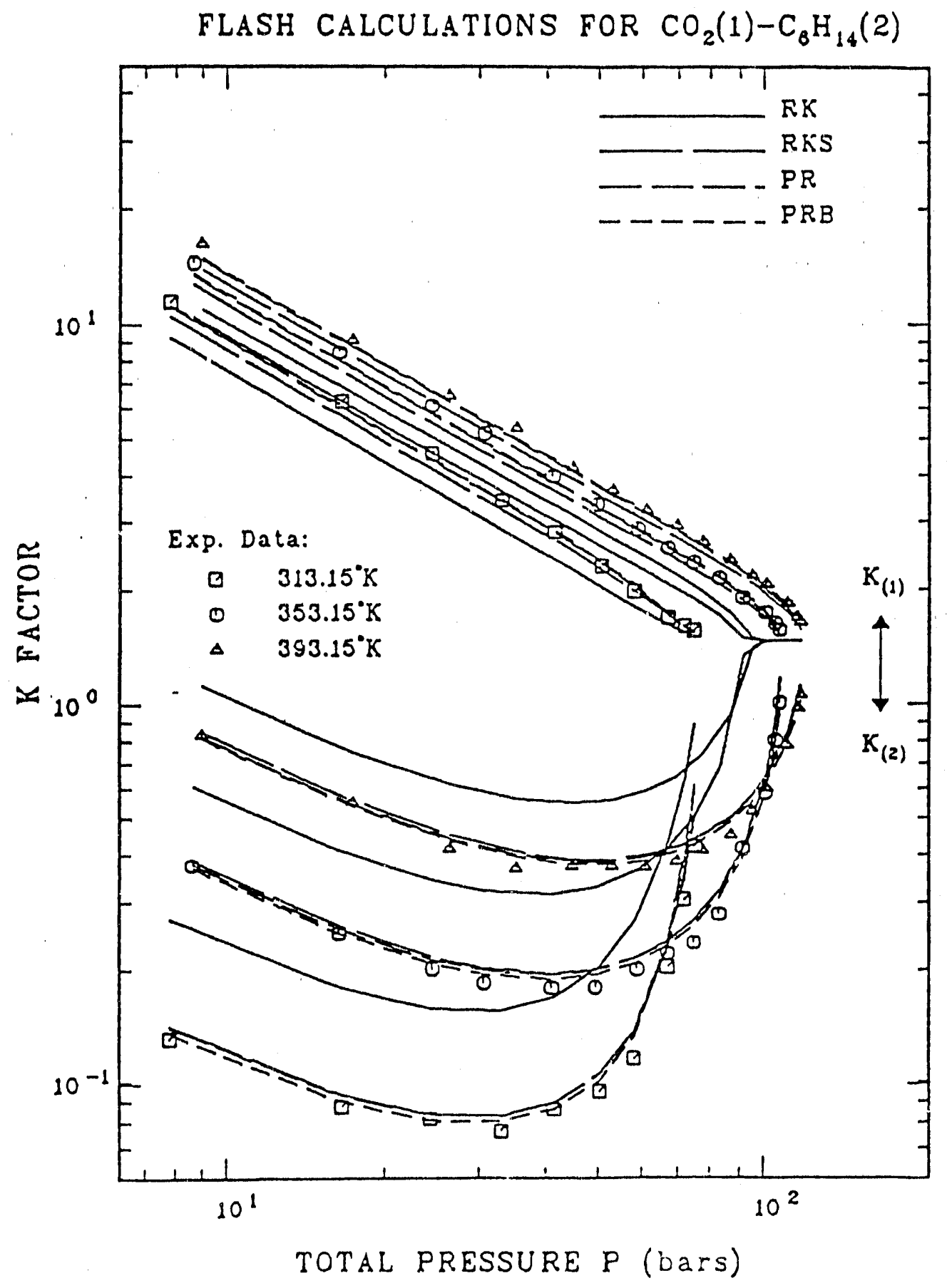

Figure 32. The results of flash calculation for carbon dioxide-hexane using RK, RKS, PR and PRB equations compared with experimental data (Li, et al., 1981). 


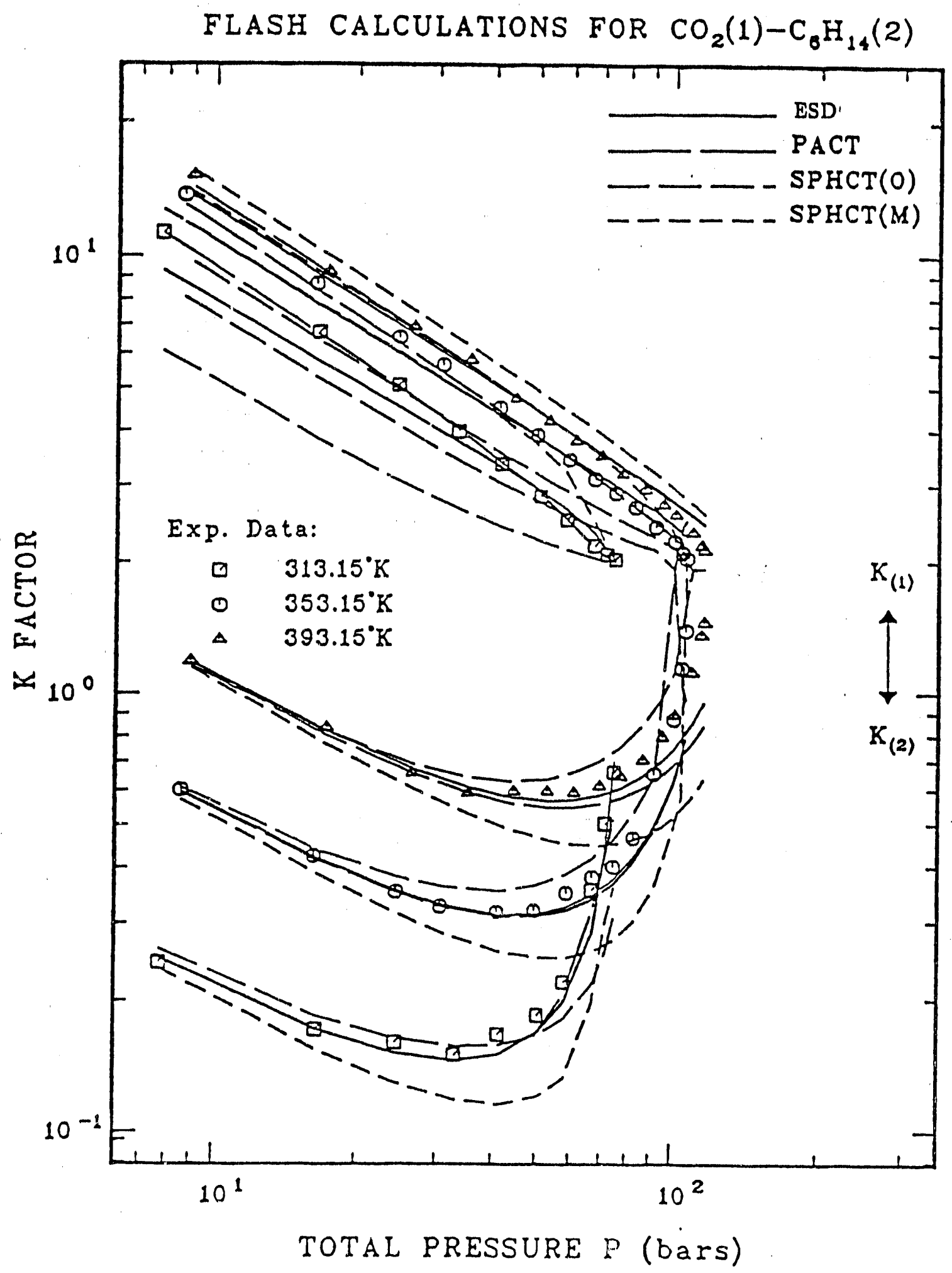

Figure 33. The results of flash calculation for carbon dioxide-hexane using ESD, SPHCT(O), SPHCT(M) and PACT equations compared with experimental data ( $\mathrm{Li}$, et al., 1981). 


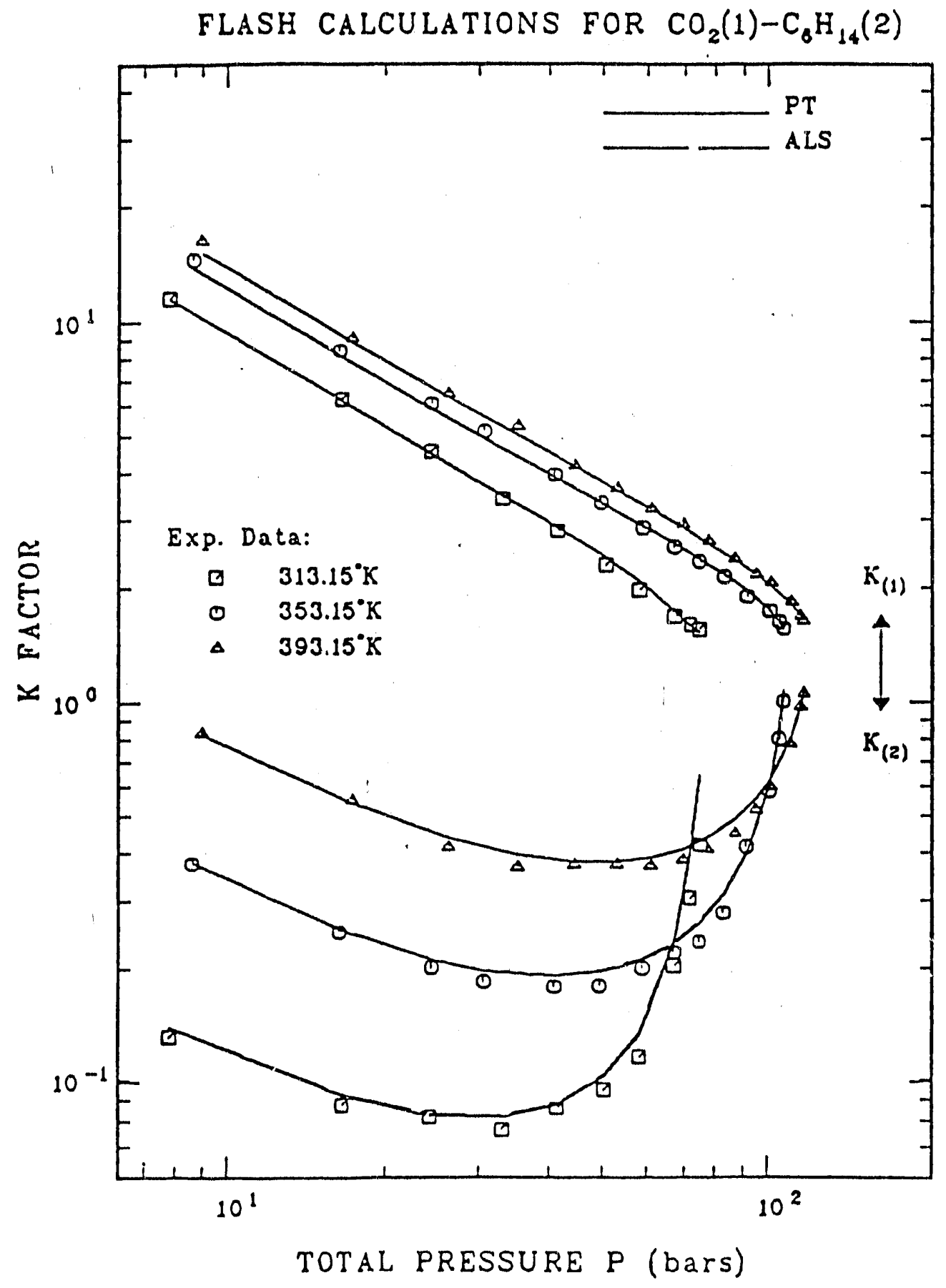

Figure 34. The results of flash calculation for carbon dioxide-hexane using PT and ALS equations compared with experimental data (Li, et al., 1981). 


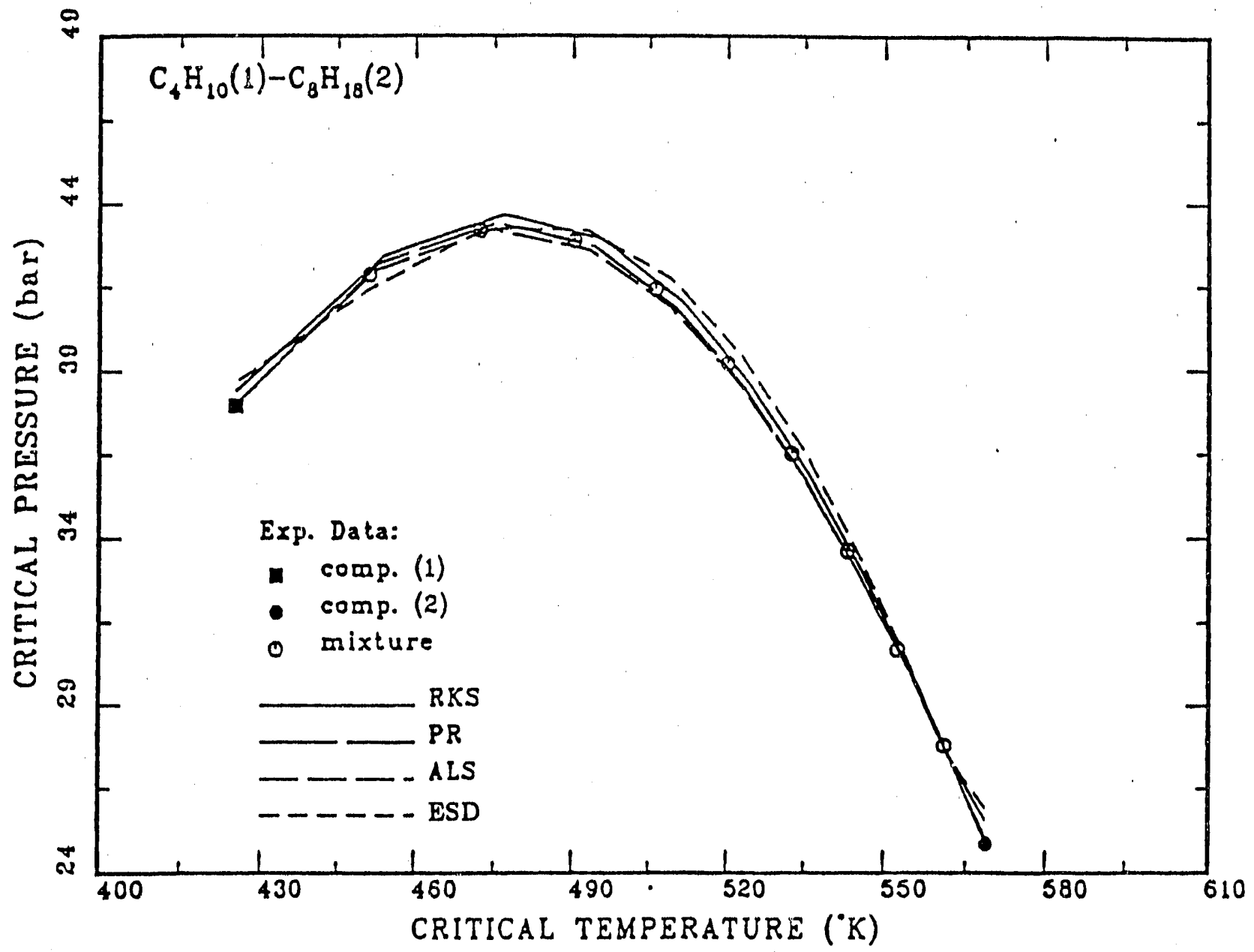

Figure 35. A comparison between calculated critical temperatures and critical pressures of $\mathrm{C}_{4} \mathrm{H}_{10}(1)-\mathrm{C}_{8} \mathrm{H}_{18}(2)$ using the RKS, PR, ALS and ESD equations of state and experimental data (Hicks and Young, 1975). 


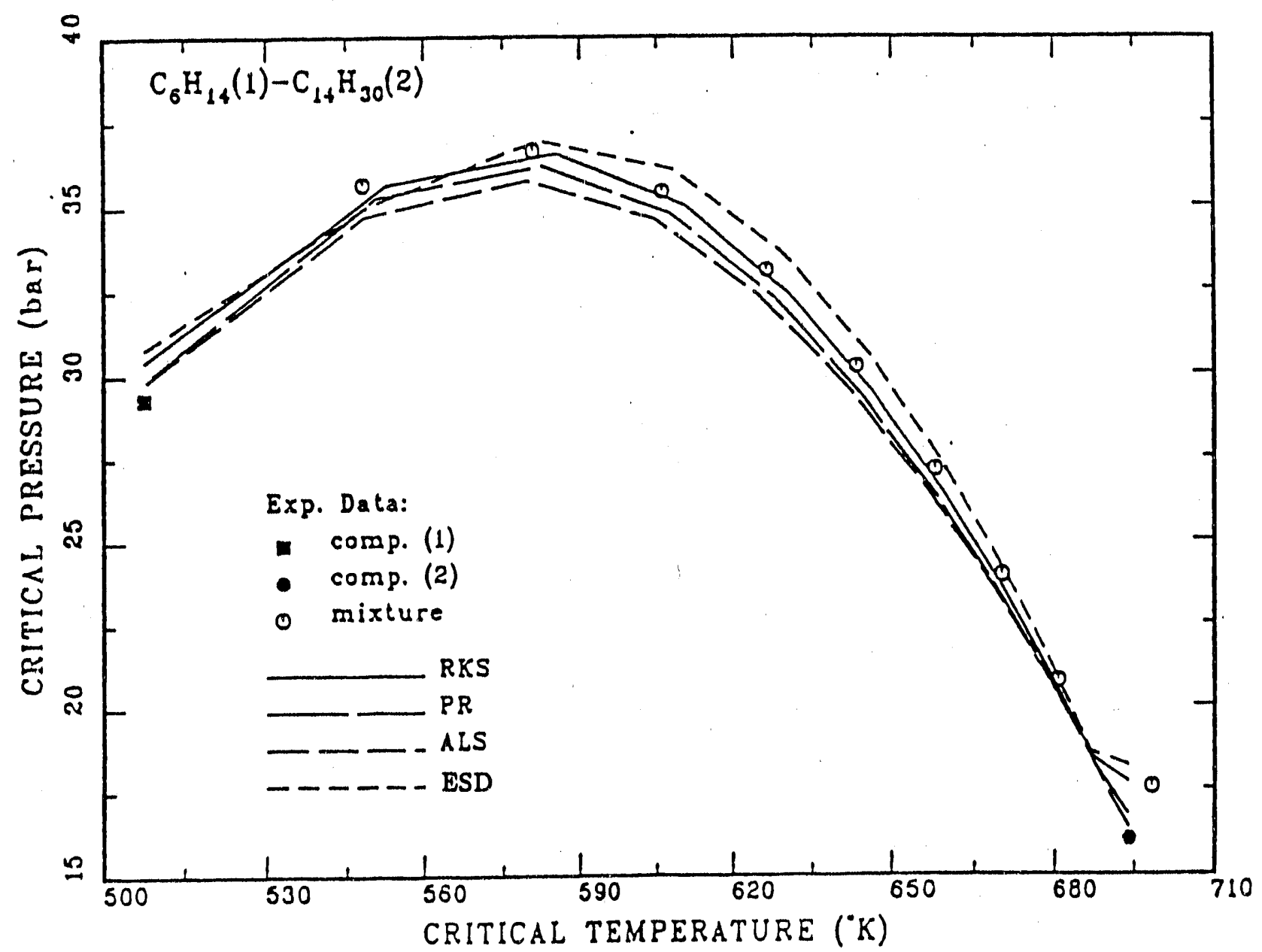

Figure 36. A comparison between calculated critical temperatures and critical pressures of $\mathrm{C}_{0} \mathrm{H}_{14}(1)-\mathrm{C}_{14} \mathrm{H}_{30}(2)$ using the RKS, PR, ALS and ESD equations of state and experimental data (Hicks and Young, 1975). 


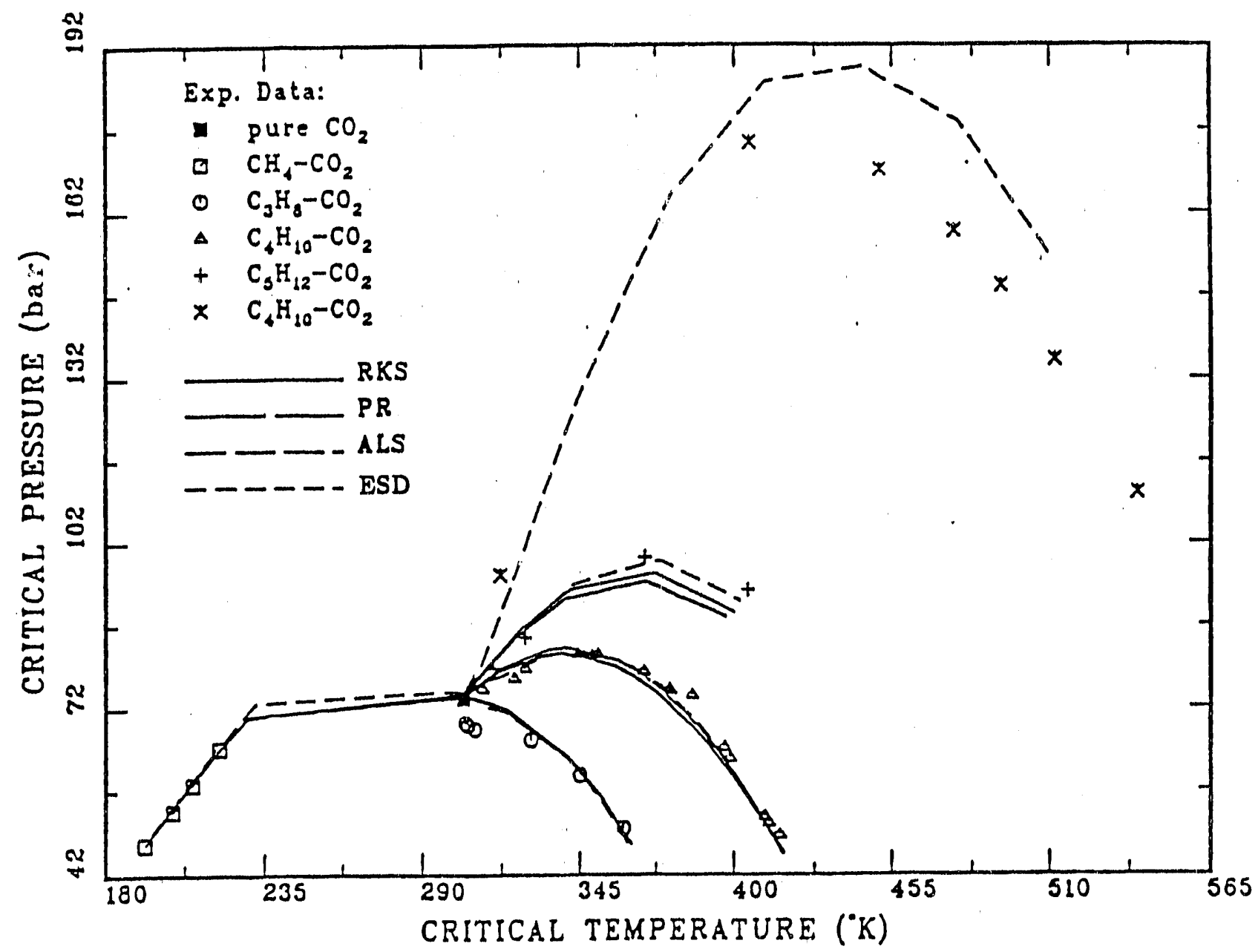

Figure 37. A comparison between calculated critical temperatures and critical pressures of five binary mixtures containing $\mathrm{CO}_{2}$ using the RKS, PR, ALS and ESD equations of state and experimental data (Hicks and Young, 1975). 


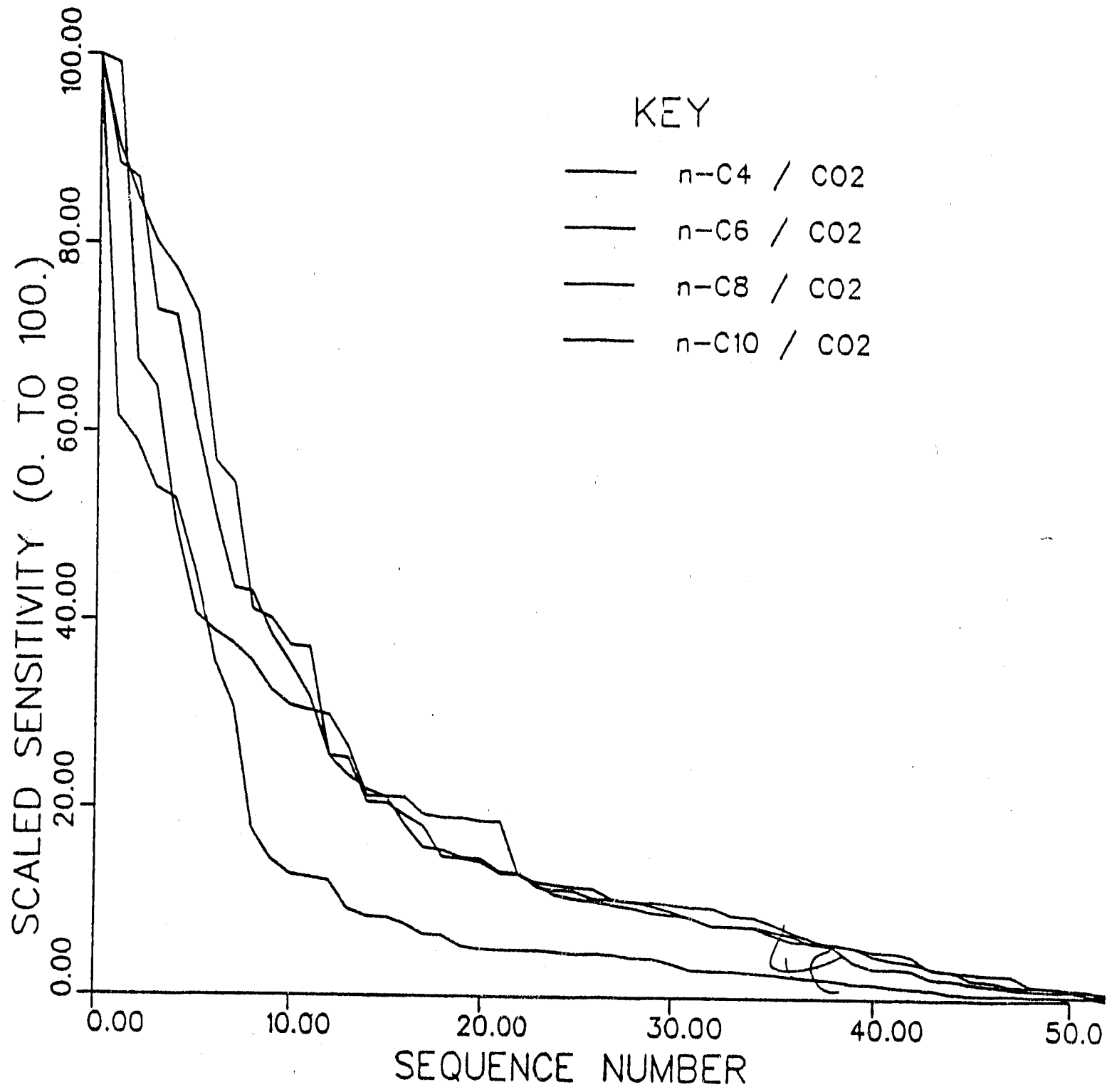

Figure 38 A. A verage Absolute Errors Among Mixtures (Liquid Density) - ESD EOS 


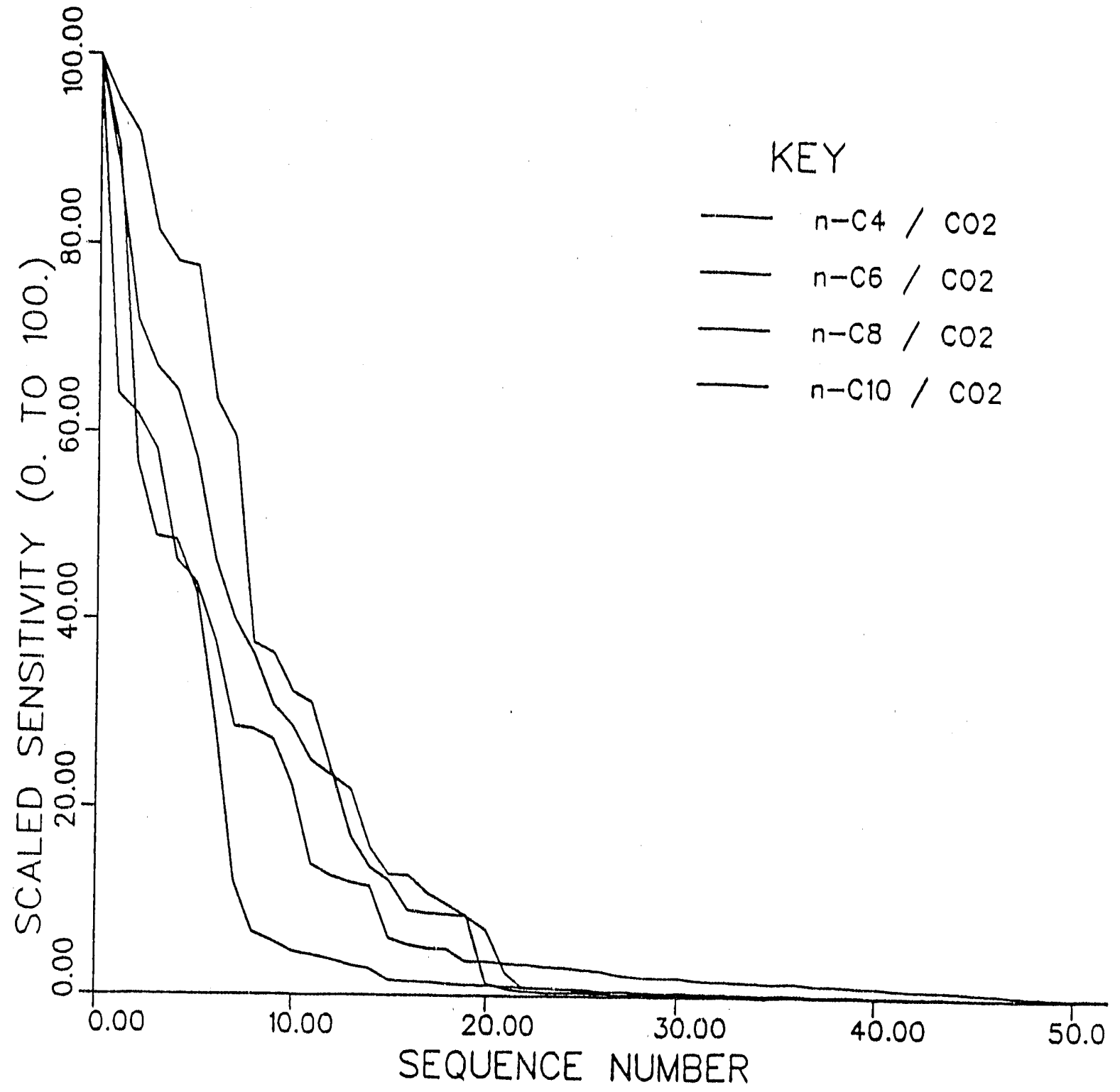

Figure 38 B. Bias Among Mixtures (Liquid Density) - ESD EOS 


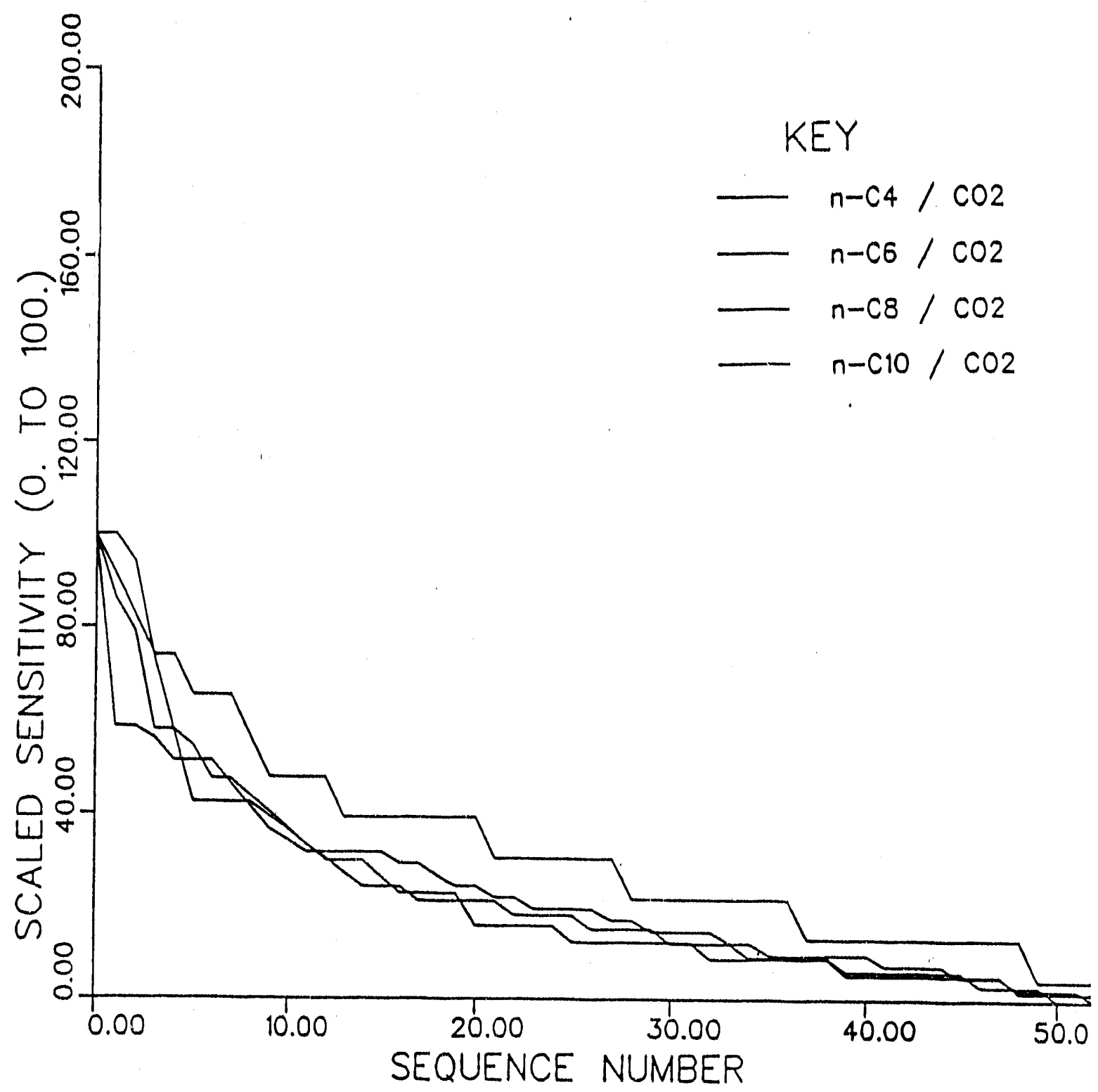

Figure 38 C. CPU Time Among Mixtures (Liquid Density) - ESD EOS 


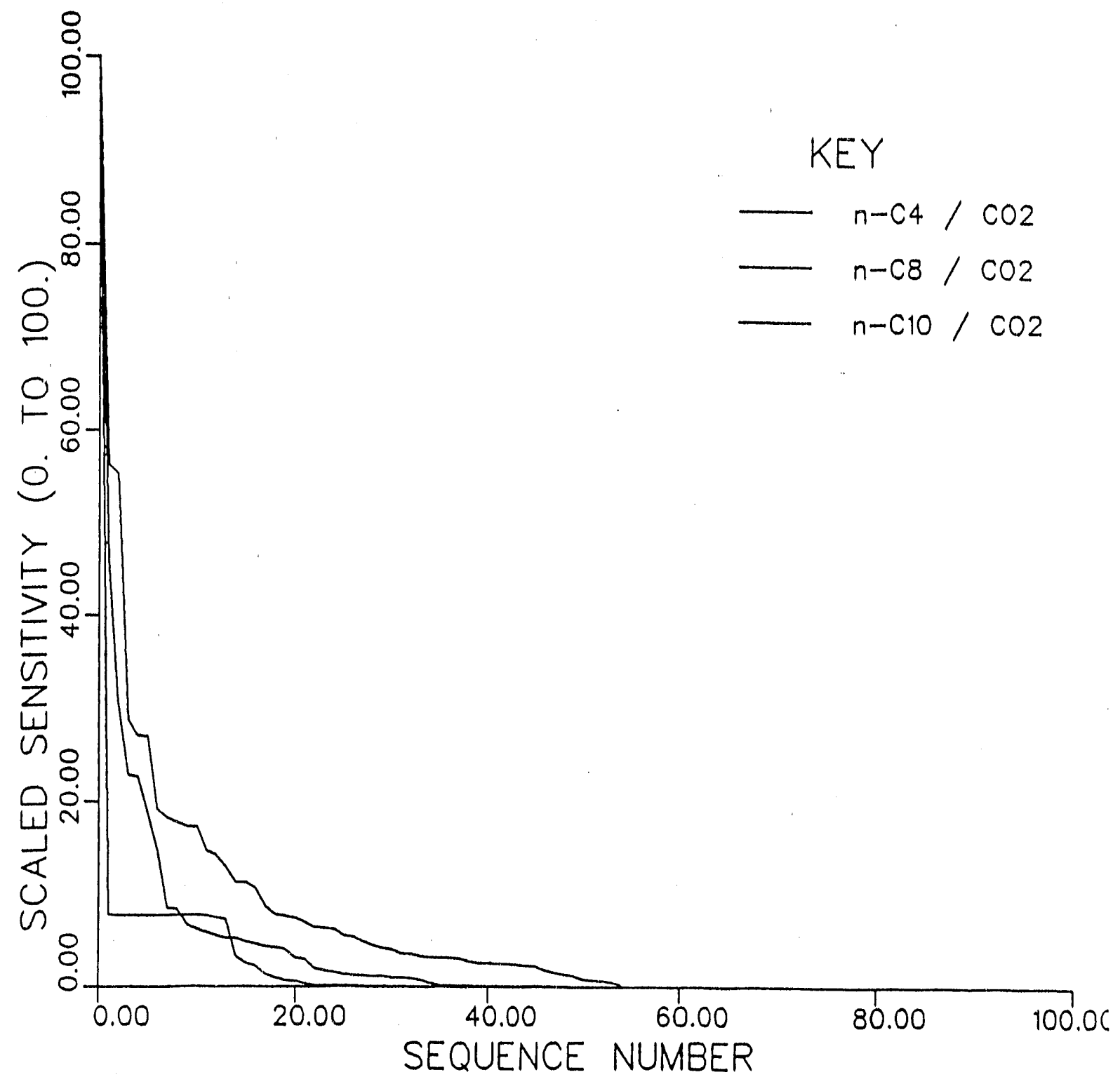

Figure 39 A. Average Absolute Errors Among Mixtures (Liquid Density) - ALS EOS 


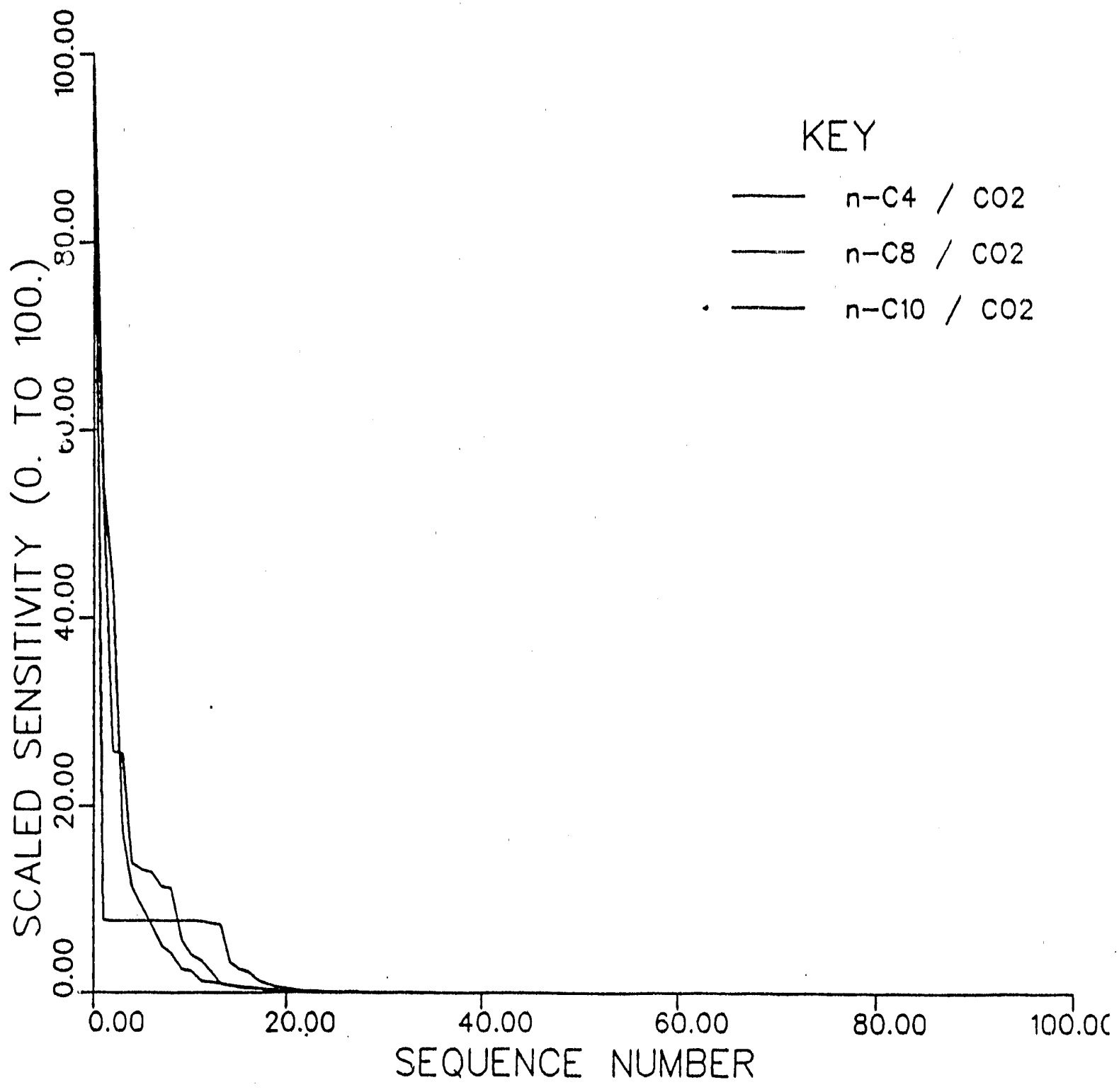

Figure 39 B. Bias Among Mixtures (Liquid Density) - ALS EOS 


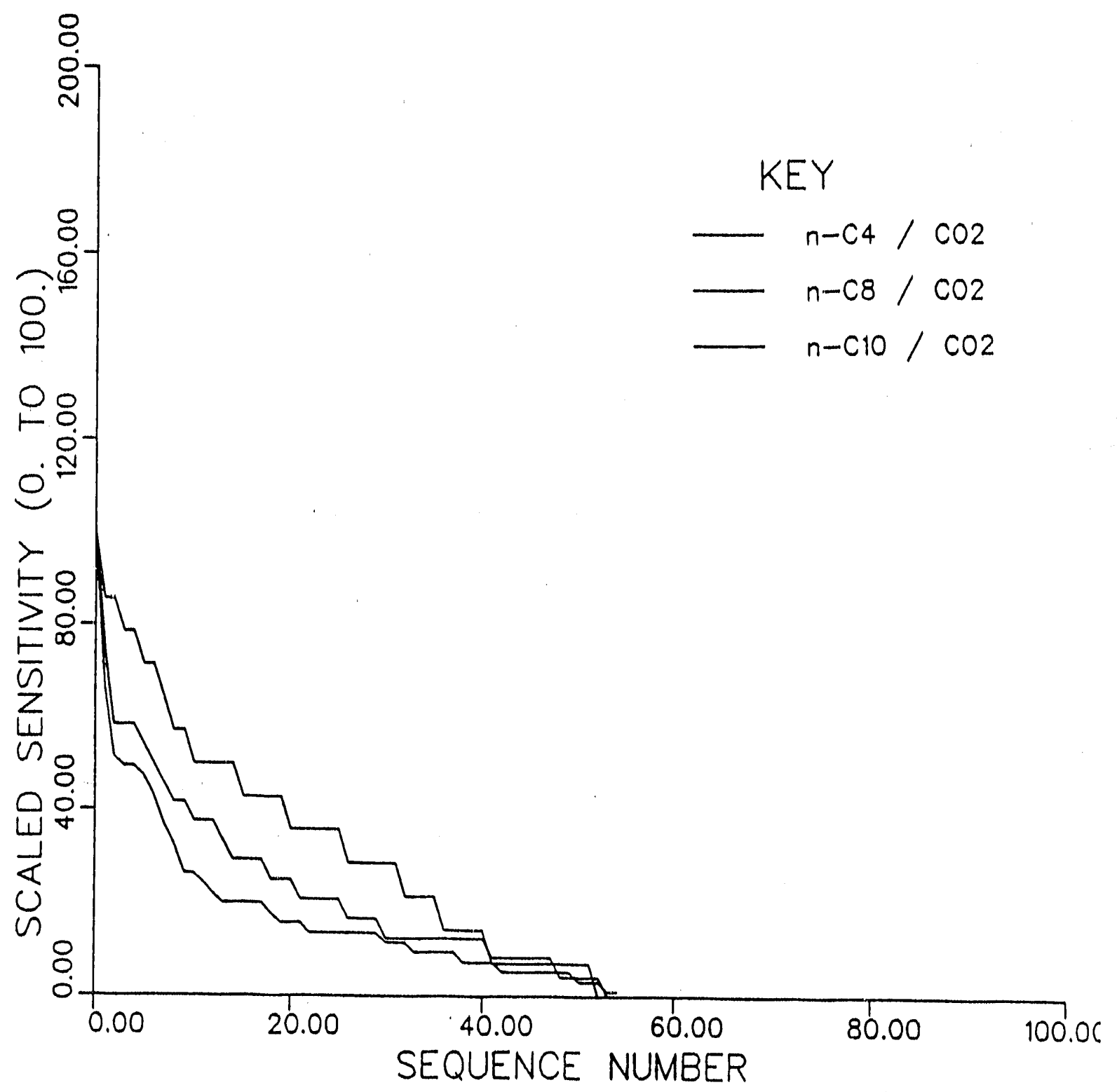

Figure 39 C. CPU Time Among Mixtures (Liquid Density) - ALS EOS 


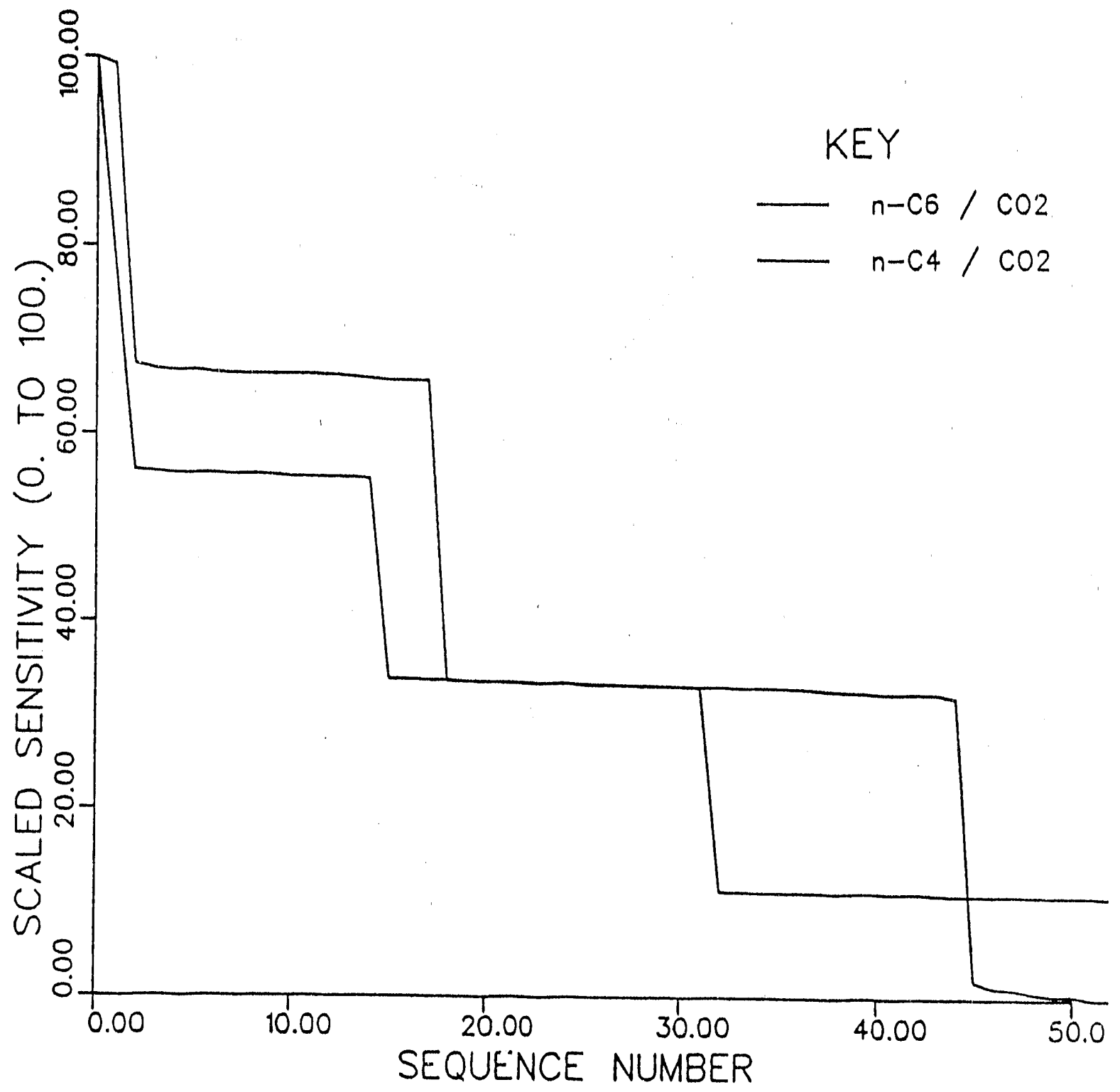

Figure $40 \mathrm{~A}$. Average Absolute Error Among Mixtures (Critical Line Locus) - ESD EOS 


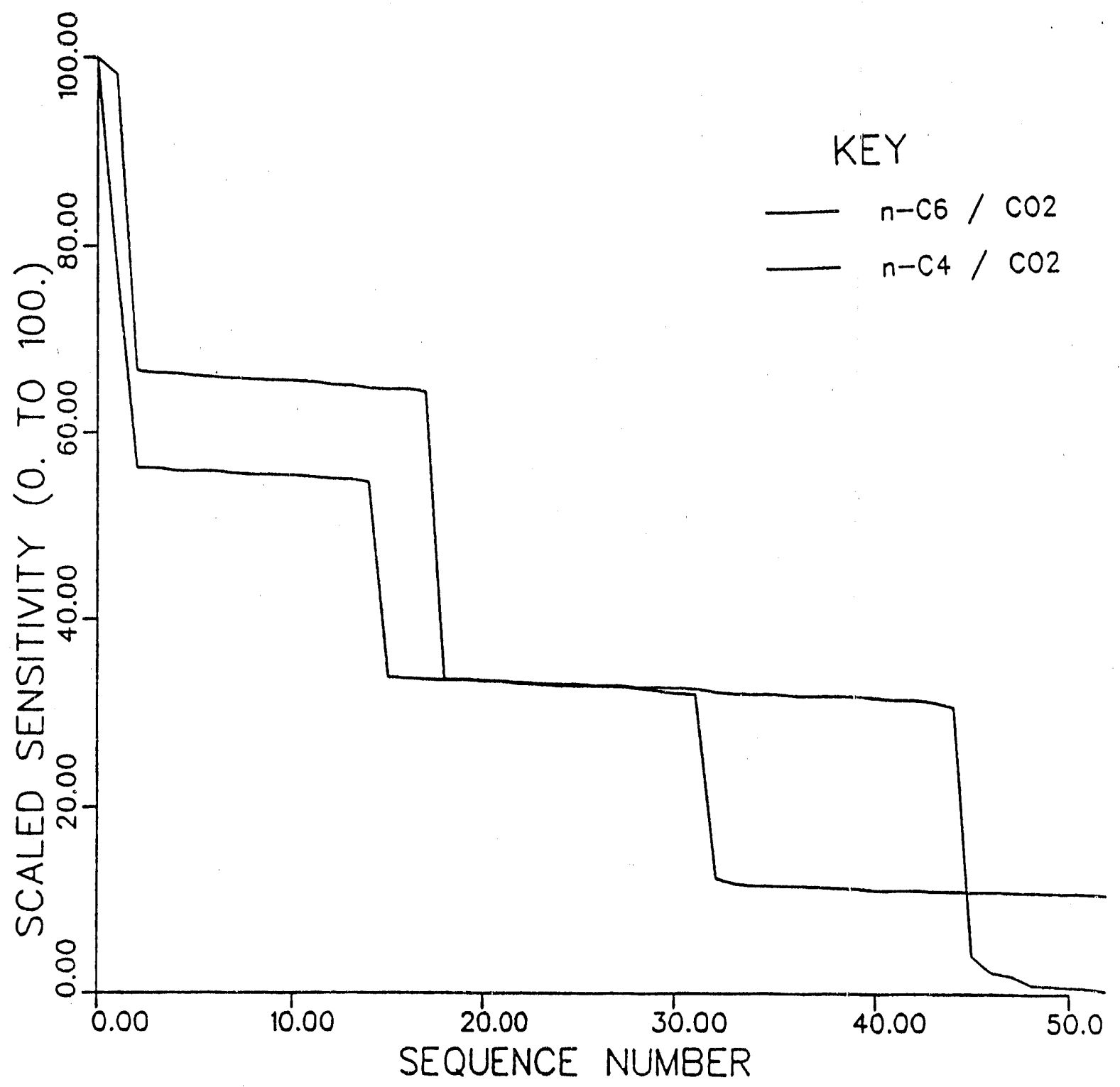

Figure $40 \mathrm{~B}$. Bias Among Mixtures (Critical Line Locus) - ESD EOS 


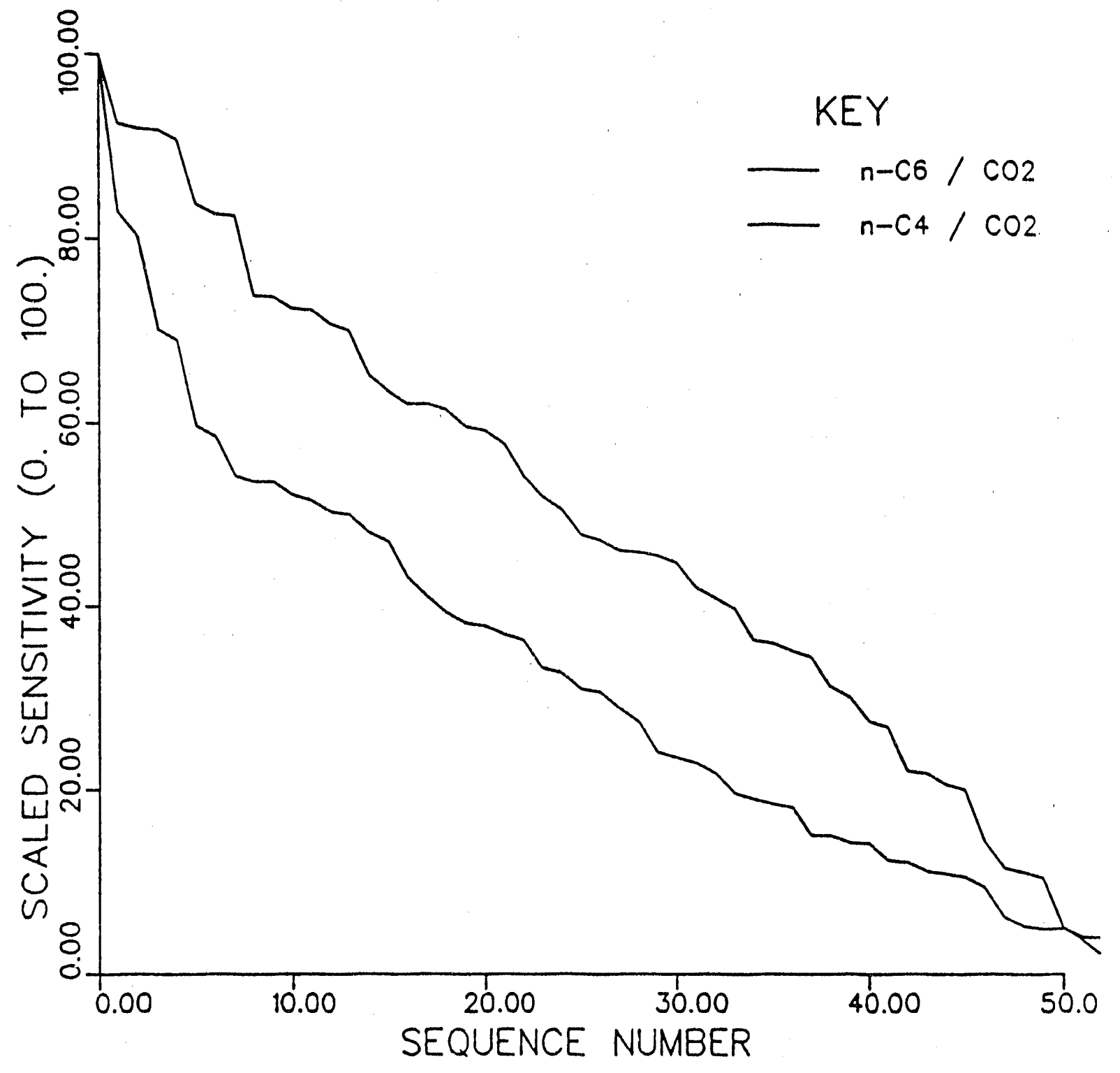

Figure 40 C. CPU Time Among Mixtures (Critical Line Locus) - ESD EOS 


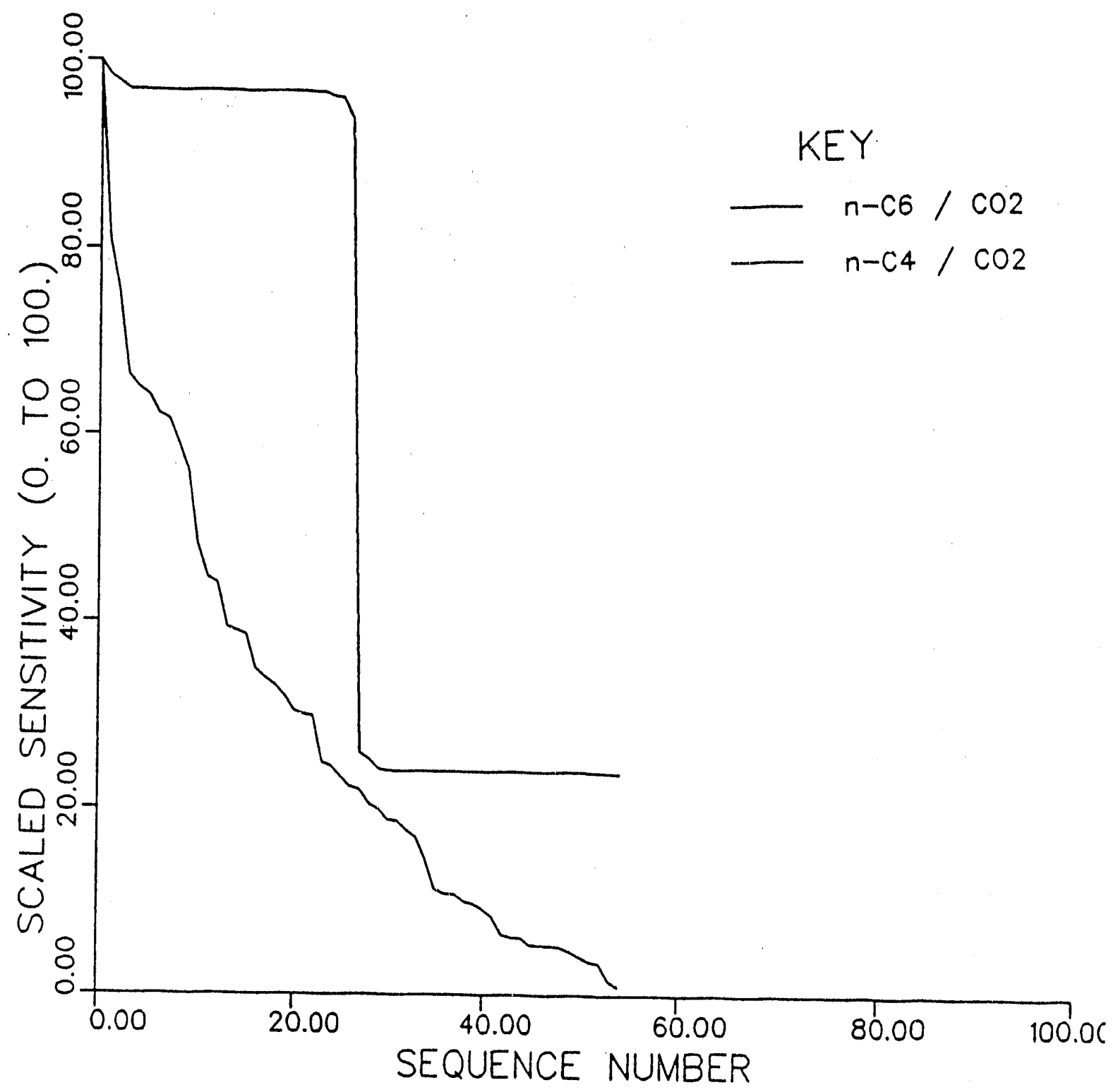

Figure $41 \mathrm{~A}$. Average Absolute Error Among Mixtures (Critical Line Locus) - ALS EOS 


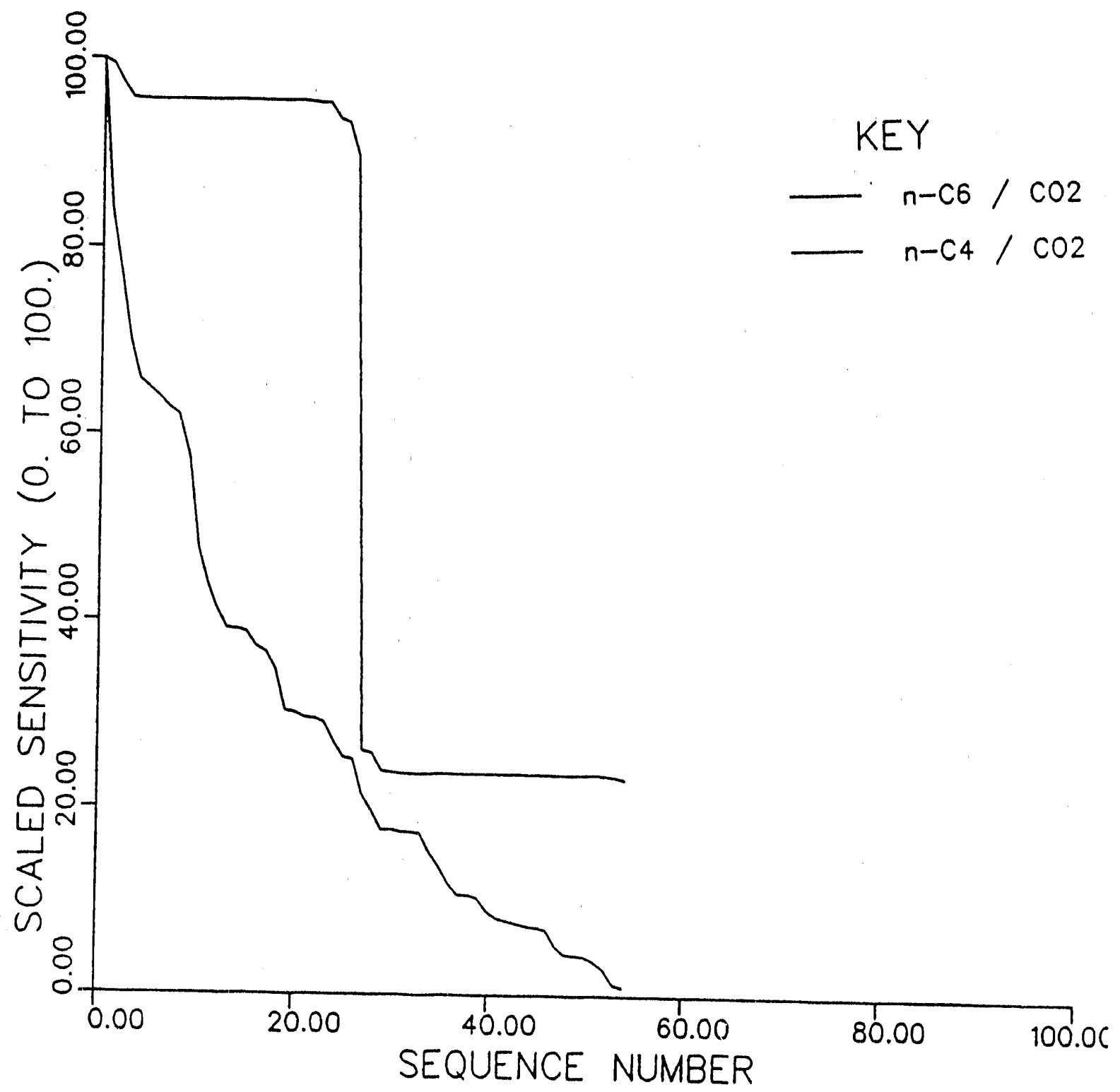

Figure $41 \mathrm{~B}$. Bias Among Mixtures (Critical Line Locus) - ALS EOS 


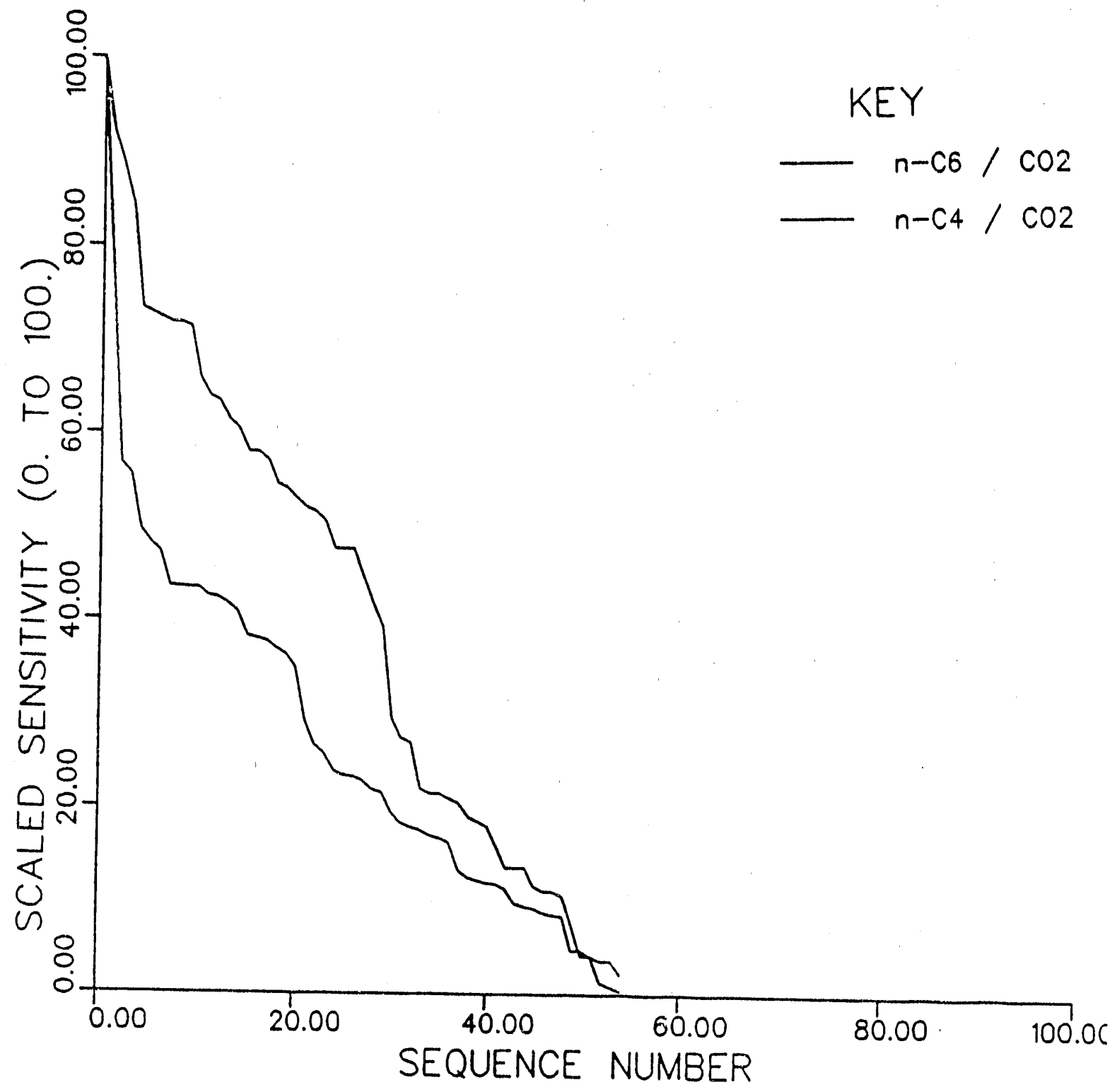

Figure 41 C. CPU Time Among Mixtures (Critical Line Locus) - ALS EOS 


\section{References}

Adachi, Y.; Lu, B. C.-Y.; Sugie, H. "A Four-Parameter Equation of State", Fluid Phase Equilibria, 11, 29-48, 1983.

Angus, S.; Armstrong, B.; Reuck, K. M. de; Altunin, V. V.; Gadetskii, O. G.; Chapela, G. A.; Rowlinson, J. S. "International Thermodynamic Tables of the Fluid State, Carbon Dioxide", UIPAC, Pergamon Press, Oxford, 1976.

Bokis, C.; Konstantopoulos, K.; Tassios, D. "A Modified Peng-Robinson Equation of State", National Technical University of Athens, Athens, Greece, unpublished, 1989.

Box, G.E.P.; W.G. Hunter; J.S. Hunter "Statistics for Experimenters", Wiley, 429, 1978.

Chaback, J. J.; Turek, E. A. "Phase Behavior of Mixtures of San Andres Formation Oils with Acid Gases", In "Equation of State Theories and Applications", K. C. Chao and R. L. Robinson, Jr. Eds., ACS Symposium Series 900, ACS, Washington, D.C., $406-433,1985$.

Chang, S. D.; Lu, B. C.-Y. "Prediction of Partial Molar Volumes of Normal Fluid Mixtures", Can. J. Chem. Eny., 48, 261-266, 1970.

Chao, K. C., Purdue University, West Lafayette, IN, personal communication, San Francisco, CA, Nov. 1989.

Close, D.J. "A Design Approach for Solar Processes", Solar Energy 11, 112, 1967.

Cotterman, R. L.; Prausnitz, J. M. "Molecular Thermodynamics for Fluids at Low and High Densities", AIChE J., 32, 1799-1812, 1986.

Donohue, M. D.; Prausnitz, J. M. "Perturbed Hard Chain Theory for Fluid Mixtures", AIChE J., 24, 849-860, 1978. 
Ekiner, O.; Thodos, G. "Critical Temperatures and Pressures of Ethane-PentaneHeptane System", J. Chem. Eng. Data, 11, 457-460, 1966.

Elliott, Jr., J. R., University of Akron, Akron, OH, personal communication, 1990.

Elliott, Jr., J. R.; Daubert, T. E. "Evaluation of an Equation of State Method for Calculating the Critical Properties of Mixtures", Ind. Eng. Chem. Res., 26, 1686$1691,1987$.

Elliott, Jr., J. R.; Suresh, S. J.; Donohue, M. D. "A Simple Equation of State for NonSpherical and Associating Molecules", Ind. \& Eng. Chem. Res., 29, 1476-1485, 1989.

Etter, D. O.; Kay, W. B. "Critical Properties of Mixtures of Normal Paraffin Hydrocarbons", ibid, 6, 409-414, 1961.

Froning, H. R.; Fussell, D. D.; Heffern, E. W. "Enhanced Oil Recovery", Encyclopedia of Chemical Technology, 3rd Ed., vol. 17, 168-182, 1982.

Gani, R.; Fredenslund, A. "Prediction of Properties of Petroleum Mixtures: Improvement in Reliability and Validity of Tuned Parameters", Fluid Phase Equilibria, 29, $575-582,1986$.

Gibbs, J. W. "On the Equilibrium of Heterogeneous Substances, Part I", Thransactions of the Connecticutt Academy, 3, 108, 1876, as reprinted in The Scientific Papers of J. Willard Gibbs, vol. 1, Dover, New York, 1961.

Heidemann, R. A.; Khalil, A. M. "The Calculation of Critical Points", AIChE J., 26(5), $769-779,1980$.

Hicks, C. P.; Young, C. L. "The Gas-Liquid Critical Properties of Binary Mixtures", Chem. Rev., 75(2), 119-175, 1975.

Hohmann, E. C., Gas Processors Association Research Report RR-38, Tulsa, OK., 1979. 
Joffe, J.; Schroeder, G. M.; Zudkevitch, D. "Vapor-Liquid Equilibria with the RedlichKwong Equation of State", AIChE J., 16, 496-498, 1970.

Katz, D. L.; Lee, R. L. Natural Gas Engineering, McGraw-Hill, New York, 1990.

Kim, C. H.; Vimalchand, P.; Donohue, M. D.; Sandler, S. I. "Local Composition Model for Chainlike Molecules: A New Simplified Version of the Perturbed Hard Chain Theory", J. AIChE, 32(10), 1726-1734, 1986.

Knapp, H.; Doring, R.; Oellrich, L.; Plocker, U.; Prausnitz, J. M. "Vapor-Liquid Equilibria for Mixtures of Low Boiling Substances", Chemistry Data Series, VI, DECHEMA, pp607, 1982.

Li, Ying-Hsiao; Dillard, K. H.; Robinson, Jr., R. L. "Vapor-Liquid Phase Equilibrium for Carbon Dioxide-n-Hexane at 40, 80, and $120^{\circ} \mathrm{C}^{\prime \prime}, J$. Chem. Eng. Data, 26, 53-55, 1981.

Lin, H. M.; Kim, H.; Guo, T. M.; Chao, K. C. Fluid Phase Equilibria, 13, 143-152, 1983.

Martin, J. J. "Equations of State", Ind. Eng. Chem., 59(12), 34-52, 1967.

Michelsen, M. L.; Heidemann, R. A. "Calculation of Critical Points from Cubic TwoConstant Equations of State", AIChE J., 27(3), 521-523, 1981.

Morris, R. W.; Turek, E. A. "Optimal Themperature-Dependent Parameters for the Redlich-Kwong Equation of State", In "Equation of State Theories and Applications", K. C. Chao and R. L. Robinson, Jr. Eds., ACS Symposium Series 900, ACS, Washington, D.C., 389-405, 1985.

Nagarajan, N. R., Mobil Res. \& Dev. Co., Dallas, TX, personal communication, San Francisco, CA, Nov. 1989. 
O'Connell, J. P., University of Virginia, Charlottsville, VA, personal communication, San Francisco, CA, Nov. 1989.

Patel, N. C.; Teja, A. S. "A New Cubic Equation of State for Fluids and Fluid Mixtures", Chem. Eng. Sci., 37(3), 463-473, 1982.

Pedersen, K. S.; Thomassen, P.; Fredenslund, A. "SRK-EOS Calculation for Crude Oils", Fluid Phase Equilibria, 14, 209-218, 1983.

Peng, D. Y., University of Saskatchewan, Canada, personal communication, San Francisco, CA, Nov. 1989.

Peng, D. Y.; Robinson, D. B. "A New Two-Constant Equation of State", Ind. Eng. Fundam., 15, 59-64, 1976.

Peng, D. Y.; Robinson, D. B. "A Regorous Method for Predicting the Critical Properties of Multicomponent Systems from an Equation of State", AIChE J., 23(2), 137-144, 1977.

Prausnitz, J. M.; Lichtenthaler, R. N.; Azevedo, E. G. Molecular Thermodynamics of Fluid-Phase Equilibria, 2nd ed., Prentice-Hall: New Jersey, 1986.

Redlich, O.; Kwong, J. N. S. "On the Thermodynamics of Solutions. V", Chem. Rev., 44, 233-244, 1948.

Soave, G. "Equilibrium Constants from A Modified Redlich-Kwong Equation of State", Chem. Eng. Sci., 27, 1197-1203, 1972.

Spencer, C. F.; Daubert, T. E. "A Critical Review of Correlations for The Critical Properties of Defined Mixtures", AIChE J., 19, 522-527, 1973.

Teja, A. S., Georgia Institute of Technology, Atlanta, GA, personal communication, San Francisco, CA, Nov. 1989. 
Trebble, M. A.; Bishnoi, P. R. "Accuracy and Consistency Comparisons of Ten Cubic Equations of State for Polar and Non-Polar Compounds", Fluid Phase Equilibria, 29, 465-474, 1986.

Tsonopoulos, C., Exxon Research and Engizeering Co., Florham Park, NJ, personal communication, 1989.

Usdin, E.; McAuliffe, J. C. "A One Parameter Family of Equations of State", Chem. Eng. Sci., 31(11), 1077-.1984, 1976.

van der Waals, J. D. Doctoral Dissertation, Leiden Holland, 1873.

Vimalchand, P.; Donohue, M. D. "Effect of Fluid Structure on Equation of State Calculations", Submitted to Fluid Phase Equilibria, 1990.

Vimalchand, P. "Thermodynamics of Multi-polar Molecu'es", Ph.D. Dissertation, The Johns Hopkins University: Baltimore, Maryland, 1986.

Vimalchan I, P.; Donohue, M. D.; Celmins, I. "Thermodynamics of dipolar molecules: The Perturbed-Anisotropic-Chain Theory"; Chao, K. C., Robinson, Jr., R. L., Ed.; Equations of State: Theories and Applications; American Chemical Society: Washington, DC, pp 297-313, 1985.

Vogel, J. L.; Turek, E. A.; Metcalfe, R. S.; Bergman, D. F.; Morris, R. W. "Applications of Equations of State To Calculate Reservoir Fluid Properties", Fluid Phase Equilibria, 14, 103-116, 1983.

Walas, S. M. "Phase Equilibria in Chemical Engineering", Butterworth Publishers, Boston, 1985.

Warner, H.K.; J.H. Hardy; N. Robertson; A.L. Barnes "University Block 31 Field Study: Part 1 - Middle Devonian Reservoir History Match", J. Pet. Technol. (Aug.), 962970,1979 , 
Warner, H.R.; J.H. Hardy; N. Robertson; A.L. Barnes "University Block 31 Field Study: Part 2 - Reservoir and Gas Plant Performance Projections", J. Pet. Technol. (Aug.), 971-978, 1979.

Yarborough, L. "Application of a Generalized Equation of State to Petroleum Reservoir Fluids", Chao, K. C., Robinson, Jr., R. L., Ed.; Equations of State in Engineering and Research; American Chemical Society: Washington, DC, 182, pp385-439, 1979.

Zudkevitch, D.; Joffe, J. "Correlation and Prediction of Vapor-Liquid Equilibria with the Redlich-Kwong Equation of State", AIChE J., 16(1), 112-119, 1970. 


\section{APPENDIX A}

In selecting equations of state, temperature and pressure ranges and thermodynamic variables relevant to enhanced oil recovery, we have consulted with experts in both industry and academia. The experts are listed below:

Dr. K. R. Cox, Shell Development Company, Houston, TX

Dr. K. C. Chao, Purdue University, West Lafayette, IN

Dr. R. P. Danner, The Pennsylvania State University, University Park, PA

Dr. C. A. Eckert, University of Illinois, Urbana, IL

Dr. J. R. Elliott, Jr., University of Akron, Akron, OH

Dr. N. R. Nagarajan, Mobil Research and Development Co., Dallas, TX

Dr. J. P. O'Connell, University of Virginia, Charlottesville, VA

Dr. D. Y. Peng, University of Saskatchewan, Canada

Dr. A. S. Teja, Georgia Institute of Technology, Atlanta, GA

Dr. G. Thomas, Exxon Production Research Company, Houston, TX

Dr. C. Tsonopoulos, Exxon Resea'ch and Engineering Co., Florham Park, NJ

In the following list, we state their opinions in four topics: (1) Temperature range, (2) Pressure Range, (3) Properties which are important to EOR processes, and (4) considerations in selecting an equation of state for EOR operation.

\begin{tabular}{lllll} 
Expert & Topic $(1)$ & Topic (2) & Topic (3) & Topic (4) \\
\hline Chao & room tempera- & up to few hun- & miscibility of the reliability, pre- \\
& ture to $200^{\circ} \mathrm{C}$ for & dreds bars & fluids $\quad$ in diction of phase \\
& $\mathrm{CO}_{2}$ oil recovery & & an oil reservoir, & behavior of sys- \\
& & & density & tems \\
\hline
\end{tabular}




\begin{tabular}{|c|c|c|c|c|}
\hline Elliott & & & & $\begin{array}{l}\text { simplicity, } \\
\text { use cubic equa- } \\
\text { tions of state }\end{array}$ \\
\hline Nagarajan & $\begin{array}{l}80^{\circ} \mathrm{F}-200^{\circ} \mathrm{F} \text { for } \\
\mathrm{CO}_{2} \mathrm{EOR}\end{array}$ & $\begin{array}{l}\text { above minimum } \\
\text { miscible pres- } \\
\text { sure of the fluids } \\
\text { in reservoir }\end{array}$ & $\begin{array}{l}\text { density, viscos- } \\
\text { ity and surface } \\
\text { tension }\end{array}$ & $\begin{array}{l}\text { simplicity (cubic } \\
\text { equations of } \\
\text { state), accuracy } \\
\text { and reliability of } \\
\text { an EOS should } \\
\text { be optimized }\end{array}$ \\
\hline O'Connell & $\begin{array}{l}25^{\circ} \mathrm{C}-100^{\circ} \mathrm{C} \text { in } \\
\text { most reservoir in } \\
\text { U.S. and higher } \\
\text { temperature in } \\
\text { North Sea fields }\end{array}$ & $\begin{array}{l}\text { 100-200bar, } \\
\text { above the crit- } \\
\text { ical pressure of } \\
\text { the mixture flu- } \\
\text { ids in reservoir }\end{array}$ & $\begin{array}{l}\text { density, viscos- } \\
\text { ity and interfa- } \\
\text { ial } \\
\text { tension in which } \\
\text { only density can } \\
\text { be calculated us- } \\
\text { ing EOS }\end{array}$ & $\begin{array}{l}\text { reliability is as } \\
\text { important as ac- } \\
\text { curacy }\end{array}$ \\
\hline Peng & $25^{\circ} \mathrm{C}-150^{\circ} \mathrm{C}$ & $\begin{array}{l}\text { few hundreds } \\
\text { bars, } 60 \text { bars is a } \\
\text { typical pressure } \\
\text { in an oil reser- } \\
\text { voir }\end{array}$ & $\begin{array}{l}\text { density and vis- } \\
\text { cosity }\end{array}$ & \\
\hline Teja & $\begin{array}{l}25^{\circ} \mathrm{C} \text { to several } \\
\text { hundreds }{ }^{\circ} \mathrm{C}\end{array}$ & $\begin{array}{l}5,000 \text { psia (345 } \\
\text { bars) }\end{array}$ & $\begin{array}{l}\text { density of the } \\
\text { fluids in reser- } \\
\text { voir }\end{array}$ & $\begin{array}{l}\text { simplicity (such } \\
\text { as the PR equa- } \\
\text { tion) and accu- } \\
\text { acy }\end{array}$ \\
\hline
\end{tabular}




\begin{tabular}{lll}
\hline Tsonopoulos & $\Delta v$ the change of accuracy, use \\
molar volume of & the \\
systems & perturbed chain \\
& theory such as \\
& PHCT, simple \\
& cubic EOS can- \\
not gives accu- & rate predictions \\
& of $\Delta v$
\end{tabular}

Other people contacted did not provide information beyond that listed above. 


\section{APPENDIX B \\ The Peng-Robinson-Bokis (PRB) Equation of State}

Although the Peng-Robinson (PR) equation of state (Peng and Robinson 1976) gives good predictions in vapor-liquid equilibrium calculations, it gives large errors in volumetric properties, especially in liquid density. To reduce errors in calculated liquid density, Bokis et al. recently modified the PR equation by recorrelating parameters $a$ and $b$ in the original PR equation and introducing a new volumetric correction term $\Delta v$ to the equation (Bokis, et al. 1989). Since this work has not been published, we describe their resulting equation here.

The Peng-Robinson-Bokis (PRB) equation of state can be written in the same form as the original PR equation by replacing molar volume $v$ by a corrected molar volume $v+\Delta v$. The resulting form is given as

$$
P=\frac{R T}{(v+\Delta v)-b}-\frac{a(T)}{(v+\Delta v)(v+\Delta v+b)+b(v+\Delta v-b)} \quad(B-1)
$$

where $\Delta v$ is a translation factor which corrects the calculated molar volume. This factor is defined as a deviation between experimental molar volume, $v^{e: c p}$, and calculated molar volume using the original PR equation, $v_{P R}^{c a l}$.

$$
\Delta v=v^{e x p}-v_{P R}^{c a l}
$$

The regressed values of $\Delta v$ at different temperatures and pressures are fitted as a function of temperature, acentric factor and critical properties. The result is

$$
\Delta v=\frac{R T_{c}}{P_{c}}\left[\Delta v_{0}+\left(\Delta v_{c}-\Delta v_{0}\right)\right] \exp \left(\beta\left|1-T_{r}\right|\right) \quad(B-3)
$$

where

$$
\Delta v_{0}=-0.01265443+0.05111279 \omega-0.03564982 \omega^{2}+0.01278032 \omega^{3} \quad(B-4)
$$




$$
\Delta v_{c}=0.3074-\not Z_{c}
$$

and

$$
\beta=-10.2447-28.6312 \omega
$$

In equations (B-3) to (B-6), $T_{r}$ is the reduced temperature $\left(=T / T_{c}\right) ; \omega$ is the acentric factor, $T_{c}, P_{c}$ and $Z_{c}$ are critical temperature, critical pressure and critical compressibility, respectively.

The parameters $a(T)$ and $b$ in the original PR equation also have been modified. New forms are

$$
a=0.457235 \frac{R^{2} T_{c}^{2}}{P_{c}}\left[1+m\left(1-T_{r}^{0.5}\right]^{2}\right.
$$

and

$$
b=0.07779608 \frac{R T_{c}}{P_{c}}
$$

The Soave coefficient $m$ in equation (B-7) also was recorrelated. The resulting form is given as

$$
m=0.3784397+1.614629 \omega-0.4280519 \omega^{2}+0.1560361 \omega^{3} \quad(B-9)
$$

For mixture calculations, the mixing rules for parameters $a_{m i x}$ and $b_{m i x}$ are the same as that given by original PR equation. These mixing rules have been given by equations (46) to (48). The mixing rule for $\Delta v_{m i x}$ is given as

$$
\Delta v_{m i x}=\sum_{i} x_{i} \Delta v_{i}
$$




\section{APPENDIX C \\ The Elliott-Suresh-Donohue (ESD) Equation of State}

The Elliott-Suresh-Donohue (ESD) equation of state is derived based on a semiempirical model which provides a good representation of available molecular simulation data for hard chains, hard spherocylinders, square-well spheres and Lennard-Jones chains (Elliott, et al., 1989). The equation is cubic and, therefore, can easily be used in industrial applications. Since this equation of state has not been published, a brief description of this equation for both pure components and mixtures is given in this section.

\section{Pure Components}

For pure components, the ESD equation of state can be written as

$$
P=\frac{R T}{v}+P^{r e p}+P^{a t t}
$$

where $P$ is the total pressure of system, $v$ is molar volume, $T$ is temperature and $R$ is the universal gas constant. The first term in equation (C-1) is the ideal gas term. The second term, $P^{r e p}$, and the third term, $P^{a t t}$ are the repulsive and attractive terms, respectively. They are given by

$$
P^{r e p}=\frac{4 c v^{*} R T}{v\left(v-1.9 v^{*}\right)}
$$

and

$$
P^{a t t}=-\frac{9.49 q v^{*} Y R T}{v\left(v+1.7745 v^{*} Y\right)}
$$

where $c$ is a shape factor, $v^{*}$ is a characteristic size parameter, $q$ is a parameter which accounts for the effect of shape on attractive interactions, and $Y$ is an attractive interaction parameter. These parameters have been fitted as functions of acentric factor and critical properties. The resulting forms are given as

$$
c=1+3.535 \omega+0.533 \omega^{2}
$$




$$
\begin{gathered}
v^{*}=\frac{R T_{c}}{P_{c}} \frac{0.0312+0.087(c-1)+0.008(c-1)^{2}}{1+2.455(c-1)+0.732(c-1)^{2}} \\
q=1+1.90476(c-1)
\end{gathered}
$$

and

$$
Y=\exp \left(\frac{\epsilon}{k T}\right)-1.0617
$$

where $k$ is the Boltzmann's constant and $\epsilon / k$ is given by

$$
\frac{\epsilon}{k}=T_{c} \frac{1+0.945(c-1)+0.134(c-1)^{2}}{1.023+2.225(c-1)+0.478(c-1)^{2}}
$$

\section{Mixtures}

The equation of state for mixtures can be written as follows

$$
P=\frac{R T}{v}+\frac{4 R T<c v^{*}>}{v\left(v-1.9<v^{*}>\right)}-\frac{9.49 R T<q v^{*} Y>}{v\left(v+1.7745<v^{*} Y>\right)}
$$

The mixing rules used in equation (C-9) are given by

$$
\begin{aligned}
<v^{*}>=\sum_{i} x_{i} v_{i}^{*} \\
<c v^{*}>=\frac{1}{2} \sum_{i} \sum_{j} x_{i} x_{j}\left(c_{i} v_{j}^{*}+c_{j} v_{i}^{*}\right) \\
<q v^{*} Y>=\frac{1}{2} \sum_{i} \sum_{j} x_{i} x_{j} Y_{i j}\left(q_{i} v_{j}^{*}+q_{j} v_{i}^{*}\right) \\
<v^{*} Y>=\frac{<q v^{*} Y>}{\sum_{i} x_{i} q_{i}}
\end{aligned}
$$

The cross parameter $Y_{i j}$ in equation (C-12) is given by

$$
Y_{i j}=\exp \left(\frac{\epsilon_{i j}}{k T}\right)-1.0617
$$

where $\epsilon_{i j}$ can be obtained using geometric mean

$$
\epsilon_{i j}=\left(\epsilon_{i i} \epsilon_{j j}\right)^{0.5}\left(1-k_{i j}\right)
$$

where $k_{i j}$ is a binary interaction parameter. 


\section{APPENDIX D \\ The Heidemann-Khalil-Michelsen (HKM) Algorithm and}

\section{The Derivatives of Helmholtz Free Energy Using Equations of State}

The calculations of mixture critical points using equations of state require trial and error solutions for the two variables $T_{c}$ and $v_{c}$. These two variables can be obtained by solving equations (54) and (55). A special algorithm for solving the equations has been developed by Heidemann and Khalil (1980) and Michelsen and Heidemann (1981). The Heidemann-Khalil-Michelsen (HKM) algorithm makes the convergence of the iterations in the calculation much faster and therefore significantly reduces computer time.

\section{The HKM Algorithm}

The strategy of the HKM algorithm is to iterate on variables $T_{c}$ and $v_{c}$ separately instead solving equations (54) and (55) simultaneously as in a typical process of solving multiple nonlinear equations. The procedure of this algorithm is given as follows

(1) Set an initial guess of $v_{c}$. For example, $v_{c}=4 b_{1}$, where $b_{1}$ is the parameter in equation (1).

(2) Calculate $T_{c}$ by solving equation (54). The initial value of $T_{c}$ can be obtained using

$$
T_{c}^{0}=\sum_{j} x_{j} T_{c, j}
$$

where subscript $j$ denotes component. The new value of $T_{c}$ substituting into equation (54) in each iteration is calculated by

$$
T_{c}^{i+1}=T_{c}^{i}+\Delta T_{c}^{i}
$$

where superscript $i$ is a iteration number and $\Delta T_{c}^{i}$ is given as

$$
\Delta T_{c}^{i}=\delta T_{c}^{i} \frac{\operatorname{det}|\mathbf{Q}|_{a t} T_{c}^{i}}{\left[\operatorname{det}|\mathbf{Q}|_{a t} T_{c}^{i}-\operatorname{det}|\mathbf{Q}|_{a t} T_{c}^{i}+\delta T_{c}^{i}\right]}
$$


where matrix $\mathbf{Q}$ is defined by equation (56) and $\delta T_{c}^{i}$ is set to be $1 \times 10^{-5} T_{c}^{i}$ in this study.

(3) Calculate $\Delta \mathrm{n}=\left(\Delta n_{1}, \Delta n_{2}, \cdots, \Delta n_{m}\right)^{T}$, where $m$ is number of component in system, by solving

$$
\mathbf{Q} \Delta \mathbf{n}=0
$$

Since $\operatorname{det}(\mathbf{Q})=0$, following procedure is used to solve equation (D-4):

(a) reduce matrix $\mathbf{Q}$ to upper triangular iorm;

(b) take $\Delta n_{m}^{\prime}=1.0$;

(c) use back substitution to find $\Delta n_{1}^{\prime}, \Delta n_{2}^{\prime}, \cdots, \Delta n_{m-1}^{\prime}$;

(d) calculate $\Delta n_{j}$ by following normalization

$$
\Delta n_{j}=\frac{\Delta n_{j}^{\prime}}{\left[\sum_{k+1}^{m}\left(\Delta n_{k}^{\prime}\right)^{2}\right]^{0.5}}
$$

(4) Calculate $v_{c}$ by solving equation (55).

(5) Set criterion $\left|\Delta v_{c}^{i-1}\right| / v_{c}^{i}<1 \times 10^{-4}$. If the criterion is not satisfied, back to step (2) with

$$
v_{c}^{i+1}=v_{c}^{i}+\Delta v_{c}^{i}
$$

where

$$
\Delta v_{c}^{i}=\delta v_{c}^{i} \frac{C_{a t v_{c}^{i}}}{\left[C_{a t} v_{c}^{i}-C_{a t} v_{c}^{i}+\delta v_{c}^{i}\right]}
$$

where $C$ is defined by equation (55) and $\delta v_{c}^{i}$ is set to be $1 \times 10^{-5} v_{c}^{i}$ in this study.

(6) Calculate $P_{c}$ using an equation of state.

In equations (55) and (56), the second and the third derivatives of the Helmholtz free energy with respect to the mole number $n_{i}$ can be written in terms of fugacity $f$ and determined using an equation of state.

$$
\begin{gathered}
\left(\frac{\partial^{2} A}{\partial n_{i} \partial n_{j}}\right)_{T, V}=R T n_{T}\left(\frac{\partial \ln f_{i}}{\partial n_{j}}\right)_{T, V} \\
\left(\frac{\partial^{3} A}{\partial n_{i} \partial n_{j} \partial n_{k}}\right)_{T, V}=R T n_{T}^{2}\left(\frac{\partial^{2} \ln f_{i}}{\partial n_{j} \partial n_{k}}\right)_{T, V}
\end{gathered}
$$


The resulting forms of $\ln f_{i},\left(\partial \ln f_{i} / \partial n_{j}\right)_{T, V}$ and $\left(\partial^{2} \ln f_{i} / \partial n_{j} \partial n_{k}\right)_{T, V}$ obtained from the RKS, PR, ALS and ESD equations of state are presented as follows.

\section{The Fugacity and Its First and Second Derivatives} with respect to Mole Number

Fugacity of component $i, f_{i}$, in a mixture can be given in terms of independent variables $T$ and $V$ using a thermodynamic relation (Prausnitz, et al., 1986).

$$
\ln f_{i}=\frac{1}{R T} \int_{v}^{\infty}\left[\left(\frac{\partial P}{\partial n_{i}}\right)_{T, V}-\frac{R T}{V}\right] \mathrm{d} V+\ln \frac{R T n_{i}}{V}
$$

where the partial derivative of pressure $P$ with respect to mole number $n_{i}$ at constant temperature, $T$, and constant volume, $V$, can be determined using an equation of state. (1) Using the RKS, PR and ALS Equations of State

A general form of the RKS, PR and ALS equations of state has been given by equation (1). This general form can also be written as

$$
P=\frac{R T}{v-b_{1}}-\frac{a}{\left(v+\lambda_{1} d_{2}\right)\left(v+\lambda_{2} b_{3}\right)}
$$

where $\lambda_{1}$ and $\lambda_{2}$ are constants given by each equation of state, $a$ is a mixture interaction parameter, and $b_{1}, b_{2}$ and $b_{3}$ are volumetric parameters for a mixture. These parameters can be calculated using the mixing rules given by equations (46) and (48), respectively. The values of the constants $\lambda_{1}$ and $\lambda_{2}$ and the volumetric parameters $b_{1}, b_{2}$ and $b_{3}$ for the RKS, PR and ALS equations of state are given as:

for the RKS equation, $b_{1}=b_{2}=b_{3}=b_{(\mathrm{RKS})}, \lambda_{1}=1$ and $\lambda_{2}=0$;

for the PR equation, $b_{1}=b_{2}=b_{3}=b_{(\mathrm{PR})}, \lambda_{1}=1+\sqrt{2}$ and $\lambda_{2}=1-\sqrt{2}$;

for the ALS equation, $b_{1}=b_{1(\mathrm{ALS})}, b_{2}=b_{2(\mathrm{ALS})}, b_{3}=b_{3(\mathrm{ALS})}, \lambda_{1}=-1$ and $\lambda_{2}=1$. Using equations (D-10) and (D-11), a fugacity of component $i$ can be written as

$$
\ln f_{i}=\ln \frac{x_{i} R T}{v-b_{1}}+\beta_{1, i}+\frac{a}{R T B}\left(\beta_{2, i}-\beta_{3, i}+\alpha_{i} D-B_{i} D\right)
$$


with

$$
\begin{gathered}
B=\lambda_{2} b_{3}-\lambda_{1} b_{2} \\
B_{i}=\frac{\left(\lambda_{2} b_{3, i}-\lambda_{1} b_{2, i}\right)}{B} \\
D=\ln \frac{v+\lambda_{1} b_{2}}{v+\lambda_{2} b_{3}} \\
\alpha_{i}=\frac{2}{a} \sum_{j} x_{j} a_{i j} \\
\beta_{1, i}=\frac{b_{1, i}}{v-b_{1}} \\
\beta_{2, i}=\frac{\lambda_{1} b_{2, i}}{v+\lambda_{1} b_{2}}
\end{gathered}
$$

and

$$
\beta_{3, i}=\frac{\lambda_{2} b_{3, i}}{v+\lambda_{2} b_{3}}
$$

where $x_{i}$ is mole fraction of component $i, v$ is molar volume of mixture, and cross parameter $a_{i j}$ in equation (D-15) is given by equation (47). In the above equations, the parameters with subscript $i$ are the parameters for pure component $i$.

From equation (D-12), the first and the second derivatives of $\ln f_{i}$ with respect to mole number can then be derived. The resulting equations are

$$
\begin{aligned}
n_{T}\left(\frac{\partial \ln f_{i}}{\partial n_{j}}\right)_{T, V}= & \frac{\Delta_{i j}}{x_{i}}+\beta_{1, i}+\beta_{1, j}+\beta_{1, i} \beta 1, j+\frac{a}{R T B}\left[\left(\alpha_{i}-B_{i}\right) \gamma_{j}+\left(\alpha_{j}-B_{j}\right) \gamma_{i}-\right. \\
& \left.-\Gamma_{i j}-\left(\alpha_{i} B_{j}+\alpha_{j} B_{i}-2 B_{i} B_{j}-\frac{2 a_{i j}}{a}\right) D\right]
\end{aligned}
$$

and

$$
\begin{aligned}
n_{T}^{2}\left(\frac{\partial^{2} \ln f_{i}}{\partial n_{j} \partial n_{k}}\right)_{T, V}= & -\frac{\Delta_{i j k}}{x_{i}^{2}}+\beta_{1, i} \beta_{1, j}+\beta_{1, i} \beta_{1, k}+\beta_{1, j} \beta_{1, k}+2 \beta_{1, i} \beta_{1, j} \beta_{1, k}+ \\
& +\frac{a}{R T B}\left\{2\left[\left(\gamma_{i}-B_{i} D\right) a_{j k}\left(\gamma_{j}-B_{j} D\right) a_{i k}+\left(\gamma_{k}-B_{k} D\right) a_{i j}\right] / a+\right. \\
& +2\left[\left(\gamma_{i}+\alpha_{i} D\right) B_{j} B_{k}+\left(\gamma_{j}+\alpha_{j} D\right) B_{i} B_{k}+\left(\gamma_{k}+\alpha_{k} D\right) B_{i} B_{j}\right]- \\
& -\left[\alpha_{i}\left(B_{j} \gamma_{k}+B_{k} \gamma_{j}\right)+\alpha_{j}\left(B_{i} \gamma_{k}+B_{k} \gamma_{i}\right)+\alpha_{k}\left(B_{i} \gamma_{j}+B_{j} \gamma_{i}\right)\right]+ \\
& +\left[\left(B_{i}-\alpha_{i}\right) \Gamma_{j k}+\left(B_{j}-\alpha_{j}\right) \Gamma_{i k}+\left(B_{k}-\alpha_{k}\right) \Gamma_{i j}\right)+ \\
& \left.+2\left(\beta_{2, i} \beta_{2, j} \beta_{2, k}-\beta_{3, i} \beta_{3, j} \beta_{3, k}\right)-6 B_{i} B_{j} B_{k} D\right\}
\end{aligned}
$$


where $n_{T}$ is total number of moles in system, $\gamma_{i}, \Gamma_{i j}, \Delta_{i j}$ and $\Delta_{i j k}$ are given by

$$
\begin{gathered}
\gamma_{i}=\beta_{2, i}-\text { beta }_{3, i} \\
\Gamma_{i j}=\beta_{2, i} \beta_{2, j}-\beta_{3, i} \beta_{3, j} \\
\Delta_{i j}= \begin{cases}1 & i=j \\
0 & \text { otherwise }\end{cases}
\end{gathered}
$$

and

$$
\Delta_{i j k}= \begin{cases}1 & i=j=k \\ 0 & \text { otherwise }\end{cases}
$$

(2) Using the ESD equation of state, $\ln f_{i},\left(\partial \ln f_{i} / \partial n_{j}\right)_{T, V}$ and $\left(\partial^{2} \ln f_{i} / \partial n_{j} \partial n_{k}\right)_{T, V}$ can be obtained as follows

$$
\begin{aligned}
\ln f_{i}= & \frac{9.49}{1.7745}\left(\frac{q_{i} C-C_{i}^{\prime} Q}{F}+q_{i} \ln \frac{v Q}{F}\right)+ \\
& +\frac{4 A}{B}\left[\frac{B_{i}^{\prime}}{E}+\left(\frac{A_{i}^{\prime}}{A}-\frac{B_{i}^{\prime}}{B}\right) \ln \frac{v}{E}\right]+\ln \frac{x_{i} R T}{v} \quad(D-25) \\
n_{T}\left(\frac{\partial \ln f_{i}}{\partial n_{j}}\right)_{T, V}= & \frac{\Delta_{i j}}{x_{i}}+\frac{9.49}{1.7745}\left[\frac{C_{i}^{\prime} C_{j}^{\prime \prime} Q-C\left(Q_{i j} v+C_{i}^{\prime} q_{j}+C_{j}^{\prime} q_{i}\right)}{F^{2}}-\frac{C_{i j}^{\prime \prime} Q+Q_{i j} v}{F}+\right. \\
& \left.+\frac{Q_{i j}}{Q}\right]+\frac{4 A}{B E}\left(\frac{A_{i}^{\prime} B_{j}^{\prime}+A_{j}^{\prime} B_{i}^{\prime}}{A}-\frac{2 B_{i j} v}{B E}+\frac{3 B_{i j}}{E}\right)+ \\
& +\frac{4}{B}\left(A_{i j}^{\prime \prime}-\frac{A_{i}^{\prime} B_{j}^{\prime}+A_{j}^{\prime} B_{i}^{\prime}}{B}+\frac{2 B_{i j} A}{B^{2}}\right) \ln \frac{v}{E} \quad(D-26)
\end{aligned}
$$

and

$$
\begin{aligned}
n_{T}^{2}\left(\frac{\partial^{2} \ln f_{i}}{\partial n_{j} \partial n_{k}}\right)_{T, V}= & -\frac{\Delta_{i j k}}{x_{i}^{2}}+\frac{9.49}{1.7745}\left[\frac{Q W_{1}-C W_{2}+Q_{i j k} v^{2}}{F^{2}}+\right. \\
& \left.+\frac{2 v C\left(W_{3}+Q_{i j k} v\right)+W_{4}(C-v Q)-2 C_{i j k}}{F^{3}}-\frac{Q_{i j k}}{Q^{2}}\right]+ \\
& +\frac{4}{B E}\left[W_{5}+\frac{W_{6}}{B E}(2 B-E-v)+\frac{A B_{i j k}}{B E}\left(\frac{2 E}{B}+\frac{2 v}{B}+\frac{2 v^{2}}{B E}-9 .\right)\right]- \\
& -\frac{4}{B^{2}}\left[W_{5}-\frac{2 W_{6}}{B}+\frac{6 A B_{i j k}}{B^{2}}\right] \ln \frac{v}{E} \quad(D-27)
\end{aligned}
$$


where $v$ is molar volume of mixture, $x_{i}$ is mole fraction of component $i, \Delta_{i j}$ and $\Delta_{i j k}$ are given by equations (D-23) and (D-24), respectively. The other parameters in the above equations are given as

$$
\begin{aligned}
& A=\left\langle c v^{*}\right\rangle \\
& A_{i}^{\prime}=\sum_{j} x_{j}\left(c_{i} v_{j}^{*}+c_{j} v_{i}^{*}\right) \\
& A_{i j}^{\prime \prime}=c_{i} v_{j}^{*}+c_{j} v_{i}^{*} \\
& B=1.9\left\langle v^{*}\right\rangle \\
& B_{i}^{\prime}=1.9 v^{*} \\
& B_{i j}=B_{i}^{\prime} B_{j}^{\prime} \\
& B_{i j k}=B_{i}^{\prime} B_{j}^{\prime} B_{k}^{\prime} \\
& C=1.7745\left\langle q v^{*} Y\right\rangle \\
& C_{i}^{\prime}=1.7745 \sum_{j} x_{j} Y_{i j}\left(q_{i} v_{j}^{*}+q_{j} v_{i}^{*}\right) \\
& C_{i j}^{\prime \prime}=1.7745 Y_{i j}\left(q_{i} v_{j}^{*}+q_{j} v_{i}^{*}\right) \\
& C_{i j k}=C_{i}^{\prime} C_{j}^{\prime} C_{k}^{\prime} \\
& E=v-B \\
& F=v Q+C \\
& Q=\sum_{i} x_{i} q_{i} \\
& Q_{i j}=q_{i} q_{j} \\
& Q_{i j k}=q_{i} q_{i} q_{k} \\
& W_{1}=C_{i}^{\prime} C_{j k}^{\prime \prime}+C_{j}^{\prime} C_{i k}^{\prime \prime}+C_{k}^{\prime} C_{i j}^{\prime \prime} \\
& W_{2}=q_{i} C_{j k}^{\prime \prime}+q_{j} C_{i k}^{\prime \prime}+q_{k} C_{i j}^{\prime \prime}
\end{aligned}
$$




$$
\begin{array}{rlr}
W_{3} & =C_{i}^{\prime} Q_{j k}+C_{j}^{\prime} Q_{i k}+C_{k}^{\prime} Q_{i j} & \\
W_{4}=q_{i} C_{j}^{\prime} C_{k}^{\prime}+q_{j} C_{i}^{\prime} C_{k}^{\prime}+q_{k} C_{i}^{\prime} C_{j}^{\prime} & (D-46) \\
W_{5}=B_{i}^{\prime} A_{j k}^{\prime \prime}+B_{j}^{\prime} A_{i k}^{\prime \prime}+B_{k}^{\prime} A_{i j}^{\prime \prime} & (D-47) \\
W_{0}=A_{i}^{\prime} B_{j k}+A_{j}^{\prime} B_{i k}+A_{k}^{\prime} B_{i j} & (D-49)
\end{array}
$$

where $v_{i}^{*}, c_{i}$ and $q_{i}$ are the ESD parameters for purc component $i$. The mixture parameters, $Y_{i j},\left\langle c v^{*}\right\rangle,\left\langle v^{*}\right\rangle$ and $\left\langle q v^{*} Y\right\rangle$, are given in Appendix C. 


\section{APPENDIX E Test Calculation Results}

This Appendix contains listings of the results of the test calculations for all of the mixtures and responses studied. The sets are organized by EOS into liquid density (ascending alkane carbon number) and critical locus groups. A complete listing of the test plan in the actual value of the factors is included before the liquid density results for the $\mathrm{n}-\mathrm{C} 4$ / CO2 mixture in each EOS set. 
LIQUID DENSITY $n-C 4$ / $\mathrm{CO} 2$ ALS DRES $=4$ SFACT $=0.5 \%$ 
LIQUID DENSITY $n-C 4 / C O 2$ ALS DRES $=4 \quad S F A C T=0.5 \%$

TABLE 2.TEST PATTERN IN ACTUAL FACTORS

1 OF 10

\begin{tabular}{|c|c|c|c|c|c|}
\hline TEST & & & & $\mathrm{C}$ & \\
\hline 1 & $.8974 \mathrm{E}-01$ & $-.3452 \mathrm{E}-01$ & $0.3300 \mathrm{E}-02$ & $0.3686 \mathrm{E}-01$ & $0.4050 \mathrm{E}-02$ \\
\hline 2 & $0.9019 \mathrm{E}-01$ & $3452 E-01$ & $0.3300 \mathrm{E}-02$ & $0.3686 \mathrm{E}-01$ & $0.4050 E-02$ \\
\hline 3 & $0.8974 \mathrm{E}-01$ & $-.3469 \mathrm{E}-01$ & $0.3300 \mathrm{E}-02$ & $0.3686 \mathrm{E}-01$ & $0.4050 E-02$ \\
\hline 4 & $0.9019 \mathrm{E}-01$ & $-.3469 \mathrm{E}-01$ & $0.3300 \mathrm{E}-02$ & $0.3686 \mathrm{E}-01$ & $0.4050 E-02$ \\
\hline 5 & $0.8974 E-01$ & $-.3452 \mathrm{E}-01$ & $0.3316 E-02$ & $0.3686 \mathrm{E}-01$ & $0.4050 \mathrm{E}-02$ \\
\hline 6 & $0.9019 E-01$ & $-.3452 \mathrm{E}-01$ & $0.3316 \mathrm{E}-02$ & $0.3686 \mathrm{E}-01$ & $0.4050 E-02$ \\
\hline 7 & $0.8974 \mathrm{E}-01$ & $-.3469 E-01$ & $0.3316 \mathrm{E}-02$ & $0.3686 \mathrm{E}-01$ & $0.4050 E-02$ \\
\hline 8 & $0.9019 \mathrm{E}-01$ & $-.3469 E-01$ & $0.3316 \mathrm{E}-02$ & $0.3686 \mathrm{E}-01$ & $0.4050 E-02$ \\
\hline 9 & $0.8974 \mathrm{E}-01$ & $-.3452 \mathrm{E}-01$ & $0.3300 \mathrm{E}-02$ & $0.3704 E-01$ & $0.4050 E-02$ \\
\hline 10 & $0.9019 \mathrm{E}-01$ & $-.3452 \mathrm{E}-01$ & $0.3300 E-02$ & $0.3704 \mathrm{E}-01$ & $0.4050 E-02$ \\
\hline 11 & $0.8974 \mathrm{E}-01$ & $-.3469 \mathrm{E}-01$ & $0.3300 E-02$ & $0.3704 \mathrm{E}-01$ & $0.4050 E-02$ \\
\hline 12 & $0.9019 \mathrm{E}-01$ & $-.3469 E-01$ & $0.3300 E-02$ & $0.3704 \mathrm{E}-01$ & $0.4050 \mathrm{E}-02$ \\
\hline 13 & $0.8974 E-01$ & $-.3452 E-01$ & $0.3316 \mathrm{E}-02$ & $0.3704 \mathrm{E}-01$ & $0.4050 \mathrm{E}-02$ \\
\hline 14 & $0.9019 E-01$. & $-.3452 \mathrm{E}-01$ & $0.3316 \mathrm{E}-02$ & $0.3704 E-01$ & $0.4050 \mathrm{E}-02$ \\
\hline 15 & $0.8974 E-01$ & $-.3469 E-01$ & $0.331 .6 \mathrm{E}-02$ & $0.3704 \mathrm{E}-01$ & $0.4050 E-02$ \\
\hline 16 & $0.9019 E-01$ & $-.3469 \mathrm{E}-01$ & $0.3316 \mathrm{E}-02$ & $0.3704 \mathrm{E}-01$ & $0.4050 \mathrm{E}-02$ \\
\hline 17 & $0.8974 \mathrm{E}-01$ & $-.3452 \mathrm{E}-01$ & $0.3300 \mathrm{E}-02$ & $0.3686 \mathrm{E}-01$ & $0.4070 E-02$ \\
\hline 18 & $0.9019 \mathrm{E}-01$ & $-.3452 \mathrm{E}-01$ & $0.3300 \mathrm{E}-02$ & $0.3686 E-01$ & $0.4070 E-02$ \\
\hline 19 & $0.8974 E-01$ & $-.3469 \mathrm{E}-01$ & $0.3300 \mathrm{E}-02$ & $0.3686 E-01$ & $0.4070 \mathrm{E}-02$ \\
\hline 20 & $0.9019 \mathrm{E}-01$ & $-.3469 \mathrm{E}-01$ & $0.3300 \mathrm{E}-02$ & $0.3686 \mathrm{E}-0 \mathrm{I}$ & $0.4070 \mathrm{E}-02$ \\
\hline 21 & $0.8974 \mathrm{E}-01$ & $-.3452 \mathrm{E}-01$ & $0.3316 E-02$ & $0.3686 \mathrm{E}-01$ & $0.4070 \mathrm{E}-02$ \\
\hline 22 & $0.9019 \mathrm{E}-01$ & $-.3452 \mathrm{E}-01$ & $0.3316 E-02$ & $0.3686 \mathrm{E}-01$ & $0.4070 \mathrm{E}-02$ \\
\hline 23 & $0.8974 \mathrm{E}-01$ & $-.3469 \mathrm{E}-01$ & $0.3316 E-02$ & $0.3686 \mathrm{E}-01$ & $0.4070 E-02$ \\
\hline 24 & $0.9019 E-01$ & $-.3469 \mathrm{E}-01$ & $0.3316 \mathrm{E}-02$ & $0.3686 \mathrm{E}-01$ & $0.4070 E-02$ \\
\hline 25 & $0.8974 \mathrm{E}-01$ & $-.3452 \mathrm{E}-01$ & $0.3300 \mathrm{E}-02$ & $0.3704 \mathrm{E}-01$ & $0.4070 \mathrm{E}-02$ \\
\hline 26 & $0.9019 E-01$ & $-.3452 \mathrm{E}-01$ & $0.3300 E-02$ & $0.3704 \mathrm{E}-01$ & $0.4070 \mathrm{E}-02$ \\
\hline 27 & $0.8974 \mathrm{E}-01$ & $-.3469 \mathrm{E}-01$ & $0.3300 \mathrm{E}-02$ & $0.3704 \mathrm{E}-01$ & $0.4070 \mathrm{E}-02$ \\
\hline 28 & $0.9019 E-01$ & $-.3469 E-01$ & $0.3300 \mathrm{E}-02$ & $0.3704 \mathrm{E}-01$ & $0.4070 \mathrm{E}-02$ \\
\hline 29 & $0.8974 \mathrm{E}-01$ & $-.3452 \mathrm{E}-01$ & $0.3316 \mathrm{E}-02$ & $0.3704 \mathrm{E}-01$ & $0.4070 \mathrm{E}-02$ \\
\hline 30 & $0.9019 \mathrm{E}-01$ & $-.3452 \mathrm{E}-01$ & $0.3316 E-02$ & $0.3704 \mathrm{E}-01$ & $0.4070 \mathrm{E}-02$ \\
\hline 31 & $0.8974 \mathrm{E}-01$ & $-.3469 \mathrm{E}-01$ & $0.3316 \mathrm{E}-02$ & $0.3704 \mathrm{E}-01$ & $0.4070 \mathrm{E}-0$ \\
\hline 32 & $0.9019 \mathrm{E}-01$ & $-.3469 \mathrm{E}-01$ & $0.3316 \mathrm{E}-02$ & $0.3704 \mathrm{E}-01$ & $0.4070 \mathrm{E}-0$ \\
\hline
\end{tabular}


LIQUID DENSITY $n-C 4 / C O 2$ ALS DRES $=4$ SFACT $=0.5 \%$

TABLE 2. (CONTINUED)

2 OF 10

\begin{tabular}{|c|c|c|c|c|c|}
\hline 'IES'I & 1 & 2 & & & \\
\hline 33 & $974 E-01$ & $3452 \mathrm{E}-0$ & $0.3300 \mathrm{E}-02$ & $0.3686 \mathrm{E}-$ & $0.4050 E-02$ \\
\hline 34 & $19 E-01$ & $3452 \mathrm{E}-01$ & $300 E-02$ & $0.3686 \mathrm{E}-\mathrm{C}$ & $050 E-02$ \\
\hline 5 & $74 \mathrm{E}-01$ & $.3469 E-01$ & $3300 E-02$ & $0.3686 \mathrm{E}-01$ & 0 \\
\hline 6 & $19 E-01$ & $.3469 \mathrm{E}-01$ & $3300 \mathrm{E}-02$ & $0.3686 \mathrm{E}-01$ & $c-c+3$ \\
\hline 7 & $974 \mathrm{E}-01$ & $.3452 \mathrm{E}-01$ & $0.3316 \mathrm{E}-02$ & $0.3686 E-01$ & O5OE- \\
\hline 8 & $019 \mathrm{E}-01$ & $.3452 \mathrm{E}-01$ & $0.3316 \mathrm{E}-02$ & $0.3686 \mathrm{E}-01$ & O50E-02 \\
\hline 9 & $974 E-01$ & $.3469 E-01$ & $0.3316 E-02$ & $0.3686 \mathrm{E}-01$ & $0.4050 \mathrm{E}-02$ \\
\hline 0 & $019 E-01$ & $.3469 E-01$ & $0.3316 \mathrm{E}-02$ & $0.3686 \mathrm{E}-01$ & $0.4050 E-02$ \\
\hline 1 & $974 E-\cup 1$ & $.3452 E-01$ & $0.3300 \mathrm{E}-02$ & $0.3704 \mathrm{E}-01$ & $0.4050 E-02$ \\
\hline 2 & $019 E-01$ & $-.3452 \mathrm{E}-01$ & $0.3300 \mathrm{E}-02$ & $0.3704 \mathrm{E}-01$ & $0.4050 E-02$ \\
\hline 3 & $974 \mathrm{E}-01$ & $-.3469 E-01$ & $0.330 \cap E-02$ & $0.3704 \mathrm{E}-01$ & $0.4050 \mathrm{E}-02$ \\
\hline 4 & $0.9019 \mathrm{E}-01$ & $-.3469 E-01$ & $0.3300 \mathrm{E}-02$ & $0.3704 \mathrm{E}-01$ & $0.4050 E-02$ \\
\hline 45 & $974 \mathrm{E}-01$ & $-.3452 \mathrm{E}$ & $0.3 .316 \mathrm{E}-02$ & $0.3704 \mathrm{E}-01$ & $0.4050 \mathrm{E}-02$ \\
\hline 46 & $019 E-01$ & $-.3452 \mathrm{E}$ & $0.3316 \mathrm{E}-02$ & $0.3704 \mathrm{E}-01$ & $0.4050 E-02$ \\
\hline$\$ 7$ & $774 \mathrm{E}-01$ & $-.3469 \mathrm{E}$ & $0.3316 \mathrm{~F}-02$ & $0.3704 \mathrm{E}-01$ & $0.4050 E-02$ \\
\hline 48 & $019 E-01$ & $-.3469 E$ & $0.3316 \mathrm{E}-02$ & $0.3704 \mathrm{E}-01$ & $0.4050 \mathrm{E}-02$ \\
\hline 49 & 14 & -.3452 & $0.3300 \mathrm{E}$ & $0.3686 \mathrm{E}$ & $70 E-02$ \\
\hline 50 & 19 & -.3452 & $0.3300 \mathrm{E}-02$ & $0.3686 \mathrm{E}$ & $0.4070 E-02$ \\
\hline 51 & 14 & -.346 & 3300 & $0.3686 \mathrm{E}$ & $0.4070 E-02$ \\
\hline 52 & 19 & -.346 & 0.3300 & $0.3686 \mathrm{E}$ & $0.4070 \mathrm{E}-02$ \\
\hline 53 & 74 & -.345 & 0.3316 & 0.3686 & $0.4070 \mathrm{E}-02$ \\
\hline 54 & 0 & -.345 & 331 & 0. & $070 E-02$ \\
\hline 55 & 74 & -.3 & 31 & 0. & $070 E-02$ \\
\hline 56 & 0 . & -. & 31 & 0 & $070 E-02$ \\
\hline 57 & 0 & - & 330 & 0.3704 & $0.4070 \mathrm{E}-02$ \\
\hline 58 & 0 & -.3 & 0.3300 & 0.3704 & $0.4070 \mathrm{E}-0 \%$ \\
\hline 59 & 74 & $-.3469 \mathrm{E}$ & $0.3300 \mathrm{E}-$ & $0.3704 \mathrm{E}$ & $0.4070 \mathrm{E}-02$ \\
\hline 60 & 0.9019 & $-.3469 \mathrm{E}$ & $0.3300 E-02$ & $0.3704 \mathrm{E}$ & $0.4070 \mathrm{E}-02$ \\
\hline 61 & $0.8974 \mathrm{E}-0$ & $-.3452 E-01$ & $0.3316 \mathrm{E}-02$ & $0.3704 \mathrm{E}$ & $0.4070 \mathrm{E}-02$ \\
\hline 62 & $0.9019 E-0$ & $-.3452 \mathrm{E}-01$ & $0.3316 \mathrm{E}-02$ & $0.3704 \mathrm{E}$ & $0.4070 \mathrm{E}-02$ \\
\hline 63 & $0.8974 \mathrm{E}-0$ & $.3469 E-01$ & $0.3315 \mathrm{E}-02$ & $0.3704 \mathrm{E}$ & $0.4070 \mathrm{E}-02$ \\
\hline 54 & $0.9019 E-01$ & $-.3469 \mathrm{E}-01$ & $0.3316 \mathrm{E}-02$ & 0.3704 & 0.407 \\
\hline
\end{tabular}


TABLE 2. (CONTINUED)

3 OF 10

\begin{tabular}{|c|c|c|c|c|c|}
\hline$T$ & 6 & C & C & $\mathrm{C}$ & \\
\hline 1 & $1073 E-01$ & $1570 \mathrm{E}-02$ & $1540 \mathrm{E}+00$ & $1412 \mathrm{E}+00$ & $20 F^{-1}$ \\
\hline 2 & $073 E-01$ & $578 E-02$ & $1548 E+00$ & $1419 E+00$ & -.2 \\
\hline 3 & $073 E-01$ & $78 E-02$ & $548 \mathrm{E}+00$ & $1419 E+00$ & $E-02$ \\
\hline 4 & $-.1073 E-01$ & $570 E-02$ & $0.1540 \mathrm{E}+00$ & $412 \mathrm{E}+00$ & $0 E-02$ \\
\hline 5 & $-.1073 E-01$ & $0.1578 \mathrm{E}-02$ & $0.1540 E+00$ & $0.1412 \mathrm{E}+00$ & $-.2720 \mathrm{E}-02$ \\
\hline 6 & $-.1073 \mathrm{E}-$ & $570 E-$ & $E+00$ & $419 E+00$ & $-.2734 \mathrm{E}-0$ \\
\hline 7 & $73 \mathrm{E}-$ & $70 E-$ & 0 & $419 \mathrm{E}+$ & -.27 \\
\hline 8 & $-.1073 \mathrm{E}-$ & $0.1578 \mathrm{E}-$ & 154 & $0.1412 \mathrm{E}+00$ & $-.2720 \mathrm{E}-0$ \\
\hline 9 & $.1073 \mathrm{E}-$ & $0.1570 \mathrm{E}-$ & 0 & $0.1412 E+00$ & $-.27 i$ \\
\hline 10 & $-.1073 \mathrm{E}-$ & $0.1578 \mathrm{E}-$ & 15 & $0.1419 \mathrm{E}-$ & -.27 \\
\hline 11 & $-.1073 E-01$ & $0.1578 \mathrm{E}-$ & 0.15 & $0.1419 \mathrm{E}+00$ & -.2 \\
\hline 12 & $-.1073 E-$ & $0.1570 E-$ & 0.15 & $0.1412 \mathrm{E}+$ & -.27 \\
\hline 13 & $-.1073 E-$ & $578 \mathrm{E}-$ & 0.19 & $0.1412 \mathrm{E}+00$ & -.27 \\
\hline 14 & $-.1073 \mathrm{E}-$ & $0.1570 \mathrm{E}-02$ & 00 & $0.1419 \mathrm{E}+00$ & $-.2734 \mathrm{E}-0$ \\
\hline 15 & $-.1073 \mathrm{E}-$ & $0.1570 \mathrm{E}-$ & 0.15 & $0.1419 E+00$ & $-.2734 E-0$ \\
\hline 16 & $-.1073 E-$ & $0.1578 \mathrm{E}-$ & 0.154 & $0.1412 \mathrm{E}+0 \mathrm{U}$ & $-.2720 \mathrm{E}-0$ \\
\hline 17 & $-.1073 E-01$ & $0.1570 \mathrm{E}-$ & 0.154 & $0.1419 \mathrm{E}+00$ & $-.2720 \mathrm{E}-0$ \\
\hline 18 & $-.1073 E-01$ & $578 \mathrm{E}-$ & 0 & $0.1412 \mathrm{E}+00$ & $-.2734 \mathrm{E}-0$ \\
\hline 19 & $-.1073 E-01$ & $0.1578 \mathrm{E}-02$ & 0.154 & $0.1412 \mathrm{E}+00$ & $-.2734 \mathrm{E}-0$ \\
\hline 20 & $-.1073 \mathrm{E}-01$ & $0.1570 E-02$ & 0.1540 & $0.1419 E+00$ & $-.2720 E-0$ \\
\hline 21 & $-.1073 E-01$ & $0.1578 \mathrm{E}-02$ & $0.1540 E+00$ & $0.1419 \mathrm{E}+00$ & $-.2720 E-0$ \\
\hline 22 & $-.1073 E-01$ & $0.1570 \mathrm{E}-02$ & $0.1548 E+00$ & $0.1412 \mathrm{E}+00$ & $-.2734 \mathrm{E}-\mathrm{O}$ \\
\hline 23 & $-.1073 \mathrm{E}-01$ & $0.1570 \mathrm{E}-02$ & $0.1548 \mathrm{E}+00$ & $0.1412 \mathrm{E}+00$ & $-.2734 \mathrm{E}-0$ \\
\hline 24 & $-.1073 E-01$ & $0.1578 \mathrm{E}-02$ & $0.1540 E+00$ & $0.1419 E+00$ & $-.2720 \mathrm{E}-0$ \\
\hline 25 & $-.1073 E-C$ & $0.1570 \mathrm{E}-02$ & $0.1548 \mathrm{E}+00$ & $0.1419 \mathrm{E}+00$ & $-.2720 \mathrm{E}-\mathrm{C}$ \\
\hline 26 & $-.1073 E-C$ & C. $1578 \mathrm{E}-02$ & 0.1540 & $0.1412 \mathrm{E}+00$ & $-.2734 E-C$ \\
\hline 27 & $-.1073 E-$ & $0.1578 \mathrm{E}-02$ & 0.1540 & $0.1412 \mathrm{E}+00$ & $-.2734 \mathrm{E}-\mathrm{C}$ \\
\hline 28 & $-.1073 \mathrm{E}-$ & $0.1570 \mathrm{E}-02$ & $0.1548 \mathrm{E}+00$ & $0.1419 E+00$ & $-.2720 \mathrm{E}-0$ \\
\hline 29 & $-.1073 E-$ & $0.1578 \mathrm{E}-02$ & $0.1548 E+00$ & $0.1419 E+00$ & $-.2720 \mathrm{E}-\mathrm{C}$ \\
\hline 30 & $-.1073 \mathrm{E}-$ & $0.1570 \mathrm{E}-02$ & 0.1540 & $0.1412 \mathrm{E}+00$ & $-.2734 E-C$ \\
\hline 31 & $-.1073 E-$ & $70 E-02$ & 0.1540 & $0.1412 E+00$ & $-.2734 \mathrm{E}-\mathrm{C}$ \\
\hline 32 & $-.1073 E-01$ & $0.1578 \mathrm{E}-02$ & $0.1548 E+00$ & $0.1419 \mathrm{E}+00$ & $-.2720 \mathrm{E}-\mathrm{C}$ \\
\hline
\end{tabular}


TABLE 2. (CONTINUED)

4 OF 10

\begin{tabular}{|c|c|c|c|c|c|}
\hline TEST & C & C & C & & C 10 \\
\hline 33 & $-.1078 E-01$ & $0.1570 \mathrm{E}-02$ & $0.1540 \mathrm{E}+00$ & $0.1412 \mathrm{E}+00$ & $-.2734 \mathrm{E}-02$ \\
\hline 34 & $-.1078 \mathrm{E}-01$ & $0.1578 \mathrm{E}-02$ & $548 E+00$ & $419 E+00$ & $20 E-02$ \\
\hline 35 & $.1078 \mathrm{E}-01$ & $0.1578 \mathrm{E}-02$ & $1548 E+00$ & $1419 E+00$ & $-.2720 \mathrm{E}-02$ \\
\hline 36 & $-.1078 \mathrm{E}-01$ & $570 E-02$ & $540 E+00$ & $1412 \mathrm{E}+00$ & $-.2734 \mathrm{E}-02$ \\
\hline 37 & $-.1078 \mathrm{E}-01$ & $578 E-02$ & $40 E+$ & $1412 \mathrm{E}+00$ & $-.2734 \mathrm{E}-02$ \\
\hline 38 & $.1078 \mathrm{E}-01$ & $570 E-02$ & $48 E+00$ & $1419 E+00$ & $-.2720 \mathrm{E}-02$ \\
\hline 9 & $.1078 \mathrm{E}-01$ & $570 E-02$ & $48 E+00$ & $1419 E+00$ & $-.2720 \mathrm{E}-02$ \\
\hline 0 & $.1078 \mathrm{E}-01$ & $578 E-02$ & $1540 E+00$ & $0.1412 \mathrm{E}+00$ & -.273 \\
\hline 1 & $.1078 \mathrm{E}-01$ & $570 E-02$ & $548 \mathrm{E}+$ & $0.1412 \mathrm{E}+00$ & -.2734 \\
\hline 2 & $-.1078 E-01$ & $578 E-02$ & $1.540 \mathrm{E}+$ & $0.1419 \mathrm{E}+00$ & $-.2720 \mathrm{E}-02$ \\
\hline 3 & $-.1078 E-01$ & $0.1578 \mathrm{E}-02$ & $1540 \mathrm{Et}$ & $0.1419 \mathrm{E}+00$ & $-.2720 \mathrm{E}-02$ \\
\hline 44 & $-.1078 E-01$ & $0.1570 E-02$ & $0.1548 \mathrm{E}+$ & $0.1412 \mathrm{E}+00$ & $-.2734 \mathrm{E}-02$ \\
\hline 45 & $-.1078 \mathrm{E}-01$ & $0.1578 \mathrm{E}-02$ & $0.1548 \mathrm{E}+$ & $0.1412 \mathrm{E}+00$ & -.273 \\
\hline 46 & $-.1078 E-01$ & $0.1570 \mathrm{E}-02$ & $0.1540 \mathrm{E}+00$ & $0.1419 \mathrm{E}+00$ & -.272 \\
\hline 47 & $-.1078 \mathrm{E}-01$ & $0.1570 \mathrm{E}-02$ & $0.1540 \mathrm{E}+00$ & $0.141 .9 \mathrm{E}+00$ & $-.2720 \mathrm{E}-02$ \\
\hline 48 & $-.1078 \mathrm{E}-01$ & $0.1578 \mathrm{E}-02$ & $0.1548 \mathrm{E}+$ & $0.1412 \mathrm{E}+00$ & -.273 \\
\hline 49 & $-.1078 \mathrm{E}-01$ & $0.1570 \mathrm{E}-02$ & $0.1540 \mathrm{E}+$ & $0.1419 E+00$ & -.273 \\
\hline 50 & $-.1078 \mathrm{E}-01$ & $0.1578 \mathrm{E}-02$ & $0.1548 \mathrm{E}+$ & $0.1412 \mathrm{E}+00$ & -.272 \\
\hline 51 & $-.1078 \mathrm{E}-01$ & $0.1578 \mathrm{E}-02$ & $0.1548 \mathrm{E}+$ & $0.1412 E+00$ & -.272 \\
\hline 52 & $-.1078 \mathrm{E}-01$ & $0.1570 \mathrm{E}-02$ & $0.1540 \mathrm{Et}$ & $0.1419 E+00$ & -.273 \\
\hline 53 & $-.1078 \mathrm{E}-01$ & $0.1578 E-02$ & 0.1540 & $0.1419 \mathrm{E}+00$ & -.273 \\
\hline 54 & $.1078 \mathrm{E}-01$ & $0.1570 \mathrm{E}-02$ & 0.1548 & $0.1412 \mathrm{E}+00$ & $-.2720 \mathrm{E}-02$ \\
\hline 55 & $-.1078 \mathrm{E}-01$ & $0.1570 E-02$ & 0.1548 & $0.1412 \mathrm{E}+00$ & $-.2720 \mathrm{E}-02$ \\
\hline 56 & $.1078 E-01$ & $0.1578 \mathrm{E}$ & 0.1540 & $0.1419 E+00$ & $-.2734 \mathrm{E}-02$ \\
\hline 57 & $.1078 E-01$ & $0.1570 \mathrm{E}$ & 0.1548 & $0.1419 E+00$ & $-.2734 \mathrm{E}-02$ \\
\hline 58 & $.1078 E-01$ & $0.1578 \mathrm{E}-02$ & 0.1540 & $0.1412 \mathrm{E}+00$ & $-.2720 \mathrm{E}-02$ \\
\hline 59 & $-.1078 \mathrm{E}-01$ & $0.1578 \mathrm{E}$ & $0.1540 \mathrm{E}+$ & $0.1412 \mathrm{E}+00$ & $-.2720 \mathrm{E}-02$ \\
\hline 60 & $.1078 \mathrm{E}-$ & $0.1570 \mathrm{E}$ & $0.1548 \mathrm{E}+$ & $0.1419 \mathrm{E}+00$ & $-.2734 \mathrm{E}-02$ \\
\hline 61 & $-.1078 \mathrm{E}$ & $0.1578 \mathrm{E}-02$ & $0.1548 \mathrm{E}+00$ & $0.1419 \mathrm{E}+00$ & $-.2734 \mathrm{E}-02$ \\
\hline 62 & $-.1078 E-01$ & $0.1570 \mathrm{E}-02$ & $0.1540 \mathrm{E}+00$ & $0.1412 \mathrm{E}+00$ & $-.2720 \mathrm{E}-02$ \\
\hline 6.3 & $-.1078 E-01$ & $0.1570 \mathrm{E}-02$ & $0.1540 \mathrm{E}+00$ & $0.1412 \mathrm{E}+00$ & $-.2720 E-02$ \\
\hline 64 & $-.1078 E-01$ & $0.1578 \mathrm{E}-02$ & $0.1548 \mathrm{E}+00$ & $0.1419 E+00$ & $-.2734 \mathrm{E}-02$ \\
\hline
\end{tabular}


TABLE 2. (CONTINUED)

5 OF 10

\begin{tabular}{|c|c|c|c|c|c|}
\hline EST & 11 & 12 & 13 & 14 & \\
\hline 1 & $40 E-02$ & $4487 E+00$ & $4024 \mathrm{E}-01$ & $11 \mathrm{E}-01$ & $E-02$ \\
\hline 2 & $-.4864 \mathrm{E}-02$ & $509 E+00$ & $044 \mathrm{E}-01$ & $1117 \mathrm{E}-01$ & $9 E-02$ \\
\hline 3 & $0 E-02$ & $7 \mathrm{E}+00$ & $24 \mathrm{E}-$ & $1111 \mathrm{E}-01$ & $E-02$ \\
\hline 4 & E-02 & $509 E+00$ & $044 \mathrm{E}-$ & $1117 \mathrm{E}-$ & $9 E-02$ \\
\hline 5 & E-02 & $9 E+00$ & $44 \mathrm{E}$ & $1111 \mathrm{E}-$ & $-.5760 E-02$ \\
\hline 6 & $D E-02$ & $7 E+00$ & $4024 \mathrm{E}$ & $1117 \mathrm{E}$ & $9 E-02$ \\
\hline 7 & $E-02$ & $99 E+00$ & $4044 \mathrm{E}$ & $1111 \mathrm{E}$ & $-.5760 \mathrm{E}-02$ \\
\hline 8 & $-.4840 \mathrm{E}-02$ & $7 E+00$ & $0.4024 \mathrm{E}$ & $0.1117 \mathrm{E}$ & $-.5789 \mathrm{E}-02$ \\
\hline 9 & $4 \mathrm{E}-02$ & $7 E+00$ & $0.4024 \mathrm{E}$ & $0.1117 \mathrm{E}$ & $-.5789 \mathrm{E}-02$ \\
\hline 0 & $-.4840 \mathrm{E}-02$ & $99 E+00$ & $0.4044 \mathrm{E}-$ & $0.1111 \mathrm{E}$ & $-.5760 \mathrm{E}-02$ \\
\hline & $-.4864 \mathrm{E}-02$ & $7 E+00$ & $0.4024 \mathrm{E}$ & $0.1117 \mathrm{E}$ & $-.5789 E-02$ \\
\hline ? & $-.4840 \mathrm{E}-02$ & $0.4509 E+00$ & 1 & $0.111 .1 \mathrm{E}$ & $-.5760 \mathrm{E}-02$ \\
\hline ? & $-.4840 \mathrm{E}-02$ & $9 E+00$ & 0.4 & $0.1117 \mathrm{E}$ & $-.5789 E-02$ \\
\hline 14 & $-.4864 \mathrm{E}-02$ & $0.4487 \mathrm{E}+00$ & 0.4 & $0.1111 \mathrm{E}$ & $E-02$ \\
\hline$r$ & -.484 & $09 E+00$ & 0.4 & $0.1117 \mathrm{E}$ & $9 E-02$ \\
\hline 16 & -.486 & $7 E+00$ & 0.4 & 0.1 & $E-02$ \\
\hline 1 & $-.4840 \mathrm{E}-02$ & $99 E+00$ & 0.4 & $0.1117 \mathrm{E}$ & $-.5760 \mathrm{E}-02$ \\
\hline 8 & -.486 & $E+00$ & 0.4 & $0.1111 \mathrm{E}$ & $-.5789 \mathrm{E}-02$ \\
\hline 1 & $O E-02$ & $9 E+00$ & 0.4 & $0.1117 \mathrm{E}$ & $-.5760 \mathrm{E}-02$ \\
\hline & $-.4864 \mathrm{E}-02$ & $7 \mathrm{E}+00$ & $44 \mathrm{E}$ & $0.1111 \mathrm{E}$ & $-.5789 E-02$ \\
\hline 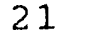 & $-.4864 \mathrm{E}-$ & $7 E+00$ & $0.4044 \mathrm{E}-$ & $0.1117 \mathrm{E}$ & $-.5760 \mathrm{E}-02$ \\
\hline 22 & $-.4840 \mathrm{E}-$ & $0.4509 E+00$ & $0.4024 \mathrm{E}-01$ & $0.1111 \mathrm{E}-$ & $-.5789 E-02$ \\
\hline & $-.4864 \mathrm{E}-$ & $0.4487 \mathrm{E}+00$ & $0.4044 \mathrm{E}-01$ & $0.1117 \mathrm{E}-01$ & $-.5760 \mathrm{E}-02$ \\
\hline & $-.484 \mathrm{OE}-02$ & $0.4509 \mathrm{E}+00$ & $0.4024 \mathrm{E}-01$ & $0.1111 \mathrm{E}-01$ & $-.5789 E-02$ \\
\hline 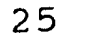 & $-.4864 \mathrm{E}-02$ & $0.4509 E+00$ & $0.4024 \mathrm{E}-01$ & $0.1111 \mathrm{E}-$ & $-.5789 E-02$ \\
\hline . & $-.4840 \mathrm{E}-02$ & $0.4487 \mathrm{E}+00$ & $0.4044 \mathrm{E}-01$ & $0.1117 \mathrm{E}-$ & $-.5760 \mathrm{E}-02$ \\
\hline 7 & $-.4864 \mathrm{E}-$ & $0.4509 \mathrm{E}+00$ & $0.4024 \mathrm{E}-01$ & $0.1111 \mathrm{E}-01$ & $-.5789 E-02$ \\
\hline 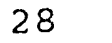 & $-.4840 \mathrm{E}-02$ & $0.4487 \mathrm{E}+00$ & $0.4044 \mathrm{E}-01$ & $0.1117 \mathrm{E}-$ & $-.5760 \mathrm{E}-02$ \\
\hline & $-.4840 \mathrm{E}-02$ & $0.4487 \mathrm{E}+00$ & $0.4044 \mathrm{E}-01$ & $0.1111 \mathrm{E}-01$ & $-.5789 E-02$ \\
\hline & $-.4864 \mathrm{E}-02$ & $0.4509 E+00$ & $0.4024 \mathrm{E}-01$ & $0.1117 \mathrm{E}$ & $-.5760 \mathrm{E}-02$ \\
\hline & $O E-$ & $87 \mathrm{E}+00$ & $0.4044 \mathrm{E}$ & $0.1111 \mathrm{E}$ & $39 E-02$ \\
\hline & $-.4864 \mathrm{E}-02$ & $0.4509 E+00$ & $0.4024 E-01$ & $0.1117 \mathrm{E}-01$ & $-.5760 E-0$ \\
\hline
\end{tabular}


TABLE 2. (CONTINUED)

6 OF 10

\begin{tabular}{|c|c|c|c|c|c|}
\hline TES & 1 & C 12 & 13 & 14 & \\
\hline 33 & $4840 \mathrm{E}-02$ & $.4487 \mathrm{E}+00$ & $4044 \mathrm{E}-01$ & $0.1111 E-01$ & $89 E-C$ \\
\hline 34 & $.4864 \mathrm{E}-02$ & $9 E+$ & $4024 \mathrm{E}-01$ & $1117 E-01$ & $O E-02$ \\
\hline 35 & $E-02$ & $7 \mathrm{E}+00$ & $44 E-01$ & $111 \mathrm{E}-$ & $E-02$ \\
\hline 36 & $\mathrm{E}-02$ & $09 E+00$ & $24 E-01$ & $117 E-01$ & $E-0$ \\
\hline 37 & $E-02$ & $9 \mathrm{E}+00$ & $024 \mathrm{E}-01$ & $111 \mathrm{E}-$ & $E-02$ \\
\hline 38 & $E-02$ & $E+00$ & $44 E-01$ & $1117 \mathrm{E}-$ & $J E-0 Z$ \\
\hline 39 & $4 \mathrm{E}-02$ & $09 E+00$ & $24 E-$ & $1111 \mathrm{E}-01$ & $9 E-02$ \\
\hline 40 & $\mathrm{DE}-02$ & $7 \mathrm{E}+00$ & $0.4044 E-01$ & $0.1117 \mathrm{E}-01$ & $E-0$ \\
\hline 1 & $E-02$ & $7 \mathrm{E}+00$ & $0.4044 \mathrm{E}-$ & $0.1117 \mathrm{E}-01$ & $E-0$ \\
\hline 42 & $E-02$ & $E+00$ & $24 E-$ & $0.1111 \mathrm{E}-$ & $E-0$ \\
\hline 3 & $E-02$ & $E+00$ & $44 E-$ & $0.1117 \mathrm{E}-$ & $E-0$ \\
\hline 44 & $a-02$ & $9 \mathrm{E}+00$ & $0.4024 \mathrm{E}-$ & $0.1111 \mathrm{E}-01$ & $9 \mathrm{E}-0$ \\
\hline 5 & $E-02$ & $\mathrm{E}+00$ & 0.4 & $0.1117 \mathrm{E}-01$ & $E-0$ \\
\hline 6 & $8-02$ & $E+00$ & 0.2 & $0.1111 \mathrm{E}-$ & $E-02$ \\
\hline 47 & -.48 & $E+00$ & 0.4 & $0.1117 \mathrm{E}-$ & $E-02$ \\
\hline 48 & -.48 & $E+00$ & 0.2 & $0.1111 \mathrm{E}$ & $E-02$ \\
\hline 49 & -.48 & $E+00$ & 0.5 & $\mathrm{E}-$ & $E-02$ \\
\hline 50 & -.48 & $E+00$ & 0.4 & 0.1 & $\mathrm{OE}-02$ \\
\hline 51 & -.48 & $E+00$ & 0. & $\mathrm{E}-$ & $3 E-02$ \\
\hline 52 & -.48 & $E+00$ & 0 . & $0.1:$ & E-02 \\
\hline 53 & -.486 & $\mathrm{E}+00$ & 0. & $0.1:$ & $9 E-02$ \\
\hline 54 & -.48 & $E+00$ & 0.4 & 0.11 & $O E-02$ \\
\hline 55 & -.48 & $E+00$ & 0.4 & $0.1117 \mathrm{E}-01$ & $-.5789 E-02$ \\
\hline 56 & $-.4840 \mathrm{E}-02$ & $9 \mathrm{E}+00$ & $0.4044 \mathrm{E}$ & $0.1111 \mathrm{E}-01$ & $-.5760 \mathrm{E}-02$ \\
\hline 57 & $-.4864 \mathrm{E}-$ & $0.4509 \mathrm{E}+00$ & $0.4044 \mathrm{E}-$ & $0.1111 \mathrm{E}-01$ & $-.5760 \mathrm{E}-02$ \\
\hline 58 & $-.4840 \mathrm{E}-02$ & $0.4487 \mathrm{E}+00$ & $0.4024 \mathrm{E}-01$ & $0.1117 \mathrm{E}-01$ & $-.5789 E-0$ \\
\hline 59 & $-.4864 \mathrm{E}-02$ & $0.4509 \mathrm{E}+00$ & $0.4044 \mathrm{E}-01$ & $0.1111 \mathrm{E}-01$ & $-.5760 \mathrm{E}-0$ \\
\hline 60 & $-.4840 \mathrm{E}-02$ & $0.4487 \mathrm{E}+00$ & $0.4024 \mathrm{E}-$ & $0.1117 \mathrm{E}-01$ & $-.5789 E-0$ \\
\hline 61 & $-.4840 \mathrm{E}-02$ & $0.4487 \mathrm{E}+00$ & $0.4024 \mathrm{E}-$ & $0.1111 \mathrm{E}-01$ & $-.5760 E-0$ \\
\hline 62 & $-.4864 \mathrm{E}-02$ & $0.4509 \mathrm{E}+00$ & $0.4044 \mathrm{E}-01$ & $0.1117 \mathrm{E}-01$ & $-.5789 E-0$ \\
\hline 67 & $-.4840 \mathrm{E}-$ & $0.4487 E+00$ & $0.4024 \mathrm{E}-$ & $0.1111 \mathrm{E}-$ & $-.5760 \mathrm{E}-0$ \\
\hline 64 & $-.4864 \mathrm{E}-02$ & $0.4509 E+00$ & $0.4044 \mathrm{E}-01$ & $0.1117 \mathrm{E}-01$ & $-.5789 E-0$ \\
\hline
\end{tabular}


TABLE 2. (CONTINUED)

7 OF 10

\begin{tabular}{cc} 
TEST & C 16 \\
1 & $0.4070 \mathrm{E}+00$ \\
2 & $0.4090 \mathrm{E}+00$ \\
3 & $0.4070 \mathrm{E}+00$ \\
4 & $0.4090 \mathrm{E}+00$ \\
5 & $0.4070 \mathrm{E}+00$ \\
6 & $0.4090 \mathrm{E}+00$ \\
7 & $0.4070 \mathrm{E}+00$ \\
8 & $0.4090 \mathrm{E}+00$ \\
9 & $0.4070 \mathrm{E}+00$ \\
10 & $0.4090 \mathrm{E}+00$ \\
11 & $0.4070 \mathrm{E}+00$ \\
12 & $0.4090 \mathrm{E}+00$ \\
13 & $0.4070 \mathrm{E}+00$ \\
14 & $0.4090 \mathrm{E}+00$ \\
15 & $0.4070 \mathrm{E}+00$ \\
16 & $0.4090 \mathrm{E}+00$ \\
17 & $0.4090 \mathrm{E}+00$ \\
13 & $0.4070 \mathrm{E}+00$ \\
19 & $0.4090 \mathrm{E}+00$ \\
20 & $0.4070 \mathrm{E}+00$ \\
21 & $0.4090 \mathrm{E}+00$ \\
22 & $0.4070 \mathrm{E}+00$ \\
23 & $0.4090 \mathrm{E}+00$ \\
24 & $0.4070 \mathrm{E}+00$ \\
25 & $0.4090 \mathrm{E}+00$ \\
26 & $0.4070 \mathrm{E}+00$ \\
27 & $0.4090 \mathrm{E}+00$ \\
28 & $0.4070 \mathrm{E}+00$ \\
29 & $0.4090 \mathrm{E}+00$ \\
30 & $0.4070 \mathrm{E}+00$ \\
31 & $0.4090 \mathrm{E}+00$ \\
32 & $0.4070 \mathrm{E}+00$ \\
& \\
\hline
\end{tabular}

\begin{tabular}{|c|c|}
\hline 17 & \\
\hline 0 & $2933 E+00$ \\
\hline & $E+00$ \\
\hline & $-.2948 E+00$ \\
\hline & $E+00$ \\
\hline & -.2 \\
\hline+01 & $-.2948 E+00$ \\
\hline+01 & $-.2933 E+00$ \\
\hline+01 & $E+00$ \\
\hline+01 & $-.2933 E+00$ \\
\hline 01 & $E+00$ \\
\hline+01 & $8 E+00$ \\
\hline+01 & $8 E+00$ \\
\hline$E+01$ & $3 E+00$ \\
\hline$E+01$ & $-.2948 \mathrm{E}+00$ \\
\hline $6 E+01$ & $-.2933 \mathrm{E}+00$ \\
\hline $36 E+01$ & $-.2933 E+00$ \\
\hline $379 E+01$ & $-.2948 E+00$ \\
\hline $1379 E+01$ & $-.2948 E+00$ \\
\hline $386 E+01$ & $-.2933 E+00$ \\
\hline $386 E+01$ & $-.2933 E+00$ \\
\hline $1386 \mathrm{E}+01$ & $-.2933 E+00$ \\
\hline $1386 E+01$ & $-.2933 E+00$ \\
\hline $1379 E+01$ & $-.2948 E+00$ \\
\hline $1379 E+01$ & $-.2948 E+00$ \\
\hline $1386 E+01$ & $-.2948 E+00$ \\
\hline $1386 E+01$ & $-.2948 E+00$ \\
\hline $1379 E+01$ & $3 E+00$ \\
\hline $9 \mathrm{E}+01$ & $3 E+00$ \\
\hline$E+01$ & $3 E+0$ \\
\hline $9 \mathrm{E}+0$ & $3 E+O C$ \\
\hline & $8 E+0$ \\
\hline$E+C$ & $8 \mathrm{E}+0$ \\
\hline
\end{tabular}

TC 1

$0.4252 E+03$

$0.4252 \mathrm{E}+03$

$0.4273 \mathrm{E}+03$

$0.4273 \mathrm{E}+03$

$0.4273 \mathrm{E}+03$

$0.4273 \mathrm{E}+03$

$0.4252 \mathrm{E}+03$

$0.4252 \mathrm{E}+03$

$0.4252 \mathrm{E}+03$

$0.4252 \mathrm{E}+03$

$0.4273 \mathrm{E}+03$

$0.4273 \mathrm{E}+03$

$0.4273 \mathrm{E}+03$

$0.4273 \mathrm{E}+03$

$0.4252 \mathrm{E}+03$

$0.4252 \mathrm{E}+03$

$0.4252 \mathrm{E}+03$

$0.4252 \mathrm{E}+03$

$0.4273 \mathrm{E}+03$

$0.4273 E+03$

$0.4273 \mathrm{E}+03$

$0.4273 \mathrm{E}+03$

$0.4252 \mathrm{E}+03$

$0.4252 \mathrm{E}+03$

$0.4252 \mathrm{E}+03$

$0.4252 \mathrm{E}+03$

$0.4273 \mathrm{E}+03$

$0.4273 E+03$

$0.4273 \mathrm{E}+03$

$0.4273 \mathrm{E}+03$

$0.4252 \mathrm{E}+03$

$0.4252 \mathrm{E}+03$
PC 1

$0.3797 E+02$

$0.3797 \mathrm{E}+02$

$0.3816 \mathrm{E}+02$

$0.3816 \mathrm{E}+02$

$0.3797 \mathrm{E}+02$

$0.3797 \mathrm{E}+02$

$0.3816 \mathrm{E}+02$

$0.3816 E+02$

$0.3816 \pi+02$

$0.3816 \mathrm{E}+02$

$0.3797 \mathrm{E}+02$

$0.3797 \mathrm{E}+02$

$0.3816 \mathrm{E}+02$

$0.3816 \mathrm{E}+02$

$0.3797 \mathrm{E}+02$

$0.3797 \mathrm{E}+02$

$0.3816 \mathrm{E}+02$

$0.3816 \mathrm{E}+02$

$0.3797 E+02$

$0.3797 \mathrm{E}+02$

$0.3816 \mathrm{E}+02$

$0.3816 \mathrm{E}+02$

$0.3797 \mathrm{E}+02$

$0.3797 \mathrm{E}+02$

$0.3797 \mathrm{E}+02$

$0.3797 \mathrm{E}+02$

$0.3816 \mathrm{E}+02$

$0.3816 \mathrm{E}+02$

$0.3797 \mathrm{E}+02$

$0.3797 \mathrm{E}+02$

$0.3816 \mathrm{E}+02$

$0.381 .6 \mathrm{E}+02$ 
LIQUID DENSITY $n-C 4 / \mathrm{CO} 2$ ALS DRES $=4 \quad \mathrm{SFACT}=0.5 \%$

TABLE 2. (CONTINUED)

8 OF 10

\begin{tabular}{|c|c|c|c|c|c|}
\hline EST & & & & 2 & 2 \\
\hline 3 & +00 & $379 E+01$ & $2933 E+00$ & $73 E+03$ & \\
\hline 4 & $D E+O O$ & $379 E+01$ & $2933 E+00$ & $73 E+03$ & $7 E+02$ \\
\hline 5 & $E+00$ & $386 E+01$ & $-.2948 \mathrm{E}+00$ & $52 \mathrm{E}+03$ & $16 \mathrm{E}+02$ \\
\hline 6 & $O E+00$ & $1386 E+01$ & $-.2948 E+00$ & $52 \mathrm{E}$ & +02 \\
\hline 7 & +00 & $0.1386 \mathrm{E}+01$ & $E+00$ & $52 E+03$ & $1+02$ \\
\hline 8 & +00 & $0.1386 \mathrm{E}+01$ & $E+00$ & $52 E+03$ & +02 \\
\hline 9 & +00 & $0.1379 \mathrm{E}+01$ & $-.2933 E+00$ & $73 E+03$ & +02 \\
\hline 0 & +00 & $0.1379 \mathrm{E}+01$ & $-.2933 E+00$ & $73 E+03$ & +02 \\
\hline 1 & +00 & 0.138 & $-.2933 E+00$ & $73 E+03$ & +02 \\
\hline 2 & +00 & 0.138 & $-.2933 E+00$ & $73 E+03$ & +02 \\
\hline 43 & +00 & 0.137 & $-.2948 E+00$ & $52 E+03$ & $7 E+02$ \\
\hline 4 & +00 & 0.137 & $-.2948 E+00$ & $52 \mathrm{E}+03$ & $97 E+02$ \\
\hline 15 & 00 & 0.137 & $-.2948 E+00$ & $52 \mathrm{E}+03$ & $16 E+02$ \\
\hline 6 & 00 & 0.137 & $-.2948 E+00$ & $52 \mathrm{E}$ & $16 E+02$ \\
\hline 47 & 00 & 0.138 & $-.2933 E+00$ & $73 \mathrm{E}$ & $97 \mathrm{E}+02$ \\
\hline 48 & 00 & 0.13 & $E+00$ & $73 E+03$ & $97 \mathrm{E}+02$ \\
\hline 49 & & 0.13 & -.29 & $73 E+03$ & $16 \mathrm{E}+02$ \\
\hline 50 & 0. & 0.137 & -.29 & $73 E+03$ & $16 E+02$ \\
\hline 51 & $0.2-3$. & 0.138 & -.29 & $52 \mathrm{E}+03$ & $97 E+02$ \\
\hline 52 & 0. & 0.138 & $3 E+00$ & $52 E+03$ & $97 E+02$ \\
\hline 53 & 0.4 & 0.138 & $3 E+00$ & $52 E+03$ & $16 E+02$ \\
\hline 54 & 0.4 & 0.13 & $3 E+00$ & $52 E+03$ & $316 E+02$ \\
\hline 55 & 0.4 & $0.13^{\circ}$ & $-.2948 E+00$ & $73 E+03$ & $0.3797 E+02$ \\
\hline 56 & 0.4 & 0.137 & $-.2948 \mathrm{E}+00$ & $0.4273 \mathrm{E}+03$ & $0.3797 \mathrm{E}+02$ \\
\hline 57 & $E+00$ & $0.1386 \mathrm{E}+01$ & $-.2948 E+00$ & $0,4273 \mathrm{E}+03$ & $0.3797 E+02$ \\
\hline 58 & $0 E+00$ & $0.1386 \mathrm{E}+01$ & $-.2948 E+00$ & $0.4273 \mathrm{E}+03$ & $0.3797 \mathrm{E}+02$ \\
\hline 59 & $0.4070 \mathrm{E}+00$ & $0.1379 E+01$ & $-.2933 E+00$ & $0.4252 \mathrm{E}+0.3$ & $0.3816 E+02$ \\
\hline 60 & $0.4090 E+00$ & $0.1379 E+01$ & $-.2933 E+00$ & $0.4252 \mathrm{E}+03$ & $0.3816 \mathrm{E}+02$ \\
\hline 61 & $0.4070 \mathrm{E}+00$ & $0.1379 E+01$ & $-.2933 \mathrm{E}+00$ & $0.4252 \mathrm{E}+0.3$ & $0.3797 E+02$ \\
\hline 62 & $0.4090 \mathrm{E}+00$ & $0.1379 E+01$ & $-.2933 E+00$ & $0.4252 \mathrm{E}+03$ & $0.3797 E+02$ \\
\hline 63 & $1070 F+00$ & $0.1386 \mathrm{E}+01$ & $-.2948 E+00$ & $0.4273 E+03$ & $0.3816 \mathrm{E}+02$ \\
\hline 64 & $0.4090 \mathrm{E}+00$ & $0.1386 \mathrm{E}+01$ & $-.2948 E+00$ & $0.4273 E+03$ & $0.3816 \mathrm{E}+0$ \\
\hline
\end{tabular}


TABLE 2. (CONTINUED)

9 OF 10

\begin{tabular}{|c|c|c|c|c|c|}
\hline IEST & OM & TC & PC & OM & 12 \\
\hline 1 & $93 E+00$ & $3042 \mathrm{E}+03$ & $382 \mathrm{E}+02$ & $2250 E+00$ & $.000 \mathrm{E}-\mathrm{C}$ \\
\hline 2 & $93 E+00$ & $42 E+03$ & $382 E+02$ & $0.2250 \mathrm{E}+00$ & $0.1000 \mathrm{E}-01$ \\
\hline 3 & $03 E+00$ & $57 E+03$ & $382 \mathrm{E}+02$ & $2250 E+00$ & . $1000 \mathrm{E}-01$ \\
\hline 4 & $003 E+00$ & $57 E+03$ & $82 E+02$ & $2250 E+00$ & $.1000 \mathrm{E}-01$ \\
\hline 5 & $1993 E+00$ & $42 E+03$ & $19 E+02$ & $0.2261 E+00$ & $.1005 \mathrm{E}-01$ \\
\hline 6 & $1993 E+00$ & $42 E+03$ & $19 E+02$ & $2261 E+00$ & $.1005 E-01$ \\
\hline 7 & $.2003 E+00$ & $57 E+03$ & $19 E+02$ & $2261 E+00$ & $.1005 \mathrm{E}-01$ \\
\hline 8 & $.2003 E+00$ & $57 E+03$ & $19 E+02$ & $0.2261 E+00$ & $9-01$ \\
\hline 9 & $.2003 E+00$ & $42 \mathrm{E}+03$ & $19 E+02$ & $0.2261 \mathrm{E}+00$ & -01 \\
\hline LO & $.2003 E+00$ & $42 E+03$ & $19 E+02$ & $0.2261 E+00$ & OE-01 \\
\hline 11 & $.1993 E+00$ & $57 E+03$ & $19 E+02$ & $2261 E+00$ & $.1000 \mathrm{E}-01$ \\
\hline 12 & $0.1993 E+00$ & $0.3057 \mathrm{E}+03$ & $419 E+02$ & $0.2261 E+00$ & $.1000 \mathrm{E}-01$ \\
\hline 13 & $0.2003 E+00$ & $0.3042 \mathrm{E}+03$ & $0.7382 \mathrm{E}+02$ & $0.2250 \mathrm{E}+00$ & $0.1005 \mathrm{E}-01$ \\
\hline 14 & $0.2003 E+00$ & $0.3042 \mathrm{E}+03$ & $0.7382 E+02$ & $0.2250 E+00$ & 0.1 \\
\hline 15 & $0.1993 E+00$ & $0.3057 E+03$ & $0.7382 \mathrm{E}+02$ & $0.2250 \mathrm{E}+00$ & 0.1 \\
\hline 16 & $0.1993 E+00$ & $0.3057 \mathrm{E}+03$ & $0.7382 \mathrm{E}+02$ & $0.2250 \mathrm{E}+00$ & 0.1 \\
\hline 17 & $0.1993 E+00$ & $57 E+03$ & $0.7419 E+02$ & $0.2250 \mathrm{E}+00$ & $0.1005 E-01$ \\
\hline 18 & $0.1993 E+00$ & $57 E+03$ & $0.7419 E+02$ & $0.2250 \mathrm{E}+00$ & 0.1 \\
\hline 19 & $0.2003 E+00$ & $42 E+03$ & $19 E+02$ & 0.2250 & $0.1005 \mathrm{E}-01$ \\
\hline 20 & $0.2003 E+00$ & $42 E+03$ & $19 E+02$ & $0.2250 E+00$ & 0.1 \\
\hline 2$]$ & $0.1993 E+00$ & $57 E+03$ & $82 E+02$ & $0.2261 \mathrm{E}+00$ & $0.1000 \mathrm{E}-01$ \\
\hline $2:$ & $0.1993 E+00$ & $0.3057 E+03$ & $382 E+02$ & $0.2261 \mathrm{E}+00$ & 0.10 \\
\hline $2:$ & $0.2003 E+00$ & $0.3042 E+03$ & $382 \mathrm{E}+02$ & $0.2261 \mathrm{E}+00$ & 0.10 \\
\hline 24 & $0.2003 E+00$ & $0.3042 E+03$ & $382 E+02$ & $0.2261 \mathrm{E}+00$ & $0.1000 \mathrm{E}-0$ \\
\hline 25 & $0.2003 E+00$ & $0.3057 E+03$ & $0.7382 E+02$ & $0.2261 \mathrm{E}+00$ & $0.1005 \mathrm{E}-0$ \\
\hline 26 & $0.2003 E+00$ & $0.3057 E+03$ & $0.7382 E+02$ & $0.2261 E+00$ & $0.1005 \mathrm{E}-0$ \\
\hline 27 & $0.1993 E+00$ & $0.3042 E+03$ & $0.7382 E+02$ & $0.2261 \mathrm{E}+00$ & $0.1005 E-0$ \\
\hline 28 & $0.1993 E+00$ & $0.3042 \mathrm{E}+03$ & $0.7382 \mathrm{E}+02$ & $0.2261 E+00$ & $0.1005 E-0$ \\
\hline 29 & $0.2003 E+00$ & $0.3057 \mathrm{E}+03$ & $0.7419 E+02$ & $0.2250 E+00$ & $0.1000 \mathrm{E}-01$ \\
\hline 30 & $0.2003 E+00$ & $0.3057 E+03$ & $0.7419 E+02$ & $0.2250 E+00$ & $0.1000 \mathrm{E}-01$ \\
\hline 3 & $0.1993 E+00$ & $0.3042 \mathrm{E}+03$ & $0.7419 E+02$ & $0.2250 \mathrm{E}+00$ & $0.1000 E-01$ \\
\hline 3 & $0.1993 E+00$ & $0.3042 E+03$ & $0.7419 E+02$ & $0.2250 E+00$ & $0.1000 E-0$ \\
\hline
\end{tabular}




\begin{tabular}{|c|c|c|c|c|c|}
\hline TEST & OM & TC & PC & OM & \\
\hline 33 & $2003 E+00$ & $057 E+03$ & 0. & $2261 E$ & 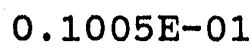 \\
\hline 34 & $03 E+00$ & $57 E+03$ & $82 E+02$ & $261 E+00$ & \\
\hline 35 & $3 E+00$ & $42 \mathrm{E}+03$ & $82 E+02$ & $2261 E+00$ & -01 \\
\hline 36 & $3 E+00$ & $42 \mathrm{E}+03$ & $82 \mathrm{E}+02$ & $2261 E+00$ & -01 \\
\hline 37 & $03 E+00$ & $57 \mathrm{E}+03$ & $19 E+02$ & .22501 & 01 \\
\hline 38 & $03 E+00$ & $57 E+03$ & $19 \mathrm{E}+02$ & $.2250 \mathrm{~B}$ & .01 \\
\hline 39 & $993 E+00$ & $2 E+03$ & $19 E+02$ & $0.2250 E+00$ & 01 \\
\hline 40 & $993 E+00$ & $42 E+03$ & $419 E+02$ & $0.2250 E+00$ & 01 \\
\hline 41 & $93 E+00$ & $57 E+03$ & $119 E+02$ & $0.2250 \mathrm{E}+00$ & \\
\hline 42 & $93 \mathrm{E}+00$ & $57 E+03$ & $19 E+02$ & $0.2250 \mathrm{E}+00$ & \\
\hline 43 & $2003 E+00$ & $42 E+03$ & $419 E+02$ & 0.2250 & 1 \\
\hline 44 & $2003 E+00$ & $42 E+03$ & $419 E+02$ & $0.2250 \mathrm{E}+00$ & 1 \\
\hline 45 & $1993 E+00$ & $57 E+03$ & $382 E+02$ & 0.2261 & 0 \\
\hline 46 & $1993 E+00$ & $57 E+03$ & $382 E+02$ & 0.2261 & $\mathrm{c}$ \\
\hline 47 & $0.2003 E+00$ & $42 E+03$ & $382 E+02$ & $0.2261 E+00$ & 0 \\
\hline 48 & $0.2003 E+00$ & $42 E+03$ & $382 E+02$ & $0.2261 \mathrm{E}+00$ & 0 \\
\hline 49 & $0.2003 E+00$ & $42 \mathrm{E}+03$ & $19 \mathrm{E}+02$ & 0.2261 & 0 \\
\hline 50 & $0.2003 \mathrm{E}+00$ & $42 E+03$ & $0.7419 \mathrm{E}+02$ & $0.2261 \mathrm{E}+00$ & 0.1 \\
\hline 51 & $0.1993 \mathrm{E}$ & $7 \mathrm{E}+$ & $19 E+02$ & $1 E+00$ & 0.1 \\
\hline 52 & 0.1 & $7 \mathrm{E}+$ & $19 E+02$ & $0.2261 \mathrm{E}+00$ & $0 E-01$ \\
\hline 53 & 0 . & $2 \mathrm{E}+$ & $32 E+02$ & $0.2250 \mathrm{E}+00$ & $5 E-01$ \\
\hline 54 & $0.2003 E+00$ & $42 E+03$ & $0.7382 E+02$ & $0.2250 \mathrm{E}+00$ & $0.1005 \mathrm{E}-0$ \\
\hline 55 & $0.1993 E+00$ & $57 E+03$ & $0.7382 \mathrm{E}+02$ & $0.2250 \mathrm{E}+00$ & $0.1005 \mathrm{E}-01$ \\
\hline 56 & $0.1993 \mathrm{E}+00$ & $0.3057 \mathrm{E}+03$ & $0.7382 \mathrm{E}+02$ & $0.2250 \mathrm{E}+00$ & $0.1005 \mathrm{E}-01$ \\
\hline 57 & $0.1993 \mathrm{E}+50$ & $0.3042 \mathrm{E}+03$ & $0.7382 \mathrm{E}+02$ & $0.2250 E+00$ & $0.1000 \mathrm{E}-01$ \\
\hline 58 & $0.1993 \mathrm{E}+00$ & $0.3042 \mathrm{E}+03$ & $0.7382 \mathrm{E}+02$ & $0.2250 \mathrm{E}+00$ & $0.1000 E-01$ \\
\hline 59 & $0.2003 E+00$ & $0.3057 E+03$ & $0.7382 E+02$ & $0.2250 E+00$ & $0.1000 \mathrm{E}-0$ \\
\hline 60 & $0.2003 E+00$ & $0.3057 E+03$ & $0.7382 E+02$ & $0.2250 \mathrm{E}+00$ & $0.1000 \mathrm{E}-01$ \\
\hline 61 & $0.1993 \mathrm{E}+00$ & $0.3042 E+03$ & $0.7419 E+02$ & $0.2261 \mathrm{E}+00$ & $0.1005 \mathrm{E}-01$ \\
\hline 62 & $0.1993 E+00$ & $0.3042 \mathrm{E}+03$ & $0.7419 \mathrm{E}+02$ & $0.2261 \mathrm{E}+00$ & $0.1005 E-01$ \\
\hline 63 & $0.2003 \mathrm{E}+00$ & $0.3057 E+03$ & $0.7419 E+02$ & $0.2261 \mathrm{E}+00$ & $0.1005 \mathrm{E}-0$ \\
\hline 64 & $0.2003 E+00$ & $0.3057 E+03$ & $0.7419 E+02$ & $0.2261 E+00$ & $0.1005 \mathrm{E}-0$ \\
\hline
\end{tabular}


TABLE 3. RESPONSES $\quad 1$ OF 2

\begin{tabular}{|c|c|c|c|}
\hline TEST & $S M A B \quad E R$ & BIAS & CPU TIME \\
\hline 1 & $0.5631 \mathrm{E}+01$ & $0.5631 E+01$ & $0.8100 \mathrm{E}+02$ \\
\hline 2 & $0.2106 \mathrm{E}+02$ & $0.2106 E+02$ & $0.7600 \mathrm{E}+02$ \\
\hline 3 & $0.6255 \mathrm{E}+01$ & $0.6255 \mathrm{E}+01$ & $0.7700 \mathrm{E}+02$ \\
\hline 4 & $0.2194 \mathrm{E}+02$ & $0.2194 \mathrm{E}+02$ & $0.7300 \mathrm{E}+02$ \\
\hline 5 & $0.6343 E+01$ & $0.6343 E+01$ & $0.8400 \mathrm{E}+02$ \\
\hline 6 & $0.2065 \mathrm{E}+02$ & $0.2065 E+02$ & $0.7800 \mathrm{E}+02$ \\
\hline 7 & $0.6724 \mathrm{E}+01$ & $0.6724 \mathrm{E}+01$ & $0.8000 \mathrm{E}+02$ \\
\hline 8 & $0.1302 \mathrm{E}+02$ & $0.1302 \mathrm{E}+02$ & $0.8000 \mathrm{E}+02$ \\
\hline 9 & $0.6014 \mathrm{E}+01$ & $0.6014 \mathrm{E}+01$ & $0.8200 E+02$ \\
\hline 10 & $0.2186 \mathrm{E}+02$ & $0.2186 E+02$ & $0.7900 \mathrm{E}+02$ \\
\hline 11 & $0.6347 \mathrm{E}+01$ & $0.6347 \mathrm{E}+01$ & $0.8300 E+02$ \\
\hline 12 & $0.2155 \mathrm{E}+02$ & $0.2155 E+02$ & $0.8000 \mathrm{E}+02$ \\
\hline 13 & $0.6549 E+01$ & $0.6549 \mathrm{E}+01$ & $0.8300 \mathrm{E}+02$ \\
\hline 14 & $0.2125 E+02$ & $0.2125 \mathrm{E}+02$ & $0.8300 \mathrm{E}+02$ \\
\hline 15 & $0.6671 \mathrm{E}+01$ & $0.6671 \mathrm{E}+01$ & $0.8200 E+02$ \\
\hline 16 & $0.2114 \mathrm{E}+02$ & $0.2114 \mathrm{E}+02$ & $0.8100 \mathrm{E}+02$ \\
\hline 17 & $0.6841 \mathrm{E}+01$ & $0.6841 \mathrm{E}+01$ & $0.8200 E+02$ \\
\hline 18 & $0.2146 \mathrm{E}+02$ & $0.2146 \mathrm{E}+02$ & $0.8500 E+02$ \\
\hline 19 & $0.6240 \mathrm{E}+01$ & $0.6240 E+01$ & $0.8600 \mathrm{E}+02$ \\
\hline 20 & $0.2089 E+02$ & $0.2089 E+02$ & $0.7900 E+02$ \\
\hline 21 & $0.6399 \mathrm{E}+01$ & $0.6399 \mathrm{E}+01$ & $0.7700 E+02$ \\
\hline 22 & $0.2169 \mathrm{E}+02$ & $0.2169 \mathrm{E}+02$ & $0.7600 E+02$ \\
\hline 23 & $0.5550 E+01$ & $0.5550 \mathrm{E}+01$ & $0.8100 E+02$ \\
\hline 24 & $0.1267 \mathrm{E}+02$ & $0.1267 \mathrm{E}+02$ & $0.7700 E+02$ \\
\hline 25 & $0.6436 \mathrm{E}+01$ & $0.6436 E+01$ & $0.7800 \mathrm{E}+02$ \\
\hline 26 & $0.2129 \mathrm{E}+02$ & $0.2129 E+02$ & $0.80001+02$ \\
\hline 27 & $0.6816 E+01$ & $0.6816 E+01$ & $0.7600 \mathrm{E}+02$ \\
\hline 28 & $0.2111 \mathrm{E}+02$ & $0.2111 \mathrm{~F}+02$ & $0.8000 \mathrm{E}+02$ \\
\hline 29 & $0.6118 E+01$ & $0.6118 \mathrm{E}+01$ & $0.7800 \mathrm{E}+02$ \\
\hline 30 & $0.2167 \mathrm{E}+02$ & $0.2167 \mathrm{E}+02$ & $0.7500 E+02$ \\
\hline 31 & $0.6292 \mathrm{E}+01$ & $0.6292 \mathrm{E}+01$ & $0.8100 E+02$ \\
\hline 32 & $0.2169 E+02$ & $1.2169 E+02$ & $0.7400 \mathrm{E}+02$ \\
\hline
\end{tabular}


LIQUID DENSITY $n-C 4 / \mathrm{CO}$ ALS DRES $=4 \quad \mathrm{SFACT}=0.5 \%$

TABLE 4 A. CONTRASTS LISTED BY FACTORS

1 OF 1

FACTOR

$\begin{array}{lll}\text { C } & 1 & 0.1399 \mathrm{E}+02 \\ \mathrm{E} & 1 & -.1086 \mathrm{E}+01 \\ \mathrm{E} & 20 & -.1083 \mathrm{E}+01 \\ \mathrm{C} & 3 & -.1076 \mathrm{E}+01 \\ \mathrm{E} & 27 & -.1075 \mathrm{E}+01 \\ \mathrm{C} & 15 & -.1075 \mathrm{E}+01 \\ \mathrm{C} & 7 & -.1074 \mathrm{E}+01 \\ \mathrm{E} & 2 & -.1074 \mathrm{E}+01 \\ \mathrm{E} & 23 & -.1074 \mathrm{E}+01 \\ \mathrm{E} & 14 & -.1074 \mathrm{E}+01 \\ \mathrm{E} & 6 & -.1074 \mathrm{E}+01 \\ \mathrm{OM} & 2 & -.1070 \mathrm{E}+01 \\ \mathrm{OM} & 1 & -.1044 \mathrm{E}+01 \\ \mathrm{C} & 2 & -.1016 \mathrm{E}+01 \\ \mathrm{C} & 12 & 0.4581 \mathrm{E}+00 \\ \mathrm{PC} & 1 & 0.3564 \mathrm{E}+00 \\ \text { TC } & 2 & 0.3150 \mathrm{E}+00 \\ \mathrm{C} & 8 & -.1893 \mathrm{E}+00 \\ \mathrm{C} & 4 & 0.1432 \mathrm{E}+00 \\ \mathrm{E} & 18 & -.9611 \mathrm{E}-01 \\ \mathrm{PC} & 2 & 0.8116 \mathrm{E}-01 \\ \mathrm{E} & 11 & -.4642 \mathrm{E}-01 \\ \mathrm{E} & 19 & 0.3697 \mathrm{E}-01 \\ \mathrm{C} & 9 & -.3523 \mathrm{E}-01 \\ \mathrm{E} & 7 & 0.31 \\ \mathrm{E} & 21 & -.224 \mathrm{E}-01 \\ \mathrm{TC} & 1 & 0.1785 \mathrm{E}-01 \\ \mathrm{C} & 13 & 0.1565 \mathrm{E}-01 \\ \mathrm{E} & 3 & -.1500 \mathrm{E}-01 \\ \mathrm{C} & 17 & 0.1429 \mathrm{E}-01 \\ \mathrm{C} & 16 & 0.1065 \mathrm{E}-01 \\ \mathrm{E} & 8 & 0.7628 \mathrm{E}-02\end{array}$

\section{FACTOR}

$\begin{array}{cc}\text { E } & 5 \\ \text { C } & 10 \\ \text { E } & 10 \\ \text { C } & 11 \\ \text { E } & 9 \\ C & 5 \\ \text { E } & 12 \\ \text { E } & 16\end{array}$

C 6

E 15

E 22

K 12

E 25

E 30

E 29

E 31 .

E 17

C 18

E 4

E 13

E 28

E 24

C 14

E 26
SM AB ER

$0.7378 \mathrm{E}-02$

$0.7082 \mathrm{E}-02$

$0.6986 \mathrm{E}-02$

$0.6973 \mathrm{E}-02$

$0.6923 \mathrm{E}-02$

$0.5918 \mathrm{E}-02$

$0.5891 \mathrm{E}-02$

$0.5504 \mathrm{E}-02$

$0.5153 \mathrm{E}-02$

$-.5036 E-02$

$0.4028 \mathrm{E}-02$

$-.3674 E-02$

$0.3567 \mathrm{E}-02$

$0.3073 E-02$

$0.2743 \mathrm{E}-02$

$0.2650 E-02$

$0.2576 \mathrm{E}-02$

$0.2424 \mathrm{E}-02$

$0.1770 \mathrm{E}-02$

$-.1584 \mathrm{E}-02$

$-.1535 \mathrm{E}-02$

$-.1219 \mathrm{E}-02$

$-.1092 \mathrm{E}-02$

$-.4480 \mathrm{E}-03$ 


\section{LIQUID DENSITY $n-C 4 / \mathrm{CO} 2$ ALS DRES $=4$ SFACT $=0.5 \%$}

TABLE 3. (CONTINUED)

BIAS

CPU TIME

$\begin{array}{llll}33 & 0.6144 \mathrm{E}+01 & 0.6144 \mathrm{E}+01 & 0.7500 \mathrm{E}+02 \\ 34 & 0.2127 \mathrm{E}+02 & 0.2127 \mathrm{E}+02 & 0.7300 \mathrm{E}+02 \\ 35 & 0.5786 \mathrm{E}+01 & 0.5786 \mathrm{E}+01 & 0.7900 \mathrm{E}+02 \\ 36 & 0.2170 \mathrm{E}+02 & 0.2170 \mathrm{E}+02 & 0.7600 \mathrm{E}+02 \\ 37 & 0.6606 \mathrm{E}+01 & 0.6606 \mathrm{E}+01 & 0.7700 \mathrm{E}+02 \\ 38 & 0.2105 \mathrm{E}+02 & 0.2105 \mathrm{E}+02 & 0.7600 \mathrm{E}+02 \\ 39 & 0.6463 \mathrm{E}+01 & 0.6463 \mathrm{E}+01 & 0.7600 \mathrm{E}+02 \\ 40 & 0.2129 \mathrm{E}+02 & 0.2129 \mathrm{E}+02 & 0.7700 \mathrm{E}+02 \\ 41 & 0.6445 \mathrm{E}+01 & 0.6445 \mathrm{E}+01 & 0.8400 \mathrm{E}+02 \\ 42 & 0.2202 \mathrm{E}+02 & 0.2202 \mathrm{E}+02 & 0.8100 \mathrm{E}+02 \\ 43 & 0.5953 \mathrm{E}+01 & 0.5953 \mathrm{E}+01 & 0.8300 \mathrm{E}+02 \\ 44 & 0.2134 \mathrm{E}+02 & 0.2134 \mathrm{E}+02 & 0.8100 \mathrm{E}+02 \\ 45 & 0.6745 \mathrm{E}+01 & 0.6745 \mathrm{E}+01 & 0.8200 \mathrm{E}+02 \\ 46 & 0.2162 \mathrm{E}+02 & 0.2162 \mathrm{E}+02 & 0.8000 \mathrm{E}+02 \\ 47 & 0.6497 \mathrm{E}+01 & 0.6497 \mathrm{E}+01 & 0.8300 \mathrm{E}+02 \\ 48 & 0.1223 \mathrm{E}+02 & 0.1222 \mathrm{E}+02 & 0.8200 \mathrm{E}+02 \\ 49 & 0.6657 \mathrm{E}+01 & 0.6657 \mathrm{E}+01 & 0.8200 \mathrm{E}+02 \\ 50 & 0.2110 \mathrm{E}+02 & 0.2110 \mathrm{E}+02 & 0.8000 \mathrm{E}+02 \\ 51 & 0.6423 \mathrm{E}+01 & 0.6423 \mathrm{E}+01 & 0.8200 \mathrm{E}+02 \\ 52 & 0.2126 \mathrm{E}+02 & 0.2126 \mathrm{E}+02 & 0.8000 \mathrm{E}+02 \\ 53 & 0.5952 \mathrm{E}+01 & 0.5952 \mathrm{E}+01 & 0.8200 \mathrm{E}+02 \\ 54 & 0.2151 \mathrm{E}+02 & 0.2151 \mathrm{E}+02 & 0.8100 \mathrm{E}+02 \\ 55 & 0.5928 \mathrm{E}+01 & 0.5928 \mathrm{E}+01 & 0.8300 \mathrm{E}+02 \\ 56 & 0.2148 \mathrm{E}+02 & 0.2148 \mathrm{E}+02 & 0.7900 \mathrm{E}+02 \\ 57 & 0.6163 \mathrm{E}+01 & 0.6163 \mathrm{E}+01 & 0.8100 \mathrm{E}+02 \\ 58 & 0.2088 \mathrm{E}+02 & 0.2088 \mathrm{E}+02 & 0.7900 \mathrm{E}+02 \\ 59 & 0.7068 \mathrm{E}+01 & 0.7068 \mathrm{E}+01 & 0.8100 \mathrm{E}+02 \\ 60 & 0.2152 \mathrm{E}+02 & 0.2152 \mathrm{E}+02 & 0.8100 \mathrm{E}+02 \\ 61 & 0.5602 \mathrm{E}+01 & 0.5602 \mathrm{E}+01 & 0.8200 \mathrm{E}+02 \\ 62 & 0.2147 \mathrm{E}+02 & 0.2147 \mathrm{E}+02 & 0.7900 \mathrm{E}+02 \\ 63 & 0.6756 \mathrm{E}+01 & 0.6756 \mathrm{E}+01 & 0.8200 \mathrm{E}+02 \\ 64 & 0.1341 \mathrm{E}+02 & 0.1341 \mathrm{E}+02 & 0.8100 \mathrm{E}+02\end{array}$


LIQUID DENSITY $n-C 4 / C O 2$ ALS DRES $=4 \quad S F A C T=0.5 \%$

TABLE 4 B. CONTRASTS LISTED BY FACTORS 1 OF 1

FACTOR

$\begin{array}{lcc}\mathrm{C} & 1 & 0.1399 \mathrm{E}+02 \\ \mathrm{E} & 1 & -.1086 \mathrm{E}+01 \\ \mathrm{E} & 20 & -.1084 \mathrm{E}+01 \\ \mathrm{C} & 3 & -.1076 \mathrm{E}+01 \\ \mathrm{E} & 27 & -.1075 \mathrm{E}+01 \\ \mathrm{C} & 15 & -.1075 \mathrm{E}+01 \\ \mathrm{C} & 7 & -.1075 \mathrm{E}+01 \\ \mathrm{E} & 2 & -.1074 \mathrm{E}+01 \\ \mathrm{E} & 23 & -.1074 \mathrm{E}+01 \\ \mathrm{E} & 14 & -.1074 \mathrm{E}+01 \\ \mathrm{E} & 6 & -.1074 \mathrm{E}+01 \\ \mathrm{OM} & 2 & -.1070 \mathrm{E}+01 \\ \mathrm{OM} & 1 & -.1044 \mathrm{E}+01 \\ \mathrm{C} & 2 & -.1016 \mathrm{E}+01 \\ \mathrm{C} & 12 & 0.4583 \mathrm{E}+00 \\ \mathrm{PC} & 1 & 0.3566 \mathrm{E}+00 \\ \text { TC } & 2 & 0.3152 \mathrm{E}+00 \\ \mathrm{C} & 8 & -.1895 \mathrm{E}+00 \\ \mathrm{C} & 4 & 0.1430 \mathrm{E}+00 \\ \mathrm{E} & 18 & -.9633 \mathrm{E}-01 \\ \mathrm{PC} & 2 & 0.8138 \mathrm{E}-01 \\ \mathrm{E} & 11 & -.4620 \mathrm{E}-01 \\ \mathrm{E} & 19 & 0.3719 \mathrm{E}-01 \\ \mathrm{C} & 9 & -.3501 \mathrm{E}-01 \\ \mathrm{E} & 7 & 0.3132 \mathrm{E}-01 \\ \mathrm{E} & 21 & -.2222 \mathrm{E}-01 \\ \mathrm{TC} & 1 & 0.1764 \mathrm{E}-01 \\ \mathrm{C} & 13 & 0.1544 \mathrm{E}-01 \\ \mathrm{E} & 3 & -.1522 \mathrm{E}-01 \\ \mathrm{C} & 17 & 0.1407 \mathrm{E}-01 \\ \mathrm{C} & 16 & 0.1087 \mathrm{E}-01 \\ \mathrm{E} & 8 & 0.7845 \mathrm{E}-02\end{array}$

\section{FACTOR}

\section{BIAS}

$\begin{array}{lccc}\text { E } & 5 & 0.7160 \mathrm{E}-02 \\ \mathrm{C} & 10 & 0.6864 \mathrm{E}-02 \\ \mathrm{E} & 10 & 0.6768 \mathrm{E}-02 \\ \mathrm{C} & 11 & 0.6755 \mathrm{E}-02 \\ \mathrm{E} & 9 & 0.6705 \mathrm{E}-02 \\ \mathrm{C} & 5 & 0.6135 \mathrm{E}-02 \\ \mathrm{E} & 12 & 0.5673 \mathrm{E}-02 \\ \mathrm{E} & 16 & 0.5286 \mathrm{E}-02 \\ \mathrm{C} & 6 & 0.4935 \mathrm{E}-02 \\ \mathrm{E} & 15 & -.4818 \mathrm{E}-02 \\ \mathrm{E} & 22 & 0.4245 \mathrm{E}-02 \\ \mathrm{E} & 25 & 0.3784 \mathrm{E}-02 \\ \mathrm{~K} & 12 & -.3456 \mathrm{E}-02 \\ \mathrm{E} & 30 & 0.3291 \mathrm{E}-02 \\ \mathrm{E} & 29 & 0.2961 \mathrm{E}-02 \\ \mathrm{E} & 31 & 0.2868 \mathrm{E}-02 \\ \mathrm{E} & 17 & 0.2794 \mathrm{E}-02 \\ \mathrm{C} & 18 & 0.2642 \mathrm{E}-02 \\ \mathrm{E} & 4 & 0.1988 \mathrm{E}-02 \\ \mathrm{E} & 13 & -.1366 \mathrm{E}-02 \\ \mathrm{E} & 28 & -.1318 \mathrm{E}-02 \\ \mathrm{E} & 24 & -.1001 \mathrm{E}-02 \\ \mathrm{C} & 14 & -.8738 \mathrm{E}-03 \\ \mathrm{E} & 26 & -.2303 \mathrm{E}-03\end{array}$


LIQUID DENSTTY $n-C 4$ / $\mathrm{CO} 2$ ALS DRES $=4 \quad \mathrm{SFACT}=0.5 \%$

TABLE 4 C. CONTRASTS LISTED BY FACTORS

1 OF 1

FACTOP

$\begin{array}{lll}\text { E } & 13 & -.2969 E+01 \\ \mathrm{C} & 1 & -.1969 \mathrm{E}+01 \\ \mathrm{~K} & 12 & 0.1531 \mathrm{E}+01 \\ \mathrm{C} & 4 & 0.1469 \mathrm{E}+01 \\ \mathrm{E} & 15 & 0.1469 \mathrm{E}+01 \\ \mathrm{E} & 11 & -.1406 \mathrm{E}+01 \\ \mathrm{E} & 14 & 0.1281 \mathrm{E}+01 \\ \mathrm{C} & 12 & -.1094 \mathrm{E}+01 \\ \mathrm{PC} & 2 & 0.9688 \mathrm{E}+00 \\ \mathrm{E} & 19 & 0.7813 \mathrm{E}+00 \\ \mathrm{E} & 27 & 0.7813 \mathrm{E}+00 \\ \mathrm{C} & 9 & -.7188 \mathrm{E}+00 \\ \mathrm{E} & 29 & 0.6563 \mathrm{E}+00 \\ \mathrm{TC} & 2 & -.5938 \mathrm{E}+00 \\ \mathrm{E} & 9 & 0.5938 \mathrm{E}+00 \\ \mathrm{E} & 16 & -.5938 \mathrm{E}+00 \\ \mathrm{E} & 21 & 0.5938 \mathrm{E}+00 \\ \mathrm{E} & 30 & -.5938 \mathrm{E}+00 \\ \mathrm{C} & 11 & -.5313 \mathrm{E}+00 \\ \mathrm{TC} & 1 & -.4688 \mathrm{E}+00 \\ \mathrm{E} & 22 & -.4688 \mathrm{E}+00 \\ \mathrm{E} & 24 & 0.4688 \mathrm{E}+00 \\ \mathrm{C} & 5 & 0.4063 \mathrm{E}+00 \\ \mathrm{C} & 6 & 0.4063 \mathrm{E}+00 \\ \mathrm{C} & 10 & 0.4063 \mathrm{E}+00 \\ \mathrm{C} & 13 & 0.4063 \mathrm{E}+00 \\ \mathrm{C} & 15 & -.4063 \mathrm{E}+00 \\ \mathrm{C} & 18 & 0.4063 \mathrm{E}+00 \\ \mathrm{E} & 3 & 0.4063 \mathrm{E}+00 \\ \mathrm{E} & 31 & 0.4063 \mathrm{E}+00 \\ \mathrm{C} & 8 & 0.3438 \mathrm{E}+00 \\ \mathrm{C} & 16 & -.3438 \mathrm{E}+00\end{array}$

FACTOR

CPU TIME

$\begin{array}{lrr}\text { E } & 12 & 0.3438 \mathrm{E}+00 \\ \mathrm{OM} & 2 & -.2813 \mathrm{E}+00 \\ \mathrm{E} & 17 & -.2813 \mathrm{E}+00 \\ \mathrm{E} & 23 & 0.2813 \mathrm{E}+00 \\ \mathrm{E} & 25 & 0.2813 \mathrm{E}+00 \\ \mathrm{E} & 28 & -.2813 \mathrm{E}+00 \\ \mathrm{E} & 4 & 0.2188 \mathrm{E}+00 \\ \mathrm{E} & 5 & 0.2188 \mathrm{E}+00 \\ \mathrm{E} & 6 & 0.2188 \mathrm{E}+00 \\ \mathrm{E} & 8 & 0.2188 \mathrm{E}+00 \\ \mathrm{C} & 2 & 0.1563 \mathrm{E}+00 \\ \mathrm{C} & 7 & 0.1563 \mathrm{E}+00 \\ \mathrm{OM} & 1 & -.1563 \mathrm{E}+00 \\ \mathrm{E} & 1 & -.1563 \mathrm{E}+00 \\ \mathrm{E} & 2 & -.1563 \mathrm{E}+00 \\ \mathrm{E} & 7 & 0.1563 \mathrm{E}+00 \\ \mathrm{E} & 18 & -.1563 \mathrm{E}+00 \\ \mathrm{E} & 20 & 0.1563 \mathrm{E}+00 \\ \mathrm{C} & 3 & -.9375 \mathrm{E}-01 \\ \mathrm{C} & 14 & 0.9375 \mathrm{E}-01 \\ \mathrm{E} & 26 & -.9375 \mathrm{E}-01 \\ \mathrm{C} & 17 & -.3125 \mathrm{E}-01 \\ \mathrm{PC} & 1 & -.3125 \mathrm{E}-01 \\ \mathrm{E} & 10 & 0.3125 \mathrm{E}-01\end{array}$


LIQUID DENSITY $n-C 6 / \mathrm{CO} 2$ ALS DRES $=4 \quad \mathrm{SFACT}=0.5 \%$ 
LIQUID DENSITY $n-C 6 / C O 2$ ALS DRES $=4$ SFACT $=0.5 \%$

TABLE 3. RESPONSES

TEST

1

$$
2
$$$$
3
$$

SM AB ER

BIAS

$0.2821 E+00$

$0.5247 \mathrm{E}+00$

$0.7185 \mathrm{E}+00$

$0.6222 \mathrm{E}+00$

$0.6981 \mathrm{E}+00$

$0.6960 \mathrm{E}+00$

$0.1196 \mathrm{E}+01$

$0.2833 \mathrm{E}+00$

$0.7886 \mathrm{E}+00$

$0.4555 \mathrm{E}+00$

$0.7016 \mathrm{E}+00$

$0.7763 \mathrm{E}+00$

$0.9270 \mathrm{E}+00$

$0.3479 \mathrm{E}+00$

$0.9671 \mathrm{E}+00$

$0.5611 \mathrm{E}+00$

$0.1205 \mathrm{E}+01$

$0.2914 \mathrm{E}+00$

$0.6683 \mathrm{E}+00$

$0.5505 \mathrm{E}+00$

$0.7534 \mathrm{E}+00$

$0.6126 \mathrm{E}+00$

$0.3123 \mathrm{E}+00$

$0.4096 \mathrm{E}+00$

$0.8193 \mathrm{E}+00$

$0.5575 \mathrm{E}+00$

$0.1122 \mathrm{E}+01$

$0.3482 \mathrm{E}+00$

$0.5928 \mathrm{E}+00$

$0.8126 \mathrm{E}+00$

$0.9850 \mathrm{E}+00$

$0.2960 \mathrm{E}+00$
$0.2821 \mathrm{E}+00$

$-.3472 \mathrm{E}+00$

$0.7185 \mathrm{E}+00$

$0.4459 \mathrm{E}+00$

$0.6354 \mathrm{E}+00$

$-.6478 \mathrm{E}+00$

$0.1196 \mathrm{E}+01$

$0.2800 \mathrm{E}+00$

$0.7886 \mathrm{E}+00$

$0.4555 \mathrm{E}+00$

$0.6357 \mathrm{E}+00$

$0.7107 \mathrm{E}-01$

$0.9270 E+00$

$-.6185 \mathrm{E}-01$

$0.9671 E+00$

$-.2370 \mathrm{E}+00$

$0.1205 \mathrm{E}+01$

$-.2968 \mathrm{E}-01$

$0.5489 \mathrm{E}+00$

$-.3612 \mathrm{E}+00$

$0.7534 \mathrm{E}+00$

$0.1383 \mathrm{E}+00$

$0.3123 \mathrm{E}+00$

$-.7264 \mathrm{E}-01$

$0.8193 \mathrm{E}+00$

$-.1107 \mathrm{E}+00$

$0.1122 \mathrm{E}+01$

$-.1866 \mathrm{E}+00$

$0.4877 E+00$

$0.1648 \mathrm{E}+00$

$0.9850 \mathrm{E}+00$

$0.2960 E+00$
1 OF 2

CPU TIME

$0.8500 \mathrm{E}+\mathrm{C} 2$

$0.8200 \mathrm{E}+02$

$0.8000 E+02$

$0.8200 \mathrm{E}+02$

$0.8000 E+02$

$0.8300 \mathrm{E}+02$

$0.8000 \mathrm{E}+02$

$0.8000 \mathrm{E}+02$

$0.8300 \mathrm{E}+02$

$0.7900 \mathrm{E}+02$

$0.8100 \mathrm{E}+02$

$0.7300 \mathrm{E}+02$

$0.8200 \mathrm{E}+02$

$0.8400 \mathrm{E}+02$

$0.8300 \mathrm{E}+02$

$0.8300 \mathrm{E}+02$

$0.8400 \mathrm{E}+02$

$0.8400 \mathrm{E}+02$

$0.8200 \mathrm{E}+02$

$0.8200 \mathrm{E}+02$

$0.8200 \mathrm{E}+02$

$0.8100 \mathrm{E}+02$

$0.8200 \mathrm{E}+02$

$0.8100 \mathrm{E}+02$

$0.8100 \mathrm{E}+02$

$0.8200 \mathrm{E}+02$

$0.8100 \mathrm{E}+02$

$0.8100 \mathrm{E}+02$

$0.7700 \mathrm{E}+02$

$0.7800 \mathrm{E}+02$

$0.7700 \mathrm{E}+02$

$0.7600 \mathrm{E}+02$ 
LIQUID DENSITY $n-C 6 / \mathrm{CO} 2$ ALS DRES $=4 \quad \mathrm{SFACT}=0.5 \%$

TABLE 3. (CONTINUED)

BIAS

CPU TIME

$\begin{array}{lll}33 & 0.5997 \mathrm{E}+00 & 0.4646 \mathrm{E}+00 \\ 34 & 0.8606 \mathrm{E}+00 & -.2200 \mathrm{E}+00 \\ 35 & 0.5872 \mathrm{E}+00 & 0.5872 \mathrm{E}+00 \\ 36 & 0.2791 \mathrm{E}+00 & 0.2791 \mathrm{E}+00 \\ 37 & 0.9506 \mathrm{E}+00 & 0.9506 \mathrm{E}+00 \\ 38 & 0.5689 \mathrm{E}+00 & -.3518 \mathrm{E}+00 \\ 39 & 0.8822 \mathrm{E}+00 & 0.8822 \mathrm{E}+00 \\ 40 & 0.3139 \mathrm{E}+00 & -.5568 \mathrm{E}-01 \\ 41 & 0.8421 \mathrm{E}+00 & 0.8421 \mathrm{E}+00 \\ 42 & 0.6616 \mathrm{E}+00 & 0.4443 \mathrm{E}+00 \\ 43 & 0.6233 \mathrm{E}+00 & 0.6233 \mathrm{E}+00 \\ 44 & 0.3435 \mathrm{E}+00 & 0.1049 \mathrm{E}-01 \\ 45 & 0.1130 \mathrm{E}+01 & 0.1130 \mathrm{E}+01 \\ 46 & 0.3253 \mathrm{E}+00 & 0.1201 \mathrm{E}+00 \\ 47 & 0.8009 \mathrm{E}+00 & 0.7951 \mathrm{E}+00 \\ 48 & 0.6079 \mathrm{E}+00 & -.4337 \mathrm{E}+00 \\ 49 & 0.1009 \mathrm{E}+01 & 0.1009 \mathrm{E}+01 \\ 50 & 0.3055 \mathrm{E}+00 & -.2076 \mathrm{E}+00 \\ 51 & 0.8293 \mathrm{E}+00 & 0.8293 \mathrm{E}+00 \\ 52 & 0.5104 \mathrm{E}+00 & -.1563 \mathrm{E}+00 \\ 53 & 0.6884 \mathrm{E}+00 & 0.6884 \mathrm{E}+00 \\ 54 & 0.1798 \mathrm{E}+00 & 0.1292 \mathrm{E}+00 \\ 55 & 0.5272 \mathrm{E}+00 & 0.3087 \mathrm{E}+00 \\ 56 & 0.8512 \mathrm{E}+00 & -.3484 \mathrm{E}-01 \\ 57 & 0.5738 \mathrm{E}+00 & 0.4874 \mathrm{E}+00 \\ 58 & 0.6638 \mathrm{E}+00 & -.4219 \mathrm{E}+00 \\ 59 & 0.1414 \mathrm{E}+01 & 0.1414 \mathrm{E}+01 \\ 60 & 0.2407 \mathrm{E}+00 & 0.1309 \mathrm{E}+00 \\ 61 & 0.3032 \mathrm{E}+00 & 0.3032 \mathrm{E}+00 \\ 62 & 0.4263 \mathrm{E}+00 & 0.4828 \mathrm{E}-01 \\ 63 & 0.1118 \mathrm{E}+01 & 0.1118 \mathrm{E}+01 \\ 64 & 0.6026 \mathrm{E}+00 & 0.4787 \mathrm{E}+00\end{array}$

$0.7100 E+02$

$0.7300 E+02$

$0.7400 \mathrm{E}+02$

$0.7300 \mathrm{E}+02$

$0.7100 \mathrm{E}+02$

$0.7200 \mathrm{E}+02$

$0.8200 \mathrm{E}+02$

$0.7800 \mathrm{E}+02$

$0.7800 \mathrm{E}+02$

$0.7900 \mathrm{E}+02$

$0.82 .00 E+02$

$0.8400 \mathrm{E}+02$

$0.8200 \mathrm{E}+02$

$0.8000 \mathrm{E}+02$

$0.7600 \mathrm{E}+02$

$0.7400 \mathrm{E}+02$

$0.7700 \mathrm{E}+02$

$0.8100 \mathrm{E}+02$

$0.8100 \mathrm{E}+02$

$0.8000 \mathrm{E}+02$

$0.8000 \mathrm{E}+02$

$0.7900 \mathrm{E}+02$

$0.8200 E+02$

$0.8000 \mathrm{E}+02$

$0.7900 \mathrm{E}+02$

$0.8100 \mathrm{E}+02$

$0.8300 E+02$

$0.8400 E+02$

$0.8400 \mathrm{E}+02$

$0.8000 \mathrm{E}+02$

$0.7900 \mathrm{E}+02$

$0.7900 \mathrm{E}+02$ 
LIQUID DENSITY $n-\mathrm{C} 6 / \mathrm{CO} 2$ ALS DRES $=4 \quad$ SFACT $=0.5 \%$

TABLE 4 A. CONTRASTS LISTED BY FACTORS 1 OF 1

\section{FACTOR}

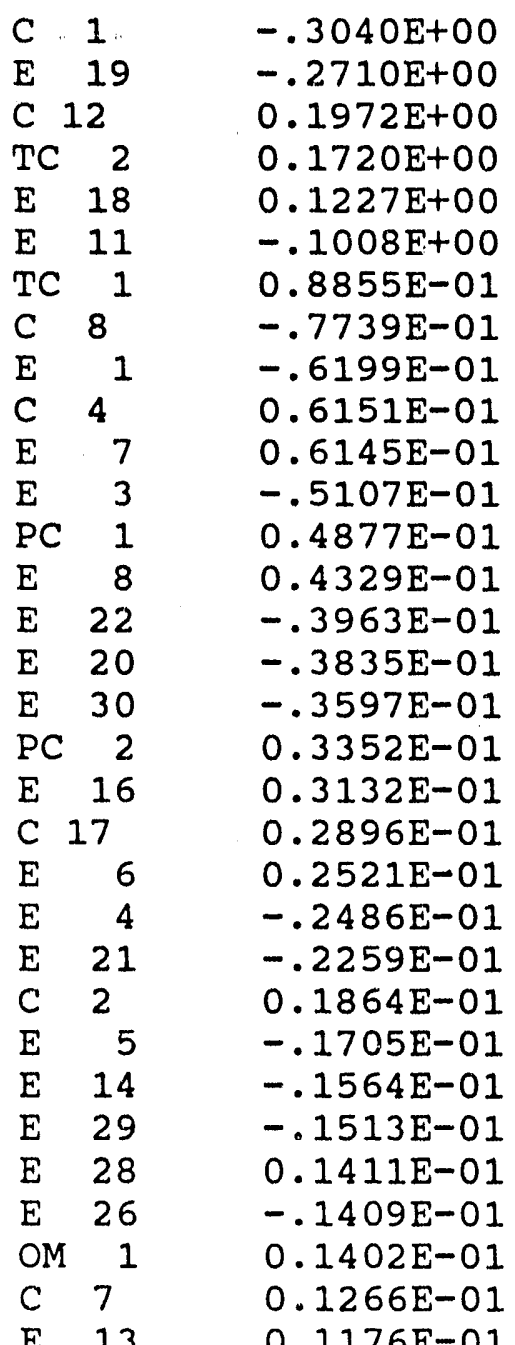

FACTOR

SM AB ER

E $24 \quad-.1165 \mathrm{E}-01$

C $5-.1071 \mathrm{E}-01$

C $16 \quad 0.1062 \mathrm{E}-01$

C $13 \quad 0.1008 \mathrm{E}-01$

E $27 \quad-.9623 \mathrm{E}-02$

E $23 \quad 0.9602 \mathrm{E}-02$

E $90.9091 \mathrm{E}-02$

C $6 \quad-.8211 \mathrm{E}-02$

C $14 \quad 0.7412 \mathrm{E}-02$

E $10 \quad-.6508 \mathrm{E}-02$

C $15 \quad 0.5479 \mathrm{E}-02$

E $17 \quad-.5255 \mathrm{E}-02$

C $9-.4993 \mathrm{E}-02$

OM $20.4586 \mathrm{E}-02$

E $25 \quad 0.4557 \mathrm{E}-02$

E $2-.4507 \mathrm{E}-02$

C $18 \quad 0.4457 \mathrm{E}-02$

C $11 \quad 0.4127 \mathrm{E}-02$

C $10 \quad 0.3935 \mathrm{E}-02$

E $12-.2700 \mathrm{E}-02$

E $15 \quad 0.2362 \mathrm{E}-02$

C $3 \quad-.1517 \mathrm{E}-02$

K $12-.1164 \mathrm{E}-02$

E $31 \quad 0.7696 \mathrm{E}-03$ 
LIQUID DENSITY $n-C 6 / \mathrm{CO} 2$ ALS DRES $=4 \quad \mathrm{SFACT}=0.5 \%$

TABLE 4 B. CONTRASTS LISTED BY FACTORS 1 OF 1

FACTOR

\begin{tabular}{|c|c|}
\hline C 1 & $-.7925 E+00$ \\
\hline PC 1 & $0.3616 \mathrm{E}+00$ \\
\hline C 12 & $0.3168 E+00$ \\
\hline TC & $0.1711 \mathrm{E}+00$ \\
\hline $\mathrm{C}$ & $-.1485 \mathrm{E}+00$ \\
\hline C & $0.1236 \mathrm{E}+00$ \\
\hline C & $0.8507 \mathrm{E}-01$ \\
\hline PC & $0.8390 E-01$ \\
\hline TC & $-.8056 \mathrm{E}-01$ \\
\hline OM & $0.6254 \mathrm{E}-01$ \\
\hline 9 & $-.3705 \mathrm{E}-01$ \\
\hline C 17 & $0.2030 \mathrm{E}-01$ \\
\hline C 16 & $0.1857 \mathrm{E}-01$ \\
\hline C 13 & $0.7597 \mathrm{E}-02$ \\
\hline OM & $0.6094 \mathrm{E}-02$ \\
\hline 5 & $0.3735 \mathrm{E}-02$ \\
\hline 11 & $-.3149 E-02$ \\
\hline 6 & $-.2782 \mathrm{E}-02$ \\
\hline 18 & $-.2709 \mathrm{E}-02$ \\
\hline 3 & $-.2291 \mathrm{E}-02$ \\
\hline 19 & $-.1807 \mathrm{E}-02$ \\
\hline 21 & $-.1615 \mathrm{E}-02$ \\
\hline 25 & $0.1541 \mathrm{E}-02$ \\
\hline 26 & $0.1428 \mathrm{E}-02$ \\
\hline C 18 & $-.1356 \mathrm{E}-02$ \\
\hline 22 & $-.1297 \mathrm{E}-02$ \\
\hline 23 & $0.1116 \mathrm{E}-02$ \\
\hline 27 & $0.9319 \mathrm{E}-03$ \\
\hline 16 & $0.7649 E-03$ \\
\hline 14 & ก. 6917E-03 \\
\hline $\mathrm{E}$ & $0.6050 \mathrm{E}-03$ \\
\hline 17 & $-.5769 E-0.3$ \\
\hline
\end{tabular}

\section{FACTOR}

BIAS

$\begin{array}{lrll}\mathrm{C} & 14 & 0.5734 \mathrm{E}-03 \\ \mathrm{E} & 1 & -.5430 \mathrm{E}-03 \\ \mathrm{E} & 8 & 0.4390 \mathrm{E}-03 \\ \mathrm{E} & 4 & -.4273 \mathrm{E}-03 \\ \mathrm{E} & 5 & 0.4176 \mathrm{E}-03 \\ \mathrm{E} & 7 & 0.3652 \mathrm{E}-03 \\ \mathrm{E} & 20 & -.3207 \mathrm{E}-03 \\ \mathrm{E} & 24 & 0.2964 \mathrm{E}-03 \\ \mathrm{C} & 10 & 0.2095 \mathrm{E}-03 \\ \mathrm{E} & 3 & 0.2088 \mathrm{E}-03 \\ \mathrm{E} & 12 & -.1904 \mathrm{E}-03 \\ \mathrm{E} & 15 & -.1634 \mathrm{E}-03 \\ \mathrm{E} & 31 & -.1221 \mathrm{E}-03 \\ \mathrm{E} & 28 & 0.1020 \mathrm{E}-03 \\ \mathrm{C} & 11 & 0.9774 \mathrm{E}-04 \\ \mathrm{C} & 7 & 0.9346 \mathrm{E}-04 \\ \mathrm{E} & 30 & 0.8191 \mathrm{E}-04 \\ \mathrm{C} & 15 & -.7754 \mathrm{E}-04 \\ \mathrm{E} & 13 & -.7342 \mathrm{E}-04 \\ \mathrm{E} & 29 & 0.5805 \mathrm{E}-04 \\ \mathrm{E} & 2 & 0.5130 \mathrm{E}-04 \\ \mathrm{E} & 10 & -.4685 \mathrm{E}-04 \\ \mathrm{E} & 9 & 0.3309 \mathrm{E}-04 \\ \mathrm{~K} & 1 & 2 & -.4910 \mathrm{E}-05\end{array}$


LIQUID DENSITY $\mathrm{n}-\mathrm{C} 6 / \mathrm{CO} 2$ ALS DRES $=4 \quad \mathrm{SFACT}=0.5 \%$

TABLE 4 C. CONTRASTS LISTED BY FACTORS

1 OF 1

\begin{tabular}{|c|c|c|c|c|}
\hline FAC & TOR & CPU TIME & FACTOR & CPU TIME \\
\hline $\mathrm{E}$ & 14 & $0.2469 E+01$ & C 18 & $-.4063 E+00$ \\
\hline C & 6 & $-.2281 E+01$ & E $\quad 18$ & $0.4063 E+00$ \\
\hline $\mathrm{E}$ & 15 & $0.2156 \mathrm{E}+01$ & $\mathrm{E} \quad 12$ & $0.3438 \mathrm{E}+00$ \\
\hline $\mathrm{E}$ & 13 & $-.1656 \mathrm{E}+01$ & E 16 & $0.3438 \mathrm{E}+00$ \\
\hline $\mathrm{C}$ & 5 & $0.1594 \mathrm{E}+01$ & 26 & $0.3438 E+00$ \\
\hline $\mathrm{E}$ & 27 & $-.1531 E+01$ & 31 & $0.3438 E+00$ \\
\hline$E$ & 30 & $0.1531 \mathrm{E}+01$ & C 15 & $0.2813 E+00$ \\
\hline $\mathrm{C}$ & 17 & $-.1469 \mathrm{E}+01$ & 2 & $-.2813 E+00$ \\
\hline $\mathrm{E}$ & 9 & $0.1469 E+01$ & 3 & $-.2813 E+00$ \\
\hline OM & 2 & $-.1281 E+01$ & 4 & $0.2813 \mathrm{E}+00$ \\
\hline $\mathrm{C}$ & 13 & $-.1219 E+01$ & $\mathrm{C}$ & $0.2188 E+00$ \\
\hline & 11 & $-.1156 \mathrm{E}+01$ & C 11 & $0.2188 E+00$ \\
\hline TC & 1 & $-.1031 E+01$ & 6 & $-.2188 \mathrm{E}+00$ \\
\hline $\mathrm{OM}$ & 1 & $-.9063 E+00$ & 22 & $-.2188 E+00$ \\
\hline $\mathrm{K}$ & 12 & $0.9063 E+00$ & C & $0.1563 E+00$ \\
\hline$E$ & 20 & $0.9063 E+00$ & C 16 & $-.1563 E+00$ \\
\hline$E$ & 7 & $-.8438 E+00$ & 5 & $0.1563 \mathrm{E}+00$ \\
\hline $\mathrm{C}$ & 12 & $-.7188 \mathrm{E}+00$ & 19 & $0.1563 E+0$ \\
\hline$C$ & 4 & $0.6563 E+00$ & C 10 & $-.9375 E-01$ \\
\hline PC & 1 & $0.5938 E+00$ & 21 & $0.9375 \mathrm{E}-01$ \\
\hline TC & 2 & $-.5938 E+00$ & C & $0.3125 \mathrm{E}-01$ \\
\hline $\mathrm{E}$ & 10 & $-.5938 E+00$ & $\mathrm{C}$ & $-.3125 E-01$ \\
\hline & 24 & $0.5938 E+00$ & $E$ & $-.3125 E-01$ \\
\hline PC & 2 & $-.5313 \mathrm{E}+00$ & 28 & $0.3125 \mathrm{E}-0$ \\
\hline $\mathrm{E}$ & 1 & $-.5313 E+00$ & & \\
\hline$E$ & 17 & $0.5313 E+00$ & & \\
\hline $\mathrm{E}$ & 25 & $-.5313 E+00$ & & \\
\hline$E$ & 29 & $0.5313 E+00$ & & \\
\hline C & 3 & $-.4688 E+00$ & & \\
\hline & 23 & $-.4688 \mathrm{E}+00$ & & \\
\hline & 1 & $-.4063 E+00$ & & \\
\hline & 14 & $0.4063 E+00$ & & \\
\hline
\end{tabular}


LIQUID DENSITY $n-C 8 / \mathrm{CO} 2$ ALS DRES $=4 \quad \mathrm{SFACT}=0.5 \%$ 


\section{LIQUID DENSITY n-C8 / CO2 ALS DRES $=4$ SFACT $=0.5 \%$}

TABLE 3. RESPONSES

TEST

1

2

3

4

5

6
SM AB ER

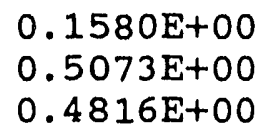

$0.3308 \mathrm{E}+00$

$0.4094 \mathrm{E}+00$

$0.7618 \mathrm{E}+00$

$0.9341 \mathrm{E}+00$

$0.1390 \mathrm{E}+00$

$0.7471 \mathrm{E}+00$

$0.2865 \mathrm{E}+00$

$0.3851 \mathrm{E}+00$

$0.5930 \mathrm{E}+00$

$0.6333 \mathrm{E}+00$

$0.2994 \mathrm{E}+00$

$0.5885 \mathrm{E}+00$

$0.4617 \mathrm{E}+00$

$0.8527 \mathrm{E}+00$

$0.2115 \mathrm{E}+00$

$0.4096 \mathrm{E}+00$

$0.5430 \mathrm{E}+00$

$0.4237 \mathrm{E}+00$

$0.4373 E+00$

$0.2773 \mathrm{E}+00$

$0.3159 \mathrm{E}+00$

$0.4975 \mathrm{E}+00$

$0.4284 \mathrm{E}+00$

$0.7768 \mathrm{E}+00$

$0.3272 \mathrm{E}+00$

$0.3323 \mathrm{E}+00$

$0.6018 \mathrm{E}+00$

$0.8775 \mathrm{E}+00$

$0.1788 \mathrm{E}+00$
BIAS

$0.1580 E+00$

$-.4874 \mathrm{E}+00$

$0.4816 \mathrm{E}+00$

$0.8000 \mathrm{E}-01$

$0.2758 \mathrm{E}+00$

$-.7618 \mathrm{E}+00$

$0.9341 \mathrm{E}+00$

$0.1390 \mathrm{E}+00$

$0.7471 \mathrm{E}+00$

$0.2865 \mathrm{E}+00$

$0.2706 \mathrm{E}+00$

$-.3194 \mathrm{E}+00$

$0.6333 \mathrm{E}+00$

$-.2197 \mathrm{E}+00$

$0.5885 \mathrm{E}+00$

$-.3924 \mathrm{E}+00$

$0.8527 \mathrm{E}+00$

$-.1552 \mathrm{E}+00$

$0.3822 \mathrm{E}+00$

$-.4929 \mathrm{E}+00$

$0.4237 \mathrm{E}+00$

$-.2114 \mathrm{E}+00$

$0.2773 \mathrm{E}+00$

$-.2276 \mathrm{E}+00$

$0.4975 \mathrm{E}+00$

$-.3157 \mathrm{E}+00$

$0.7768 \mathrm{E}+00$

$-.2898 \mathrm{E}+00$

$0.1732 \mathrm{E}+00$

$-.2727 \mathrm{E}+00$

$0.8775 \mathrm{E}+00$

$0.1788 \mathrm{E}+00$
1. OF 2

CPU TIME

$0.8000 \mathrm{E}+02$

$0.7900 \mathrm{E}+02$

$0.7900 \mathrm{E}+02$

$0.7900 \mathrm{E}+02$

$0.7900 \mathrm{E}+02$

$0.8200 \mathrm{E}+02$

$0.7900 \mathrm{E}+02$

$0.8000 E+02$

$0.8000 \mathrm{E}+02$

$0.7900 E+02$

$0.8100 E+02$

$0.8000 \mathrm{E}+02$

$0.8300 \mathrm{E}+02$

$0.8300 \mathrm{E}+02$

$0.8400 \mathrm{E}+02$

$0.8000 \mathrm{E}+02$

$0.8000 \mathrm{E}+02$

$0.8000 \mathrm{E}+02$

$0.8000 \mathrm{E}+02$

$0.8100 \mathrm{E}+02$

$0.8100 \mathrm{E}+02$

$0.7900 E+02$

$0.7800 \mathrm{E}+02$

$0.8200 \mathrm{E}+02$

$0.8000 \mathrm{E}+02$

$0.8200 \mathrm{E}+02$

$0.8000 \mathrm{E}+02$

$0.7900 E+02$

$0.8100 \mathrm{E}+02$

$0.8000 \mathrm{E}+02$

$0.8000 \mathrm{E}+02$

$0.8000 E+02$ 
LIQUID DENSITY $n-\mathrm{C} 8 / \mathrm{CO} 2$ ALS DRES $=4 \quad \mathrm{SFACT}=0.5 \%$

TABLE 3. (CONTINUED)

2 OF 2

$\begin{array}{lccc}\text { TEST } & \text { SM AB ER } & \text { BIAS } & \text { CPU TIME } \\ 33 & 0.3225 E+00 & 0.1203 E+00 & 0.8100 E+02 \\ 34 & 0.7168 E+00 & -.5713 E+00 & 0.7900 E+02 \\ 35 & 0.5706 E+00 & 0.5706 E+00 & 0.8000 E+02 \\ 36 & 0.1276 E+00 & 0.1276 \mathrm{I}+00 & 0.8100 \mathrm{E}+02 \\ 37 & 0.5911 \mathrm{E}+00 & 0.5911 \mathrm{E}+00 & 0.8000 \mathrm{E}+02 \\ 38 & 0.5043 \mathrm{E}+00 & -.4736 \mathrm{E}+00 & 0.8100 \mathrm{E}+02 \\ 39 & 0.6190 \mathrm{E}+00 & 0.6190 \mathrm{E}+00 & 0.7900 \mathrm{E}+02 \\ 40 & 0.2825 \mathrm{E}+00 & -.1960 \mathrm{E}+00 & 0.7900 \mathrm{E}+02 \\ 41 & 0.5299 \mathrm{E}+00 & 0.5299 \mathrm{E}+00 & 0.8000 \mathrm{E}+02 \\ 42 & 0.3959 \mathrm{E}+00 & 0.2139 \mathrm{E}-01 & 0.8000 \mathrm{E}+02 \\ 43 & 0.5137 \mathrm{E}+00 & 0.5137 \mathrm{E}+00 & 0.7900 \mathrm{E}+02 \\ 44 & 0.2548 \mathrm{E}+00 & -.1236 \mathrm{E}+00 & 0.7900 \mathrm{E}+02 \\ 45 & 0.7975 \mathrm{E}+00 & 0.7975 \mathrm{E}+00 & 0.7900 \mathrm{E}+02 \\ 46 & 0.1977 \mathrm{E}+00 & -.8069 \mathrm{E}-01 & 0.8000 \mathrm{E}+02 \\ 47 & 0.4689 \mathrm{E}+00 & 0.4557 \mathrm{E}+00 & 0.8000 \mathrm{E}+02 \\ 48 & 0.5840 \mathrm{E}+00 & -.5469 \mathrm{E}+00 & 0.8000 \mathrm{E}+02 \\ 49 & 0.6956 \mathrm{E}+00 & 0.6956 \mathrm{E}+00 & 0.7900 \mathrm{E}+02 \\ 50 & 0.3126 \mathrm{E}+00 & -.2875 \mathrm{E}+00 & 0.7900 \mathrm{E}+02 \\ 51 & 0.5218 \mathrm{E}+00 & 0.5218 \mathrm{E}+00 & 0.8000 \mathrm{E}+02 \\ 52 & 0.4190 \mathrm{E}+00 & -.3303 \mathrm{E}+00 & 0.7900 \mathrm{E}+02 \\ 53 & 0.6198 \mathrm{E}+00 & 0.6198 \mathrm{E}+00 & 0.7700 \mathrm{E}+02 \\ 54 & 0.8153 \mathrm{E}-01 & 0.2976 \mathrm{E}-01 & 0.8000 \mathrm{E}+02 \\ 55 & 0.2992 \mathrm{E}+00 & 0.1355 \mathrm{E}-01 & 0.7400 \mathrm{E}+02 \\ 56 & 0.6684 \mathrm{E}+00 & -.4453 \mathrm{E}+00 & 0.7400 \mathrm{E}+02 \\ 57 & 0.3308 \mathrm{E}+00 & 0.1614 \mathrm{E}+00 & 0.7700 \mathrm{E}+02 \\ 58 & 0.6817 \mathrm{E}+00 & -.6262 \mathrm{E}+00 & 0.7600 \mathrm{E}+02 \\ 59 & 0.1069 \mathrm{E}+01 & 0.1069 \mathrm{E}+01 & 0.8000 \mathrm{E}+02 \\ 60 & 0.9196 \mathrm{E}-01 & 0.2298 \mathrm{E}-01 & 0.8000 \mathrm{E}+02 \\ 61 & 0.2161 \mathrm{E}+00 & 0.2161 \mathrm{E}+00 & 0.7800 \mathrm{E}+02 \\ 62 & 0.3456 \mathrm{E}+00 & -.1797 \mathrm{E}+00 & 0.7500 \mathrm{E}+02 \\ 63 & 0.7929 \mathrm{E}+00 & 0.7929 \mathrm{E}+00 & 0.7700 \mathrm{E}+02 \\ 64 & 0.3230 \mathrm{E}+00 & 0.1411 \mathrm{E}+00 & 0.8000 \mathrm{E}+02\end{array}$


LIQUID DENSITY $n-C 8 / C O 2$ ALS DRES $=4$ SFACT $=0.5 \%$

TABLE 4 A. CONTRASTS LISTED BY FACTORS

1 OF 1

\section{FACTOR}

$\begin{array}{lrr}\text { E } & 19 & -.2957 \mathrm{E}+00 \\ \mathrm{C} & 1 & -.1666 \mathrm{E}+00 \\ \mathrm{E} & 18 & 0.1634 \mathrm{E}+00 \\ \mathrm{E} & 11 & -.8529 \mathrm{E}-01 \\ \mathrm{C} & 12 & 0.8023 \mathrm{E}-01 \\ \mathrm{E} & 1 & -.7995 \mathrm{E}-01 \\ \mathrm{E} & 20 & -.5666 \mathrm{E}-01 \\ \mathrm{TC} & 2 & 0.5396 \mathrm{E}-01 \\ \mathrm{E} & 3 & -.5248 \mathrm{E}-01 \\ \mathrm{E} & 7 & 0.5100 \mathrm{E}-01 \\ \mathrm{E} & 30 & -.5091 \mathrm{E}-01 \\ \mathrm{TC} & 1 & 0.4329 \mathrm{E}-01 \\ \mathrm{E} & 8 & 0.4209 \mathrm{E}-01 \\ \mathrm{E} & 22 & -.3803 \mathrm{E}-01 \\ \mathrm{C} & 4 & 0.3323 \mathrm{E}-01 \\ \mathrm{C} & 8 & -.3322 \mathrm{E}-01 \\ \mathrm{PC} & 2 & 0.3150 \mathrm{E}-01 \\ \mathrm{E} & 6 & 0.2554 \mathrm{E}-01 \\ \mathrm{PC} & 1 & 0.2298 \mathrm{E}-01 \\ \mathrm{E} & 4 & -.2245 \mathrm{E}-01 \\ \mathrm{E} & 16 & 0.2192 \mathrm{E}-01 \\ \mathrm{C} & 17 & 0.2055 \mathrm{E}-01 \\ \mathrm{E} & 5 & -.1906 \mathrm{E}-01 \\ \mathrm{E} & 29 & -.1870 \mathrm{E}-01 \\ \mathrm{E} & 14 & -.1814 \mathrm{E}-01 \\ \mathrm{E} & 28 & 0.1615 \mathrm{E}-01 \\ \mathrm{E} & 13 & 0.1570 \mathrm{E}-01 \\ \mathrm{E} & 21 & -.1455 \mathrm{E}-01 \\ \mathrm{E} & 24 & -.1341 \mathrm{E}-01 \\ \mathrm{E} & 10 & -.1232 \mathrm{E}-01 \\ \mathrm{E} & 27 & -.1184 \mathrm{E}-01 \\ \mathrm{C} & 7 & 0.1055 \mathrm{E}-01 \\ & & \end{array}$

FAC'LUR

$S M \quad A B \quad E R$

$\begin{array}{lll}\text { C } & 13 & 0.1052 \mathrm{E}-01 \\ \mathrm{E} & 26 & -.9813 \mathrm{E}-02 \\ \mathrm{E} & 17 & -.9372 \mathrm{E}-02 \\ \mathrm{C} & 2 & 0.9341 \mathrm{E}-02 \\ \mathrm{E} & 12 & -.9336 \mathrm{E}-02 \\ \mathrm{E} & 23 & 0.9046 \mathrm{E}-02 \\ \mathrm{C} & 6 & -.8187 \mathrm{E}-02 \\ \mathrm{OM} & 1 & 0.7722 \mathrm{E}-02 \\ \mathrm{C} & 15 & 0.7625 \mathrm{E}-02 \\ \mathrm{C} & 18 & 0.7407 \mathrm{E}-02 \\ \mathrm{E} & 9 & 0.7356 \mathrm{E}-02 \\ \mathrm{C} & 5 & -.7284 \mathrm{E}-02 \\ \mathrm{E} & 15 & 0.6806 \mathrm{E}-02 \\ \mathrm{C} & 11 & 0.6707 \mathrm{E}-02 \\ \mathrm{C} & 16 & 0.5430 \mathrm{E}-02 \\ \mathrm{OM} & 2 & 0.4698 \mathrm{E}-02 \\ \mathrm{E} & 31 & -.3943 \mathrm{E}-02 \\ \mathrm{E} & 25 & 0.3644 \mathrm{E}-02 \\ \mathrm{C} & 10 & 0.2640 \mathrm{E}-02 \\ \mathrm{C} & 14 & 0.2210 \mathrm{E}-02 \\ \mathrm{E} & 2 & -.1976 \mathrm{E}-02 \\ \mathrm{~K} & 112 & -.1548 \mathrm{E}-02 \\ \mathrm{C} & 3 & -.8476 \mathrm{E}-03 \\ \mathrm{C} & 9 & -.6153 \mathrm{E}-03\end{array}$


LIQUID DENSITY $\mathrm{n}-\mathrm{C8} / \mathrm{CO} 2$ ALS DRES $=4 \quad \mathrm{SFACT}=0.5 \%$

TABLE 4 B. CONTRASTS LISTED BY FACTORS 1 OF 1

FACTOR

$\begin{array}{lr}\text { C } & 1 \\ \text { PC } & 1 \\ \text { C } & 12 \\ \text { TC } & 1 \\ \text { C } & 2 \\ \text { C } & 4 \\ \text { C } & 8\end{array}$

PC 2

OM 1

TC 2

C 9

C 17

C 16

OM 2

C 13

C 5

C 6

C 3

C 18

E 19

E 11

E 21

E 1

E 22

C 14

E 26

E 3

E 23

E 20

E 6

E 16

E 8

\section{BIAS}

$-.7381 \mathrm{E}+00$

$0.3862 \mathrm{E}+00$

$0.1906 \mathrm{E}+00$

$-.1889 \mathrm{E}+00$

$0.1026 \mathrm{E}+00$

$0.9725 \mathrm{E}-01$

$-.9504 \mathrm{E}-01$

$0.8365 \mathrm{E}-01$

$0.8272 \mathrm{E}-01$

$0.4161 \mathrm{E}-01$

$-.3031 \mathrm{E}-01$

$0.2512 \mathrm{E}-01$

$0.1745 \mathrm{E}-01$

$0.7271 \mathrm{E}-02$

$0.5739 \mathrm{E}-02$

$0.3775 \mathrm{E}-02$

$-.3704 \mathrm{E}-02$

$-.3684 \mathrm{E}-02$

$-.2205 E-02$

$-.1593 E-02$

$-.1016 \mathrm{E}-02$

$-.7850 \mathrm{E}-03$

$-.6839 E-03$

$-.6368 \mathrm{E}-03$

$0.5682 \mathrm{E}-03$

$0.5057 \mathrm{E}-03$

$-.4942 \mathrm{E}-03$

$0.4864 \mathrm{E}-03$

$-.4849 \mathrm{E}-03$

$0.4445 E-03$

$0.3640 \mathrm{E}-03$

$0.3559 \mathrm{E}-03$
FACTOR

BIAS

$\begin{array}{lrll}\mathrm{E} & 25 & 0.3533 \mathrm{E}-03 \\ \mathrm{E} & 18 & 0.3322 \mathrm{E}-03 \\ \mathrm{E} & 7 & 0.3247 \mathrm{E}-03 \\ \mathrm{E} & 30 & -.2825 \mathrm{E}-03 \\ \mathrm{E} & 4 & -.2552 \mathrm{E}-03 \\ \mathrm{E} & 14 & 0.2375 \mathrm{E}-03 \\ \mathrm{C} & 10 & 0.2148 \mathrm{E}-03 \\ \mathrm{C} & 7 & 0.2037 \mathrm{E}-03 \\ \mathrm{E} & 29 & -.1825 \mathrm{E}-03 \\ \mathrm{E} & 24 & 0.1710 \mathrm{E}-03 \\ \mathrm{E} & 5 & 0.1653 \mathrm{E}-03 \\ \mathrm{E} & 17 & -.1 .596 \mathrm{E}-03 \\ \mathrm{E} & 12 & -.1443 \mathrm{E}-03 \\ \mathrm{C} & 11 & 0.1429 \mathrm{E}-03 \\ \mathrm{C} & 15 & -.1 .086 \mathrm{E}-03 \\ \mathrm{E} & 28 & 0.9623 \mathrm{E}-04 \\ \mathrm{E} & 13 & 0.8313 \mathrm{E}-04 \\ \mathrm{E} & 9 & 0.5962 \mathrm{E}-04 \\ \mathrm{E} & 2 & 0.5873 \mathrm{E}-04 \\ \mathrm{E} & 10 & -.5846 \mathrm{E}-04 \\ \mathrm{E} & 31 & -.3707 \mathrm{E}-04 \\ \mathrm{E} & 15 & 0.6541 \mathrm{E}-05 \\ \mathrm{E} & 27 & 0.3425 \mathrm{E}-05 \\ \mathrm{~K} & 12 & -.3549 \mathrm{E}-06\end{array}$


LIQUID DENSITY $n-\mathrm{C} 8 / \mathrm{CC} / 2$ ALS DRES $=4 \quad \mathrm{SFACT}=0.5 \%$

TABLE $4 \mathrm{C}$. CONTRASTS LISTED BY FACTORS

1 OF 1

\begin{tabular}{|c|c|c|c|c|}
\hline FACI & TOR & CPU TIME & FACTOR & CPU TIME \\
\hline C & 6 & $-.1500 E+01$ & C 16 & $-.1875 E+00$ \\
\hline C & 5 & $-.1125 E+01$ & C 18 & $-.1875 \mathrm{E}+00$ \\
\hline $\mathrm{E}$ & 11 & $-.8750 E+00$ & TC 2 & $0.1875 \mathrm{E}+00$ \\
\hline $\mathrm{E}$ & 12 & $-.8750 E+00$ & $E$ & $0.1875 E+00$ \\
\hline $\mathrm{E}$ & 15 & $-.8750 E+00$ & $\mathrm{E}$ & $0.1875 \mathrm{E}+00$ \\
\hline $\mathrm{E}$ & 29 & $0.8125 \mathrm{E}+00$ & $\mathrm{E}$ & $0.1875 \mathrm{E}+00$ \\
\hline $\mathrm{E}$ & 17 & $0.7500 E+00$ & E 23 & $0.1875 \mathrm{E}+00$ \\
\hline OM & 1 & $0.6875 \mathrm{E}+00$ & E 24 & $0.1875 \mathrm{E}+00$ \\
\hline $\mathrm{E}$ & 10 & $0.6250 E+00$ & E 26 & $0.1875 \mathrm{E}+00$ \\
\hline $\mathrm{E}$ & 20 & $0.6250 E+00$ & C 3 & $-.1250 \mathrm{E}+00$ \\
\hline$E$ & 6 & $-.5625 \mathrm{E}+00$ & C 8 & $-.1250 E+00$ \\
\hline $\mathrm{E}$ & 7 & $0.5625 \mathrm{E}+0 \mathrm{D}$ & C 10 & $0.1250 \mathrm{E}+00$ \\
\hline $\mathrm{E}$ & 28 & $-.5625 E+00$ & OM 2 & $0.1250 \mathrm{E}+00$ \\
\hline$E$ & 14 & $-.5000 E+00$ & $E$ & $0.1250 E+00$ \\
\hline C 1 & 11 & $-.4375 \mathrm{E}+00$ & E 18 & $-.1250 E+00$ \\
\hline C 1 & 17 & $0.4375 \mathrm{E}+00$ & E 31 & $0.1250 E+00$ \\
\hline$E$ & 3 & $-.4375 E+00$ & C 1 & $0.6250 \mathrm{E}-01$ \\
\hline $\mathrm{E}$ & 27 & $0.4375 E+00$ & C 12 & $-.6250 E-01$ \\
\hline C & 4 & $0.3750 \mathrm{E}+00$ & C 14 & $0.6250 \mathrm{E}-01$ \\
\hline C & 9 & $0.3750 E+00$ & $\mathrm{E}$ & $0.6250 E-01$ \\
\hline $\mathrm{E}$ & 30 & $0.3750 E+00$ & E 22 & $0.6250 E-01$ \\
\hline C 1 & 15 & $0.3125 E+00$ & C 7 & $0.0000 E+00$ \\
\hline $\mathrm{TC}$ & 1 & $-.3125 \mathrm{E}+00$ & PC 2 & $0.0000 E+00$ \\
\hline PC & 1 & $0.3125 \mathrm{E}+00$ & K 12 & $0.0000 E+00$ \\
\hline $\mathrm{E}$ & 2 & $0.3125 E+00$ & & \\
\hline$E$ & 25 & $-.3125 E+00$ & & \\
\hline$E$ & 13 & $-.2500 E+00$ & & \\
\hline $\mathrm{E}$ & 16 & $0.2500 E+00$ & & \\
\hline $\mathrm{E}$ & 19 & $0.2500 E+00$ & & \\
\hline $\bar{E}$ & 21 & $-.2500 \mathrm{E}+00$ & & \\
\hline $\mathrm{C}$ & 2 & $-.1875 E+00$ & & \\
\hline C 1 & 13 & $0.1875 E+00$ & & \\
\hline
\end{tabular}


LIQUID DENSITY $n-C 10 / C O 2$ ALS DRES $=4 \quad S F A C T=0.5 \%$ 


\section{LIQUID DENSITY n-C10 / CO2 ALS DRES $=4$ SFACT $=0.5 \%$}

TABLE 3. RESPONSES

TEST

1

2

3

4

5

6

7

8

9
$S M A B$ ER

$0.6048 E+01$

$0.5936 \mathrm{E}+01$

$0.4787 \mathrm{E}+01$

$0.5093 E+01$

$0.5605 E+01$

$0.6996 \mathrm{E}+01$

$0.5121 \mathrm{E}+01$

$0.5076 E+01$

$0.6380 E+01$

$0.5881 \mathrm{E}+01$

$0.5147 \mathrm{E}+01$

$0.5130 E+01$

$0.5373 \mathrm{E}+01$

$0.6351 \mathrm{E}+01$

$0.5417 \mathrm{E}+01$

$0.5017 \mathrm{E}+01$

$0.5269 E+01$

$0.5325 E+01$

$0.5783 E+01$

$0.6711 E+01$

$0.4963 E+01$

$0.4928 \mathrm{E}+01$

$0.6166 \mathrm{E}+01$

$0.5694 \mathrm{E}+01$

$0.5178 E+01$

$0.4829 E+01$

$0.5139 E+01$

$0.6483 \mathrm{E}+01$

$0.4950 \mathrm{E}+01$

$0.5272 \mathrm{E}+01$

$0.6159 \mathrm{E}+01$

$0.6063 E+01$
BIAS

$-.4162 \mathrm{E}+01$

$-.4353 \mathrm{E}+01$

$-.2040 \mathrm{E}+01$

$-.1342 \mathrm{E}+01$

$-.3125 \mathrm{E}+01$

$-.5679 E+01$

$-.1092 \mathrm{E}+01$

$-.2832 \mathrm{E}+01$

$-.4387 \mathrm{E}+01$

$-.3826 \mathrm{E}+01$

-.1892E+01

$-.1969 E+01$

$-.2839 \mathrm{E}+01$

$-.4747 \mathrm{E}+01$

$-.4786 E+00$

$-.2963 E+01$

$-.8925 \mathrm{E}+00$

$-.3363 E+01$

$-.3381 E+01$

$-.5178 E+01$

$-.1787 \mathrm{E}+01$

$-.1910 E+01$

$-.4346 \mathrm{E}+01$

$-.3837 \mathrm{E}+01$

$-.9475 E+00$

$-.2595 \mathrm{E}+01$

$-.2386 E+01$

$-.4993 E+01$

$-.2329 E+01$

$-.1659 \mathrm{E}+01$.

$-.3936 \mathrm{E}+01$.

$-.4170 \mathrm{E}+01$
1 OF 2

CPU TIME

$0.6200 \mathrm{E}+02$

$0.6000 \mathrm{E}+02$

$0.6000 \mathrm{E}+02$

$0.6200 \mathrm{E}+02$

$0.6100 \mathrm{E}+02$

$0.5900 \mathrm{E}+02$

$0.6000 \mathrm{E}+02$

$0.6100 \mathrm{E}+02$

$0.6200 \mathrm{E}+02$

$0.6100 \mathrm{E}+02$

$0.6100 E+02$

$0.6100 \mathrm{E}+02$

$0.6000 \mathrm{E}+02$

$0.6000 \mathrm{E}+02$

$0.5700 \mathrm{E}+02$

$0.5800 \mathrm{E}+02$

$0.6000 \mathrm{E}+02$

$0.5600 E+02$

$0.6100 \mathrm{E}+02$

$0.6100 E+02$

$0.6000 E+02$

$0.6100 \mathrm{E}+02$

$0.6000 \mathrm{E}+02$

$0.6100 \mathrm{E}+02$

$0.6200 E+02$

$0.6100 \mathrm{E}+02$

$0.6100 E+02$

$0.6000 \mathrm{E}+02$

$0.6000 E+02$

$0.6000 E+02$

$0.6200 E+02$

$0.6000 \mathrm{E}+02$ 
LIQUID DENSITY $n-\mathrm{C} 10 / \mathrm{CO} 2$ ALS DRES $=4$ SFACT $=0.5 \%$

TABLE 3. (CONTINUED)

2 OF 2

$\begin{array}{lccc}\text { TEST } & \text { SM AB ER } & \text { BIAS } & \text { CPU TIME } \\ 33 & 0.5040 E+01 & -.1828 E+01 & 0.5900 E+02 \\ 34 & 0.4931 E+01 & -.2134 E+01 & 0.6000 E+02 \\ 35 & 0.6165 E+01 & -.4327 E+01 & 0.6000 E+02 \\ 36 & 0.5611 E+01 & -.3628 E+01 & 0.5900 E+02 \\ 37 & 0.5346 E+01 & -.9092 E+00 & 0.6100 E+02 \\ 38 & 0.5414 E+01 & -.3562 E+01 & 0.6100 E+02 \\ 39 & 0.5794 E+01 & -.3409 E+01 & 0.6100 E+02 \\ 40 & 0.6626 E+01 & -.5019 E+01 & 0.6000 E+02 \\ 41 & 0.4933 E+01 & -.2115 E+01 & 0.6000 E+02 \\ 42 & 0.5189 E+01 & -.1641 E+01 & 0.6000 E+02 \\ 43 & 0.6242 E+01 & -.4158 E+01 & 0.6000 E+02 \\ 44 & 0.6066 E+01 & -.4210 E+01 & 0.6200 E+02 \\ 45 & 0.5169 E+01 & -.7725 E+00 & 0.5900 E+02 \\ 46 & 0.4835 E+01 & -.2618 E+01 & 0.6000 E+02 \\ 47 & 0.5233 E+01 & -.2593 E+01 & 0.6000 E+02 \\ 48 & 0.6490 E+01 & -.5014 E+01 & 0.6400 E+02 \\ 49 & 0.5576 E+01 & -.3019 E+01 & 0.6300 E+02 \\ 50 & 0.6876 E+01 & -.5407 E+01 & 0.6000 E+02 \\ 51 & 0.5223 E+01 & -.1190 E+01 & 0.5800 E+02 \\ 52 & 0.5190 E+01 & -.3121 E+01 & 0.5700 E+02 \\ 53 & 0.6017 E+01 & -.4029 E+01 & 0.6000 E+02 \\ 54 & 0.5828 E+01 & -.4045 E+01 & 0.6000 E+02 \\ 55 & 0.4887 E+01 & -.2178 E+01 & 0.5900 E+02 \\ 56 & 0.5118 E+01 & -.1668 E+01 & 0.6200 E+02 \\ 57 & 0.5489 E+01 & -.3133 E+01 & 0.6100 E+02 \\ 58 & 0.6387 E+01 & -.4848 E+01 & 0.6100 E+02 \\ 59 & 0.5403 E+01 & -.1809 E+00 & 0.6100 E+02 \\ 60 & 0.4984 E+01 & -.2855 E+01 & 0.6200 E+02 \\ 61 & 0.6485 E+01 & -.4705 E+01 & 0.6100 E+02 \\ 62 & 0.5908 E+01 & -.3959 E+01 & 0.6400 E+02 \\ 63 & 0.5129 E+01 & -.1570 E+01 & 0.6400 E+02 \\ 64 & 0.5034 E+01 & -.1836 E+01 & 0.6000 E+02\end{array}$


LIQUID DENSITY $n-C 10 / C O 2$ ALS DRES $=4 \quad S F A C T=0.5 \%$

TABLE 4 A. CONTRASTS LISTED BY FACTORS 1 OF 1

\section{FACTOR}

$\begin{array}{lr}\text { TC } & 2 \\ \mathrm{E} & 18 \\ \mathrm{C} & 12 \\ \mathrm{PC} & 2 \\ \mathrm{E} & 21 \\ \mathrm{C} & 1 \\ \mathrm{E} & 25 \\ \mathrm{E} & 27 \\ \mathrm{E} & 11\end{array}$

TC 1

C 8

PC 1

OM 2

E 7

E 22

E 3

C 4

E 23

E 26

E 29

E 5

E 15

E 17

C 2

E 1

C 9

OM 1

K 12

C 6

C 7

E 6

E 8
SM AB ER

$-.9448 \mathrm{E}+00$

$0.4350 \mathrm{E}+00$

$-.2902 \mathrm{E}+00$

$0.2159 \mathrm{E}+00$

$-.2141 \mathrm{E}+00$

$0.1769 \mathrm{E}+00$

$-.1402 \mathrm{E}+00$

$-.8045 \mathrm{E}-01$

$-.7899 \mathrm{E}-01$

$-.6251 \mathrm{E}-01$

$0.5874 \mathrm{E}-01$

$-.5532 \mathrm{E}-01$

$-.5205 \mathrm{E}-01$

$0.4942 \mathrm{E}-01$

$0.4904 \mathrm{E}-01$

$-.4594 \mathrm{E}-01$

$-.4368 \mathrm{E}-01$

-.4083E-01

$. .4040 \mathrm{E}-01$

$0.3778 \mathrm{E}-01$

$-.2962 \mathrm{E}-01$

$0.2808 \mathrm{E}-01$

$0.1921 \mathrm{E}-01$

$-.1642 \mathrm{E}-01$

$-.1469 \mathrm{E}-01$

$0.1239 \mathrm{E}-01$

$-.1189 \mathrm{E}-01$

$-.1182 \mathrm{E}-01$

$0.1089 \mathrm{E}-01$

$-.1087 E-01$

$-.9623 \mathrm{E}-02$

$0.9415 \mathrm{E}-02$

\section{FACTOR}

E 24

C 16

C 18

E 13

E 9

E 14

C 10

E 16

C 17

C 14

E 20

C 13

E 19

C 3

E 4

E 31

E 12

E 30

C 11

E 28

E 2

E 10

C 15

C 5
$S M \quad A B E R$

$-.8592 \mathrm{E}-02$

$-.6821 \mathrm{E}-02$

$0.4125 \mathrm{E}-02$

$0.2394 \mathrm{E}-02$

$0.2220 \mathrm{E}-02$

$0.2208 \mathrm{E}-02$

$-.2060 \mathrm{E}-02$

$0.1623 \mathrm{E}-02$

$0.1489 \mathrm{E}-02$

-.1476E-02

-.1389E-02

$0.1373 \mathrm{E}-02$

-. $1310 \mathrm{E}-02$

$0.1227 \mathrm{E}-02$

$-.9877 \mathrm{E}-03$

$0.9284 \mathrm{E}-03$

$-.7492 \mathrm{E}-03$

$-.6802 E-03$

$0.4803 \mathrm{E}-03$

$0.4749 \mathrm{E}-03$

$0.3452 \mathrm{E}-03$

$0.2808 \mathrm{E}-03$

$-.2172 \mathrm{E}-03$

$0.1559 \mathrm{E}-03$ 
LIQUID DENSITY $n-C 10 / C O 2$ ALS DRES $=4$ SFACT $=0.5 \%$

TABLE 4 B. CONTRASTS LISTED BY FACTORS

1 OF 1

FACTOR

$\begin{array}{lcl}\text { TC } & 2 & 0.2205 \mathrm{E}+01 \\ \mathrm{C} & 12 & 0.1189 \mathrm{E}+01 \\ \mathrm{C} & 1 & -.9639 \mathrm{E}+00 \\ \mathrm{C} & 8 & -.3809 \mathrm{E}+00 \\ \text { PC } & 2 & -.2489 \mathrm{E}+00 \\ \mathrm{C} & 4 & 0.2022 \mathrm{E}+00 \\ \text { PC } & 1 & 0.1591 \mathrm{E}+00 \\ \mathrm{C} & 2 & 0.1104 \mathrm{E}+00 \\ \mathrm{C} & 9 & -.9326 \mathrm{E}-01 \\ \text { TC } & 1 & 0.5580 \mathrm{E}-01 \\ \text { OM } & 1 & 0.5188 \mathrm{E}-01 \\ \mathrm{E} & 21 & -.2770 \mathrm{E}-01 \\ \mathrm{E} & 11 & -.2608 \mathrm{E}-01 \\ \mathrm{C} & 13 & 0.2111 \mathrm{E}-01 \\ \mathrm{E} & 27 & 0.1923 \mathrm{E}-01 \\ \mathrm{C} & 17 & 0.1581 \mathrm{E}-01 \\ \mathrm{OM} & 2 & 0.1455 \mathrm{E}-01 \\ \mathrm{E} & 29 & 0.1202 \mathrm{E}-01 \\ \mathrm{E} & 26 & -.9579 \mathrm{E}-02 \\ \mathrm{E} & 7 & 0.9461 \mathrm{E}-02 \\ \mathrm{E} & 25 & -.9162 \mathrm{E}-02 \\ \mathrm{C} & 6 & -.7647 \mathrm{E}-02 \\ \mathrm{C} & 5 & 0.6527 \mathrm{E}-02 \\ \mathrm{E} & 22 & 0.6344 \mathrm{E}-02 \\ \mathrm{E} & 15 & 0.5524 \mathrm{E}-02 \\ \mathrm{C} & 3 & -.3588 \mathrm{E}-02 \\ \mathrm{E} & 3 & -.3564 \mathrm{E}-02 \\ \mathrm{E} & 1 & -.3514 \mathrm{E}-02 \\ \mathrm{~K} & 12 & 0.2308 \mathrm{E}-02 \\ \mathrm{C} & 14 & 0.2304 \mathrm{E}-02 \\ \mathrm{E} & 8 & 0.2203 \mathrm{E}-02 \\ \mathrm{E} & 6 & -.2080 \mathrm{E}-02\end{array}$

FACTOR BIAS

$\begin{array}{ccc}\text { E } & 12 & -.2077 \mathrm{E}-02 \\ \mathrm{E} & 14 & 0.1947 \mathrm{E}-02 \\ \mathrm{E} & 23 & 0.1931 \mathrm{E}-02 \\ \mathrm{E} & 17 & 0.1767 \mathrm{E}-02 \\ \mathrm{E} & 19 & -.1723 \mathrm{E}-02 \\ \mathrm{E} & 5 & -.1704 \mathrm{E}-02 \\ \mathrm{E} & 13 & 0.9411 \mathrm{E}-03 \\ \mathrm{C} & 10 & 0.8568 \mathrm{E}-03 \\ \mathrm{E} & 16 & 0.7319 \mathrm{E}-03 \\ \mathrm{E} & 4 & -.7221 \mathrm{E}-03 \\ \mathrm{E} & 20 & -.3952 \mathrm{E}-03 \\ \mathrm{C} & 11 & -.3514 \mathrm{E}-03 \\ \mathrm{C} & 16 & -.2735 \mathrm{E}-03 \\ \mathrm{E} & 2 & 0.2633 \mathrm{E}-03 \\ \mathrm{E} & 24 & 0.2358 \mathrm{E}-03 \\ \mathrm{E} & 18 & 0.2131 \mathrm{E}-03 \\ \mathrm{C} & 18 & -.2121 \mathrm{E}-03 \\ \mathrm{E} & 31 & 0.2067 \mathrm{E}-03 \\ \mathrm{E} & 30 & 0.1845 \mathrm{E}-03 \\ \mathrm{E} & 10 & 0.1781 \mathrm{E}-03 \\ \mathrm{E} & 9 & 0.1466 \mathrm{E}-03 \\ \mathrm{E} & 28 & -.7013 \mathrm{E}-04 \\ \mathrm{C} & 7 & 0.6116 \mathrm{E}-04 \\ \mathrm{C} & 15 & -.4098 \mathrm{E}-04\end{array}$


LIQUID DENSITY $n-\mathrm{C} 10 / \mathrm{CO} 2$ ALS DRES $=4 \quad \mathrm{SFACT}=0.5 \%$

TABLE 4 C. CONTRASTS LISTED BY FACTORS 1 OF 1

\begin{tabular}{|c|c|c|c|c|}
\hline FAC & TOR & CPU TIME & FACTOR & CPU TIME \\
\hline$O M$ & 1 & $0.8750 \mathrm{E}+00$ & C 18 & $0.1875 \mathrm{E}+00$ \\
\hline TC & 2 & $-.7500 E+00$ & PC 2 & $0.1875 \mathrm{E}+00$ \\
\hline $\mathrm{E}$ & 12 & $0.7500 \mathrm{E}+00$ & E 15 & $0.1875 \mathrm{E} \cdot 00$ \\
\hline $\mathrm{E}$ & 13 & $0.6875 E+00$ & $\mathrm{E} \quad 16$ & $-.1875 \mathrm{E}+00$ \\
\hline$E$ & 22 & $-.6875 E+00$ & 6 & $0.1 \div 50 \mathrm{E}+00$ \\
\hline C & 4 & $0.6250 \mathrm{E}+00$ & 21 & $0.1250 \mathrm{E}+00$ \\
\hline $\mathrm{E}$ & 19 & $-.6250 E+00$ & 25 & $-.1250 \mathrm{E}+0$ \\
\hline$E$ & 14 & $0.5625 \mathrm{E}+00$ & 28 & $0.1250 E+0$ \\
\hline $\mathrm{E}$ & 10 & $-.5000 E+00$ & 30 & $0.1250 \mathrm{E}+00$ \\
\hline $\mathrm{E}$ & 11 & $0.5000 E+00$ & $C$ & $-.6250 E-0$ \\
\hline C & 8 & $-.4375 E+00$ & $\mathrm{C}$ & $0.6250 \mathrm{E}-01$ \\
\hline TC & 1 & $0.4375 \mathrm{E}+00$ & C 16 & $-.6250 E-0$ \\
\hline$K$ & 12 & $-.4375 E+00$ & C 17 & $-.6250 E-0$ \\
\hline $\mathrm{E}$ & 2 & $0.4375 E+00$ & 7 & $0.62 .50 \mathrm{E}-0$ \\
\hline$E$ & 17 & $-.4375 E+00$ & 8 & $-.6250 \mathrm{E}-0$ \\
\hline $\mathrm{C}$ & 7 & $-.3750 E+00$ & 9 & $-.6250 E-0$ \\
\hline$E$ & 1 & $0.3750 E+00$ & 18 & $0.6250 \mathrm{E}-0$ \\
\hline $\mathrm{E}$ & 4 & $-.3750 \mathrm{E}+00$ & 23 & $-.6250 \mathrm{E}-0$ \\
\hline$E$ & 5 & $0.3750 E+00$ & 24 & $-.6250 \mathrm{E}-0$ \\
\hline $\mathrm{E}$ & 27 & $-.3750 E+00$ & 29 & $-.6250 \mathrm{E}-0$ \\
\hline $\mathrm{C}$ & 9 & $-.3125 \mathrm{E}+00$ & C 2 & $0.0000 E+0$ \\
\hline $\mathrm{C}$ & 10 & $-.3125 E+00$ & C 13 & $0.0000 \mathrm{E}+0$ \\
\hline $\mathrm{C}$ & 14 & $-.3125 \mathrm{E}+00$ & PC 1 & $0.0000 \mathrm{E}+0$ \\
\hline C & 15 & $0.3125 \mathrm{E}+00$ & E 26 & $0.0000 \mathrm{E}+0$ \\
\hline OM & 2 & $0.3125 E+00$ & & \\
\hline $\mathrm{E}$ & 31 & $-.3125 E+00$ & & \\
\hline C & 5 & $0.2500 \mathrm{E}+00$ & & \\
\hline C & 6 & $0.2500 E+00$ & & \\
\hline $\mathrm{C}$ & 11 & $-.2500 E+00$ & & \\
\hline C & 12 & $0.2500 E+00$ & & \\
\hline $\mathrm{E}$ & 3 & $0.2500 \mathrm{E}+00$ & & \\
\hline $\mathrm{E}$ & 20 & $0.2500 E+00$ & & \\
\hline
\end{tabular}


CRITICAL LINE OUTPUT ALS CO2 / C4 DRES=4 . 09/20/90 
CRITICAL LINE OUTPUT ALS CO2 / C4 DRES $=4$. 09/20/90

TABLE 3. RESPONSES

TEST

1 .

2

\section{RT SQ ER}

$0.6457 E+01$

$0.6819 \mathrm{E}+01$

$0.6524 \mathrm{E}+01$

$0.6600 \mathrm{E}+01$

$0.6647 \mathrm{E}+01$

$0.6550 \mathrm{E}+01$

$0.6777 \mathrm{E}+01$

$0.6200 E+01$

$0.6307 \mathrm{E}+01$

$0.6463 \mathrm{E}+01$

$0.6768 \mathrm{E}+01$

$0.6069 \mathrm{E}+01$

$0.6303 \mathrm{E}+01$

$0.65+2 \mathrm{E}+01$

$0.7068 \mathrm{E}+01$

$0.1238 E+03$

$0.6969 \mathrm{E}+01$

$0.6240 \mathrm{E}+01$

$0.6518 \mathrm{E}+01$

$0.6403 \mathrm{E}+01$

$0.6748 \mathrm{E}+01$

$0.6526 \mathrm{E}+01$

$0.6671 E+01$

$0.6698 \mathrm{E}+01$

$0.6960 \mathrm{E}+01$

$0.5919 \mathrm{E}+01$

$0.6414 E+01$

$0.6583 E+01$

$0.6700 \mathrm{E}+01$

$0.6043 E+01$

$0.6444 E+01$
$0.6168 \mathrm{E}+01$
RMS BIAS

$-.4845 \mathrm{E}+01$

$-.5468 \mathrm{E}+01$

$-.4894 \mathrm{E}+01$

$-.4910 \mathrm{E}+01$

$-.4039 E+01$

$-.5097 \mathrm{E}+01$

$-.4844 \mathrm{E}+01$

$-.5367 \mathrm{E}+01$

$-.4449 E+01$

$-.4558 \mathrm{E}+01$

$-.4526 E+01$

$-.5037 \mathrm{E}+01$

$-.4086 E+01$

$-.4650 \mathrm{E}+01$

$-.4824 E+01$

$-.5861 E+01$

$-.1222 \mathrm{E}+03$

$-.5727 \mathrm{E}+01$

$-.4194 \mathrm{E}+01$

$-.4816 \mathrm{E}+01$

$-.4653 \mathrm{E}+01$

$-.5234 \mathrm{E}+01$

$-.4996 E+01$

$-.5172 \mathrm{E}+01$

$-.5069 E+01$

$-.5645 \mathrm{E}+01$

$-.3785 \mathrm{E}+01$

$-.4883 \mathrm{E}+01$

$-.4776 \mathrm{E}+01$

$-.4866 \mathrm{E}+01$

$-.4131 \mathrm{E}+01$

$-.4826 \mathrm{E}+01$
1 OF 2

CPU TIME

$0.2510 E+03$

$0.2580 \mathrm{E}+03$

$0.2570 \mathrm{E}+03$

$0.2520 \mathrm{E}+03$

$0.2630 \mathrm{E}+03$

$0.2560 \mathrm{E}+03$

$0.2600 \mathrm{E}+03$

$0.2540 \mathrm{E}+03$

$0.2540 E+03$

$0.2550 \mathrm{E}+03$

$0.2530 E+03$

$0.2630 \mathrm{E}+03$

$0.2620 \mathrm{E}+03$

$0.2650 E+03$

$0.2580 \mathrm{E}+03$

$0.2580 \mathrm{E}+03$

$0.3570 \mathrm{E}+03$

$0.2660 \mathrm{E}+03$

$0.2550 \mathrm{E}+03$

$0.2600 \mathrm{E}+03$

$0.2630 \mathrm{E}+03$

$0.2630 \mathrm{E}+03$

$0.2580 \mathrm{E}+03$

$0.2560 \mathrm{E}+03$

$0.2730 \mathrm{E}+03$

$0.269 \mathrm{CE}+03$

$0.2730 \mathrm{E}+03$

$0.2850 \mathrm{E}+03$

$0.2700 \mathrm{E}+03$

$0.2610 \mathrm{E}+03$

$0.2730 \mathrm{E}+03$

$0.2630 \mathrm{E}+03$ 
CRITICAL LINE OUTPUT ALS CO2 / C4 DRES $=4$. 09/20/90

TABLE 3. (CONTINUED)

2 OF 2

$\begin{array}{lccc}\text { TEST } & \text { RT SQ ER } & \text { RMS BIAS } & \text { CPU TIME } \\ 33 & 0.6587 \mathrm{E}+01 & -.4844 \mathrm{E}+01 & 0.2540 \mathrm{E}+03 \\ 34 & 0.6974 \mathrm{E}+01 & -.5516 \mathrm{E}+01 & 0.2700 \mathrm{E}+03 \\ 35 & 0.6341 \mathrm{E}+01 & -.4814 \mathrm{E}+01 & 0.2650 \mathrm{E}+03 \\ 36 & 0.6442 \mathrm{E}+01 & -.4893 \mathrm{E}+01 & 0.2610 \mathrm{E}+03 \\ 37 & 0.6611 \mathrm{E}+01 & -.4781 \mathrm{E}+01 & 0.2460 \mathrm{E}+03 \\ 38 & 0.7180 \mathrm{E}+01 & -.5975 \mathrm{E}+01 & 0.2650 \mathrm{E}+03 \\ 39 & 0.6088 \mathrm{E}+01 & -.4058 \mathrm{E}+01 & 0.2650 \mathrm{E}+03 \\ 40 & 0.6323 \mathrm{E}+01 & -.4588 \mathrm{E}+01 & 0.2730 \mathrm{E}+03 \\ 41 & 0.6359 \mathrm{E}+01 & -.4502 \mathrm{E}+01 & 0.2620 \mathrm{E}+03 \\ 42 & 0.6517 \mathrm{E}+01 & -.4685 \mathrm{E}+01 & 0.2490 \mathrm{E}+03 \\ 43 & 0.6273 \mathrm{E}+01 & -.4417 \mathrm{E}+01 & 0.2610 \mathrm{E}+03 \\ 44 & 0.6630 \mathrm{E}+01 & -.5019 \mathrm{E}+01 & 0.2390 \mathrm{E}+03 \\ 45 & 0.6505 \mathrm{E}+01 & -.4839 \mathrm{E}+01 & 0.2690 \mathrm{E}+03 \\ 46 & 0.6810 \mathrm{E}+01 & -.5512 \mathrm{E}+01 & 0.2680 \mathrm{E}+03 \\ 47 & 0.6116 \mathrm{E}+01 & -.4018 \mathrm{E}+01 & 0.2560 \mathrm{E}+03 \\ 48 & 0.6577 \mathrm{E}+01 & -.5032 \mathrm{E}+01 & 0.2670 \mathrm{E}+03 \\ 49 & 0.6005 \mathrm{E}+01 & -.3873 \mathrm{E}+01 & 0.2710 \mathrm{E}+03 \\ 50 & 0.6436 \mathrm{E}+01 & -.4833 \mathrm{E}+01 & 0.2640 \mathrm{E}+03 \\ 51 & 0.2015 \mathrm{E}+03 & -.2001 \mathrm{E}+03 & 0.4150 \mathrm{E}+03 \\ 52 & 0.6991 \mathrm{E}+01 & -.5629 \mathrm{E}+01 & 0.2730 \mathrm{E}+03 \\ 53 & 0.6259 \mathrm{E}+01 & -.4630 \mathrm{E}+01 & 0.2600 \mathrm{E}+03 \\ 54 & 0.6576 \mathrm{E}+01 & -.5158 \mathrm{E}+01 & 0.2460 \mathrm{E}+03 \\ 55 & 0.6714 \mathrm{E}+01 & -.5103 \mathrm{E}+01 & 0.2700 \mathrm{E}+03 \\ 56 & 0.6828 \mathrm{E}+01 & -.5207 \mathrm{E}+01 & 0.2550 \mathrm{E}+03 \\ 57 & 0.6272 \mathrm{E}+01 & -.4336 \mathrm{E}+01 & 0.2690 \mathrm{E}+03 \\ 58 & 0.6470 \mathrm{E}+01 & -.4815 \mathrm{E}+01 & 0.2460 \mathrm{E}+03 \\ 59 & 0.6388 \mathrm{E}+01 & -.4564 \mathrm{E}+01 & 0.2650 \mathrm{E}+03 \\ 60 & 0.6903 \mathrm{E}+01 & -.5692 \mathrm{E}+01 & 0.2520 \mathrm{E}+03 \\ 61 & 0.6438 \mathrm{E}+01 & -.4757 \mathrm{E}+01 & 0.2460 \mathrm{E}+03 \\ 62 & 0.6499 \mathrm{E}+01 & -.4768 \mathrm{E}+01 & 0.2820 \mathrm{E}+03 \\ 63 & 0.6231 \mathrm{E}+01 & -.4227 \mathrm{E}+01 & 0.2660 \mathrm{E}+03 \\ 64 & 0.6574 \mathrm{E}+01 & -.4825 \mathrm{E}+01 & 0.2690 \mathrm{E}+03\end{array}$


CRITICAL LINE OUTPUT ALS CO2 / C4 DRES $=4$. 09/20/90

TABLE 4 A. CONTRASTS IISTED BY FACTORS 1 OF 1

\begin{tabular}{|c|c|c|c|c|c|}
\hline \multicolumn{2}{|c|}{ FACTOR } & RT SQ ER & \multicolumn{2}{|c|}{ FACTOR } & RT SQ ER \\
\hline $\mathrm{TC}$ & 2 & $0.1007 E+02$ & $\mathrm{E}$ & 30 & $0.2421 E+01$ \\
\hline $\mathrm{TC}$ & 1 & $-.9909 E+01$ & $\mathrm{E}$ & 1 & $-.2421 E+01$ \\
\hline C & 4 & $-.9849 E+01$ & $E$ & 8 & $0.2420 E+01$ \\
\hline $\mathrm{E}$ & 11 & $-.9761 E+01$ & C & 6 & $0.2420 \mathrm{E}+01$ \\
\hline$O M$ & 1 & $-.9758 E+01$ & $\mathrm{E}$ & 6 & $-.2420 E+01$ \\
\hline $\mathrm{E}$ & 27 & $0.9757 \mathrm{E}+01$ & $\mathrm{E}$ & 17 & $0.2420 \mathrm{E}+01$ \\
\hline $\mathrm{E}$ & 4 & $-.9754 \mathrm{E}+01$ & C & 15 & $0.2419 \mathrm{E}+01$ \\
\hline $\mathrm{E}$ & 10 & $0.9754 \mathrm{E}+01$ & $\mathrm{E}$ & 5 & $-.2419 E+01$ \\
\hline C & 11 & $-.9754 \mathrm{E}+01$ & C & 7 & $0.2419 \mathrm{E}+01$ \\
\hline C & 10 & $-.9754 \mathrm{E}+01$ & $\mathrm{E}$ & 15 & $0.2419 \mathrm{E}+01$ \\
\hline $\mathrm{E}$ & 2 & $0.9754 \mathrm{E}+01$ & $\mathrm{E}$ & 25 & $0.2419 \mathrm{E}+01$ \\
\hline $\mathrm{E}$ & 9 & $0.9754 \mathrm{E}+01$ & C & 18 & $-.2419 E+01$ \\
\hline C & 14 & $0.9754 \mathrm{E}+01$ & $\mathrm{E}$ & 14 & $-.2418 E+01$ \\
\hline $\mathrm{E}$ & 28 & $-.9754 \mathrm{E}+01$ & C & 13 & $0.2418 E+01$ \\
\hline $\mathrm{E}$ & 20 & $0.9754 \mathrm{E}+01$ & $\mathrm{E}$ & 19 & $0.2418 \mathrm{E}+01$ \\
\hline $\mathrm{E}$ & 31 & $-.9753 E+01$ & $\mathrm{E}$ & 16 & $-.2418 \mathrm{E}+01$ \\
\hline C & 3 & $-.9753 \mathrm{E}+01$ & $\mathrm{E}$ & 12 & $-.2418 E+01$ \\
\hline $\mathrm{E}$ & 13 & $-.9753 \mathrm{E}+01$ & $\mathrm{E}$ & 24 & $0.2418 \mathrm{E}+01$ \\
\hline $\mathrm{E}$ & 29 & $-.9753 E+01$ & $\mathrm{E}$ & 7 & $-.2415 E+01$ \\
\hline C & 5 & $0.9752 \mathrm{E}+01$ & $\mathrm{E}$ & 26 & $0.2414 \mathrm{E}+01$ \\
\hline $\mathrm{E}$ & 3 & $0.9752 \mathrm{E}+01$ & OM & 2 & $0.2410 \mathrm{E}+01$ \\
\hline $\mathrm{E}$ & 22 & $-.9752 \mathrm{E}+01$ & $\mathrm{C}$ & 17 & $0.2405 E+01$ \\
\hline $\mathrm{E}$ & 21 & $-.9743 \mathrm{E}+01$ & $\mathrm{C}$ & 9 & $-.2398 E+01$ \\
\hline $\mathrm{E}$ & 18 & $0.9743 E+01$ & C & 2 & $0.2397 \mathrm{E}+01$ \\
\hline $\mathrm{PC}$ & 2 & $0.9696 \mathrm{E}+01$ & & & \\
\hline C & 12 & $0.9683 \mathrm{E}+01$ & & & \\
\hline $\mathrm{C}$ & 1 & $-.9452 \mathrm{E}+01$ & & & \\
\hline PC & 1 & $-.2615 E+01$ & & & \\
\hline C & 8 & $0.2540 \mathrm{E}+01$ & & & \\
\hline $\mathrm{C}$ & 16 & $-.2438 E+01$ & & & \\
\hline $\mathrm{E}$ & 23 & $-.2425 E+01$ & & & \\
\hline K 1 & 2 & $-.2421 E+01$ & & & \\
\hline
\end{tabular}


CRITICAL LINE OUTPUT ALS CO2 / C4 DRES $=4$. 09/20/90

TABLE 4 B. CONTRASTS LISTED BY FACTORE 1 OF 1

\begin{tabular}{lrrrrr}
\multicolumn{1}{l}{ FACTOR } & RMS BIAS & FACTOR & RMS BIAS \\
& & & & & \\
TC & 2 & $-.1020 \mathrm{E}+02$ & $\mathrm{C}$ & 6 & $-.2423 \mathrm{E}+01$ \\
$\mathrm{TC}$ & 1 & $0.1014 \mathrm{E}+02$ & $\mathrm{E}$ & 1 & $0.2423 \mathrm{E}+01$ \\
$\mathrm{C}$ & 4 & $0.9952 \mathrm{E}+01$ & $\mathrm{E}$ & 8 & $-.2422 \mathrm{E}+01$ \\
$\mathrm{OM}$ & 1 & $0.9782 \mathrm{E}+01$ & $\mathrm{E}$ & 25 & $-.2422 \mathrm{E}+01$ \\
$\mathrm{E}$ & 11 & $0.978 \mathrm{E}+01$ & $\mathrm{E}$ & 6 & $0.2422 \mathrm{E}+01$ \\
$\mathrm{E}$ & 27 & $-.9776 \mathrm{E}+01$ & $\mathrm{C}$ & 15 & $-.2422 \mathrm{E}+01$ \\
$\mathrm{E}$ & 4 & $0.9771 \mathrm{E}+01$ & $\mathrm{~K}$ & 1 & $0.2422 \mathrm{E}+01$ \\
$\mathrm{E}$ & 10 & $-.9770 \mathrm{E}+01$ & $\mathrm{E}$ & 17 & $-.2421 \mathrm{E}+01$ \\
$\mathrm{C}$ & 11 & $0.9770 \mathrm{E}+01$ & $\mathrm{C}$ & 7 & $-.2421 \mathrm{E}+01$ \\
$\mathrm{C}$ & 10 & $0.9770 \mathrm{E}+01$ & $\mathrm{E}$ & 5 & $0.2421 \mathrm{E}+01$ \\
$\mathrm{E}$ & 2 & $-.9770 \mathrm{E}+01$ & $\mathrm{E}$ & 14 & $0.2421 \mathrm{E}+01$ \\
$\mathrm{E}$ & 9 & $-.9770 \mathrm{E}+01$ & $\mathrm{E}$ & 15 & $-.2421 \mathrm{E}+01$ \\
$\mathrm{E}$ & 28 & $0.9770 \mathrm{E}+01$ & $\mathrm{E}$ & 16 & $0.2420 \mathrm{E}+01$ \\
$\mathrm{E}$ & 20 & $-.9770 \mathrm{E}+01$ & $\mathrm{C}$ & 18 & $0.2420 \mathrm{E}+01$ \\
$\mathrm{C}$ & 14 & $-.9770 \mathrm{E}+01$ & $\mathrm{E}$ & 19 & $-.2420 \mathrm{E}+01$ \\
$\mathrm{E}$ & 31 & $0.9770 \mathrm{E}+01$ & $\mathrm{E}$ & 24 & $-.2420 \mathrm{E}+01$ \\
$\mathrm{E}$ & 3 & $-.9770 \mathrm{E}+01$ & $\mathrm{E}$ & 12 & $0.2420 \mathrm{E}+01$ \\
$\mathrm{E}$ & 13 & $0.9769 \mathrm{E}+01$ & $\mathrm{C}$ & 13 & $-.2417 \mathrm{E}+01$ \\
$\mathrm{C}$ & 3 & $0.9769 \mathrm{E}+01$ & $\mathrm{E}$ & 26 & $-.2416 \mathrm{E}+01$ \\
$\mathrm{E}$ & 22 & $0.9768 \mathrm{E}+01$ & $\mathrm{E}$ & 7 & $0.2416 \mathrm{E}+01$ \\
$\mathrm{E}$ & 29 & $0.9768 \mathrm{E}+01$ & $\mathrm{OM}$ & 2 & $-.2412 \mathrm{E}+01$ \\
$\mathrm{C}$ & 5 & $-.9766 \mathrm{E}+01$ & $\mathrm{C}$ & 17 & $-.2399 \mathrm{E}+01$ \\
$\mathrm{E}$ & 21 & $0.9756 \mathrm{E}+01$ & $\mathrm{C}$ & 2 & $-.2376 \mathrm{E}+01$ \\
$\mathrm{E}$ & 18 & $-.9752 \mathrm{E}+01$ & $\mathrm{C}$ & 9 & $0.2375 \mathrm{E}+01$ \\
$\mathrm{PC}$ & 2 & $-.9579 \mathrm{E}+01$ & & & \\
$\mathrm{C}$ & 12 & $-.9533 \mathrm{E}+01$ & & & \\
$\mathrm{C}$ & 1 & $0.9184 \mathrm{E}+01$ & & & \\
$\mathrm{C}$ & 8 & $-.2672 \mathrm{E}+01$ & & & \\
$\mathrm{PC}$ & 1 & $0.2644 \mathrm{E}+01$ & & & \\
$\mathrm{C}$ & 16 & $0.2453 \mathrm{E}+01$ & & & \\
$\mathrm{E}$ & 23 & $0.2428 \mathrm{E}+01$ & & & \\
$\mathrm{E}$ & 30 & $-.2423 \mathrm{E}+01$ & & & \\
& & & & &
\end{tabular}


CRITICAL LINE OUTPUT ALS CO2 / C4 DRES $=4$. 09/20/90

TABLE 4 C. CONTRASTS LISTED BY FACTORS

1 OF 1

FACTOR

$\begin{array}{lrl}\mathrm{C} & 5 & 0.1266 \mathrm{E}+02 \\ \mathrm{E} & 11 & -.1166 \mathrm{E}+02 \\ \mathrm{OM} & 1 & -.1122 \mathrm{E}+02 \\ \mathrm{E} & 10 & 0.1066 \mathrm{E}+02 \\ \mathrm{C} & 14 & 0.9281 \mathrm{E}+01 \\ \mathrm{E} & 31 & -.9219 \mathrm{E}+01 \\ \mathrm{E} & 9 & 0.9156 \mathrm{E}+01 \\ \mathrm{C} & 10 & -.9094 \mathrm{E}+01 \\ \mathrm{E} & 4 & -.9094 \mathrm{E}+01 \\ \mathrm{E} & 2 & 0.9031 \mathrm{E}+01 \\ \mathrm{TC} & 2 & 0.8344 \mathrm{E}+01 \\ \mathrm{E} & 28 & -.8094 \mathrm{E}+01 \\ \mathrm{C} & 1 & -.8031 \mathrm{E}+01 \\ \mathrm{E} & 29 & -.7781 \mathrm{E}+01 \\ \mathrm{E} & 21 & -.765 \mathrm{E}+01 \\ \mathrm{PC} & 2 & 0.7344 \mathrm{E}+01 \\ \mathrm{E} & 18 & 0.7344 \mathrm{E}+01 \\ \mathrm{E} & 27 & 0.7219 \mathrm{E}+01 \\ \mathrm{C} & 13 & 0.6906 \mathrm{E}+01 \\ \mathrm{E} & 3 & 0.6844 \mathrm{E}+01 \\ \mathrm{C} & 12 & 0.6719 \mathrm{E}+01 \\ \mathrm{C} & 3 & -.6594 \mathrm{E}+01 \\ \mathrm{E} & 20 & 0.6531 \mathrm{E}+01 \\ \mathrm{OM} & 2 & 0.6406 \mathrm{E}+01 \\ \mathrm{E} & 14 & -.6031 \mathrm{E}+01 \\ \mathrm{E} & 22 & -.6031 \mathrm{E}+01 \\ \mathrm{E} & 25 & 0.6031 \mathrm{E}+01 \\ \mathrm{C} & 4 & -.5656 \mathrm{E}+01 \\ \mathrm{TC} & 1 & -.5281 \mathrm{E}+01 \\ \mathrm{E} & 13 & -.4969 \mathrm{E}+01 \\ \mathrm{C} & 11 & -.3719 \mathrm{E}+01 \\ \mathrm{E} & 30 & 0.3469 \mathrm{E}+01 \\ & & \end{array}$

FACTOR CPU TIME

$\begin{array}{lrl}\mathrm{E} & 16 & -.3406 \mathrm{E}+01 \\ \mathrm{E} & 23 & 0.2781 \mathrm{E}+01 \\ \mathrm{C} & 16 & -.2719 \mathrm{E}+01 \\ \mathrm{E} & 8 & 0.2719 \mathrm{E}+01 \\ \mathrm{C} & 8 & 0.2656 \mathrm{E}+01 \\ \mathrm{E} & 1 & -.2594 \mathrm{E}+01 \\ \mathrm{C} & 2 & 0.2406 \mathrm{E}+01 \\ \mathrm{E} & 7 & -.2344 \mathrm{E}+01 \\ \mathrm{E} & 26 & 0.2281 \mathrm{E}+01 \\ \mathrm{E} & 5 & -.2031 \mathrm{E}+01 \\ \mathrm{C} & 6 & 0.1719 \mathrm{E}+01 \\ \mathrm{E} & 17 & -.1719 \mathrm{E}+01 \\ \mathrm{E} & 24 & 0.1719 \mathrm{E}+01 \\ \mathrm{E} & 15 & -.1469 \mathrm{E}+01 \\ \mathrm{~K} & 12 & -.1406 \mathrm{E}+01 \\ \mathrm{E} & 6 & -.1406 \mathrm{E}+01 \\ \mathrm{C} & 18 & -.1344 \mathrm{E}+01 \\ \mathrm{PC} & 1 & 0.9688 \mathrm{E}+00 \\ \mathrm{C} & 9 & -.5313 \mathrm{E}+00 \\ \mathrm{E} & 19 & -.5313 \mathrm{E}+00 \\ \mathrm{C} & 15 & 0.4688 \mathrm{E}+00 \\ \mathrm{E} & 12 & -.4688 \mathrm{E}+00 \\ \mathrm{C} & 17 & -.2813 \mathrm{E}+00 \\ \mathrm{C} & 7 & 0.2188 \mathrm{E}+00\end{array}$


CRITICAL LOCUS OUTPUT. CO2 / C6 ALS EOS DRES $=4$, RFACT $=0.0025$ 
CRITICAL LOCUS OUTPUT. CO2 / C6 ALS EOS DRES=4, RFACT=0.0025

TABLE 3. RESPONSES 1 OF 2

$\begin{array}{cccc}\text { TEST } & \text { RT SQ ER } & \text { RMS BIAS } & \text { CPU TIME } \\ 1 & 0.9590 \mathrm{E}+02 & -.6430 \mathrm{E}+02 & 0.6310 \mathrm{E}+03 \\ 2 & 0.1002 \mathrm{E}+03 & -.5917 \mathrm{E}+02 & 0.6110 \mathrm{E}+03 \\ 3 & 0.1122 \mathrm{E}+02 & 0.6568 \mathrm{E}+01 & 0.3310 \mathrm{E}+03 \\ 4 & 0.1119 \mathrm{E}+02 & 0.6573 \mathrm{E}+01 & 0.3920 \mathrm{E}+03 \\ 5 & 0.9738 \mathrm{E}+02 & -.6234 \mathrm{E}+02 & 0.6570 \mathrm{E}+03 \\ 6 & 0.1107 \mathrm{E}+02 & 0.6410 \mathrm{E}+01 & 0.3360 \mathrm{E}+03 \\ 7 & 0.1120 \mathrm{E}+02 & 0.6511 \mathrm{E}+01 & 0.3790 \mathrm{E}+03 \\ 8 & 0.1103 \mathrm{E}+02 & 0.6199 \mathrm{E}+01 & 0.3250 \mathrm{E}+03 \\ 9 & 0.9419 \mathrm{E}+02 & -.6660 \mathrm{E}+02 & 0.8890 \mathrm{E}+03 \\ 10 & 0.9489 \mathrm{E}+02 & -.6563 \mathrm{E}+02 & 0.3450 \mathrm{E}+03 \\ 11 & 0.1128 \mathrm{E}+02 & 0.6744 \mathrm{E}+01 & 0.3400 \mathrm{E}+03 \\ 12 & 0.5645 \mathrm{E}+03 & -.5492 \mathrm{E}+03 & 0.4750 \mathrm{E}+03 \\ 13 & 0.1155 \mathrm{E}+02 & 0.7119 \mathrm{E}+01 & 0.3430 \mathrm{E}+03 \\ 14 & 0.9732 \mathrm{E}+02 & -.6246 \mathrm{E}+02 & 0.6240 \mathrm{E}+03 \\ 15 & 0.6360 \mathrm{E}+03 & -.6159 \mathrm{E}+03 & 0.4140 \mathrm{E}+03 \\ 16 & 0.1085 \mathrm{E}+02 & 0.5927 \mathrm{E}+01 & 0.3370 \mathrm{E}+03 \\ 17 & 0.9490 \mathrm{E}+02 & -.6561 \mathrm{E}+02 & 0.9110 \mathrm{E}+03 \\ 18 & 0.1089 \mathrm{E}+02 & 0.5955 \mathrm{E}+01 & 0.3540 \mathrm{E}+03 \\ 19 & 0.9659 \mathrm{E}+02 & -.6334 \mathrm{E}+02 & 0.6600 \mathrm{E}+03 \\ 20 & 0.1022 \mathrm{E}+03 & -.5800 \mathrm{E}+02 & 0.3960 \mathrm{E}+03 \\ 21 & 0.9763 \mathrm{E}+02 & -.6441 \mathrm{E}+\mathrm{C}-1 & 0.6030 \mathrm{E}+03 \\ 22 & 0.1003 \mathrm{E}+03 & -.5902 \mathrm{E}+02 & 0.3100 \mathrm{E}+03 \\ 23 & 0.1022 \mathrm{E}+03 & -.5806 \mathrm{E}+02 & 0.3840 \mathrm{E}+03 \\ 24 & 0.1107 \mathrm{E}+02 & 0.6328 \mathrm{E}+01 & 0.3670 \mathrm{E}+03 \\ 25 & 0.1112 \mathrm{E}+02 & 0.6405 \mathrm{E}+01 & 0.3620 \mathrm{E}+03 \\ 26 & 0.9584 \mathrm{E}+02 & -.6440 \mathrm{E}+02 & 0.3690 \mathrm{E}+03 \\ 27 & 0.1022 \mathrm{E}+03 & -.5684 \mathrm{E}-02 & 0.3490 \mathrm{E}+03 \\ 28 & 0.5514 \mathrm{E}+03 & -.5353 \mathrm{E}+03 & 0.4270 \mathrm{E}+03 \\ 29 & 0.9586 \mathrm{E}+02 & -.6431 \mathrm{E}+02 & 0.6050 \mathrm{E}+03 \\ 30 & 0.9650 \mathrm{E}+02 & -.6347 \mathrm{E}+02 & 0.1059 \mathrm{E}+04 \\ 31 & 0.5491 \mathrm{E}+03 & -.5328 \mathrm{E}+03 & 0.4250 \mathrm{E}+03 \\ 32 & 0.1123 \mathrm{E}+02 & 0.6545 \mathrm{E}+01 & 0.3640 \mathrm{E}+03 \\ & & & \\ 13 & & & \end{array}$


CRITICAL LOCUS OUTPUT. $\mathrm{CO} 2$ / C6 ALS EOS DRES $=4$, RFACT $=0.0025$

TABLE 3. (CONTINUED)

$2 \mathrm{OF} \quad 2$

\begin{tabular}{|c|c|c|c|}
\hline EST & RT SQ ER & RMS BIAS & CPU TIME \\
\hline 33 & $0.9419 \mathrm{E}+02$ & $-.6660 \mathrm{E}+02$ & $0.3440 \mathrm{E}+\mathrm{C}$ \\
\hline 34 & $0.1089 E+02$ & $0.6130 E+01$ & $0.334 .0 E+03$ \\
\hline 35 & $0.9451 E+02$ & $-.6615 E+02$ & $0.4160 E+03$ \\
\hline 36 & $0.1124 E+02$ & $0.6535 E+01$ & $0.3290 \mathrm{E}+03$ \\
\hline 37 & $0.1021 \mathrm{E}+03$ & $-.5804 E+02$ & $0.3280 \mathrm{E}+03$ \\
\hline 38 & $0.1074 E+02$ & $0.5797 E+01$ & $0.3500 E+03$ \\
\hline 39 & $0.1152 \mathrm{E}+02$ & $0.7100 \mathrm{E}+01$ & $0 E+03$ \\
\hline 40 & $0.1129 E+02$ & $0.6722 \mathrm{E}+0.1$ & $E+03$ \\
\hline 41 & $0.1029 E+03$ & $-.5847 E+02$ & $E+03$ \\
\hline 42 & $0.1122 \mathrm{E}+02$ & $0.6643 \mathrm{E}+01$ & $0 E+03$ \\
\hline 43 & $0.526 C E+03$ & $-.5082 E+03$ & 0.36 \\
\hline 44 & $0.5534 E+03$ & $-.5376 \mathrm{E}+03$ & $0.4350 \mathrm{E}+0$ \\
\hline 45 & $0.1125 \mathrm{E}+02$ & $0.6547 \mathrm{E}+01$ & $0.3990 \mathrm{E}+0$ \\
\hline 46 & $0.1099 E+02$ & $0.6093 \mathrm{E}+01$ & $0.3360 \mathrm{E}+0$ \\
\hline 47 & $0.9742 \mathrm{E}+02$ & $-.6229 \mathrm{E}+02$ & $0.3760 \mathrm{E}+0$ \\
\hline 48 & $0.1115 \mathrm{E}+02$ & $0.6510 \mathrm{E}+01$ & $0.4120 \mathrm{E}+0$ \\
\hline 49 & $0.1031 E+03$ & $-.5808 E+02$ & $0.3690 \mathrm{E}+0$ \\
\hline 50 & $0.1122 \mathrm{E}+02$ & $0.6594 \mathrm{E}+01$ & $0.4210 \mathrm{E}+0$ \\
\hline 51 & $0.9419 \mathrm{E}+02$ & $-.6660 \mathrm{E}+02$ & $0.7070 E+0$ \\
\hline 52 & $0.1088 E+02$ & $0.6018 \mathrm{E}+01$ & $0.3570 \mathrm{E}+0$ \\
\hline 53 & $0.3443 \mathrm{ErO} 3$ & $-.3225 \mathrm{E}+03$ & $0.5380 \mathrm{E}+0$ \\
\hline 54 & $0.9588 E+02$ & $-.6434 \mathrm{E}+02$ & $0.3980 \mathrm{E}+0$ \\
\hline 55 & $0.9449 \mathrm{E}+02$ & $-.6617 \mathrm{E}+02$ & $0.8120 \mathrm{E}+0$ \\
\hline 56 & $0.9582 E+02$ & $-.6436 \mathrm{E}+02$ & $0.6190 \mathrm{E}+0$ \\
\hline 57 & $0.1829 \mathrm{E}+03$ & $-.1658 \mathrm{E}+03$ & $0.3770 E+0$ \\
\hline 58 & $0.9727 E+02$ & $-.6253 E+02$ & $0.8240 E+0$ \\
\hline 59 & $0.9491 \mathrm{E}+02$ & $-.6559 E+02$ & $0.5720 \mathrm{E}+0$ \\
\hline 60 & $0.1020 E+03$ & $-.5845 E+02$ & $0.6040 \mathrm{E}+0$ \\
\hline 61 & $0.1123 \mathrm{E}+02$ & $0.6557 \mathrm{E}+01$ & $0.3560 E+0$ \\
\hline 62 & $0.1119 \mathrm{E}+02$ & $0.6559 E+01$ & $0.3100 E+0$ \\
\hline 63 & $0.9533 \mathrm{E}+02$ & $-.6501 E+02$ & $0.1123 \mathrm{E}+0$ \\
\hline 64 & $0.9654 E+02$ & $-.6343 E+02$ & $0.5880 E+03$ \\
\hline
\end{tabular}


CRITICAL LOCUS OUTPUT. CO2 / C6 ALS EOS DRES=4, RFACT=0.0025

TABLE 4 A. CONTRASTS LISTED BY FACTORS 1 OF 1

\begin{tabular}{|c|c|c|c|c|c|}
\hline \multicolumn{2}{|c|}{ FACTOR } & RT SQ ER & \multicolumn{2}{|c|}{ FACTOR } & RT SQ ER \\
\hline$E$ & 7 & $0.1118 \mathrm{E}+03$ & C & 8 & $-.1976 \mathrm{E}+02$ \\
\hline C & 4 & $0.9025 E+02$ & $\mathrm{OM}$ & 1 & $-.1889 E+02$ \\
\hline $\mathrm{E}$ & 16 & $-.8445 E+02$ & $E$ & 29 & $-.1616 \mathrm{E}+02$ \\
\hline C & 2 & $0.7428 \mathrm{E}+02$ & $E$ & 30 & $0.1272 \mathrm{E}+02$ \\
\hline C & 13 & $0.7268 E+02$ & $E$ & 15 & $-.1215 E+02$ \\
\hline $\mathrm{E}$ & 18 & $0.7172 \mathrm{E}+02$ & C & 18 & $0.1209 E+02$ \\
\hline C & 7 & $-.6949 E+02$ & $\mathrm{E}$ & 27 & $-.1118 \mathrm{E}+02$ \\
\hline$E$ & 2 & $-.6890 \mathrm{E}+02$ & $\mathrm{E}$ & 17 & $0.1094 \mathrm{E}+02$ \\
\hline $\mathrm{E}$ & 23 & $0.6619 E+02$ & $\mathrm{E}$ & 21 & $0.1030 \mathrm{E}+02$ \\
\hline C & 11 & $-.6279 E+02$ & C & 14 & $0.9516 \mathrm{E}+01$ \\
\hline OM & 2 & $-.5393 E+02$ & $\mathrm{C}$ & 17 & $-.7258 \mathrm{E}+01$ \\
\hline$E$ & 10 & $-.4991 \mathrm{E}+02$ & $\mathrm{E}$ & 1 & $0.6932 \mathrm{E}+01$ \\
\hline$E$ & 28 & $0.4927 \mathrm{E}+02$ & $\mathrm{PC}$ & 2 & $0.6894 \mathrm{E}+01$ \\
\hline $\mathrm{E}$ & 13 & $-.4407 E+02$ & $\mathrm{E}$ & 4 & $-.6041 E+01$ \\
\hline TC & 2 & $-.4354 \mathrm{E}+02$ & C & 15 & $0.5997 \mathrm{E}+01$ \\
\hline $\mathrm{E}$ & 11 & $0.4319 E+02$ & $\mathrm{E}$ & 22 & $0.5941 E+01$ \\
\hline $\mathrm{E}$ & 14 & $-.3905 E+02$ & $\mathrm{E}$ & 20 & $0.5861 E+01$ \\
\hline $\mathrm{E}$ & 26 & $0.3802 \mathrm{E}+02$ & $E$ & 25 & $0.5333 E+01$ \\
\hline K 1 & 2 & $0.3720 \mathrm{E}+02$ & $E$ & 24 & $-.4616 E+01$ \\
\hline C & 1 & $-.3583 E+02$ & C & 5 & $0.4045 E+01$ \\
\hline C & 3 & $-.3399 E+02$ & C & 9 & $0.3946 E+01$ \\
\hline$E$ & 6 & $-.3360 E+02$ & C & 12 & $0.1808 E+01$ \\
\hline $\mathrm{PC}$ & 1 & $-.3356 \mathrm{E}+02$ & $\mathrm{E}$ & 19 & $-.1126 E+01$ \\
\hline$E$ & 12 & $-.2786 \mathrm{E}+02$ & $\mathrm{C}$ & 16 & $0.3755 \mathrm{E}+00$ \\
\hline$E$ & 8 & $-.2740 E+02$ & & & \\
\hline $\mathrm{TC}$ & 1 & $-.2626 \mathrm{E}+02$ & & & \\
\hline $\mathrm{E}$ & 9 & $-.2495 E+02$ & & & \\
\hline C & 6 & $-.2458 E+02$ & & & \\
\hline $\mathrm{C}$ & 10 & $0.2286 E+02$ & & & \\
\hline$E$ & 3 & $0.2228 E+02$ & & & \\
\hline$E$ & 5 & $-.2096 E+02$ & & & \\
\hline $\mathrm{E}$ & 31 & $-.2095 E+02$ & & & \\
\hline
\end{tabular}


CRITICAL LOCUS OUTPUT. CO2 / C6 ALS EOS DRES $=4$, RFACT $=0.0025$

TABLE 4 B. CONTRASTS LISTED BY FACTORS

1 OF 1

\begin{tabular}{|c|c|c|c|c|c|}
\hline FACT & COR & RMS BIAS & FACI & COR & RMS BIAS \\
\hline$E$ & 7 & $-.1102 E+03$ & C & 10 & $-.1927 E+02$ \\
\hline C & 4 & $-.9235 \mathrm{E}+02$ & $\mathrm{E}$ & 5 & $0.1918 \mathrm{E}+02$ \\
\hline$E$ & 16 & $0.8510 E+02$ & C & 8 & $0.1686 \mathrm{E}+02$ \\
\hline C & 2 & $-.7712 \mathrm{E}+02$ & C & 18 & $-.1531 \mathrm{E}+02$ \\
\hline $\mathrm{E}$ & 18 & $-.7258 E+02$ & $E$ & 27 & $0.1323 E+02$ \\
\hline C & 13 & $-.7151 E+02$ & $\mathrm{E}$ & 17 & $-.1185 \mathrm{E}+02$ \\
\hline $\mathrm{E}$ & 23 & $-.7054 \mathrm{E}+02$ & $\mathrm{E}$ & 30 & $-.1183 E+02$ \\
\hline $\mathrm{E}$ & 2 & $0.6929 E+02$ & $\mathrm{E}$ & 15 & $0.1153 \mathrm{E}+02$ \\
\hline C & 7 & $0.6840 \mathrm{E}+02$ & $E$ & 21 & $-.9901 E+01$ \\
\hline C & 11 & $0.6324 E+02$ & $E$ & 22 & $-.9169 E+01$ \\
\hline $\mathrm{OM}$ & 2 & $0.5258 E+02$ & PC & 2 & $-.8829 \mathrm{E}+01$ \\
\hline $\mathrm{E}$ & 10 & $0.4836 \mathrm{E}+02$ & C & 17 & $0.8591 \mathrm{E}+01$ \\
\hline$E$ & 28 & $-.4540 E+02$ & $\mathrm{E}$ & 4 & $0.8224 \mathrm{E}+01$ \\
\hline$E^{\prime}$ & 13 & $0.4320 \mathrm{E}+02$ & C & 14 & $-.8118 E+01$ \\
\hline$E$ & 11 & $-.4309 E+02$ & $E$ & 1 & $-.7767 \mathrm{E}+01$ \\
\hline TC & 2 & $0.4276 E+02$ & C & 9 & $-.5940 \mathrm{E}+01$ \\
\hline $\mathrm{E}$ & 14 & $0.4106 \mathrm{E}+02$ & C & 15 & $-.4938 E+01$ \\
\hline & 26 & $-.4041 \mathrm{E}+02$ & $\mathrm{E}$ & 20 & $-.4814 \mathrm{E}+01$ \\
\hline $\mathrm{K} 1$ & 2 & $-.3843 E+02$ & $\mathrm{E}$ & 24 & $0.4726 \mathrm{E}+01$ \\
\hline PC & 1 & $0.3351 \mathrm{E}+02$ & C & 16 & $0.4047 E+01$ \\
\hline $\mathrm{E}$ & 6 & $0.3334 \mathrm{E}+02$ & $\mathrm{E}$ & 25 & $-.3155 E+01$ \\
\hline C & 3 & $0.3270 E+02$ & $\mathrm{E}$ & 19 & $0.1384 \mathrm{E}+01$ \\
\hline $\mathrm{E}$ & 8 & $0.3258 E+02$ & C & 5 & $0.1105 \mathrm{E}+01$ \\
\hline C & 1 & $0.3221 E+02$ & C & 12 & $-.7836 \mathrm{E}+00$ \\
\hline TC & 1 & $0.2980 E+02$ & & & \\
\hline $\mathrm{E}$ & 9 & $0.2802 E+02$ & & & \\
\hline $\mathrm{E}$ & 12 & $0.2780 E+02$ & & & \\
\hline $\mathrm{C}$ & 6 & $0.2367 E+02$ & & & \\
\hline OM & 1. & $0.2184 E+02$ & & & \\
\hline $\mathrm{E}$ & 31 & $0.1954 \mathrm{E}+02$ & & & \\
\hline $\mathrm{E}$ & 3 & $-.1944 \mathrm{E}+02$ & & & \\
\hline & 29 & $0.1930 E+02$ & & & \\
\hline
\end{tabular}


CRITICAL LOCUS OUTPUT. CO2 / C6 ALS EOS DRES=4, RFACT $=0.0025$

TABLE 4 C. CONTRASTS LISTED BY FACTORS 1 OF 1

\begin{tabular}{|c|c|c|c|c|}
\hline \multicolumn{2}{|c|}{ FACTOR } & CPU TIME & FACTOR & CPU TIME \\
\hline$E$ & 9 & $0.1476 \mathrm{E}+03$ & 12 & $0.264 .1 E+02$ \\
\hline C & 5 & $0.1142 \mathrm{E}+03$ & 29 & $0.2591 E+02$ \\
\hline$E$ & 28 & $0.8372 \mathrm{E}+02$ & $E$ & $-.2509 E+02$ \\
\hline$E$ & 15 & $0.8191 \mathrm{E}+02$ & C & $0.2472 \mathrm{E}+02$ \\
\hline $\mathrm{E}$ & 3 & $0.7334 \mathrm{E}+02$ & $E$ & $0.2403 E+02$ \\
\hline$E$ & 16 & $-.7109 \mathrm{E}+02$ & 14 & $0.1972 E+02$ \\
\hline $\mathrm{E}$ & 30 & $-.6978 \mathrm{E}+02$ & OM 1 & $0.1847 \mathrm{E}+02$ \\
\hline$E$ & 23 & $-.6422 \mathrm{E}+02$ & C 16 & $-.1797 \mathrm{E}+02$ \\
\hline E & 22 & $-.6416 \mathrm{E}+02$ & C & $-.1766 E+02$ \\
\hline C & 13 & $-.6409 E+02$ & C & $-.1753 E+02$ \\
\hline $\mathrm{C}$ & 10 & $-.6403 E+02$ & 12 & $-.1666 \mathrm{E}+02$ \\
\hline C & 9 & $-.6272 E+02$ & $E$ & $-.1441 \mathrm{E}+02$ \\
\hline OM & 2 & $-.6247 E+02$ & E $\quad 17$ & $0.1397 E+02$ \\
\hline $\mathrm{C}$ & 1 & $-.6153 E+02$ & $\mathrm{PC} \quad 1$ & $-.1359 E+02$ \\
\hline C & 14 & $0.6028 \mathrm{E}+02$ & $\mathrm{E}$ & $0.1297 \mathrm{E}+02$ \\
\hline$E$ & 18 & $0.5641 \mathrm{E}+02$ & 11 & $-.1259 \mathrm{E}+02$ \\
\hline $\mathrm{E}$ & 27 & $0.5603 \mathrm{E}+02$ & $\mathrm{~K} 12$ & $-.1241 \mathrm{E}+02$ \\
\hline $\mathrm{E}$ & 24 & $-.5553 \mathrm{E}+02$ & C & $0.7031 E+01$ \\
\hline $\mathrm{E}$ & 8 & $0.5441 \mathrm{E}+02$ & 13 & $0.7031 \mathrm{E}+01$ \\
\hline $\mathrm{E}$ & 19 & $-.5347 \mathrm{E}+02$ & 17 & $-.6094 \mathrm{E}+01$ \\
\hline TC & 1 & $0.5153 \mathrm{E}+02$ & 18 & $0.2094 \mathrm{E}+01$ \\
\hline $\mathrm{E}$ & $1 \overline{1}$ & $0.4309 E+02$ & 26 & $-.1281 \mathrm{E}+01$ \\
\hline TC & 2 & $0.3928 \mathrm{E}+02$ & $\mathrm{E}$ & $-.5938 \mathrm{E}+00$ \\
\hline$C$ & 8 & $-.3791 E+02$ & $\bar{E}$ & $0.5938 \mathrm{E}+00$ \\
\hline c & 15 & $-.3528 E+02$ & & \\
\hline C & 7 & $-.3447 \mathrm{E}+02$ & & \\
\hline PC & 2 & $0.3434 \mathrm{E}+02$ & & \\
\hline $\mathrm{E}$ & 10 & $0.3378 E+02$ & & \\
\hline $\mathrm{E}$ & 25 & $-.3222 \mathrm{E}+02$ & & \\
\hline $\mathrm{E}$ & 31 & $-3178 E+02$ & & \\
\hline $\mathrm{E}$ & 21 & $-.2872 E+02$ & & \\
\hline $\mathrm{E}$ & 20 & $0.2709 E+02$ & & \\
\hline
\end{tabular}


LIQUID DENSITY OUTPUT $n-C 4 / C O 2$ DRES $=4$ SFACT $=0.5 \%$ 
TABLE 2.TEST PATTERN IN ACTUAL FACTORS

$\begin{array}{cc}\text { TEST } & C \quad 1 \\ 1 & 0.1000 \mathrm{E}+01 \\ 2 & 0.1005 \mathrm{E}+01 \\ 3 & 0.1000 \mathrm{E}+01 \\ 4 & 0.1005 \mathrm{E}+01 \\ 5 & 0.1000 \mathrm{E}+01 \\ 6 & 0.1005 \mathrm{E}+01 \\ 7 & 0.1000 \mathrm{E}+01 \\ 8 & 0.1005 \mathrm{E}+01 \\ 9 & 0.1000 \mathrm{E}+01 \\ 10 & 0.1005 \mathrm{E}+01 \\ 11 & 0.1000 \mathrm{E}+01 \\ 12 & 0.1005 \mathrm{E}+01 \\ 13 & 0.1000 \mathrm{E}+01 \\ 14 & 0.1005 \mathrm{E}+01 \\ 15 & 0.1000 \mathrm{E}+01 \\ 16 & 0.1005 \mathrm{E}+01 \\ 17 & 0.1000 \mathrm{E}+01 \\ 18 & 0.1005 \mathrm{E}+01 \\ 19 & 0.1000 \mathrm{E}+01 \\ 20 & 0.1005 \mathrm{E}+01 \\ 21 & 0.1000 \mathrm{E}+01 \\ 22 & 0.1005 \mathrm{E}+01 \\ 23 & 0.1000 \mathrm{E}+01 \\ 24 & 0.1005 \mathrm{E}+01 \\ 25 & 0.1000 \mathrm{E}+01 \\ 26 & 0.1005 \mathrm{E}+01 \\ 27 & 0.1000 \mathrm{E}+01 \\ 28 & 0.1005 \mathrm{E}+01 \\ 29 & 0.1000 \mathrm{E}+01 \\ 30 & 0.1005 \mathrm{E}+01 \\ 31 & 0.1000 \mathrm{E}+01 \\ 32 & 0.1005 \mathrm{E}+01\end{array}$

$\begin{array}{cc}C & 2 \\ 0.3535 E+01 \\ 0.3535 E+01 \\ 0.3553 E+01 \\ 0.3553 E+01 \\ 0.3535 E+01 \\ 0.3535 E+01 \\ 0.3553 E+01 \\ 0.3553 E+01 \\ 0.3535 E+01 \\ 0.3535 E+01 \\ 0.3553 E+01 \\ 0.3553 E+01 \\ 0.3535 E+01 \\ 0.3535 E+01 \\ 0.3553 E+01 \\ 0.3553 E+01 \\ 0.3535 E+01 \\ 0.3535 E+01 \\ 0.3553 E+01 \\ 0.3553 E+01 \\ 0.3535 E+01 \\ 0.3535 E+01 \\ 0.3553 E+01 \\ 0.3553 E+01 \\ 0.3535 E+01 \\ 0.3535 E+01 \\ 0.3553 E+01 \\ 0.3553 E+01 \\ 0.3535 E+01 \\ 0.3535 E+01 \\ 0.3553 E+01 \\ 0.3553 E+01 \\ \end{array}$

$C \quad 3$
$0.5330 E+00$
$0.5330 E+00$
$0.5330 E+00$
$0.5330 E+00$
$0.5357 E+00$
$0.5357 E+00$
$0.5357 E+00$
$0.5357 E+00$
$0.5330 E+00$
$0.5330 E+00$
$0.5330 E+00$
$0.5330 E+00$
$0.5357 E+00$
$0.5357 E+00$
$0.5357 E+00$
$0.5357 E+00$
$0.5330 E+00$
$0.5330 E+00$
$0.5330 E+00$
$0.5330 E+00$
$0.5357 E+00$
$0.5357 E+00$
$0.5357 E+00$
$0.5357 E+00$
$0.5330 E+00$
$0.5330 E+00$
$0.5330 E+00$
$0.5330 E+00$
$0.5357 E+00$
$0.5357 E+00$
$0.5357 E+00$
$0.5357 E+00$

C 4
$0.1000 E+01$

$0.1000 E+01$

$0.1000 \mathrm{E}+01$

$0.1000 \mathrm{E}+01$

$0.1000 E+01$

$0.1000 E+01$

$0.1000 \mathrm{E}+01$

$0.1000 \mathrm{E}+01$

$0.1005 \mathrm{E}+01$

$0.1005 \mathrm{E}+01$

$0.1005 \mathrm{E}+01$

$0.1005 E+01$

$0.1005 E+01$

$0.1005 E+01$

$0.1005 \mathrm{E}+01$

$0.1005 \mathrm{E}+01$

$0.1000 E+01$

$0.1000 E+01$

$0.1000 E+01$

$0.1000 E+01$

$0.1000 E+01$

$0.1000 \mathrm{E}+01$

$0.1000 E+01$

$0.1000 E+01$

$0.1005 \mathrm{E}+01$

$0.1005 \mathrm{E}+01$

$0.1005 \mathrm{E}+01$

$0.1005 \mathrm{E}+01$

$0.1005 \mathrm{E}+01$

$0.1005 E+01$

$0.1005 E+01$

$0.1005 E+01$
1 OF 10

C 5

$0.9450 \mathrm{E}+00$

$0.9450 \mathrm{E}+00$

$0.9450 \mathrm{E}+00$

$0.9450 \mathrm{E}+00$

$0.9450 E+00$

$0.9450 \mathrm{E}+00$

$0.9450 \mathrm{E}+00$

$0.9450 \mathrm{E}+00$

$0.9450 \mathrm{E}+00$

$0.9450 \mathrm{E}+00$

$0.9450 \mathrm{E}+00$

$0.9450 \mathrm{E}+00$

$0.9450 \mathrm{E}+00$

$0.9450 \mathrm{E}+00$

$0.9450 \mathrm{E}+00$

$0.9450 \mathrm{E}+00$

$0.9497 \mathrm{E}+00$

$0.9497 \mathrm{E}+00$

$0.9497 \mathrm{E}+00$

$0.9497 \mathrm{E}+00$

$0.9497 \mathrm{E}+00$

$0.9497 \mathrm{E}+00$

$0.9497 \mathrm{E}+00$

$0.9497 \mathrm{E}+00$

$0.9497 \mathrm{E}+00$

$0.9497 \mathrm{E}+00$

$0.9497 \mathrm{E}+00$

$0.9497 \mathrm{E}+00$

$0.9497 \mathrm{E}+00$

$0.9497 \mathrm{E}+00$

$0.9497 \mathrm{E}+00$

$0.9497 \mathrm{E}+00$ 
TABLE 2. (CONTINUED)

2 OF 10

\begin{tabular}{|c|c|c|c|c|c|}
\hline PST & C & C & & & \\
\hline 33 & $0.1000 E+01$ & $3535 E+01$ & $0.5330 \mathrm{E}+00$ & $0.1000 E+01$ & $450 \mathrm{E}+00$ \\
\hline 34 & $1005 E+01$ & $3535 E+01$ & $330 E+00$ & $0.1000 E+01$ & $E+00$ \\
\hline 35 & $000 E+01$ & $553 E+01$ & $330 E+00$ & $0.1000 E+01$ & $E+00$ \\
\hline 36 & $005 E+01$ & $553 E+01$ & $330 E+00$ & $0.1 C J 0 E+01$ & $E+00$ \\
\hline 37 & $000 E+01$ & $535 E+01$ & $357 E+00$ & $0.1000 E+01$ & $E+00$ \\
\hline 38 & $005 E+01$ & $535 E+01$ & $357 E+00$ & $0.1000 \mathrm{E}+01$ & $D E+00$ \\
\hline 39 & $000 E+01$ & $553 E+01$ & $357 E+00$ & $0.1000 \mathrm{E}+01$ & $0 E+00$ \\
\hline 40 & $0.1005 E+01$ & $0.3553 \mathrm{E}+01$ & $5357 E+00$ & $0.1000 E+01$ & $0.9450 E+00$ \\
\hline 41 & $0.1000 E+01$ & $3535 E+01$ & $5330 E+00$ & $0.1005 \mathrm{E}+01$ & $0.9450 E+00$ \\
\hline 42 & $0.1005 \mathrm{E}+01$ & $3535 E+01$ & $5330 E+00$ & $0.1005 E+01$ & $0.9450 E+00$ \\
\hline 43 & $0.1000 \mathrm{E}+01$ & $0.3553 \mathrm{E}+01$ & $5330 E+00$ & $0.1005 E+01$ & $0.9450 \mathrm{E}+00$ \\
\hline 44 & $0.1005 E+01$ & $0.3553 \mathrm{E}+01$ & $0.5330 E+00$ & $0.1005 \mathrm{E}+01$ & $0.9450 E+00$ \\
\hline 45 & $0.1000 E+01$ & $0.3535 E+01$ & $0.5357 \mathrm{E}+00$ & $0.1005 E+01$ & $0.9450 E+00$ \\
\hline 46 & $0.1005 E+01$ & $0.3535 E+01$ & $0.5357 E+00$ & $0.1005 E+01$ & $0.9450 E+00$ \\
\hline 47 & $0.1000 E+01$ & $0.3553 \mathrm{E}+01$ & $0.5357 \mathrm{E}+00$ & $0.1005 E+01$ & $0.9450 \mathrm{E}+00$ \\
\hline 48 & $0.1005 E+01$ & $0.3553 E+01$ & $0.5357 E+00$ & $0.1005 \mathrm{E}+01$ & $0.9450 E+00$ \\
\hline 49 & $0.1000 E+01$ & $0.3535 E+01$ & $0.5330 E+00$ & $0.1000 \mathrm{E}+01$ & $0.9497 \mathrm{E}+00$ \\
\hline 50 & $0.1005 \mathrm{E}+01$ & $0.3535 \mathrm{E}+01$ & $0.5330 \mathrm{E}+00$ & $0.1000 E+01$ & $0.9497 E+00$ \\
\hline 51 & $0.1000 E+01$ & $0.3553 E+01$ & $0.5330 \mathrm{E}+00$ & $0.1000 E+01$ & $0.9497 E+00$ \\
\hline 52 & $0.1005 E+01$ & $0.3553 E+01$ & $0.5330 \mathrm{E}+00$ & $0.1000 E+01$ & $0.9497 \mathrm{E}+00$ \\
\hline 53 & $0.1000 E+01$ & $0.3535 E+01$ & $0.5357 \mathrm{E}+00$ & $0.1000 \mathrm{E}+01$ & $0.9497 E+00$ \\
\hline 54 & $0.1005 E+01$ & $0.3535 E+01$ & $0.5357 \mathrm{E}+00$ & $0.1000 E+01$ & $0.9497 E+00$ \\
\hline 55 & $0.1000 \mathrm{E}+01$ & $0.3553 \mathrm{E}+01$ & $0.5357 \mathrm{E}+00$ & $0.1000 E+01$ & $0.9497 \mathrm{E}+00$ \\
\hline 56 & $0.1005 E+01$ & $0.3553 E+01$ & $0.5357 \mathrm{E}+00$ & $0.1000 E+01$ & $0.9497 \mathrm{E}+00$ \\
\hline 57 & $0.1000 \mathrm{E}+01$ & $0.3535 E+01$ & $0.5330 \mathrm{E}+00$ & $0.1005 E+01$ & $0.9497 \mathrm{E}+00$ \\
\hline 58 & $0.1005 E+01$ & $0.3535 E+01$ & $0.5330 \mathrm{E}+00$ & $0.1005 E+01$ & $0.9497 E+00$ \\
\hline 59 & $0.1000 E+01$ & $0.3553 \mathrm{E}+01$ & $0.5330 \mathrm{E}+00$ & $0.1005 E+01$ & $0.9497 \mathrm{E}+00$ \\
\hline 60 & $0.1005 E+01$ & $0.3553 E+01$ & $0.5330 \mathrm{E}+00$ & $0.1005 \mathrm{E}+01$ & $0.9497 \mathrm{E}+00$ \\
\hline 61 & $0.1000 E+01$ & $0.3535 E+01$ & $0.5357 \mathrm{E}+00$ & $0.1005 E+01$ & $0.9497 \mathrm{E}+00$ \\
\hline 62 & $0.1005 E+01$ & $0.3535 E+01$ & $0.5357 \mathrm{E}+00$ & $0.1005 E+01$ & $0.9497 \mathrm{E}+00$ \\
\hline 63 & $0.1000 E+01$ & $0.3553 \mathrm{E}+01$ & $0.5357 \mathrm{E}+00$ & $0.1005 \mathrm{E}+01$ & $0.9497 \mathrm{E}+00$ \\
\hline 64 & $0.1005 E+01$ & $0.3553 E+01$ & $0.5357 \mathrm{E}+00$ & $0.1005 E+01$ & $0.9497 E+00$ \\
\hline
\end{tabular}


LIQUID DENSITY OUTPUT $\mathrm{n}-\mathrm{C} 4$ / $\mathrm{CO} 2$ DRES $=4 \quad \mathrm{SFACT}=0.5 \%$

TABLE 2. (CONTINUED)

3 or 10

\begin{tabular}{|c|c|c|c|c|c|}
\hline EST & & C & $\mathrm{C}$ & 9 & C 10 \\
\hline 1 & $.1340 \mathrm{E}+00$ & $0.1023 \mathrm{E}+01$ & $0.2225 \mathrm{E}+01$ & $0.4780 \mathrm{E}+00$ & $0.3120 E-01$ \\
\hline 2 & $340 E+00$ & $1028 E+01$ & $0.2236 \mathrm{E}+01$ & $0.4804 \mathrm{E}+00$ & $.3136 \mathrm{E}-01$ \\
\hline 3 & $340 E+00$ & $1028 \mathrm{E}+01$ & $0.2236 \mathrm{E}+01$ & $0.4804 \mathrm{E}+00$ & $0.3136 \mathrm{E}-01$ \\
\hline 4 & $340 \mathrm{E}+00$ & $1023 E+01$ & $0.2225 \mathrm{E}+01$ & $0.4780 \mathrm{E}+00$ & $0.3120 E-01$ \\
\hline 5 & $340 E+00$ & $0.1028 E+01$ & $0.2225 \mathrm{E}+01$ & $0.4780 \mathrm{E}+00$ & $0.3120 \mathrm{E}-01$ \\
\hline 6 & $340 E+00$ & $0.1023 E+01$ & $0.2236 \mathrm{E}+01$ & $0.4804 \mathrm{E}+00$ & $0.3136 \mathrm{E}-01$ \\
\hline 7 & $340 E+00$ & $0.1023 E+01$ & $0.2236 \mathrm{E}+01$ & $0.4804 \mathrm{E}+00$ & $0.3136 \mathrm{E}-01$ \\
\hline 8 & $.1340 E+00$ & $0.1028 E+01$ & $0.2225 \mathrm{E}+01$ & $0.4780 \mathrm{E}+00$ & $0.312 .0 E-01$ \\
\hline 9 & $.1340 E+00$ & $0.1023 \mathrm{E}+01$ & $0.2236 E+01$ & $0.4780 \mathrm{E}+00$ & $0.3120 \mathrm{E}-01$ \\
\hline 10 & $.1340 \mathrm{E}+00$ & $0.1028 \mathrm{E}+01$ & $0.2225 \mathrm{E}+01$ & $0.4804 \mathrm{E}+00$ & $.3136 \mathrm{E}-01$ \\
\hline 11 & $.1340 \mathrm{E}+00$ & $0.1028 E+01$ & $0.2225 \mathrm{E}+01$ & $0.4804 \mathrm{E}+00$ & $.3136 \mathrm{E}-01$ \\
\hline 12 & $.1340 E+00$ & $0.1023 \mathrm{E}+01$ & $0.2236 \mathrm{E}+01$ & $0.4780 \mathrm{E}+00$ & $.31 .20 \mathrm{E}-01$ \\
\hline 13 & $.1340 \mathrm{E}+00$ & $0.1028 \mathrm{E}+01$ & $0.2236 \mathrm{E}+01$ & $0.4780 \mathrm{E}+00$ & $0.3120 E-01$ \\
\hline 14 & $0.1340 \mathrm{E}+00$ & $0.1023 E+01$ & $0.2225 \mathrm{E}+01$ & $0.4804 \mathrm{E}+00$ & $0.3136 \mathrm{E}-01$ \\
\hline 15 & $0^{\circ} .1340 \mathrm{E}+00$ & $0.1023 \mathrm{E}+01$ & $0.2225 \mathrm{E}+01$ & $0.4804 \mathrm{E}+00$ & $0.3136 \mathrm{E}-01$ \\
\hline 16 & $0.1340 \mathrm{E}+00$ & $0.1028 \mathrm{E}+01$ & $0.2236 \mathrm{E}+01$ & $0.4780 \mathrm{E}+00$ & $0.3120 \mathrm{E}-01$ \\
\hline 17 & $0.1340 \mathrm{E}+00$ & $0.1023 E+01$ & $0.2225 \mathrm{E}+01$ & $0.4804 \mathrm{E}+00$ & $0.3120 \mathrm{E}-01$ \\
\hline 18 & $0.1340 \mathrm{E}+00$ & $0.1028 E+01$ & $0.2236 \mathrm{E}+01$ & $0.4780 \mathrm{E}+00$ & $0.3136 \mathrm{E}-01$ \\
\hline 19 & $0.1340 E+00$ & $0.1028 \mathrm{E}+01$ & $0.2236 \mathrm{E}+01$ & 0.4780 & $6 E-01$ \\
\hline 20 & $0.1340 E+00$ & $0.1023 E+01$ & $0.2225 E+01$ & +00 & $20 E-01$ \\
\hline 21 & $0.1340 \mathrm{E}+00$ & $0.1028 \mathrm{E}+01$ & $0.2225 \mathrm{E}+01$ & +00 & $0.3120 E-01$ \\
\hline 22 & $0.1340 E+00$ & $0.1023 E+01$ & $0.2236 \mathrm{E}+01$ & +00 & $0.3136 \mathrm{E}-01$ \\
\hline 23 & $.1340 \mathrm{E}$ & $0.1023 \mathrm{E}+01$ & $0.2236 E+01$ & +00 & $0.3136 \mathrm{E}-01$ \\
\hline 24 & $.1340 E+00$ & $0.1028 \mathrm{E}+01$ & $0.2225 E+01$ & 0.480 & $0.3120 \mathrm{E}-01$ \\
\hline 25 & .13401 & $0.1023 E+01$ & $0.2236 E+01$ & $804 E+00$ & $0.3120 \mathrm{E}-01$ \\
\hline 26 & .13401 & $0.1028 \mathrm{E}+01$ & $0.2225 \mathrm{E}+01$ & $0.4780 \mathrm{E}+00$ & $0.3136 \mathrm{E}-01$ \\
\hline 27 & $.1340 \mathrm{I}$ & $0.1028 E+01$ & $0.2225 \mathrm{E}+01$ & $0.4780 \mathrm{E}+00$ & $0.3136 \mathrm{E}-01$ \\
\hline 28 & $1340 E+00$ & $0.1023 \mathrm{E}+01$ & $0.2236 \mathrm{E}+01$ & $0.4804 E+00$ & $0.3120 \mathrm{E}-01$ \\
\hline 29 & $1340 E+00$ & $0.1028 E+01$ & $0.2236 E+01$ & $0.4804 \mathrm{E}+00$ & $0.3120 \mathrm{E}-01$ \\
\hline 30 & $1340 E+00$ & $0.1023 E+01$ & $0.2225 \mathrm{E}+01$ & $0.4780 \mathrm{E}+00$ & $0.3136 \mathrm{E}-01$ \\
\hline 31 & $.1340 \mathrm{E}+00$ & $0.1023 E+01$ & $0.2225 \mathrm{E}+01$ & $0.4780 \mathrm{E}+00$ & $0.3136 \mathrm{E}-01$ \\
\hline 0 & $.1340 E+00$ & $0.1028 E+01$ & $0.2236 \mathrm{E}+01$ & $0.4804 \mathrm{E}+00$ & $0.3120 \mathrm{E}-01$ \\
\hline
\end{tabular}


TABLE 2. (CONTINUED)

$\begin{array}{lc}\text { TEST } & \text { C } 6 \\ 33 & 0.1347 \mathrm{E}+00 \\ 34 & 0.1347 \mathrm{E}+00 \\ 35 & 0.1347 \mathrm{E}+00 \\ 36 & 0.1347 \mathrm{E}+00 \\ 37 & 0.1347 \mathrm{E}+00 \\ 38 & 0.1347 \mathrm{E}+00 \\ 39 & 0.1347 \mathrm{E}+00 \\ 40 & 0.1347 \mathrm{E}+00 \\ 41 & 0.1347 \mathrm{E}+00 \\ 42 & 0.1347 \mathrm{E}+00 \\ 43 & 0.1347 \mathrm{E}+00 \\ 44 & 0.1347 \mathrm{E}+00 \\ 45 & 0.1347 \mathrm{E}+00 \\ 46 & 0.1347 \mathrm{E}+00 \\ 47 & 0.1347 \mathrm{E}+00 \\ 48 & 0.1347 \mathrm{E}+00 \\ 49 & 0.1347 \mathrm{E}+00 \\ 50 & 0.1347 \mathrm{E}+00 \\ 51 & 0.1347 \mathrm{E}+00 \\ 52 & 0.1347 \mathrm{E}+00 \\ 53 & 0.1347 \mathrm{E}+00 \\ 54 & 0.1347 \mathrm{E}+00 \\ 55 & 0.1347 \mathrm{E}+00 \\ 56 & 0.1347 \mathrm{E}+00 \\ 57 & 0.1347 \mathrm{E}+00 \\ 58 & 0.1347 \mathrm{E}+00 \\ 59 & 0.1347 \mathrm{E}+00 \\ 60 & 0.1347 \mathrm{E}+00 \\ 61 & 0.1347 \mathrm{E}+00 \\ 62 & 0.1347 \mathrm{E}+00 \\ 63 & 0.1347 \mathrm{E}+00 \\ 64 & 0.1347 \mathrm{E}+00\end{array}$

C 7

$0.1023 E+01$

$0.1028 \mathrm{E}+01$

$0.1028 \mathrm{E}+01$

$0.1023 \mathrm{E}+01$

$0.1028 \mathrm{E}+01$.

$0.1028 \mathrm{E}+01$

$0.1023 E+01$

$0.1028 \mathrm{E}+01$

$0.1028 \mathrm{E}+01$.

$0.1023 \mathrm{E}+01$

$0.1028 \mathrm{E}+01$

$0.1023 E+01$

$0.1023 E+01$

$0.1028 \mathrm{E}+01$

$0.1023 \mathrm{E}+01$

$0.1028 \mathrm{E}+01$

$0.1028 \mathrm{E}+01$

$0.1023 \mathrm{E}+01$

$0.1028 \mathrm{E}+01$

$0.1023 E+01$

$0.102 .3 \mathrm{E}+01$

$0.1028 \mathrm{E}+01$

$0.1023 E+01$

$0.1028 \mathrm{E}+01$

$0.1028 \mathrm{E}+01$

$0.1023 \mathrm{E}+01$

$0.1028 \mathrm{E}+01$

$0.1023 \mathrm{E}+01$

$0.1023 \mathrm{E}+01$

$0.1028 E+01$
$0.1023 \mathrm{E}+01$

$0.1023 \mathrm{E}+01$
C 8

$0.2225 E+01$

$0.2236 \mathrm{E}+01$

$0.2236 \mathrm{E}+01$

$0.2225 \mathrm{E}+01$

$0.2225 \mathrm{E}+01$

$0.2236 \mathrm{E}+01$

$0.2236 \mathrm{E}+01$

$0.2225 \mathrm{E}+01$

$0.2236 \mathrm{E}+01$

$0.2225 \mathrm{E}+01$

$0.2225 \mathrm{E}+01$

$0.2236 \mathrm{E}+01$

$0.2236 \mathrm{E}+01$

$0.2225 \mathrm{E}+01$

$0.2225 \mathrm{E}+01$

$0.2236 \mathrm{E}+01$

$0.2225 \mathrm{E}+01$

$0.2236 \mathrm{E}+01$

$0.2236 \mathrm{E}+01$

$0.2225 E+01$

$0.2225 \mathrm{E}+01$

$0.2236 \mathrm{E}+01$

$0.2236 \mathrm{E}+01$

$0.2225 \mathrm{E}+01$

$0.2236 \mathrm{E}+01$

$0.2225 \mathrm{E}+01$

$0.2225 \mathrm{E}+01$

$0.2236 \mathrm{E}+01$

$0.2236 \mathrm{E}+01$

$0.2225 \mathrm{E}+01$

$0.2225 \mathrm{E}+01$

$0.2236 \mathrm{E}+01$

4 OF 10

C 9

$0.4780 E+00$

$0.4804 \mathrm{E}+00$

$0.4804 \mathrm{E}+00$

$0.4780 \mathrm{E}+00$

$0.4780 \mathrm{E}+00$

$0.4804 \mathrm{E}+00$

$0.4804 \mathrm{E}+00$

$0.4780 \mathrm{E}+00$

$0.4780 \mathrm{E}+00$

$0.4804 \mathrm{E}+00$

$0.4804 \mathrm{E}+00$

$0.4780 \mathrm{E}+00$

$0.4780 \mathrm{E}+00$

$0.4804 \mathrm{E}+00$

$0.4804 \mathrm{E}+00$

$0.4780 E+00$

$0.4804 \mathrm{E}+00$

$0.4780 \mathrm{E}+00$

$0.4780 \mathrm{E}+00$

$0.4804 \mathrm{E}+00$

$0.4804 \mathrm{E}+00$

$0.4780 E+00$

$0.4780 E+00$

$0.4804 \mathrm{E}+00$

$0.4804 \mathrm{E}+00$

$0.4780 \mathrm{E}+00$

$0.4780 \mathrm{E}+00$

$0.4804 \mathrm{E}+00$

$0.4804 \mathrm{E}+00$

$0.4780 E+00$

$0.4780 \mathrm{E}+00$

$0.4804 \mathrm{E}+00$
C 10

$0.3136 \mathrm{E}-01$

$0.3120 \mathrm{E}-01$

$0.3120 \mathrm{E}-0.1$

$0.3136 \mathrm{E}-01$

$0.3136 \mathrm{E}-01$

$0.3120 E-01$

$0.3120 \mathrm{E}-01$

$0.3136 \mathrm{E}-01$

$0.3136 \mathrm{E}-01$

$0.3120 \mathrm{E}-01$

$0.3120 \mathrm{E}-01$

$0.3136 \mathrm{E}-01$

$0.3136 \mathrm{E}-01$

$0.3120 \mathrm{E}-01$

$0.3120 \mathrm{E}-01$

$0.3136 \mathrm{E}-01$

$0.3136 E-01$

$0.3120 \mathrm{E}-01$

$0.3120 \mathrm{E}-01$

$0.3136 \mathrm{E}-01$

$0.3136 \mathrm{E}-01$

$0.3120 \mathrm{E}-01$

$0.3120 \mathrm{E}-01$

$0.3136 \mathrm{E}-01$

$0.3136 \mathrm{E}-01$

$0.3120 \mathrm{E}-01$

$0.3120 \mathrm{E}-01$

$0.3136 \mathrm{E}-01$

$0.3136 \mathrm{E}-01$

$0.3120 \mathrm{E}-01$

$0.3120 \mathrm{E}-01$

$0.3136 E-01$ 
LIQUID DENSITY OUTPUT $n-C 4 ! C O 2$ DRES $=4 \quad S F A C T=0.5{ }^{\circ}$

TABLE 2. (CONTINUED)

5 OF 10

\begin{tabular}{|c|c|c|c|c|c|}
\hline TEST & C 11 & C 12 & C 13 & 14 & \\
\hline 1 & $0.8700 \mathrm{E}-01$ & $0.8000 \mathrm{E}-02$ & $0.1000 E+01$ & $0.2455 E+01$ & $0.7320 \mathrm{E}+00$ \\
\hline 2 & $0.8743 \mathrm{E}-01$ & $0.8040 \mathrm{E}-02$ & $0.1005 E+01$ & $0.2467 E+01$ & $0.7357 \mathrm{E}+00$ \\
\hline 3 & $0.8700 \mathrm{E}-01$ & $0.8000 \mathrm{E}-02$ & $0.1000 E+01$ & $0.2455 \mathrm{E}+01$ & $0.7320 \mathrm{E}+00$ \\
\hline 4 & $8743 E-01$ & $0.8040 \mathrm{E}-02$ & $0.1005 E+01$ & $0.2467 \mathrm{E}+01$ & $0.7357 \mathrm{E}+00$ \\
\hline 5 & $0.8743 \mathrm{E}-01$ & $0.8040 \mathrm{E}-02$ & $0.1005 \mathrm{E}+01$ & $0.2455 E+01$ & $0.7320 \mathrm{E}+00$ \\
\hline 6 & $0.8700 \mathrm{E}-01$ & $0.8000 E-02$ & $0.1000 E+01$ & $0.2467 \mathrm{E}+01$ & $0.7357 \mathrm{E}+00$ \\
\hline 7 & $0.8743 \mathrm{E}-01$ & $0.8040 \mathrm{E}-02$ & $0.1005 \mathrm{E}+01$ & $0.2455 \mathrm{E}+01$ & $0.7320 E+00$ \\
\hline 8 & $0.8700 \mathrm{E}-01$ & $0.8000 \mathrm{E}-02$ & $0.1000 E+01$ & $0.2467 E+01$ & $0.7357 \mathrm{E}+00$ \\
\hline 9 & $0.8743 \mathrm{E}-01$ & $0.8000 \mathrm{E}-02$ & $0.1000 E+01$ & $0.2467 E+01$ & $0.7357 \mathrm{E}+00$ \\
\hline 10 & $0.8700 \mathrm{E}-01$ & $0.8040 \mathrm{E}-02$ & $0.1005 \mathrm{E}+01$ & $0.2455 E+0.1$ & $0.7320 E+00$ \\
\hline 11 & $0.8743 \mathrm{E}-01$ & $0.8000 E-02$ & $0.1000 \mathrm{E}+01$ & $0.2467 E+01$ & $0.7357 \mathrm{E}+00$ \\
\hline 12 & $0.87007-01$ & $0.8040 E-02$ & $0.1005 E+01$ & $0.2455 E+01$ & $0.7320 \mathrm{E}+00$ \\
\hline 13 & $0.8700 \mathrm{E}-01$ & $0.8040 E-02$ & $0.1005 \mathrm{E}+01$ & $7 E+01$ & $0.7357 \mathrm{E}+00$ \\
\hline 14 & $0.8743 \mathrm{E}-01$ & $0.8000 \mathrm{E}-02$ & $0.1000 E+01$ & $5 E+01$ & $0.7320 \mathrm{E}+0$ \\
\hline 15 & $0.8700 \mathrm{E}-01$ & $0.8040 \mathrm{E}-02$ & $0.1005 E+01$ & $7 E+01$ & $0.7357 \mathrm{E}+00$ \\
\hline 16 & $0.8743 \mathrm{E}-01$ & $0.8000 \mathrm{E}-02$ & $0.1000 \mathrm{E}+01$ & $5 E+01$ & $0.7320 \mathrm{E}+00$ \\
\hline 17 & $0.8700 \mathrm{E}-01$ & $0.8040 \mathrm{E}-02$ & $0.1000 \mathrm{E}+01$ & $7 E+01$ & $0.7320 \mathrm{E}+00$ \\
\hline 18 & $0.8743 E-01$ & $0.8000 \mathrm{E}-02$ & $0.1005 E+01$ & $5 E+01$ & $0.7357 \mathrm{E}+0$ \\
\hline 19 & $0.8700 \mathrm{E}-01$ & $0.8040 \mathrm{E}-02$ & $0.1000 E+01$ & $7 E+01$ & $0.7320 E+00$ \\
\hline 20 & $0.8743 \mathrm{E}-01$ & $0.8000 \mathrm{E}-02$ & $0.1005 E+01$ & $5 E+01$ & $0.7357 \mathrm{E}+00$ \\
\hline 21 & $0.8743 \mathrm{E}-01$ & $0.8000 \mathrm{E}-02$ & $0.1005 E+01$ & $7 E+01$ & $0.7320 E+00$ \\
\hline 22 & $0.8700 E-01$ & $0.8040 \mathrm{E}-02$ & $0.1000 E+01$ & $5 E+01$ & $0.7357 \mathrm{E}+00$ \\
\hline 23 & $0.8743 E-01$ & $0.8000 \mathrm{E}-02$ & $0.1005 E+01$ & $7 E+01$ & $0.7320 E+00$ \\
\hline 24 & $0.8700 E-01$ & $0.8040 E-02$ & $0.1000 E+01$ & $5 E+01$ & $0.7357 \mathrm{E}+0$ \\
\hline 25 & $0.8743 E-01$ & $0.8040 \mathrm{E}-02$ & $0.1000 \mathrm{E}+01$ & $5 E+01$ & $0.7357 \mathrm{E}+0$ \\
\hline 26 & $0.8700 \mathrm{E}-$ & $0.8000 \mathrm{E}-02$ & $0.1005 E+01$ & $0.2467 \mathrm{E}+01$ & $0.7320 \mathrm{E}+0$ \\
\hline 27 & $0.8743 E-01$ & $0.8040 E-02$ & $0.1000 E+01$ & $0.2455 E+01$ & $0.7357 E+00$ \\
\hline 28 & $0.8700 E-01$ & $0.8000 \mathrm{E}-02$ & $0.1005 E+01$ & $0.2467 \mathrm{E}+01$ & $0.7320 E+00$ \\
\hline 29 & $0.8700 \mathrm{E}-01$ & $0.8000 E-02$ & $0.1005 \mathrm{E}+01$ & $0.2455 \mathrm{E}+01$ & $0.7357 \mathrm{E}+0$ \\
\hline 30 & $0.8743 E-01$ & $0.8040 E-02$ & $0.1000 E+01$ & $0.2467 E+01$ & $0.7320 \mathrm{E}+00$ \\
\hline 31 & $0.8700 E-01$ & $0.8000 E-02$ & $0.1005 E+01$ & $55 E+01$ & $0.7357 \mathrm{E}+0$ \\
\hline 32 & $0.8743 E-01$ & $0.8040 E-02$ & $0.1000 E+01$ & $0.2467 \mathrm{E}+01$ & $0.7320 \mathrm{E}+0$ \\
\hline
\end{tabular}


TABLE 2. (CONTINUED)

$\begin{array}{lcc}\text { TEST } & \text { C 11 } & \text { C 12 } \\ 33 & 0.8700 E-01 & 0.8000 E-02 \\ 34 & 0.8743 E-01 & 0.8040 E-02 \\ 35 & 0.8700 E-01 & 0.8000 E-02 \\ 36 & 0.8743 E-01 & 0.8040 E-02 \\ 37 & 0.8743 E-01 & 0.8040 E-02 \\ 38 & 0.8700 E-01 & 0.8000 E-02 \\ 39 & 0.8743 E-01 & 0.8040 E-02 \\ 40 & 0.8700 E-01 & 0.8000 E-02 \\ 41 & 0.8743 E-01 & 0.8000 E-02 \\ 42 & 0.8700 E-01 & 0.8040 E-02 \\ 43 & 0.8743 E-01 & 0.8000 E-02 \\ 44 & 0.8700 E-01 & 0.8040 E-02 \\ 45 & 0.8700 E-01 & 0.8040 E-02 \\ 46 & 0.8743 E-01 & 0.8000 E-02 \\ 47 & 0.8700 E-01 & 0.8040 E-02 \\ 48 & 0.8743 E-01 & 0.8000 E-02 \\ 49 & 0.8700 E-01 & 0.8040 E-02 \\ 50 & 0.8743 E-01 & 0.8000 E-02 \\ 51 & 0.8700 E-01 & 0.8040 E-02 \\ 52 & 0.8743 E-01 & 0.8000 E-02 \\ 53 & 0.8743 E-01 & 0.8000 E-02 \\ 54 & 0.8700 E-01 & 0.8040 E-02 \\ 55 & 0.8743 E-01 & 0.8000 E-02 \\ 56 & 0.8700 E-01 & 0.8040 E-02 \\ 57 & 0.8743 E-01 & 0.8040 E-02 \\ 58 & 0.8700 E-01 & 0.8000 E-02 \\ 59 & 0.8743 E-01 & 0.8040 E-02 \\ 60 & 0.8700 E-01 & 0.8000 E-02 \\ 61 & 0.8700 E-01 & 0.8000 E-02 \\ 62 & 0.8743 E-01 & 0.8040 E-02 \\ 63 & 0.8700 E-01 & 0.8000 E-02 \\ 64 & 0.8743 E-01 & 0.8040 E-02\end{array}$

C 13

$0.1005 \mathrm{E}+0.1$

$0.1000 E+01$

$0.1005 \mathrm{E}+01$

$0.1000 \mathrm{E}+01$

$0.1000 \mathrm{E}+01$

$0.1005 \mathrm{E}+01$

$0.1000 \mathrm{E}+01$

$0.1005 \mathrm{E}+01$

$0.1005 \mathrm{E}+01$

$0.1000 \mathrm{E}+01$

$0.1005 \mathrm{E}+01$

$0.1000 \mathrm{E}+01$

$0.1000 \mathrm{E}+01$

$0.1005 \mathrm{E}+01$.

$0.1000 \mathrm{E}+01$

$0.1005 \mathrm{E}+01$

$0.1005 E+01$

$0.1000 \mathrm{E}+01$

$0.1005 \mathrm{E}+01$

$0.1000 E+01$

$0.1000 \mathrm{E}+01$

$0.1005 \mathrm{E}+01$

$0.1000 E+01$

$0.1005 \mathrm{E}+01$

$0.1005 E+01$

$0.1000 E+01$

$0.1005 \mathrm{E}+01$

$0.1000 \mathrm{E}+01$

$0.1000 \mathrm{E}+01$

$0.1005 \mathrm{E}+01$

$0.1000 E+01$

$0.1005 \mathrm{E}+01$
6 OF 10
C 14
C 15

$0.2455 E+01$

$0.2467 \mathrm{E}+01$

$0.2455 \mathrm{E}+01$

$0.2467 \mathrm{E}+01$

$0.2455 \mathrm{E}+01$

$0.2467 \mathrm{E}+01$

$0.2455 \mathrm{E}+01$

$0.2467 E+01$

$0.2467 \mathrm{E}+01$

$0.2455 \mathrm{E}+01$

$0.2467 \mathrm{E}+01$

$0.2455 \mathrm{E}+01$

$0.2467 \mathrm{E}+01$

$0.2455 \mathrm{E}+01$

$0.2467 \mathrm{E}+01$

$0.2455 \mathrm{E}+01$

$0.2467 \mathrm{E}+01$

$0.2455 \mathrm{E}+01$

$0.2467 \mathrm{E}+01$

$0.2455 \mathrm{E}+01$

$0.2467 \mathrm{E}+01$

$0.2455 \mathrm{E}+01$

$0.2467 \mathrm{E}+01$

$0.2455 \mathrm{E}+01$

$0.2455 \mathrm{E}+01$

$0.2467 \mathrm{E}+01$

$0.2455 \mathrm{E}+0.1$

$0.2467 \mathrm{E}+01$

$0.2455 \mathrm{E}+01$

$0.2467 \mathrm{E}+0.1$

$0.2455 \mathrm{E}+01$

$0.2467 \mathrm{E}+01$
$0.7357 \mathrm{E}+00$

$0.7320 E+00$

$0.7357 \mathrm{E}+00$

$0.7320 \mathrm{E}+00$

$0.7357 \mathrm{E}+00$

$0.7320 \mathrm{E}+00$

$0.7357 \mathrm{E}+00$

0.732 $\mathrm{E}+00$

$0.7320 \mathrm{E}+00$

$0.7357 E+00$

$0.7320 \mathrm{E}+00$

$0.7357 \mathrm{E}+00$

$0.7320 \mathrm{E}+00$

$0.7357 \mathrm{E}+00$

$0.7320 \mathrm{E}+00$

$0.7357 \mathrm{E}+00$

$0.7357 \mathrm{E}+00$

$0.7320 \mathrm{E}+00$

$0.7357 \mathrm{E}+00$

$0.7320 \mathrm{E}+00$

$0.7357 \mathrm{E}+00$

$0.7320 \mathrm{E}+00$

$0.7357 \mathrm{E}+00$

$0.7320 \mathrm{E}+00$

$0.7320 \mathrm{E}+00$

$0.7357 \mathrm{E}+00$

$0.7320 \mathrm{E}+00$

$0.7357 \mathrm{E}+00$

$0.7320 \mathrm{E}+00$

$0.7357 \mathrm{E}+00$

$0.7320 \mathrm{E}+00$

$0.7357 \mathrm{E}+00$ 
TABLE 2. (CONTINUED)

$\begin{array}{cc}\text { TEST } & \mathrm{C} 16 \\ 1 & 0.1062 \mathrm{E}+01 \\ 2 & 0.1067 \mathrm{E}+01 \\ 3 & 0.1062 \mathrm{E}+01 \\ 4 & 0.1067 \mathrm{E}+01 \\ 5 & 0.1062 \mathrm{E}+01 \\ 6 & 0.1067 \mathrm{E}+01 \\ 7 & 0.1062 \mathrm{E}+01 \\ 8 & 0.1067 \mathrm{E}+01 \\ 9 & 0.1062 \mathrm{E}+01 \\ 10 & 0.1067 \mathrm{E}+01 \\ 11 & 0.1062 \mathrm{E}+01 \\ 12 & 0.1057 \mathrm{E}+01 \\ 13 & 0.1062 \mathrm{E}+01 \\ 14 & 0.1067 \mathrm{E}+01 \\ 15 & 0.1062 \mathrm{E}+01 \\ 16 & 0.1067 \mathrm{E}+01 \\ 17 & 0.1067 \mathrm{E}+01 \\ 18 & 0.1062 \mathrm{E}+01 \\ 19 & 0.1067 \mathrm{E}+01 \\ 20 & 0.1062 \mathrm{E}+01 \\ 21 & 0.1067 \mathrm{E}+01 \\ 22 & 0.1062 \mathrm{E}+01 \\ 23 & 0.1067 \mathrm{E}+01 \\ 24 & 0.1062 \mathrm{E}+01 \\ 25 & 0.1067 \mathrm{E}+01 \\ 26 & 0.1062 \mathrm{E}+01 \\ 27 & 0.1067 \mathrm{E}+01 \\ 28 & 0.1062 \mathrm{E}+01 \\ 29 & 0.1067 \mathrm{E}+01 \\ 30 & 0.1062 \mathrm{E}+01 \\ 31 & 0.1067 \mathrm{E}+01 \\ 32 & 0.1062 \mathrm{E}+01\end{array}$

\begin{abstract}
C 17
\end{abstract}
$0.1905 \mathrm{E}+01$

$0.1905 \mathrm{E}+01$

$0.1914 \mathrm{E}+01$

$0.1914 \mathrm{E}+01$

$0.1914 \mathrm{E}+01$

$0.1914 \mathrm{E}+01$

$0.1905 \mathrm{E}+01$

$0.1905 \mathrm{E}+01$

$0.1914 \mathrm{E}+01$

$0.1914 \mathrm{E}+01$

$0.1905 \mathrm{E}+01$

$0.1905 \mathrm{E}+01$

$0.1905 \mathrm{E}+01$

$0.1905 E+01$

$0.1914 \mathrm{E}+01$

$0.1914 \mathrm{E}+01$

$0.1905 \mathrm{E}+01$

$0.1905 \mathrm{E}+01$

$0.1914 \mathrm{E}+01$

$0.1914 E+01$

$0.1914 \mathrm{E}+01$

$0.1914 \mathrm{E}+01$

$0.1905 \mathrm{E}+01$

$0.1905 \mathrm{E}+01$

$0.1914 \mathrm{E}+01$

$0.1914 \mathrm{E}+01$

$0.1905 \mathrm{E}+01$

$0.1905 E+01$

$0.1905 E+01$

$0.1905 \mathrm{E}+01$

$0.1914 \mathrm{E}+01$

$0.1914 \mathrm{E}+01$
TC 1

$0.4252 \mathrm{E}+03$

$0.4252 \mathrm{E}+03$

$0.4273 \mathrm{E}+03$

$0.4273 \mathrm{E}+03$

$0.4273 \mathrm{E}+03$

$0.4273 E+03$

$0.4252 \mathrm{E}+03$

$0.4252 \mathrm{E}+03$

$0.4252 \mathrm{E}+03$

$0.4252 \mathrm{E}+03$

$0.4273 \mathrm{E}+03$

$0.4273 \mathrm{E}+03$

$0.4273 E+03$

$0.4273 \mathrm{E}+03$

$0.4252 \mathrm{E}+03$

$0.4252 \mathrm{E}+03$

$0.4273 E+03$

$0.4273 \mathrm{E}+03$

$0.4252 \mathrm{E}+03$

$0.4252 \mathrm{E}+03$

$0.4252 \mathrm{E}+03$

$0.4252 \mathrm{E}+03$

$0.4273 \mathrm{E}+03$

$0.4273 \mathrm{E}+03$

$0.4273 E+03$

$0.4273 \mathrm{E}+03$

$0.4252 \mathrm{E}+03$

$0.4252 \mathrm{E}+03$

$0.4252 \mathrm{E}+03$

$0.4252 \mathrm{E}+03$

$0.4273 \mathrm{E}+03$

$0.4273 \mathrm{E}+03$
PC 1

$0.3797 \mathrm{E}+02$

$0.3797 \mathrm{E}+02$

$0.3816 \mathrm{E}+02$

$0.3816 \mathrm{E}+02$

$0.3816 \mathrm{E}+02$

$0.3816 \mathrm{E}+02$

$0.3797 \mathrm{E}+02$

$0.3797 \mathrm{E}+02$

$0.3797 \mathrm{E}+02$

$0.3797 \mathrm{E}+02$

$0.3816 \mathrm{E}+02$

$0.3816 \mathrm{E}+02$

$0.3816 \mathrm{E}+02$

$0.3816 \mathrm{E}+02$

$0.3797 \mathrm{E}+02$

$0.3797 \mathrm{E}+02$

$0.3797 \mathrm{E}+02$

$0.3797 \mathrm{E}+02$

$0.3816 \mathrm{E}+02$

$0.3816 \mathrm{E}+02$

$0.3816 \mathrm{E}+02$

$0.3816 \mathrm{E}+02$

$0.3797 E+02$

$0.3797 \mathrm{E}+02$

$0.3797 \mathrm{E}+02$

$0.3797 \mathrm{E}+02$

$0.3816 \mathrm{E}+02$

$0.3816 \mathrm{E}+02$

$0.3816 \mathrm{E}+02$

$0.3816 \mathrm{E}+02$

$0.3797 \mathrm{E}+02$

$0.3797 \mathrm{E}+02$
7 OF 10

OM 1

$0.1995 \mathrm{~F}+00$

$0.1993 \mathrm{E}+00$

$0.2003 E+00$

$0.2003 E+00$

$0.1993 \mathrm{E}+00$

$0.1993 E+00$

$0.2003 E+00$

$0.2003 \mathrm{E}+00$

$0.2003 E+00$

$0.2003 \mathrm{E}+00$

$0.1993 \mathrm{E}+00$

$0.1993 \mathrm{E}+00$

$0.2003 E+00$

$0.2003 \mathrm{E}+00$

$0.1993 \mathrm{E}+00$

$0.1993 \mathrm{E}+00$

$0.2003 \mathrm{E}+00$

$0.2003 E+00$

$0.1993 E+00$

$0.1993 \mathrm{E}+00$

$0.2003 \mathrm{E}+00$

$0.2003 \mathrm{E}+00$

$0.1993 E+00$

$0.1993 \mathrm{E}+00$

$0.1993 \mathrm{E}+00$

$0.1993 \mathrm{E}+00$

$0.2003 \mathrm{E}+00$

$0.2003 E+00$

$0.1993 E+00$

$0.1993 E+00$

$0.2003 E+00$

$0.2003 \mathrm{E}+00$ 
TABLE 2. (CONTINUED)

8 OF 10

\begin{tabular}{|c|c|c|c|c|c|}
\hline E & 16 & 17 & $\mathrm{TC}$ & PC & $\mathrm{OM}$ \\
\hline 3 & $67 E+01$ & $0.1905 \mathrm{E}+01$ & $0.4252 E+03$ & $0.3816 \mathrm{E}+02$ & $1993 \mathrm{E}+00$ \\
\hline & $62 E+01$ & $.1905 \mathrm{E}+01$ & $252 E+03$ & $0.3816 \mathrm{E}+02$ & $993 E+00$ \\
\hline & $067 E+01$ & $914 E+01$ & $273 E+03$ & $0.3797 \mathrm{E}+02$ & $0.2003 E+0$ \\
\hline & $062 \mathrm{E}+01$ & $1914 \mathrm{E}+01$ & $73 E+03$ & $0.3797 \mathrm{E}+02$ & $0.2003 E+0$ \\
\hline 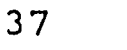 & $067 \mathrm{E}+01$ & $914 \mathrm{E}+01$ & $273 E+03$ & $0.3797 \mathrm{E}+02$ & $0.1993 \mathrm{E}+0$ \\
\hline 38 & $1052 \mathrm{E}+01$ & $.1914 E+01$ & $4273 E+03$ & $0.3797 E+02$ & $0.1993 \mathrm{E}+0$ \\
\hline קב & $1067 \mathrm{E}+01$ & $0.1905 \mathrm{E}+01$ & $4252 E+03$ & $0.3816 E+02$ & $03 E+0$ \\
\hline 0 & $0.1062 \mathrm{E}+01$ & $0.1905 \mathrm{E}+01$ & $0.4252 \mathrm{E}+03$ & $0.3816 \mathrm{E}+02$ & $0.2003 \mathrm{E}+0$ \\
\hline 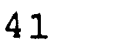 & $0.1067 E+01$ & $0.1914 \mathrm{E}+01$ & $0.4252 \mathrm{E}+03$ & $0.3816 \mathrm{E}+02$ & $0.2003 E+0$ \\
\hline 2 & $E+01$ & $0.1914 \mathrm{E}+01$ & $0.4252 \mathrm{E}+03$ & $0.3816 \mathrm{E}+02$ & $03 E+0$ \\
\hline 43 & $E+01$ & $5 E+01$ & $0.4273 E+03$ & $0.3797 \mathrm{~F}+02$ & $3 E+0$ \\
\hline 44 & $E+01$ & $8+01$ & $0.4273 F+03$ & $0.3797 \mathrm{E}+02$ & $3 E+0$ \\
\hline- & $E+01$ & +01 & $0.4273 E+03$ & $0.3797 \mathrm{E}+02$ & 0 . \\
\hline 6 & $0.1062 \mathrm{E}+01$ & $0.1905 \mathrm{E}+01$ & $0.4273 E+03$ & $0.3797 \mathrm{E}+02$ & 0 . \\
\hline 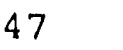 & $0.1067 \mathrm{E}+01$ & $0.1914 \mathrm{E}+01$ & $0.4252 E+03$ & $0.3816 E+02$ & 0. \\
\hline 48 & $0.1062 \mathrm{E}+01$ & 0.191 & $4252 E+03$ & $0.3816 \mathrm{E}+02$ & \\
\hline 4 & $E+01$ & 0.19 & $273 E+03$ & $0.3816 \mathrm{E}+02$ & \\
\hline & $E+01$ & $0.1905 \mathrm{E}+01$ & $273 E+03$ & $0.3816 E+02$ & $03 E+0$ \\
\hline & $0.1062 \mathrm{E}+01$ & $0.1914 E+01$ & $252 E+03$ & $0.3797 E+02$ & $93 E+0$ \\
\hline & $0.1067 E+01$ & $0.1914 \mathrm{E}+01$ & $0.4252 \mathrm{E}+03$ & $0.3797 \mathrm{E}+02$ & $93 \mathrm{E}$ \\
\hline 3 & $0.1062 \mathrm{E}+01$ & $0.1914 \mathrm{E}+01$ & $0.4252 \mathrm{E}+03$ & $0.3797 E+02$ & $3 \mathrm{E}$ \\
\hline 34 & $0.1067 \mathrm{E}+01$ & 0.191 & $52 E+03$ & $0.3797 E+02$ & $3 E$ \\
\hline 55 & $0.1062 \mathrm{E}+01$ & 0.19 & $73 E+03$ & $6 E+02$ & $93 E+0$ \\
\hline 56 & $0.1067 \mathrm{E}+0$ & 0.19 & $73 E+03$ & $6 \mathrm{E}+02$ & $93 E+0$ \\
\hline 57 & $0.1062 \mathrm{E}+0$ & 0.19 & $73 E$ & $6 \mathrm{E}+02$ & $3 E+0$ \\
\hline 58 & $0.1067 \mathrm{E}+$ & 0.19 & $73 \mathrm{E}$ & $0.3816 E+02$ & $93 E+0$ \\
\hline 59 & $0.1062 \mathrm{E}+\mathrm{C}$ & 0.19 & $52 \mathrm{E}+03$ & $0.3797 E+02$ & $0.2003 E+0$ \\
\hline 60 & $0.1067 \mathrm{E}+01$ & 0.19 & $52 \mathrm{E}+03$ & $0.3797 \mathrm{E}+02$ & $0.2003 E+0$ \\
\hline 61 & $0.1062 E+01$ & 0.19 & $252 E+03$ & $0.3797 E+02$ & $0.1993 \mathrm{E}+0$ \\
\hline 62 & $E+C$ & 0.19 & $+252 E+03$ & $0.3797 E+02$ & $0.1993 E+0$ \\
\hline 6 & $E+C$ & 0.19 & $273 E+03$ & $0.3816 \mathrm{E}+02$ & $03 E+0$ \\
\hline 54 & $E+01$ & $.1914 \mathrm{E}+01$ & $273 E+03$ & $0 ; 3816 \mathrm{E}+02$ & $0.2003 E+0$ \\
\hline
\end{tabular}


TABLE 2. (CONTINUED)

\begin{tabular}{|c|c|c|c|c|}
\hline TEST & TC & PC & OM & 2 \\
\hline 1 & $0.3042 \mathrm{E}+03$ & $0.7382 \mathrm{E}+02$ & $0.2250 \mathrm{E}+00$ & $0.1000 E-01$ \\
\hline 2 & $0.3042 E+03$ & $0.7382 \mathrm{E}+02$ & $0.2250 \mathrm{E}+00$ & 1 \\
\hline 3 & $0.3057 E+03$ & $0.7419 \mathrm{E}+02$ & $0.2250 \mathrm{E}+00$ & 1 \\
\hline 4 & $0.3057 E+03$ & $0.7419 \mathrm{E}+02$ & $0.2250 \mathrm{E}+00$ & 1 \\
\hline 5 & $0.3042 E+03$ & $0.7382 \mathrm{E}+02$ & $0.2261 E+00$ & 1 \\
\hline 6 & $0.3042 \mathrm{E}+03$ & $0.7382 E+02$ & $0.2261 \mathrm{E}+00$ & -01 \\
\hline 7 & $0.3057 \mathrm{E}+03$ & $0.7419 \mathrm{E}+02$ & $0.2261 E+00$ & -01 \\
\hline 8 & $0.3057 \mathrm{E}+03$ & $0.7419 \mathrm{E}+02$ & $0.2261 \mathrm{E}+00$ & $5 E-01$ \\
\hline 9 & $0.3057 \mathrm{E}+03$ & $0.7382 \mathrm{E}+02$ & $0.2261 \mathrm{E}$ & $5 E-01$ \\
\hline 10 & $0.3057 E+03$ & $0.7382 \mathrm{E}+02$ & $0.2261 \mathrm{E}$ & $5 E-01$ \\
\hline 11 & $0.3042 E+03$ & $0.7419 E+02$ & $0.2261 \mathrm{E}$ & $E-01$ \\
\hline 12 & $0.3042 E+03$ & $0.7419 E+02$ & $0.2261 \mathrm{E}$ & $5 E-01$ \\
\hline 13 & $0.3057 E+03$ & $0.7382 \mathrm{E}+02$ & $0.2250 \mathrm{E}$ & $E-01$ \\
\hline 14 & $0.3057 \mathrm{E}+03$ & $0.7382 E+02$ & $0.2250 \mathrm{E}$ & $E-01$ \\
\hline 15 & $0.3042 \mathrm{E}+03$ & $0.7419 \mathrm{E}+02$ & 0.2250 & 0.10 \\
\hline 16 & $0.3042 E+03$ & $0.7419 \mathrm{E}+02$ & 0.2250 & $2-01$ \\
\hline 17 & $0.3042 E+03$ & $0.7419 \mathrm{E}+02$ & 0.226 & 0.10 \\
\hline 18 & $0.3042 \mathrm{E}+03$ & $0.7419 E+02$ & 0.226 & -01 \\
\hline 19 & $0.3057 \mathrm{E}+03$ & $0.7382 E+02$ & 0.226 & 0.10 \\
\hline 20 & $0.3057 \mathrm{E}+03$ & $0.7382 E+02$ & 0.22 & 0.10 \\
\hline 21 & $0.3042 E+03$ & $0.7419 E+02$ & 0.225 & 0.10 \\
\hline 22 & $0.3042 E+03$ & $0.7419 \mathrm{E}+02$ & 0.225 & 0.100 \\
\hline 23 & $0.3057 E+03$ & $0.7382 \mathrm{E}+02$ & $0.2250 E+00$ & $0.1005 \mathrm{E}-01$ \\
\hline 24 & $0.3057 E+03$ & $0.7382 \mathrm{E}+02$ & $0.2250 \mathrm{E}+00$ & $0.1005 \mathrm{E}-01$ \\
\hline 25 & $0.3057 E+03$ & $0.7419 \mathrm{E}+02$ & $0.2250 \mathrm{E}+00$ & $0.1005 \mathrm{E}-01$ \\
\hline 26 & $0.3057 \mathrm{E}+03$ & $0.7419 E+02$ & $0.2250 E+00$ & $0.1005 \mathrm{E}-01$ \\
\hline 27 & $0.3042 E+03$ & $0.7382 E+02$ & $0.2250 \mathrm{E}+00$ & $0.1005 \mathrm{E}-01$ \\
\hline 28 & $0.3042 E+03$ & $0.7382 E+02$ & $0.2250 E+00$ & $0.1005 E-01$ \\
\hline 29 & $0.3057 E+03$ & $0.7419 E+02$ & $0.2261 E+00$ & $0.1000 \mathrm{E}-01$ \\
\hline 30 & $0.3057 \mathrm{E}+03$ & $0.7419 E+02$ & $0.2261 \mathrm{E}+00$ & $0.1000 \mathrm{E}-01$ \\
\hline 31 & $0.3042 E+03$ & $0.7382 E+02$ & $0.2261 E+00$ & $0.1000 \mathrm{E}-01$ \\
\hline 32 & $0.3042 E+03$ & $0.7382 E+02$ & $0.2261 \mathrm{E}+00$ & $0.1000 \mathrm{E}-01$ \\
\hline
\end{tabular}


TABLE 2. (CONTINUED)

\begin{tabular}{|c|c|c|c|c|}
\hline TEST & $\mathrm{TC}$ & PC & $O M$ & 12 \\
\hline 33 & $3057 E+03$ & $0.7419 E+02$ & $0.2250 \mathrm{E}+00$ & $0.1005 \mathrm{E}-01$ \\
\hline 34 & $057 E+03$ & $419 E+02$ & $0.2250 \mathrm{E}+00$ & $0.1005 \mathrm{E}-01$ \\
\hline 35 & $0.3042 \mathrm{E}+03$ & $382 E+02$ & $2250 E+00$ & $.1005 \mathrm{E}-01$ \\
\hline 36 & $0.3042 E+03$ & $382 E+02$ & $0.2250 E+00$ & $0.1005 \mathrm{E}-01$ \\
\hline 37 & $0.3057 E+03$ & $7419 E+02$ & $0.2261 E+00$ & $0.1000 \mathrm{E}-01$ \\
\hline 38 & $57 E+03$ & $419 \mathrm{E}+02$ & $61 E+00$ & $0.1000 \mathrm{E}-01$ \\
\hline 9 & $42 E+03$ & $382 \mathrm{E}+02$ & $61 \mathrm{E}+00$ & $0.1000 E-01$ \\
\hline 0 & $2 E+03$ & $82 \mathrm{E}+02$ & $61 \mathrm{E}+00$ & $0.1000 \mathrm{E}-01$ \\
\hline 1 & $42 E+03$ & $0.7419 \mathrm{E}+02$ & $61 \mathrm{E}+00$ & $0.1000 \mathrm{E}-01$ \\
\hline 42 & $42 E+03$ & $0.7419 \mathrm{E}+02$ & $61 E+00$ & $0.1000 \mathrm{E}-01$ \\
\hline 43 & $57 E+03$ & $0.7382 \mathrm{E}+02$ & $61 E+00$ & $0.1000 E \cdots 01$ \\
\hline 4 & $57 E+03$ & $0.7382 \mathrm{E}+02$ & $0.2261 \mathrm{E}+00$ & $0.1000 \mathrm{E}-01$ \\
\hline & $2 E+03$ & $0.7419 E+02$ & $0.2250 \mathrm{E}+00$ & $0.1005 \mathrm{E}-01$ \\
\hline & $2 E+03$ & $0.7419 \mathrm{E}+02$ & $0.2250 E+00$ & $0.1005 E-01$ \\
\hline & $57 E+03$ & $0.7382 \mathrm{E}+02$ & $0.2250 E+00$ & $0.1005 \mathrm{E}-01$ \\
\hline & $57 E+03$ & $0.7382 \mathrm{E}+02$ & $0.2250 E+00$ & $0.1005 \mathrm{E}-01$ \\
\hline & $0.3057 \mathrm{E}+03$ & $0.7382 \mathrm{E}+02$ & $0.2261 E+00$ & $0.1005 \mathrm{E}-01$ \\
\hline & $0.3057 E+03$ & $0.7 .382 \mathrm{E}+02$ & $0.2261 E+00$ & $0.1005 \mathrm{E}-01$ \\
\hline & $0.3042 \mathrm{E}+03$ & $0.7419 E+02$ & $0.2261 \mathrm{E}+00$ & $0.1005 \mathrm{E}-01$ \\
\hline & $0.3042 \mathrm{E}+03$ & $0.7419 \mathrm{E}+02$ & $0.2261 E+00$ & $0.1005 \mathrm{E}-01$ \\
\hline & $0.3057 E+03$ & $0.7382 E+02$ & $0.2250 E+00$ & $0.1000 \mathrm{E}-01$ \\
\hline & $0.3057 E+03$ & $0.7382 E+02$ & $0.2250 E+00$ & $0.1000 E-01$ \\
\hline 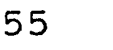 & $0.3042 E+03$ & $0.7419 E+02$ & $0.2250 E+00$ & $0.1000 \mathrm{E}-01$ \\
\hline 56 & $0.3042 E+03$ & $0.7419 E+02$ & $0.2250 \mathrm{E}+00$ & $0.1000 \mathrm{E}-01$ \\
\hline 57 & $0.3042 E+03$ & $0.7382 \mathrm{E}+02$ & $0.2250 E+00$ & $0.1000 \mathrm{E}-01$ \\
\hline & $0.3042 E+03$ & $0.7382 \mathrm{E}+02$ & $0.2250 E+00$ & $0.1000 E-01$ \\
\hline & $0.3057 \mathrm{E}+03$ & $0.7419 E+02$ & $0.2250 E+00$ & $0.1000 E-01$ \\
\hline & $0.3057 E+03$ & $0.7419 E+02$ & $0.2250 E+00$ & $0.1000 \mathrm{E}-01$ \\
\hline & $0.3042 E+03$ & $0.7382 \mathrm{E}+02$ & $0.2261 E+00$ & $0.1005 \mathrm{E}-01$ \\
\hline & $0.3042 E+03$ & $0.7382 \mathrm{E}+02$ & $0.2261 E+00$ & $0.1005 \mathrm{E}-01$ \\
\hline & $0.3057 E+03$ & $0.7419 E+02$ & $0.2261 E+00$ & $0.1005 \mathrm{E}-01$ \\
\hline & $0.3057 E+03$ & $0.7419 E+02$ & $0.2261 E+00$ & $0.1005 E-01$ \\
\hline
\end{tabular}


LIQUID DENSITY OUTPUT $n-C 4 / C O 2$ DRES $=4$ SFACT $=0.5 \%$

TABLE 3. RESPONSES

TEST

$\begin{array}{rl}1 & 0.7720 \mathrm{E}+01 \\ 2 & 0.6543 \mathrm{E}+01 \\ 3 & 0.8004 \mathrm{E}+01 \\ 4 & 0.8158 \mathrm{E}+01 \\ 5 & 0.8086 \mathrm{E}+01 \\ 6 & 0.7354 \mathrm{E}+01 \\ 7 & 0.7571 \mathrm{E}+01 \\ 8 & 0.7378 \mathrm{E}+01 \\ 9 & 0.8464 \mathrm{E}+01 \\ 10 & 0.7826 \mathrm{E}+01 \\ 11 & 0.8142 \mathrm{E}+01 \\ 12 & 0.7493 \mathrm{E}+01 \\ 13 & 0.8262 \mathrm{E}+01 \\ 14 & 0.7859 \mathrm{E}+01 \\ 15 & 0.8668 \mathrm{E}+01 \\ 16 & 0.7182 \mathrm{E}+01 \\ 17 & 0.7641 \mathrm{E}+01 \\ 18 & 0.7575 \mathrm{E}+01 \\ 19 & 0.7559 \mathrm{E}+01 \\ 20 & 0.8869 \mathrm{E}+01 \\ 21 & 0.7612 \mathrm{E}+01 \\ 22 & 0.8077 \mathrm{E}+01 \\ 23 & 0.7494 \mathrm{E}+01 \\ 24 & 0.8305 \mathrm{E}+01 \\ 25 & 0.8169 \mathrm{E}+01 \\ 26 & 0.9599 \mathrm{E}+01 \\ 27 & 0.7442 \mathrm{E}+01 \\ 28 & 0.8183 \mathrm{E}+01 \\ 29 & 0.7634 \mathrm{E}+01 \\ 30 & 0.9048 \mathrm{E}+01 \\ 31 & 0.8333 \mathrm{E}+01 \\ 32 & 0.8239 \mathrm{E}+01\end{array}$

BIAS

$0.7013 E+01$

$0.5328 \mathrm{E}+01$

$0.7397 \mathrm{E}+01$

$0.7720 \mathrm{E}+01$

$0.7558 \mathrm{E}+01$

$0.6706 \mathrm{E}+01$

$0.6611 \mathrm{E}+01$

$0.6599 \mathrm{E}+01$

$0.7940 \mathrm{E}+01$

$0.7122 \mathrm{E}+01$

$0.7659 \mathrm{E}+01$

$0.6892 \mathrm{E}+01$

$0.7852 \mathrm{E}+01$

$0.7101 \mathrm{E}+01$

$0.8316 \mathrm{E}+01$

$0.6238 \mathrm{E}+01$

$0.7015 \mathrm{E}+01$

$0.6714 \mathrm{E}+01$

$0.6896 \mathrm{E}+01$

$0.8494 \mathrm{E}+01$

$0.7079 E+01$

$0.7621 \mathrm{E}+01$

$0.6574 \mathrm{E}+01$

$0.7681 E+01$

$0.7476 \mathrm{E}+01$

$0.9295 \mathrm{E}+01$

$0.6677 \mathrm{E}+01$

$0.7844 \mathrm{E}+01$

$0.7030 E+01$

$0.8668 \mathrm{E}+01$

$0.7888 \mathrm{E}+01$

$0.7706 E+01$
1 OF 2

CPU TIME

$0.8400 \mathrm{E}+02$

$0.8000 \mathrm{E}+02$

$0.7700 \mathrm{E}+02$

$0.7700 \mathrm{E}+02$

$0.7700 \mathrm{E}+02$

$0.8000 \mathrm{E}+02$

$0.7600 \mathrm{E}+02$

$0.7800 \mathrm{E}+02$

$0.7900 \mathrm{E}+02$

$0.7700 E+02$

$0.8100 \mathrm{E}+02$

$0.8100 E+02$

$0.8300 E+02$

$0.8000 \mathrm{E}+02$

$0.7900 E+02$

$0.7800 \mathrm{E}+02$

$0.7800 \mathrm{E}+02$

$0.8300 E+02$

$0.7800 \mathrm{E}+02$

$0.7800 \mathrm{E}+02$

$0.8300 \mathrm{E}+02$

$0.8200 \mathrm{E}+02$

$0.8200 \mathrm{E}+02$

$0.8300 \mathrm{E}+02$

$0.7700 \mathrm{E}+02$

$0.7900 \mathrm{E}+02$

$0.8200 \mathrm{E}+02$

$0.7700 \mathrm{E}+02$

$0.8300 \mathrm{E}+02$

$0.8200 \mathrm{E}+\mathrm{O} 2$

$0.7600 \mathrm{E}+02$

$0.7800 \mathrm{E}+02$ 
LIQUID DENSITY OUTPUT $\mathrm{n}-\mathrm{C} 4 / \mathrm{CO} 2$ DRES $=4$ SFACT $=0.5 \%$

TABLE 3 - (CONTINUED)

2 OF 2

\begin{tabular}{|c|c|c|c|}
\hline TEST & $S M A B \quad E R$ & BIAS & CPU TIME \\
\hline 33 & $0.7616 \mathrm{E}+01$ & $0.6973 \mathrm{E}+01$ & $0.8000 E+02$ \\
\hline 34 & $0.7538 \mathrm{E}+01$ & $0.6781 E+01$ & $0.8300 E+02$ \\
\hline 35 & $0.7070 \mathrm{E}+01$ & $0.6176 \mathrm{E}+01$ & $0.8100 \mathrm{E}+02$ \\
\hline 36 & $0.8242 E+01$ & $0.7665 \mathrm{E}+01$ & $0.8300 E+02$ \\
\hline 37 & $0.7729 E+01$ & $0.6803 \mathrm{E}+01$ & $0.7700 \mathrm{E}+02$ \\
\hline 38 & $0.8462 \mathrm{E}+01$ & $0.7994 E+01$ & $0.7600 \mathrm{E}+02$ \\
\hline 39 & $0.6751 \mathrm{E}+01$ & $0.5705 E+01$ & $0.7700 E+02$ \\
\hline 40 & $0.7729 E+01$ & $0.7220 E+01$ & $0.7600 E+02$ \\
\hline 41 & $0.7607 \mathrm{E}+01$ & $0.7042 \mathrm{E}+01$ & $0.7700 \mathrm{E}+02$ \\
\hline 42 & $0.8430 E+01$ & $0.8076 \mathrm{E}+01$ & $0.7700 E+02$ \\
\hline 43 & $0.7780 E+01$ & $0.7026 \mathrm{E}+01$ & $0.7700 E+02$ \\
\hline 44 & $0.8334 \mathrm{E}+01$ & $0.7666 \mathrm{E}+01$ & $0.7700 \mathrm{E}+02$ \\
\hline 45 & $0.7193 E+01$ & $0.6320 \mathrm{E}+01$ & $0.7700 E+02$ \\
\hline 46 & $0.8297 \mathrm{E}+01$ & $0.7770 \mathrm{E}+01$ & $0.7800 E+02$ \\
\hline 47 & $0.8314 E+01$ & $0.7941 \mathrm{E}+01$ & $0.7800 E+02$ \\
\hline 48 & $0.8229 E+01$ & $0.7678 \mathrm{E}+01$ & $0.7900 E+02$ \\
\hline 49 & $0.8841 \mathrm{E}+01$ & $0.8510 E+01$ & $0.7300 E+02$ \\
\hline 50 & $0.7299 E+01$ & $0.6347 \mathrm{E}+01$ & $0.7400 E+02$ \\
\hline 51 & $0.8037 E+01$ & $0.7564 \mathrm{E}+01$ & $0.7200 E+02$ \\
\hline 52 & $0.7606 \mathrm{E}+01$ & $0.6796 \mathrm{E}+01$ & $0.7100 E+02$ \\
\hline 53 & $0.8433 E+01$ & $0.7855 \mathrm{E}+01$ & $0.7500 E+02$ \\
\hline 54 & $0.7692 \mathrm{E}+01$ & $0.6983 E+01$ & $0.7700 E+02$ \\
\hline 55 & $0.8005 E+01$ & $0.7519 \mathrm{E}+01$ & $0.8100 E+02$ \\
\hline 56 & $0.7455 E+01$ & $0.6796 \mathrm{E}+01$ & $0.8000 E+02$ \\
\hline 57 & $0.8562 E+01$ & $0.8133 \mathrm{E}+01$ & $0.7900 E+02$ \\
\hline 58 & $0.8337 E+01$ & $0.8033 \mathrm{E}+01$ & $0.7800 \mathrm{E}+02$ \\
\hline 59 & $0.8614 \mathrm{E}+01$ & $0.8067 E+01$ & $0.7600 E+02$ \\
\hline 60 & $0.7741 E+01$ & $0.7089 E+01$ & $0.7800 E+02$ \\
\hline 6.1 & $0.7695 E+01$ & $0.6922 \mathrm{E}+01$ & $0.8000 E+02$ \\
\hline 62 & $0.7824 \mathrm{E}+01$ & $0.7251 E+01$ & $0.7900 E+02$ \\
\hline 63 & $0.1012 \mathrm{E}+02$ & $0.9911 E+01$ & $0.7800 \mathrm{E}+02$ \\
\hline 64 & $0.8121 E+01$ & $0.7632 \mathrm{E}+01$ & $0.7800 E+02$ \\
\hline
\end{tabular}


TABLE 4 A. CONTRASTS LISTED BY FACTORS 1 OF 1

\begin{tabular}{|c|c|c|c|}
\hline FACTOR & $S M A B \quad E R$ & FACTOR & $S M A B \quad E R$ \\
\hline C 16 & $-.7050 E+00$ & C 12 & $-.2065 \mathrm{E}-01$ \\
\hline & $-.4348 E+00$ & 22 & $-.2059 E-01$ \\
\hline C 4 & $0.4151 \mathrm{E}+00$ & E $\quad 18$ & $-.1890 \mathrm{E}-01$ \\
\hline C 17 & $0.3808 E+00$ & OM 1 & $-.1840 \mathrm{E}-01$ \\
\hline TC 2 & $0.3728 E+00$ & E 16 & $-.1676 \mathrm{E}-01$ \\
\hline C 5 & $0.3164 \mathrm{E}+00$ & 10 & $0.1481 \mathrm{E}-01$ \\
\hline 7 & $-.2511 E+00$ & 17 & $-.1417 \mathrm{E}-01$ \\
\hline TC 1 & $0.2164 \mathrm{E}+00$ & 27 & $0.1151 \mathrm{E}-01$ \\
\hline C 11 & $-.1252 \mathrm{E}+00$ & $\mathrm{E}$ & $-.1151 \mathrm{E}-01$ \\
\hline PC 1 & $0.1023 E+00$ & 4 & $-.9097 \mathrm{E}-02$ \\
\hline C 14 & $0.9087 \mathrm{E}-01$ & K 12 & $0.8649 \mathrm{E}-02$ \\
\hline PC 2 & $0.8909 \mathrm{E}-01$ & E 21 & $-.7862 \mathrm{E}-02$ \\
\hline C 9 & $-.8627 \mathrm{E}-01$ & C 15 & $0.4794 \mathrm{E}-02$ \\
\hline 19 & $-.6493 \mathrm{E}-01$ & C & $0.4477 \mathrm{E}-02$ \\
\hline $\mathrm{E}$ & $-.5992 E-01$ & 20 & $-.4470 \mathrm{E}-02$ \\
\hline 29 & $0.5919 \mathrm{E}-01$ & C & $-.4427 \mathrm{E}-02$ \\
\hline C 10 & $-.5422 \mathrm{E}-01$ & 7 & $0.3458 \mathrm{E}-02$ \\
\hline C 13 & $0.4673 \mathrm{E}-01$ & C & $0.3285 \mathrm{E}-02$ \\
\hline 25 & $-.4648 \mathrm{E}-01$ & 14 & $0.2839 \mathrm{E}-02$ \\
\hline C & $0.3762 \mathrm{E}-01$ & 11 & $-.2701 E-02$ \\
\hline $\mathrm{E}$ & $-.3591 E-01$ & 24 & $-.8133 E-03$ \\
\hline 31. & $-.3537 E-01$ & 8 & $0.6165 E-03$ \\
\hline $\mathrm{E}$ & $-.3523 E-01$ & 15 & $-.3916 E-03$ \\
\hline $\mathrm{E}$ & $0.3512 \mathrm{E}-01$ & & \\
\hline 30 & $0.3392 E-01$ & & \\
\hline 28 & $0.3278 E-01$ & & \\
\hline $\mathrm{E}$ & $0.3251 E-01$ & & \\
\hline 13 & $0.3211 \mathrm{E}-01$ & & \\
\hline OM & $0.3004 \mathrm{E}-01$ & & \\
\hline 26 & $0.2952 \mathrm{E}-01$ & & \\
\hline 23 & $-.2612 \mathrm{E}-01$ & & \\
\hline 12 & $0.2107 \mathrm{E}-01$ & & \\
\hline
\end{tabular}


LIQUID DENSITY OUTPUT $n-C 4 / C O 2$ DRES $=4 \quad \mathrm{SFACT}=0.5 \%$

TABLE 4 B. CONTRASTS LISTED BY FACTORS 1 OF 1

\begin{tabular}{|c|c|c|c|}
\hline FACTOR & BIAS & FACTOR & BIAS \\
\hline C 16 & $-.8890 E+00$ & 16 & $-.1524 E-01$ \\
\hline 8 & $-.5689 E+00$ & 23 & $-.1507 \mathrm{E}-01$ \\
\hline 4 & $0.5489 E+00$ & 1 & $-.1495 \mathrm{E}-01$ \\
\hline C 17 & $0.5160 \mathrm{E}+00$ & 22 & $-.1409 \mathrm{E}-01$ \\
\hline C 5 & $0.411 .8 \mathrm{E}+00$ & $\mathrm{OM}$ & $-.1387 \mathrm{E}-01$ \\
\hline $\mathrm{TC}$ & $0.3898 \mathrm{E}+00$ & 30 & $0.1258 \mathrm{E}-01$ \\
\hline C 7 & $-.3344 \mathrm{E}+00$ & 4 & $-.1212 \mathrm{E}-01$ \\
\hline TC & $0.2536 \mathrm{E}+00$ & 3 & $-.1058 \mathrm{E}-01$ \\
\hline PC & $0.2508 E+00$ & C & $0.1038 \mathrm{E}-01$ \\
\hline C 11 & $-.2416 \mathrm{E}+00$ & 10 & $0.8552 \mathrm{E}-02$ \\
\hline C 14 & $0.1977 \mathrm{E}+00$ & 21 & $-.8160 \mathrm{E}-02$ \\
\hline PC 2 & $0.1241 \mathrm{E}+00$ & 17 & $-.7952 \mathrm{E}-02$ \\
\hline C 10 & $-.1129 \mathrm{E}+00$ & 11 & $0.705 .1 \mathrm{E}-02$ \\
\hline C 9 & $-.1068 \mathrm{E}+00$ & K 12 & $0.6046 \mathrm{E}-02$ \\
\hline C 13 & $0.1033 \mathrm{E}+00$ & 14 & $0.5477 \mathrm{E}-02$ \\
\hline E 19 & $-.5416 \mathrm{E}-01$ & 27 & $0.3977 E-02$ \\
\hline 6 & $0.4788 \mathrm{E}-01$ & $\mathrm{C}$ & $0.3221 \mathrm{E}-02$ \\
\hline 5 & $-.4486 \mathrm{E}-01$ & 20 & $-.2597 \mathrm{E}-02$ \\
\hline E 29 & $0.4459 \mathrm{E}-01$ & 8 & $0.2275 \mathrm{E}-02$ \\
\hline C 15 & $0.3277 \mathrm{E}-01$ & $\mathrm{E}$ & $0.1952 \mathrm{E}-02$ \\
\hline E 25 & $-.3269 E-01$ & $\mathrm{C}$ & $0.1873 \mathrm{E}-02$ \\
\hline 9 & $0.3068 \mathrm{E}-01$ & 15 & $-.1140 \mathrm{E}-02$ \\
\hline OM 2 & $0.3036 \mathrm{E}-01$ & 24 & $-.6691 \mathrm{E}-03$ \\
\hline E 31 & $-.2814 \mathrm{E}-01$ & & \\
\hline C 12 & $-.2781 \mathrm{E}-01$ & & \\
\hline E 26 & $0.2502 \mathrm{E}-01$ & & \\
\hline 13 & $0.2469 \mathrm{E}-01$ & & \\
\hline 6 & $0.2103 \mathrm{E}-01$ & & \\
\hline 12 & $0.1919 \mathrm{E}-01$ & & \\
\hline 18 & $-.1889 \mathrm{E}-01$ & & \\
\hline 28 & $0.1865 \mathrm{E}-01$ & & \\
\hline 2 & $-.1689 \mathrm{E}-01$ & & \\
\hline
\end{tabular}


LIQUID DENSITY OUTPUT $n-C 4 / \mathrm{CO} 2$ DRES $=4$ SFACT $=0.5 \%$

TABLE 4 C. CONTRASTS LISTED BY FACTORS 1 OF 1

FACTOR

$\begin{array}{lcc}\text { C } & 6 & -.2063 \mathrm{E}+01 \\ \mathrm{E} & 11 & 0.2063 \mathrm{E}+01 \\ \mathrm{OM} & 2 & -.1938 \mathrm{E}+01 \\ \mathrm{C} & 17 & -.1500 \mathrm{E}+01 \\ \mathrm{E} & 15 & -.1188 \mathrm{E}+01 \\ \mathrm{PC} & 1 & 0.8750 \mathrm{E}+00 \\ \mathrm{OM} & 1 & -.8750 \mathrm{E}+00 \\ \mathrm{E} & 9 & 0.8750 \mathrm{E}+00 \\ \mathrm{E} & 27 & 0.8750 \mathrm{E}+00 \\ \mathrm{E} & 30 & -.8125 \mathrm{E}+00 \\ \mathrm{C} & 2 & -.7500 \mathrm{E}+00 \\ \mathrm{C} & 3 & 0.6875 \mathrm{E}+00 \\ \mathrm{TC} & 2 & -.6250 \mathrm{E}+00 \\ \mathrm{E} & 17 & 0.5625 \mathrm{E}+00 \\ \mathrm{E} & 3 & -.5000 \mathrm{E}+00 \\ \mathrm{E} & 26 & -.5000 \mathrm{E}+00 \\ \mathrm{E} & 28 & 0.5000 \mathrm{E}+00 \\ \mathrm{C} & 9 & -.4375 \mathrm{E}+00 \\ \mathrm{E} & 13 & 0.4375 \mathrm{E}+00 \\ \mathrm{E} & 14 & 0.4375 \mathrm{E}+00 \\ \mathrm{E} & 18 & -.4375 \mathrm{E}+00 \\ \mathrm{E} & 21 & 0.4375 \mathrm{E}+00 \\ \mathrm{C} & 13 & -.3750 \mathrm{E}+00 \\ \mathrm{C} & 15 & 0.3750 \mathrm{E}+00 \\ \mathrm{C} & 16 & -.3750 \mathrm{E}+00 \\ \mathrm{E} & 24 & 0.3750 \mathrm{E}+00 \\ \mathrm{C} & 5 & -.3125 \mathrm{E}+00 \\ \mathrm{C} & 7 & 0.3125 \mathrm{E}+00 \\ \mathrm{C} & 8 & 0.3125 \mathrm{E}+00 \\ \mathrm{E} & 20 & 0.3125 \mathrm{E}+00 \\ \mathrm{TC} & 1 & 0.2500 \mathrm{E}+00 \\ \mathrm{E} & 5 & 0.2500 \mathrm{E}+00\end{array}$

FACTOR

CPU TIME

$\begin{array}{lrrr}\mathrm{E} & 22 & 0.2500 \mathrm{E}+00 \\ \mathrm{E} & 23 & 0.2500 \mathrm{E}+00 \\ \mathrm{C} & 4 & 0.1875 \mathrm{E}+00 \\ \mathrm{~K} & 1 & 2 & 0.1875 \mathrm{E}+00 \\ \mathrm{E} & 10 & 0.1875 \mathrm{E}+00 \\ \mathrm{E} & 16 & 0.1875 \mathrm{E}+00 \\ \mathrm{E} & 19 & 0.1875 \mathrm{E}+00 \\ \mathrm{C} & 1 & 0.1250 \mathrm{E}+00 \\ \mathrm{C} & 11 & 0.1250 \mathrm{E}+00 \\ \mathrm{C} & 12 & -.1250 \mathrm{E}+00 \\ \mathrm{PC} & 2 & -.1250 \mathrm{E}+00 \\ \mathrm{E} & 4 & 0.1250 \mathrm{E}+00 \\ \mathrm{E} & 8 & -.1250 \mathrm{E}+00 \\ \mathrm{E} & 29 & 0.1250 \mathrm{E}+00 \\ \mathrm{C} & 10 & -.6250 \mathrm{E}-01 \\ \mathrm{E} & 1 & -.6250 \mathrm{E}-01 \\ \mathrm{E} & 12 & -.6250 \mathrm{E}-01 \\ \mathrm{E} & 31 & 0.6250 \mathrm{E}-01 \\ \mathrm{C} & 14 & 0.0000 \mathrm{E}+00 \\ \mathrm{E} & 2 & 0.0000 \mathrm{E}+00 \\ \mathrm{E} & 6 & 0.0000 \mathrm{E}+00 \\ \mathrm{E} & 7 & 0.0000 \mathrm{E}+00 \\ \mathrm{E} & 25 & 0.0000 \mathrm{E}+00\end{array}$


LIQUID DENSITY $n-C 6 / C O 2$ ESD DRES $=4$ SFACT $=0.5 \%$ 


\section{LIQUID DENSITY $\mathrm{n}-\mathrm{C} 6$ / CO2 ESD DRES $=4 \quad \mathrm{SFACT}=0.5 \%$}

TABLE 3. RESPONSES

\section{TEST}

1

2

3

4
$S M A B E R$
$0.3803 E+00$
$0.4014 \mathrm{E}+00$
$0.6043 \mathrm{E}+00$
$0.9656 \mathrm{E}+00$
$0.5864 \mathrm{E}+00$
$0.5449 \mathrm{E}+00$
$0.2945 E+00$
$0.5217 \mathrm{E}+00$
$0.7178 E+00$
$0.4218 \mathrm{E}+00$
$0.7862 \mathrm{E}-00$
$0.5374 \mathrm{E}+00$
$0.9807 \mathrm{E}+00$
$0.2423 E+00$
$0.1299 E+01$
$0.1697 \mathrm{E}+00$
$0.5346 \mathrm{E}+00$
$0.3605 \mathrm{E}+00$
$0.6587 \mathrm{E}+00$
$0.1120 \mathrm{E}+01$
$0.8765 \mathrm{E}+00$
$0.1054 \mathrm{E}+01$
$0.2580 \mathrm{E}+00$
$0.5229 \mathrm{E}+00$
$0.4675 \mathrm{E}+00$
$0.1365 \mathrm{E}+01$
$0.3405 E+00$
$0.1266 \mathrm{E}+01$
$0.7030 E+00$
$0.1150 \mathrm{E}+01$
$0.8336 \mathrm{E}+00$
$0.6076 \mathrm{E}+00$

BIAS

$0.3803 E+00$

$-.4014 \mathrm{E}+00$

$0.6043 \mathrm{E}+00$

$0.9656 \mathrm{E}+00$

$0.5864 \mathrm{E}+00$

$0.5449 \mathrm{E}+00$

$-.6002 \mathrm{E}-01$

$0.5217 \mathrm{E}+00$

$0.7178 \mathrm{E}+00$

$0.4218 \mathrm{E}+00$

$0.7862 \mathrm{E}+00$

$0.5374 \mathrm{E}+00$

$0.9807 \mathrm{E}+00$

$0.1492 \mathrm{E}+00$

$0.1299 \mathrm{E}+01$

$0.1096 \mathrm{E}-01$

$0.5346 \mathrm{E}+00$

$0.1014 \mathrm{E}+00$

$0.6587 \mathrm{E}+00$

$0.1120 \mathrm{E}+01$

$0.8765 \mathrm{E}+00$

$0.1054 \mathrm{E}+01$

$-.5184 \mathrm{E}-01$

$0.5089 \mathrm{E}+00$

$0.2719 \mathrm{E}+00$

$0.1365 \mathrm{E}+01$

$0.3405 \mathrm{E}+00$

$0.1266 \mathrm{E}+01$

$0.7030 \mathrm{E}+00$

$0.1150 E+01$

$0.8336 \mathrm{E}+00$

$0.5995 \mathrm{E}+00$
1 OF 2

CPU TIME

$0.7800 \mathrm{E}+02$

$0.7600 \mathrm{E}+02$

$0.7600 \mathrm{E}+02$

$0.7500 \mathrm{E}+02$

$0.7600 \mathrm{E}+02$

$0.7500 \mathrm{E}+02$

$0.7500 \mathrm{E}+02$

$0.7500 E+02$

$0.7500 E+02$

$0.7700 \mathrm{E}+02$

$0.7600 \mathrm{E}+02$

$0.7500 \mathrm{E}+02$

$0.7600 \mathrm{E}+02$

$0.7600 \mathrm{E}+02$

$0.7500 E+02$

$0.7700 E+02$

$0.7600 \mathrm{E}+02$

$0.7500 \mathrm{E}+02$

$0.7600 \mathrm{E}+02$

$0.7100 \mathrm{E}+02$

$0.7300 \mathrm{E}+02$

$0.7500 \mathrm{E}+02$

$0.7500 \mathrm{E}+02$

$0.7500 \mathrm{E}+02$

$0.7800 \mathrm{E}+02$

$0.7700 \mathrm{E}+02$

$0.7700 \mathrm{E}+02$

$0.7600 \mathrm{E}+02$

$0.7500 E+02$

$0.7500 \mathrm{E}+02$

$0.7400 \mathrm{E}+02$

$0.750 \cup E+02$ 
LIQUID DENSITY $n-C 6 / C O 2$ ESD DRES $=4 \quad \mathrm{SFACT}=0.5 \%$

TABLE 3. (CONTINUED)

2 OF 2

TEST

$\begin{array}{ll}33 & 0.6408 \mathrm{E}+00 \\ 34 & 0.4079 \mathrm{E}+00 \\ 35 & 0.1175 \mathrm{E}+00 \\ 36 & 0.5649 \mathrm{E}+00 \\ 37 & 0.4104 \mathrm{E}+00 \\ 38 & 0.9022 \mathrm{E}+00 \\ 39 & 0.1653 \mathrm{E}+00 \\ 40 & 0.9723 \mathrm{E}+00 \\ 41 & 0.7293 \mathrm{E}+00 \\ 42 & 0.1302 \mathrm{E}+01 \\ 43 & 0.2937 \mathrm{E}+00 \\ 44 & 0.4383 \mathrm{E}+00 \\ 45 & 0.2073 \mathrm{E}+00 \\ 46 & 0.6151 \mathrm{E}+00 \\ 47 & 0.1147 \mathrm{E}+01 \\ 48 & 0.7102 \mathrm{E}+00 \\ 49 & 0.1224 \mathrm{E}+01 \\ 50 & 0.1651 \mathrm{E}+00 \\ 51 & 0.1098 \mathrm{E}+01 \\ 52 & 0.3290 \mathrm{E}+00 \\ 53 & 0.6979 \mathrm{E}+00 \\ 54 & 0.4564 \mathrm{E}+00 \\ 55 & 0.8209 \mathrm{E}+00 \\ 56 & 0.5239 \mathrm{E}+00 \\ 57 & 0.7117 \mathrm{E}+00 \\ 58 & 0.1308 \mathrm{E}+01 \\ 59 & 0.6901 \mathrm{E}+00 \\ 60 & 0.6549 \mathrm{E}+00 \\ 61 & 0.2927 \mathrm{E}+00 \\ 62 & 0.6401 \mathrm{E}+00 \\ 63 & 0.1617 \mathrm{E}+01 \\ 64 & 0.8644 \mathrm{E}+00\end{array}$

BIAS

$0.6408 \mathrm{E}+00$

$0.4079 \mathrm{E}+00$

$0.8625 \mathrm{E}-01$

$0.5373 \mathrm{E}+00$

$-.2044 \mathrm{E}-01$

$0.9022 \mathrm{E}+00$

$-.1474 E+00$

$0.9723 \mathrm{E}+00$

$0.7293 E+00$

$0.1302 \mathrm{E}+01$

$0.1938 \mathrm{E}+00$

$0.3620 \mathrm{E}+00$

$0.1203 \mathrm{E}+00$

$0.5786 \mathrm{E}+00$

$0.1147 \mathrm{E}+01$

$0.7102 \mathrm{E}+00$

$0.1224 \mathrm{E}+01$

$-.5210 \mathrm{E}-01$

$0.1098 \mathrm{E}+01$.

$0.2637 \mathrm{E}+00$

$0.6979 E+00$

$0.4564 \mathrm{E}+00$

$0.8209 \mathrm{E}+00$

$0.5239 \mathrm{E}+00$

$0.7117 \mathrm{E}+00$

$0.1308 E+01$

$0.6901 \mathrm{E}+00$

$0.6549 \mathrm{E}+00$

$0.2786 \mathrm{E}+00$

$0.6401 \mathrm{E}+00$

$0.1617 \mathrm{E}+01$

$0.8644 \mathrm{E}+00$
CPU TIME

$0.7500 \mathrm{E}+02$

$0.7500 \mathrm{E}+02$

$0.7400 \mathrm{E}+02$

$0.7600 \mathrm{E}+02$

$0.7800 \mathrm{E}+02$

$0.7500 \mathrm{E}+02$

$0.7700 E+02$

$0.7600 \mathrm{E}+02$

$0.7600 \mathrm{E}+02$

$0.7600 \mathrm{E}+02$

$0.7600 \mathrm{E}+02$

$0.7700 \mathrm{E}+02$

$0.7600 \mathrm{E}+02$

$0.7600 \mathrm{E}+02$

$0.7600 \mathrm{E}+02$

$0.7500 \mathrm{E}+02$

$0.7600 \mathrm{E}+02$

$0.7600 \mathrm{E}+02$

$0.7700 \mathrm{E}+02$

$0.7600 \mathrm{E}+02$

$0.7600 \mathrm{E}+02$

$0.7600 \mathrm{E}+02$

$0.7600 \mathrm{E}+02$

$0.7600 \mathrm{E}+02$

$0.7500 \mathrm{E}+02$

$0.7500 \mathrm{E}+02$

$0.7500 \mathrm{E}+02$

$0.7600 \mathrm{E}+02$

$0.7600 \mathrm{E}+02$

$0.7500 \mathrm{E}+02$

$0.7400 \mathrm{E}+02$

$0.7600 \mathrm{E}+02$ 
LIQUID DENSITY $n-C 6 / C O 2$ ESD DRES $=4 \quad$ SFACT $=0.5 \%$

TABLE 4 A. CONTRASTS LISTED BY FACTORS

1 OF 1

FACTOR

\begin{tabular}{|c|c|c|c|}
\hline C 16 & $-.2822 \mathrm{E}+00$ & $\mathrm{E}$ & 29 \\
\hline PC! 1 & $0.2551 E+00$ & $\mathrm{E}$ & 16 \\
\hline C 14 & $0.2388 E+00$ & $\mathrm{E}$ & 13 \\
\hline C 17 & $0.2257 \mathrm{E}+00$ & $\mathrm{E}$ & 12 \\
\hline C II & $-.2178 E+00$ & $\mathrm{E}$ & 26 \\
\hline C 8 & $-.2048 E+00$ & $\mathrm{E}$ & 14 \\
\hline 5 & $0.1604 \mathrm{E}+00$ & $\mathrm{E}$ & 7 \\
\hline 4 & $0.1540 E+00$ & $O M$ & 2 \\
\hline PC 2 & $0.1161 \mathrm{E}+00$ & $\mathrm{E}$ & 9 \\
\hline C 13 & $0.1133 E+00$ & C & 2 \\
\hline C 15 & $0.1051 \mathrm{E}+00$ & $\mathrm{E}$ & 31 \\
\hline 7 & $-.1048 E+00$ & $\mathrm{E}$ & 4 \\
\hline 2 & $-.7196 \mathrm{E}-01$ & $\mathrm{E}$ & 15 \\
\hline C 10 & $-.6612 \mathrm{E}-01$ & $\mathrm{E}$ & 18 \\
\hline 30 & $0.6199 \mathrm{E}-01$ & C & 6 \\
\hline 20 & $-.5981 E-01$ & $\mathrm{E}$ & 19 \\
\hline 6 & $0.5127 \mathrm{E}-01$ & $\mathrm{E}$ & 8 \\
\hline 1 & $-.4469 \mathrm{E}-01$ & $\mathrm{E}$ & 24 \\
\hline C 9 & $-.4395 \mathrm{E}-01$ & $\mathrm{C}$ & 3 \\
\hline TC 2 & $0.4179 \mathrm{E}-01$ & & 12 \\
\hline $\mathrm{TC}$ & $-.4147 \mathrm{E}-01$ & $\mathrm{OM}$ & 1 \\
\hline E 23 & $-.3760 E-01$ & $\mathrm{E}$ & 3 \\
\hline 27 & $0.3753 E-01$ & $\mathrm{E}$ & 10 \\
\hline 22 & $-.3298 E-01$ & & \\
\hline 21 & $-.3221 \mathrm{E}-01$ & & \\
\hline 28 & $0.3210 \mathrm{E}-01$ & & \\
\hline 25 & $-.2961 \mathrm{E}-01$ & & \\
\hline 5 & $-.2929 E-01$ & & \\
\hline 1 & $0.2875 E-01$ & & \\
\hline K 12 & $-.2773 E-01$ & & \\
\hline 11 & $-.2603 E-01$ & & \\
\hline 17 & $-.2414 \mathrm{E}-01$ & & \\
\hline
\end{tabular}

SM AB ER

$0.2179 E-01$

$-.2169 E-01$

$0.2168 \mathrm{E}-01$

$0.2029 E-01$

$0.1901 \mathrm{E}-01$

$0.1626 \mathrm{E}-01$

$0.1584 \mathrm{E}-01$

$0.1111 \mathrm{E}-01$

$0.9289 \mathrm{E}-02$

$0.9209 \mathrm{E}-02$

-.8867E-02

$0.6393 \mathrm{E}-02$

$-.6066 \mathrm{E}-02$

$0.5079 \mathrm{E}-02$

$0.4572 \mathrm{E}-02$

$-.3511 \mathrm{E}-02$

$0.3146 \mathrm{E}-02$

$-.2739 \mathrm{~s}-02$

$0.2672 \mathrm{E}-02$

$0.1860 \mathrm{E}-02$

$0.1283 \mathrm{E}-02$

$0.9871 \mathrm{E}-03$

$0.4663 \mathrm{E}-03$ 
LIQUID DENSITY $n-C 6 / C O 2$ ESD DRES $=4 \quad \mathrm{SFACT}=0.5 \%$

TABLE 4 B. CONTRASTS LISTED BY FACTORS 1 OF 1

FACTOR

BIAS

\begin{tabular}{|c|c|}
\hline C 16 & $-.3451 E+00$ \\
\hline PC 1 & $0.3284 \mathrm{E}+00$ \\
\hline C 11 & $-.3162 E+00$ \\
\hline C 17 & $0.2807 E+00$ \\
\hline C & $-.2692 \mathrm{E}+00$ \\
\hline C 14 & $0.2677 \mathrm{E}+00$ \\
\hline C & $0.2182 \mathrm{E}+00$ \\
\hline C 5 & $0.2050 E+00$ \\
\hline PC 2 & $0.1292 \mathrm{E}+00$ \\
\hline C 7 & $-.1252 \mathrm{E}+00$ \\
\hline C 10 & $-.1113 \mathrm{E}+00$ \\
\hline C 13 & $0.1073 E+00$ \\
\hline C 15 & $0.8248 E-01$ \\
\hline C 9 & $-.5837 \mathrm{E}-01$ \\
\hline TC 1 & $-.4703 E-01$ \\
\hline TC 2 & $0.4217 \mathrm{E}-01$ \\
\hline C 1 & $0.3115 \mathrm{E}-01$ \\
\hline C 2 & $0.3040 E-01$ \\
\hline C 12 & $-.3015 E-01$ \\
\hline C 6 & $0.2949 \mathrm{E}-01$ \\
\hline OM 1 & $0.2442 \mathrm{E}-01$ \\
\hline$O M$ & $0.8558 \mathrm{E}-02$ \\
\hline $\mathrm{E} \quad 19$ & $0.3079 E-02$ \\
\hline 29 & $-.2964 \mathrm{E}-02$ \\
\hline 5 & $0.2861 \mathrm{E}-02$ \\
\hline 18 & $0.2824 \mathrm{E}-02$ \\
\hline 25 & $0.2121 \mathrm{E}-0$ \\
\hline 13 & $-.2089 E-02$ \\
\hline 26 & $-.1894 \mathrm{E}-02$ \\
\hline 3 & $0.1286 \mathrm{E}-0$ \\
\hline 22 & $0.1244 \mathrm{E}-0$ \\
\hline 4 & $0.1223 \mathrm{E}$ \\
\hline
\end{tabular}

FACTOR

BIAS

\begin{tabular}{|c|c|c|}
\hline $\mathrm{E}$ & 31 & $0.1197 \mathrm{E}-02$ \\
\hline $\mathrm{E}$ & 9 & $-.1107 \mathrm{E}-02$ \\
\hline $\mathrm{E}$ & 12 & $-.1041 \mathrm{E}-02$ \\
\hline $\mathrm{E}$ & 6 & $-.9500 E-03$ \\
\hline $\mathrm{E}$ & 16 & $0.7687 \mathrm{E}-03$ \\
\hline $\mathrm{E}$ & 28 & $-.7354 \mathrm{E}-03$ \\
\hline $\mathrm{E}$ & 27 & $0.5871 E-03$ \\
\hline $\mathrm{E}$ & 2 & $0.5425 \mathrm{E}-03$ \\
\hline $\mathrm{E}$ & 23 & $-.5169 \mathrm{E}-03$ \\
\hline $\mathrm{E}$ & 15 & $-.3571 E-03$ \\
\hline $\mathrm{E}$ & 21 & $-.3540 \mathrm{E}-03$ \\
\hline $\mathrm{E}$ & 20 & $-.3288 E-03$ \\
\hline$E$ & 1 & $0.2893 E-03$ \\
\hline $\mathrm{E}$ & 10 & $0.2335 \mathrm{E}-03$ \\
\hline$E$ & 14 & $0.2115 \mathrm{E}-03$ \\
\hline $\mathrm{E}$ & 11 & $-.1949 E-03$ \\
\hline $\mathrm{E}$ & 7 & $-.1420 \mathrm{E}-03$ \\
\hline $\mathrm{E}$ & 17 & $-.1333 E-03$ \\
\hline$E$ & 24 & $-.1069 \mathrm{E}-03$ \\
\hline $\mathrm{E}$ & 30 & $-.8404 \mathrm{E}-04$ \\
\hline $\mathrm{E}$ & 3 & $0.7979 E-04$ \\
\hline K 1 & 12 & $0.4207 \mathrm{E}-04$ \\
\hline & 8 & $0.6541 \mathrm{E}-05$ \\
\hline
\end{tabular}


LIQUID DENSITY $n-C 6 / C O 2$ ESD DRES $=4$ SFACT $=0.5 \%$

TABLE 4 C. CONTRASTS LISTED BY FACTORS

1 OF 1

FACTOR

CPU TIME

FACTOR

CPU TIME

$\begin{array}{lcclrl}\mathrm{E} & 30 & 0.7188 \mathrm{E}+00 & \mathrm{E} & 5 & 0.1563 \mathrm{E}+00 \\ \mathrm{C} & 13 & -.6563 \mathrm{E}+00 & \mathrm{E} & 6 & 0.1563 \mathrm{E}+00 \\ \mathrm{E} & 19 & 0.5938 \mathrm{E}+00 & \mathrm{E} & 21 & 0.1563 \mathrm{E}+00 \\ \mathrm{C} & 12 & 0.5313 \mathrm{E}+00 & \mathrm{E} & 25 & 0.1563 \mathrm{E}+00 \\ \mathrm{E} & 14 & -.5313 \mathrm{E}+00 & \mathrm{E} & 28 & -.1563 \mathrm{E}+00 \\ \mathrm{PC} & 1 & -.4688 \mathrm{E}+00 & \mathrm{C} & 11 & -.9375 \mathrm{E}-01 \\ \mathrm{E} & 3 & 0.4688 \mathrm{E}+00 & \mathrm{C} & 14 & -.9375 \mathrm{E}-01 \\ \mathrm{E} & 12 & 0.4688 \mathrm{E}+00 & \mathrm{TC} & 1 & 0.9375 \mathrm{E}-01 \\ \mathrm{C} & 5 & -.4063 \mathrm{E}+00 & \mathrm{TC} & 2 & -.9375 \mathrm{E}-01 \\ \mathrm{C} & 10 & 0.3438 \mathrm{E}+00 & \mathrm{~K} & 1 & -.9375 \mathrm{E}-01 \\ \mathrm{E} & 9 & 0.3438 \mathrm{E}+00 & \mathrm{E} & 1 & 0.9375 \mathrm{E}-01 \\ \mathrm{E} & 10 & -.3438 \mathrm{E}+00 & \mathrm{E} & 16 & 0.9375 \mathrm{E}-01 \\ \mathrm{E} & 11 & -.3438 \mathrm{E}+00 & \mathrm{E} & 17 & 0.9375 \mathrm{E}-01 \\ \mathrm{C} & 2 & -.2813 \mathrm{E}+00 & \mathrm{E} & 20 & -.9375 \mathrm{E}-01 \\ \mathrm{C} & 3 & -.2813 \mathrm{E}+00 & \mathrm{E} & 26 & -.9375 \mathrm{E}-01 \\ \mathrm{C} & 6 & 0.2813 \mathrm{E}+00 & \mathrm{E} & 27 & 0.9375 \mathrm{E}-01 \\ \mathrm{C} & 7 & 0.2813 \mathrm{E}+00 & \mathrm{E} & 29 & 0.9375 \mathrm{E}-01 \\ \mathrm{C} & 17 & -.2813 \mathrm{E}+00 & \mathrm{PC} & 2 & 0.3125 \mathrm{E}-01 \\ \mathrm{E} & 22 & -.2813 \mathrm{E}+00 & \mathrm{E} & 4 & -.3125 \mathrm{E}-01 \\ \mathrm{E} & 23 & 0.2813 \mathrm{E}+00 & \mathrm{E} & 7 & 0.3125 \mathrm{E}-01 \\ \mathrm{E} & 31 & 0.2813 \mathrm{E}+00 & \mathrm{E} & 8 & 0.3125 \mathrm{E}-01 \\ \mathrm{C} & 1 & -.2188 \mathrm{E}+00 & \mathrm{E} & 13 & 0.3125 \mathrm{E}-01 \\ \mathrm{C} & 4 & 0.2188 \mathrm{E}+00 & \mathrm{E} & 24 & 0.3125 \mathrm{E}-01 \\ \mathrm{C} & 9 & -.2188 \mathrm{E}+00 & & & \\ \mathrm{C} & 16 & 0.2188 \mathrm{E}+00 & & & \\ \mathrm{E} & 2 & 0.2188 \mathrm{E}+00 & & & \\ \mathrm{E} & 15 & 0.2188 \mathrm{E}+00 & & & \\ \mathrm{E} & 18 & -.2188 \mathrm{E}+00 & & & \\ \mathrm{C} & 8 & 0.1563 \mathrm{E}+00 & & & \\ \mathrm{C} & 15 & -.1563 \mathrm{E}+00 & & & \\ \text { OM } & 1 & -.1563 \mathrm{E}+00 & & & \\ \mathrm{OM} & 2 & -.1563 \mathrm{E}+00 & & & \\ & & & & & \end{array}$


LIQUID DENSITY $n-C 8$ / CO2 ESD DRES $=4$ SFACT $=0.5 \%$ 


\section{LIQUID DENSITY $n-C 8 / C O 2$ ESD DRES $=4 \quad \mathrm{SFACT}=0.5 \%$}

TABLE 3. RESPONSES

TEST

1
SM AB ER

$0.2728 \mathrm{E}+00$

$0.2787 \mathrm{E}+00$

$0.5063 \mathrm{E}+00$

$0.8200 \mathrm{E}+00$

$0.3890 E+00$

$0.5424 \mathrm{E}+00$

$0.2388 \mathrm{E}+00$

$0.5577 \mathrm{E}+00$

$0.5401 \mathrm{E}+00$

$0.3149 E+00$

$0.6252 \mathrm{E}+00$

$0.4388 \mathrm{E}+00$

$0.8177 \mathrm{E}+00$

$0.1550 \mathrm{E}+00$

$0.1066 \mathrm{E}+01$

$0.1459 E+00$

$0.4384 \mathrm{E}+00$

$0.3023 \mathrm{E}+00$

$0.6836 \mathrm{E}+00$

$0.9277 \mathrm{E}+00$

$0.8370 \mathrm{E}+00$

$0.9936 E+00$

$0.2466 \mathrm{E}+00$

$0.3772 E+00$

$0.3551 \mathrm{E}+00$

$0.1042 E+01$

$0.3721 \mathrm{E}+00$

$0.1148 \mathrm{E}+01$

$0.6646 \mathrm{E}+00$

$0.9058 E+00$

$0.6865 \mathrm{E}+00$

$0.4366 \mathrm{E}+00$
BIAS

$0.2728 E+00$

$-.2787 \mathrm{E}+00$

$0.5063 \mathrm{E}+00$

$0.8200 \mathrm{E}+00$

$0.3890 \mathrm{E}+00$

$0.5424 \mathrm{E}+00$

$-.1364 \mathrm{E}+00$

$0.5577 \mathrm{E}+00$

$0.5383 E+00$

$0.3^{\top} .30 \mathrm{E}+00$

$0.6 \angle 52 \mathrm{E}+00$

$0.4388 \mathrm{E}+00$

$0.8159 \mathrm{E}+00$

$0.2865 \mathrm{E}-01$

$0.1066 \mathrm{E}+01$

$0.1607 \mathrm{E}-03$

$0.3922 \mathrm{E}+00$

$0.4941 E-02$

$0.6836 \mathrm{E}+00$

$0.9277 \mathrm{E}+00$

$0.8370 \mathrm{E}+00$

$0.9936 \mathrm{E}+00$

$-.1479 \mathrm{E}+00$

$0.3191 \mathrm{E}+00$

$0.4091 \mathrm{E}-01$

$0.9937 \mathrm{E}+00$

$0.3721 \mathrm{E}+00$

$0.1148 \mathrm{E}+01$

$0.6646 \mathrm{E}+00$

$0.9058 \mathrm{E}+00$

$0.6385 \mathrm{E}+00$

$0.3791 \mathrm{E}+00$
1 OF 2

CPU TIME

$0.7400 \mathrm{E}+02$

$0.7200 \mathrm{E}+02$

$0.7300 \mathrm{E}+02$

$0.7400 E+02$

$0.7800 \mathrm{E}+02$

$0.7800 \mathrm{E}+02$

$0.8000 \mathrm{E}+02$

$0.7500 \mathrm{E}+02$

$0.7300 E+02$

$0.7700 \mathrm{E}+02$

$0.7300 \mathrm{E}+02$

$0.7800 E+02$

$0.6900 E+02$

$0.8100 \mathrm{E}+02$

$0.7900 \mathrm{E}+02$

$0.8200 \mathrm{E}+02$

$0.7700 \mathrm{E}+02$

$0.7900 \mathrm{E}+02$

$0.7900 \mathrm{E}+02$

$0.7800 \mathrm{E}+02$

$0.7700 \mathrm{E}+02$

$0.7400 \mathrm{E}+02$

$0.7500 \mathrm{E}+02$

$0.7500 E+02$

$0.7100 \mathrm{E}+02$

$0.7200 E+02$

$0.7600 \mathrm{E}+02$

$0.7700 \mathrm{E}+\mathrm{J} 2$

$0.7400 \mathrm{E}+02$

$0.7200 \mathrm{E}+02$

$0.7400 \mathrm{E}+02$

$0.7700 \mathrm{E}+02$ 
TABLE 3. (CONTINUED)

2 OF 2

$\begin{array}{lccc}\text { TEST } & \text { SM AB ER } & \text { BIAS } & \text { CPU TIME } \\ 33 & 0.6289 E+00 & 0.6289 E+00 & 0.7600 E+02 \\ 34 & 0.3711 E+00 & 0.3711 E+00 & 0.7500 E+02 \\ 35 & 0.1569 E+00 & 0.1024 E+00 & 0.7500 E+02 \\ 36 & 0.4130 E+00 & 0.3438 E+00 & 0.7100 E+02 \\ 37 & 0.3508 E+00 & -.1363 E+00 & 0.7100 E+02 \\ 38 & 0.6858 E+00 & 0.6378 E+00 & 0.7000 E+02 \\ 39 & 0.1253 E+00 & 0.1395 E-02 & 0.7100 E+02 \\ 40 & 0.9714 E+00 & 0.9714 E+00 & 0.7400 E+02 \\ 41 & 0.6884 E+00 & 0.6884 E+00 & 0.7200 E+02 \\ 42 & 0.1164 E+01 & 0.1164 E+01 & 0.7200 E+02 \\ 43 & 0.2333 E+00 & 0.2053 E-01 & 0.7400 E+02 \\ 44 & 0.3008 E+00 & 0.1720 E+00 & 0.7600 E+02 \\ 45 & 0.2043 E+00 & 0.8178 E-01 & 0.7600 E+02 \\ 46 & 0.4295 E+00 & 0.3231 E+00 & 0.7500 E+02 \\ 47 & 0.1009 E+01 & 0.1009 E+01 & 0.7600 E+02 \\ 48 & 0.6192 E+00 & 0.6192 E+00 & 0.7500 E+02 \\ 49 & 0.1007 E+01 & 0.1006 E+01 & 0.7500 E+02 \\ 50 & 0.1206 E+00 & -.5270 E-01 & 0.7500 E+02 \\ 51 & 0.9847 E+00 & 0.9847 E+00 & 0.7200 E+02 \\ 52 & 0.2426 E+00 & 0.1949 E+00 & 0.7400 E+02 \\ 53 & 0.5684 E+00 & 0.5666 E+00 & 0.6900 E+02 \\ 54 & 0.3861 E+00 & 0.3843 E+00 & 0.7000 E+02 \\ 55 & 0.6961 E+00 & 0.6961 E+00 & 0.7400 E+02 \\ 56 & 0.4716 E+00 & 0.4716 E+00 & 0.7200 E+02 \\ 57 & 0.4468 E+00 & 0.4468 E+00 & 0.6800 E+02 \\ 58 & 0.1150 E+01 & 0.1150 E+01 & 0.6800 E+02 \\ 59 & 0.4633 E+00 & 0.4633 E+00 & 0.7300 E+02 \\ 60 & 0.6195 E+00 & 0.6195 E+00 & 0.7500 E+02 \\ 61 & 0.2010 E+00 & 0.1907 E+00 & 0.7400 E+02 \\ 62 & 0.5047 E+00 & 0.5047 E+00 & 0.7500 E+02 \\ 63 & 0.1275 E+01 & 0.1275 E+01 & 0.7300 E+02 \\ 64 & 0.7385 E+00 & 0.7385 E+00 & 0.7500 E+02\end{array}$


LIQUID DENSITY $n-C 8 / C O 2$ ESD DRES $=4$ SFACT $=0.5 \%$

TABLE 4 A. CONTRASTS LISTED BY FACTORS

1 OF 1

\section{FACTOR}

$\begin{array}{lr}\text { PC } & 1 \\ \text { C } & 14 \\ \text { C } & 11 \\ \text { C } & 16 \\ \text { C } & 17 \\ \text { C } & 8 \\ \text { C } & 5 \\ \text { C } & 15 \\ \text { PC } & 2 \\ \text { C } & 4\end{array}$

C 13

TC 1

C 7

E 20

E 2

E 30

C 9

C 10

E 23

E 6

E 15

E 27

E 2

E 21

E 28

E 11

C 2

E 17

C 1

E 22

E 18

TC 2
$S M A B$ ER

$0.2583 E+00$

$0.2284 \mathrm{E}+00$

$-.2243 E+00$

$-.1881 \mathrm{E}+00$

$0.1868 \mathrm{E}+00$

$-.1558 \mathrm{E}+00$

$0.1323 \mathrm{E}+00$

$0.1121 \mathrm{E}+00$

$0.1109 E+00$

$0.9902 \mathrm{E}-01$

$0.9110 \mathrm{E}-01$

-.8236E-01

$-.6609 E-01$

$-.6535 \mathrm{E}-01$

$-.5329 E-01$

$0.5319 \mathrm{E}-01$

$-.4967 \mathrm{E}-01$.

$-.4658 E-01$

$-.3847 \mathrm{E}-01$

$0.3782 \mathrm{E}-01$

$-.3698 E-01$

$0.3389 \mathrm{E}-01$

$-.3359 E-01$

$-.3076 E-01$

$0.2818 E-01$

$-.2715 \mathrm{E}-01$

$0.2626 \mathrm{E}-01$

$-.2561 \mathrm{E}-01$

$0.2487 \mathrm{E}-01$

$-.2348 E-01$

$0.2298 E-01$

$0.2209 E-01$

\section{FACTOR}

$\begin{array}{lr}\mathrm{OM} & 1 \\ \mathrm{E} & 16\end{array}$

C 12

E 14

K 12

E 5

E 19

E 26

E 25

E 7

E 10

E 13

E 12

C 3

E 29

OM 2

E 3

E 31

C 6

E 4

E 24

E 8

E 9
SM AB ER

$0.2001 E-01$

-.1995E-01

-.1938E-01

$0.1764 \mathrm{E}-01$

$-.1577 \mathrm{E}-01$

$-.1557 \mathrm{E}-01$

$0.1543 E-01$

$0.1414 \mathrm{E}-01$

$-.1194 \mathrm{E}-01$

$0.1086 E-01$

$-.1021 \mathrm{E}-01$

$0.8867 \mathrm{E}-02$

$0.7856 \mathrm{E}-02$

$0.7476 \mathrm{E}-02$

$0.7207 \mathrm{E}-02$

$0.6857 \mathrm{E}-02$

$0.4210 \mathrm{E}-02$

$0.3698 \mathrm{E}-02$

$0.3197 \mathrm{E}-02$

$0.2636 \mathrm{E}-02$

$-.2229 \mathrm{E}-02$

$0.1649 \mathrm{E}-02$

$-.4989 \mathrm{E}-03$ 
LIQUID DENSITY $n-C 8 / C O 2$ ESD DRES $=4$ SFACT $=0.5 \%$

TABLE 4 B. CONTRASTS LISTED BY FACTORS 1 OF 1

FACTOR

\begin{tabular}{|c|c|c|}
\hline & 1 & $0.3586+00$ \\
\hline & 11 & $-.3156 \mathrm{E}+00$ \\
\hline & 14 & $0.2576 \mathrm{E}+00$ \\
\hline & 16 & $-.2397 E+00$ \\
\hline & 17 & $0.2308 \mathrm{E}+00$ \\
\hline & 8 & $-.2043 E+00$ \\
\hline & 5 & $0.1655 \mathrm{E}+00$ \\
\hline & 4 & $0.1431 E+00$ \\
\hline & 1 & $-.1301 E+00$ \\
\hline & 2 & $0.1105 E+00$ \\
\hline & 15 & $0.1027 \mathrm{E}+00$ \\
\hline & 10 & $-.8944 E-01$ \\
\hline & 13 & $0.8376 E-01$ \\
\hline & 7 & $-.7847 E-01$ \\
\hline & 9 & $-.5667 E-01$ \\
\hline M & 1 & $0.4630 \mathrm{E}-01$ \\
\hline & 2 & $0.4601 E-01$ \\
\hline & 12 & $-.3909 E-01$ \\
\hline & 1 & $0.3517 E-01$ \\
\hline & 6 & $0.3083 \mathrm{E}-01$ \\
\hline & 2 & $0.5002 E-02$ \\
\hline & 3 & $0.2649 \mathrm{E}-02$ \\
\hline & 18 & $0.1938 \mathrm{E}-02$ \\
\hline & 19 & $0.1630 E-02$ \\
\hline & 29 & $-.1619 E-02$ \\
\hline & 5 & $0.1618 E-02$ \\
\hline & 13 & $-.1219 \mathrm{E}-02$ \\
\hline & 25 & $0.1132 \mathrm{E}-02$ \\
\hline & 26 & $-.9952 E-03$ \\
\hline & 22 & $0.7721 E-03$ \\
\hline & 12 & $-.6816 E-03$ \\
\hline & 4 & $0.6682 \mathrm{E}-03$ \\
\hline
\end{tabular}

FACTOR

BIAS

$\begin{array}{lrrr}\mathrm{E} & 31 & 0.6590 \mathrm{E}-03 \\ \mathrm{E} & 23 & -.5986 \mathrm{E}-03 \\ \mathrm{TC} & 2 & 0.5125 \mathrm{E}-03 \\ \mathrm{E} & 6 & -.4897 \mathrm{E}-03 \\ \mathrm{E} & 9 & -.4825 \mathrm{E}-03 \\ \mathrm{E} & 27 & 0.4471 \mathrm{E}-03 \\ \mathrm{E} & 15 & -.4205 \mathrm{E}-03 \\ \mathrm{E} & 20 & -.3180 \mathrm{E}-03 \\ \mathrm{E} & 21 & -.2962 \mathrm{E}-03 \\ \mathrm{E} & 14 & 0.2863 \mathrm{E}-03 \\ \mathrm{E} & 2 & 0.2567 \mathrm{E}-03 \\ \mathrm{E} & 17 & -.2435 \mathrm{E}-03 \\ \mathrm{E} & 16 & 0.2379 \mathrm{E}-03 \\ \mathrm{E} & 28 & -.2105 \mathrm{E}-03 \\ \mathrm{E} & 30 & 0.2004 \mathrm{E}-03 \\ \mathrm{E} & 10 & 0.1657 \mathrm{E}-03 \\ \mathrm{E} & 7 & -.1115 \mathrm{E}-03 \\ \mathrm{E} & 11 & -.5289 \mathrm{E}-04 \\ \mathrm{E} & 24 & -.4005 \mathrm{E}-04 \\ \mathrm{E} & 8 & -.3817 \mathrm{E}-04 \\ \mathrm{E} & 3 & -.2746 \mathrm{E}-04 \\ \mathrm{~K} & 1 & 2 & 0.1320 \mathrm{E}-04 \\ \mathrm{E} & 1 & -.2698 \mathrm{E}-05\end{array}$


LIQUID DENSITY $n-C 8$ / CO2 ESD DRES $=4$ SFACT $=0.5 \%$

TABLE 4 C. CONTRASTS LISTED BY FACTORS

1 OF 1

\begin{tabular}{|c|c|c|c|}
\hline FACTOR & CPU TIME & FACTOR & CPU TIME \\
\hline 6 & $-.2563 E+01$ & 31 & $0.3750 \mathrm{E}+00$ \\
\hline 10 & $0.1500 E+01$ & C 10 & $0.3125 \mathrm{E}+00$ \\
\hline 27 & $-.1500 E+01$ & E 28 & $0.3125 \mathrm{E}+00$ \\
\hline 2 & $0.1438 E+01$ & C 7 & $-.2500 \mathrm{E}+00$ \\
\hline E & $0.1313 E+01$ & $\mathrm{E}$ & $-.2500 \mathrm{E}+00$ \\
\hline$E$ & $0.1313 E+01$ & $E$ & $-.2500 E+00$ \\
\hline 13 & $-.1313 \mathrm{E}+01$ & 15 & $-.2500 \mathrm{E}+00$ \\
\hline $\mathrm{K} 12$ & $0.1188 \mathrm{E}+01$ & 16 & $-.2500 \mathrm{E}+00$ \\
\hline E 11 & $-.1063 E+01$ & E 22 & $0.2500 E+00$ \\
\hline C 17 & $-.9375 E+00$ & C 11 & $0.1875 \mathrm{E}+00$ \\
\hline C 15 & $-.8750 E+00$ & PC 2 & $0.1875 \mathrm{E}+00$ \\
\hline$C$ & $-.8125 E+00$ & E 18 & $0.1875 \mathrm{E}+00$ \\
\hline C 16 & $0.8125 \mathrm{E}+00$ & E 20 & $0.1875 \mathrm{E}+00$ \\
\hline OM & $0.8125 E+00$ & $\mathrm{C}$ & $0.1250 E+00$ \\
\hline E 14 & $0.8125 E+00$ & C 13 & $0.1250 E+00$ \\
\hline $\mathrm{E}$ & $0.8125 E+00$ & OM & $0.1250 E+00$ \\
\hline C 14 & $-.7500 E+00$ & $E$ & $-.6250 E-01$ \\
\hline$E$ & $-.7500 E+00$ & $\mathrm{E}$ & $0.6250 E-01$ \\
\hline C & $0.6875 E+00$ & 24 & $0.6250 \mathrm{E}-01$ \\
\hline $\mathrm{TC}$ & $-.6250 E+00$ & 29 & $-.6250 E-01$ \\
\hline E 23 & $-.6250 E+00$ & C 4 & $0.0000 E+00$ \\
\hline$E$ & $-.5625 E+00$ & PC 1 & $0.0000 E+00$ \\
\hline$E$ & $0.5625 \mathrm{E}+00$ & $\mathrm{E}$ & $0.0000 E+00$ \\
\hline $\mathrm{C}$ & $0.5000 E+00$ & & \\
\hline$E$ & $-.5000 E+00$ & & \\
\hline 21 & $-.5000 E+00$ & & \\
\hline 30 & $-.5000 E+00$ & & \\
\hline $\mathrm{C}$ & $0.4375 E+00$ & & \\
\hline E 19 & $0.4375 E+00$ & & \\
\hline C 12 & $-.3750 E+00$ & & \\
\hline TC 2 & $-.3750 E+00$ & & \\
\hline$E$ & $0.3750 E+00$ & & \\
\hline
\end{tabular}


IIQUID DENSITY $n-C 10 / C O 2$ ESD DRES $=4$ SFACT $=0.5 \%$ 
LIQUID DENSITY $\mathrm{n}-\mathrm{C} 10 / \mathrm{CO} 2$ ESD DRES $=4$ SFACT $=0.5 \%$

TABLE 3. RESPONSES

1 OF 2

TEST

SM $A B \quad E R$

BIAS

CPU TIME

1

$0.8184 \mathrm{E}+01$

$0.1016 \mathrm{E}+02$

$-.2382 \mathrm{E}+01$

$-.6204 \mathrm{E}+01$

$0.6200 \mathrm{E}+02$

$0.8326 \mathrm{E}+01$

$-.1481 \mathrm{E}+01$

$0.5900 \mathrm{E}+02$

$0.8460 \mathrm{E}+01$

$-.1536 \mathrm{E}+01$

$0.6000 \mathrm{E}+02$

$0.8274 \mathrm{E}+01$

$-.1881 \mathrm{E}+01$

$0.6000 \mathrm{E}+02$

$0.9536 \mathrm{E}+01$

$-.4484 \mathrm{E}+01$

$0.6100 \mathrm{E}+02$

$0.8118 \mathrm{E}+01$

$-.2083 E+01$

$0.6100 \mathrm{E}+02$

$0.8641 \mathrm{E}+01$

$-.3122 \mathrm{E}+01$

$0.6100 \mathrm{E}+02$

$0.8413 \mathrm{E}+01$

$-.5177 \mathrm{E}-01$

$0.6000 \mathrm{E}+02$

$0.8195 \mathrm{E}+01$

$0.8347 \mathrm{E}+01$

$0.9594 \mathrm{E}+01$

$0.8393 E+01$

$0.8021 \mathrm{E}+01$

$0.8868 \mathrm{E}+01$

$0.9159 \mathrm{E}+01$

$0.9260 \mathrm{E}+01$

$0.8764 \mathrm{E}+01$

$0.8028 \mathrm{E}+01$

$0.8450 \mathrm{E}+01$

$0.9003 \mathrm{E}+01$

$0.8449 \mathrm{E}+01$

$0.8126 \mathrm{E}+01$

$0.8408 \mathrm{E}+01$

$0.8435 E+01$

$0.9038 \mathrm{E}+01$

$0.8324 \mathrm{E}+01$

$0.8359 E+01$

$0.8311 \mathrm{E}+01$

$0.8622 \mathrm{E}+01$

$0.8493 E+01$

$-.1538 \mathrm{E}+01$

$0.5900 \mathrm{E}+02$

$-.2079 \mathrm{E}+01$

$0.6000 \mathrm{E}+02$

$-.4420 \mathrm{E}+01$

$0.5900 E+02$

$-.1189 \mathrm{E}+01$

$0.6000 \mathrm{E}+02$

$-.1695 \mathrm{E}+01$

$0.5800 \mathrm{E}+02$

$-.8741 \mathrm{E}+00$

$0.5600 \mathrm{E}+02$

$0.5600 \mathrm{E}+02$

$-.4164 \mathrm{E}+01$

$0.5400 \mathrm{E}+02$

-.3722E+01

$0.5300 E+02$

$-.3166 \mathrm{E}+01$

$-.2316 \mathrm{E}+01$

$0.5800 \mathrm{E}+02$

$0.7163 \mathrm{E}+00$

$0.5800 \mathrm{E}+02$

$-.3338 \mathrm{E}+01$

$0.5900 \mathrm{E}+02$

-.1683E+01

$0.6100 E+02$

$-.2725 \mathrm{E}+01$

$0.6000 \mathrm{E}+02$

$-.6726 \mathrm{E}+00$

$0.5800 E+02$

$-.1298 \mathrm{E}+01$

$0.6100 \mathrm{E}+02$

$0.1154 \mathrm{E}+01$

$0.6100 \mathrm{E}+02$

$-.2939 \mathrm{E}+01$

$0.6000 \mathrm{E}+02$

$-.1808 \mathrm{E}+01$

$0.6000 \mathrm{E}+02$

$-.2612 \mathrm{E}+01$

$0.6100 \mathrm{E}+02$

$0.8206 \mathrm{E}+00$

$0.5700 \mathrm{E}+02$

$-.1533 E+01$

$0.6000 \mathrm{E}+02$

$0.8437 E+01$

$-.1518 \mathrm{E}+01$

$0.5800 E+02$

$0.5400 E+02$ 
LIQUID DENSITY $n-C 10 / C O 2$ ESD DRES $=4 \quad \mathrm{SFACT}=0.5 \%$

TABLE 3. (CONTINUED)

2 OF 2

\begin{tabular}{|c|c|c|c|}
\hline TEST & $S M A B \quad E R$ & BIAS & CPU TIME \\
\hline 33 & $0.8161 \mathrm{E}+01$ & $-.2392 \mathrm{E}+01$ & $0.5300 E+02$ \\
\hline 34 & $0.8085 E+01$ & $-.2380 E+01$ & $0.5500 \mathrm{E}+02$ \\
\hline 35 & $0.9835 \mathrm{E}+01$ & $-.5092 \mathrm{E}+01$ & $0.5600 \mathrm{E}+02$ \\
\hline 36 & $0.8437 E+01$ & $-.1315 E+01$ & $0.5400 \mathrm{E}+02$ \\
\hline 37 & $0.8216 \mathrm{E}+01$ & $-.2107 E+01$ & $0.5700 E+02$ \\
\hline 38 & $0.8715 E+01$ & $-.9934 E+00$ & $0.5800 E+02$ \\
\hline 39 & $0.9662 \mathrm{E}+01$ & $-.5406 \mathrm{E}+01$ & $0.5500 \mathrm{E}+02$ \\
\hline 40 & $0.8575 \mathrm{E}+01$ & $-.2647 E+01$ & $0.5300 \mathrm{E}+02$ \\
\hline 41 & $0.8896 E+01$ & $-.3358 E+01$ & $0.5400 E+02$ \\
\hline 42 & $0.8670 \mathrm{E}+01$ & $-.1177 \mathrm{E}+01$ & $0.5400 E+02$ \\
\hline 43 & $0.8128 \mathrm{E}+01$ & $-.2221 \mathrm{E}+01$ & $0.5700 E+02$ \\
\hline 44 & $0.8351 \mathrm{E}+01$ & $-.7157 \mathrm{E}+00$ & $0.5600 \mathrm{E}+02$ \\
\hline 45 & $0.9886 \mathrm{E}+01$ & $-.4985 E+01$ & $0.5800 E+02$ \\
\hline 46 & $0.8550 E+01$ & $-.1597 \mathrm{E}+01$ & $0.5700 E+02$ \\
\hline 47 & $0.8412 \mathrm{E}+01$ & $-.7908 E+00$ & $0.5900 \mathrm{E}+02$ \\
\hline 48 & $0.8249 E+01$ & $-.3562 E+00$ & $0.5900 \mathrm{E}+02$ \\
\hline 49 & $0.8626 \mathrm{E}+01$ & $0.6295 \mathrm{E}-01$ & $0.5900 E+02$ \\
\hline 50 & $0.8225 E+01$ & $-.3039 E+01$ & $0.5900 E+02$ \\
\hline 51 & $0.8660 \mathrm{E}+01$ & $-.2228 E+01$ & $0.5800 E+02$ \\
\hline 52 & $0.8402 E+01$ & $-.2741 E+01$ & $0.5800 E+02$ \\
\hline 53 & $0.8390 E+01$ & $0.1977 \mathrm{E}+00$ & $0.5700 E+02$ \\
\hline 54 & $0.8165 E+01$ & $-.1835 E+01$ & $0.5800 E+02$ \\
\hline 55 & $0.8567 \mathrm{E}+01$ & $-.2398 E+01$ & $0.5800 E+02$ \\
\hline 56 & $0.9403 E+01$ & $-.4085 E+01$ & $0.5900 \mathrm{E}+02$ \\
\hline 57 & $0.8458 E+01$ & $-.8661 . E+00$ & $0.5800 E+02$ \\
\hline 58 & $0.8478 E+01$ & $-.1829 E+01$ & $0.5800 E+02$ \\
\hline 59 & $0.8573 E+01$ & $0.2674 \mathrm{E}+00$ & $0.5800 \mathrm{E}+02$ \\
\hline 60 & $0.8241 E+01$ & $-.2030 E+01$ & $0.6000 E+02$ \\
\hline 61 & $0.8128 E+01$ & $-.2374 \mathrm{E}+01$ & $0.5800 \mathrm{E}+02$ \\
\hline 62 & $0.8291 E+01$ & $-.2437 E+01$ & $0.5800 \mathrm{E}+02$ \\
\hline 63 & $0.8907 E+01$ & $0.1932 \mathrm{E}+01$ & $0.5800 \mathrm{E}+02$ \\
\hline 64 & $0.8415 E+01$ & $-.1534 \mathrm{E}+01$ & $0.5900 \mathrm{E}+02$ \\
\hline
\end{tabular}


LIQUID DENSITY $n-C 10 / \mathrm{CO} 2$ ESD DRES $=4 \quad \mathrm{SFACT}=0.5 \%$

TABLE 4 A. CONTRASTS IISTED BY FACTORS 1 OF 1

\begin{tabular}{|c|c|c|c|}
\hline FACTOR & $S M A B \quad E R$ & FACTOR & $S M A B \quad E R$ \\
\hline TC 2 & $-.4458 \mathrm{E}+00$ & $\mathrm{E}$ & $-.4295 \mathrm{E}-01$ \\
\hline E 19 & $-.4418 E+00$ & $\mathrm{OM}$ & $-.3922 \mathrm{E}-01$ \\
\hline 5 & $-.3014 \mathrm{E}+00$ & $1 \overline{1}$ & $-.3870 \mathrm{E}-01$ \\
\hline 29 & $0.2886 \mathrm{E}+00$ & $\bar{C}$ & $0.3559 \mathrm{E}-01$ \\
\hline C 16 & $0.2241 \mathrm{E}+00$ & 24 & $0.3143 E-01$ \\
\hline 9 & $0.1812 \mathrm{E}+00$ & $\mathrm{~K} 12$ & $-.2810 E-01$ \\
\hline PC & $0.1729 E+00$ & C & $0.2585 \mathrm{E}-01$ \\
\hline E 25 & $-.1668 E+00$ & 30 & $-.2457 \mathrm{E}-01$ \\
\hline C & $-.1588 E+00$ & $\bar{E}$ & $-.2267 \mathrm{E}-01$ \\
\hline C 11 & $-.1450 E+00$ & 15 & $-.2234 \mathrm{E}-01$ \\
\hline E 31 & $-.1375 \mathrm{E}+00$ & 18 & $0.2038 E-01$ \\
\hline C 8 & $0.1356 \mathrm{E}+00$ & 10 & $0.1436 \mathrm{E}-01$ \\
\hline TC 1 & $0.1333 \mathrm{E}+00$ & $\mathrm{C}$ & $-.1378 E-01$ \\
\hline $\mathrm{E} \quad 12$ & $0.1184 \mathrm{E}+00$ & 14 & $-.9804 \mathrm{E}-02$ \\
\hline C 14 & $0.9484 \mathrm{E}-01$ & E 20 & $0.9280 \mathrm{E}-02$ \\
\hline $\mathrm{E} \quad 6$ & $0.9458 \mathrm{E}-01$ & C 17 & $-.6867 E-02$ \\
\hline 28 & $0.9422 \mathrm{E}-01$ & E 21 & $0.6643 E-02$ \\
\hline 9 & $0.8623 \mathrm{E}-01$ & $\mathbf{E}$ & $0.5921 \mathrm{E}-02$ \\
\hline C 12 & $0.8492 \mathrm{E}-01$ & 26 & $0.5516 \mathrm{E}-02$ \\
\hline E 23 & $-.8444 \mathrm{E}-01$ & 13 & $-.5068 E-02$ \\
\hline $\bar{C}$ & $-.8332 \mathrm{E}-01$ & 27 & $-.2412 \mathrm{E}-02$ \\
\hline C 13 & $0.8299 \mathrm{E}-01$ & C & $-.1362 \mathrm{E}-02$ \\
\hline $\mathrm{E} \quad 16$ & $-.5808 \mathrm{E}-01$ & 17 & $-9700 \mathrm{E}-03$ \\
\hline 3 & $-.5448 \mathrm{E}-01$ & & \\
\hline 22 & $0.5302 E-01$ & & \\
\hline C 15 & $0.5246 E-01$ & & \\
\hline 8 & $-.5160 \mathrm{E}-01$ & & \\
\hline C & $0.4680 E-01$ & & \\
\hline OM 1 & $0.4578 \mathrm{E}-01$ & & \\
\hline C 10 & $-.4536 \mathrm{E}-01$ & & \\
\hline & $-.4440 E-01$ & & \\
\hline PC & $-.4328 E-01$ & & \\
\hline
\end{tabular}


LIQUID DENSITY $n-C 10 / C O 2$ ESD DRES $=4$ SFACT $=0.5 \%$

TABLE 4 B. CONTRASTS LISTED BY FACTORS 1 OF 1

FACTOR

$\begin{array}{lr}\text { C } & 16 \\ \text { TC } & 2 \\ \text { C } & 8 \\ \text { C } & 4 \\ \text { C } & 17 \\ \text { C } & 5 \\ \text { C } & 7 \\ \text { C } & 9\end{array}$

PC 1

C 6

E 19

PC 2

TC 1

E 5

E 29

E 9

C 12

E 25

C 15

E 12

E 30

E 31

C 11

C 14

C 2

E 28

C 13

E 18

OM 1

E 23

OM 2

E 24
BIAS

$0.1661 \mathrm{E}+01$

-.1040E+01

$0.8955 \mathrm{E}+00$

$0.8892 \mathrm{E}+00$

$0.7856 \mathrm{E}+00$

$-.5319 \mathrm{E}+00$

$-.2231 \mathrm{E}+00$

$0.1225 \mathrm{E}+00$

$0.1048 \mathrm{E}+00$

$0.8500 \mathrm{E}-01$

$-.7900 \mathrm{E}-01$

$0.6994 \mathrm{E}-01$

$0.5822 \mathrm{E}-01$

$-.5196 \mathrm{E}-01$

$-.3018 E-01$

-.2734E-01

$0.2678 \mathrm{E}-01$

$0.2382 \mathrm{E}-01$

$-.2135 \mathrm{E}-01$

$0.2055 \mathrm{E}-01$

$0.1954 \mathrm{E}-01$

$-.1770 \mathrm{E}-01$

$0.1674 \mathrm{E}-01$

$0.1444 \mathrm{E}-01$

-.1346E-01

-.1277E-01

-.1252E-01

$0.1175 \mathrm{E}-01$

$0.1086 \mathrm{E}-01$

$0.1080 \mathrm{E}-01$

$-.9728 \mathrm{E}-02$

\section{FACTOR}

E 16

E 22

E 8

C 1

E 1

E 26

K 12

E 4

C 10

E 20

E 27

E 2

E 13

E 15

E 10

C 3

E 17

E 7

E 6

E 21

E 3

E 11

E 14
BIAS

$0.8654 \mathrm{E}-02$

$-.8309 \mathrm{E}-02$

$0.7533 \mathrm{E}-02$

$0.7186 \mathrm{E}-02$

$-.6135 \mathrm{E}-02$

$-.5635 \mathrm{E}-02$

$-.5593 E-02$

$0.5586 \mathrm{E}-02$

$0.4752 \mathrm{E}-02$

$-.4083 E-02$

$0.3538 \mathrm{E}-02$

$0.3150 E-02$

$0.231 .5 \mathrm{E}-02$

$0.1734 \mathrm{E}-02$

$0.1549 \mathrm{E}-02$

$-.1450 \mathrm{E}-02$

$0.1273 \mathrm{E}-02$

$0.1121 E-02$

$-.9247 \mathrm{E}-03$

$-.9041 \mathrm{E}-03$

$0.7403 \mathrm{E}-03$

$0.6339 \mathrm{E}-03$

$-.3594 \mathrm{E}-03$ 
LIQUID DENSITY $n-C 10 / C O 2$ ESD DRES $=4$ SiACT $=0.5 \%$

TABLE 4 C. CONTRASTS LISTED BY FACTORS

1 OF 1

\begin{tabular}{|c|c|c|c|c|}
\hline \multicolumn{2}{|c|}{ FACTOR } & CPU TIME & FACTOR & CPU TIME \\
\hline C & 6 & $-.1781 \mathrm{E}+01$ & C & $-.1563 \mathrm{E}+00$ \\
\hline & 12 & $0.1531 \mathrm{E}+01$ & C 16 & $-.1563 \mathrm{E}+00$ \\
\hline$E$ & 15 & $0.1406 \mathrm{E}+01$ & TC 1 & $0.1563 \mathrm{E}+00$ \\
\hline$E$ & 10 & $-.1031 E+01$ & PC & $-.1563 \mathrm{E}+00$ \\
\hline $\mathrm{E}_{1}$ & 14 & $0.1031 \mathrm{E}+01$ & $\mathrm{E}$ & $0.1563 \mathrm{E}+00$ \\
\hline & 5 & $0.9688 \mathrm{E}+00$ & 8 & $0.1563 \mathrm{E}+00$ \\
\hline TC & 2 & $0.8438 E+00$ & 26 & $-.1563 E+00$ \\
\hline & 12 & $0.8438 \mathrm{E}+00$ & C 1 & $0.9375 E-01$ \\
\hline $\mathrm{E}$ & 6 & $-.7813 E+00$ & $\mathrm{C}$ & $-.9375 \mathrm{E}-01$ \\
\hline & 28 & $0.7188 \mathrm{E}+00$ & C & $0.9375 \mathrm{E}-01$ \\
\hline $\mathrm{E}$ & 4 & $0.6563 E+00$ & C & $0.9375 E-01$ \\
\hline & 31 & $0.5938 \mathrm{E}+00$ & 23 & $0.9375 \mathrm{E}-01$ \\
\hline & 1 & $-.5313 \mathrm{E}+00$ & 24 & $0.9375 \mathrm{E}-01$ \\
\hline & 20 & $0.5313 \mathrm{E}+00$ & 27 & $0.9375 \mathrm{E}-01$ \\
\hline & 21 & $0.5313 E+00$ & 29 & $-.9375 \mathrm{E}-01$ \\
\hline & 2 & $-.4688 E+00$ & 30 & $0.9375 E-01$ \\
\hline & 9 & $0.4063 E+00$ & C & $-.3125 \mathrm{E}-01$ \\
\hline & 13 & $0.4063 E+00$ & $\mathrm{C}$ & $0.3125 \mathrm{E}-\mathrm{C} 1$ \\
\hline & 16 & $-.4063 E+00$ & C 10 & $-.3125 E-J 1$ \\
\hline & 25 & $0.4063 E+00$ & C 12 & $0.3125 \mathrm{E}-01$ \\
\hline & 13 & $0.2813 E+00$ & C 17 & $0.3125 \mathrm{E}-01$ \\
\hline $\mathrm{C}$ & 14 & $-.2813 E+00$ & $\mathrm{E}$ & $0.3125 E-01$ \\
\hline $\mathrm{PC}$ & 1 & $0.2813 E+00$ & 17 & $-.3125 E-01$ \\
\hline $\mathrm{E}$ & 2 & $-.2813 E+00$ & & \\
\hline $\mathrm{E}$ & 11 & $-.2813 E+00$ & & \\
\hline C & 11 & $-.2188 \mathrm{E}+00$ & & \\
\hline $\mathrm{C}$ & 15 & $0.2188 \mathrm{E}+00$ & & \\
\hline $\mathrm{E}$ & 1 & $-.2188 E+00$ & & \\
\hline $\mathrm{E}$ & 3 & $-.2188 \mathrm{E}+00$ & & \\
\hline$E$ & 18 & $0.2188 E+00$ & & \\
\hline$E$ & 19 & $-.2188 \mathrm{E}+00$ & & \\
\hline $\mathrm{E}$ & 22 & $0.2188 E+00$ & & \\
\hline
\end{tabular}


CRIT LOCUS OUTPUT. CO2 / C4 ESD. WITH ERROR HANDLING 
CRIT LOCUS OUTPUT. CO2 / C4 ESD. WITH ERROR HANDLING

TABLE 3. RESPONSES TEST

1

2

3

4

5

6

7

8

9

10

11

12

13

14

15

16

17

18

19

20

21

22

23

24

25

26

27

28

29

30

31

32 RT SQ ER

$0.1260 E+02$

$0.1261 E+02$

$0.1259 \mathrm{E}+02$

$0.1235 \mathrm{E}+02$

$0.1248 \mathrm{E}+02$

$0.1223 E+02$

$0.1293 \mathrm{E}+02$

$0.1256 \mathrm{E}+02$

$0.9597 \mathrm{E}+02$

$0.1264 \mathrm{E}+02$

$0.1249 \mathrm{E}+02$

$0.1228 \mathrm{E}+02$

$0.1233 \mathrm{E}+02$

$0.1266 \mathrm{E}+02$

$0.1241 \mathrm{E}+02$

$0.9533 E+02$

$0.1245 \mathrm{E}+02$

$0.1276 \mathrm{E}+02$

$0.1243 E+02$

$0.1255 \mathrm{E}+02$

$0.1219 \mathrm{E}+02$

$0.1234 \mathrm{E}+02$

$0.1275 \mathrm{E}+02$

$0.1278 \mathrm{E}+02$

$0.1282 \mathrm{E}+02$

$0.1267 \mathrm{E}+02$

$0.1245 \mathrm{E}+02$

$0.1210 \mathrm{E}+02$

$0.1241 \mathrm{E}+02$

$0.1264 \mathrm{E}+02$

$0.9419 E+02$

$0.1261 \mathrm{E}+02$
RMS BIAS

-.6720E+01

-.7115E+01

$-.6612 E+01$

-.6182E+01

$-.6322 \mathrm{E}+01$

-.6141E+01

$-.7345 E+01$

$-.6812 \mathrm{E}+01$

$-.6888 E+02$

$-.6936 E+01$

$-.6142 E+01$

$-.6028 E+01$

$-.6318 \mathrm{E}+01$

$-.6896 E+01$

$-.5966 \mathrm{E}+01$

$-.6816 \mathrm{E}+02$

$-.6096 E+01$.

$-.6618 E+01$

$-.6837 \mathrm{E}+01$

$-.6640 E+01$

$-.6152 E+01$

$-.6233 E+01$

$-.6994 E+01$

$-.6786 \mathrm{E}+01$

$-.6692 \mathrm{E}+01$

$-.6111 \mathrm{E}+01$

$-.6739 E+01$

$-.6021 E+01$

$-.6645 E+01$

$-.6579 E+01$

$-.6660 \mathrm{E}+02$

$-.6300 E+01$
1 OF 2

CPU TIME

$0.4250 \mathrm{E}+03$

$0.4770 \mathrm{E}+03$

$0.4380 \mathrm{E}+03$

$0.4410 \mathrm{E}+03$

$0.4300 \mathrm{E}+03$

$0.4430 \mathrm{E}+03$

$0.4740 \mathrm{E}+03$

$0.4480 \mathrm{E}+03$

$0.5360 \mathrm{E}+03$

$0.4520 \mathrm{E}+03$

$0.4640 \mathrm{E}+03$

$0.4570 \mathrm{E}+03$

0.4 ? $00 \mathrm{E}+03$

$0.4260 \mathrm{E}+03$

$0.4920 \mathrm{E}+03$

$0.5470 \mathrm{E}+03$

$0.4360 \mathrm{E}+03$

$0.4440 \mathrm{E}+03$

$0.4590 \mathrm{E}+03$

$0.4410 \mathrm{E}+03$

$0.4370 \mathrm{E}+03$

$0.4110 \mathrm{E}+03$

$0.4480 \mathrm{E}+03$

$0.4580 \mathrm{E}+03$

$0.4690 \mathrm{E}+03$

$0.4520 \mathrm{E}+03$

$0.4930 \mathrm{E}+03$

$0.4560 \mathrm{E}+03$

$0.4450 \mathrm{E}+03$

$0.4170 \mathrm{E}+03$

$0.5590 \mathrm{E}+03$

$0.4410 \mathrm{E}+03$ 
CRIT LOCUS OUTPUT. CO2 / C4 ESD. WITH ERROR HANDLING

TABLE 3. (CONTINUED)

2 OF 2

$\begin{array}{lccc}\text { TEST } & \text { RT SQ ER } & \text { RMS BIAS } & \text { CPU TIME } \\ 33 & 0.1246 \mathrm{E}+02 & -.6743 \mathrm{E}+01 & 0.4450 \mathrm{E}+03 \\ 34 & 0.1259 \mathrm{E}+02 & -.7008 \mathrm{E}+01 & 0.4420 \mathrm{E}+03 \\ 35 & 0.1241 \mathrm{E}+02 & -.6430 \mathrm{E}+01 & 0.4510 \mathrm{E}+03 \\ 36 & 0.1265 \mathrm{E}+02 & -.6394 \mathrm{E}+01 & 0.4640 \mathrm{E}+03 \\ 37 & 0.1294 \mathrm{E}+02 & -.6999 \mathrm{E}+01 & 0.4590 \mathrm{E}+03 \\ 38 & 0.1260 \mathrm{E}+02 & -.6286 \mathrm{E}+01 & 0.4360 \mathrm{E}+03 \\ 39 & 0.1247 \mathrm{E}+02 & -.6979 \mathrm{E}+01 & 0.4530 \mathrm{E}+03 \\ 40 & 0.1223 \mathrm{E}+02 & -.6324 \mathrm{E}+01 & 0.4620 \mathrm{E}+03 \\ 41 & 0.1227 \mathrm{E}+02 & -.6229 \mathrm{E}+01 & 0.4570 \mathrm{E}+03 \\ 42 & 0.1230 \mathrm{E}+02 & -.6030 \mathrm{E}+01 & 0.4490 \mathrm{E}+03 \\ 43 & 0.1270 \mathrm{E}+02 & -.6781 \mathrm{E}+01 & 0.4260 \mathrm{E}+03 \\ 44 & 0.1286 \mathrm{E}+02 & -.6864 \mathrm{E}+01 & 0.4930 \mathrm{E}+03 \\ 45 & 0.1251 \mathrm{E}+02 & -.6368 \mathrm{E}+01 & 0.4680 \mathrm{E}+03 \\ 46 & 0.9536 \mathrm{E}+02 & -.6806 \mathrm{E}+02 & 0.4990 \mathrm{E}+03 \\ 47 & 0.1228 \mathrm{E}+02 & -.6397 \mathrm{E}+01 & 0.4150 \mathrm{E}+03 \\ 48 & 0.1259 \mathrm{E}+02 & -.6917 \mathrm{E}+01 & 0.4500 \mathrm{E}+03 \\ 49 & 0.1243 \mathrm{E}+02 & -.6267 \mathrm{E}+01 & 0.4870 \mathrm{E}+03 \\ 50 & 0.1265 \mathrm{E}+02 & -.7073 \mathrm{E}+01 & 0.4640 \mathrm{E}+03 \\ 51 & 0.1239 \mathrm{E}+02 & -.6120 \mathrm{E}+01 & 0.4660 \mathrm{E}+03 \\ 52 & 0.1271 \mathrm{E}+02 & -.6699 \mathrm{E}+01 & 0.4440 \mathrm{E}+03 \\ 53 & 0.1276 \mathrm{E}+02 & -.6979 \mathrm{E}+01 & 0.4330 \mathrm{E}+03 \\ 54 & 0.9531 \mathrm{E}+02 & -.6811 \mathrm{E}+02 & 0.5690 \mathrm{E}+03 \\ 55 & 0.1241 \mathrm{E}+02 & -.6095 \mathrm{E}+01 & 0.4560 \mathrm{E}+03 \\ 56 & 0.9472 \mathrm{E}+02 & -.6731 \mathrm{E}+02 & 0.5300 \mathrm{E}+03 \\ 57 & 0.1253 \mathrm{E}+02 & -.6248 \mathrm{E}+01 & 0.4540 \mathrm{E}+03 \\ 58 & 0.1213 \mathrm{E}+02 & -.5709 \mathrm{E}+01 & 0.4550 \mathrm{E}+03 \\ 59 & 0.9600 \mathrm{E}+02 & -.6891 \mathrm{E}+02 & 0.4320 \mathrm{E}+03 \\ 60 & 0.1257 \mathrm{E}+02 & -.6710 \mathrm{E}+01 & 0.4310 \mathrm{E}+03 \\ 61 & 0.1269 \mathrm{E}+02 & -.6840 \mathrm{E}+01 & 0.4600 \mathrm{E}+03 \\ 62 & 0.1245 \mathrm{E}+02 & -.6402 \mathrm{E}+01 & 0.4440 \mathrm{E}+03 \\ 63 & 0.9681 \mathrm{E}+02 & -.6969 \mathrm{E}+02 & 0.4610 \mathrm{E}+03 \\ 64 & 0.1244 \mathrm{E}+02 & -.6298 \mathrm{E}+01 & 0.4640 \mathrm{E}+03\end{array}$


CRIT LOCUS OUTPUT. CO2 / C4 ESD. WITH ERROR HANDLING

TABLE 4 A. CONTRASTS LISTED BY FACTORS 1 OF 1 FACTOR

$\begin{array}{lrllrl}\mathrm{C} & 14 & -.1564 \mathrm{E}+02 & \mathrm{E} & 11 & 0.5179 \mathrm{E}+01 \\ \mathrm{E} & 22 & -.1553 \mathrm{E}+02 & \mathrm{C} & 5 & 0.5171 \mathrm{E}+01 \\ \mathrm{PC} & 1 & -.1054 \mathrm{E}+02 & \mathrm{C} & 8 & -.5166 \mathrm{E}+01 \\ \mathrm{E} & 25 & -.1045 \mathrm{E}+02 & \mathrm{C} & 2 & 0.5163 \mathrm{E}+01 \\ \mathrm{E} & 15 & 0.1044 \mathrm{E}+02 & \mathrm{C} & 17 & 0.5157 \mathrm{E}+01 \\ \mathrm{E} & 20 & -.1044 \mathrm{E}+02 & \mathrm{E} & 13 & -.5137 \mathrm{E}+01 \\ \mathrm{C} & 9 & -.1040 \mathrm{E}+02 & \mathrm{OM} & 2 & -.5116 \mathrm{E}+01 \\ \mathrm{C} & 10 & -.1039 \mathrm{E}+02 & \mathrm{E} & 31 & 0.5092 \mathrm{E}+01 \\ \mathrm{C} & 4 & 0.1038 \mathrm{E}+02 & \mathrm{E} & 14 & -.5082 \mathrm{E}+01 \\ \mathrm{E} & 3 & -.1038 \mathrm{E}+02 & \mathrm{E} & 21 & 0.5081 \mathrm{E}+01 \\ \mathrm{E} & 19 & -.1038 \mathrm{E}+02 & \mathrm{~K} 12 & -.5081 \mathrm{E}+01 \\ \mathrm{E} & 8 & 0.1038 \mathrm{E}+02 & \mathrm{E} & 5 & 0.5077 \mathrm{E}+01 \\ \mathrm{OM} & 1 & 0.1037 \mathrm{E}+02 & \mathrm{C} & 13 & 0.5009 \mathrm{E}+01 \\ \mathrm{E} & 2 & 0.1034 \mathrm{E}+02 & \mathrm{TC} & 2 & 0.2912 \mathrm{E}+00 \\ \mathrm{C} & 3 & 0.1031 \mathrm{E}+02 & \mathrm{C} & 16 & -.1909 \mathrm{E}+00 \\ \mathrm{E} & 24 & 0.1028 \mathrm{E}+02 & \mathrm{C} & 11 & 0.1686 \mathrm{E}+00 \\ \mathrm{E} & 30 & -.1028 \mathrm{E}+02 & \mathrm{E} & 9 & 0.1026 \mathrm{E}+00 \\ \mathrm{E} & 27 & -.1028 \mathrm{E}+02 & \mathrm{C} & 1 & -.7200 \mathrm{E}-01 \\ \mathrm{E} & 29 & 0.5265 \mathrm{E}+01 & \mathrm{E} & 18 & -.6926 \mathrm{E}-01 \\ \mathrm{C} & 15 & -.5262 \mathrm{E}+01 & \mathrm{TC} & 1 & 0.9914 \mathrm{E}-02 \\ \mathrm{E} & 12 & 0.5245 \mathrm{E}+01 & \mathrm{E} & 26 & 0.5650 \mathrm{E}-02 \\ \mathrm{E} & 28 & 0.5244 \mathrm{E}+01 & \mathrm{E} & 10 & -.3274 \mathrm{E}-02 \\ \mathrm{PC} & 2 & 0.5243 \mathrm{E}+01 & \mathrm{E} & 17 & 0.2416 \mathrm{E}-02 \\ \mathrm{E} & 23 & -.5242 \mathrm{E}+01 & & & \\ \mathrm{C} & 6 & 0.5238 \mathrm{E}+01 & & & \\ \mathrm{E} & 1 & -.5202 \mathrm{E}+01 & & & \\ \mathrm{E} & 4 & -.5196 \mathrm{E}+01 & & & \\ \mathrm{E} & 7 & 0.5194 \mathrm{E}+01 & & & \\ \mathrm{C} & 7 & -.5189 \mathrm{E}+01 & & & \\ \mathrm{C} & 12 & -.5187 \mathrm{E}+01 & & & \\ \mathrm{E} & 6 & 0.5185 \mathrm{E}+01 & & & \\ \mathrm{E} & 16 & -.5180 \mathrm{E}+01 & & & \end{array}$


CRIT LOCUS OUTPUT. CO2 / C4 ESD. WITH ERROR HANDLING

TABLE 4 B. CONTRASTS IIS'IED BY FACTORS 1 OF 1

FACTOR

$\begin{array}{lrl}\mathrm{C} & 14 & 0.1178 \mathrm{E}+02 \\ \mathrm{E} & 22 & 0.1157 \mathrm{E}+02 \\ \mathrm{PC} & 1 & 0.7853 \mathrm{E}+01 \\ \mathrm{E} & 20 & \mathrm{v} \\ \mathrm{E} & 25 & 0.7824 \mathrm{E}+01 \\ \mathrm{E} & 15 & -.7720 \mathrm{E}+01 \\ \mathrm{E} & 8 & -.7773 \mathrm{E}+01 \\ \mathrm{OM} & 1 & -.7760 \mathrm{E}+01 \\ \mathrm{E} & 19 & 0.7744 \mathrm{E}+01 \\ \mathrm{E} & 3 & 0.7734 \mathrm{E}+01 \\ \mathrm{C} & 9 & 0.7727 \mathrm{E}+01 \\ \mathrm{C} & 10 & 0.7712 \mathrm{E}+01 \\ \mathrm{C} & 3 & -.7679 \mathrm{E}+01 \\ \mathrm{E} & 2 & -.7664 \mathrm{E}+01 \\ \mathrm{E} & 27 & 0.7629 \mathrm{E}+01 \\ \mathrm{C} & 4 & -.7627 \mathrm{E}+01 \\ \mathrm{E} & 30 & 0.7617 \mathrm{E}+01 \\ \mathrm{E} & 24 & -.7588 \mathrm{E}+01 \\ \mathrm{C} & 15 & 0.3958 \mathrm{E}+01 \\ \mathrm{E} & 29 & -.3955 \mathrm{E}+01 \\ \mathrm{E} & 28 & -.3951 \mathrm{E}+01 \\ \mathrm{E} & 12 & -.3937 \mathrm{E}+01 \\ \mathrm{C} & 6 & -.3927 \mathrm{E}+01 \\ \mathrm{E} & 4 & 0.3913 \mathrm{E}+01 \\ \mathrm{E} & 1 & 0.3907 \mathrm{E}+01 \\ \mathrm{E} & 23 & 0.3907 \mathrm{E}+01 \\ \mathrm{E} & 16 & 0.3895 \mathrm{E}+01 \\ \mathrm{C} & 12 & 0.3892 \mathrm{E}+01 \\ \mathrm{E} & 6 & -.3875 \mathrm{E}+01 \\ \mathrm{E} & 7 & -.3871 \mathrm{E}+01 \\ \mathrm{E} & 11 & -.3854 \mathrm{E}+01 \\ \mathrm{C} & 2 & -.3852 \mathrm{E}+01\end{array}$

\section{FACTOR}

C 7

OM 2

E 13

PC 2

C 5

E 31

E 21

C 8

E 5

K 12

E 14

C 17

C 13

TC 2

C 11

TC 1

E 9

C 1

F. 18

C 16

E 10

E 26

E 17

\section{RMS BIAS}

$0.3797 E+01$

$0.3790 E+01$

$0.3783 E+01$

$-.3780 E+01$

$-.3754 \mathrm{E}+01$

$-.3752 \mathrm{E}+01$

$-.3751 E+01$

$0.3746 \mathrm{E}+01$

$-.3738 \mathrm{E}+01$

$0.3712 \mathrm{E}+01$

$0.3711 \mathrm{E}+01$

$-.3678 \mathrm{E}+01$

$-.3624 \mathrm{E}+01$

$-.4912 \mathrm{E}+00$

$-.2742 \mathrm{E}+00$

$0.2334 \mathrm{E}+00$

$-.1209 E+00$

$0.1060 E+00$

$0.8332 E-01$

$0.7442 \mathrm{E}-01$

$-.3397 E-01$

$-.3000 \mathrm{E}-01$

$-.6309 E-02$ 
CRIT LOCUS OUTPUT. CO2 / C4 ESD. WITH ERRCR HANDLING

TABLE 4 C. CONTRASTS LISTED BY FACTORS 1 OF 1

\begin{tabular}{|c|c|c|c|c|}
\hline \multicolumn{2}{|c|}{ FACTOR } & CPU TIME & FACTOR & CPU TIME \\
\hline $\mathrm{E}$ & 14 & $-.2091 E+02$ & 17 & $0.4531 \mathrm{E}+01$ \\
\hline$E$ & 22 & $-.1734 \mathrm{E} \div \mathrm{C2}$ & 29 & $0.4094 \mathrm{E}+01$ \\
\hline $\mathrm{E}$ & 5 & $0.1678 \mathrm{E}+02$ & 12 & $0.3969 E+01$ \\
\hline $\mathbf{E}$ & 9 & $-.1466 \mathrm{E}+02$ & 16 & $-.3844 \mathrm{E}+01$ \\
\hline $\mathrm{PC}$ & 1 & $-.1441 E+02$ & 18 & $-.3781 \mathrm{E}+01$ \\
\hline $\mathrm{E}$ & 6 & $0.1247 \mathrm{E}+02$ & K 12 & $-.3156 E+01$ \\
\hline $\mathrm{E}$ & 24 & $0.1222 \mathrm{E}+02$ & 8 & $0.3156 \mathrm{E}+01$ \\
\hline $\mathrm{E}$ & 13 & $-.1134 \mathrm{E}+02$ & 10 & $0.2969 E+01$ \\
\hline C & 9 & $-.1122 \mathrm{E}+02$ & 11 & $0.2969 E+01$ \\
\hline C & 14 & $-.1122 \mathrm{E}+02$ & 26 & $0.2594 \mathrm{E}+01$ \\
\hline$E$ & 28 & $0.1091 E+02$ & TC 1 & $0.2531 E+01$ \\
\hline$E$ & 3 & $-.1078 E+02$ & 30 & $-.2344 \mathrm{E}+01$ \\
\hline $\mathrm{E}$ & 19 & $-.1053 E+02$ & $\mathrm{C}$ & $-.2281 E+01$ \\
\hline $\mathrm{E}$ & 15 & $0.1047 \mathrm{E}+02$ & 1 & $0.2219 E+01$ \\
\hline$E$ & 23 & $-.1003 E+02$ & OM & $-.1969 E+01$ \\
\hline $\mathrm{E}$ & 31 & $0.9844 \mathrm{E}+01$ & 10 & $-.1281 E+01$ \\
\hline C & 13 & $0.9031 E+01$ & 20 & $-.1094 \mathrm{E}+01$ \\
\hline $\mathrm{E}$ & 27 & $-.8594 \mathrm{E}+01$ & C & $0.1031 \mathrm{E}+01$ \\
\hline C & 4 & $0.8219 E+01$ & $E$ & $0.1031 \mathrm{E}+01$ \\
\hline$E$ & 25 & $-.7959 E+01$ & $C$ & $0.8438 \mathrm{E}+00$ \\
\hline C & 8 & $0.7906 \mathrm{E}+\mathrm{U} 1$ & 17 & $-.8438 E+00$ \\
\hline C & 15 & $0.7719 E+01$ & 21 & $0.5313 \mathrm{E}+00$ \\
\hline $\mathrm{TC}$ & 2 & $-.7594 E+01$ & C & $0.2813 \mathrm{E}+00$ \\
\hline $\mathrm{C}$ & 2 & $0.6969 E+01$ & & \\
\hline C & 16 & $0.6844 \mathrm{E}+01$ & & \\
\hline$O M$ & 1 & $0.6469 \mathrm{E}+01$ & & \\
\hline C & 3 & $0.6406 \mathrm{E}+01$ & & \\
\hline $\mathrm{E}$ & 12 & $0.6031 E+01$ & & \\
\hline PC & 2 & $-.5719 E+01$ & & \\
\hline$E$ & 2 & $0.5031 E+01$ & & \\
\hline$E$ & 4 & $-.4906 E+01$ & & \\
\hline C & 11 & $-.4781 E+01$ & & \\
\hline
\end{tabular}


CRITICAL LOCUS OUTPUT. CO2 / C6 BASE GENERATED BY ESD EOS DRES=4 0.005 
CRITICAI LOCUS OUTPUT. CO2 / C6 BASE GENERATED BY ESD EOS DRES $=4 \quad 0.005$

TABLE 3. RESPONSES

TEST

1

2

3

4

5

6

7

8

9

10

11

12

13

14

15

16

17

18

19

20

21

22

23

24

25

26

27

28

29

30

31

32 RT SQ ER

$0.3611 \mathrm{E}-03$

$0.1198 \mathrm{E}+01$

$0.9419 E+02$

$0.9436 \mathrm{E}+02$

$0.1061 \mathrm{E}+01$

$0.9432 E+02$

$0.9424 \mathrm{E}+02$

$0.5957 \mathrm{E}+00$

$0.9419 \mathrm{E}+02$

$0.9419 \mathrm{E}+02$

$0.9441 E+02$

$0.9427 E+02$

$0.9449 \mathrm{E}+02$

$0.1538 \mathrm{E}+00$

$0.9444 \mathrm{E}+02$

$0.9424 \mathrm{E}+02$

$0.9419 E+02$

$0.9419 \mathrm{E}+02$

$0.4734 \mathrm{E}+00$

$0.9419 E+02$

$0.9419 E+02$

$0.9433 E+02$

$0.9419 E+02$

$0.5823 E+00$

$0.9421 E+02$

$0.1676 \mathrm{E}+01$

$0.9421 \mathrm{E}+02$

$0.9419 E+02$

$0.5179 E+00$

$0.5835 \mathrm{E}+00$

$0.9419 \mathrm{E}+02$

$0.9436 \mathrm{E}+02$
RMS BIAS

$-.2177 \mathrm{E}-03$

$-.1205 \mathrm{E}+01$

$-.6660 \mathrm{E}+02$

$-.6640 \mathrm{E}+02$

$0.1072 \mathrm{E}+01$

$-.6644 \mathrm{E}+02$

$-.6666 \mathrm{E}+02$

$-.5985 \mathrm{E}+00$

$-.6660 \mathrm{E}+02$

$-.6660 \mathrm{E}+02$

$-.6637 \mathrm{E}+02$

$-.6651 \mathrm{E}+02$

$-.6627 \mathrm{E}+02$

$-.6759 \mathrm{E}-01$

$-.663 .3 \mathrm{E}+02$

$-.6665 \mathrm{E}+02$

$-.6660 \mathrm{E}+02$

$-.6660 \mathrm{E}+02$

$0.2877 \mathrm{E}+00$

$-.6660 \mathrm{E}+02$

$-.6660 \mathrm{E}+02$

$-.6643 \mathrm{E}+02$

$-.6660 \mathrm{E}+02$

$0.5838 \mathrm{E}+00$

$-.6663 \mathrm{E}+02$

$0.1679 \mathrm{E}+01$

$-.6657 \mathrm{E}+02$

$-.6660 \mathrm{E}+02$

$-.1358 \mathrm{E}+00$

$0.3579 E+00$

$-.6660 \mathrm{E}+02$

$-.6642 \mathrm{E}+02$
1 OF 2

CPU TIME

$0.5060 \mathrm{E}+03$

$0.5070 \mathrm{E}+03$

$0.5340 \mathrm{E}+03$

$0.5750 \mathrm{E}+03$

$0.5390 \mathrm{E}+03$

$0.5860 \mathrm{E}+03$

$0.6300 \mathrm{E}+03$

$0.5540 \mathrm{E}+03$

$0.6000 \mathrm{E}+03$

$0.7150 \mathrm{E}+03$

$0.6730 \mathrm{E}+03$

$0.5200 E+03$

$0.5700 \mathrm{E}+03$

$0.6110 \mathrm{E}+03$

$0.5600 \mathrm{E}+03$

$0.6830 \mathrm{E}+03$

$0.5870 \mathrm{E}+03$

$0.6200 \mathrm{E}+03$

$0.5490 \mathrm{E}+03$

$0.5200 \mathrm{E}+03$

$0.7130 \mathrm{E}+03$

$0.6290 \mathrm{E}+03$

$0.7400 \mathrm{E}+03$

$0.5230 \mathrm{D}+03$

$0.5530 \mathrm{E}+03$

$0.5330 \mathrm{E}+03$

$0.5680 \mathrm{E}+03$

$0.1477 \mathrm{E}+04$

$0.5270 \mathrm{E}+03$

$0.5350 \mathrm{E}+03$

$0.5400 \mathrm{E}+03$

$0.5980 \mathrm{E}+03$ 
CRITICAL LOCUS OUTPUT. CO2 / C6 BASE GENERATED BY ESD EOS DRES=4 0.005

TABLE 3. (CONTINUED)

2 OF 2

$\begin{array}{lccc}\text { TEST } & \text { RT SQ ER } & \text { RMS BIAS } & \text { CPU TIME } \\ 33 & 0.9421 \mathrm{E}+02 & -.6657 \mathrm{E}+02 & 0.6610 \mathrm{E}+03 \\ 34 & 0.9425 \mathrm{E}+02 & -.6667 \mathrm{E}+02 & 0.6290 \mathrm{E}+03 \\ 35 & 0.9420 \mathrm{E}+02 & -.6662 \mathrm{E}+02 & 0.5860 \mathrm{E}+03 \\ 36 & 0.9425 \mathrm{E}+02 & -.6654 \mathrm{E}+02 & 0.6590 \mathrm{E}+03 \\ 37 & 0.9422 \mathrm{E}+02 & -.6664 \mathrm{E}+02 & 0.4470 \mathrm{E}+03 \\ 38 & 0.7842 \mathrm{E}+00 & 0.7885 \mathrm{E}+00 & 0.5780 \mathrm{E}+03 \\ 39 & 0.9425 \mathrm{E}+02 & -.6667 \mathrm{E}+02 & 0.5580 \mathrm{E}+03 \\ 40 & 0.7524 \mathrm{E}+00 & 0.7543 \mathrm{E}+00 & 0.5030 \mathrm{E}+03 \\ 41 & 0.9419 \mathrm{E}+02 & -.6660 \mathrm{E}+02 & 0.5590 \mathrm{E}+03 \\ 42 & 0.9452 \mathrm{E}+02 & -.6623 \mathrm{E}+02 & 0.7270 \mathrm{E}+03 \\ 43 & 0.9424 \mathrm{E}+02 & -.6667 \mathrm{E}+02 & 0.7540 \mathrm{E}+03 \\ 44 & 0.9436 \mathrm{E}+02 & -.6682 \mathrm{E}+02 & 0.5840 \mathrm{E}+03 \\ 45 & 0.6679 \mathrm{E}+00 & 0.5595 \mathrm{E}+00 & 0.5560 \mathrm{E}+03 \\ 46 & 0.9419 \mathrm{E}+02 & -.6660 \mathrm{E}+02 & 0.6090 \mathrm{E}+03 \\ 47 & 0.5369 \mathrm{E}+00 & 0.5058 \mathrm{E}+00 & 0.6170 \mathrm{E}+03 \\ 48 & 0.3152 \mathrm{E}+00 & -.9858 \mathrm{E}-01 & 0.5740 \mathrm{E}+03 \\ 49 & 0.9444 \mathrm{E}+02 & -.6635 \mathrm{E}+02 & 0.7280 \mathrm{E}+03 \\ 50 & 0.9419 \mathrm{E}+02 & -.6660 \mathrm{E}+02 & 0.8800 \mathrm{E}+03 \\ 51 & 0.9435 \mathrm{E}+02 & -.6639 \mathrm{E}+02 & 0.6170 \mathrm{E}+03 \\ 52 & 0.9426 \mathrm{E}+02 & -.6665 \mathrm{E}+02 & 0.5510 \mathrm{E}+03 \\ 53 & 0.9419 \mathrm{E}+02 & -.6660 \mathrm{E}+02 & 0.6540 \mathrm{E}+03 \\ 54 & 0.9424 \mathrm{E}+02 & -.6666 \mathrm{E}+02 & 0.5640 \mathrm{E}+03 \\ 55 & 0.9419 \mathrm{E}+02 & -.6660 \mathrm{E}+02 & 0.7860 \mathrm{E}+03 \\ 56 & 0.9419 \mathrm{E}+02 & -.6660 \mathrm{E}+02 & 0.7180 \mathrm{E}+03 \\ 57 & 0.1286 \mathrm{E}+01 & 0.1293 \mathrm{E}+01 & 0.6210 \mathrm{E}+03 \\ 58 & 0.9428 \mathrm{E}+02 & -.6650 \mathrm{E}+02 & 0.6410 \mathrm{E}+03 \\ 59 & 0.9421 \mathrm{E}+02 & -.6657 \mathrm{E}+02 & 0.6280 \mathrm{E}+03 \\ 60 & 0.9421 \mathrm{E}+02 & -.6663 \mathrm{E}+02 & 0.5270 \mathrm{E}+03 \\ 61 & 0.9421 \mathrm{E}+02 & -.6663 \mathrm{E}+02 & 0.6260 \mathrm{E}+03 \\ 62 & 0.4841 \mathrm{E}+00 & 0.4855 \mathrm{E}+00 & 0.4940 \mathrm{E}+03 \\ 63 & 0.9419 \mathrm{E}+02 & -.6660 \mathrm{E}+02 & 0.7760 \mathrm{E}+03 \\ 64 & 0.9419 \mathrm{E}+02 & -.6660 \mathrm{E}+02 & 0.5670 \mathrm{E}+03 \\ & & & \end{array}$


CRITICAL LOCUS OUTPUT. CO2 / C6 BASE GENERATED BY ESD EOS DRES=4 0.005

TABLE 4 A. CONTRASTS LISTED BY FACTORS 1 OF 1

FACTOR

$\begin{array}{lr}\text { OM } & 1 \\ C & 3 \\ E & 2 \\ E & 27 \\ \text { PC } & 2 \\ E & 14 \\ E & 16 \\ C & 15 \\ E & 20 \\ E & 7 \\ E & 11 \\ C & 2 \\ E & 13 \\ E & 31 \\ E & 18 \\ E & 10 \\ E & 25 \\ T C & 1 \\ C & 17 \\ T C & 2 \\ E & 12 \\ C & 9 \\ C & 8 \\ E & 9 \\ E & 15 \\ C & 5 \\ C & 6 \\ C & 11 \\ C & 1 \\ E & 29 \\ C & 7 \\ C & 14\end{array}$

$$
\text { RT SQ ER }
$$

$0.2627 \mathrm{E}+02$

$-.2055 \mathrm{E}+02$

$-.1472 \mathrm{E}+02$

$-.1468 \mathrm{E}+02$

$0.1466 \mathrm{E}+02$

$-.1465 \mathrm{E}+02$

$0.1465 \mathrm{E}+02$

$0.1464 \mathrm{E}+02$

$-.1462 \mathrm{E}+02$

$0.1461 \mathrm{E}+02$

$0.1456 \mathrm{E}+02$

$0.1456 \mathrm{E}+02$

$-.1456 \mathrm{E}+02$

$0.1455 \mathrm{E}+02$

$0.1451 \mathrm{E}+02$

$-.8884 \mathrm{E}+01$

$-.8879 E+01$

$0.8866 \mathrm{E}+01$

$0.8861 E+01$

$-.8827 E+01$

$-.8819 E+01$

$0.8793 E+01$

$0.8792 E+01$

$-.8790 E+01$

$0.8789 E+01$

$0.8783 E+01$

$0.8763 \mathrm{E}+01$

$0.8752 \mathrm{E}+01$

$-.8747 \mathrm{E}+01$

$-.872 .4 E+01$

$-.8706 E+01$

$-.8686 E+01$

\section{FACTOR}

E 30

E 19

E 23

E 1

E 28

E 22

E 24

K 12

PC 1

E 4

OM 2

C 4

E 6

E 3

E 8

E 26

E 17

C 10

E 21

E 5

C 16

C 12

C 13
RT SQ ER

$0.2966 E+01$

$-.2960 \mathrm{E}+01$

$-.2956 \mathrm{E}+01$.

$-.2954 \mathrm{E}+01$

$-.2950 E+01$

$-.2949 E+01$

$-.2936 E+01$

$0.2935 \mathrm{E}+01$

-.2932E+01

$0.2929 E+01$

$0.2923 E+01$

$-.2902 E+01$

$-.2887 E+01$

$0.2873 E+01$

$0.2872 \mathrm{E}+01$

$-.2871 E+01$

$0.2871 E+01$

$-.2869 E+01$

$-.2865 E+01$

$0.2864 E+01$

$0.2827 \mathrm{E}+01$

$-.2824 E+01$

$-.2811 \mathrm{E}+01$ 
CRITICAL LOCUS OUTPUT. CO2 / C6 BASE GENERATED BY ESD EOS DRES $=40.005$

TABLE 4 B. CONTRASTS LISTED BY FACTORS 1 OF 1

FACTOR

$\begin{array}{lr}\text { OM } & 1 \\ \text { C } & 3 \\ \text { E } & 11 \\ \text { C } & 15 \\ \text { E } & 7 \\ \text { C } & 2 \\ \text { E } & 20 \\ \text { E } & 18 \\ \text { E } & 31 \\ \text { E } & 13\end{array}$

EC 2

E 16

E 14

E 27

C 8

C 11

E 15

C 14

C 9

E 29

E 25

C 7
E

E 12

TC 2

C 1

C 6

E 9

C 5

E 10

C 17

TC 1
RMS BIAS

$-.1886 \mathrm{E}+02$

$0.1463 \mathrm{E}+02$

-.1060E+U2

$-.1060 \mathrm{E}+02$

$-.1054 \mathrm{E}+02$

$-.1053 E+02$

$0.1053 E+02$

$-.1049 \mathrm{E}+02$

$-.1046 \mathrm{E}+02$

$0.1046 \mathrm{E}+02$

$0.1045 E+02$

$-.1042 \mathrm{E}+02$

$-.1038 \mathrm{E}+02$

$0.1037 E+02$

$0.1031 E+02$

$-.6375 E+01$

$-.6366 E+01$

$-.6340 \mathrm{E}+01$

$0.6327 E+01$

$-.6324 \mathrm{E}+01$

$0.6311 \mathrm{E}+01$

$0.6311 \mathrm{E}+01$

$0.6244 \mathrm{E}+01$

$0.6243 \mathrm{E}+01$

$0.6211 \mathrm{E}+01$

$0 . F 209 E+01$

$-, 206 E+01$

$0.6197 E+01$

$-.6167 \mathrm{E}+01$

$0.6114 E+01$

$-.6062 \mathrm{E}+01$

$-.6054 \mathrm{E}+01$
FACTOR

RMS BIAS

C 16

E 21

C 13

PC 1

C 4

C 12

E 22

E 8

E 19

E 24

C 10

E 23

OM 2

E 5

E 1

E 30

E 6

E 3

E 17

E 26

K 12

E 4

E 28
$-.2335 \mathrm{E}+01$

$0.2229 E+01$

$0.2190 \mathrm{E}+01$

$0.2188 \mathrm{E}+01$

$0.2187 \mathrm{E}+01$

$0.2175 \mathrm{E}+01$

$0.2159 \mathrm{E}+01$

$-.2138 \mathrm{E}+01$

$0.2111 \mathrm{E}+01$

$0.2108 \mathrm{E}+01$

$0.2097 \mathrm{E}+01$

$0.2096 \mathrm{E}+01$

$-.2089 E+01$

$-.2085 E+01$

$0.2084 \mathrm{E}+01$

$-.2068 \mathrm{E}+01$

$0.2066 \mathrm{E}+01$

$-.2057 \mathrm{E}+01$

$-.2049 \mathrm{E}+01$

$0.2039 E+01$

-.2014E+01

$-.1952 \mathrm{E}+01$

$0.1937 E+01$ 
CRITICAL LOCUS OUTPUT. CO2 / C6 BASE GENERATED BY ESD EOS DRES=4 0.005

TABLE 4 C. CONTRASTS LISTED BY FACTORS

1 OF 1

\section{FACTOR}

E 28

C 15

K 12

C 12

PC 1

OM 1

E 19

C 5

E 2

E 26

E 20

C 10

E 7

E 25

E 29

E 10

C 3

C 17

E 14

C 9

E 5

C 16

OM 2

E 13

E 18

C 2

E 21

E 30

E 3

C 14

E 9

PC 2
CPU TIME

$0.6163 E+02$

$-.5700 \mathrm{E}+02$

$0.5663 \mathrm{E}+02$

$-.5656 \mathrm{E}+02$

$0.5588 \mathrm{E}+02$

$0.5156 \mathrm{E}+02$

$0.5088 E+02$

$0.5081 E+02$

$-.4544 E+02$

$-.4538 \mathrm{E}+02$

$-.4456 \mathrm{E}+02$

$-.4450 \mathrm{E}+02$

$0.4356 E+02$

$-.4313 E+02$

$-.4013 E+02$

$-.3906 E+02$

$-.3825 E+02$

$-.3825 \mathrm{E}+02$

$-.3781 \mathrm{E}+02$

$0.3669 E+02$

$-.3644 \mathrm{E}+02$

$-.3556 \mathrm{E}+02$

$-.3344 E+02$

$-.3213 E+02$

$0.3119 E+02$

$0.2950 \mathrm{E}+02$

$-.2913 E+02$

$0.2838 E+02$

$0.2831 E+02$

$0.2806 \mathrm{E}+02$

$-.2756 \mathrm{E}+02$

$-.2594 E+02$
FACTOR

CPU TIME

$\begin{array}{lr}\text { E } & 17 \\ \text { E } & 22 \\ C & 8 \\ \text { E } & 24 \\ \text { C } & 4 \\ \text { TC } & 2 \\ \text { E } & 8 \\ \text { E } & 31 \\ C & 13 \\ C & 7 \\ \text { E } & 6 \\ C & 1 \\ C & 6 \\ E & 1 \\ C & 11 \\ \text { E } & 12 \\ \text { E } & 27 \\ E & 23 \\ \text { E } & 4 \\ \text { E } & 15 \\ \text { E } & 16 \\ \text { TC } & 1 \\ \text { E } & 11\end{array}$

$-.2513 E+02$

$-.2450 \mathrm{E}+02$

$0.2238 \mathrm{E}+02$

$0.2213 \mathrm{E}+02$

$0.2163 \mathrm{E}+02$

$-.2125 \mathrm{E}+02$

$0.1925 \mathrm{E}+02$

$0.1850 \mathrm{E}+02$

$0.1688 \mathrm{E}+02$

$-.1650 E+02$

$0.1356 \mathrm{E}+02$

$0.1338 \mathrm{E}+02$

$0.1263 \mathrm{E}+02$

$-.1231 \mathrm{E}+02$

$-.8875 E+01$

$-.7063 \mathrm{E}+01$

$0.6813 \mathrm{E}+01$

$0.6438 \mathrm{E}+01$

$-.3125 \mathrm{E}+01$

$-.2250 E+01$

$0.1313 E+01$

$0.9375 \mathrm{E}+00$

$0.1250 \mathrm{E}+00$ 


\section{APPENDIX F Fortran Source Code}

This appendix contains listings of the two main programs used in the test calculations as well as the auxiliary subroutines which they call. The code is ANSI Fortran 77 , with rare invocations of vax extensions. 
$C$

C ADAPTATION OF JIN'S PROGRAM TO TEST WORK REQUIRED BY

C DOE CONTRACT. J.R. LOEHE AUG / SEPT 90

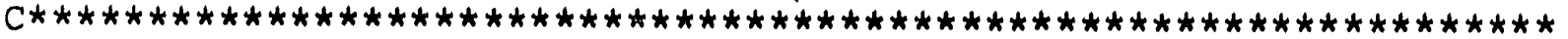

C

C

$\mathrm{C}$

MIAN PROGRAM,

SUBROUTINES: VOLUME, CONS, MIXPRP, PRES

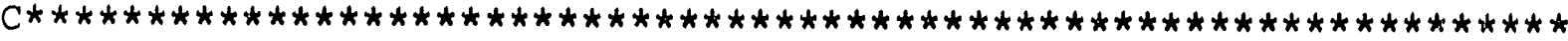

$\mathrm{C}$

$\mathrm{C}$

$\mathrm{C}$

$\mathrm{C}$

$\mathrm{C}$

C

$\mathrm{C}$

C

$\mathrm{C}$

C

C

C

$\mathrm{C}$

C

C

C

$\mathrm{C}$

C

$\mathrm{C}$

$\mathrm{C}$

PROGRAM MIXTUR (INPUT, OUTPUT)

PROGRAM VOLUME

THIS PROGRAM CALCULATES THE LIQUID VOLUMES

USING EITHER ESD OR ALS EQUATION

OF STATE FOR MULTICOMPONENT MIXTURES.

VARIABLES

NCOMP $=$ NUMBER OF COMPONENTS

NDATA $=$ NUMBER OT DATA SETS

IPRINT CONTROLS AUXILLARY PRINT STATEMENTS

KEP = BINARY INTERACTION PARAMETER

$\mathrm{T}=$ TEMPERATURE (DEGREES $\mathrm{K}$ )

$P=$ PRESSURE (BARS)

$X=$ MOLE FRACTION IN LIQUID PHASE

$\mathrm{VL}=$ LIQUID MOLAR VOLUME (LIT/MOL)

COMMON / PURE/TC (10), PC (10), OM (10)

COMMON/CONST/C74, PI, SQR2, IPRINT, RK, NCOMP

COMMON / XPROP / KEP

COMMON/MIX/AM, BM, CHEM1 (10)

COMMON/IJPROP/A $(10,10), B(10)$

COMMON/SED1/C (10), $Q(10), V S(10), E K(10)$

COMMON / SED2 / ASED, DASED (10), BSED, CSED, DCSED (10), DSED

COMMON/ALS1/BP1 (10), BP2 (10),BP3 (10), AP (10), ALPHA (10)

COMMON/EOS/NEOS, CPARA (20)

COMMON/RETDAT/ITIME

$\operatorname{REAL} \operatorname{KEP}(10,10), \operatorname{RFACT}, \operatorname{RESP}(3,128), \operatorname{TPAT}(128,127)$

REAL X(10), TITLE (20), FACT (127), FCVAL $(128,127)$

REAL TKK(100), $\operatorname{PKK}(100), \operatorname{XKK}(10,100), \operatorname{VKK}(100), \operatorname{BIAS}$

INTEGER NFACT, NBIN, NTST, ITIME

CHARACTER $\star 20$, TITIN, TITOUT, TEOS

CHARACTER FACNM $(127) * 5, \operatorname{RSPNM}(3) * 8$

C

EXTERNAL DS GETDATA

TYPE *, 'INPUT' FILE NAME'

$\operatorname{READ}(5,2000)$ TITIN

TYPE *, 'OU'TPUT FILENAME'

$\operatorname{READ}(5,2000)$ TITOUT

2000 FORMAT (A20) 


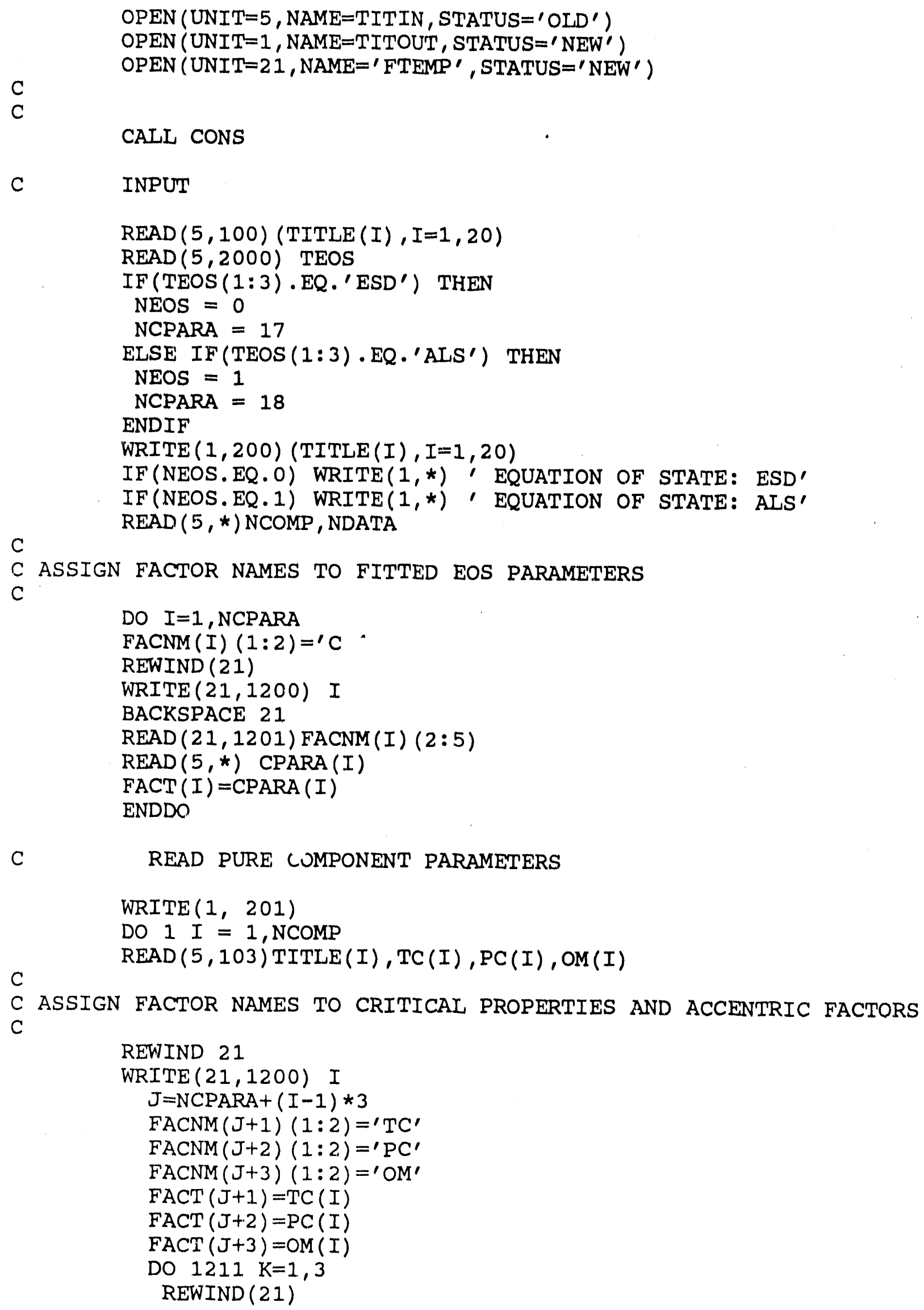


$B(I)=0.0778 * R K * T C(I) / P C(I)$

WRITE (1, 203) TITLE (I), TC (I) , PC (I), OM(I) , B (I)

IF (NEOS.EQ.0) THEN

$C(I)=\operatorname{CPARA}(1)+\operatorname{CPARA}(2) * O M(I)+\operatorname{CPARA}(3) \star O M(I) \star O M(I)$

$C 1=C(I)-1$.

$A 1=(\operatorname{CPARA}(4)+\operatorname{CPARA}(5) * \mathrm{C} 1+\operatorname{CPARA}(6) * \mathrm{C} 1 * \mathrm{C} 1) /$

(CPARA $(7)+$ CPARA $(8) * C 1+\operatorname{CPARA}(9) * C 1 * C 1)$

$\operatorname{EK}(I)=T C(I) * A 1$

$A 2=(\operatorname{CPARA}(10)+\operatorname{CPARA}(11) * \mathrm{C} 1+\mathrm{CPARA}(12) * \mathrm{C} 1 * \mathrm{C} 1) /$

1

(CPARA $(13)+$ CPARA $(14) * C 1+\operatorname{CPARA}(15) * C 1 * \mathrm{C} 1)$

$\mathrm{VS}(\mathrm{I})=\mathrm{RK} * \mathrm{TC}(\mathrm{I}) * \mathrm{~A} 2 / \mathrm{PC}(\mathrm{I})$

$Q(I)=1 .+C P A R A(17) *(C(I)-1$.

$\operatorname{WRITE}(1, *) \operatorname{VS}(I), C(I), \operatorname{EK}(I), Q(I),{ }^{\prime} \quad$ VS, C, EK, $Q^{\prime}$

ELSE

$\mathrm{AA}=\mathrm{RK} * \mathrm{TC}(I) / \mathrm{PC}(I)$

$O M M=O M(I)$

BPI (I) $=A A *(C P A R A(1)+C P A R A(2) * O M M+C P A R A(3) * O M M * O M M)$

BP2 $(I)=A A *(C P A R A(4)+C P A R A(5) * O M M+C P A R A(6) * O M M * O M M+C P A R A(7) * O M M * * 3)$

BP3 $(I)=A A *(C P A R A(8)+C P A R A(9) * O M M+C P A R A(10) * O M M * O M M$

$1+\mathrm{CPARA}(11) \star O M M * * 3)$

$A P P=C P A R A(12)+C P A R A(13) * O M M+C P A R A(14) * O M M * O M M+C P A R A(15) * O M M * * 3$

$\mathrm{AP}(I)=\mathrm{RK} * \mathrm{TC}(I) * \mathrm{AA} * \mathrm{APP}$

$\operatorname{ALPHA}(I)=C P A R A(16)+C P A R A(17) * O M M+C P A R A(18) * O M M * O M M$

1 $\operatorname{WRITE}(1, *) \operatorname{BP1}(I), \mathrm{BP2}(I), \mathrm{BP} 3(I), \mathrm{AP}(I), \mathrm{ALPHA}(I), ' \quad B 1, \mathrm{~B} 2, \mathrm{~B} 3, \mathrm{~A}$,

ENDIF

CONTINUE

C

READ BINARY INTERACTION PARAMETERS

NCOMP $=$ NCOMP -1

DO $2 \mathrm{~J}=1, \mathrm{NCOMP} 1$

$\mathrm{J} 1=\mathrm{J}+1$

$\operatorname{READ}(5,101)(\operatorname{KEP}(J, I), I=J 1, \operatorname{NCOMP})$

$\operatorname{KEP}(J, J)=0.0$

DO $2 \mathrm{I}=\mathrm{J} 1, \mathrm{NCOMP}$

$\operatorname{KEP}(I, I)=0.0$

$\operatorname{KEP}(I, J)=\operatorname{KEP}(J, I)$

$\operatorname{WRITE}(1,505) I, J, \operatorname{KEP}(I, J)$

505 FORMAT (2X,'KIJ ', 2I5, F10.5//)

2 CONIINUE

C READ DATA POINT INFORMATION

DO $35 K=1$, NDATA

$\operatorname{READ}\left(5,{ }^{*}\right) \operatorname{TKK}(K), \operatorname{PKK}(K), \operatorname{VKK}(K),(\operatorname{XKK}(J, K), J=1, \operatorname{NCOMP} 1)$

C

C CALCULATE TEST PATTERN FROM NCOMP, NFACT FOR THE

C EOS AT HAND

C

C 
C NUMBER OF BINARY INTERACTION COEFFICIENTS

$\mathrm{C}$

C

$$
\text { NBIN }=(\text { NCOMP } * \text { NCOMP } 1) / 2
$$

C ASSIGN FACTOR NAMES TO THE BINARY

C

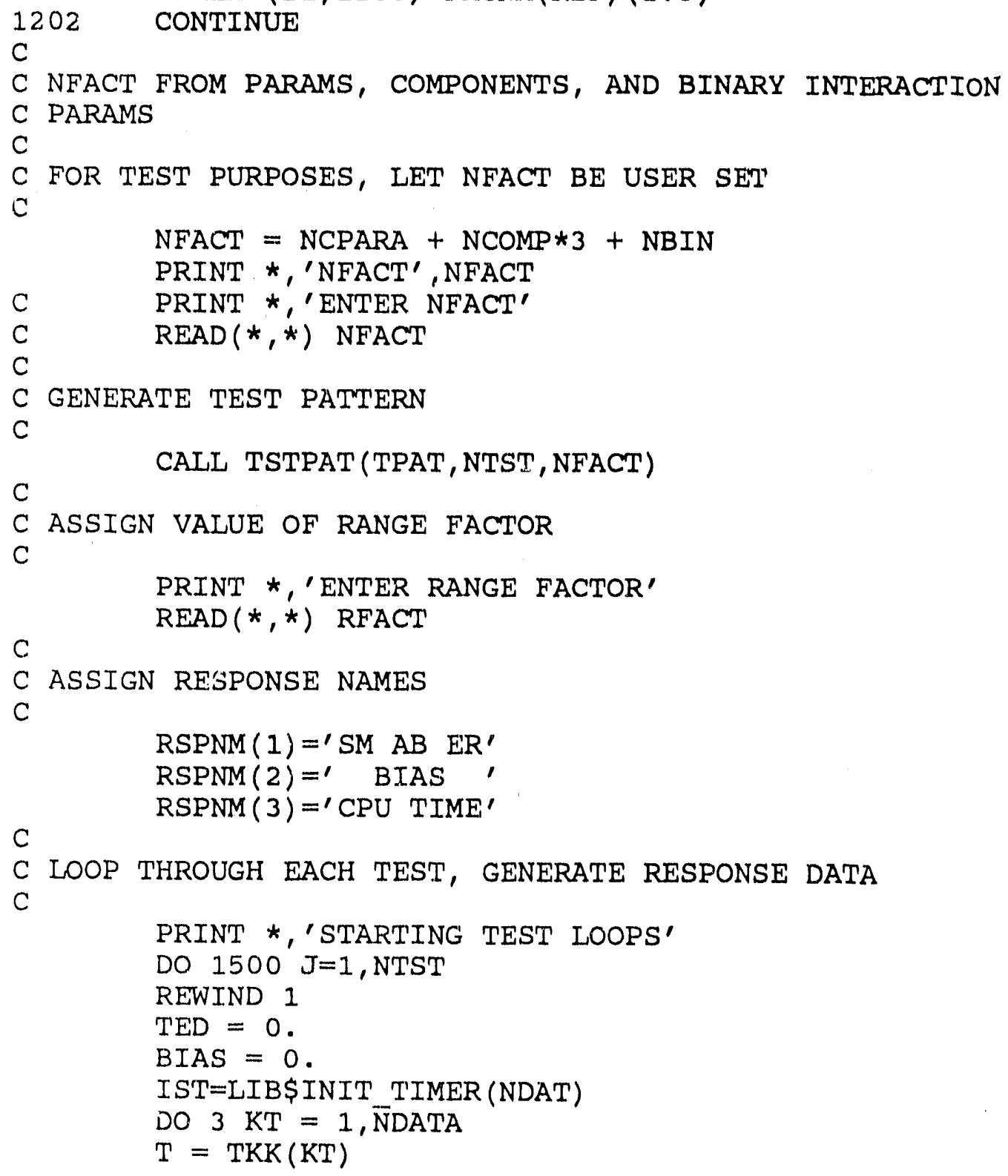

1202 CONTINUE 


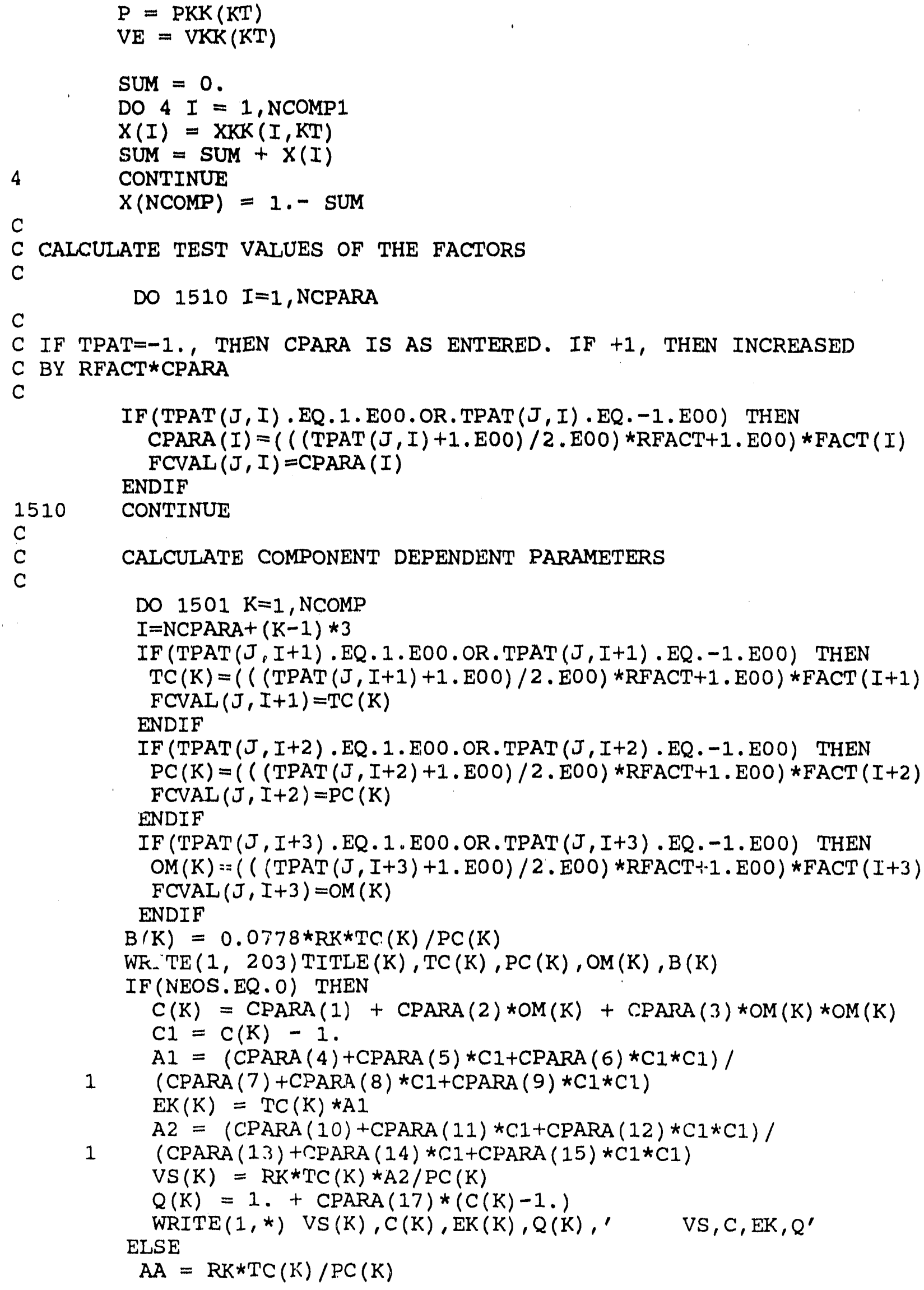




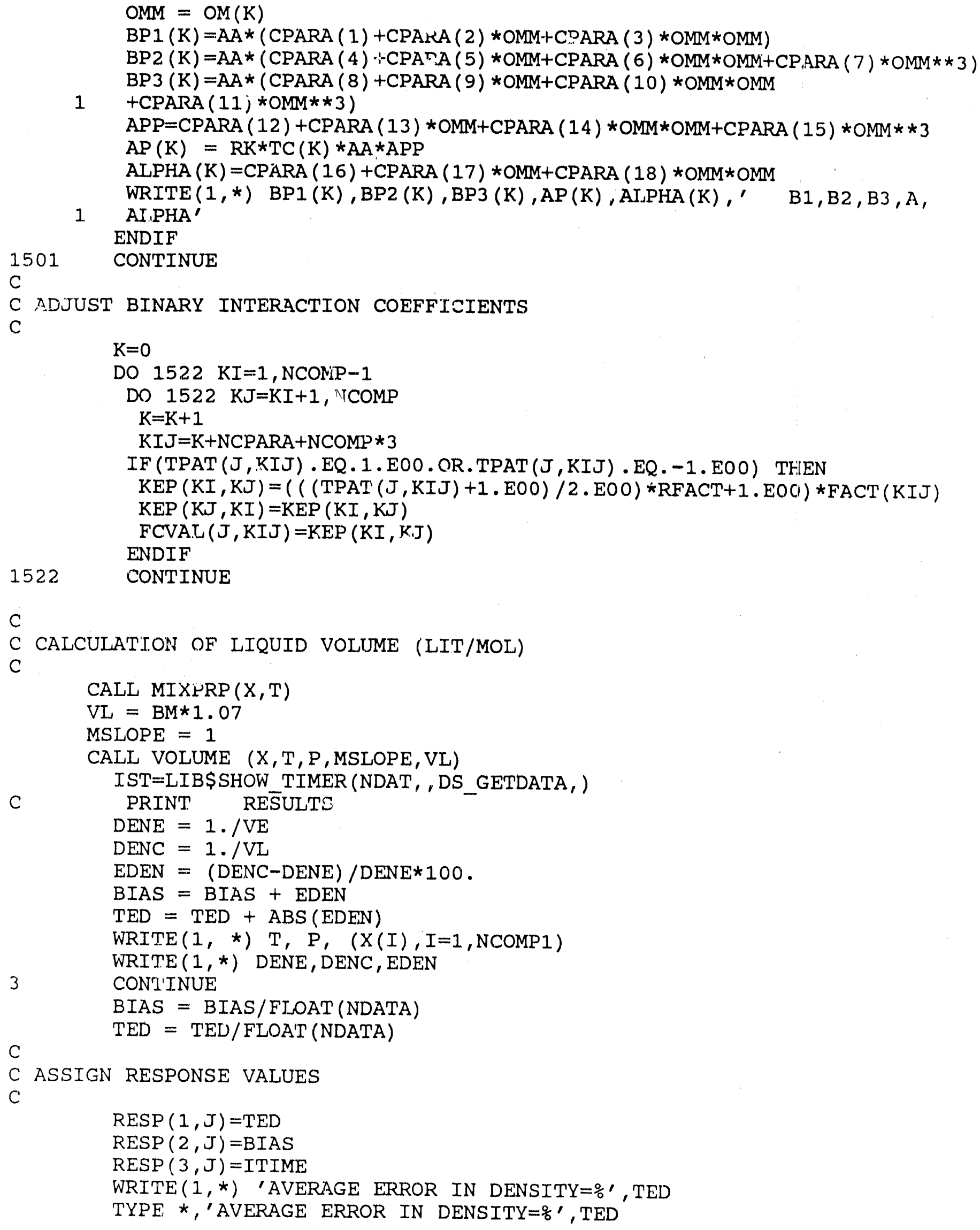




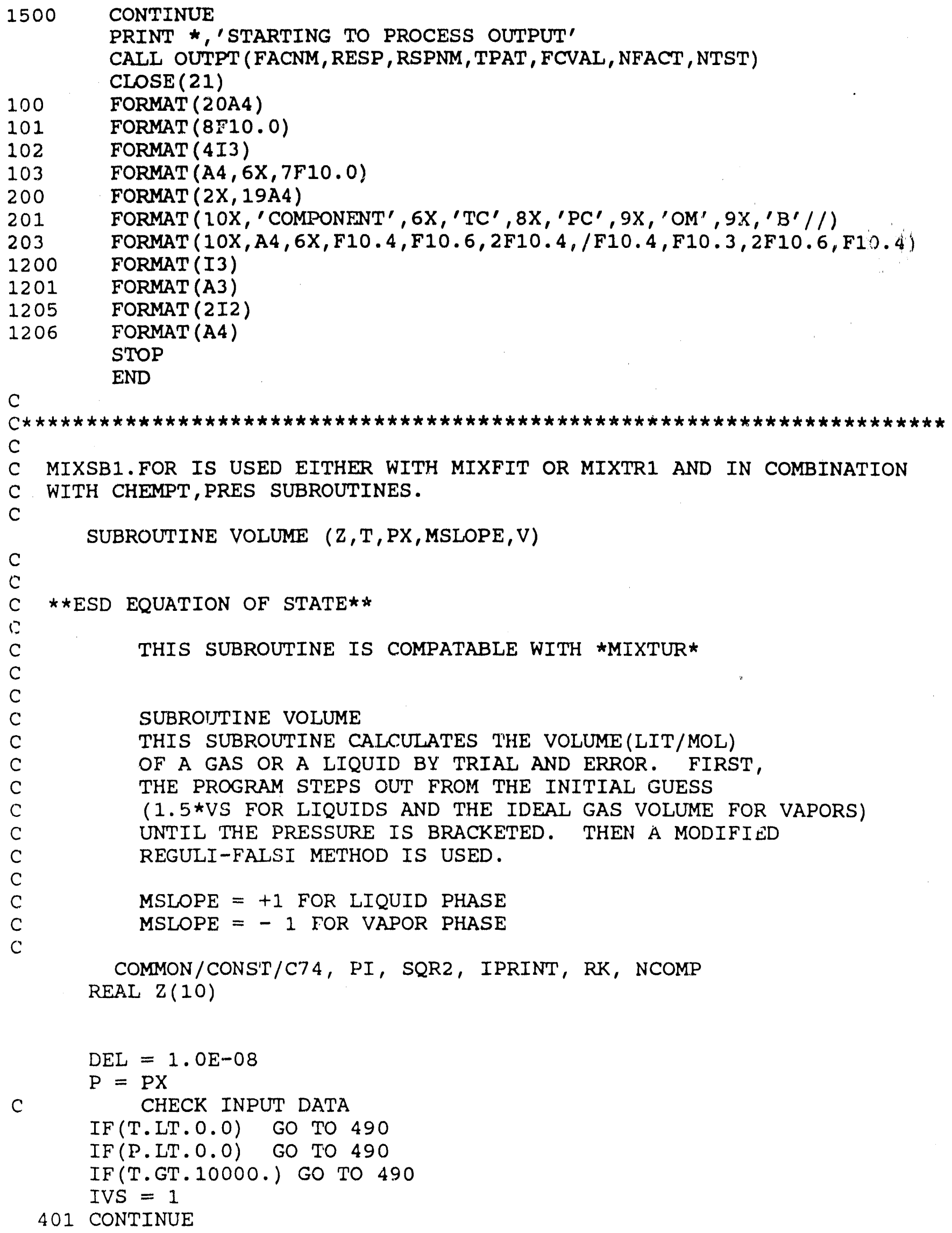







301 FORMAT $\left(10 X, ' \mathrm{P} \operatorname{EXP}=\prime, \mathrm{E} 15.4,^{\prime} \mathrm{T}=\right.$ ',E15.5,'VC=',E15.4,'MSLOPE $=$ 1, I3)

C FOR LIQUIDS V LESS THAN $1.5 \mathrm{~V}$ STAR IS VERY UNUSUAL IF (MSLOPE.GT.0) RETURN

IVS $=$ IVS +1

IF (IVS.GT.3) RETURN

$\mathrm{V}=\mathrm{V} /(1.1 * *$ MSLOPE $)$

GO TO 401

$800 \mathrm{~V}=\mathrm{V} 3$

IF (IPRINT.GE.5) GO TO 460

GO TO 660

460 CONTINUE

C $\operatorname{WRITE}(6,360) \mathrm{V}$

360 FORMAT(10X,'LEAVING SUB VOLUME, $\quad V=$ ',E15.5)

660 CONTINUE

RETURN

490 CONTINUE

C WRITE $(6,390)$ T, P,MSLOPE

390 FORMAT (10X,'SUB VOLUME, BAD INPUT DATA'/10X,'T $=$ ',F10.3,' $\mathrm{P}=$,

1 ,F10.4,'MSLOPE $=$ ', I3)

RETURN

END

C

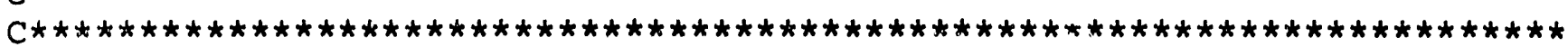

C

SUBROUTINE CONS

C

C $* *$ ESD EQUATION OF STATE**

c.

COMMON/CONST/ C74, PI, SQR2, IPRINT, RK, NCOMP

$\mathrm{SQR2}=1.414213562$

$\mathrm{PI}=3.141592654$

C RK = GAS CONSTANT (BARS*LITERS/ GM-MOLES*DEG K)

$\mathrm{RK}=0.08314572$

C7 $4=\mathrm{PI} * \mathrm{SQR} 2 / 6$.

RETURN

END

C

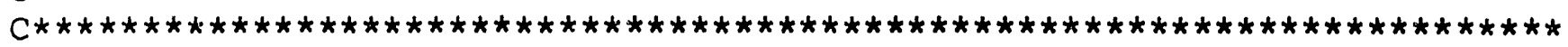

C

SUBROUTINE MIXPRP $(\mathrm{Z}, \mathrm{T})$

C

C

THIS SUBROUTINE IS COMPATABLE WITH *MIXTUR*

REAL Z(10)

$\operatorname{REAL} \operatorname{KEP}(10,10)$

COMMON / XPROP / KEP

COMMON/MIX/AM, BM, CHEM1 (10)

COMMON/IONST/C74, PI，SQR2, IPRINT, RK, NCOMP

COMMON/PURE/TC (10), PC (10), OM(10)

COMMON/SEDI/C (10), Q (10), VS(10), EK(10) 


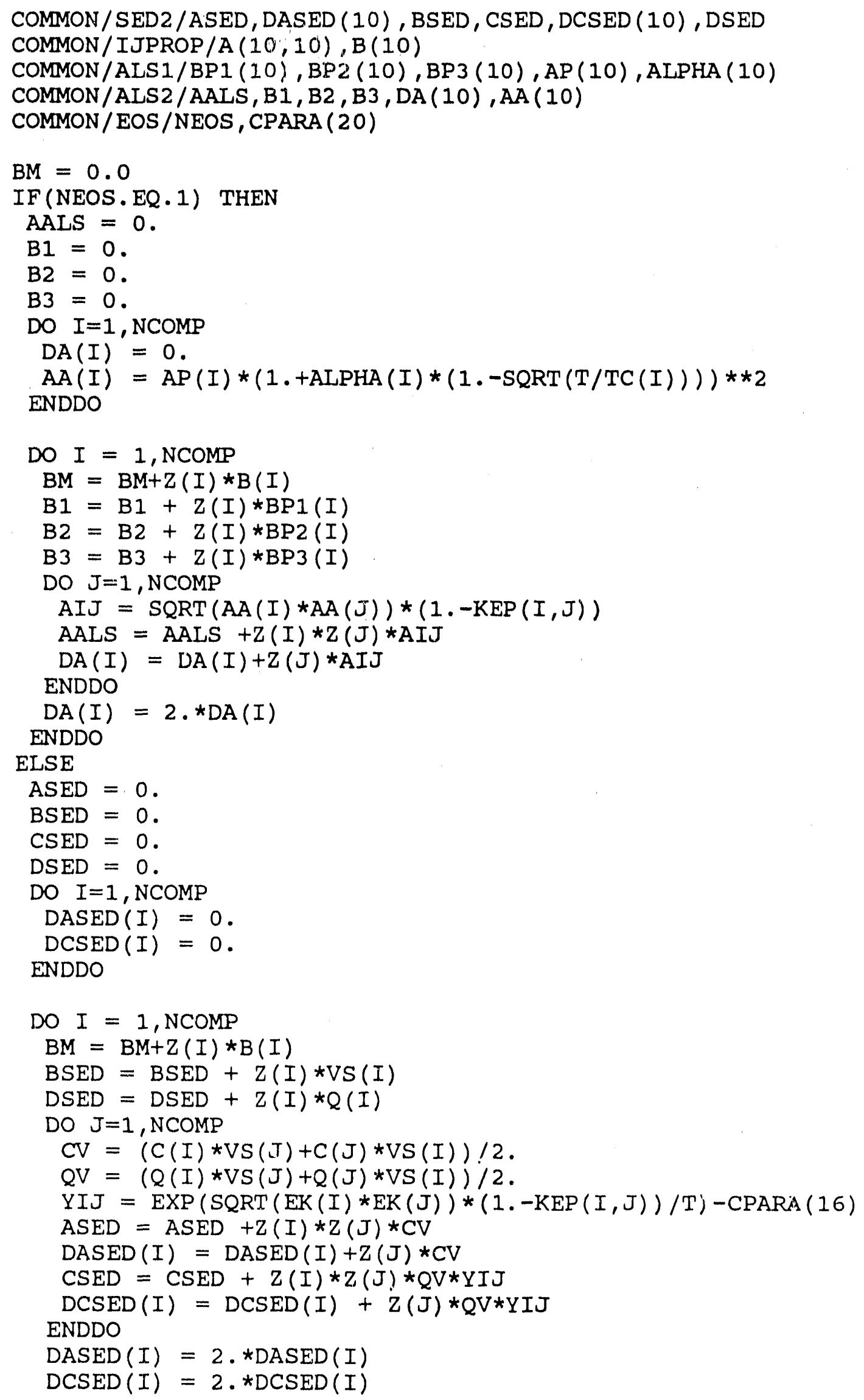




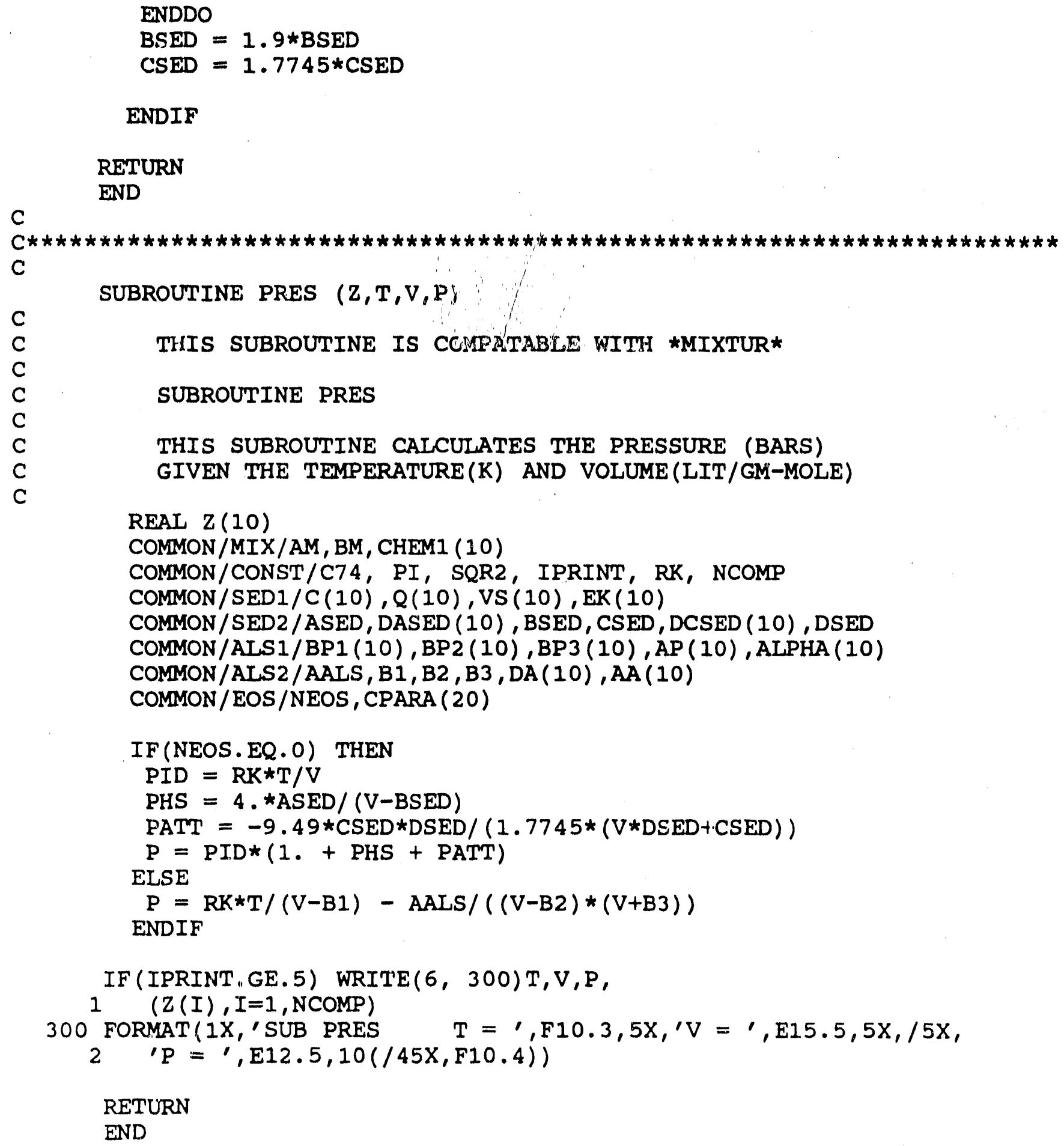


C

C ADAPTATION OF JINS PROGRAM FOR DOE CONTRACT WORK. J.R. LOEHE

C AUG/SEPT 1990

C

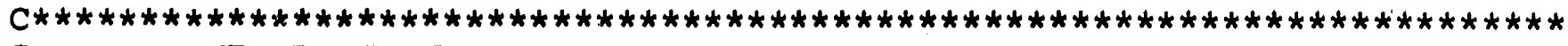

THIS PROGRAM IS TO CALCULATE CRITICAL POINTS OF MIXTURES

USING THJE TECHNIQUE DEVELOPED BY HEIDEMANN AND KHALIL (1980).

REFERENCE: Heidemann, R. A. and Khalil, A. M., "The Calculation of Critical Points", J. AIChE, vol. 26, No. 5, 1980, pp769-779.

(*) Elliott, Jr., J. R. and Daubert T. E.," Evaluation of An Equation of State Method for Calculating the Critical Properties of Mixtures", Ind. Eng. Chem. Res., vol. 26, 1987, pp1686-1691.

MAIN PROGRAM MIXCRI (INPUT, OUTPUT)

SUBROUTINES: MIXPRP, PRES, LNF1, LNF2

CRIT, EVC, EVT, UPTRI

$\operatorname{REAL} \operatorname{KEP}(20,20), \operatorname{RFACT}, \operatorname{RESP}(3,128), \operatorname{TPAT}(128,127)$

COMMON/PURE/TC (20), PC (20), OM(20)

COMMON/CONST/RK, NCOMP

COMMON / XPROP/KEP

COMMON/EOS/AO (20), BO (20), CO (20), DO (20), AMI (20), D1, D2

COMMON/SED1/C (20), Q(20), VS (20), EK(20)

COMMON/IEOS/IEOS, CPARA (20)

COMMON/RETDAT/ITIME

REAL X(20), TITLE(20), $\operatorname{FACT}(127), \operatorname{FCVAL}(128,127)$

REAL TKK (100), PKK(100), XKK(20, 200), VKK(100), BIAS

INTEGER NFACT, NBIN, NTST, ITIME, IERR, IEH

CHARACTER *20, TITIN, TITOUT, TEOS

CHARACTER FACNM $(127) * 5, \operatorname{RSPNM}(3) * 8$

C

EXTERNAL DS_GETDATA

TYPE *,' INPUT FILE NAME'

$\operatorname{READ}(*, 220)$ TITIN

TYPE *, 'OUTPUT FILE NAME'

$\operatorname{READ}(*, 220)$ TITOUT

OPEN (UNIT $=17$, NAME $=$ ' FACCHK' , STATUS $=$ ' NEW' $)$

OPEN (UNIT $=5$, NAME $=$ TITIN , STATUS $=$ ' OLD' $)$

OPEN (UNIT $=1$, NAME=TIT'J'JT, STATUS $=$ ' NEW' )

OPEN (UNIT=21, NAME=' FTEMP' , STATUS $=$ ' NEW' $)$

$\operatorname{REAN}(5,100)$ (TITLE (I), I=1, 20)

$\operatorname{READ}(5,220)$ TEOS

$\mathrm{NC}=0$

C

$\mathrm{RK}=$ GAS CONSTANT (BARS*LITERS/ GM-MOLES*DEG K)

$R K=0.08314572$ 


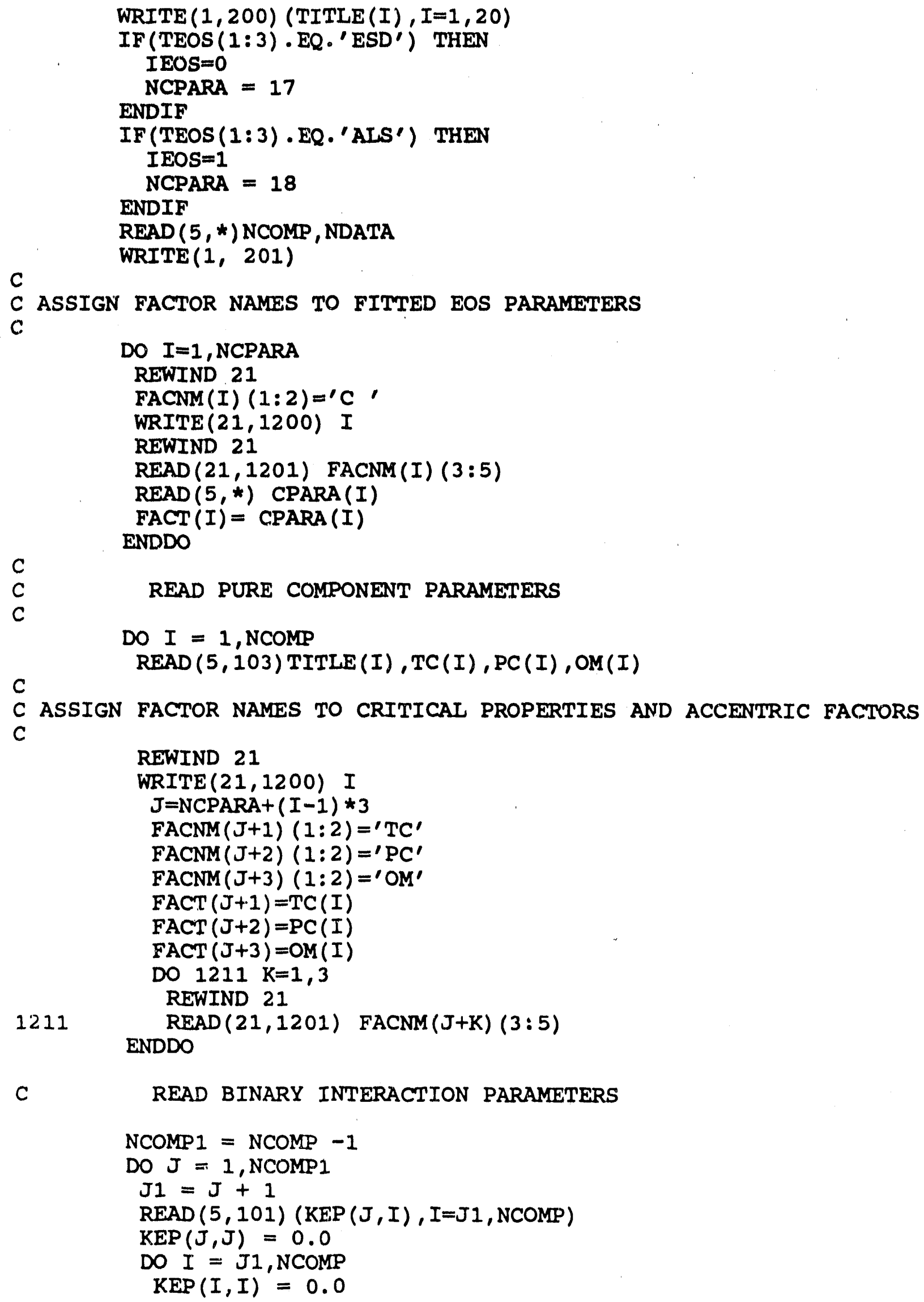




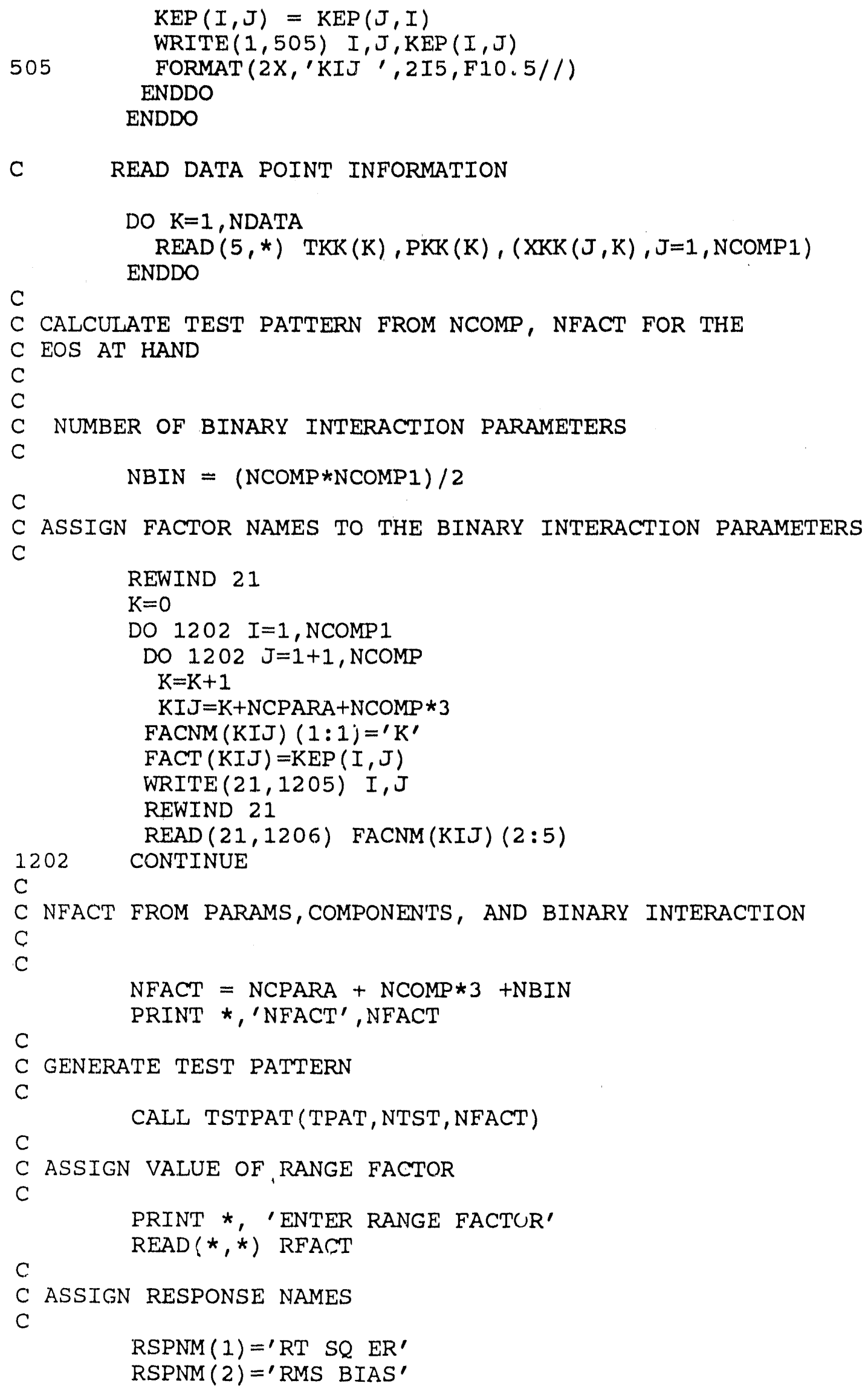




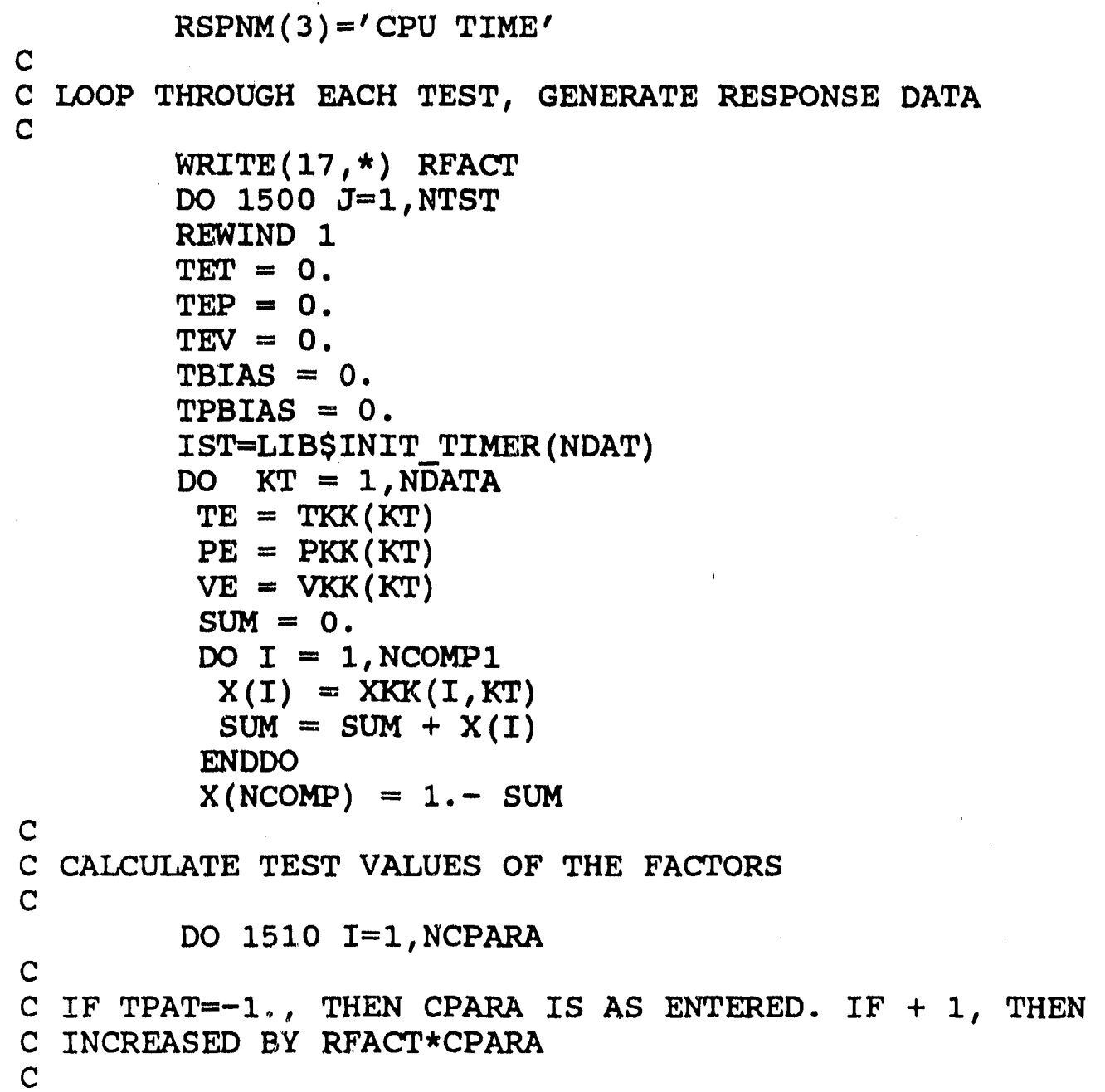

IF (TPAT (J, I) .EQ.1E00.OR.TPAT(J,I).EQ. -1.EO0) THEN $\operatorname{CPARA}(I)=(((\operatorname{TPAT}(J, I)+1 . E 00) / 2 . \mathrm{EO} 0) \star R F A C T+1 . \operatorname{EO0}) \star F A C T(I)$ $\operatorname{FCVAL}(J, I)=\operatorname{CPARA}(I)$ ENDIF

1510 CONTINUE

C

C CALCULATE COMPONENT DEPENDENT PARAMETERS

C

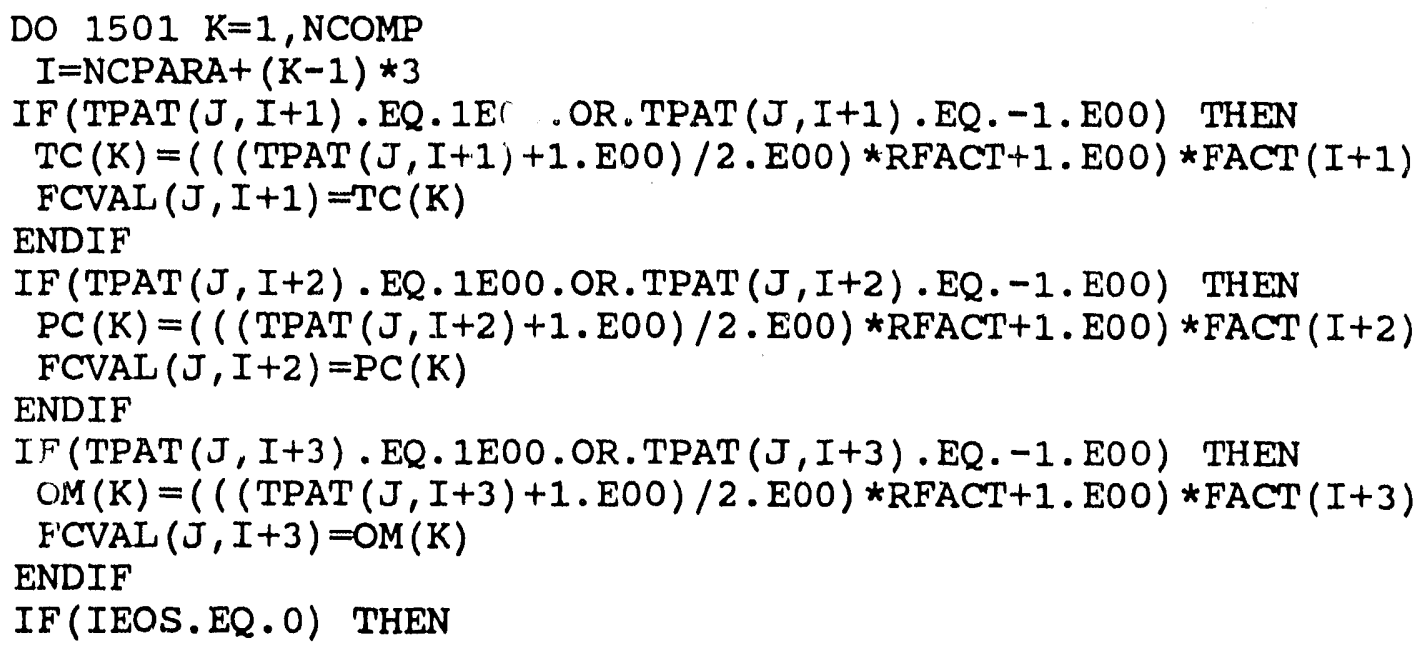




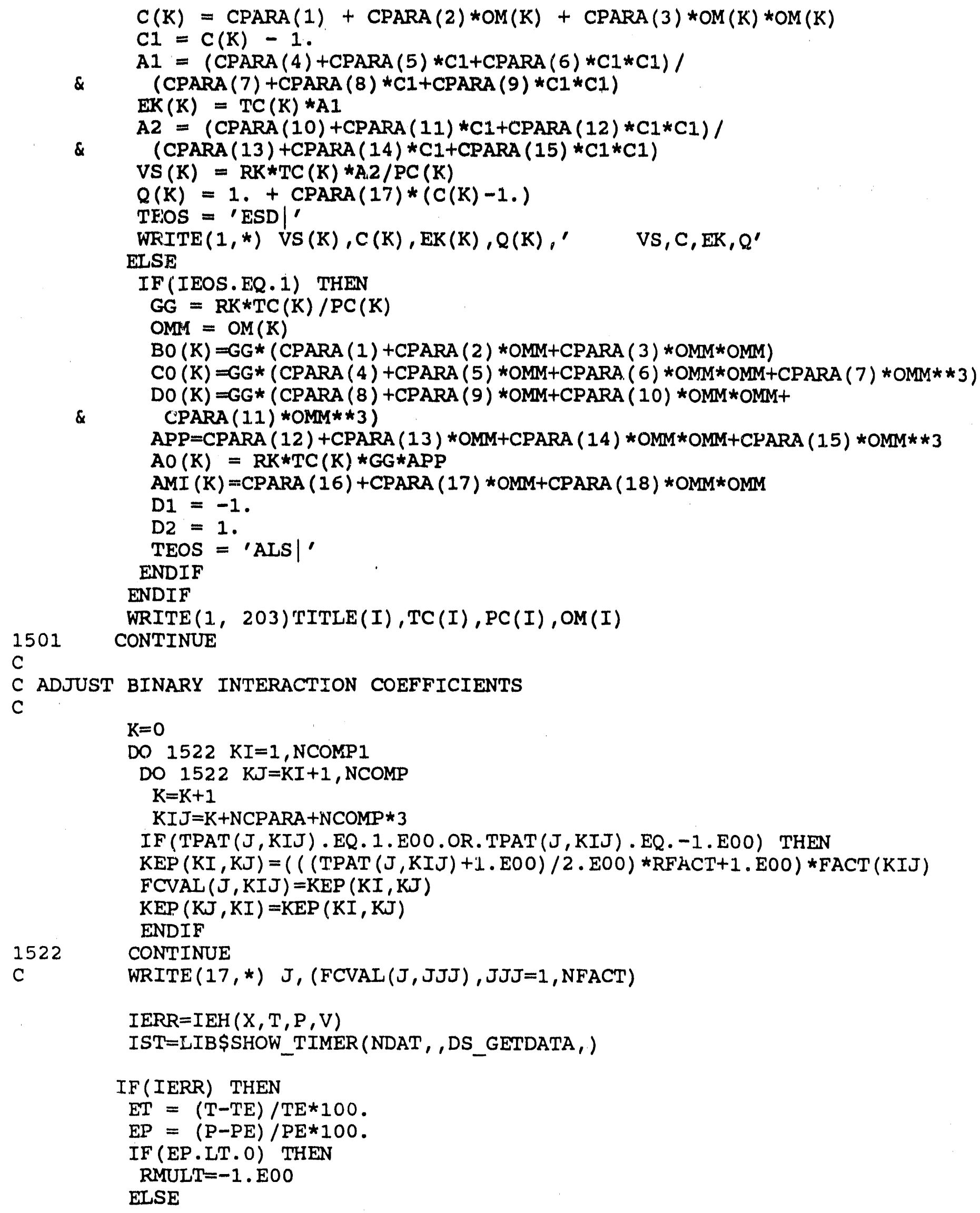




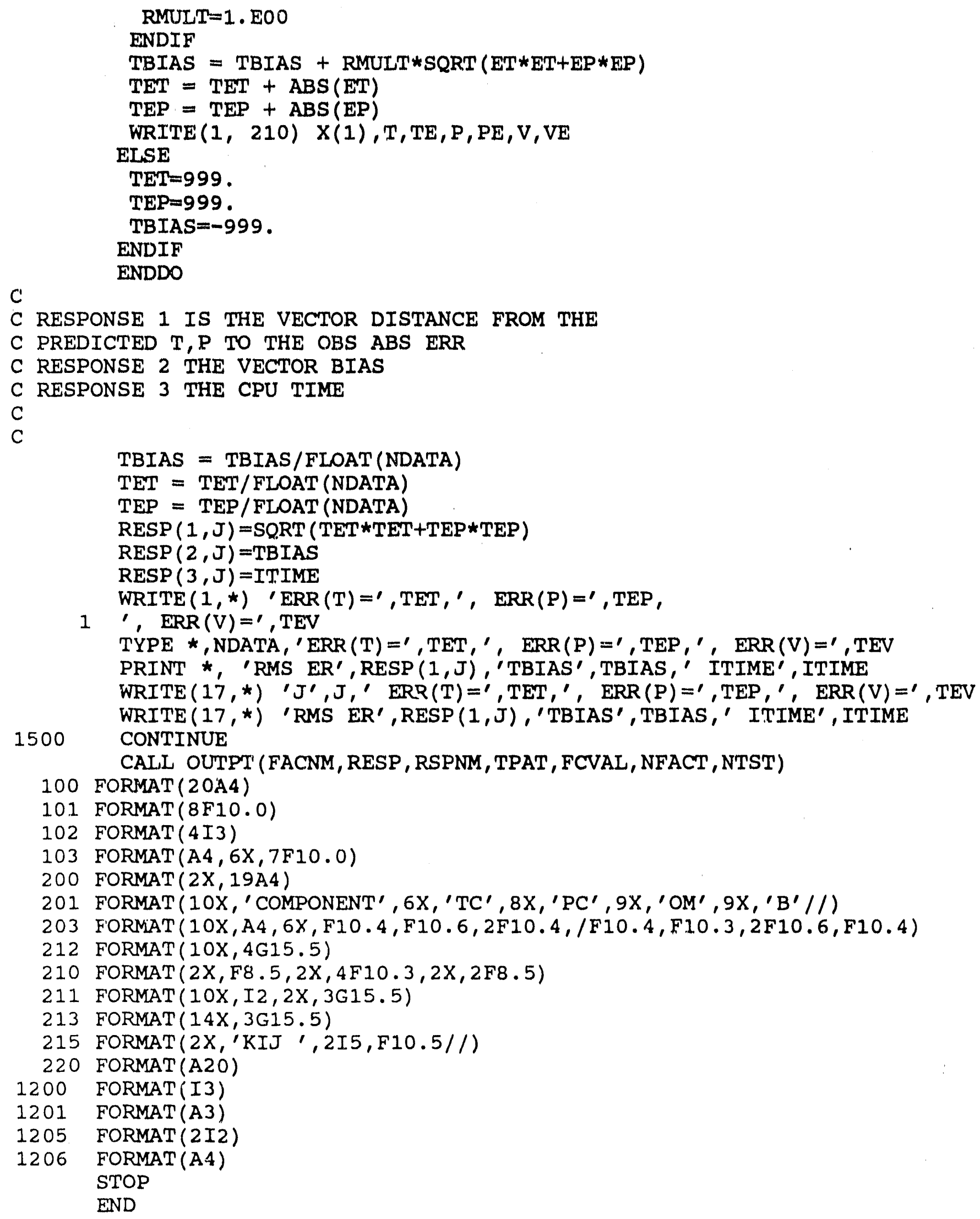


C

C
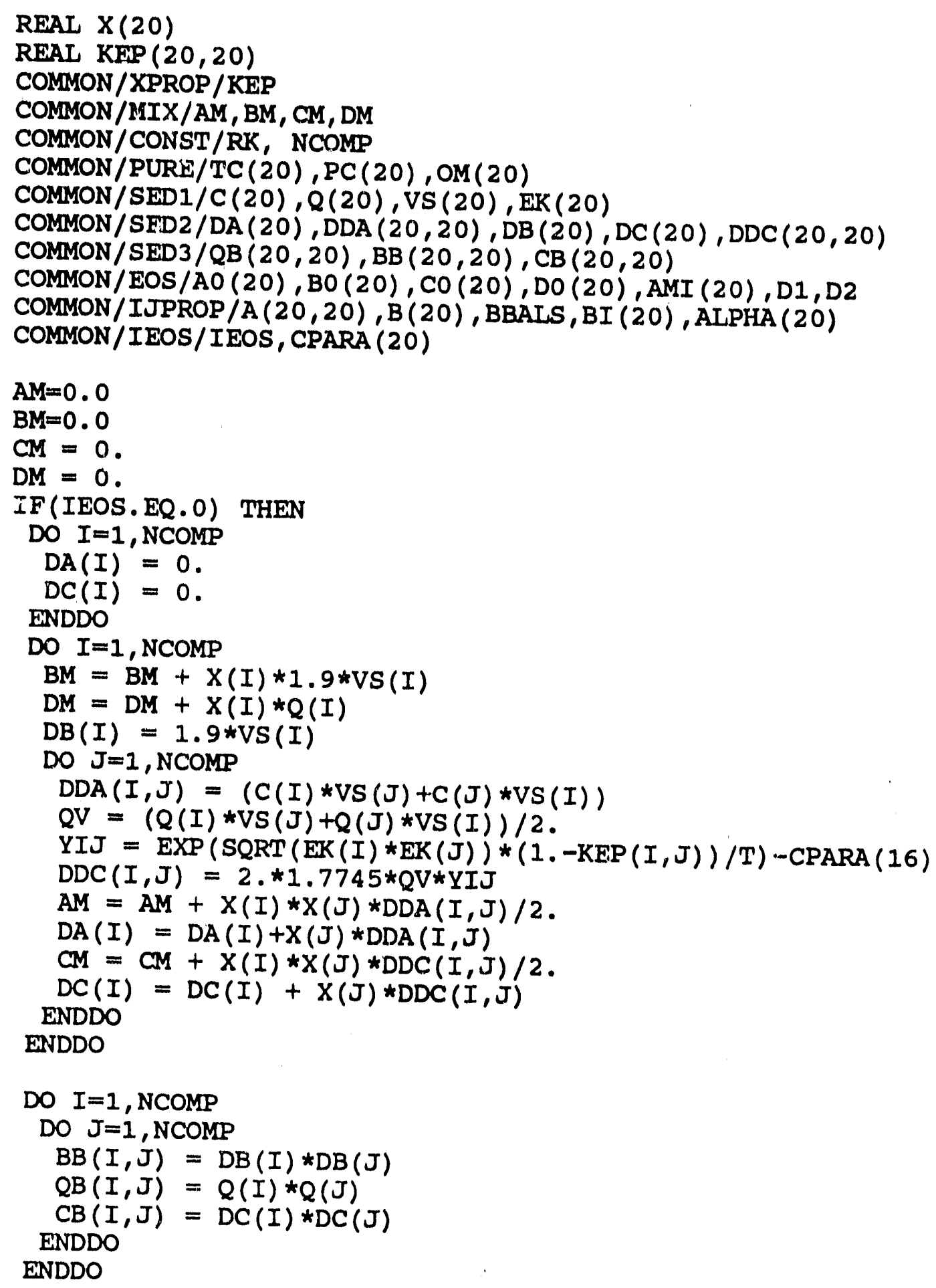

ELSE 


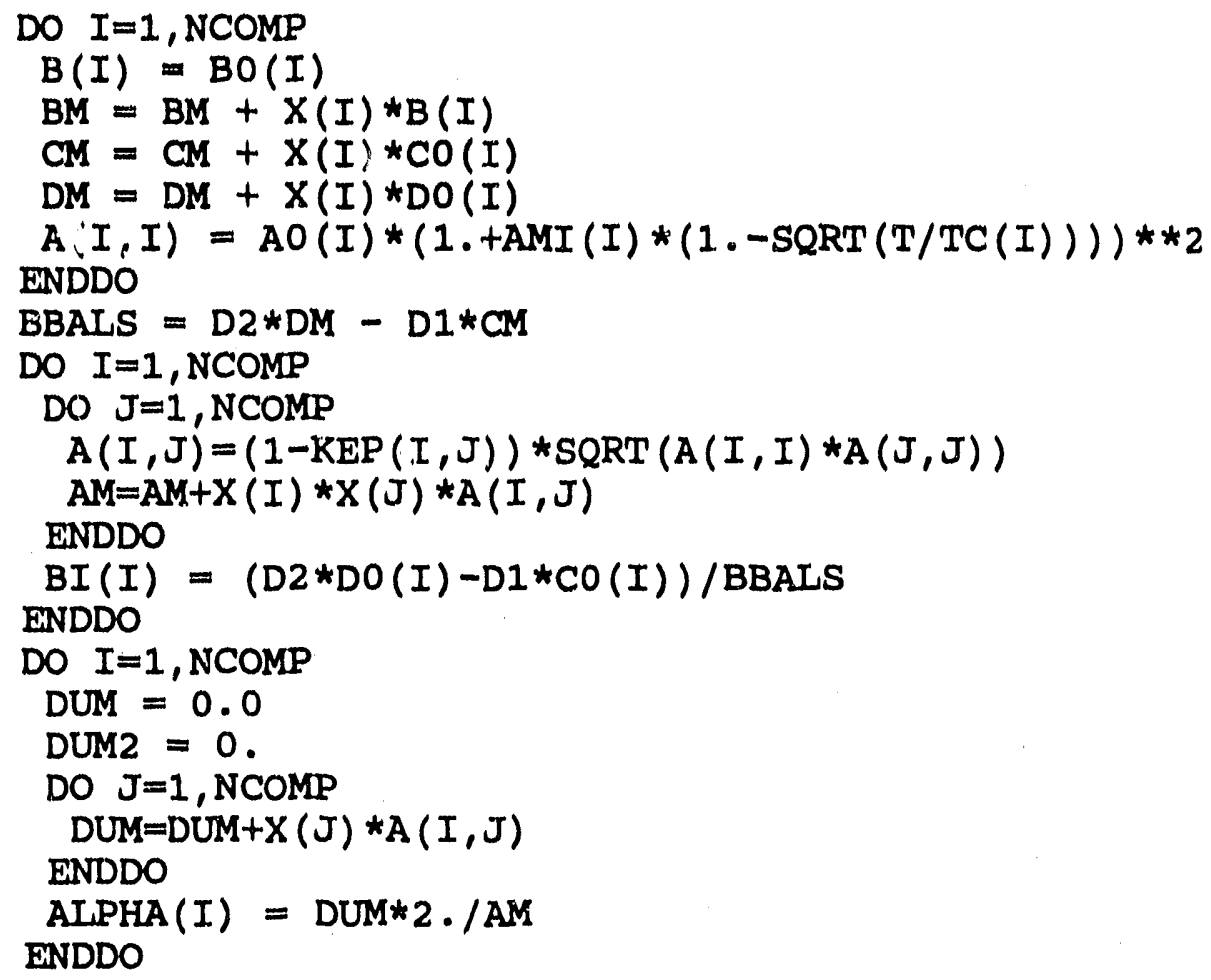

ENDIF

RETURN

END

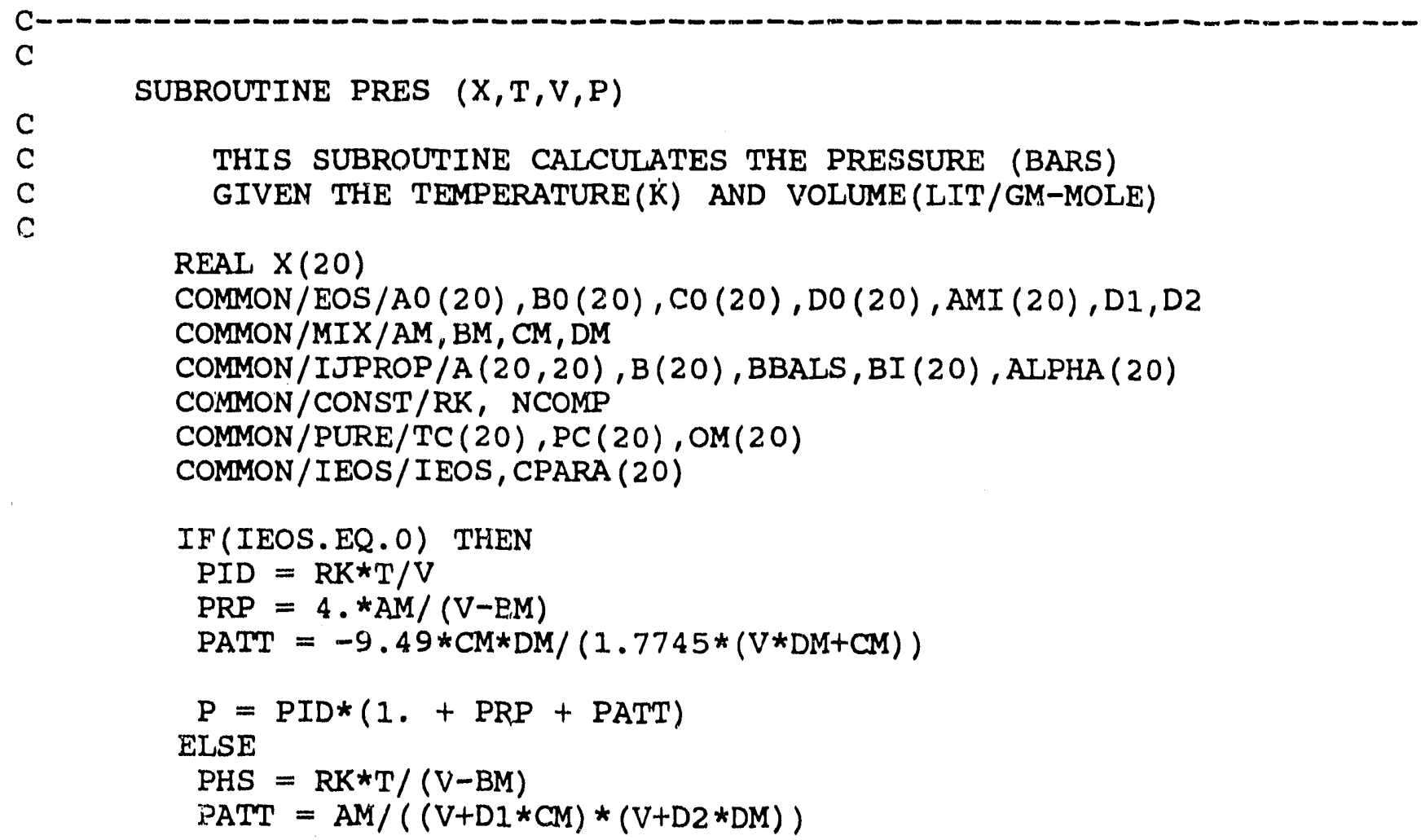




$$
P=P H S-P A T T
$$

ENDIF

RETURN

END

C

SUBROUTINE INF1 $(X, T, V, Q Q)$

DOUBLE PRECISION $Q Q(20,20)$

REAI $X(20)$

COMMON / XPROP / KEP

COMMON/MIX/AM, BM, CM, DM

COMMON/CONST/RK, NCOMP

COMMON/PURE/TC (20), PC (20), OM (20)

COMMON/SED1/C(20), $Q(20), V S(20), E K(20)$

COMMON / SED2 / DA (20), DDA $(20,20), \operatorname{DB}(20), \operatorname{DC}(20), \operatorname{DDC}(20,20)$

COMMON/SED3/QB $(20,20), \mathrm{BB}(20,20), \mathrm{CB}(20,20)$

COMMON/EOS/AO (20), BO (20), CO (20), DO (20), AMI (20), D1, D2

COMMON/IJPROP/A $(20,20), B(20)$, BBALS, BI (20), ALPHA (20)

COMMON/CRIT/BETA (20), $\operatorname{EPS}(20), \operatorname{ETA}(20), \operatorname{GMA}(20), \operatorname{OMG}(20,20), D$

COMMON/IEOS/IEOS, CPARA (20)

IF(IEOS. EQ.0) THEN

$E=V-B M$

$F=V * D M+C M$

$A E=A L O G(A B S(1 .-B M / V))$

DO $I=1$, NCOMP

DO $J=1$, NCOMP

IF (I.EQ.J) THEN

DELTA $=1$.

ELSE

DELTA $=0$.

ENDIF

$A B=D A(I) * D B(J)+D A(J) * D B(I)$

$D U M A=D C(I) * D C(J) * D M-C M *(Q B(I, J) * V+D C(I) * Q(J)+D C(J) * Q(I))$

$D U M B=D D C(I, J) * D M+Q B(I, J) * V$

DUM1 $=D E L T A / X(I)+9.49 *(D U M A / F / F-D U M B / F+Q B(I, J) / D M) / 1.7745$

DUM2 $=4 . * A M *(A B / A M-2 . * B B(I, J) * V / B M / E+3 . * B B(I, J) / E) / B M / E$

DUM3 $=-4 . * A E *(D D A(I, J)-A B / B M+2 . * B B(I, J) * A M / B M / B M) / B M$

$Q Q(I, J)=R K \star T *(D U M 1+D U M 2+D U M 3)$

ENDDO

ENDDO

ELSE

DO $I=1$, NCOMP

$\mathrm{BETA}(I)=\mathrm{BO}(\mathrm{I}) /(\mathrm{V}-\mathrm{BM})$

$\operatorname{EPS}(I)=D I * C O(I) /(V+D I * C M)$

$\operatorname{ETA}(I)=\mathrm{D} 2 * \mathrm{DO}(\mathrm{I}) /(\mathrm{V}+\mathrm{D} 2 * \mathrm{DM})$

$\operatorname{GMA}(I)=\operatorname{EPS}(I)-\operatorname{ETA}(I)$

ENDDO 


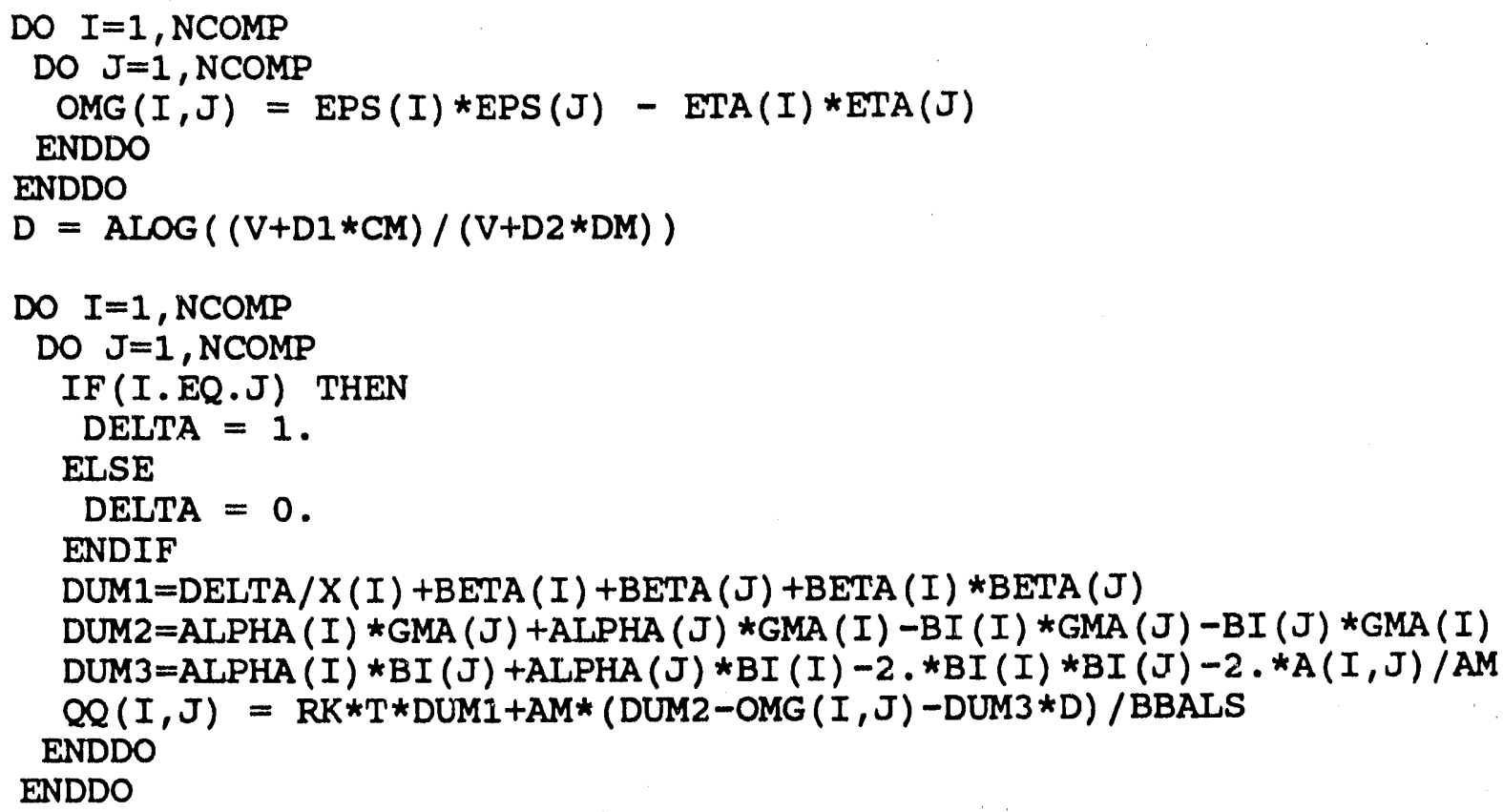




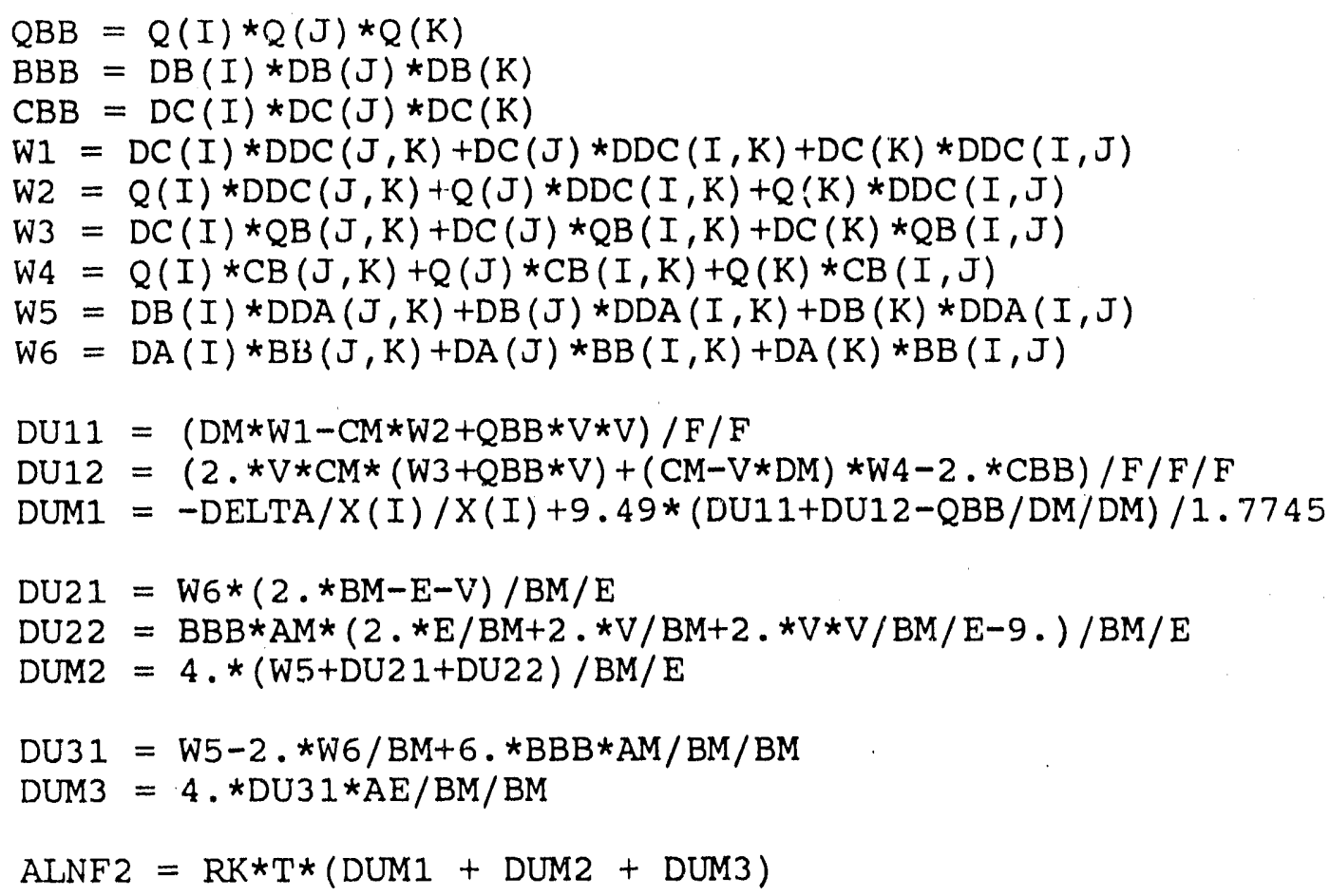


DOUBLE PRECISION $Q(20,20)$

DOUBLE PRECISION C,CC

REAL X (20), DN (20)

COMMON/MIX/AM, BM, CM, DM

COMMON/CONST/RK, NCOMP

COMMON/PURE/TC (20), PC (20), OM(20)

COMMON/SED1/C2 (20), Q2 (20), VS (20), EK(20)

COMMON/XPROP/KEP

COMMON/EOS/AO (20), BO (20), CO (20), DO (20), AMI (20),D1,D2

COMMON/IJPROP / A $(20,20), B(20), B B, B I(20)$, ALPHA (20)

COMMON/CRIT/BETA (20), EPS (20), ETA (20), GMA (20), OMG $(20,20)$, D COMMON/CHECK/CT, TQ

COMMON/IEOS/IEOS, CPARA (20)

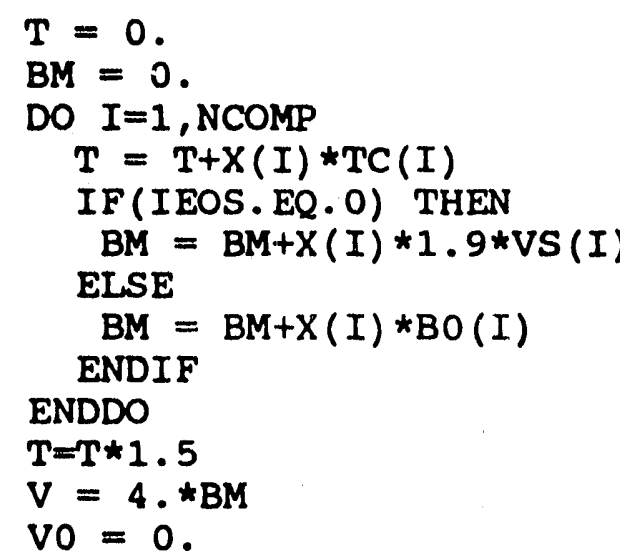

C

EVALUATE T AT GIVEN V

$100 \operatorname{CALL} \operatorname{EVT}(X, T, V, Q, D N)$

C EVALUATE V AT CALCULATED $T$

$\operatorname{CALL} \operatorname{EVC}(\mathrm{X}, \mathrm{T}, \mathrm{V}, \mathrm{DN}, \mathrm{C})$

$\mathrm{CVT}=\mathrm{ABS}(\mathrm{V}-\mathrm{VO}) / \mathrm{V}$

IF(CVT.GT. 1E-04) THEN

$\mathrm{DV}=\mathrm{V} * 1 . \mathrm{E}-05$

$V V=V+D V$

CALL $\operatorname{EVT}(X, T, V V, Q, D N)$

CALL EVC $(X, T, V, D N, C C)$

$\mathrm{DDV}=(\mathrm{CC}-\mathrm{C}) /(\mathrm{VV}-\mathrm{V})$

$\mathrm{VO}=\mathrm{V}$

$V:=A B S(V-C / D D V)$

GO TO 100

ENDIF

CALL PRES $(X, T, V, P)$

RETURN

END 


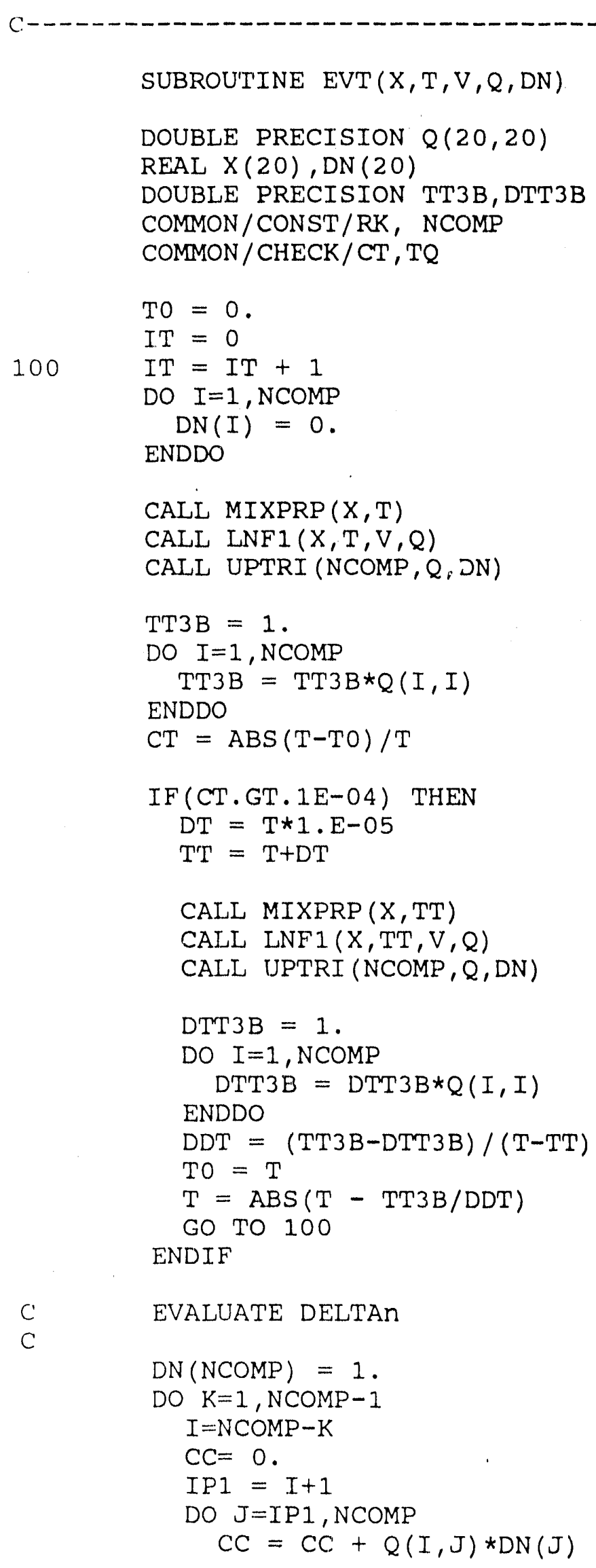


ENDDO

ENDDO

$D N(I)=(D N(I)-C C) / Q(I, I)$

$D=0$.

DO $I=1$, NCOMP

$D=D+D N(I) * D N(I)$

ENDDO

$D=\operatorname{SQRT}(D)$

DO $I=1$, NCOMP

$D N(I)=D N(I) / D$

ENDDO

$\mathrm{TQ}=0$.

DO $I=1$, NCOMP

$\mathrm{TQI}=0$.

DO $J=1$, NCOMP

$\mathrm{TQI}=\mathrm{TQI}+\mathrm{Q}(\mathrm{I}, \mathrm{J}) * \mathrm{DN}(\mathrm{J})$

ENDDO

$\mathrm{TQ}=\mathrm{TQ}+\mathrm{ABS}(\mathrm{TQI})$

ENDDO

RETURN

END

SUBROUTINE EVC (X, T, V, DN, CSUM)

DOUBLE PRECISION CSUM, ALNF2

REAL X (20), DN (20)

COMMON/CONST/RK, NCOMP

CSUM $=0$.

DO $I=1, N C O M P$

DO $\mathrm{J}=1, \mathrm{NCOMP}$

DO $K=1$, NCOMP

CALL $\operatorname{LNF2}(I, J, K, X, T, V, \operatorname{ALNF2})$

CSUM $=$ CSUM $+A L N F 2 * D N(I) * D N(K) * D N(J)$

ENDDO

ENDDO

ENDDO

RETURN

END

SUBROUTINE UPTRI ( $N, A A, B B)$

DOUBLE PRECISION AA $(20,20), A F, B B(20)$

DO $K=1, N-1$

DO $\mathrm{J}=\mathrm{K}+1, \mathrm{~N}$

$A F=A A(J, K) / A A(K, K)$ 


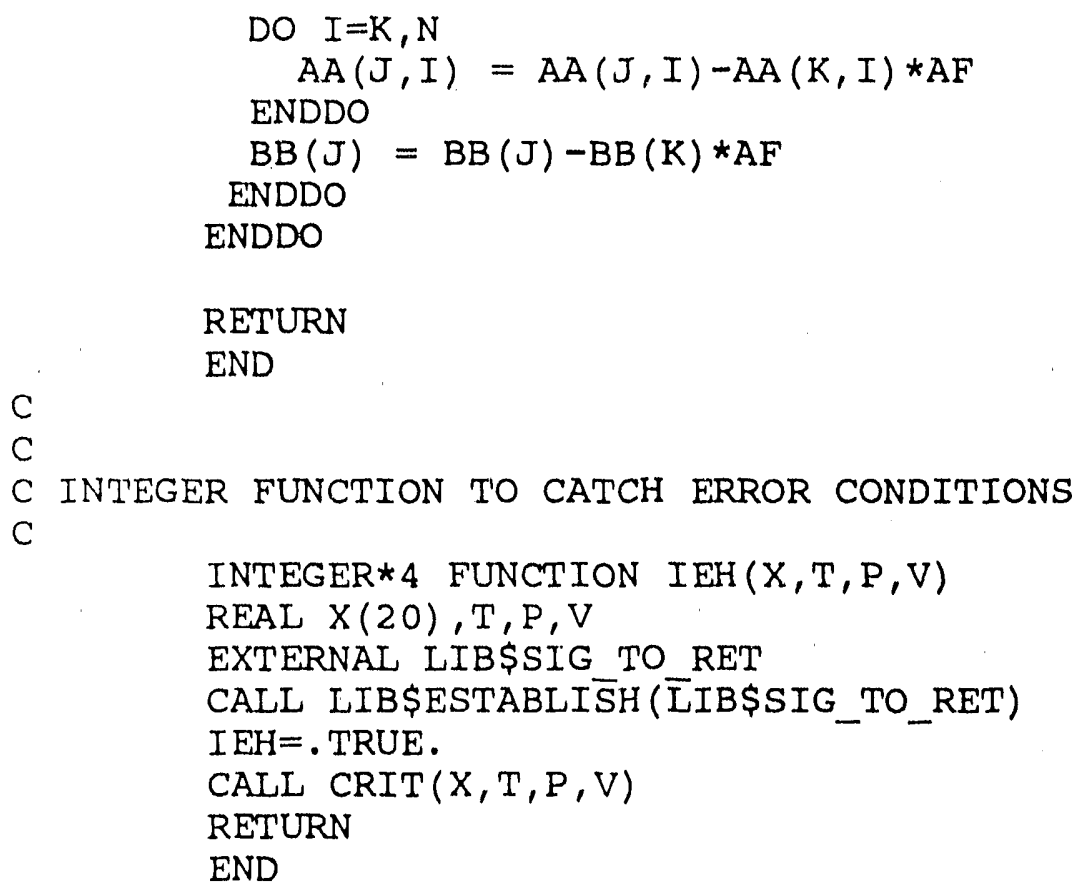


$\mathrm{C}$

C GENERATES TEST PATTERNS FOR DOE CONTRACT

C

SUBROUTINE TSTPAT (TFAT, NTST, NFACT')

INTEGER I (7) , J, K, L, M, N , O, LLIM(6), NR, ULIM(7)

INTEGER IALG $(7,128), \operatorname{REJ}(7,64), \operatorname{TRIAL}(7,64), \operatorname{DRES}, \mathrm{NZCNT}$

INTEGER NTST, NFACT, NREJ

REAL TPAT $(128,127)$

LOGICAL DECTST

COMMON / ALG / NR, DRES, NREJ , IALG, REJ

C

PRINT *,'ENTER NR (=LOGBASE2 (NO.RUNS))'

C

C

PRINT *,'NR MUST BE AN INT'EGER BETWEEN 2 AND 7'

$\operatorname{READ}(*, *) \quad \mathrm{NR}$

PRINT *,'ENTER DESIGN RESOLUTION (3, 4, OR 5)'

$\operatorname{READ}(*, *)$ DRES

$C$

DECTST $=$. TRUE.

C ASSIGN NUMBER OF TESTS FROM DESIGN RESOLUTION AND NUMBER OF

C FACTORS TO BE TESTED

$\mathrm{C}$

IF (DRES.EQ.3) THEN

NTST $=$ NFACT +1

DO $1200 \quad I I=2,7$

ITST $=2 *$ *II

IF (NTST.LE.ITST.AND.DECTST) THEN

NTST $=$ ITST

$\mathrm{NR}=\mathrm{II}$

DECTST $=$. FALSE.

ENDIF

1200 CONTINUE

ENDIF

IF (DRES.EQ.4) THEN

DO 1201 II $=2,7$

ITST $=2 * \star I I$

IF (NFACT.LE. (ITST/2). AND.DECTST) THEN

NTST =ITST

$N R=I I$

DECTST $=$. FALSE.

ENDIF

1201 CONTINUE

ENDIF

IF (DRES.EQ.5) THEN

DO 1202 II $=2,7$

IF (NFACT.EQ.II+1.AND.DECTST) THEN

$\mathrm{NTST}=2 * * I I$

$\mathrm{NR}=\mathrm{II}$

DECTST $=$, FALSE

ENDIF

1202 CONTINUE

ENDIF

IF (DECTST) THEN

PRINT *,'NIMBER OF FACTORS INCONSISTENT WITH DESIGN'

PRINT *,' RESOLUTION AND NUMBER OF AVAILABIE TESTS'

PRINT *,'PROGRAM WILL END' 


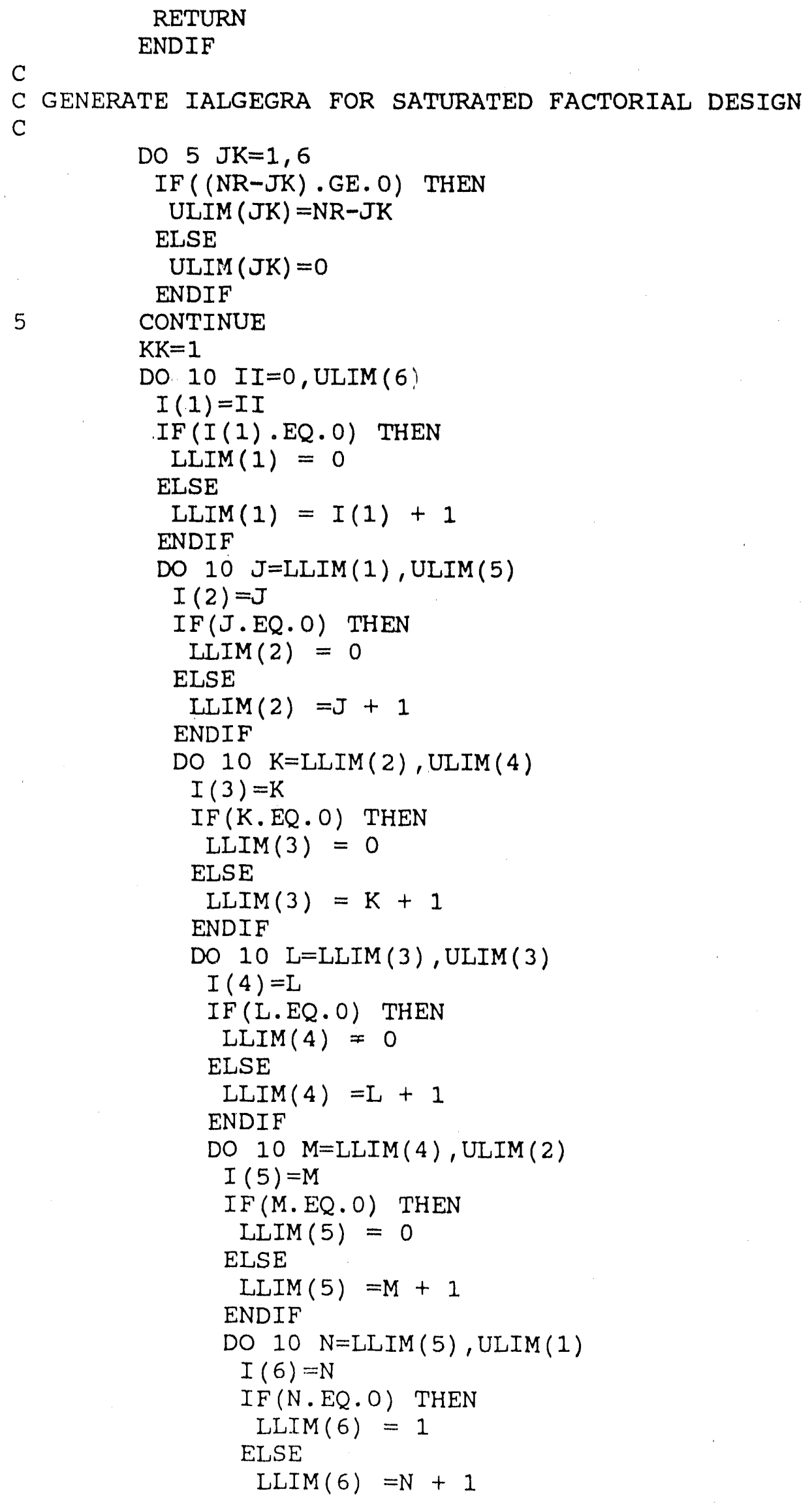




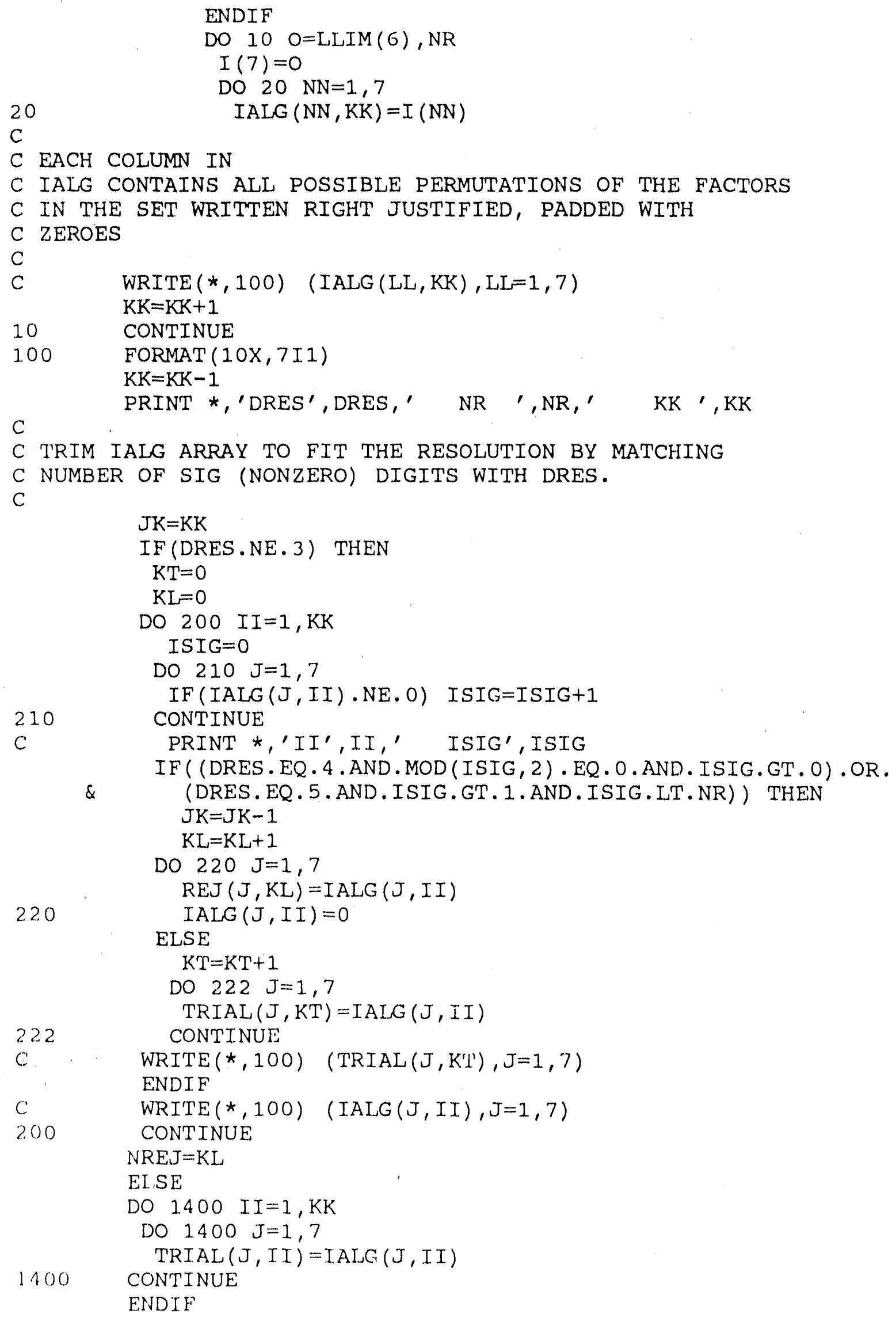


C

C REPLACE IALG BY TRIAL

C

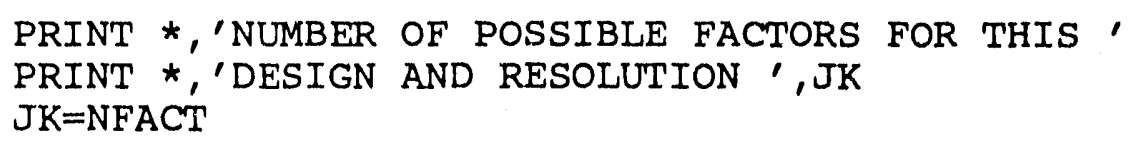

C

C GENERATE TEST PATTERN COLUMN BY COLUMN

C

$$
\begin{aligned}
& \text { DO } 30 \mathrm{KK}=1, \mathrm{NR} \\
& \text { DO } 40 \mathrm{JJ}=1,2 * *(\mathrm{KK}-1)
\end{aligned}
$$

40

$\operatorname{TPAT}(\mathrm{JJ}, \mathrm{KK})=-1.0 \mathrm{EOO}$

$$
\mathrm{MM}=2 * *(\mathrm{KK}-1)+1
$$

50 IF (MM.LE. $(2 * *$ NR)) THEN

DO $60 \mathrm{LI}=1,2 * *(\mathrm{KK}-1)$

$\operatorname{TPAT}(\mathrm{MM}, \mathrm{KK})=-\operatorname{TPAT}((\mathrm{MM}-2 * *(\mathrm{KK}-1)), \mathrm{KK})$

$6 \mathrm{C}$

$\mathrm{MM}=\mathrm{MM}+1$

GO TO 50

ENDIF

30 CONTINUE

DO $70 \quad K K=N R+1,(2 * * N R)-1$

IF (KK.LE.JK) THEN

DO $71 \mathrm{JJ}=1,2 * \star N R$

$\operatorname{TPAT}(J J, K K)=1.0 \mathrm{E} 00$

DO 71 II $=1,7$

IF (IALG (II, KK) .NE.0) THEN

$\operatorname{TPAT}(J J, K K)=\operatorname{TPAT}(J J, K K) * \operatorname{TPAT}(J J, \operatorname{IALG}(I I, K K))$ ENDIF

71 CONTINUE

\section{ENDIF}

70

c

CONTINUE

WRITE $(*, 110)$

FORMAT $(15(2 \mathrm{X}, \mathrm{F} 3.0))$

RETURN

END 
$\mathrm{C}$

C CALCULATES CONTRASTS, SETS UP AND PRINTS OUT TEST AND

C RESPONSE DATA FOR THE DOE CONTRACT

$\mathrm{C}$

SUBROUTINE OUTPT ( FACNM , RESP, RSPNM, TPAT, FCVAL, NFACT, NTST)

$\operatorname{REAL} \operatorname{RESP}(3,128), \operatorname{TPAT}(128,127), \operatorname{FCVAL}(128,127)$

REAL CNTR $(3,127), \operatorname{CHD}(127)$

DOUBLE PRECISION SUMHI, SUMLO

INTEGER IPNT $(120), \operatorname{DRES}, \operatorname{IALG}(7,128), \operatorname{REJ}(7,64), \operatorname{INCTR}(3,127)$

INTEGER NREJ , NR, NFACT, NTST, NPG , NCOLM, NROWS, IHD (127)

CHARACTER FACNM $(127) * 5, \operatorname{PNTR}(120) * 23, \operatorname{ALIST}(64,127) * 8$

CHARACTER OUTFILE*25, RNTITLE*80, RSPNM (3) *8, TABDES*2, PROD*23

LOGICAL MATCH

COMMON /ALG/NR, DRES, NREJ , IALG , REJ

$\mathrm{C}$

C

PRINT *,'ENTER DESCRIPTIVE TITLE FOR THE RUN OUTPUT'

$\operatorname{READ}(*, 5000)$ RNTITLE

PRINT *,'ENTER OUTFILE NAME'

READ (*,5001) OUTFILE

$5000 \quad$ FORMAT $($ A80)

5001 FORMAT (A25)

$\operatorname{OPEN}\left(9\right.$, FILE=OUTFILE, STATUS $={ }^{\prime}$ NEW' $)$

$\mathrm{C}$

C GENERATE CONTRASTS FOR MAIN EFFECTS

C

20

DO $10 \quad I=1,3$

DO $10 \mathrm{~J}=1, \mathrm{NFACT}$

SUMHI $=0$. DOO

SUMLO $=0$. DOO

DO $20 \mathrm{~K}=1$, NTST

$\operatorname{IF}(\operatorname{TPAT}(K, J) \cdot E Q \cdot-1 \cdot E O O) \quad \operatorname{SUMLO}=\operatorname{SUMLO}+\mathrm{RESP}(I, K)$

$\operatorname{IF}(\operatorname{TPAT}(K, J) \cdot E Q \cdot 1 . \mathrm{EOO}) \quad \operatorname{SUMHI}=\operatorname{SUMHI}+\operatorname{RESP}(I, K)$

CONTINUE

SUMHI $=$ SUMH I / FLOAT $($ NTST $/ 2)$

SUMLO $=$ SUMLO / FLOAT $($ NTST $/ 2)$

$\operatorname{INCTR}(I, J)=J$

$\operatorname{CNTR}(I, J)=$ SUMHI - SUMLO

C PRINT *,' I',I,' $\operatorname{CNTR}(I, J) ', \operatorname{CNTR}(I, J)$

10 CONTINUE

C

C GENERATE CONTRASTS FOR $2 \mathrm{FI}$

$\mathrm{C}$

C FOR LEVEL V RESOLUTION DESIGNS

C THERE WILL BE CONFOUNDED $2 F I$ AS FACTORS FACT + 1 TO NTST - 1

C FOR LEVEL IV DESIGNS,

C AND THERE WILL BE 2 FI CONFOUNDED WITH ME FOK LEVEL III DESIGNS.

$\mathrm{C}$

C NREJ IS THE NUMBER OF IALG STRINGS REJECTED DURING THE

C RESOLUTION ASSIGNMENT IN TSTPAT

C REJ CONTAINS THE STRINGS THEMSELVES.

C

C GENERATE 'THE PATTERN AT THE END OF TPAT'

C 


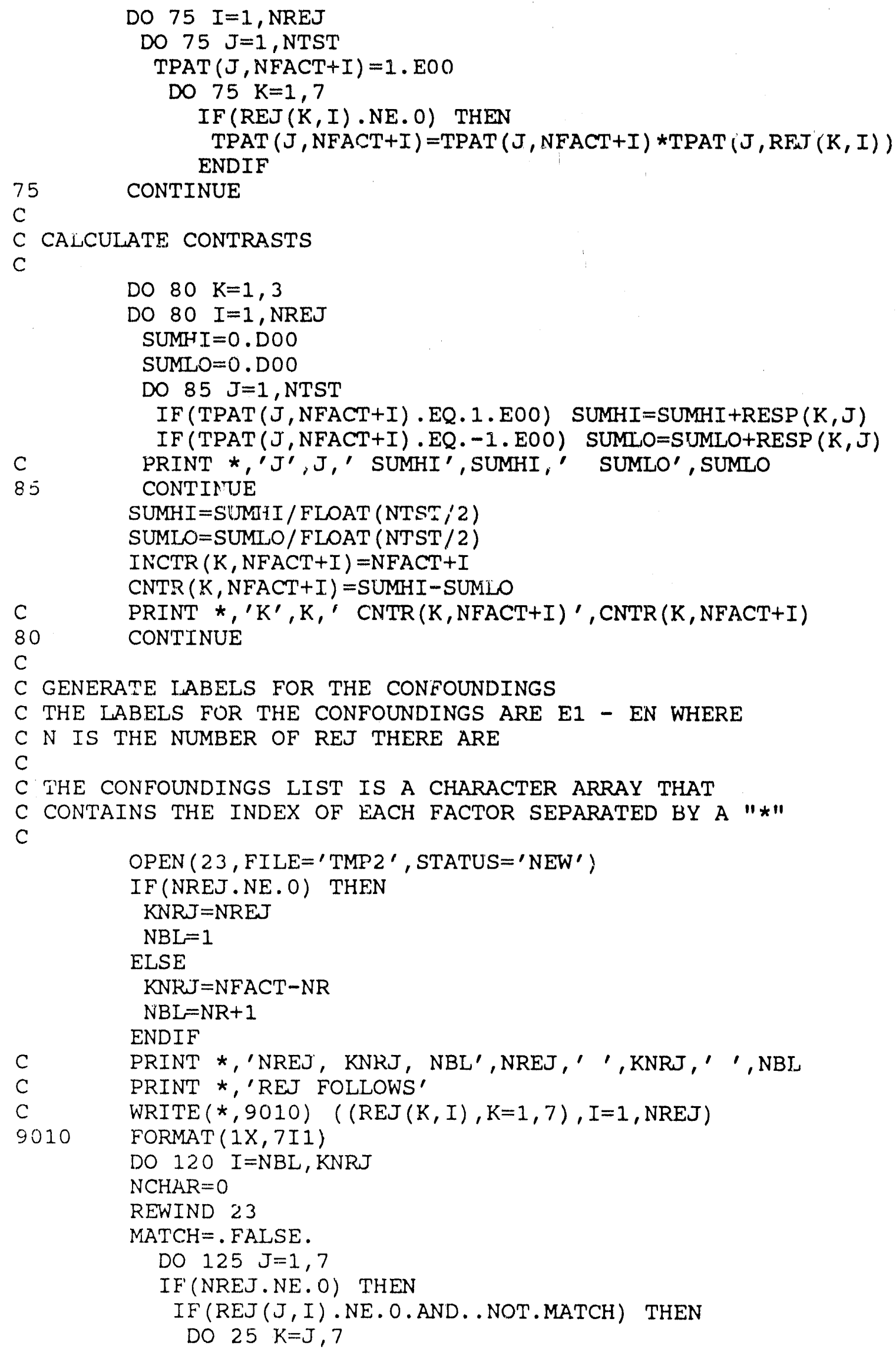


C

25

26

125

200

130

C

C

C

C THE LARGEST POSSIBLE REJ IS 6 INTEGERS LONG, WITH

C THREE NUMBERS PER INDEX (AT MOST), AND $5 \prime \star \prime$

$\mathrm{C}$

201

50

C

C EVALIAS RETURNS THE ALIAS ALGEBRA PRODUCT OF I AND J

C IN THE SANE (UNAMBIGUOUS) FORM AS PNTR. ALIST CONTAINS

$C$ 'THE LIST OF CONFOUNDED 2FI. NOTE THAT PNTR CAN CONTAIN

C UP TO $6 \mathrm{FI}$

C.

CALI EVALIAS (IALG, PROD, I, J,NR)

C PRINT *, ' J',J,' I', I,' PROD ', PROD

C PAUSE

DO $40 \mathrm{~K}=1$, NREJ

IF (PROD.EQ.PNTR(K) ! THEN

WRITE $(23,210) \quad I, J$

BACKSPACE 23

$\operatorname{IPNT}(K)=\operatorname{IPNT}(K)+1$

$\operatorname{READ}(23,202, \operatorname{END}=9002) \operatorname{ALIST}(\operatorname{IPNT}(K), K)$ 


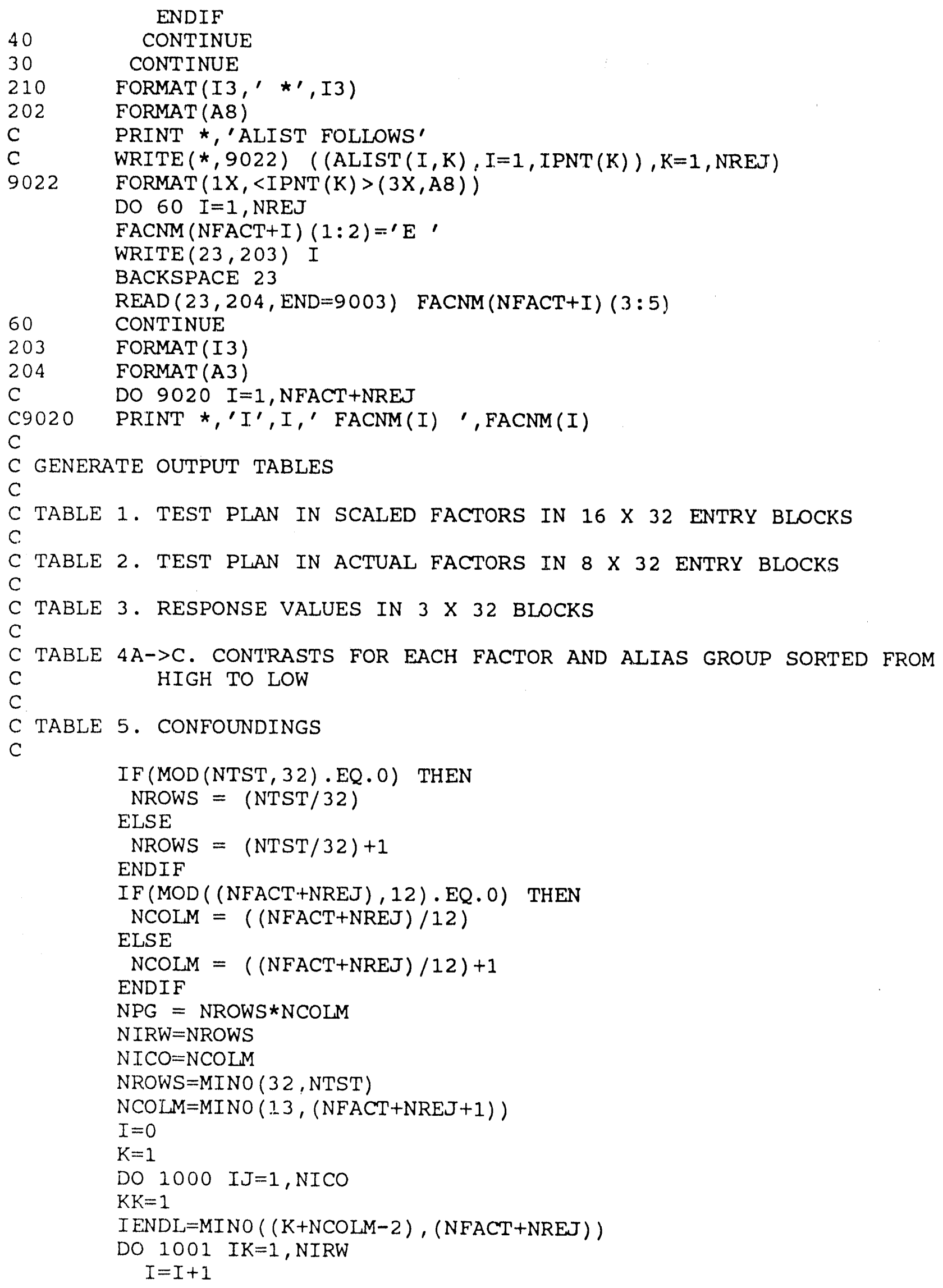


C

C WRITE PAGE HEADER

$\mathrm{C}$

IF (I.EQ.1) THEN

WRITE $(9,2000)$ RNTITLE, I, NPG, (FACNM (J) , J=K, K+NCOLM-2)

ELSE

WRITE $(9,2002)$ RNTITLE, I, NPG, (FACNM (J) , J=K, IENDL)

ENDIF

$\mathrm{C}$

C FILL IN TABLE 1 ENTRIES

C

DO $1010 \mathrm{~L}=\mathrm{KK}, \mathrm{KK}+\mathrm{NROWS}-1$

$\operatorname{WRITE}(9,2001) \quad \mathrm{L},(\operatorname{TPAT}(\mathrm{L}, \mathrm{J}), \mathrm{J}=\mathrm{K}, \mathrm{IENDL})$

1010 CONTINUE

$\mathrm{KK}=\mathrm{KK}+\mathrm{NROWS}$

1001. CONTINUE

$\mathrm{K}=\mathrm{K}+\mathrm{NCOLM}-1$

1000 CONTINUE

2000 FORMAT ('1'///1X,A80////15X,' TABLE 1. TEST PATTERN IN SCALED',

\& ' FACTORS', 5X,I2,' OF ',I2//1X,' TEST',15(1X,A5)/)

2.001 FORMAT $(2 \mathrm{X}, \mathrm{I} 2,3 \mathrm{X}, 15(1 \mathrm{X}, \mathrm{F} 3.0,2 \mathrm{X}))$

2002 FORMAT (' 1 '///1X,A80////15X,' TABLE 1. (CONTINUED)',27X

$\left.\& \quad, I 2,{ }^{\prime} \mathrm{OF}, \mathrm{I} 2 / / 1 \mathrm{X},{ }^{\prime} \mathrm{TEST}, 15(1 \mathrm{X}, \mathrm{A} 5) /\right)$

$\mathrm{C}$

C TABLE 2

C

IF (MOD (NFACT, 5) . EQ . 0) THEN

NCOLM $=($ NFACT $/ 5)$

ELSE

NCOLM $=($ NFACT $/ 5)+1$

ENDIF

NPG $=N I R W * N C O L M$

$\mathrm{NICO}=\mathrm{NCOLM}$

NCOLM $=$ MINO $(6$, NFACT +1$)$

$I=0$

$\mathrm{K}=1$

DO 1100 IK $=1$, NICO

$\mathrm{KK}=1$

IENDL $=$ MINO $((K+$ NCOLM-2), NFACT $)$

DO 1101 IJ $=1$, NIRW

$I=I+1$

$\mathrm{C}$

C WRITE PAGE HEADER

C

IF (I.EQ.1) THEN

WRITE $(9,2100)$ RNTITLE, I, NPG, (FACNM (J) , J=K, K+NCOLM-2)

EISE

WRITE $(9,2102)$ RNTITLE, I, NPG, (FACNM(J), J=K, IENDL)

$\mathrm{C}$

ENDIF

C FILL IN TABLE 2 ENTRIES

C

DO $1110 \quad \mathrm{~L}=\mathrm{KK}, \mathrm{KK}+\mathrm{NROWS}-1$

$\operatorname{WRITE}(9,2101) \quad \mathrm{L},(\operatorname{FCVAL}(\mathrm{L}, \mathrm{J}), \mathrm{J}=\mathrm{K}, \mathrm{IENDL})$ 


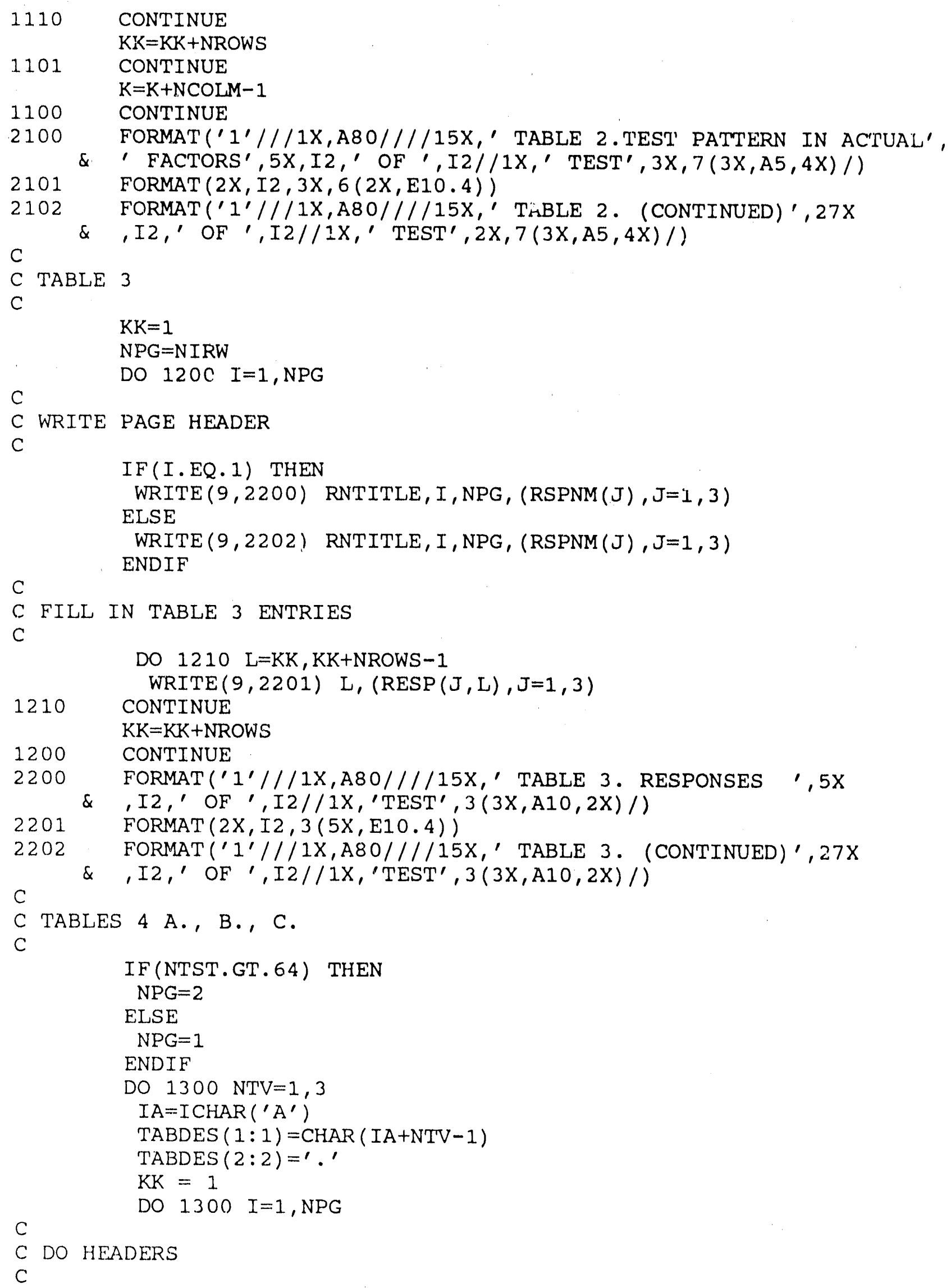




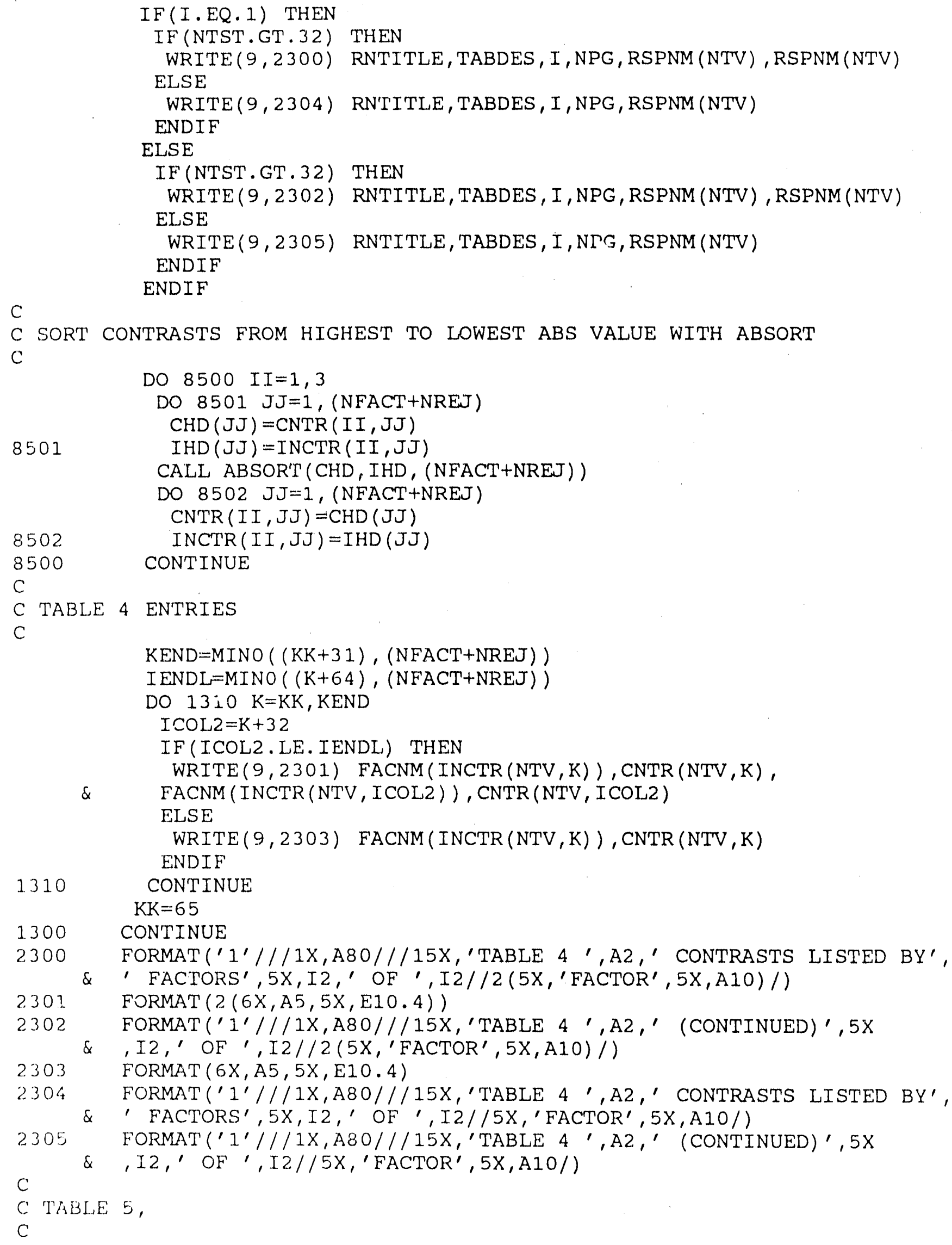




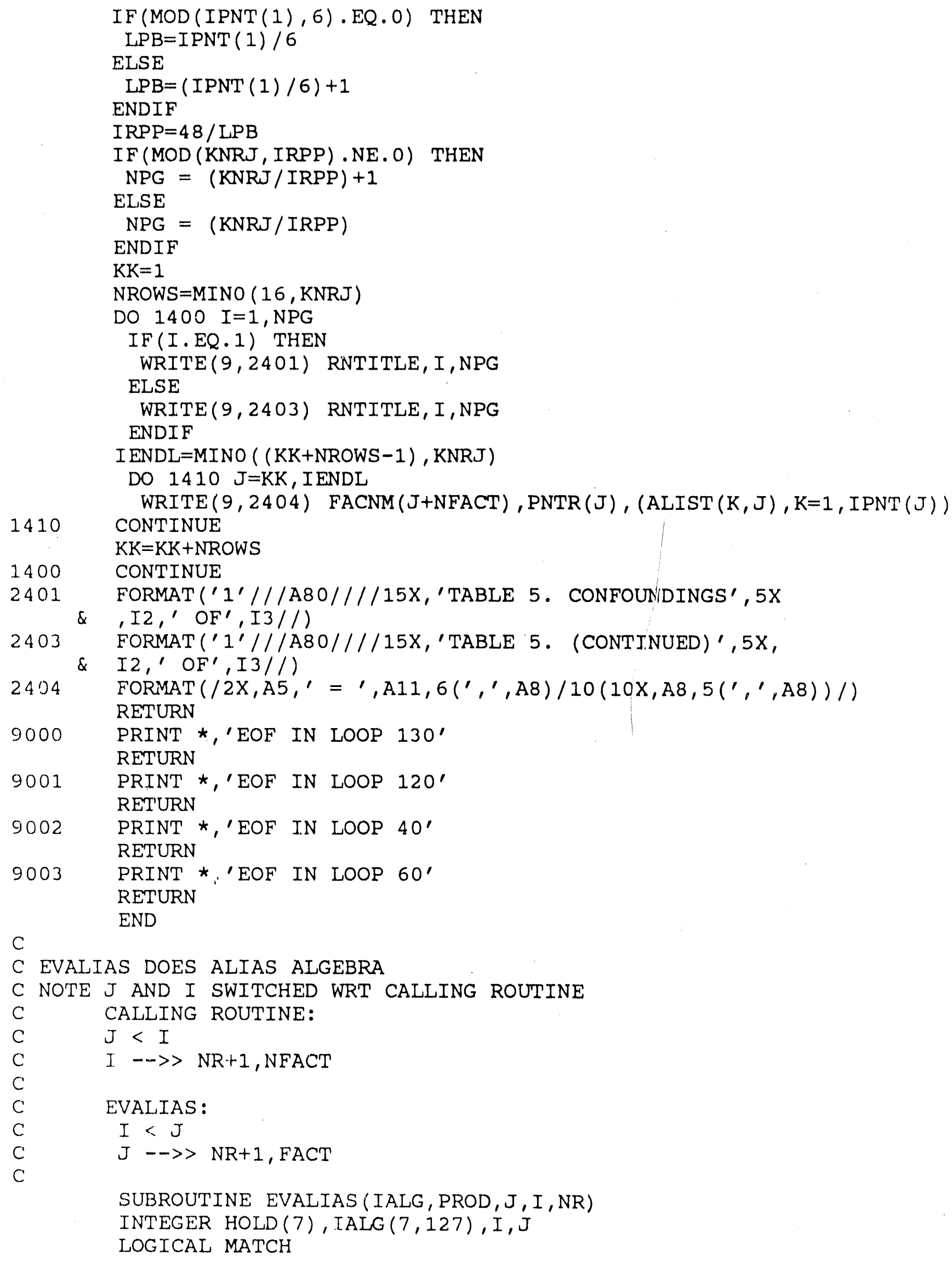




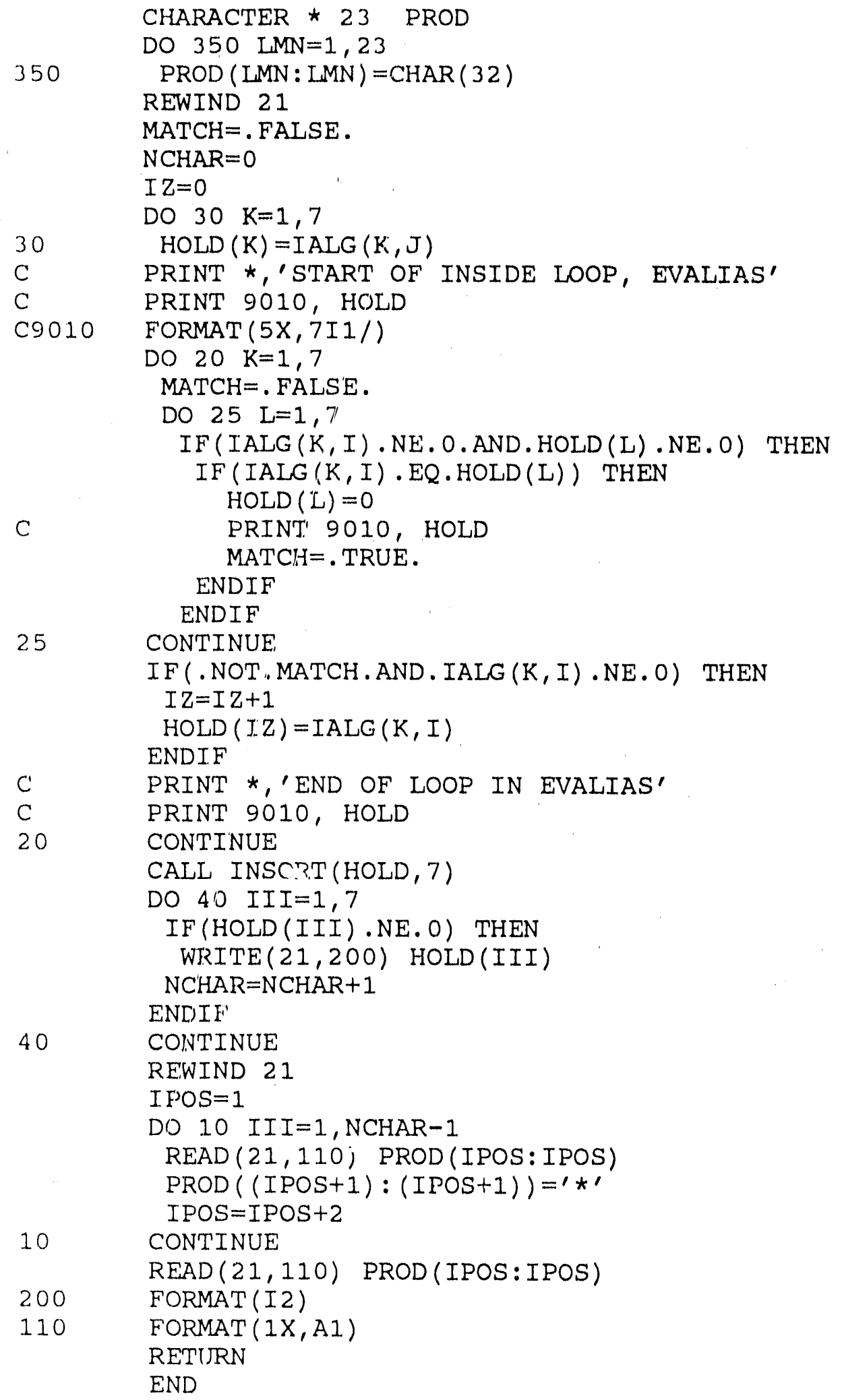




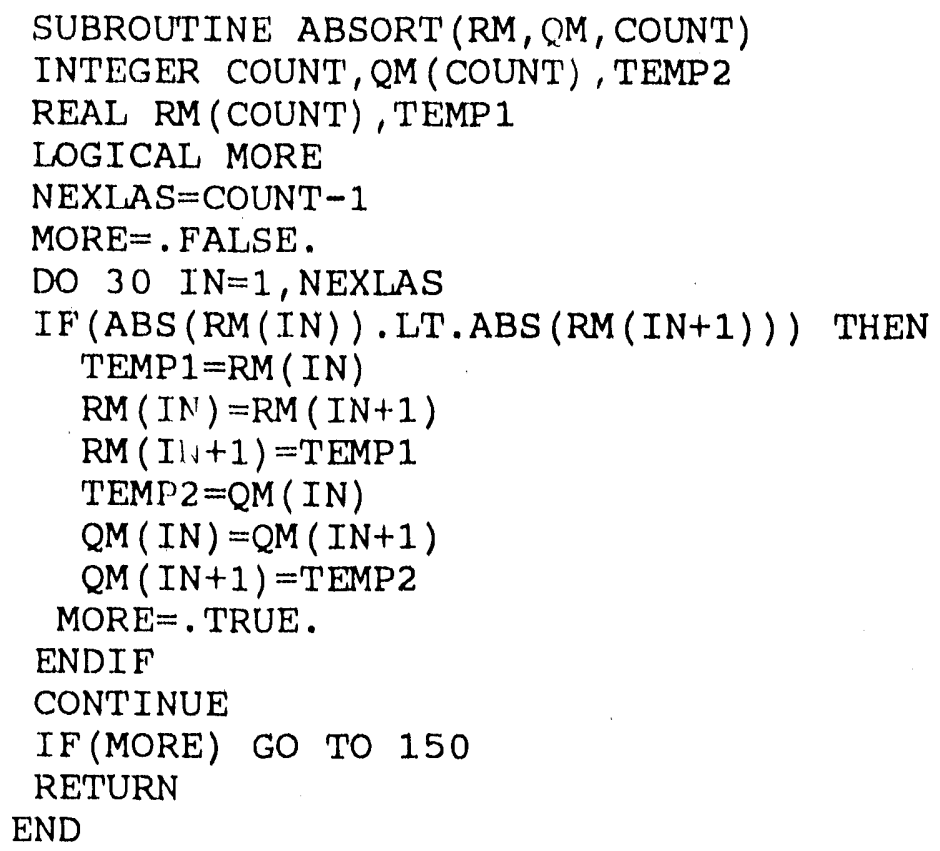




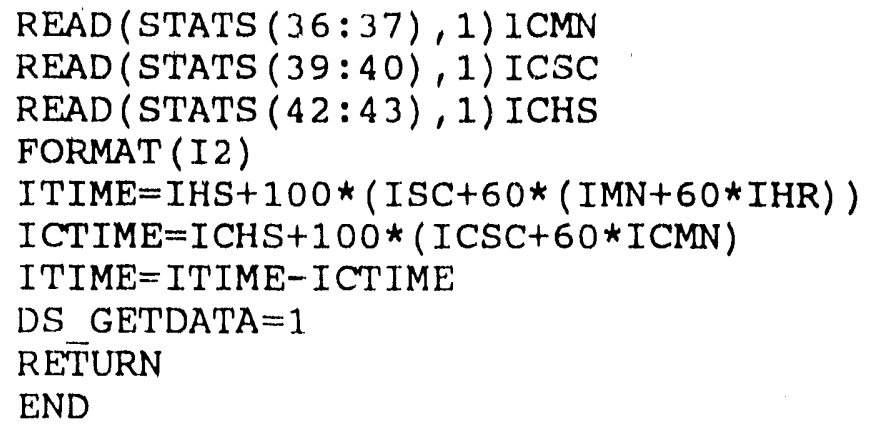

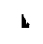



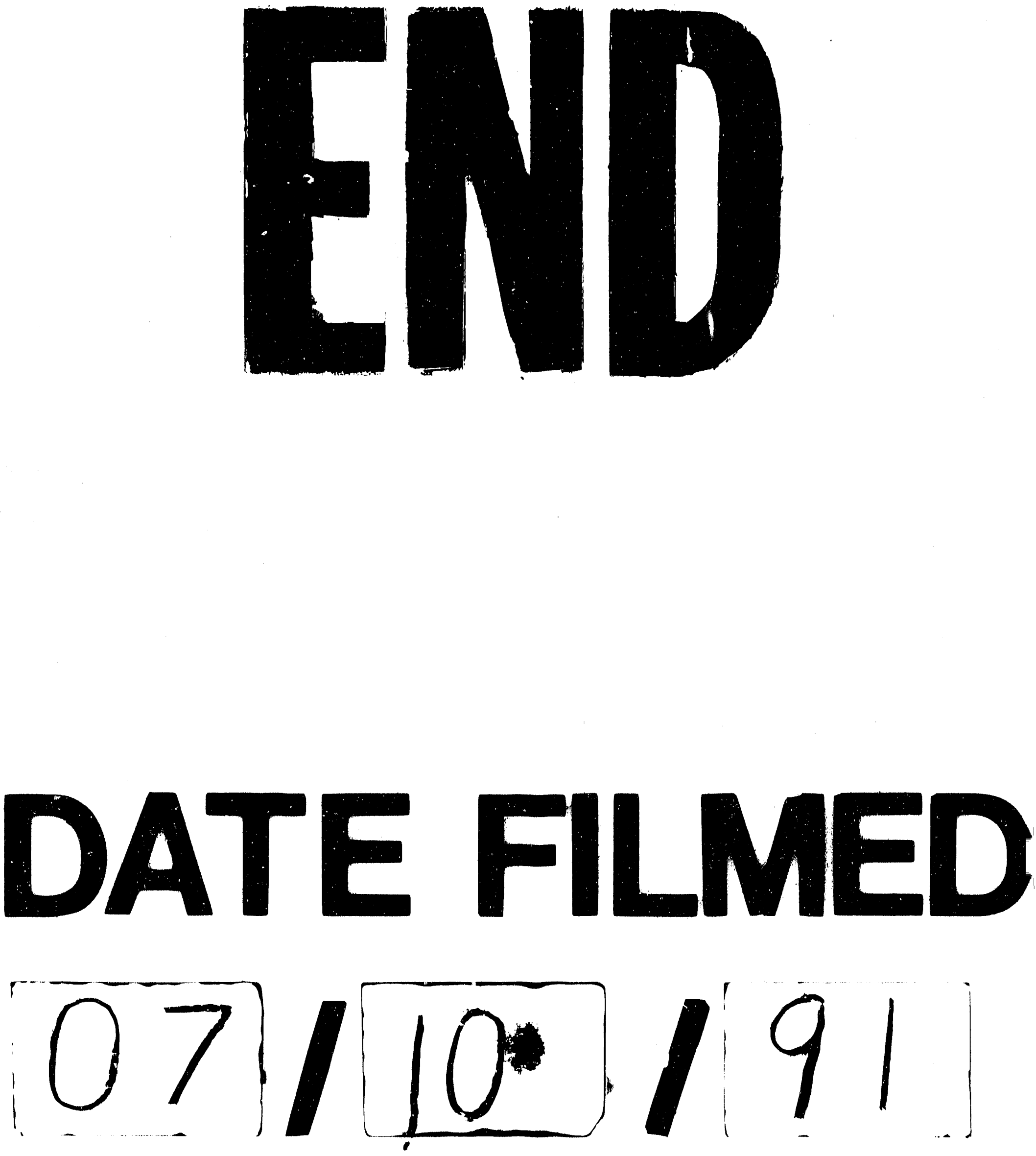
